\title{
Nutritional supplementation for nonalcohol-related fatty liver disease: a network meta-analysis (Review)
}

Komolafe O, Buzzetti E, Linden A, Best LMJ, Madden AM, Roberts D, Chase TJG, Fritche D, Freeman SC, Cooper NJ, Sutton AJ, Milne EJ, Wright K, Pavlov CS, Davidson BR, Tsochatzis E, Gurusamy KS

Komolafe O, Buzzetti E, Linden A, Best LMJ, Madden AM, Roberts D, Chase TJG, Fritche D, Freeman SC, Cooper NJ, Sutton AJ, Milne EJ, Wright K, Pavlov CS, Davidson BR, Tsochatzis E, Gurusamy KS.

Nutritional supplementation for nonalcohol-related fatty liver disease: a network meta-analysis.

Cochrane Database of Systematic Reviews 2021, Issue 7. Art. No.: CD013157.

DOI: 10.1002/14651858.CD013157.pub2.

www.cochranelibrary.com 
TABLE OF CONTENTS

HEADER 1

ABSTRACT

PLAIN LANGUAGE SUMMARY

SUMMARY OF FINDINGS

Figure 1.

BACKGROUND

OBJECTIVES

METHODS

Figure 2.

RESULTS

Figure 3.

Figure 4.

Figure 5.

DISCUSSION

AUTHORS' CONCLUSIONS

ACKNOWLEDGEMENTS

REFERENCES

CHARACTERISTICS OF STUDIES

ADDITIONAL TABLES

APPENDICES

HISTORY

CONTRIBUTIONS OF AUTHORS

DECLARATIONS OF INTEREST

SOURCES OF SUPPORT

DIFFERENCES BETWEEN PROTOCOL AND REVIEW

NOTES 
[Intervention Review]

\section{Nutritional supplementation for nonalcohol-related fatty liver disease: a network meta-analysis}

Oluyemi Komolafe1, Elena Buzzetti2 ${ }^{2}$, Audrey Linden³ ${ }^{3}$ Lawrence MJ Best ${ }^{3}$, Angela M Madden 4 , Danielle Roberts ${ }^{3}$, Thomas JG Chase ${ }^{5}$, Dominic Fritche ${ }^{6}$, Suzanne C Freeman 7 , Nicola J Cooper 7 , Alex J Sutton 7 , Elisabeth Jane Milne ${ }^{8}$, Kathy Wright ${ }^{9}$, Chavdar S Pavlov ${ }^{10}$, Brian R Davidson³, Emmanuel Tsochatzis², Kurinchi Selvan Gurusamy3,10

1University College London, London, UK. 2Sheila Sherlock Liver Centre, Royal Free Hospital and the UCL Institute of Liver and Digestive Health, London, UK. ${ }^{3}$ Division of Surgery and Interventional Science, University College London, London, UK. ${ }^{4}$ School of Life and Medical Sciences, University of Hertfordshire, Hatfield, UK. ${ }^{5}$ Department of General Surgery, Homerton University Hospital NHS Foundation Trust, London, UK. 6UCL Medical School, London, UK. ${ }^{7}$ Department of Health Sciences, University of Leicester, Leicester, UK. ${ }^{8}$ Centre for Trust, Peace and Social Relations, Coventry University, Coventry, UK. ${ }^{9}$ Cochrane Hepato-Biliary Group, Copenhagen Trial Unit, Centre for Clinical Intervention Research, The Capital Region of Denmark, Rigshospitalet, Copenhagen University Hospital, Copenhagen, Denmark. 10Department of Therapy, I.M. Sechenov First Moscow State Medical University, Moscow, Russian Federation

Contact address: Kurinchi Selvan Gurusamy, k.gurusamy@ucl.ac.uk.

Editorial group: Cochrane Hepato-Biliary Group.

Publication status and date: New, published in Issue 7, 2021.

Citation: Komolafe O, Buzzetti E, Linden A, Best LMJ, Madden AM, Roberts D, Chase TJG, Fritche D, Freeman SC, Cooper NJ, Sutton AJ, Milne EJ, Wright K, Pavlov CS, Davidson BR, Tsochatzis E, Gurusamy KS. Nutritional supplementation for nonalcohol-related fatty liver disease: a network meta-analysis. Cochrane Database of Systematic Reviews 2021, Issue 7. Art. No.: CD013157. DOI: 10.1002/14651858.CD013157.pub2.

Copyright @ 2021 The Cochrane Collaboration. Published by John Wiley \& Sons, Ltd.

\section{A B S T R A C T}

\section{Background}

The prevalence of non-alcohol-related fatty liver disease (NAFLD) varies between 19\% and 33\% in different populations. NAFLD decreases life expectancy and increases risks of liver cirrhosis, hepatocellular carcinoma, and the requirement for liver transplantation. Uncertainty surrounds relative benefits and harms of various nutritional supplements in NAFLD. Currently no nutritional supplement is recommended for people with NAFLD.

\section{Objectives}

- To assess the benefits and harms of different nutritional supplements for treatment of NAFLD through a network meta-analysis

- To generate rankings of different nutritional supplements according to their safety and efficacy

\section{Search methods}

We searched the Cochrane Central Register of Controlled Trials, MEDLINE, Embase, Science Citation Index Expanded, Conference Proceedings Citation Index-Science, the World Health Organization International Clinical Trials Registry Platform, and trials registers until February 2021 to identify randomised clinical trials in people with NAFLD.

\section{Selection criteria}

We included only randomised clinical trials (irrespective of language, blinding, or status) for people with NAFLD, irrespective of method of diagnosis, age and diabetic status of participants, or presence of non-alcoholic steatohepatitis (NASH). We excluded randomised clinical trials in which participants had previously undergone liver transplantation. 


\section{Data collection and analysis}

We performed a network meta-analysis with OpenBUGS using Bayesian methods whenever possible and calculated differences in treatments using hazard ratios (HRs), odds ratios (ORs), and rate ratios with 95\% credible intervals (Crls) based on an available-case analysis, according to National Institute of Health and Care Excellence Decision Support Unit guidance.

\section{Main results}

We included in the review a total of 202 randomised clinical trials (14,200 participants). Nineteen trials were at low risk of bias. A total of 32 different interventions were compared in these trials. A total of 115 trials (7732 participants) were included in one or more comparisons. The remaining trials did not report any of the outcomes of interest for this review.

Follow-up ranged from 1 month to 28 months. The follow-up period in trials that reported clinical outcomes was 2 months to 28 months. During this follow-up period, clinical events related to NAFLD such as mortality, liver cirrhosis, liver decompensation, liver transplantation, hepatocellular carcinoma, and liver-related mortality were sparse.

We did not calculate effect estimates for mortality because of sparse data (zero events for at least one of the groups in the trial). None of the trials reported that they measured overall health-related quality of life using a validated scale. The evidence is very uncertain about effects of interventions on serious adverse events (number of people or number of events).

We are very uncertain about effects on adverse events of most of the supplements that we investigated, as the evidence is of very low certainty. However, people taking PUFA (polyunsaturated fatty acid) may be more likely to experience an adverse event than those not receiving an active intervention (network meta-analysis results: OR 4.44, 95\% Crl 2.40 to 8.48 ; low-certainty evidence; 4 trials, 203 participants; direct evidence: OR 4.43, 95\% Crl 2.43 to 8.42). People who take other supplements (a category that includes nutritional supplements other than vitamins, fatty acids, phospholipids, and antioxidants) had higher numbers of adverse events than those not receiving an active intervention (network meta-analysis: rate ratio $1.73,95 \% \mathrm{Crl} 1.26$ to $2.41 ; 6$ trials, 291 participants; direct evidence: rate ratio $1.72,95 \% \mathrm{Crl} 1.25$ to 2.40 ; low-certainty evidence).

Data were sparse (zero events in all groups in the trial) for liver transplantation, liver decompensation, and hepatocellular carcinoma. So, we did not perform formal analysis for these outcomes. The evidence is very uncertain about effects of other antioxidants (antioxidants other than vitamins) compared to no active intervention on liver cirrhosis (HR 1.68, 95\% Crl 0.23 to 15.10; 1 trial, 99 participants; very lowcertainty evidence).

The evidence is very uncertain about effects of interventions in any of the remaining comparisons, or data were sparse (with zero events in at least one of the groups), precluding formal calculations of effect estimates.

Data were probably because of the very short follow-up period ( 2 months to 28 months). It takes follow-up of 8 to 28 years to detect differences in mortality between people with NAFLD and the general population. Therefore, it is unlikely that differences in clinical outcomes are noted in trials providing less than 5 to 10 years of follow-up.

\section{Authors' conclusions}

The evidence indicates considerable uncertainty about effects of nutritional supplementation compared to no additional intervention on all clinical outcomes for people with non-alcohol-related fatty liver disease.

Accordingly, high-quality randomised comparative clinical trials with adequate follow-up are needed. We propose registry-based randomised clinical trials or cohort multiple randomised clinical trials (study design in which multiple interventions are trialed within large longitudinal cohorts of patients to gain efficiencies and align trials more closely to standard clinical practice) comparing interventions such as vitamin E, prebiotics/probiotics/synbiotics, PUFAs, and no nutritional supplementation. The reason for the choice of interventions is the impact of these interventions on indirect outcomes, which may translate to clinical benefit. Outcomes in such trials should be mortality, health-related quality of life, decompensated liver cirrhosis, liver transplantation, and resource utilisation measures including costs of intervention and decreased healthcare utilisation after minimum follow-up of 8 years (to find meaningful differences in clinically important outcomes).

\section{PLAIN LANGUAGESUMMARY}

\section{Nutritional supplementation for people with non-alcohol-related fatty liver disease}

\section{What is the aim of this Cochrane Review?}

To find out if any form of nutritional supplementation decreases effects of non-alcohol-related fatty liver disease on lifespan, health-related quality of life, chronic liver disease, and its complications, and whether nutritional supplementation causes any harm.

Non-alcoholic fatty liver disease (NAFLD) is an accumulation of fat in the liver of people who have no history of significant alcohol consumption, use of medicines, disease such as hepatitis $C$ virus infection, or other conditions such as starvation that can damage the liver. Fatty liver can lead to liver damage resulting in inflammation (non-alcohol-related steatohepatitis, or NASH) or liver scarring (liver cirrhosis). Various medical treatments have been tried for treatment of NAFLD. However, no current evidence suggests that any of them 
work. Nutritional supplementation has the potential to decrease liver damage, but whether this occurs is currently unclear. The authors of this review collected and analysed all relevant randomised clinical trials with the aim of finding out what is the best treatment. They found 202 randomised clinical trials (studies where participants are randomly assigned to one of two treatment groups). During analysis of data, review authors used standard Cochrane methods, which allow comparison of only two treatments at a time. In addition, review authors used advanced techniques that allow comparison of multiple treatments at the same time (usually referred as 'network (or indirect) metaanalysis').

\section{Date of literature search}

February 2021.

\section{Key messages}

Only 19 trials were at low risk of bias, and because of this, uncertainty about the findings of this review is considerable. Studies that reported clinically important liver damage or its complications studied participants for a period of 2 months to 28 months. During this period, clinically important outcomes related to NAFLD such as death, liver-related complications such as liver cirrhosis (scarring of the liver), liver decompensation (complications caused by scarring of the liver), liver transplantation, hepatocellular carcinoma (liver cancer), and death due to liver disease were rare, even without any treatment. No evidence suggests that any nutritional supplementation decreased these. A possible reason for complications of liver disease being rare in trial participants may be the short follow-up period given in these trials (participants were followed only for a period of 2 months to 28 months). Liver-related complications due to NAFLD develop over 8 to 28 years. Therefore, it is unlikely that differences in clinical outcomes can be noted in trials with less than 5 to 10 years of follow-up.

\section{What was studied in the Review?}

This Review looked at people of any sex, age, and ethnic origin, with non-alcohol-related liver disease. Review authors excluded studies of people with previous liver transplantation. The average age of participants, when reported, ranged from 7 to 61 years. Participants were given different treatments including various vitamins and other nutritional supplements. Review authors wanted to gather and analyse data on death, quality of life, serious and non-serious adverse events, severe liver damage, complications resulting from severe liver damage, liver cancer, and death due to liver damage ('clinical outcomes').

\section{What were the main results of the Review?}

The 202 studies included 14,200 participants. Study data were sparse. In all, 115 studies with 7732 participants provided data for analyses. Follow-up of trial participants ranged from 1 month to 28 months ( 2 months to 28 months for trials that reported clinical outcomes). The Review shows the following.

- The evidence indicates considerable uncertainty about effects of interventions on all clinical outcomes.

- Well-designed trials that collect data over longer follow-up times are needed in the future to find out the best nutritional supplementation (if any) for people with NAFLD. 


\begin{tabular}{|c|c|c|c|c|c|c|}
\hline \multicolumn{7}{|c|}{ Summary of findings 1. Nutritional supplementation for non-alcohol-related fatty liver disease } \\
\hline \multicolumn{7}{|c|}{$\begin{array}{l}\text { Patient or population: people with non-alcohol-related fatty liver disease (NAFLD) } \\
\text { Settings: community or primary care } \\
\text { Intervention: various interventions } \\
\text { Comparison: no active intervention } \\
\text { Follow-up period: } 2 \text { months to } 28 \text { months } \\
\text { Network geometry plots: Figure } 1\end{array}$} \\
\hline \multirow[t]{2}{*}{ Interventions } & \multirow{2}{*}{$\begin{array}{l}\text { Relative effect } \\
(95 \% \mathrm{Crl})\end{array}$} & \multicolumn{3}{|c|}{ Anticipated absolute effect ${ }^{\star}(95 \% \mathrm{Crl})$} & \multirow{2}{*}{$\begin{array}{l}\text { Quality of evi- } \\
\text { dence }\end{array}$} & \multirow[t]{2}{*}{ Comments } \\
\hline & & $\begin{array}{l}\text { No active in- } \\
\text { tervention }\end{array}$ & $\begin{array}{l}\text { Various inter- } \\
\text { ventions }\end{array}$ & Difference & & \\
\hline \multicolumn{7}{|c|}{$\begin{array}{l}\text { Mortality } \\
\text { Total studies: } 52 \\
\text { Total participants: } 3372 \\
\text { Follow-up period: } 2 \text { to } 28 \text { months }\end{array}$} \\
\hline No active intervention & \multicolumn{5}{|l|}{ Reference } & $\begin{array}{l}\text { There were no deaths in the reference } \\
\text { group }\end{array}$ \\
\hline $\begin{array}{l}\text { Other supplements } \\
\text { (12 trials; } 650 \text { participants) }\end{array}$ & Not estimable & 0 per 1000 & Not estimable & Not estimable & $\begin{array}{l}\text { Very low cer- } \\
\text { tainty a,b,c }\end{array}$ & All trials had zero events in both groups \\
\hline $\begin{array}{l}\text { Prebiotics/Probiotics/Syn- } \\
\text { biotics } \\
\text { (15 trials; } 763 \text { participants) }\end{array}$ & Not estimable & 0 per 1000 & Not estimable & Not estimable & $\begin{array}{l}\text { Very low cer- } \\
\text { tainty a,b,c }\end{array}$ & All trials had zero events in both groups \\
\hline $\begin{array}{l}\text { PUFA } \\
\text { (9 trials; } 750 \text { participants) }\end{array}$ & Not estimable & 0 per 1000 & Not estimable & Not estimable & $\begin{array}{l}\text { Very low cer- } \\
\text { tainty a,b,c }\end{array}$ & All trials had zero events in both groups \\
\hline $\begin{array}{l}\text { Vitamin E } \\
\text { (4 trials; } 379 \text { participants) }\end{array}$ & Not estimable & 0 per 1000 & Not estimable & Not estimable & $\begin{array}{l}\text { Very low cer- } \\
\text { tainty a,b,c }\end{array}$ & $\begin{array}{l}\text { There was } 1 \text { death in the intervention group } \\
(1 / 84 ; 1.2 \%) \text { in } 1 \text { trial, and there were } 2 \\
\text { deaths in the intervention group }(2 / 36 ; \\
5.6 \%) \text { in another trial. Effect estimates } \\
\text { could not be calculated because of zero } \\
\text { events in the reference group in all trials }\end{array}$ \\
\hline $\begin{array}{l}\text { Other antioxidants } \\
\text { ( } 3 \text { trials; } 191 \text { participants) }\end{array}$ & Not estimable & 0 per 1000 & Not estimable & Not estimable & $\begin{array}{l}\text { Very low cer- } \\
\text { tainty } a, b, c\end{array}$ & All trials had zero events in both groups \\
\hline
\end{tabular}




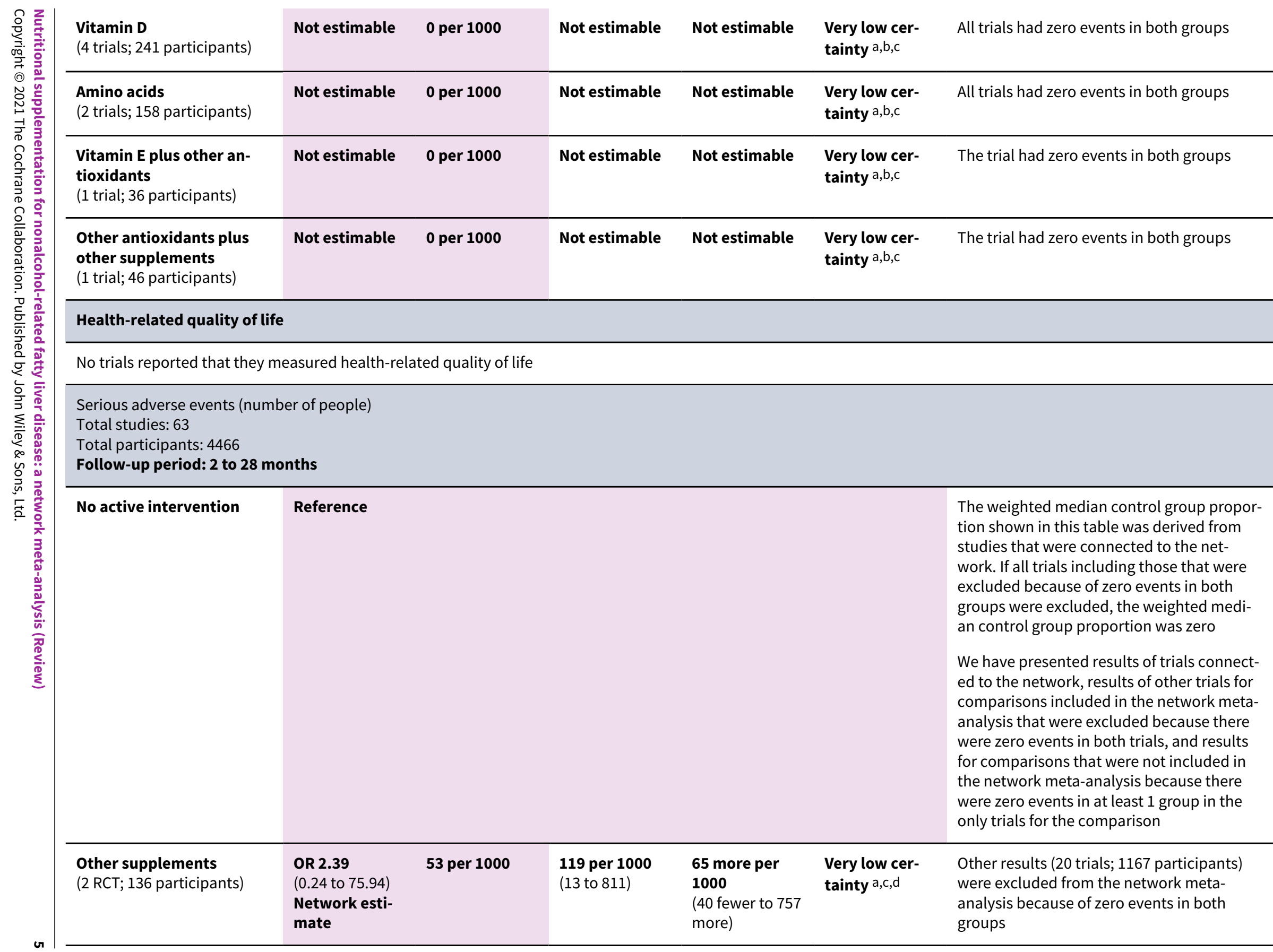




\begin{tabular}{|c|c|c|c|c|c|c|}
\hline $\begin{array}{l}\text { PUFA } \\
\text { (3 RCT; } 301 \text { participants) }\end{array}$ & $\begin{array}{l}\text { OR } 1.31 \\
(0.49 \text { to } 4.00) \\
\text { Network esti- } \\
\text { mate }\end{array}$ & 53 per 1000 & $\begin{array}{l}\mathbf{6 9} \text { per } 1000 \\
(27 \text { to } 184)\end{array}$ & $\begin{array}{l}\mathbf{1 6} \text { more per } \\
\mathbf{1 0 0 0} \\
(26 \text { fewer to } 131 \\
\text { more) }\end{array}$ & $\begin{array}{l}\text { Very low cer- } \\
\text { tainty a,c,d }\end{array}$ & $\begin{array}{l}\text { Other results ( } 3 \text { trials; } 240 \text { participants) } \\
\text { were excluded from the network meta- } \\
\text { analysis because of zero events in both } \\
\text { groups }\end{array}$ \\
\hline $\begin{array}{l}\text { Vitamin E } \\
\text { (2 RCT; } 235 \text { participants) }\end{array}$ & $\begin{array}{l}\text { OR } 0.79 \\
(0.35 \text { to } 1.77) \\
\text { Network esti- } \\
\text { mate }\end{array}$ & 53 per 1000 & $\begin{array}{l}\mathbf{4 3} \text { per } \mathbf{1 0 0 0} \\
(19 \text { to } 91)\end{array}$ & $\begin{array}{l}\mathbf{1 1} \text { fewer per } \\
\mathbf{1 0 0 0} \\
\text { (34 fewer to } 37 \\
\text { more) }\end{array}$ & $\begin{array}{l}\text { Very low cer- } \\
\text { tainty a,c,d }\end{array}$ & $\begin{array}{l}\text { Other results ( } 3 \text { trials; } 248 \text { participants) } \\
\text { were excluded from the network meta- } \\
\text { analysis because of zero events in both } \\
\text { groups }\end{array}$ \\
\hline $\begin{array}{l}\text { Vitamin D } \\
\text { (1 RCT; } 18 \text { participants) }\end{array}$ & $\begin{array}{l}\text { OR } 1.30 \\
(0.03 \text { to } 58.38) \\
\text { Network esti- } \\
\text { mate }\end{array}$ & 53 per 1000 & $\begin{array}{l}68 \text { per } 1000 \\
(2 \text { to } 767)\end{array}$ & $\begin{array}{l}\mathbf{1 5} \text { more per } \\
\mathbf{1 0 0 0} \\
\text { (52 fewer to } 714 \\
\text { more) }\end{array}$ & $\begin{array}{l}\text { Very low cer- } \\
\text { tainty a,c,d }\end{array}$ & $\begin{array}{l}\text { Other results ( } 2 \text { trials; } 371 \text { participants) } \\
\text { were excluded from the network meta- } \\
\text { analysis because of zero events in both } \\
\text { groups }\end{array}$ \\
\hline $\begin{array}{l}\text { Other antioxidants } \\
\text { (1 RCT; } 99 \text { participants) }\end{array}$ & $\begin{array}{l}\text { OR } 1.67 \\
(0.24 \text { to } 14.73) \\
\text { Network esti- } \\
\text { mate }\end{array}$ & 53 per 1000 & $\begin{array}{l}\mathbf{8 6} \text { per } \mathbf{1 0 0 0} \\
(13 \text { to } 454)\end{array}$ & $\begin{array}{l}32 \text { more per } \\
1000 \\
\text { (40 fewer to } 400 \\
\text { more) }\end{array}$ & $\begin{array}{l}\text { Very low cer- } \\
\text { tainty a,c,d }\end{array}$ & $\begin{array}{l}\text { Other results ( } 3 \text { trials; } 152 \text { participants) } \\
\text { were excluded from the network meta- } \\
\text { analysis because of zero events in both } \\
\text { groups }\end{array}$ \\
\hline $\begin{array}{l}\text { Prebiotics/Probiotics/Syn- } \\
\text { biotics } \\
\text { (14 trials; } 649 \text { participants) }\end{array}$ & Not estimable & 53 per 1000 & Not estimable & Not estimable & $\begin{array}{l}\text { Very low cer- } \\
\text { tainty a,b,c }\end{array}$ & All trials had zero events in both groups \\
\hline $\begin{array}{l}\text { Amino acids } \\
\text { ( } 2 \text { trials; } 98 \text { participants) }\end{array}$ & Not estimable & 53 per 1000 & Not estimable & Not estimable & $\begin{array}{l}\text { Very low cer- } \\
\text { tainty a,b,c }\end{array}$ & $\begin{array}{l}\text { Only } 1 \text { serious adverse event was reported } \\
\text { in the amino acid group in } 1 \text { trial. Effect esti- } \\
\text { mates could not be calculated as there were } \\
\text { zero events in the reference group in both } \\
\text { trials }\end{array}$ \\
\hline $\begin{array}{l}\text { Vitamin E plus other an- } \\
\text { tioxidants } \\
\text { (1 trial; } 36 \text { participants) }\end{array}$ & Not estimable & 53 per 1000 & Not estimable & Not estimable & $\begin{array}{l}\text { Very low cer- } \\
\text { tainty a,b,c }\end{array}$ & The trial had zero events in both groups \\
\hline $\begin{array}{l}\text { Other antioxidants plus } \\
\text { other supplements } \\
\text { (1 trial; } 46 \text { participants) }\end{array}$ & Not estimable & 53 per 1000 & Not estimable & Not estimable & $\begin{array}{l}\text { Very low cer- } \\
\text { tainty a,b,c }\end{array}$ & The trial had zero events in both groups \\
\hline $\begin{array}{l}\text { PUFA plus vitamin E } \\
\text { (1 trial; } 70 \text { participants) }\end{array}$ & Not estimable & 53 per 1000 & Not estimable & Not estimable & $\begin{array}{l}\text { Very low cer- } \\
\text { tainty a,b,c }\end{array}$ & The trial had zero events in both groups \\
\hline $\begin{array}{l}\text { Vitamin C plus vitamin E } \\
\text { (1 trial; } 88 \text { participants) }\end{array}$ & Not estimable & 53 per 1000 & Not estimable & Not estimable & $\begin{array}{l}\text { Very low cer- } \\
\text { tainty } a, b, c\end{array}$ & The trial had zero events in both groups \\
\hline
\end{tabular}




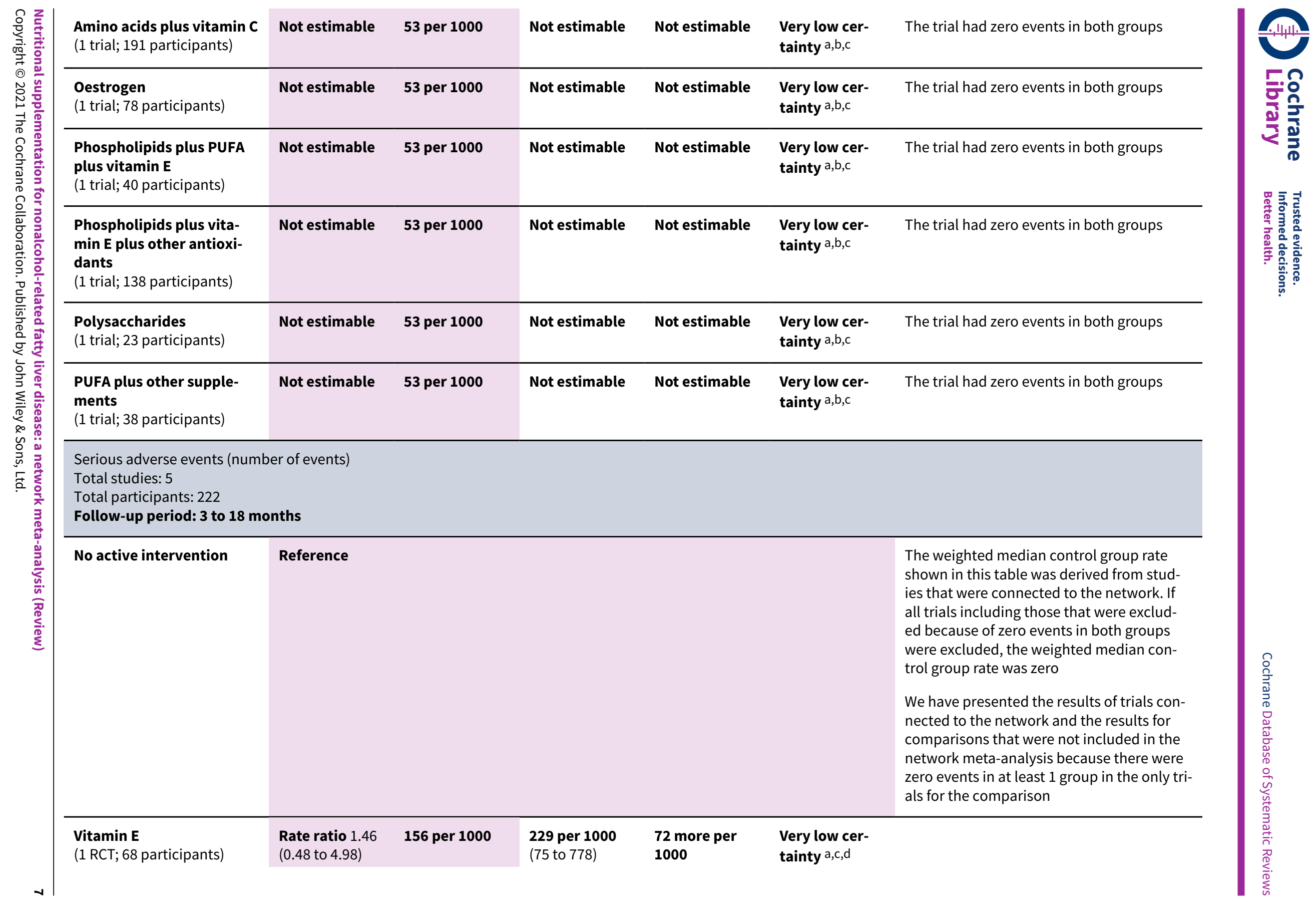




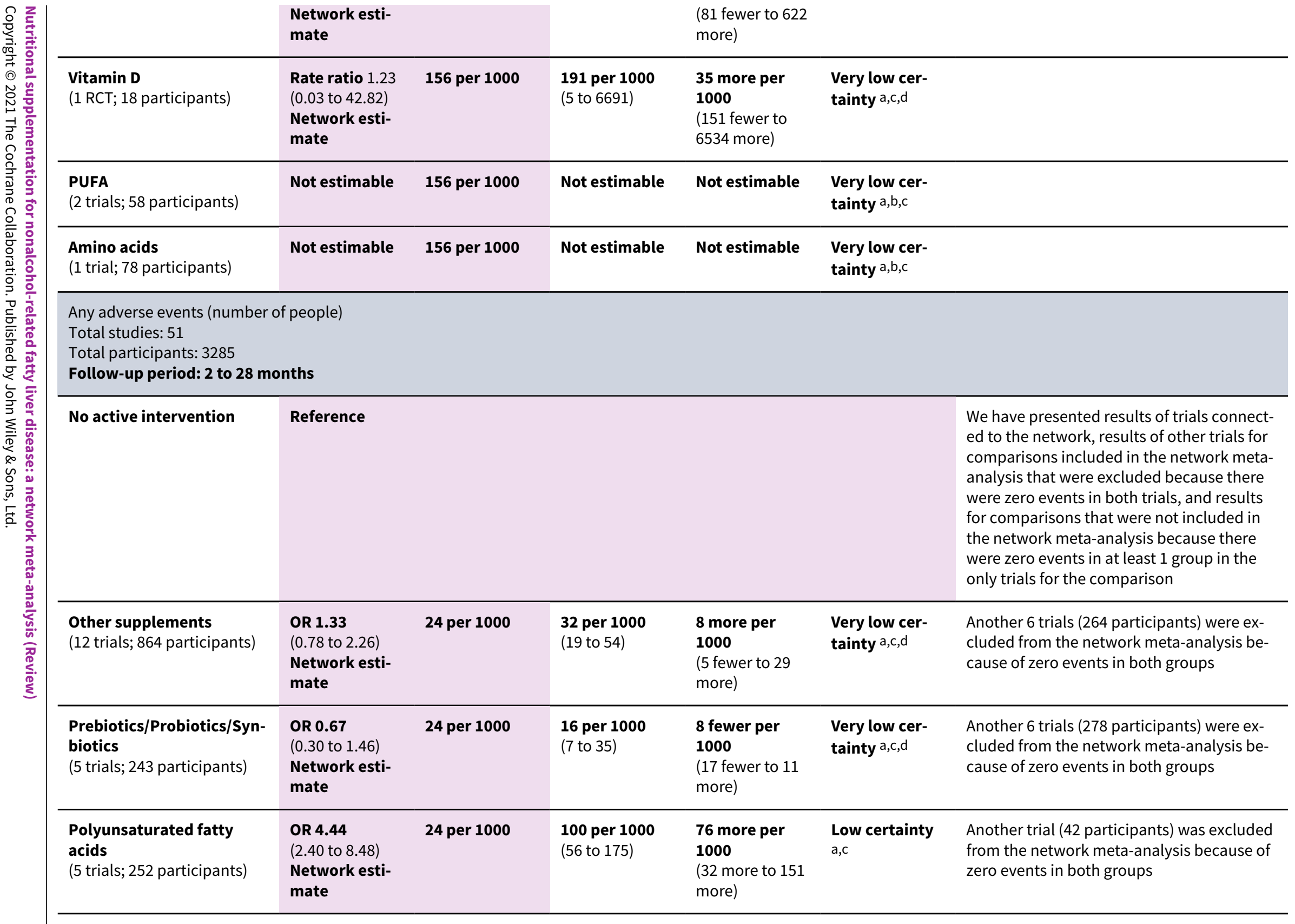




\begin{tabular}{|c|c|c|c|c|c|c|}
\hline $\begin{array}{l}\text { Vitamin E } \\
\text { (1 RCT; } 97 \text { participants) }\end{array}$ & $\begin{array}{l}\text { OR } 0.83 \\
(0.36 \text { to } 1.91) \\
\text { Network esti- } \\
\text { mate }\end{array}$ & 24 per 1000 & $\begin{array}{l}20 \text { per } 1000 \\
(9 \text { to } 46)\end{array}$ & $\begin{array}{l}\mathbf{4} \text { fewer per } \\
\mathbf{1 0 0 0} \\
\text { (16 fewer to } 21 \\
\text { more) }\end{array}$ & $\begin{array}{l}\text { Very low cer- } \\
\text { tainty a,c,d }\end{array}$ & $\begin{array}{l}\text { Another } 2 \text { trials ( } 151 \text { participants) were ex- } \\
\text { cluded from the network meta-analysis be- } \\
\text { cause of zero events in both groups }\end{array}$ \\
\hline $\begin{array}{l}\text { Other antioxidants } \\
\text { (1 RCT; } 99 \text { participants) }\end{array}$ & $\begin{array}{l}\text { OR } 1.67 \\
(0.64 \text { to } 4.50) \\
\text { Network esti- } \\
\text { mate }\end{array}$ & 24 per 1000 & $\begin{array}{l}\mathbf{4 0} \text { per } \mathbf{1 0 0 0} \\
(16 \text { to } 101)\end{array}$ & $\begin{array}{l}\mathbf{1 6} \text { more per } \\
\mathbf{1 0 0 0} \\
\text { (9 fewer to } 77 \\
\text { more) }\end{array}$ & $\begin{array}{l}\text { Very low cer- } \\
\text { tainty a,c,d }\end{array}$ & $\begin{array}{l}\text { Another } 2 \text { trials ( } 147 \text { participants) were ex- } \\
\text { cluded from the network meta-analysis be- } \\
\text { cause of zero events in both groups }\end{array}$ \\
\hline $\begin{array}{l}\text { Amino acids } \\
\text { (2 RCT; } 152 \text { participants) }\end{array}$ & $\begin{array}{l}\text { OR } 0.61 \\
(0.28 \text { to } 1.28) \\
\text { Network esti- } \\
\text { mate }\end{array}$ & 24 per 1000 & $\begin{array}{l}\mathbf{1 5} \text { per } 1000 \\
(7 \text { to } 31)\end{array}$ & $\begin{array}{l}\mathbf{9} \text { fewer per } \\
\mathbf{1 0 0 0} \\
\text { (17 fewer to } 7 \\
\text { more) }\end{array}$ & $\begin{array}{l}\text { Very low cer- } \\
\text { tainty a,c,d }\end{array}$ & \\
\hline $\begin{array}{l}\text { Phospholipids } \\
\text { (No direct RCT) }\end{array}$ & $\begin{array}{l}\text { OR } 0.37 \\
(0.04 \text { to } 2.01) \\
\text { Network esti- } \\
\text { mate }\end{array}$ & 24 per 1000 & $\begin{array}{l}\mathbf{9} \text { per } \mathbf{1 0 0 0} \\
(1 \text { to } 48)\end{array}$ & $\begin{array}{l}\mathbf{1 5} \text { fewer per } \\
\mathbf{1 0 0 0} \\
(23 \text { fewer to } 24 \\
\text { more) }\end{array}$ & $\begin{array}{l}\text { Very low cer- } \\
\text { tainty a,c,d }\end{array}$ & \\
\hline $\begin{array}{l}\text { Amino acids + vitamin C } \\
\text { (1 RCT; } 191 \text { participants) }\end{array}$ & $\begin{array}{l}\text { OR } 1.76 \\
(0.62 \text { to } 5.53) \\
\text { Network esti- } \\
\text { mate }\end{array}$ & 24 per 1000 & $\begin{array}{l}\mathbf{4 2} \text { per } \mathbf{1 0 0 0} \\
(15 \text { to } 121)\end{array}$ & $\begin{array}{l}\mathbf{1 8} \text { more per } \\
\mathbf{1 0 0 0} \\
\text { (9 fewer to } 97 \\
\text { more) }\end{array}$ & $\begin{array}{l}\text { Very low cer- } \\
\text { tainty a,c,d }\end{array}$ & \\
\hline $\begin{array}{l}\text { Oestrogen } \\
\text { (1 RCT; } 78 \text { participants) }\end{array}$ & $\begin{array}{l}\text { OR } 1.11 \\
(0.03 \text { to } 44.88) \\
\text { Network esti- } \\
\text { mate }\end{array}$ & 24 per 1000 & $\begin{array}{l}\mathbf{2 7} \text { per } \mathbf{1 0 0 0} \\
\text { (1 to } 529)\end{array}$ & $\begin{array}{l}\mathbf{3} \text { more per } \\
\mathbf{1 0 0 0} \\
\text { (24 fewer to } 504 \\
\text { more) }\end{array}$ & $\begin{array}{l}\text { Very low cer- } \\
\text { tainty a,c,d }\end{array}$ & \\
\hline $\begin{array}{l}\text { Vitamin } \mathbf{D} \\
\text { (1 trial; } 60 \text { participants) }\end{array}$ & Not estimable & 24 per 1000 & Not estimable & Not estimable & $\begin{array}{l}\text { Very low cer- } \\
\text { tainty } a, b, c\end{array}$ & \\
\hline $\begin{array}{l}\text { Vitamin E plus other an- } \\
\text { tioxidants } \\
\text { (1 trial; } 36 \text { participants) }\end{array}$ & Not estimable & 24 per 1000 & Not estimable & Not estimable & $\begin{array}{l}\text { Very low cer- } \\
\text { tainty a,b,c }\end{array}$ & \\
\hline $\begin{array}{l}\text { Other antioxidants plus } \\
\text { other supplements } \\
\text { (1 trial; } 46 \text { participants) }\end{array}$ & Not estimable & 24 per 1000 & Not estimable & Not estimable & $\begin{array}{l}\text { Very low cer- } \\
\text { tainty a,b,c }\end{array}$ & \\
\hline $\begin{array}{l}\text { PUFA plus vitamin E } \\
\text { (1 trial; } 70 \text { participants) }\end{array}$ & Not estimable & 24 per 1000 & Not estimable & Not estimable & $\begin{array}{l}\text { Very low cer- } \\
\text { tainty } a, b, c\end{array}$ & \\
\hline
\end{tabular}




\begin{tabular}{|c|c|c|c|c|c|c|}
\hline 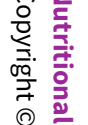 & $\begin{array}{l}\text { PUFA plus other supple- } \\
\text { ments } \\
\text { (1 trial; } 38 \text { participants) }\end{array}$ & Not estimable & 24 per 1000 & Not estimable & Not estimable & $\begin{array}{l}\text { Very low cer- } \\
\text { tainty a,b,c }\end{array}$ \\
\hline 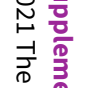 & $\begin{array}{l}\text { Vitamin C plus vitamin E } \\
\text { (1 trial; } 88 \text { participants) }\end{array}$ & Not estimable & 24 per 1000 & Not estimable & Not estimable & $\begin{array}{l}\text { Very low cer- } \\
\text { tainty a,b,c }\end{array}$ \\
\hline 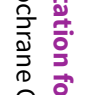 & $\begin{array}{l}\text { Polysaccharides } \\
\text { (1 trial; } 23 \text { participants) }\end{array}$ & Not estimable & 24 per 1000 & Not estimable & Not estimable & $\begin{array}{l}\text { Very low cer- } \\
\text { tainty } a, b, c\end{array}$ \\
\hline 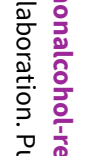 & $\begin{array}{l}\text { Any adverse events (numbe } \\
\text { Total studies: } 13 \\
\text { Total participants: } 971 \\
\text { Follow-up: } 2 \text { to } 28 \text { months }\end{array}$ & of events) & & & & \\
\hline 可 & No active intervention & Reference & & & & \\
\hline 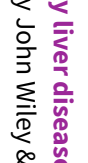 & $\begin{array}{l}\text { Other supplements } \\
\text { (6 RCTs; } 291 \text { participants) }\end{array}$ & $\begin{array}{l}\text { Rate ratio } 1.72 \\
(1.25 \text { to } 2.40) \\
\text { Direct esti- } \\
\text { mate }\end{array}$ & 602 per 1000 & $\begin{array}{l}\mathbf{1 0 3 6} \text { per } \mathbf{1 0 0 0} \\
\text { (753 to } 1446)\end{array}$ & $\begin{array}{l}\mathbf{4 3 4} \text { more per } \\
\mathbf{1 0 0 0} \\
\text { (151 more to } \\
843 \text { more) }\end{array}$ & $\begin{array}{l}\text { Low certainty } \\
\text { a,c }\end{array}$ \\
\hline 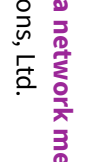 & $\begin{array}{l}\text { Polyunsaturated fatty } \\
\text { acids } \\
\text { (2 RCTs; } 252 \text { participants) }\end{array}$ & $\begin{array}{l}\text { Rate ratio } 0.90 \\
(0.68 \text { to } 1.21) \\
\text { Network esti- } \\
\text { mate }\end{array}$ & 602 per 1000 & $\begin{array}{l}\mathbf{5 4 4} \text { per } \mathbf{1 0 0 0} \\
\text { (409 to } 728)\end{array}$ & $\begin{array}{l}\mathbf{5 9} \text { fewer per } \\
\mathbf{1 0 0 0} \\
(193 \text { fewer to } \\
126 \text { more })\end{array}$ & $\begin{array}{l}\text { Very low cer- } \\
\text { tainty a,c,d,e }\end{array}$ \\
\hline 竞 & $\begin{array}{l}\text { Vitamin E } \\
\text { (3 RCTs; } 332 \text { participants) }\end{array}$ & $\begin{array}{l}\text { Rate ratio } 0.91 \\
(0.71 \text { to } 1.18) \\
\text { Network esti- } \\
\text { mate }\end{array}$ & 602 per 1000 & $\begin{array}{l}\mathbf{5 4 9} \text { per } \mathbf{1 0 0 0} \\
\text { (425 to } 708)\end{array}$ & $\begin{array}{l}\mathbf{5 4} \text { fewer per } \\
\mathbf{1 0 0 0} \\
(178 \text { fewer to } \\
106 \text { more })\end{array}$ & $\begin{array}{l}\text { Very low cer- } \\
\text { tainty a,c,d,e }\end{array}$ \\
\hline & $\begin{array}{l}\text { Vitamin D } \\
\text { (1 RCT; } 18 \text { participants) }\end{array}$ & $\begin{array}{l}\text { Rate ratio } 1.63 \\
(0.60 \text { to } 4.64) \\
\text { Network esti- } \\
\text { mate }\end{array}$ & 602 per 1000 & $\begin{array}{l}\mathbf{9 8 1} \text { per } \mathbf{1 0 0 0} \\
\text { (360 to } 2793 \text { ) }\end{array}$ & $\begin{array}{l}\mathbf{3 7 8} \text { more per } \\
\mathbf{1 0 0 0} \\
(242 \text { fewer to } \\
2191 \text { more })\end{array}$ & $\begin{array}{l}\text { Very low cer- } \\
\text { tainty a,c,d,e }\end{array}$ \\
\hline & $\begin{array}{l}\text { Amino acids } \\
\text { (1 RCT; } 78 \text { participants) }\end{array}$ & $\begin{array}{l}\text { Rate ratio } 0.81 \\
(0.42 \text { to } 1.54) \\
\text { Network esti- } \\
\text { mate }\end{array}$ & 602 per 1000 & $\begin{array}{l}\mathbf{4 8 6} \text { per } \mathbf{1 0 0 0} \\
\text { (252 to } 927)\end{array}$ & $\begin{array}{l}\mathbf{1 1 6} \text { fewer per } \\
\mathbf{1 0 0 0} \\
\text { (351 fewer to } \\
325 \text { more) }\end{array}$ & $\begin{array}{l}\text { Very low cer- } \\
\text { tainty a,c,d,e }\end{array}$ \\
\hline 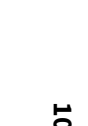 & $\begin{array}{l}\text { Liver transplantation } \\
\text { Total studies: } 20 \\
\text { Total participants: } 1204\end{array}$ & & & & & \\
\hline
\end{tabular}




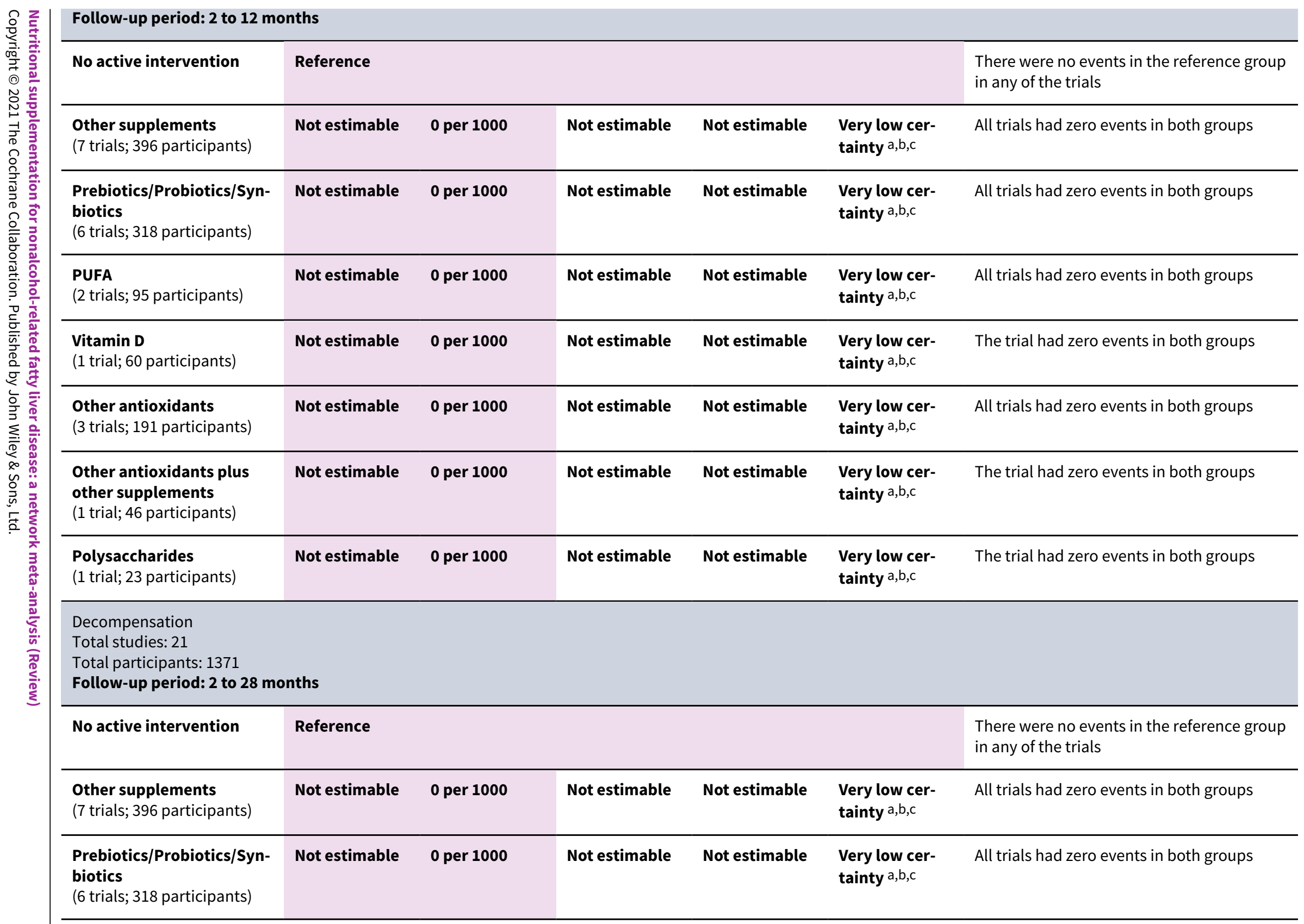




\begin{tabular}{|c|c|c|c|c|c|c|c|}
\hline 垈 & $\begin{array}{l}\text { PUFA } \\
\text { (2 trials; } 95 \text { participants) }\end{array}$ & Not estimable & 0 per 1000 & Not estimable & Not estimable & $\begin{array}{l}\text { Very low cer- } \\
\text { tainty } a, b, c\end{array}$ & All trials had zero events in both groups \\
\hline 茎 & $\begin{array}{l}\text { Vitamin D } \\
\text { (1 trial; } 60 \text { participants) }\end{array}$ & Not estimable & 0 per 1000 & Not estimable & Not estimable & $\begin{array}{l}\text { Very low cer- } \\
\text { tainty } a, b, c\end{array}$ & The trial had zero events in both groups \\
\hline 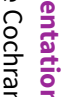 & $\begin{array}{l}\text { Other antioxidants } \\
\text { ( } 3 \text { trials; } 191 \text { participants) }\end{array}$ & Not estimable & 0 per 1000 & Not estimable & Not estimable & $\begin{array}{l}\text { Very low cer- } \\
\text { tainty a,b,c }\end{array}$ & All trials had zero events in both groups \\
\hline 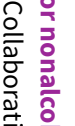 & $\begin{array}{l}\text { Other antioxidants plus } \\
\text { other supplements } \\
\text { (1 trial; } 46 \text { participants) }\end{array}$ & Not estimable & 0 per 1000 & Not estimable & Not estimable & $\begin{array}{l}\text { Very low cer- } \\
\text { tainty } a, b, c\end{array}$ & The trial had zero events in both groups \\
\hline 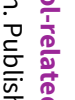 & $\begin{array}{l}\text { Polysaccharides } \\
\text { (1 trial; } 23 \text { participants) }\end{array}$ & Not estimable & 0 per 1000 & Not estimable & Not estimable & $\begin{array}{l}\text { Very low cer- } \\
\text { tainty a,b,c }\end{array}$ & The trial had zero events in both groups \\
\hline 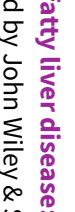 & $\begin{array}{l}\text { Vitamin E } \\
\text { (1 trial; } 167 \text { participants) }\end{array}$ & Not estimable & 0 per 1000 & Not estimable & Not estimable & $\begin{array}{l}\text { Very low cer- } \\
\text { tainty a,b,c }\end{array}$ & $\begin{array}{l}1 / 84(1.2 \%) \text { in the vitamin E group and } 0 / 83 \\
(0 \%) \text { in the no active intervention group de- } \\
\text { veloped decompensation. Effect estimates } \\
\text { could not be calculated because of zero } \\
\text { events in the reference group }\end{array}$ \\
\hline 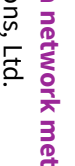 & $\begin{array}{l}\text { Cirrhosis } \\
\text { Total studies: } 19 \\
\text { Total participants: } 1172 \\
\text { Follow-up period: } \mathbf{2} \text { to } \mathbf{2 8}\end{array}$ & nths & & & & & \\
\hline 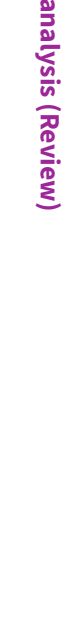 & No active intervention & Reference & & & & & $\begin{array}{l}\text { The weighted median control group pro- } \\
\text { portion shown in this table was from the } \\
\text { only study in which a formal analysis was } \\
\text { performed. If all trials including those that } \\
\text { were excluded because of zero events in } \\
\text { both groups were excluded, the weighted } \\
\text { median control group proportion was zero } \\
\text { We have presented results of trials connect- } \\
\text { ed to the network, results of other trials for } \\
\text { comparisons included in the network meta- } \\
\text { analysis that were excluded because there } \\
\text { were zero events in both trials, and results } \\
\text { for comparisons that were not included in } \\
\text { the network meta-analysis because there } \\
\text { were zero events in at least } 1 \text { group in the } \\
\text { only trials for the comparison }\end{array}$ \\
\hline
\end{tabular}




\begin{tabular}{|c|c|c|c|c|c|c|}
\hline $\begin{array}{l}\text { Other antioxidants } \\
\text { (1 RCT; } 99 \text { participants) }\end{array}$ & $\begin{array}{l}\text { HR } 0.25 \\
(0.01 \text { to } 2.44) \\
\text { Network esti- } \\
\text { mate }\end{array}$ & 60 per 1000 & $\begin{array}{l}15 \text { per } 1000 \\
(0 \text { to } 146)\end{array}$ & $\begin{array}{l}\mathbf{4 5} \text { fewer per } \\
\mathbf{1 0 0 0} \\
(60 \text { fewer to } 86 \\
\text { more) }\end{array}$ & $\begin{array}{l}\text { Very low cer- } \\
\text { tainty a,c,d }\end{array}$ & $\begin{array}{l}\text { Another } 2 \text { trials ( } 92 \text { participants) were ex- } \\
\text { cluded from the network meta-analysis be- } \\
\text { cause of zero events in both groups }\end{array}$ \\
\hline $\begin{array}{l}\text { Other supplements } \\
\text { (7 trials; } 396 \text { participants) }\end{array}$ & Not estimable & 60 per 1000 & Not estimable & Not estimable & $\begin{array}{l}\text { Very low cer- } \\
\text { tainty a,b,c }\end{array}$ & All trials had zero events in both groups \\
\hline $\begin{array}{l}\text { Prebiotics/Probiotics/Syn- } \\
\text { biotics } \\
\text { (6 trials; } 296 \text { participants) }\end{array}$ & Not estimable & 60 per 1000 & Not estimable & Not estimable & $\begin{array}{l}\text { Very low cer- } \\
\text { tainty } a, b, c\end{array}$ & All trials had zero events in both groups \\
\hline $\begin{array}{l}\text { PUFA } \\
\text { (2 trials; } 95 \text { participants) }\end{array}$ & Not estimable & 60 per 1000 & Not estimable & Not estimable & $\begin{array}{l}\text { Very low cer- } \\
\text { tainty a,b,c }\end{array}$ & All trials had zero events in both groups \\
\hline $\begin{array}{l}\text { Vitamin E } \\
\text { (1 trial; } 167 \text { participants) }\end{array}$ & Not estimable & 60 per 1000 & Not estimable & Not estimable & $\begin{array}{l}\text { Very low cer- } \\
\text { tainty a,b,c }\end{array}$ & $\begin{array}{l}1 / 84(1.2 \%) \text { in the vitamin E group devel- } \\
\text { oped cirrhosis and } 0 / 83(0 \%) \text { in the no ac- } \\
\text { tive intervention group developed decom- } \\
\text { pensation. Effect estimates could not be } \\
\text { calculated because of zero events in the ref- } \\
\text { erence group }\end{array}$ \\
\hline $\begin{array}{l}\text { Other antioxidants plus } \\
\text { other supplements } \\
\text { (1 trial; } 46 \text { participants) }\end{array}$ & Not estimable & 60 per 1000 & Not estimable & Not estimable & $\begin{array}{l}\text { Very low cer- } \\
\text { tainty a,b,c }\end{array}$ & The trial had zero events in both groups \\
\hline $\begin{array}{l}\text { Polysaccharides } \\
\text { (1 trial; } 23 \text { participants) }\end{array}$ & Not estimable & 60 per 1000 & Not estimable & Not estimable & $\begin{array}{l}\text { Very low cer- } \\
\text { tainty a,b,c }\end{array}$ & The trial had zero events in both groups \\
\hline $\begin{array}{l}\text { Hepatocellular carcinoma } \\
\text { Total studies: } 18 \\
\text { Total participants: } 1058 \\
\text { Follow-up period: } \mathbf{2} \text { to } \mathbf{1 2} \mathbf{~ m o}\end{array}$ & nths & & & & & \\
\hline No active intervention & Reference & & & & & $\begin{array}{l}\text { There were no events in the reference group } \\
\text { in any of the trials }\end{array}$ \\
\hline $\begin{array}{l}\text { Other supplements } \\
\text { (7 trials; } 396 \text { participants) }\end{array}$ & Not estimable & 0 per 1000 & Not estimable & Not estimable & $\begin{array}{l}\text { Very low cer- } \\
\text { tainty a,b,c }\end{array}$ & All trials had zero events in both groups \\
\hline $\begin{array}{l}\text { Prebiotics/Probiotics/Syn- } \\
\text { biotics } \\
\text { ( } 5 \text { trials; } 289 \text { participants) }\end{array}$ & Not estimable & 0 per 1000 & Not estimable & Not estimable & $\begin{array}{l}\text { Very low cer- } \\
\text { tainty a,b,c }\end{array}$ & All trials had zero events in both groups \\
\hline
\end{tabular}




\begin{tabular}{|c|c|c|c|c|c|c|c|}
\hline \multirow{2}{*}{\multicolumn{2}{|c|}{ 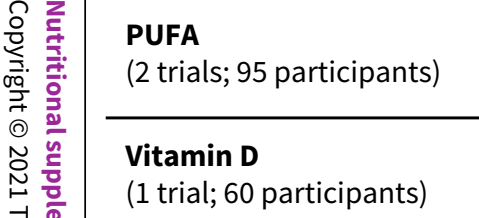 }} & Not estimable & 0 per 1000 & Not estimable & Not estimable & $\begin{array}{l}\text { Very low cer- } \\
\text { tainty a,b,c }\end{array}$ & All trials had zero events in both groups \\
\hline & & Not estimable & 0 per 1000 & Not estimable & Not estimable & $\begin{array}{l}\text { Very low cer- } \\
\text { tainty a,b,c }\end{array}$ & The trial had zero events in both groups \\
\hline 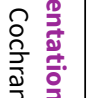 & $\begin{array}{l}\text { Other antioxidants } \\
\text { ( } 3 \text { trials; } 191 \text { participants) }\end{array}$ & Not estimable & 0 per 1000 & Not estimable & Not estimable & $\begin{array}{l}\text { Very low cer- } \\
\text { tainty a,b,c }\end{array}$ & All trials had zero events in both groups \\
\hline 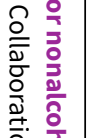 & $\begin{array}{l}\text { Other antioxidants plus } \\
\text { other supplements } \\
\text { ( } 1 \text { trial; } 46 \text { participants) }\end{array}$ & Not estimable & 0 per 1000 & Not estimable & Not estimable & $\begin{array}{l}\text { Very low cer- } \\
\text { tainty a,b,c }\end{array}$ & The trial had zero events in both groups \\
\hline 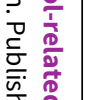 & $\begin{array}{l}\text { Polysaccharides } \\
\text { (1 trial; } 23 \text { participants) }\end{array}$ & Not estimable & 0 per 1000 & Not estimable & Not estimable & $\begin{array}{l}\text { Low certainty } \\
b, c\end{array}$ & The trial had zero events in both groups \\
\hline 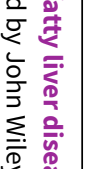 & \multicolumn{7}{|c|}{$\begin{array}{l}\text { Liver-related mortality } \\
\text { Total studies: } 52 \\
\text { Total participants: } 3372 \\
\text { Follow-up period: } 2 \text { to } \mathbf{2 8} \text { months }\end{array}$} \\
\hline$\stackrel{2}{=}$ & \multicolumn{7}{|c|}{ All 3 deaths mentioned in the mortality section may be related to the liver disease } \\
\hline 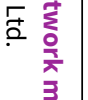 & \multicolumn{7}{|c|}{$\begin{array}{l}\text { ^Ranking was not provided because of considerable uncertainty in the ranking. } \\
\text { Crl: credible interval; HR: hazard ratio; OR: odds ratio; PUFA: polyunsaturated fatty acid. }\end{array}$} \\
\hline 总. & \multicolumn{7}{|c|}{$\begin{array}{l}\text { GRADE Working Group grades of evidence. } \\
\text { High certainty: we are very confident that the true effect lies close to that of the estimate of the effect. } \\
\text { Moderate certainty: we are moderately confident in the effect estimate; the true effect is likely to be close to the estimate of the effect, but there is a possibility that it is } \\
\text { substantially different. } \\
\text { Low certainty: our confidence in the effect estimate is limited; the true effect may be substantially different from the estimate of the effect. } \\
\text { Very low certainty: we have very little confidence in the effect estimate; the true effect is likely to be substantially different from the estimate of effect. }\end{array}$} \\
\hline & \multicolumn{7}{|c|}{$\begin{array}{l}\text { General notes: the overall number of trials included under the outcome may not match the sum of the number of trials included under each comparison. This is because of multi- } \\
\text { arm trials and trials not involving 'no active intervention' as the control group. } \\
\text { aDowngraded one level for risk of bias because trial(s) included in the analysis was/were at high risk of bias. } \\
\text { bDowngraded one level for imprecision because there were no events. } \\
\text { cDowngraded one level for imprecision because the sample size was small. } \\
\text { dDowngraded one level for imprecision because credible intervals were wide (included clinical benefits and harms). } \\
\text { eDowngraded one level for heterogeneity because of significant heterogeneity (when network estimate is indicated, heterogeneity refers to heterogeneity in the network meta- } \\
\text { analysis, not in the direct comparison). }\end{array}$} \\
\hline
\end{tabular}




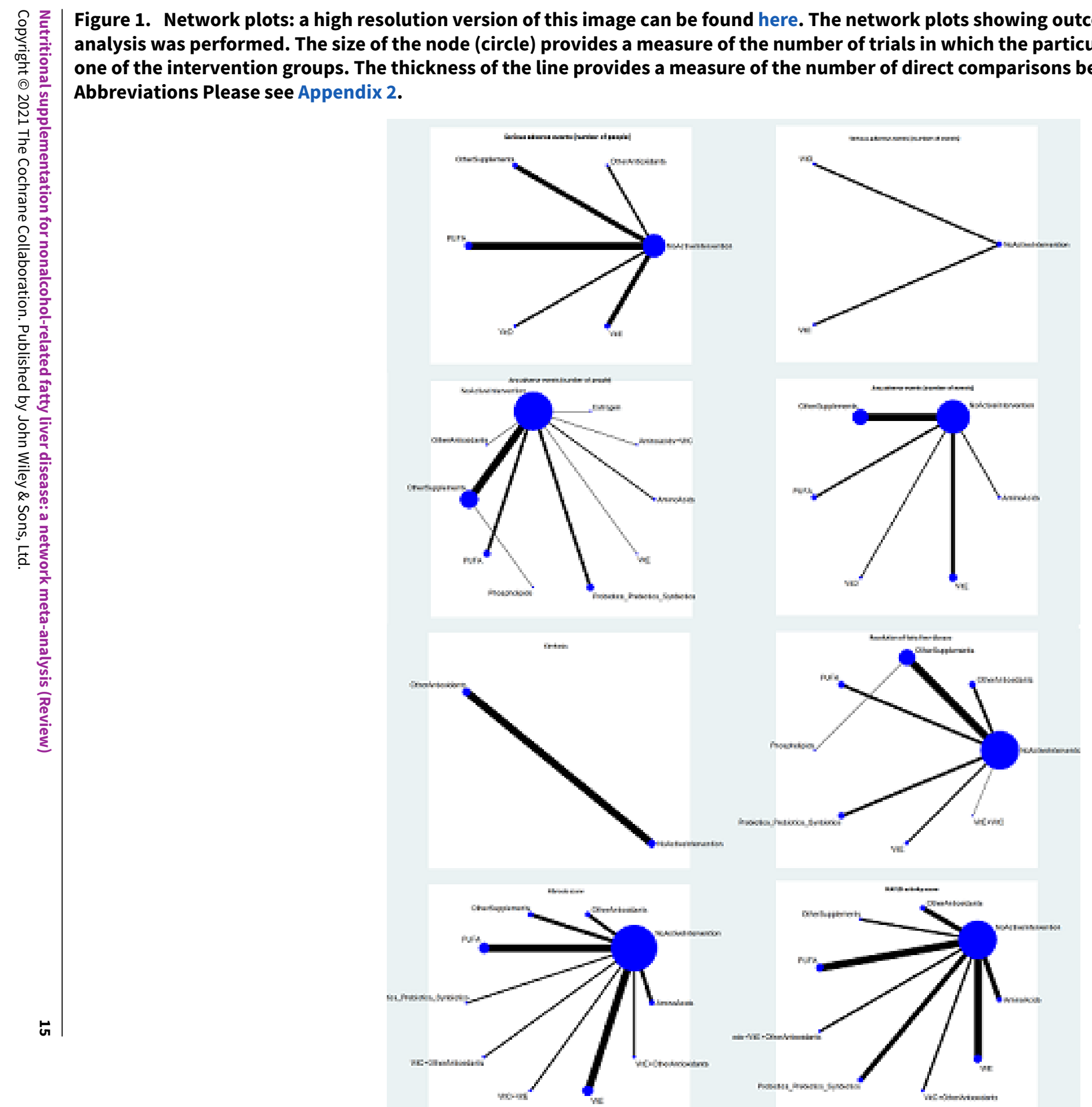




\section{B A C K G R O U N D}

\section{Description of the condition}

Fatty liver disease is steatosis (accumulation of fat, usually triglycerides) in the liver parenchymal cells (NCBI 2018). Nonalcohol-related fatty liver disease (also called non-alcoholic fatty liver disease) (NAFLD) is liver steatosis in the absence of significant alcohol consumption; use of medications such as methotrexate, tamoxifen, or steroids; or other disorders such as hepatitis $C$ virus infection, Wilson's disease, starvation, and lecithin cholesterol acyltransferase (LCAT) deficiency resulting in fat accumulation (Angulo 2002; Chalasani 2012). Fatty liver disease includes a spectrum of disorders ranging from simple steatosis or nonalcoholic fatty liver (NAFL) (fat accumulation without evidence of liver parenchymal cell injury), to non-alcoholic steatohepatitis (NASH) (fat accumulation with liver parenchymal injury but without cirrhosis), to NASH cirrhosis (advanced liver fibrosis with current or previous NAFL or NASH) (Chalasani 2012; Rinella 2015). However, it must be noted that existing non-invasive tests to distinguish NAFLD from alcohol-related liver disease (ALD) are only about $75 \%$ to $90 \%$ accurate, and some individuals with ALD may be mis-classified as having NAFLD (Cerovic 2013; Wang 2016).

Prevalence of NAFLD varies between $19 \%$ and 33\% in different populations, depending upon ethnicity, region of origin (also among people of similar ethnicity), overweight or obesity, and presence of other disorders such as diabetes mellitus or hypertension (Bedogni 2005; Park 2006; Dassanayake 2009; Koehler 2012; Lazo 2013; Fleischman 2014; Li 2014; Shen 2014; Nishioji 2015). Major risk factors associated with increased prevalence of NAFLD are obesity, being male, increasing age, ethnicity (e.g. Mexican-Americans have higher prevalence of fatty liver than other ethnic groups), genetic susceptibility (e.g. genetic variation in patatin-like phospholipase domain-containing 3 protein coding gene (PNPLA3)), hypertension, hypercholesterolaemia, diabetes mellitus, lower socio-economic level, lower-level educational attainment, poor sleep pattern, and lower physical activity (Bedogni 2005; Park 2006; Dassanayake 2009; Sookoian 2011; Koehler 2012; Lazo 2013; Fleischman 2014; Shen 2014; Bernsmeier 2015; Lonardo 2015).

The mean age of people with NAFLD varies between 40 and 60 years (Bedogni 2005; Dassanayake 2009; Shen 2014). In studies with long-term follow-up, the mean age of people with NAFLD ranged between 45 and 50 years (Adams 2005; Bedogni 2007; Soderberg 2010; Onnerhag 2014). After a mean follow-up period of 8 to 28 years, the presence of NAFLD was noted to increase overall longterm mortality compared to the general population without NAFLD (Adams 2005; Bedogni 2007; Ong 2008; Soderberg 2010; Onnerhag 2014).

People with NAFLD are at risk of dying before reaching the mean life expectancy at birth (Adams 2005; Bedogni 2007; Ong 2008; Soderberg 2010; Onnerhag 2014). It is widely believed that people with simple steatosis rarely progress to advanced liver disease, but people with NASH may develop cirrhosis (Chalasani 2012). In people with NAFLD, liver fibrosis was the only histological feature associated with increased mortality and requirement for liver transplantation (Angulo 2015; Ekstedt 2015). In a study that followed people with simple steatosis and NASH for a mean of 28 years, similar rates of mortality were observed between participants with simple steatosis and NASH groups, but higher mortality rates were noted in people with severe fibrosis regardless of whether they had bland steatosis or NASH (Soderberg 2010). It is noteworthy that NAFLD is associated with metabolic syndrome (presence of three of the following factors: hypertension, raised triglycerides, lowered high-density lipoprotein cholesterol, raised fasting glucose, central obesity) (Alberti 2009; Ballestri 2016). Therefore increased mortality among people with NAFLD may be related to metabolic syndrome, rather than to NAFLD alone. Furthermore, ALD has a worse prognosis than NAFLD (Dam-Larsen 2005); difficulty involved in distinguishing NAFLD from ALD may also contribute to the higher mortality observed in NAFLD.

Non-alcohol-related fatty liver disease is currently one of the most common reasons for liver transplantation: from 2008, NAFLD was the second or third most common reason for liver transplantation each year; the number of people who underwent liver transplantation was similar to the number with alcoholrelated liver disease since 2008 (Cholankeril 2017). Risk of hepatocellular carcinoma (HCC) is increased in people with NASH cirrhosis compared to people with NAFLD without cirrhosis and compared to the general population: approximately $2 \%$ to $13 \%$ of people with NASH cirrhosis develop HCC in three to seven years (White 2012). However, HCC can also occur in people with NAFLD without cirrhosis (Piscaglia 2016).

Fat accumulates within the liver cells when there is an imbalance between mechanisms that reduce fat in cells (such as oxidation of fatty acids or secretion of lipoproteins) and mechanisms that increase fat in cells (such as increased uptake of fat and increased production of fat). The accumulation of fat leading to NAFLD is believed to be mediated by insulin resistance because insulin resistance promotes the breakdown of peripheral adipose tissue with resultant increased influx of free fatty acids; promotes synthesis of new triglycerides within the liver; and decreases oxidation of free fatty acids (Abdelmalek 2007; Buzzetti 2016). The accumulation of fat in the liver causes injury due to proinflammatory cytokines (Riley 2007). However, the mechanism by which only a proportion of people develop advanced liver fibrosis or primary liver cancer (HCC) is unclear (Abdelmalek 2007). A 'multiple parallel hits' model involving nutrition, gut bacteria, and accumulation of fat leading to liver inflammation has been proposed as an explanation for development and progression of NAFLD (Tilg 2010; Buzzetti 2016).

Ultrasound is widely used for screening the general population for NAFLD; however it is operator-dependent, and 15 people with fatty liver disease out of every 100 people screened may be missed (Hernaez 2011). Ultrasound may yield false-positive results in 7 out of 100 people without fatty liver disease (Hernaez 2011). Although liver biopsy can be considered the definitive investigation to confirm the diagnosis, it is invasive and is not suitable for screening the general population.

\section{Description of the intervention}

Various interventions have been used in attempts to treat people with NAFLD, including nutritional supplementation (probiotics, prebiotics, synbiotics, vitamin supplementation, polyunsaturated fatty acid supplementation) (Nabavi 2014; Sharifi 2014; Li 2015; Nogueira 2016; Mofidi 2017); lifestyle modifications such as dietary changes and exercise training (not included in this review) (Abenavoli 2015; Shojaee-Moradie 2016; Zhang 2016; Houghton 2017); pharmacological interventions (not included in this review) 
(Lombardi 2017); and weight reduction surgery (bariatric surgery) (not included in this review) for obese people with NAFLD (Adorini 2012; Anstee 2012; Chalasani 2012; Paschos 2012; Abenavoli 2013).

\section{How the intervention might work}

Nutritional supplementation (the main focus of this review) may work in different ways, for example, vitamin E decreases oxidative damage to liver cells (Chalasani 2012); the effect of vitamin D supplementation may be mediated through its ability to decrease inflammatory markers and lipid peroxidation (Sharifi 2014); that of probiotics may be mediated through its ability to decrease inflammatory markers and alter lipid profile (Al-Muzafar 2017); and that of polyunsaturated fatty acids may be mediated through ability to alter the lipid profile (Chalasani 2012). This may lead to resolution or decreased progression of fatty liver disease.

\section{Why it is important to do this review}

Research on treatments to decrease NAFLD and NASH has been identified as a top priority by patients, carers, and healthcare professionals involved in the treatment of liver diseases in the UK (Gurusamy 2019). Nutritional supplementation has the potential to result in resolution or decreased progression of fatty liver disease. Network meta-analysis enables direct and indirect evidence to be combined and different interventions to be ranked in terms of different outcomes (Salanti 2011; Salanti 2012). As no previous Cochrane Review has examined this topic, it is important to identify the benefits and harms of nutritional supplementation for treatment of people with NAFLD. With this systematic review and network meta-analysis, we aim to provide the best level of evidence for benefits and harms of nutritional supplementation for people with NAFLD. We have presented results from direct comparisons whenever possible and have performed the network meta-analysis.

\section{OB JECTIVES}

- To assess the benefits and harms of different nutritional supplements for treatment of NAFLD through a network metaanalysis

- To generate rankings of different nutritional supplements according to their safety and efficacy

\section{METHODS}

\section{Criteria for considering studies for this review}

\section{Types of studies}

We considered only randomised clinical trials (including crossover and cluster-randomised clinical trials) for this network metaanalysis, irrespective of language, publication status, or date of publication. We excluded studies of other designs (including quasirandomised trials) because of the risk of bias in such studies. We excluded trials in which participants without NAFLD were included but no separate data were available for those with NAFLD.

\section{Types of participants}

We included randomised clinical trials with participants who have non-alcohol-related fatty liver disease (NAFLD), irrespective of method of diagnosis, age and diabetic status of participants, or presence of non-alcoholic steatohepatitis (NASH). We excluded randomised clinical trials in which participants had previously undergone liver transplantation or had chronic kidney disease.

\section{Types of interventions}

We included any of the following nutritional supplements for comparison with one another, given alone or in combination.

- Vitamin E supplementation.

- Vitamin D supplementation.

- Vitamin C supplementation.

- Multi-vitamin and micronutrient supplementation.

- Other antioxidants including milk thistle.

- Prebiotics/Probiotics/Synbiotics.

- Polyunsaturated fatty acids such as omega-3 fatty acids.

- Phospholipids.

- Amino acids.

- Other nutritional supplements.

- No active intervention (no intervention or placebo).

We updated the list mentioned in the protocol to include any nutritional supplementation used primarily for treatment of NAFLD. We reported findings for all these interventions (including those not mentioned in the protocol) in the Results and Discussion sections of the review.

We considered no active intervention as the reference group. We considered each of the above subcategories as a 'treatment node'. We considered variations in sub-categories, for example, different doses or durations of nutritional supplementation, as the same treatment node. We treated each different combination of categories as a different treatment node. All of the above interventions were considered the 'decision set', that is, all of the above interventions were of direct interest.

We included trials in which the above interventions were combined with other interventions aimed at decreasing NAFLD (but were considered these as potential effect modifiers), provided these cointerventions were administered equally in both arms. We included in a different review modifications in lifestyle such as dietary modifications that alter nutritional intake (e.g. more fruits and vegetables) (Buzzetti 2021).

We evaluated the plausibility of the network meta-analysis transitivity assumption by looking at inclusion and exclusion criteria in all studies. The transitivity assumption means that participants included in different trials with different treatments (in this case, for NAFLD) can be considered as part of a multiarm randomised clinical trial and could potentially have been randomised to any of the interventions (Salanti 2012). In other words, any participant who meets the inclusion criteria is, in principle, equally likely to be randomised to any of the above eligible interventions. The transitivity assumption also means that potential effect modifiers are not systematically different across comparisons. This necessitates that information on potential effect modifiers such as diabetic status and co-intervention status is similar across trials of different comparisons. Because of the inclusion criteria and the nature of interventions considered in this review, we had no obvious concerns about the transitivity assumption with relation to these effect modifiers, although we cannot rule this out completely. 


\section{Types of outcome measures}

\section{Primary outcomes}

- All-cause mortality at maximal follow-up

- Health-related quality of life, as defined in the included trials, based on a validated scale such as the EuroQoL Group Quality of Life Questionnaire based on 5 dimensions (EQ-5D) or the 36Item Short Form Health Survey (SF-36) at maximal follow-up (EuroQol 2018; Optum 2018)

- Serious adverse events (during or within six months after cessation of intervention). We defined a serious adverse event as any event that would increase mortality; is life-threatening; requires hospitalisation; results in persistent or significant disability; is a congenital anomaly/birth defect; or is any important medical event that might jeopardise the person or require intervention for prevention (ICH-GCP 1997). However, none of the trial authors defined serious adverse events. Therefore, we used the list provided by trial authors for serious adverse events (as indicated in the protocol)

* Proportion of trial participants with one or more serious adverse event

* Number of serious adverse events reported per participant

\section{Secondary outcomes}

- Any adverse events (during or within six months after cessation of intervention). We defined an adverse event as any untoward medical occurrence not necessarily having a causal relationship with the intervention but resulting in a dose reduction or discontinuation of the intervention (any time after commencement of the intervention) (ICH-GCP 1997). However, none of the trial authors defined 'adverse event'. Therefore, we used the list provided by trial authors for adverse events (as indicated in the protocol)

* Proportion of trial participants with any adverse event

* Numbers of any adverse events per participant

- Liver transplantation (time to liver transplantation at maximal follow-up)

- Decompensation (time to decompensation at maximal followup)

- Cirrhosis (time to cirrhosis at maximal follow-up)

- Liver-related mortality (time to liver-related death at maximal follow-up)

\section{Exploratory outcomes}

- Resolution of fatty liver disease (time to resolution of fatty liver disease at maximal follow-up)

- Fibrosis score at maximal follow-up

- NAFLD activity score

- Model for end-stage liver disease (MELD) score
We had chosen outcomes based on:

- their importance to patients in a survey related to research priorities for people with liver disease (Gurusamy 2019);

- feedback from patient and public representative for this project;

- an online survey about outcomes promoted through the Cochrane Consumer Network; and

- information provided through personal communication about results of the coreNASH project (which resulted in the addition of liver-related mortality and the MELD score) (Clearfield 2021)

\section{Search methods for identification of studies}

\section{Electronic searches}

We searched the Cochrane Central Register of Controlled Trials, in the Cochrane Library, MEDLINE Ovid, Embase Ovid, Science Citation Index Expanded (Web of Science), and Conference Proceedings Citation Index-Science (Web of Science) from inception to February 2021 for randomised clinical trials comparing two or more of the above interventions, without applying any language restrictions. We searched for all possible comparisons formed by the interventions of interest. To identify further ongoing or completed trials, we also searched clinicaltrials.gov and the World Health Organization International Clinical Trials Registry Platform (ictrptest.azurewebsites.net/Default.aspx), which searches various trial registers, including International Standard Randomized Controlled Trial Number (ISRCTN) and ClinicalTrials.gov. We also searched European Medical Agency (EMA) (www.ema.europa.eu/ema/) and US Food and Drug Administration (FDA) (www.fda.gov) registries for randomised clinical trials. We have provided the search strategies along with dates of the search in Appendix 1.

\section{Searching other resources}

We searched the references of identified trials to gather additional trials for inclusion. We contacted study authors to ask about any other potential studies they were aware of.

\section{Data collection and analysis}

\section{Selection of studies}

Two review authors ( $K G$ and $O K, L B, D R$, or $A L$ ) independently identified trials for inclusion by screening titles and abstracts of articles identified by the literature search, and sought full-text articles of any records identified by at least one review author for potential inclusion. We selected trials for inclusion based on review of the full-text articles. We listed the records that we excluded and the reasons for their exclusion in the Characteristics of excluded studies table. We listed any ongoing trials identified primarily through the search of the clinical trials registers for further followup. We resolved discrepancies through discussion. We illustrated the study selection process in a PRISMA diagram (Figure 2). 
Figure 2. Study flow diagram. Date of search: 25 February 2021.

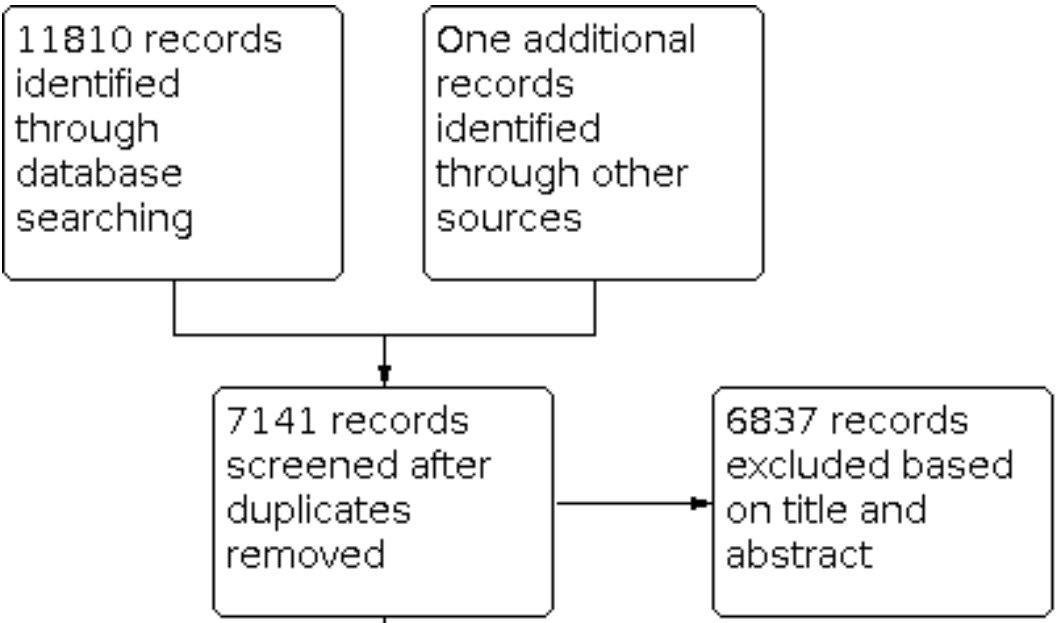

24 records (21 studies) excluded

- Reasons related to study design: 6

304 full-text records assessed for eligibility - Reasons related to interventions or co-interventions: 13

- Reasons related to participants: 1 - Other reasons: 1

\begin{tabular}{l|}
\hline \multicolumn{1}{c|}{202 studies } \\
(253 records) \\
included in \\
qualitative \\
synthesis \\
27 records on \\
ongoing studies
\end{tabular}

\section{Data extraction and management}

Two review authors ( $K G$ and $O K, L B, D R$, or $A L$ ) independently extracted the data below onto a pre-piloted Microsoft Excel-based data extraction form (after translation of non-English articles). NonEnglish articles were translated by performing optical character recognition using OmniPage 18.0 on the electronic version of the article, or on a high-resolution scanned copy of the article when electronic versions were not available, then using Google Translate to translate the words. If we found multiple records of the same trial, we collated all records related to the same study at the time of data extraction and obtained from these reports the following information as related to the study. 
- Outcome data (for each outcome and for each intervention group when applicable).

* Number of participants randomised.

* Number of participants included for analysis.

* Number of participants with events for binary outcomes, mean and standard deviation for continuous outcomes, numbers of events and mean follow-up period for count outcomes, and numbers of participants with events and mean follow-up period for time-to-event outcomes.

* Natural logarithm of hazard ratio and its standard error if this was reported rather than numbers of participants with events and mean follow-up period for time-to-event outcomes.

* Definition of outcomes or scale used if appropriate.

- Data on potential effect modifiers.

* Participant characteristics such as age, sex, diabetic status, method of diagnosis, presence of NASH.

* Details of intervention and control (including dose, frequency, and duration).

* Length of follow-up.

* Information related to risk of bias assessment (see below).

- Other data.

* Year and language of publication.

* Country in which participants were recruited.

* Year(s) in which the trial was conducted.

* Inclusion and exclusion criteria.

We collected data at maximum follow-up but also at short term (up to three months) and at medium term (from three months to five years) if these were available.

We attempted to contact trial authors in the case of unclear or missing information. If there was any doubt as to whether trials shared the same participants, completely or partially (by identifying common authors and centres), we planned to contact trial authors to clarify whether the trial report was duplicated. We resolved differences in opinion through discussion.

\section{Assessment of risk of bias in included studies}

We followed guidance provided in the Cochrane Handbook for Systematic Reviews of Interventions to assess risk of bias in included trials (Higgins 2011a). Specifically, we assessed sources of bias as defined below (Schulz 1995; Moher 1998; Kjaergard 2001; Wood 2008; Savović 2012a; Savović 2012b; Savović 2018).

\section{Allocation sequence generation}

- Low risk of bias: sequence generation was achieved using computer random number generation or a random numbers table. Drawing lots, tossing a coin, shuffling cards, and throwing dice were adequate if performed by an independent person not otherwise involved in the trial

- Unclear risk of bias: the method of sequence generation was not specified

- High risk of bias: the sequence generation method was not random

\section{Allocation concealment}

- Low risk of bias: the allocation sequence was described as unknown to investigators. Hence, participants' allocations could not have been foreseen in advance of, or during, enrolment.
Allocation was controlled by a central and independent randomisation unit, an onsite locked computer, identicallooking numbered sealed opaque envelopes, or drug bottles or containers prepared by an independent pharmacist or by an independent investigator

- Unclear risk of bias: it is unclear if allocation was hidden or if block size was relatively small and fixed, so that intervention allocations may have been foreseen in advance of, or during, enrolment

- High risk of bias: the allocation sequence was likely to be known to investigators, who assigned participants

\section{Blinding of participants and personnel}

- Low risk of bias: blinding of participants and key study personnel ensured, and it is unlikely that blinding could have been broken; or rarely, no blinding or incomplete blinding, but review authors judged that the outcome was not likely to be influenced by lack of blinding

- Unclear risk of bias: any of the following: insufficient information to permit judgement of 'low risk' or 'high risk'; or trial did not address this outcome

- High risk of bias: any of the following: no blinding or incomplete blinding and outcome was likely to be influenced by lack of blinding; or blinding of key study participants and personnel attempted, but likely that blinding could have been broken and the outcome was likely to be influenced by lack of blinding

\section{Blinded outcome assessment}

- Low risk of bias: blinding of outcome assessment ensured, and it is unlikely that blinding could have been broken; or rarely, no blinding of outcome assessment, but review authors judged that outcome measurement was not likely to be influenced by lack of blinding

- Unclear risk of bias: any of the following: insufficient information to permit judgement of 'low risk' or 'high risk'; or trial did not address this outcome

- High risk of bias: any of the following: no blinding of outcome assessment and outcome measurement was likely to be influenced by lack of blinding; or blinding of outcome assessment, but likely that blinding could have been broken and outcome measurement was likely to be influenced by lack of blinding

\section{Incomplete outcome data}

- Low risk of bias: missing data were unlikely to make treatment effects depart from plausible values. The study used sufficient methods, such as multiple imputation, to handle missing data

- Unclear risk of bias: information was insufficient to assess whether missing data in combination with the method used to handle missing data were likely to induce bias on the results

- High risk of bias: results were likely to be biased due to missing data, for example, missing data were likely to be related to the intervention and outcomes

\section{Selective outcome reporting}

- Low risk of bias: the trial reported the following pre-defined outcomes: at least one of the outcomes related to the main reason for treatment of people with NAFLD, namely, all-cause mortality or resolution of NAFLD, along with adverse events. 
If the original trial protocol was available, outcomes should have been those called for in that protocol. If we obtained the trial protocol from a trial registry (e.g. ClinicalTrials.gov), the outcomes sought should have been those enumerated in the original protocol if the trial protocol had been registered before or at the time the trial was begun. If the trial protocol was registered after the trial was begun, we did not consider those outcomes to be reliable

- Unclear risk of bias: not all pre-defined or clinically relevant and reasonably expected outcomes were reported fully, or it is unclear whether data on these outcomes were recorded

- High risk of bias: one or more pre-defined or clinically relevant and reasonably expected outcomes were not reported, despite the fact that data on these outcomes should have been available and even recorded

\section{Other bias}

- Low risk of bias: the trial appeared to be free of other components that could put it at risk of bias (e.g. inappropriate control or dose or administration of control, baseline differences, early stopping)

- Uncertain risk of bias: the trial may or may not have been free of other components that could put it at risk of bias

- High risk of bias: other factors in the trial could put it at risk of bias (e.g. baseline differences, early stopping)

We considered a trial to be at low risk of bias if we assessed the trial to be at low risk of bias across all listed bias risk domains. Otherwise, we considered the trial to be at high risk of bias. At the outcome level, we classified an outcome to be at low risk of bias if allocation sequence generation; allocation concealment; blinding of participants, healthcare professionals, and outcome assessors (at the outcome level); and incomplete outcome data (at the outcome level) were at low risk of bias for objective and subjective outcomes (Savović 2018).

\section{Measures of treatment effect}

\section{Relative treatment effects}

For dichotomous variables (e.g. proportion of participants with serious adverse events or any adverse events), we calculated the odds ratio (OR) with 95\% credible interval (Crl) (or Bayesian confidence interval) (Severini 1993). For continuous variables (e.g. fibrosis scores reported on the same scale), we calculated the mean difference (MD) with 95\% Crl. We planned to use standardised mean difference (SMD) values with $95 \% \mathrm{Crl}$ for health-related quality of life if included trials used different scales. If we calculated the SMD, we planned to convert it to a common scale, for example, EQ-5D or SF-36 (using the standard deviation of the common scale) for the purpose of interpretation. For count outcomes (e.g. number of serious adverse events or number of any adverse events), we calculated the rate ratio ( $\mathrm{RaR}$ ) with $95 \% \mathrm{Crl}$. This assumes that events are independent of each other (i.e. if a person has had an event, he or she is not at increased risk of further outcomes, which is the assumption in Poisson likelihood). For time-to-event data (e.g. all-cause mortality at maximal follow-up), we calculated hazard ratios (HRs) with $95 \%$ Crls.

\section{Relative ranking}

We estimated the ranking probabilities for all interventions of being at each possible rank for each intervention for each outcome when
NMA (network meta-analysis) was performed. We obtained the surface under the cumulative ranking curve (SUCRA) (cumulative probability), the rankogram, and the relative ranking table with $\mathrm{Crl}$ for ranking probabilities for each outcome when NMA was performed (Salanti 2011; Chaimani 2013).

\section{Unit of analysis issues}

The unit of analysis was the participant undergoing treatment for NAFLD according to the intervention group to which the participant was randomly assigned.

\section{Cluster-randomised clinical trials}

If we identified any cluster-randomised clinical trials, we planned to include cluster-randomised clinical trials, provided that the effect estimate adjusted for cluster correlation was available, or sufficient information was available to calculate the design effect (which would allow us to take clustering into account). We planned to assess additional domains of risk of bias for cluster-randomised trials according to guidance provided in the Cochrane Handbook for Systematic Reviews of Interventions (Higgins 2011b).

\section{Cross-over randomised clinical trials}

If we identified any cross-over randomised clinical trials, we planned to include only outcomes after the period of the first intervention because included treatments could have residual effects, provided the period of follow-up before the cross-over was sufficient to address the objectives of this review (Higgins 2011b), noting that the period of follow-up before the cross-over to address the objectives of this review will be around eight years (we are not aware of any cross-over trial with such a long period of follow-up before the cross-over).

\section{Trials with multiple intervention groups}

We collected data for all trial intervention groups that met the inclusion criteria. The codes that we used for analysis accounted for the correlation between effect sizes from studies with more than two groups.

\section{Dealing with missing data}

We performed an intention-to-treat analysis (including all randomised participants in the analysis according to the group to which they were randomised, regardless of the intervention they received) whenever possible (Newell 1992); otherwise, we used the data available to us. When intention-to-treat analysis was not performed and data were not missing at random (e.g. treatment was withdrawn due to adverse events, duration of treatment was shortened because of lack of response, and such participants were excluded from analysis), this could lead to biased results; therefore, we conducted best-worst case scenario analysis (assuming a good outcome in the intervention group and a bad outcome in the control group) and worst-best case scenario analysis (assuming a bad outcome in the intervention group and a good outcome in the control group) as sensitivity analyses, whenever possible (regardless of whether we considered that the data were missing at random or were not missing at random), for binary and time-toevent outcomes when binomial likelihood was used.

For continuous outcomes, we imputed the standard deviation from $P$ values, according to guidance provided in the Cochrane Handbook for Systematic Reviews of Interventions (Schünemann 2011). If the 
data were likely to be normally distributed, we used the median for meta-analysis when the mean was not available; otherwise, we planned to simply provide a median and an interquartile range of the difference in medians. If it was not possible to calculate the standard deviation from the $P$ value or from the confidence intervals, we planned to impute the standard deviation using the largest standard deviation in other trials for that outcome. This form of imputation can decrease the weight of the study for calculation of mean differences and may bias the effect estimate to no effect for calculation of standardised mean differences (Higgins 2011b).

\section{Assessment of heterogeneity}

We assessed clinical and methodological heterogeneity by carefully examining the characteristics and design of included trials. We planned to assess the presence of clinical heterogeneity by comparing effect estimates (please see Subgroup analysis and investigation of heterogeneity) in trial reports based on the presence of diabetes and NASH, and based on the co-interventions (e.g. both groups received lifestyle interventions). Different study designs and risk of bias can contribute to methodological heterogeneity.

We assessed statistical heterogeneity by comparing results of the fixed-effect model meta-analysis and the random-effects model meta-analysis, with lack of overlap of $95 \%$ credible intervals of between-study variance $\left(\operatorname{tau}^{2}\right)$ with 0 (after rounding to two decimals), and by calculating the NMA-specific I ${ }^{2}$ statistic (Jackson 2014). For direct comparisons, we assessed heterogeneity using Higgins' I (Higgins 2003). When possible, we explored substantial clinical, methodological, or statistical heterogeneity and addressed the heterogeneity in subgroup analyses (see Subgroup analysis and investigation of heterogeneity).

\section{Assessment of transitivity across treatment comparisons}

We assessed the transitivity assumption by comparing the distribution of potential effect modifiers (clinical: presence of diabetes and NASH; methodological: risk of bias, year of randomisation, duration of follow-up) across the different pairwise comparisons.

\section{Assessment of reporting biases}

For the network meta-analysis, we planned to prepare a comparison-adjusted funnel plot. However, to interpret a comparison-adjusted funnel plot, it is necessary to rank the studies in a meaningful way, as asymmetry may be due to small sample sizes in newer studies (comparing newer treatments with older treatments) or higher risk of bias in older studies (Chaimani 2012). As there was no specific change in risk of bias among studies, in sample size, or in the control group used over time (to put this in perspective, the first trial report for this review was published only in 2000), we judged the reporting bias by completeness of the search (Chaimani 2012). We also considered lack of reporting of outcomes as a form of reporting bias.

\section{Data synthesis}

We conducted network meta-analyses to compare multiple interventions simultaneously for each of the primary and secondary outcomes. When two or more interventions were combined, we considered this as a separate intervention ('node'). Network meta-analysis combines direct evidence within trials and indirect evidence across trials (Mills 2012). We obtained a network plot to ensure that trials were connected by interventions using Stata/SE 15.1 (Chaimani 2013). We excluded from network meta-analysis any trials that were not connected to the network, and we reported only the direct pairwise meta-analysis for such comparisons. We summarised the population and methodological characteristics of trials included in the network meta-analysis in a table based on pairwise comparisons. We conducted a Bayesian network meta-analysis using the Markov chain Monte Carlo method in OpenBUGS 3.2.3, according to guidance obtained from the National Institute for Health and Care Excellence (NICE) Decision Support Unit (DSU) documents (Dias 2016). We modelled the treatment contrast (i.e. log odds ratio for binary outcomes, mean difference or standardised mean difference for continuous outcomes, log rate ratio for count outcomes, and log hazard ratio for time-to-event outcomes) for any two interventions ('functional parameters') as a function of comparison between each individual intervention and the reference group ('basic parameters') using appropriate likelihood functions and links (Lu 2006). We used binomial likelihood and logit link for binary outcomes, Poisson likelihood and log link for count outcomes, binomial likelihood and complementary log-log link (a semi-parametric model that excludes censored individuals from the denominator of 'at risk' individuals at the point when they are censored) for time-to-event outcomes, and normal likelihood and identity link for continuous outcomes. We used 'no active intervention' as the reference group across networks, as there is no established 'standard of care' for lifestyle modifications in NAFLD. We performed a fixed-effect model and a random-effects model for the network meta-analysis. We reported both models for comparison with the reference group in a forest plot when the results were different between models. For each pairwise comparison in a table, we reported the fixed-effect model if the two models reported similar results; otherwise, we reported the more conservative model (i.e. usually the randomeffects model).

We used a hierarchical Bayesian model with three different sets of initial values to start the simulation-based parameter estimation to assist with assessment of convergence, employing codes provided by NICE DSU (Dias 2016). We used a normal distribution with large variance $(10,000)$ for treatment effect priors (vague or flat priors) centred at no effect. For the random-effects model, we used a prior distributed uniformly (limits: 0 to 5) for the between-trial standard deviation parameter and assumed that this variability would be the same across treatment comparisons (Dias 2016). We used a 'burn-in' of 30,000 simulations, checked for convergence (of effect estimates and between-study heterogeneity) visually (i.e. whether the values in different chains mixed very well by visualisation), and ran the models for another 10,000 simulations to obtain effect estimates. If we did not obtain convergence, we increased the number of simulations for the 'burn-in' and used the 'thin' and 'over relax' functions to decrease the autocorrelation. If we still did not obtain convergence, we planned to use alternate initial values and priors employing methods suggested by van Valkenhoef 2012. We estimated the probability that each intervention was ranked at each of the possible positions based on estimated effect sizes and their corresponding uncertainty using the NICE DSU codes (Dias 2016).

\section{Assessment of inconsistency}

We assessed inconsistency (statistical evidence of violation of the transitivity assumption) by fitting both an inconsistency model 
and a consistency model. We used inconsistency models employed in the NICE DSU manual, as we used a common betweenstudy standard deviation (Dias 2014). In addition, we planned to use design-by-treatment full interaction model and inconsistency factor (IF) plots to assess inconsistency when applicable (Higgins 2012; Chaimani 2013). We planned to use Stata/SE 15.1 to create IF plots. In the presence of inconsistency (model fit better with inconsistency models than consistency model, 95\% Crl of 'between-design' variance did not overlap 0 , and $95 \%$ confidence intervals of inconsistency factor did not overlap 0), we planned to assess whether inconsistency was due to clinical or methodological heterogeneity by performing separate analyses for each of the different subgroups mentioned in the subgroup analysis and in the investigation of heterogeneity section or by performing limited network meta-analysis of a more compatible subset of trials when possible.

\section{Direct comparison}

We performed direct comparisons in the randomised clinical trials using the same codes and the same technical details.

\section{Subgroup analysis and investigation of heterogeneity}

We planned to assess the differences in effect estimates between the following subgroups and planned to investigate heterogeneity and inconsistency using meta-regression with the help of the codes provided in NICE DSU guidance if we included a sufficient number of trials (when there were at least two trials in at least two of the subgroups) for all primary and secondary outcomes (Dias 2012a). We planned to use the following trial-level covariates for metaregression.

- Trials at low risk of bias compared to trials at high risk of bias.

- Participants with NASH compared to participants with NAFLD but without NASH.

- Participants with diabetes mellitus compared to participants without diabetes mellitus.

- Co-interventions (e.g. both groups receive some pharmacological intervention or lifestyle intervention aimed at decreasing NAFLD).

- Period of follow-up (short term: up to three months; medium term: more than three months to five years; long term: more than five years).

- Definition used by study authors for serious adverse events and any adverse events (ICH-GCP 1997 versus other definitions).

We planned to calculate a single common interaction term, which assumes that each relative treatment effect compared to a common comparator treatment (i.e. 'no active intervention') is impacted in the same way by the covariate in question when applicable (Dias 2012a). If the 95\% Crl of the interaction term did not overlap zero, we considered this statistically significant heterogeneity or inconsistency (depending upon the factor used as covariate).

\section{Sensitivity analysis}

If there were post-randomisation dropouts, we re-analysed the results using best-worst case scenario and worst-best case scenario analyses as sensitivity analyses whenever possible. We also performed a sensitivity analysis that excluded trials in which mean or standard deviation, or both, were imputed, and we used the median standard deviation in trials to impute missing standard deviations.

We considered variations in subcategories, for example, different doses or durations of nutritional supplementation, as the same treatment node. For future updates, if trials are designed to measure clinically meaningful outcomes, we will consider the subnode approach described by del Giovanni et al to assess the impact of considering different doses or durations as the same treatment node (Del Giovane 2013).

\section{Presentation of results}

We followed the PRISMA-NMA statement while reporting (Hutton 2015). We presented effect estimates with 95\% Crls for each pairwise comparison calculated from direct comparisons and network meta-analysis. We originally planned to present the cumulative probability of treatment ranks (i.e. the probability that the intervention was within the top two, the probability that the intervention was within the top three, etc.), but we did not present these in graphs (SUCRA) because of the sparse data, which can lead to misinterpretation of results due to large uncertainty in the rankings (the Crl was 0 to 1 for all ranks) (Salanti 2011). We plotted the probability that each intervention was best, second best, third best, etc., for each of the different outcomes (rankogams), which is generally considered more informative (Salanti 2011; Dias 2012b), but we did not present these because of the sparse data, which can lead to misinterpretation of results due to large uncertainty in the rankings (the $95 \% \mathrm{Crl}$ was 0 to 1 for most of the ranks). We uploaded all raw data and codes used for analysis in the European Organization for Nuclear Research open source database (Zenodo). You can find this information by clicking here.

\section{Recommendations for future research}

We provided recommendations for future research regarding population, intervention, control, outcomes, period of follow-up, and study design based on the uncertainties that we identified from existing research.

\section{Summary of findings and assessment of the certainty of the evidence}

We presented 'Summary of findings' tables for all primary and secondary outcomes (see Primary outcomes; Secondary outcomes). We followed the approach suggested by Yepes-Nunez and colleagues (Yepes-Nunez 2019). First, we calculated direct and indirect effect estimates (when possible) and 95\% Crls using the node-splitting approach (Dias 2010), that is, by calculating the direct estimate for each comparison by including only trials in which there was direct comparison of interventions and by calculating the indirect estimate for each comparison by excluding trials in which there was direct comparison of interventions (and ensuring a connected network). Next, we rated the quality of direct and indirect effect estimates using the GRADE method, which takes into account risk of bias, inconsistency (heterogeneity), directness of evidence (including incoherence, the term used in the GRADE method for inconsistency in network meta-analysis), imprecision, and publication bias (Guyatt 2011). We then presented relative and absolute estimates of the meta-analysis with the best certainty of evidence (Yepes-Nunez 2019). For illustration of absolute measures, we used weighted median control group proportion or mean (Edgeworth 1887). We did not present 'Summary of findings' tables in the second format presenting all outcomes for selected 
interventions (Yepes-Nunez 2019), as none of the interventions are routinely used in clinical practice, and there was no clinical benefit in any of the interventions, which would have warranted this approach to help balance benefits and harms of the intervention.

\section{RES U L T S}

\section{Description of studies}

\section{Results of the search}

We identified 11,810 references through electronic searches of the Cochrane Central Register of Controlled Trials $(n=2294)$, MEDLINE Ovid $(n=3654)$, Embase Ovid $(n=2311)$, Science Citation Index Expanded and Conference Proceedings Citation Index-Science (n $=2871)$, ClinicalTrials.gov $(n=393)$, World Health Organization (WHO) Trials register $(n=19)$, FDA $(n=137)$, and EMA $(n=131)$. We identified one additional reference by handsearching. After duplicate references were removed, there were 7141 references. We excluded 6837 clearly irrelevant records upon reading titles and abstracts. We retrieved a total of 304 full-text records for further detailed assessment. We excluded 24 records ( 21 studies) for the reasons stated under Characteristics of excluded studies. Twentyseven records describe ongoing trials. Thus, we included a total of 202 trials described in 253 records (Characteristics of included studies). The reference flow is shown in Figure 2.

\section{Included studies}

A total of 202 trials were included (Miglio 2000; Uygun 2000; Harrison 2003; Kugelmas 2003; Deng 2005; Chande 2006; Chou 2006; Dufour 2006; Nobili 2006; Chen 2008; Spadaro 2008; Wang 2008; Zhu 2008; Abdelmalek 2009; Gomez 2009; Hashemi 2009; Nelson 2009; Fabbrini 2010; Khoshbaten 2010b; Li 2010; Malaguarnera 2010; Ruan 2010; Sanyal 2010; Aller 2011; Lavine 2011; Tan 2011; Vajro 2011; Basu 2012; Della Corte 2012; Gonciarz 2012; Loguercio 2012; Malaguarnera 2012; Panahi 2012; Basu 2013; Ghergherehchi 2013; Gianturco 2013; Illnait 2013; Magosso 2013; Nobili 2013; Saxena 2013; Shavakhi 2013; Wong 2013a; Wong 2013b; Aliashrafi 2014; Alisi 2014; Askari 2014; Byrne 2014; Celinski 2014; Chachay 2014; Eslamparast 2014; Farhangi 2014; Foroughi 2014; Martinez-Rodriguez 2014; Sanyal 2014; Scorletti 2014; Sharifi 2014; Solhi 2014; Somi 2014; Akbarzadeh 2015; Aller 2015; Amiri-Moghadam 2015; Argo 2015; Bae 2015; Bonfrate 2015; Boyraz 2015; Chen 2015a; Chen 2015b; Dasarathy 2015; Faghihzadeh 2015; Janczyk 2015; Orr 2015; Pacifico 2015; Qin 2015; Yan 2015; Zhang 2015; Asgharian 2016; Barchetta 2016; Boonyagard 2016; Della Corte 2016; Ebrahimi-Mameghani 2016; Eghtesadi 2016; Ekhlasi 2016; Farsi 2016; Ferolla 2016; Guo 2016; Heeboll 2016; Hong 2016; Li 2016; Nabavi 2016; Naganuma 2016; Nogueira 2016; Panahi 2016; Pezeshki 2016; Rahimlou 2016; Rahmani 2016; Sepideh 2016; Yari 2016; Amiri 2017; Ashraf 2017; Behrouz 2017; Chan 2017; Chongsrisawat 2017; EbrahimiMameghani 2017; Famouri 2017a; Gavrilescu 2017; Hussain 2017; Jameshorani 2017; Javadi 2017; Jeong 2017; Kobyliak 2017; Manzhalii 2017; Mofidi 2017; Navekar 2017; Palamaru 2017; Sakpal 2017; Schattenberg 2017; Shahmohammadi 2017; Tabatabaee
2017; Wang 2017; Youshari 2017; Zohrer 2017; Ahn 2018; Amanat 2018; Amirkhizi 2018; Asghari 2018; Bakhshimoghaddam 2018; Bomhof 2018; Dabbaghmanesh 2018; Daneshi-Maskooni 2018; Eriksson 2018; Geier 2018; Ghaffari 2018; Hosseini 2018; Javanmardi 2018; Kobyliak 2018; Lewis 2018; Oscarsson 2018; Pervez 2018; Sayari 2018; Taghvaei 2018; Tobin 2018; Wang 2018; Zamani 2018; Bril 2019; Cheraghpour 2019; Duseja 2019; JazayeriTehrani 2019; Abhari 2020; Afsharinasab 2020; Afzali 2020; Babaei 2020; Bahrami 2020; Barbakadze 2020; Boonyagard 2020; Cai 2020; Cerletti 2020; Climax 2020; Dallio 2020; Farzin 2020; Fathi 2020; Fernandez-Travieso 2020; Ferro 2020; Hormoznejad 2020; Hoseini 2020; Hosseinabadi 2020; Kazemi 2020; Khutsishvili 2020; Kooshki 2020; Mansour 2020; Moradi 2020; Orang 2020; Parsi 2020; Pasdar 2020; Pervez 2020; Poparn 2020; Pour 2020; Rafie 2020; Sadrkabir 2020; Sangouni 2020; Scorletti 2020; Soleimani 2020; Song 2020; Tutunchi 2020; Yari 2020; Zanko 2020; Chiou 2021; Hong 2021; Izadi 2021; Kanoni 2021; Morvaridzadeh 2021; Poulos 2021; Soleimani 2021; EUCTR 2008-008275-34-GB; EUCTR 2009-017080-41-GB; NCT00816465; NCT00845845; NCT00941642; NCT00977730; NCT01083992; NCT01623024; NCT02690792; NCT04411862). A total of 14,200 participants were randomised to different interventions in these 202 trials. The number of participants in the trials ranged from 8 to 311 . Only a total of 7732 participants from 115 trials were included in one of more outcomes. No cluster-randomised trials or cross-over trials are included in this review.

Further details of the summary of included trials are available in Table 1. Important characteristics, potential effect modifiers, and follow-up for each trial are reported in Table 2. Overall, there do not seem to be any systematic differences between comparisons (i.e. there was no immediate overt concern about the transitivity assumption).

\section{Excluded studies}

The reasons for exclusion of studies are listed in Characteristics of excluded studies. A summary of reasons for exclusion of studies is provided here.

- Reasons related to study design: Chang 2014; Singhal 2015; Semiserin 2016; Abenavoli 2017; Famouri 2017b; NCT04281121.

- Reasons related to interventions or co-interventions: Ersoz 2005; Zhang 2008; Khoshbaten 2010a; Akcam 2011; Dela Cruz 2012; Hajiaghamohammadi 2012; Basu 2014; Han 2014; Chambers 2018; Petyaev 2018; Mahmoudi 2020; Podszun 2020; NCT00820651.

- Reasons related to participants: Saarinen 2011.

- Other reasons: Guo 2014.

\section{Risk of bias in included studies}

Risk of bias is summarised in Figure 3, Figure 4, Table 3 (domainlevel summary), and Table 4 (study-level summary ordered by comparisons). The risk of bias is presented at trial level, as assessments at outcome level for clinical outcomes were the same. 
Figure 3. Risk of bias graph: review authors' judgements about each risk of bias item presented as percentages across all included studies.

Random sequence generation (selection bias)

Allocation concealment (selection bias)

Blinding of participants and personnel (performance bias): All outcomes

Blinding of outcome assessment (detection bias): All outcomes

Incomplete outcome data (attrition bias): All outcomes

Selective reporting (reporting bias)

Other bias
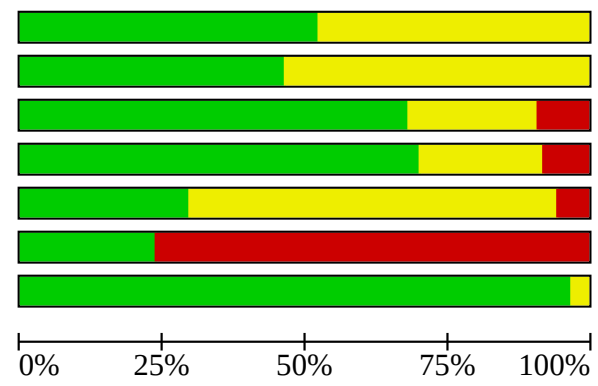

Low risk of bias $\square$ Unclear risk of bias $\square$ High risk of bias 
Figure 4. Risk of bias summary: review authors' judgements about each risk of bias item for each included study.

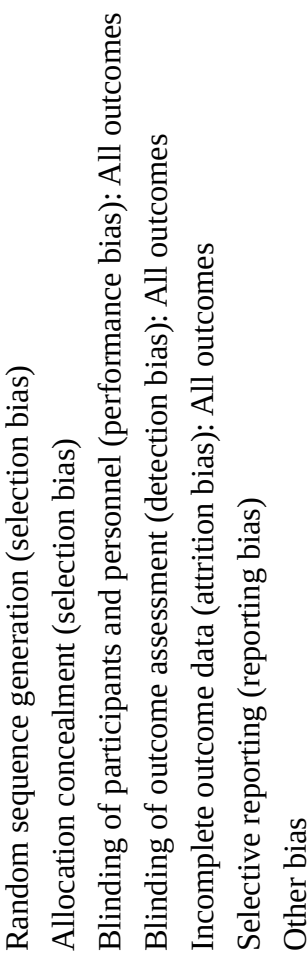

Abdelmalek 2009

Abhari 2020

Afsharinasab 2020

Afzali 2020

Ahn 2018

Akbarzadeh 2015

Aliashrafi 2014

Alisi 2014

Aller 2011

Aller 2015

Amanat 2018

Amiri 2017
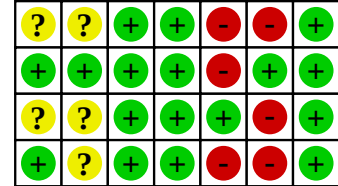

\begin{tabular}{llll|lll}
\hline$?$ & $?$ & + & + & $?$ & - & + \\
\hline
\end{tabular}

Amiri-Moghadam 2015

Amirkhizi 2018

Argo 2015

Asghari 2018

Asgharian 2016

Ashraf 2017

Askari 2014

Babaei 2020

Bae 2015

Bahrami 2020

\section{.}


Figure 4. (Continued)

Bakhshimoghaddam 2018

Barbakadze 2020

Barchetta 2016

Basu 2012

Basu 2013

Behrouz 2017

Bomhof 2018

Bonfrate 2015

Boonyagard 2016

Boonyagard 2020

Boyraz 2015

Bril 2019

Byrne 2014

Cai 2020

Celinski 2014

Cerletti 2020

Chachay 2014

Chan 2017

Chande 2006

Chen 2008

Chen 2015a

Chen 2015b

Cheraghpour 2019

Chiou 2021

Chongsrisawat 2017

Chou 2006

Climax 2020

Dabbaghmanesh 2018

Dallio 2020

Daneshi-Maskooni 2018

Dasarathy 2015

Della Corte 2012

Della Corte 2016

Deng 2005

Dufour 2006

Duseja 2019

Ebrahimi-Mameghani 2016

Ebrahimi-Mameghani 2017

Eghtesadi 2016

Ekhlasi 2016

Eriksson 2018

Eslamparast 2014

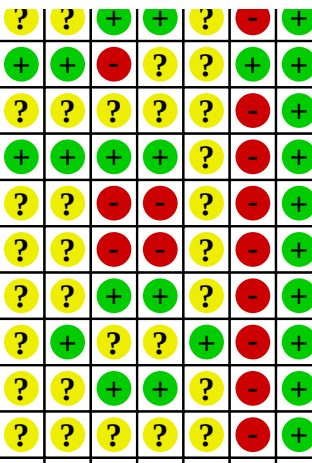

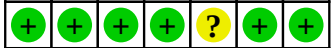

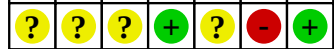

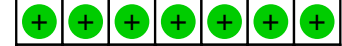

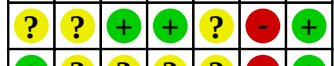

+ ? 3 ?

\begin{tabular}{|l|l|l|l|l|l|l|}
\hline$?$ & $?$ & $?$ & $?$ & $?$ & - & + \\
\hline
\end{tabular}

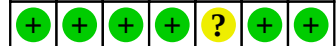

\begin{tabular}{llllll}
+ & + & + & + & + & + \\
\hline
\end{tabular}

\begin{tabular}{lllllll}
\hline+ & + & + & + & + & + & + \\
\hline
\end{tabular}

? $+4+(+$

? ? ++ ? $\odot+$

$+4+(+)$

$+4+4+$

$+4+4$ ? + ?

? ? $++\circ O+$

\begin{tabular}{llll|lll}
\hline$?$ & $?$ & + & + & ? & - & + \\
\hline
\end{tabular}

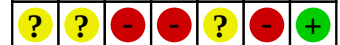

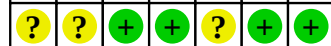

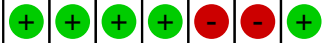

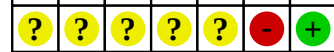

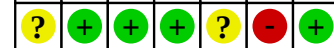

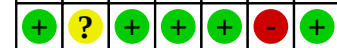

\begin{tabular}{l|l|l|l|lll}
\hline$?$ & $?$ & + & + & ? & - & + \\
\hline
\end{tabular}

\begin{tabular}{llllllll}
+ & + & + & + & $?$ & - & + \\
\hline
\end{tabular}

\begin{tabular}{llllllll}
\hline+ & $?$ & $?$ & $?$ & + & - & + \\
\hline
\end{tabular}

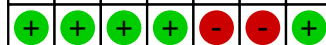

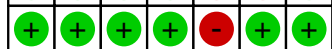

\begin{tabular}{lllllllll}
\hline ? & $?$ & $?$ & $?$ & $?$ & - & + \\
\hline
\end{tabular}

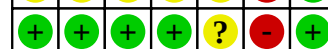

\begin{tabular}{llllllll}
\hline$?$ & $?$ & + & + & ? & - & + \\
\hline
\end{tabular}

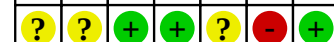

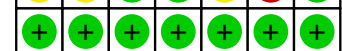

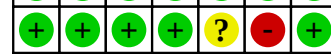

EUCTR 2008-008275-34-GB $+++_{+}++_{+}+{ }_{+}$

EUCTR 2009-017080-41-GB

Fabbrini 2010

\begin{tabular}{l|l|l|l|l|l|l|}
\hline$?$ & ? & + & + & + & - & + \\
\hline$?$ & ? & + & + & ? & & \\
\hline
\end{tabular}

\begin{tabular}{lllllllll} 
& $?$ & ? & + & + & ? & & & + \\
\cline { 2 - 6 } & + & + & + & + & ? & + & +
\end{tabular} 
Figure 4. (Continued)

Fabbrini 2010

Faghihzadeh 2015

Famouri 2017a

Farhangi 2014

Farsi 2016

Farzin 2020

Fathi 2020

Fernandez-Travieso 2020

Ferolla 2016

Ferro 2020

Foroughi 2014

Gavrilescu 2017

Geier 2018

Ghaffari 2018

Ghergherehchi 2013

Gianturco 2013

Gomez 2009

Gonciarz 2012

Guo 2016

Harrison 2003

Hashemi 2009

Heeboll 2016

Hong 2016

Hong 2021

Hormoznejad 2020

Hoseini 2020

Hosseinabadi 2020

Hosseini 2018

Hussain 2017

Illnait 2013

Izadi 2021

Jameshorani 2017

Janczyk 2015

Javadi 2017

Javanmardi 2018

Jazayeri-Tehrani 2019

Jeong 2017

Kanoni 2021

Kazemi 2020

Khoshbaten 2010b

Khutsishvili 2020

Kobyliak 2017

Kobyliak 2018

Kooshki 2020

Kugelmas 2003

Lavine 2011
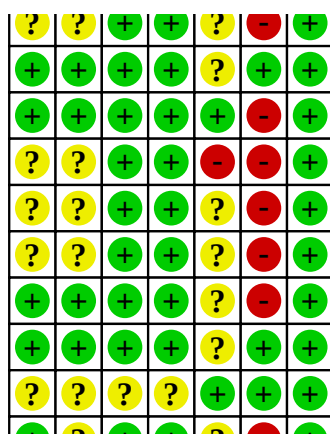

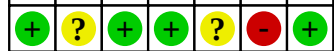

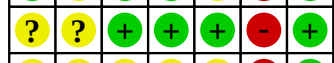

\begin{tabular}{|l|l|l|l|l|l|l|}
\hline ? & $?$ & $?$ & $?$ & $?$ & - & + \\
\hline
\end{tabular}

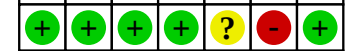
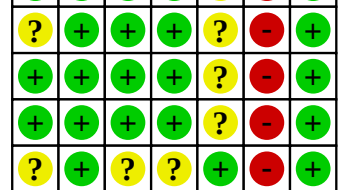

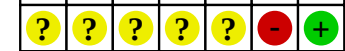

\begin{tabular}{|l|l|l|l|l|l|l|}
\hline$?$ & $?$ & $?$ & $?$ & $?$ & - & + \\
\hline
\end{tabular}

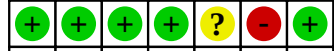


Figure 4. (Continued)

\begin{tabular}{|c|c|c|c|c|c|c|c|}
\hline Lavine 2011 & ? & |? & $\mid+1$ & +1 & ? & & + \\
\hline Lewis 2018 & + & + & + & + & + & + & + \\
\hline Li 2010 & + & \begin{tabular}{l|l}
$?$ \\
\end{tabular} & $?$ & $?$ & + & + & + \\
\hline Li 2016 & $?$ & $?$ & - & - & + & $\Theta$ & + \\
\hline Loguercio 2012 & + & \begin{tabular}{l|l}
$?$ \\
\end{tabular} & + & + & $?$ & + & + \\
\hline Magosso 2013 & + & + & ? & + & + & + & + \\
\hline Malaguarnera 2010 & + & + & + & + & $?$ & - & + \\
\hline Malaguarnera 2012 & + & + & $?$ & + & + & + & + \\
\hline Mansour 2020 & + & + & + & + & + & + & + \\
\hline Manzhalii 2017 & + & + & $-\mathrm{O}$ & $-\mathrm{C}$ & + & + & + \\
\hline Martinez-Rodriguez 2014 & + & ? & $?$ & $?$ & + & + & + \\
\hline Miglio 2000 & + & + & + & + & $?$ & & + \\
\hline Mofidi 2017 & + & + & + & + & ? & - & + \\
\hline Moradi 2020 & $?$ & + & + & + & + & E & + \\
\hline Morvaridzadeh 2021 & + & ? & + & + & $?$ & - & + \\
\hline Nabavi 2016 & + & + & + & + & + & + & + \\
\hline Naganuma 2016 & $?$ & \begin{tabular}{l|l}
$?$ \\
\end{tabular} & $?$ & $?$ & $?$ & & + \\
\hline Navekar 2017 & $?$ & ? & + & + & $?$ & & + \\
\hline NCT00816465 & $?$ & \begin{tabular}{l|l}
$?$ \\
\end{tabular} & + & + & $?$ & & + \\
\hline NCT00845845 & $?$ & \begin{tabular}{l|l}
$?$ \\
\end{tabular} & + & + & + & & + \\
\hline NCT00941642 & $?$ & ? & -0 & - & $?$ & & + \\
\hline NCT00977730 & $?$ & ? & + & + & ? & & + \\
\hline NCT01083992 & $?$ & ? & -0 & - & $?$ & & \\
\hline NCT01623024 & $?$ & ? & E & - & $?$ & & \\
\hline NCT02690792 & $?$ & $?$ & + & + & $?$ & & + \\
\hline NCT04411862 & $?$ & ? & -0 & - & $?$ & - & + \\
\hline Nelson 2009 & $?$ & $?$ & $?$ & $?$ & $?$ & - & + \\
\hline Nobili 2006 & $?$ & + & + & + & $?$ & + & + \\
\hline Nobili 2013 & + & + & + & + & + & & + \\
\hline Nogueira 2016 & + & + & + & + & $?$ & - & + \\
\hline Orang 2020 & + & + & + & + & $?$ & - & + \\
\hline Orr 2015 & $?$ & \begin{tabular}{l|l}
$?$ \\
\end{tabular} & + & + & $?$ & - & + \\
\hline Oscarsson 2018 & + & + & + & + & $?$ & + & + \\
\hline Pacifico 2015 & + & ? & + & + & $?$ & & + \\
\hline Palamaru 2017 & $?$ & $?$ & $?$ & $?$ & $?$ & & + \\
\hline Panahi 2012 & $?$ & $?$ & - & - & $?$ & & + \\
\hline Panahi 2016 & $?$ & ? & ? & $?$ & $?$ & & + \\
\hline Parsi 2020 & $?$ & \begin{tabular}{l|l}
$?$ \\
\end{tabular} & + & + & + & O & + \\
\hline Pasdar 2020 & + & \begin{tabular}{l|l}
$?$ \\
\end{tabular} & + & + & - & - & + \\
\hline Pervez 2018 & $?$ & + & + & + & $?$ & + & + \\
\hline Pervez 2020 & $?$ & + & + & + & + & 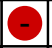 & + \\
\hline Pezeshki 2016 & + & + & + & + & $?$ & - & + \\
\hline Poparn 2020 & + & + & + & + & + & + & + \\
\hline Poulos 2021 & $?$ & $?$ & ? & $?$ & $?$ & & + \\
\hline Pour 2020 & + & + & + & + & + & & + \\
\hline Qin 2015 & + & + & + & + & $?$ & & + \\
\hline Rafie 2020 & $?$ & + & + & + & $?$ & & \\
\hline
\end{tabular}


Figure 4. (Continued)

\begin{tabular}{|c|c|c|c|c|c|c|c|}
\hline Qin 2015 & + & + & + & + & $?$ & & \\
\hline Rafie 2020 & $?$ & + & + & + & $?$ & & \\
\hline Rahimlou 2016 & + & + & + & + & $?$ & & \\
\hline Rahmani 2016 & $?$ & $?$ & + & + & $?$ & & \\
\hline Ruan 2010 & $?$ & $?$ & - & - & $?$ & & \\
\hline Sadrkabir 2020 & $?$ & $?$ & $?$ & $?$ & $?$ & & \\
\hline Sakpal 2017 & $?$ & $?$ & $?$ & $?$ & + & & \\
\hline Sangouni 2020 & + & + & + & + & $?$ & & + \\
\hline Sanyal 2010 & + & + & + & + & + & + & + \\
\hline Sanyal 2014 & + & + & + & + & + & + & + \\
\hline Saxena 2013 & $?$ & $?$ & + & + & $?$ & & \\
\hline Sayari 2018 & $?$ & $?$ & + & + & $?$ & & + \\
\hline Schattenberg 2017 & $?$ & $?$ & - & - & + & & + \\
\hline Scorletti 2014 & + & + & + & + & + & & \\
\hline Scorletti 2020 & + & + & + & + & - & & \\
\hline Sepideh 2016 & $?$ & + & + & + & $?$ & & + \\
\hline Shahmohammadi 2017 & + & + & + & + & + & & + \\
\hline Sharifi 2014 & + & + & + & + & $?$ & & + \\
\hline Shavakhi 2013 & $?$ & $?$ & + & + & $?$ & & + \\
\hline Soleimani 2020 & $?$ & + & + & + & $?$ & & + \\
\hline Soleimani 2021 & + & + & + & + & + & + & + \\
\hline Solhi 2014 & $?$ & $?$ & $?$ & $?$ & $?$ & & + \\
\hline Somi 2014 & $?$ & $?$ & $?$ & + & + & & + \\
\hline Song 2020 & + & + & + & + & $?$ & & + \\
\hline Spadaro 2008 & + & $?$ & $?$ & $?$ & $?$ & & + \\
\hline Tabatabaee 2017 & + & + & + & + & $?$ & & + \\
\hline Taghvaei 2018 & + & $?$ & $?$ & $?$ & + & & + \\
\hline Tan 2011 & $?$ & $?$ & $?$ & $?$ & $?$ & & + \\
\hline Tobin 2018 & + & + & + & + & $?$ & & $?$ \\
\hline Tutunchi 2020 & + & + & + & + & + & + & + \\
\hline Uygun 2000 & $?$ & $?$ & $?$ & $?$ & $?$ & & + \\
\hline Vajro 2011 & $?$ & $?$ & + & + & + & + & + \\
\hline Wang 2008 & $?$ & $?$ & $?$ & $?$ & + & & + \\
\hline Wang 2017 & $?$ & $?$ & $?$ & $?$ & $?$ & & + \\
\hline Wang 2018 & + & $?$ & $?$ & $?$ & $?$ & & + \\
\hline Wong 2013a & + & $?$ & $?$ & $?$ & + & + & + \\
\hline Wong 2013b & + & + & + & + & + & + & + \\
\hline Yan 2015 & + & $?$ & & & $?$ & & + \\
\hline Yari 2016 & $?$ & $?$ & & & + & & + \\
\hline Yari 2020 & + & + & - & C & $?$ & + & $?$ \\
\hline Youshari 2017 & $?$ & $?$ & + & + & $?$ & & + \\
\hline Zamani 2018 & + & + & + & + & $?$ & + & $?$ \\
\hline Zanko 2020 & + & + & + & + & + & & + \\
\hline Zhang 2015 & + & + & + & + & + & + & + \\
\hline Zhu 2008 & $?$ & $?$ & $?$ & $?$ & $?$ & + & + \\
\hline Zohrer 2017 & + & + & + & + & $?$ & & + \\
\hline
\end{tabular}


A total of 19 trials were at low risk of bias (Sanyal 2010; Wong 2013b; Sanyal 2014; Bae 2015; Chen 2015b; Zhang 2015; Heeboll 2016; Nabavi 2016; Chan 2017; Asghari 2018; Eriksson 2018; Kobyliak 2018; Lewis 2018; Bril 2019; Mansour 2020; Poparn 2020; Tutunchi 2020; Soleimani 2021; EUCTR 2008-008275-34-GB). The remaining 182 trials were at unclear or high risk of bias in at least one domain and were considered to be at high risk of bias overall.

\section{Allocation}

A total of 106 trials were at low risk of selection bias due to lack of random sequence generation; the remaining 96 trials, which did not provide sufficient information, were at unclear risk of selection bias due to lack of random sequence generation.

In all, 94 trials were at low risk of selection bias due to lack of allocation concealment; the remaining 108 trials, which did not provide sufficient information, were at unclear risk of selection bias due to lack of allocation concealment.

\section{Blinding}

A total of 138 trials were at low risk of performance bias, as participants and healthcare providers were blinded; 46 trials, which did not provide sufficient information, were at unclear risk of performance bias; the remaining 18 trials were at high risk of performance bias, as it is clear that either participants or healthcare providers, or both, were not blinded.

In all, 142 trials were at low risk of detection bias; 44 trials, which did not provide sufficient information, were at unclear risk of detection bias; the remaining 16 trials were at high risk of detection bias, as it is clear that outcome assessors were not blinded.

\section{Incomplete outcome data}

A total of 60 trials were at low risk of attrition bias, as there were no post-randomisation dropouts, or an intention-to-treat analysis was used; 129 trials were at unclear risk of attrition bias because it is not clear whether there were post-randomisation dropouts, or whether post-randomisation dropouts were related to outcomes (if there were post-randomisation dropouts); the remaining 13 trials were at high risk of attrition bias, as post-randomisation dropouts were probably related to the intervention and to outcomes.

\section{Selective reporting}

In all, 48 trials were at low risk of selective outcome reporting bias, as the important clinical outcomes expected to be reported in such trials were reported; the remaining 154 trials were at high risk of selective outcome reporting bias as outcomes were changed from the protocol published prior to recruitment without sufficient justification, or trials did not report reasonably expected clinical outcomes if no protocol was published prior to recruitment.

\section{Other potential sources of bias}

A total of 196 trials were at low risk of other bias; the remaining 6 trials were at unclear risk of other bias because there were baseline differences in important prognostic factors.

\section{Effects of interventions}

See: Summary of findings 1 Nutritional supplementation for nonalcohol-related fatty liver disease

The network plots (when relevant) are available in Figure 1. The forest plots (when relevant) are available in Figure 5. The model fit when network meta-analysis was performed is available in Table 5. The effect estimates when network meta-analysis was performed are available in Table 6. 
Figure 5. Forest plots showing the network estimates for outcomes for which a formal analysis was performed. The more conservative random-effects model was used when there were differences between fixed-effect and randomeffects models. A higher resolution image of this picture is available here. Abbreviations Please see Appendix 2.
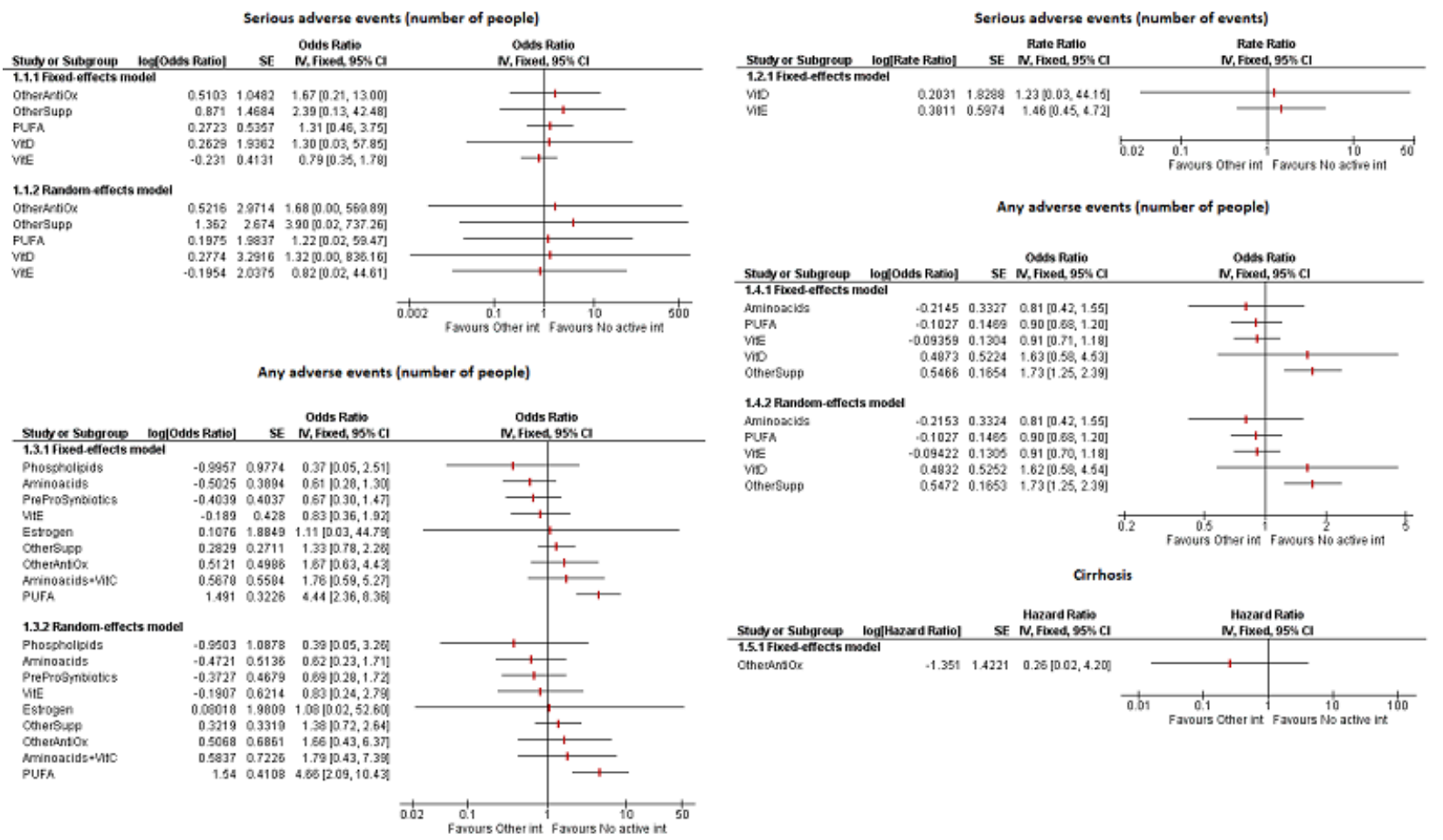

The $95 \%$ credible intervals (Crls) of probability ranks were wide and included 0 and 1 in most comparisons for all outcomes. This was probably because of the sparse data derived from small trials. Therefore, we did not present the ranking probabilities (in a table), in rankograms, and in SUCRA plots, as we considered that presenting this information would be unhelpful and potentially misleading, and it would ignore the differences in systematic errors in the trials.

The certainty of evidence was low or very low for all clinical outcomes because all trials included in the comparisons were at unclear or high risk of bias for at least one risk of bias domain at the outcome level (downgraded one level). For all direct comparisons and for network meta-analysis involving clinical outcomes, events were fewer than 300 , and we downgraded one level for imprecision. In comparisons involving clinical outcomes, the credible intervals were wide and overlapped significant clinical effect and no effect; therefore, we downgraded one more level for imprecision for comparisons with wide confidence intervals. For outcomes for which we were able to assess heterogeneity, there was evidence of heterogeneity for any adverse events (number of events); therefore, we downgraded one more level for heterogeneity for this outcome. Overall, downgrading of evidence resulted in very low certainty of evidence for all comparisons of clinical outcomes.

\section{Mortality}

A total of 52 trials (3372 participants) reported mortality at maximal follow-up of 2 to 28 months (Wang 2008; Gomez 2009; Sanyal 2010; Vajro 2011; Malaguarnera 2012; Illnait 2013; Magosso 2013; Nobili 2013; Shavakhi 2013; Wong 2013a; Chachay 2014; Foroughi

2014; Sanyal 2014; Scorletti 2014; Somi 2014; Aller 2015; Bae 2015; Chen 2015a; Dasarathy 2015; Zhang 2015; Ferolla 2016; Heeboll 2016; Li 2016; Nabavi 2016; Yari 2016; Chan 2017; Famouri 2017a; Hussain 2017; Manzhalii 2017; Sakpal 2017; Schattenberg 2017; Shahmohammadi 2017; Bakhshimoghaddam 2018; Eriksson 2018; Kobyliak 2018; Lewis 2018; Oscarsson 2018; Taghvaei 2018; Zamani 2018; Bril 2019; Cheraghpour 2019; Duseja 2019; Jazayeri-Tehrani 2019; Abhari 2020; Afzali 2020; Boonyagard 2020; Mansour 2020; Poparn 2020; Scorletti 2020; Yari 2020; Soleimani 2021; EUCTR 2008-008275-34-GB). These trials compared a total of 13 treatments (amino acids, other antioxidants, other antioxidants plus other supplements, other supplements, polysaccharides, prebiotics/ probiotics/synbiotics, PUFA, vitamin C plus other antioxidants, vitamin $D$, vitamin $E$, vitamin $E$ plus other antioxidants, vitamin E plus other antioxidants plus other supplements, no active intervention).

None of the 3137 participants in 50 trials died during followup ranging from 2 to 24 months (Wang 2008; Gomez 2009; Vajro 2011; Malaguarnera 2012; Illnait 2013; Magosso 2013; Nobili 2013; Shavakhi 2013; Wong 2013a; Chachay 2014; Foroughi 2014; Sanyal 2014; Scorletti 2014; Somi 2014; Aller 2015; Bae 2015; Chen 2015a; Dasarathy 2015; Zhang 2015; Ferolla 2016; Heeboll 2016; Li 2016; Nabavi 2016; Yari 2016; Chan 2017; Famouri 2017a; Hussain 2017; Manzhalii 2017; Sakpal 2017; Schattenberg 2017; Shahmohammadi 2017; Bakhshimoghaddam 2018; Eriksson 2018; Kobyliak 2018; Lewis 2018; Oscarsson 2018; Taghvaei 2018; Zamani 2018; Cheraghpour 2019; Duseja 2019; Jazayeri-Tehrani 2019; Abhari 2020; Afzali 2020; Boonyagard 2020; Mansour 2020; Poparn 2020; Scorletti 2020; Yari 2020; Soleimani 2021; EUCTR 
2008-008275-34-GB). In the remaining 2 trials that compared vitamin E with no active intervention, 2 of 36 (5.6\%) in Bril 2019 and 1 of $84(1.2 \%)$ in Sanyal 2010 in the intervention groups died, while none of the participants receiving no active intervention $(0 / 32 ; 0 \%$ in Bril 2019; 0/83 participants; 0\% in Sanyal 2010) died. Reasons for death were sepsis in a participant who had fibrosis in Sanyal 2010, and ischaemic and haemorrhagic stroke in two participants who had NASH in Bril 2019.

Because all trials had zero events in at least one of the intervention groups, we did not calculate effect estimates using formal statistical methods.

\section{Quality of life (maximal follow-up)}

None of the trials reported that they measured overall healthrelated quality of life using a validated scale. However, some trials measured and reported specific components of health-related quality of life (Chande 2006; Sanyal 2010; Lavine 2011).

\section{Serious adverse events}

\section{Serious adverse events (number of people)}

A total of 63 trials (4466 participants) reported serious adverse events (number of people) (Miglio 2000; Chande 2006; Chou 2006; Nobili 2006; Zhu 2008; Li 2010; Sanyal 2010; Lavine 2011; Vajro 2011; Loguercio 2012; Magosso 2013; Saxena 2013; Wong 2013a; Wong 2013b; Martinez-Rodriguez 2014; Sanyal 2014; Aller 2015; Bae 2015; Chen 2015b; Faghihzadeh 2015; Janczyk 2015; Qin 2015; Yan 2015; Zhang 2015; Ferolla 2016; Guo 2016; Nabavi 2016; Naganuma 2016; Rahimlou 2016; Sepideh 2016; Chan 2017; Manzhalii 2017; Mofidi 2017; Schattenberg 2017; Zohrer 2017; Amanat 2018; Asghari 2018; Bakhshimoghaddam 2018; Geier 2018; Kobyliak 2018; Lewis 2018; Pervez 2018; Zamani 2018; Bril 2019; Cheraghpour 2019; Duseja 2019; Jazayeri-Tehrani 2019; Abhari 2020; Boonyagard 2020; Mansour 2020; Poparn 2020; Pour 2020; Sangouni 2020; Soleimani 2020; Song 2020; Tutunchi 2020; Yari 2020; Zanko 2020; Izadi 2021; Soleimani 2021; EUCTR 2008-008275-34-GB; EUCTR 2009-017080-41-GB; NCT00845845). These trials compared a total of 19 treatments (amino acids, amino acids plus vitamin C, oestrogen, other antioxidants, other antioxidants plus other supplements, other supplements, phospholipids, phospholipids plus PUFA plus vitamin E, phospholipids plus vitamin E plus other antioxidants, polysaccharides, prebiotics/probiotics/synbiotics, PUFA, PUFA plus other supplements, PUFA plus vitamin $E$, vitamin $D$, vitamin $E$, vitamin $E$ plus other antioxidants, vitamin E plus vitamin C, no active intervention). A total of 53 trials (3599 participants) were not connected to the network because they had zero events in both arms (Miglio 2000; Chande 2006; Chou 2006; Nobili 2006; Zhu 2008; Li 2010; Lavine 2011; Vajro 2011; Loguercio 2012; Magosso 2013; Saxena 2013; Wong 2013a; Martinez-Rodriguez 2014; Aller 2015; Chen 2015b; Faghihzadeh 2015; Janczyk 2015; Qin 2015; Yan 2015; Zhang 2015; Ferolla 2016; Guo 2016; Nabavi 2016; Naganuma 2016; Rahimlou 2016; Sepideh 2016; Manzhalii 2017; Mofidi 2017; Schattenberg 2017; Zohrer 2017; Amanat 2018; Asghari 2018; Bakhshimoghaddam 2018; Kobyliak 2018; Lewis 2018; Pervez 2018; Zamani 2018; Cheraghpour 2019; Duseja 2019; Jazayeri-Tehrani 2019; Abhari 2020; Boonyagard 2020; Mansour 2020; Poparn 2020; Sangouni 2020; Soleimani 2020; Song 2020; Tutunchi 2020; Yari 2020; Zanko 2020; Izadi 2021; Soleimani 2021; EUCTR 2009-017080-41-GB); one trial was not connected to the network because it was the only trial for the comparison and had zero events in one arm (Bae 2015). We did not calculate effect estimates for these trials using formal statistical methods because of sparse data. In the remaining 9 trials, six treatments (PUFA, vitamin E, vitamin D, other supplements, other antioxidants, no active intervention) were compared and could be included in the network meta-analysis. The weighted median of the proportion of people who developed serious adverse events in the no intervention group in trials included in the analysis was 5.3\% (this was $0 \%$ across all trials including those with zero events).

\section{Direct comparisons}

There was no evidence of differences between first and second interventions in any of the comparisons (very low-certainty evidence) (Table 6).

\section{Network meta-analysis}

The fixed-effect model was used because it had similar fit and provided equivalent results as the random-effects model. Betweenstudy variance was 3.27 ( $95 \% \mathrm{Crl} 0.01$ to 22.97 ).

There were no closed loops in the network; therefore inconsistency was not checked. There was no evidence of differences between first and second interventions in any of the comparisons (very lowcertainty evidence) (Table 6).

\section{Serious adverse events (number of events)}

Five trials (222 participants) reported serious adverse events (number of events) (Bae 2015; Geier 2018; Bril 2019; EUCTR 2008-008275-34-GB; NCT00845845). A total of five treatments (PUFA, vitamin $E$, vitamin $D$, amino acids, no active intervention) were compared in these trials. Three trials were not connected to the network because they were the only trials for the comparison and had zero events in one arm (Bae 2015; EUCTR 2008-008275-34GB; NCT00845845). We did not calculate effect estimates for these trials using formal statistical methods because of sparse data. In the remaining 2 trials, three treatments (vitamin $E$, vitamin $D$, no active intervention) were compared and could be included in the network meta-analysis. The weighted median of the number of serious adverse events in the no intervention group in trials included in the analysis was 15.6 per 100 participants (this was 0 per 100 participants across all trials including those with zero events).

\section{Direct comparisons}

There was no evidence of differences between first and second interventions in any of the comparisons (very low-certainty evidence) (Table 6).

\section{Network meta-analysis}

The fixed-effect model was used because there was only one trial for each comparison. There was no evidence of differences between first and second interventions in any of the comparisons (very lowcertainty evidence) (Table 6).

\section{Any adverse events}

\section{Any adverse events (number of people)}

A total of 51 trials (3285 participants) reported any adverse events (number of people) (Miglio 2000; Nobili 2006; EUCTR 2008-008275-34-GB 2008; EUCTR 2009-017080-41-GB; NCT00845845; Khoshbaten 2010b; Li 2010; Malaguarnera 2010; Lavine 2011; Vajro 2011; Malaguarnera 2012; Magosso 2013; Saxena 2013; Wong 2013a; Wong 2013b; Martinez-Rodriguez 2014; Aller 
2015; Bae 2015; Janczyk 2015; Qin 2015; Guo 2016; Heeboll 2016; Nabavi 2016; Chan 2017; Jeong 2017; Manzhalii 2017; Schattenberg 2017; Amanat 2018; Asghari 2018; Daneshi-Maskooni 2018; Eriksson 2018; Kobyliak 2018; Lewis 2018; Oscarsson 2018; Pervez 2018; Zamani 2018; Cheraghpour 2019; Duseja 2019; Jazayeri-Tehrani 2019; Abhari 2020; Boonyagard 2020; Cerletti 2020; Fernandez-Travieso 2020; Mansour 2020; Poparn 2020; Pour 2020; Rafie 2020; Soleimani 2020; Song 2020; Yari 2020; Kanoni 2021). These trials compared a total of 18 treatments (amino acids, amino acids plus vitamin C, oestrogen, other antioxidants, other antioxidants plus other supplements, other supplements, phospholipids, polysaccharides, prebiotics/probiotics/synbiotics, PUFA, PUFA plus other supplements, PUFA plus vitamin E, vitamin $C$, vitamin $D$, vitamin $E$, vitamin $E$ plus other antioxidants, vitamin E plus vitamin C, no active intervention). In all, 22 trials were not connected to the network because they had zero events in both arms (Nobili 2006; Khoshbaten 2010b; Vajro 2011; Magosso 2013; Saxena 2013; Aller 2015; Qin 2015; Nabavi 2016; Manzhalii 2017; Schattenberg 2017; Asghari 2018; Lewis 2018; Pervez 2018; Cheraghpour 2019; Abhari 2020; Boonyagard 2020; Mansour 2020; Poparn 2020; Rafie 2020; Song 2020; Yari 2020; Kanoni 2021). We did not calculate effect estimates for these trials using formal statistical methods because of sparse data. In the remaining 29 trials (2064 participants), 10 interventions (other supplements, prebiotics/ probiotics/synbiotics, PUFA, vitamin E, other antioxidants, amino acids, phospholipids, amino acids plus vitamin C, oestrogen, no active intervention) were compared and could be included in the network meta-analysis. The weighted median of the proportion of people who developed any adverse events in the no intervention group was $2.4 \%$.

\section{Direct comparisons}

PUFA had higher adverse events (number of people) than no active intervention (odds ratio (OR) 4.43; 95\% Crl 2.43 to 8.42; low-certainty evidence; 5 trials, 252 participants). There was no evidence of differences between first and second interventions in any of the remaining comparisons (very low-certainty evidence) (Table 6).

\section{Network meta-analysis}

The fixed-effect model was used because it had similar fit and provided equivalent results as the random-effects model. Betweenstudy variance was 0.09 (95\% Crl 0.00 to 1.04$)$.

There were no closed loops in the network; therefore inconsistency was not checked.

The first intervention had higher numbers of adverse events (number of people) than the second intervention in the following comparisons (Table 6).

- PUFA versus no active intervention: OR 4.44, 95\% Crl 2.40 to 8.48; low-certainty evidence; 4 trials, 203 participants; direct evidence: OR $4.43,95 \% \mathrm{Crl} 2.43$ to 8.42 ).

- PUFA versus other supplements: OR 3.35, 95\% $\mathrm{Crl} 1.48$ to 7.71 ; low-certainty evidence; no direct evidence.

- PUFA versus prebiotics/probiotics/synbiotics: OR 6.68, 95\% Crl 2.46 to 18.67 ; low-certainty evidence; no direct evidence.

The first intervention had lower numbers of adverse events (number of people) than the second intervention in the following comparisons.
- Amino acids versus PUFA: OR $0.14,95 \% \mathrm{Crl} 0.05$ to 0.36 ; lowcertainty evidence; no direct evidence.

- Phospholipids versus PUFA: OR $0.08,95 \% \mathrm{Crl} 0.01$ to 0.51 ; lowcertainty evidence; no direct evidence.

There was no evidence of differences between first and second interventions in any of the remaining comparisons (very lowcertainty evidence) (Table 6).

\section{Any adverse events (number of events)}

Thirteen trials (971 participants) reported any adverse events (number of events) (Chande 2006; EUCTR 2009-017080-41GB 2009; NCT00845845 2009; Sanyal 2010; Lavine 2011; Sanyal 2014; Yan 2015; Bae 2015; Heeboll 2016; Jeong 2017; Geier 2018; Bril 2019; Soleimani 2020a). These trials compared a total of six treatments (amino acids, other supplements, PUFA, vitamin D, vitamin $\mathrm{E}$, no active intervention). All trials were connected to the network. The weighted median of the proportion of people who developed serious adverse events in the no intervention group was 60.2 events per 100 participants.

\section{Direct comparisons}

The number of adverse events was higher with other supplements than with no active intervention: rate ratio $1.72,95 \% \mathrm{Crl} 1.25$ to 2.40 ; 6 trials, 291 participants; low-certainty evidence.

There was no evidence of differences between first and second interventions in any of the remaining comparisons (very lowcertainty evidence) (Table 6).

\section{Network meta-analysis}

The fixed-effect model was used because it had similar fit and provided equivalent results as the random-effects model. Betweenstudy variance was 6.35 ( $95 \% \mathrm{Crl} 0.02$ to 23.79 ).

The number of adverse events was higher with other supplements than with no active intervention: rate ratio $1.73,95 \% \mathrm{Crl} 1.26$ to 2.41 ; 6 trials, 291 participants; direct evidence: rate ratio 1.72, 95\% Crl 1.25 to 2.40 ; low-certainty evidence.

The first intervention had fewer adverse events (number of people) than the second intervention in the following comparisons.

- PUFA versus other supplements: rate ratio $0.52,95 \% \mathrm{Crl} 0.34$ to 0.80; no direct evidence; low-certainty evidence.

- Vitamin E versus other supplements: rate ratio $0.53,95 \% \mathrm{Crl} 0.35$ to 0.79 ; no direct evidence; low-certainty evidence.

- Amino acids versus other supplements: rate ratio $0.47,95 \% \mathrm{Crl}$ 0.22 to 0.96 ; no direct evidence; low-certainty evidence.

There was no evidence of differences between first and second interventions in any of the remaining comparisons (very lowcertainty evidence) (Table 6).

\section{Liver transplantation}

A total of 20 trials (1204 participants) reported liver transplantation at maximal follow-up of 2 to 12 months (Zhang 2015; Heeboll 2016; Chan 2017; Schattenberg 2017; Bakhshimoghaddam 2018; Kobyliak 2018; Lewis 2018; Oscarsson 2018; Zamani 2018; Cheraghpour 2019; Jazayeri-Tehrani 2019; Abhari 2020; Afzali 2020; Boonyagard 2020; Mansour 2020; Poparn 2020; Scorletti 2020; 
Yari 2020; Soleimani 2021; EUCTR 2008-008275-34-GB). These trials compared a total of 10 treatments (other antioxidants, other antioxidants plus other supplements, other supplements, polysaccharides, prebiotics/probiotics/synbiotics, PUFA, vitamin $D$, vitamin $E$ plus other antioxidants, vitamin $E$ plus other antioxidants plus other supplements, no active intervention). None of the 1204 participants in these 20 trials underwent liver transplantation during the follow-up period. We did not calculate effect estimates using formal statistical methods for this outcome because of sparse data.

\section{Decompensation}

A total of 21 trials (1371 participants) reported liver decompensation at maximal follow-up of 2 to 28 months (Sanyal 2010; Zhang 2015; Heeboll 2016; Chan 2017; Schattenberg 2017; Bakhshimoghaddam 2018; Kobyliak 2018; Lewis 2018; Oscarsson 2018; Zamani 2018; Cheraghpour 2019; JazayeriTehrani 2019; Abhari 2020; Afzali 2020; Boonyagard 2020; Mansour 2020; Poparn 2020; Scorletti 2020; Yari 2020; Soleimani 2021; EUCTR 2008-008275-34-GB). These trials compared a total of 11 treatments (other antioxidants, other antioxidants plus other supplements, other supplements, polysaccharides, prebiotics/ probiotics/synbiotics, PUFA, vitamin $D$, vitamin $E$, vitamin $E$ plus other antioxidants, vitamin $\mathrm{E}$ plus other antioxidants plus other supplements, no active intervention). None of the 1204 participants in 20 trials developed any decompensation events during the follow-up period (Zhang 2015; Heeboll 2016; Chan 2017; Schattenberg 2017; Bakhshimoghaddam 2018; Kobyliak 2018; Lewis 2018; Oscarsson 2018; Zamani 2018; Cheraghpour 2019; Jazayeri-Tehrani 2019; Abhari 2020; Afzali 2020; Boonyagard 2020; Mansour 2020; Poparn 2020; Scorletti 2020; Yari 2020; Soleimani 2021; EUCTR 2008-008275-34-GB). In one trial (167 participants), one participant in the vitamin E group $(1 / 84 ; 1.2 \%)$ and no participants in the no active intervention group $(0 / 83 ; 0 \%)$ developed decompensation (Sanyal 2010). We did not calculate effect estimates using formal statistical methods for this outcome because of sparse data.

\section{Cirrhosis}

A total of 19 trials (1172 participants) reported liver cirrhosis at maximal follow-up of 2 to 28 months (Sanyal 2010; Zhang 2015; Heeboll 2016; Chan 2017; Schattenberg 2017; Bakhshimoghaddam 2018; Kobyliak 2018; Lewis 2018; Oscarsson 2018; Zamani 2018; Cheraghpour 2019; Duseja 2019; Jazayeri-Tehrani 2019; Abhari 2020; Mansour 2020; Scorletti 2020; Yari 2020; Soleimani 2021; EUCTR 2008-008275-34-GB). These trials compared a total of eight treatments (other antioxidants, other antioxidants plus other supplements, other supplements, polysaccharides, prebiotics/ probiotics/synbiotics, PUFA, vitamin E, no active intervention). None of 906 participants in 17 trials developed cirrhosis (Zhang 2015; Heeboll 2016; Schattenberg 2017; Zamani 2018; Bakhshimoghaddam 2018; Kobyliak 2018; Lewis 2018; Oscarsson 2018; Cheraghpour 2019; Duseja 2019; Jazayeri-Tehrani 2019; Abhari 2020; Mansour 2020; Scorletti 2020; Yari 2020; Soleimani 2021; EUCTR 2008-008275-34-GB). In one trial, 1 of 83 participants in the vitamin E group $(1 / 83 ; 1.2 \%)$ and none of 84 participants in the no active intervention group $(0 / 84 ; 0 \%$ ) developed cirrhosis (Sanyal 2010). We did not calculate effect estimates using formal statistical methods for these 18 trials because of sparse data. In the remaining trial (99 participants), there was no evidence of differences in proportions of participants who developed liver cirrhosis between other antioxidants versus no active intervention (hazard ratio (HR) $1.68,95 \% \mathrm{Crl} 0.23$ to 15.10 ; very low-certainty evidence) (Chan 2017).

\section{Hepatocellular carcinoma}

A total of 18 trials (1058 participants) reported hepatocellular carcinoma at maximum follow-up of 2 to 12 months (Zhang 2015; Heeboll 2016; Chan 2017; Bakhshimoghaddam 2018; Kobyliak 2018; Lewis 2018; Oscarsson 2018; Zamani 2018; Cheraghpour 2019; Jazayeri-Tehrani 2019; Abhari 2020; Boonyagard 2020; Mansour 2020; Poparn 2020; Scorletti 2020; Yari 2020; Soleimani 2021; EUCTR 2008-008275-34-GB). These trials compared a total of eight treatments (other antioxidants, other antioxidants plus other supplements, other supplements, polysaccharides, prebiotics/ probiotics/synbiotics, PUFA, vitamin D, no active intervention). None of 1058 participants in these 18 trials developed hepatocellular carcinoma during the follow-up period. We did not calculate effect estimates using formal statistical methods for this outcome because of sparse data.

\section{Liver-related mortality}

Of the 52 trials that reported mortality, deaths were reported in only two trials (please see above). The reasons for death were sepsis in a participant who had fibrosis in Sanyal 2010, and ischaemic and haemorrhagic stroke in two participants who had NASH in Bril 2019. All these deaths may be related to liver disease. Because these trials had zero events in the no intervention group (please see mortality; we did not calculate effect estimates using formal statistical methods).

\section{Exploratory outcomes}

\section{Resolution of fatty liver disease}

A total of 45 trials (2913 participants) reported resolution of fatty liver disease at maximal follow-up of 2 to 28 months (Nobili 2006; Spadaro 2008; Zhu 2008; Li 2010; Ruan 2010; Sanyal 2010; Loguercio 2012; Illnait 2013; Magosso 2013; Nobili 2013; Shavakhi 2013; Wong 2013b; Martinez-Rodriguez 2014; Sharifi 2014; Somi 2014; Chen 2015b; Faghihzadeh 2015; Janczyk 2015; Asgharian 2016; Farsi 2016; Heeboll 2016; Rahmani 2016; Chan 2017; Famouri 2017a; Navekar 2017; Asghari 2018; Bakhshimoghaddam 2018; Kobyliak 2018; Lewis 2018; Pervez 2018; Cheraghpour 2019; Duseja 2019; Jazayeri-Tehrani 2019; Abhari 2020; Bahrami 2020; Boonyagard 2020; Fathi 2020; Fernandez-Travieso 2020; Hormoznejad 2020; Hosseinabadi 2020; Mansour 2020; Pervez 2020; Tutunchi 2020; Yari 2020; EUCTR 2008-008275-34-GB). These trials compared a total of 13 treatments (amino acids, other antioxidants, other antioxidants plus other supplements, other supplements, phospholipids, phospholipids plus vitamin E plus other antioxidants, polysaccharides, prebiotics/probiotics/ synbiotics, PUFA, vitamin D, vitamin E, vitamin E plus vitamin C, no active intervention). None of the 646 participants in 12 trials developed resolution of fatty liver disease (Loguercio 2012; Sharifi 2014; Heeboll 2016; Navekar 2017; Asghari 2018; Lewis 2018; Pervez 2018; Duseja 2019; Abhari 2020; Boonyagard 2020; Mansour 2020; Yari 2020). One trial (80 participants) was not connected to the network because this was the only trial for the comparison and it included zero participants in one arms (Somi 2014). In this trial, 9 of $40(22.5 \%)$ participants in the amino acids group and 0 of $40(0 \%)$ participants developed fatty liver resolution. We did not calculate effect estimates using formal statistical methods for 
these trials because of sparse data. The remaining 32 trials (2187 participants) were connected to the network (Nobili 2006; Spadaro 2008; Zhu 2008; Li 2010; Ruan 2010; Sanyal 2010; Illnait 2013; Nobili 2013; Magosso 2013; Shavakhi 2013; Wong 2013b; MartinezRodriguez 2014; Chen 2015b; Faghihzadeh 2015; Janczyk 2015; Asgharian 2016; Farsi 2016; Rahmani 2016; Chan 2017; Famouri 2017a; Bakhshimoghaddam 2018; Kobyliak 2018; Cheraghpour 2019; Jazayeri-Tehrani 2019; Bahrami 2020; Fathi 2020; FernandezTravieso 2020; Hormoznejad 2020; Hosseinabadi 2020; Pervez 2020; Tutunchi 2020; EUCTR 2008-008275-34-GB).

\section{Direct comparisons}

In the following comparisons, the first intervention had higher resolution of fatty liver than the second intervention.

- Other supplements versus no active intervention: HR 3.00, 95\% $\mathrm{Crl} 2.12$ to $4.74 ; 12$ trials, 715 participants.

- Prebiotics/Probiotics/Synbiotics versus no active intervention: HR 4.50, 95\% Crl 2.76 to 7.70 ; 5 trials, 319 participants.

There was no evidence of differences between first and second interventions in any of the remaining comparisons (very lowcertainty evidence) (Table 6).

\section{Network meta-analysis}

The random-effects model was used because we could not obtain convergence for the fixed-effect model despite various measures. Between-study variance was 0.06 (95\% $\mathrm{Crl} 0.00$ to 0.56 ).

There were no closed loops in the network; therefore inconsistency was not checked.

In the following comparisons, the first intervention had higher resolution of fatty liver than the second intervention.

- Other supplements versus no active intervention: HR 3.03, 95\% $\mathrm{Crl} 2.02$ to $4.74 ; 12$ trials, 715 participants; direct evidence: 3.00 , $95 \% \mathrm{Crl} 2.12$ to 4.74 .

- Prebiotics/Probiotics/Synbiotics versus no active intervention: HR 4.64, 95\% Crl 2.58 to 9.09; 5 trials, 319 participants; direct evidence: $4.50,95 \% \mathrm{Crl} 2.76$ to 7.70 .

- PUFA versus no active intervention: HR 3.31,95\% Crl 1.67 to 7.58; 5 trials, 343 participants; direct evidence: $4.78,95 \% \mathrm{Crl} 0.99$ to 123.72 .

- Vitamin E versus no active intervention: HR 2.15, 95\% Crl 1.15 to 4.39; 3 trials, 325 participants; direct evidence: 0.55 , 95\% Crl 0.08 to 1.51 .

- Other antioxidants versus no active intervention: HR 3.43, 95\% Crl 1.37 to 9.63; 5 trials, 309 participants; direct evidence: 3.19 $95 \% \mathrm{Crl} 0.44$ to 15.50 .

There was no evidence of differences between first and second interventions in any of the remaining comparisons (Table 6).

\section{Fibrosis score}

A total of 18 trials (1429 participants) reported fibrosis score (Harrison 2003; Dufour 2006; Abdelmalek 2009; Gomez 2009; Sanyal 2010; Malaguarnera 2010; Lavine 2011; Malaguarnera 2012; Wong 2013a; Gianturco 2013; Sanyal 2014; Zhang 2015; Dasarathy 2015; Aller 2015; Li 2016; Nogueira 2016; Chan 2017; Bril 2019). These trials compared a total of 10 treatments (prebiotics/probiotics/ synbiotics, PUFA, vitamin E, amino acids, vitamin E plus other antioxidants, vitamin $\mathrm{C}$ plus other antioxidants, vitamin $\mathrm{C}$ plus vitamin $E$, other supplements, other antioxidants, no active intervention). All trials were connected to the network.

\section{Direct comparisons}

There was no evidence of differences between first and second interventions in any of the direct comparisons (Table 6).

\section{Network meta-analysis}

The random-effects model was used because it was conservative, although it had similar fit as the fixed-effect model. Between-study variance was 0.04 (95\% Crl 0.00 to 0.25 ).

There were no closed loops in the network; therefore inconsistency was not checked.

There was no evidence of differences between first and second interventions in any of the comparisons in the network metaanalysis (Table 6).

\section{NAFLD activity score}

A total of 15 trials (1279 participants) reported NAFLD activity score (Dufour 2006; Abdelmalek 2009; Gomez 2009; Malaguarnera 2010; Sanyal 2010; Lavine 2011; Loguercio 2012; Malaguarnera 2012; Gianturco 2013; Wong 2013b; Sanyal 2014; Dasarathy 2015; Nogueira 2016; Chan 2017; Bomhof 2018). These trials compared a total of nine treatments (prebiotics/probiotics/synbiotics, PUFA, vitamin E, amino acids, vitamin C plus other antioxidants, phospholipids plus vitamin $\mathrm{E}$ plus other antioxidants, other supplements, other antioxidants, no active intervention). All trials were connected to the network.

\section{Direct comparisons}

Vitamin C plus other antioxidants had lower NAFLD activity scores than no active intervention: mean difference (MD) $-1.66,95 \% \mathrm{Crl}$ -1.94 to $-1.38 ; 1$ trial, 60 participants.

There was no evidence of differences between first and second interventions in any of the direct comparisons (Table 6).

\section{Network meta-analysis}

The random-effects model was used because it was conservative, although it had similar fit as the fixed-effect model. Between-study variance was 0.31 (95\% $\mathrm{Crl} 0.00$ to 2.35 ).

There were no closed loops in the network; therefore inconsistency was not checked.

In the following comparisons, the first intervention had lower NALFD scores than the second intervention.

- Vitamin E versus no active intervention: MD -1.28, 95\% Crl -2.36 to -0.24 ; 3 trials, 270 participants; direct evidence: $-1.28,95 \% \mathrm{Crl}$ -3.14 to 0.50

- Vitamin C plus other antioxidants versus no active intervention: $-1.66,95 \% \mathrm{Crl}-3.18$ to $-0.14 ; 1$ trial, 60 participants; direct evidence: $-1.66,95 \% \mathrm{Crl}-1.94$ to -1.38 .

- Vitamin E versus PUFA: $-1.68,95 \% \mathrm{Crl}-3.13$ to -0.24 ; no direct evidence. 
- Vitamin C plus other antioxidants versus PUFA: $-2.06,95 \% \mathrm{Crl}$ -3.85 to -0.22 ; no direct evidence.

There was no evidence of differences between first and second interventions in any of the remaining comparisons in the network meta-analysis (Table 6).

\section{MELD score}

None of the trials reported that they measured MELD score.

\section{Subgroup analysis}

We did not perform any subgroup analysis because of the sparse data (as described above). However, we acknowledge the heterogeneity in trials in terms of how NAFLD was diagnosed, age groups included in the trials, and interventions and cointerventions that trial participants received.

\section{Sensitivity analysis}

\section{'Best-worst' and 'worst-best' scenario analyses}

We performed 'best-worst' and 'worst-best' scenario analyses for the sensitivity analysis related to missing outcome data. There were changes to interpretation of results for any adverse events (number of people) and for fatty liver resolution (Table 7). The 'main analysis' refers to results without any imputation of data.

Results for these outcomes should be interpreted with caution, as they are susceptible to attrition bias resulting from postrandomisation dropouts. There were no changes to interpretation of results for the remaining analyses or outcomes. These outcomes and comparisons are therefore robust to post-randomisation dropouts.

\section{Imputation of standard deviation}

\section{Fibrosis score}

Exclusion of two trials in which mean or standard deviation or both were imputed did not alter the results (Dufour 2006; Chan 2017).

\section{NAFLD activity score}

Exclusion of three trials in which mean or standard deviation or both were imputed resulted in changes in the results (Dufour 2006; Chan 2017; Bomhof 2018). There was no evidence of differences between first and second interventions in any of the comparisons in the network meta-analysis.

\section{Assessment of reporting biases}

There was no meaningful way in which to rank these studies (i.e. there was no specific change in risk of bias in studies, in sample size, or in the control group used over time, noting that first published report for this review was in 2000 and the second trial report was in 2006); therefore we were unable to perform the comparison-adjusted funnel plot. Important clinical outcomes were not reported in many trials despite high probability of being recorded. We performed a thorough search of literature including search of trial registers. Therefore, we identified most published studies and studies registered in the clinical trials register. All but one trial were published after 2006; therefore we expect that most registered trials on the topic have been identified.

\section{DISCUSSION}

\section{Summary of main results}

We performed a systematic review and network meta-analysis of lifestyle modifications for non-alcohol related fatty liver disease. We included in this review a total of 202 trials (14,200 participants). These trials compared a total of 32 interventions. A total of 115 trials including 7732 participants were included for one or more comparisons in this review (Miglio 2000; Harrison 2003; Chande 2006; Chou 2006; Dufour 2006; Nobili 2006; Spadaro 2008; Wang 2008; Zhu 2008; Abdelmalek 2009; Gomez 2009; Khoshbaten 2010b; Li 2010; Malaguarnera 2010; Ruan 2010; Sanyal 2010; Lavine 2011; Vajro 2011; Loguercio 2012; Malaguarnera 2012; Gianturco 2013; Illnait 2013; Magosso 2013; Nobili 2013; Saxena 2013; Shavakhi 2013; Wong 2013a; Wong 2013b; Chachay 2014; Eslamparast 2014; Foroughi 2014; Martinez-Rodriguez 2014; Sanyal 2014; Scorletti 2014; Sharifi 2014; Somi 2014; Aller 2015; Bae 2015; Chen 2015a; Chen 2015b; Dasarathy 2015; Faghihzadeh 2015; Janczyk 2015; Qin 2015; Yan 2015; Zhang 2015; Asgharian 2016; Farsi 2016; Ferolla 2016; Guo 2016; Heeboll 2016; Li 2016; Nabavi 2016; Naganuma 2016; Nogueira 2016; Rahimlou 2016; Rahmani 2016; Sepideh 2016; Yari 2016; Chan 2017; Famouri 2017a; Hussain 2017; Jeong 2017; Manzhalii 2017; Mofidi 2017; Navekar 2017; Sakpal 2017; Schattenberg 2017; Shahmohammadi 2017; Zohrer 2017; Amanat 2018; Asghari 2018; Bakhshimoghaddam 2018; Bomhof 2018; Daneshi-Maskooni 2018; Eriksson 2018; Geier 2018; Kobyliak 2018; Lewis 2018; Oscarsson 2018; Pervez 2018; Taghvaei 2018; Zamani 2018; Bril 2019; Cheraghpour 2019; Duseja 2019; JazayeriTehrani 2019; Abhari 2020; Afzali 2020; Bahrami 2020; Boonyagard 2020; Cerletti 2020; Climax 2020; Fathi 2020; Fernandez-Travieso 2020; Hormoznejad 2020; Hosseinabadi 2020; Mansour 2020; Pervez 2020; Poparn 2020; Pour 2020; Rafie 2020; Sangouni 2020; Scorletti 2020; Soleimani 2020; Song 2020; Tutunchi 2020; Yari 2020; Zanko 2020; Izadi 2021; Kanoni 2021; Soleimani 2021; EUCTR 2008-008275-34-GB; EUCTR 2009-017080-41-GB; NCT00845845). The remaining trials did not report any outcomes of interest for this review.

The follow-up period in trials that reported primary or secondary outcomes was 2 months to 28 months. During this follow-up period, clinical events related to non-alcohol-related fatty liver disease (NAFLD) such as mortality, liver cirrhosis, liver decompensation, and liver transplantation were sparse, probably because of the very short follow-up period ( 2 months to 28 months). It takes follow-up of 8 to 28 years to detect differences in mortality between people with NAFLD and the general population (Adams 2005; Bedogni 2007; Ong 2008; Soderberg 2010; Onnerhag 2014). Therefore, it is unlikely that differences in clinical outcomes are noted in trials with less than 5 to 10 years of follow-up.

There was no evidence of increased serious adverse events with nutritional supplements at prescribed doses. There were some differences in any adverse events in some comparisons, but the impact of these adverse events on patients is not clear in the absence of health-related quality of life information. Although there were some differences between treatments in surrogate outcomes, the implication of these differences for clinical outcomes is not known. Therefore, there appears to be considerable uncertainty about whether any of the nutritional supplements are beneficial for people with NAFLD. We note that there is also considerable uncertainty about whether any pharmacological interventions work in NAFLD (Lombardi 2017), or whether any 
lifestyle interventions work in NAFLD (Buzzetti 2021). However, this does not mean there is nothing we can do and we should ignore people with NAFLD: NAFLD decreases life expectancy and increases liver cirrhosis, hepatocellular carcinoma, and requirement for liver transplantation (Adams 2005; Bedogni 2007; Ong 2008; Soderberg 2010; White 2012; Onnerhag 2014; Angulo 2015; Ekstedt 2015; Cholankeril 2017; Piscaglia 2016).

It is unlikely that ongoing trials will provide an answer to whether any nutritional supplements improve clinical outcomes in people with NAFLD. As mentioned earlier, it is unlikely that it is possible to note any differences in important clinical outcomes before 5 to 10 years. It is important that any nutritional supplements that are proposed are affordable and sustainable over this period of time. In terms of intervention, this systematic review suggests that some surrogate outcomes such as resolution of fatty liver and NAFLD activity score may improve with prebiotics/probiotics/synbiotics, PUFA, and vitamin E. For this review, we did not explore the optimum dose and duration; additional research may be required to identify the optimum dose and duration of these interventions. In terms of outcomes, major clinical outcomes should include mortality, health-related quality of life, decompensated liver cirrhosis, and liver transplantation.

Sample size estimation for a parallel randomised controlled trial (RCT) was made on the basis of two studies of natural history of NAFLD that followed participants for a median period of around 8 years (Adams 2005; Bedogni 2007). The proportion of participants who died was approximately $6 \%$ in Bedogni 2007 and $12.6 \%$ in Adams 2005. The hazard ratio for mortality of people with NAFLD versus those without NAFLD was 1.34 in Adams 2005 and 1.47 in Bedogni 2007. Therefore, it is reasonable to expect a $20 \%$ relative reduction in mortality by intervention: even this will mean that mortality in NALFD is higher than in those without NAFLD. If we assumed a proportional hazards model, an alpha error of 0.5 , power of 0.9 , mortality of people who received standard care of $9 \%$ at 8 years, estimated $20 \%$ reduction in mortality by intervention, recruitment period of 3 years, and follow-up period of 8 years, one would need 3610 participants in each group before loss to followup (PS: power and sample size 3.1.6).

Clearly, such a trial will be expensive to conduct. Some recent and innovative trial designs may allow completion of NAFLD trials powered to detect differences in clinically important outcomes rather than relying on unvalidated surrogate outcomes. There are no national registries for NAFLD that can be used for registrybased RCTs. Existing registries for NAFLD such as European NAFLD, European paediatric NAFLD, and the TARGET-NASH study register observational studies with bio-banking facilities (Barritt 2017; Mann 2018; Hardy 2020). Establishment of national research registries for NAFLD will allow efficient large-scale RCTs (James 2015). In the absence of such registries, another efficient and innovative study design is the cohort multiple RCT (cmRCT) such as Relton 2010 (although staged-informed consent in the design is less contentious in terms of ethical concerns in Young-Afat 2016 than the originally proposed design of $\mathrm{cmRCT}$, whereby some participants did not know of their participation in an RCT such as Relton 2010). There are methodological differences in the way cmRCT is designed compared to the standard parallel RCT design, for example, sample size calculations in such cmRCTs need to take into account the proportion who consented to receive the intervention in addition to the attrition that is usually accounted for in the standard parallel RCT design (Reeves 2018). Furthermore, other than a certain proportion of participants allocated to the intervention group after consenting to undergo the intervention, the efficiency of the cmRCT is lost (Reeves 2018). Because of these methodological challenges, feasibility studies may be necessary to determine the optimal design of cmRCT. Some innovations such as follow-up based on national electronic health record data (participants should be consented for linking their details to national electronic health record data at the time they consent to trial participation) will allow assessment of outcomes such as mortality, liver transplantation, and liver cirrhosis for several decades. However, the use of national electronic health record data brings its own challenges such as data quality and validation, completeness of data capture, and heterogeneity among systems for international trials (Cowie 2017). Besides, the use of national electronic health record data does not allow the capture of healthrelated quality of life. Potential solutions include self-reported health-related quality of life and health-related quality of life measured in a sample of participants, but there is no current evidence on the validity of these approaches nor on the biases in these approaches. Therefore, nesting methodological research projects within NAFLD trials can reveal the optimal trade-off between the most valid and the most efficient study designs in trials involving people with NAFLD.

\section{Overall completeness and applicability of evidence}

Trials included only people with NAFLD with and without nonalcoholic steatohepatitis (NASH). Therefore, the results of this review are applicable in people with NAFLD with or without $\mathrm{NASH}$ who are able to undergo these interventions. The results are not applicable in people who had previously undergone liver transplantation.

Different studies used different methods of diagnosis of NAFLD. Having consensus on minimum standards for definition of NAFLD in clinical trials can help with the applicability of evidence from future trials.

It should also be noted that study investigators made the diagnosis of NAFLD based on the presence of fatty liver in the absence of excessive alcohol consumption. However, there is ongoing debate as to what excessive alcohol consumption is in the context of fatty liver (Eslam 2019). Therefore, it is possible that fatty liver may have been caused by alcohol consumption, although such alcohol consumption would be considered non-excessive according to the current definition of NAFLD. The findings of this review are applicable in people with NAFLD as per current definitions in 2021. This might change in the future if the nomenclature for fatty liver is changed.

The review provides evidence only about what happens within the first 28 months and does not provide any information on what happens beyond 28 months.

\section{Quality of the evidence}

The overall certainty (quality) of evidence was low or very low for all clinical outcomes. One of the main reasons for this was high risk of bias in all trials. To provide some information on whether it is possible to perform trials at low risk of bias, we have considered each source of bias. This can give context for interpretation of information. Randomisation can be performed by standard 
methods, for example, by web-based central randomisation; an intention-to-treat analysis can be performed; and a protocol should be published prior to recruitment. Blinding of healthcare providers, participants, and outcome assessors is possible through use of an identical placebo. Another major reason for the decreased certainty of evidence is imprecision. Clinical events were extremely sparse, resulting in difficulty undertaking a formal analysis, or in the rare instance when formal analysis was possible, the credible intervals were extremely wide for most comparisons. The designs of ongoing trials suggest that this imprecision cannot be addressed by these trials. We used clinical outcomes; therefore, there is no issue of indirectness due to outcomes. There is no suggestion that the potential effect modifiers were systematically different across comparisons (i.e. there was no concern about the transitivity assumption) for most outcomes. However, we were unable to perform a formal analysis to assess this inconsistency because of sparse data and absence of direct and indirect estimates for the same comparison. Therefore, one cannot rule out inconsistency ('incoherence' according to GRADE terminology). There was no meaningful way to rank these studies (i.e. there was no specific change in risk of bias in the studies, in sample size, or in the control group used over time, noting that the first trial dates back to only 2000 and there is no evidence that any additional intervention works); we have completed a thorough search for studies on effectiveness. However, only 52 of 202 (25.7\%) trials reported mortality; fewer trials reported other clinical outcomes, which would have been recorded in trials of this nature. Many of these outcomes were considered as core outcome measures (Clearfield 2021). We acknowledge there is no publication related to core outcomes, but we expect reporting of clinical outcomes, even if the primary outcomes of these studies were surrogate outcomes. This may suggest reporting bias for these outcomes.

\section{Potential biases in the review process}

We selected a range of databases to search without using any language restrictions and conducted the network meta-analysis according to National Institute for Health and Care Excellence Decision Support Unit (NICE DSU) guidance. In addition, we analysed by using the fixed-effect model and the random-effects model. These are the strengths of the review process. We excluded studies that compared variations in duration or dose in the different interventions. Hence, this review does not provide information on whether one variation is better than another. The potential effect modifiers in trials that reported them were broadly similar across comparisons. Therefore, concern about the transitivity assumption is low. We were unable to assess or report inconsistency, as no comparisons with direct and indirect comparisons were available for any of these outcomes. Therefore, concern about the transitivity assumption cannot be ruled out. However, this is only of academic interest because data are sparse.

Inclusion of indirect observational evidence could weaken our network meta-analysis, but this could also be viewed as a strength for assessing rare adverse events. It is well established that exclusion of non-randomised studies increases focus on potential benefits and reduces focus on risks of serious adverse events and risks of any adverse events. As stated in the protocol, we would have recommended a new systematic review of non-randomised studies for identifying true adverse event proportions and rates, but we do not recommend such a systematic review because of the findings of this review (i.e. there is uncertainty about whether any of the interventions improve clinical outcomes, and trials powered to measure such clinical outcomes are likely to identify the major harms that need to be considered in decision-making). A significant effort is required to identify non-randomised studies that report on harm. It is also challenging to assess risk of bias in these studies. As trials powered to measure clinical outcomes are likely to identify major harms that need to be considered in decision-making, a systematic review on adverse events from observational studies will likely be unnecessary.

\section{Agreements and disagreements with other studies or reviews}

This is the first network meta-analysis on the impact of nutritional supplementation on clinical outcomes in people with NAFLD. Therefore, we are unable to compare our conclusions with those of other reviews. Our conclusions differ from those of many study authors included in this review because we relied on clinical rather than surrogate outcomes.

\section{AUTHORS' CONCLUSIONS}

\section{Implications for practice}

The evidence indicates considerable uncertainty about effects of nutritional supplementation compared to no nutritional supplementation on any clinical outcomes in people with nonalcohol-related fatty liver disease.

\section{Implications for research}

Further well-designed randomised clinical trials are necessary. Some aspects of the design of these randomised clinical trials are provided here.

\section{Study design}

Registry-based RCT or cmRCT.

\section{Participants}

People with NAFLD.

\section{Intervention/control}

Vitamin E, prebiotics/probiotics/synbiotics, polyunsaturated fatty acid (PUFA), no nutritional supplementation.

\section{Outcomes}

- Primary outcome: mortality

- Secondary outcomes: health-related quality of life, decompensated liver cirrhosis, liver transplantation, resource utilisation measures including costs of intervention, decreased healthcare utilisation

- Minimum length of follow-up: 8 years

\section{Sample size}

If we assumed a proportional hazards model, an alpha error of 0.5 , power of 0.9 , mortality of people who received standard care of $9 \%$ at 8 years, estimated $20 \%$ reduction in mortality by intervention, recruitment period of 3 years, and follow-up period of 8 years, one would need 3610 participants in each group prior to loss to followup. Adjustments to sample size should be made to reflect the loss 
to follow-up and the proportion of participants who accept the intervention in $\mathrm{cmRCTs}$.

\section{Other aspects}

Trials need to be conducted and reported according to the SPIRIT (Standard Protocol Items: Recommendations for Interventional Trials) statement - Chan 2013 - and the CONSORT statement Schulz 2010. Methodological research within trials may lead to trials conducted in the optimal way.

\section{ACKNOWLEDGEMENTS}

We acknowledge the help and support of the Cochrane HepatoBiliary Group, the Cochrane Central Editorial Unit, and copyeditors. We also thank Amanda Brand, researcher at the Centre for Evidence-based Health Care, Stellenbosch University, South Africa, and co-ordinator at Cochrane Nutrition, for comments on the review.

Peer reviewers: Amanda Brand, South Africa (Cochrane Nutrition); Luca Giocaomelli, Italy; Kerry Dwan, UK.

Contact editor: Christian Gluud, Denmark. Sign-off editor: Rachel Richardson, UK.
Cochrane Review Group funding acknowledgement: the Danish State is the largest single funder of the Cochrane Hepato-Biliary Group through its investment in the Copenhagen Trial Unit, Centre for Clinical Intervention Research, Capital Region of Denmark, Rigshospitalet, Copenhagen, Denmark.

This project was funded by the National Institute for Health Research (NIHR) Systematic Reviews Programme (project number 16/114/17) and was supported by the Complex Reviews Support Unit; it was also funded by the National Institute for Health Research (project number 14/178/29).

\section{Department of Health disclaimer}

The views and opinions expressed therein are those of the review authors and do not necessarily reflect those of the $16 / 114 / 17$ or 14/178/29 Programmes, the NIHR, the NHS, or the Department of Health.

\section{Danish State and the Copenhagen Trial Unit disclaimer}

The views and opinions expressed in this protocol are those of the review authors and do not necessarily reflect those of the Danish State or The Copenhagen Trial Unit. 


\section{R E F E R E N C E S}

\section{References to studies included in this review}

Abdelmalek 2009 \{published data only\}

Abdelmalek MF, Sanderson SO, Angulo P, Soldevila-Pico C, Liu C, Peter J, et al. Betaine for nonalcoholic fatty liver disease: results of a randomized placebo-controlled trial. Hepatology (Baltimore, Md.) 2009;50(6):1818-26.

\section{Abhari 2020 \{published data only\}}

Abhari K, Saadati S, Yari Z, Hosseini H, Hedayati M, Abhari S, et al. The effects of bacillus coagulans supplementation in patients with non-alcoholic fatty liver disease: a randomized, placebo-controlled, clinical trial. Clinical Nutrition ESPEN 2020;39:53-60.

\section{Afsharinasab 2020 \{published data only\}}

Afsharinasab M, Mohammad-Sadeghipour M, Reza Hajizadeh M, Khoshdel A, Mirzaiey V, Mahmoodi M. The effect of hydroalcoholic berberis integerrima fruits extract on the lipid profile, antioxidant parameters and liver and kidney function tests in patients with nonalcoholic fatty liver disease. Saudi Journal of Biological Sciences 2020;27(8):2031-7.

Afzali 2020 \{published data only\}

Afzali N, Ebadi SS, Afzali H, Sharif MR, Vazirian M, Ebadi SA, et al. Effect of beta vulgaris extract on liver enzymes in patients with non-alcoholic fatty liver disease: a randomized clinical trial. Hepatitis Monthly 2020;20(7):1-8.

Ahn 2018 \{published data only\}

Ahn SB, Jun DW, Kim E, Oh H, Jeong JY, Sohn JH, et al. Change of microbiota in patients with improved fatty liver and obesity. Journal of Hepatology 2018;68(Suppl 1):S838-9.

\section{Akbarzadeh 2015 \{published data only\}}

Akbarzadeh Z, Nourian M, Askari G, Maracy MR, Rafiei R. The effect of psyllium on anthropometric measurements and liver enzymes in overweight or obese adults with nonalcoholic fatty liver disease (NAFLD). Journal of Isfahan Medical School 2015;33(355):1771-83.

\section{Aliashrafi 2014 \{published data only\}}

Aliashrafi S, Ebrahimi-Mameghani M, Kakaie F, Javadzadeh Y, Jafarabadi MA. The effect of microalgae chlorella vulgaris supplementation on inflammatory factors in non-alcoholic fatty liver disease: a double blind randomized clinical trial. Journal of Mazandaran University of Medical Sciences 2014;24(112):113-21.

\section{Alisi 2014 \{published data only\}}

Alisi A, Bedogni G, Baviera G, Giorgio V, Porro E, Paris C, et al. Randomised clinical trial: the beneficial effects of VSL\#3 in obese children with non-alcoholic steatohepatitis. Alimentary Pharmacology \& Therapeutics 2014;39(11):1276-85.

\section{Aller 2011 \{published data only\}}

Aller R, De Luis DA, Izaola O, Conde R, Gonzalez Sagrado M, Primo D, et al. Effect of a probiotic on liver aminotransferases in nonalcoholic fatty liver disease patients: a double blind randomized clinical trial. European Review for Medical and Pharmacological Sciences 2011;15(9):1090-5.

\section{Aller 2015 \{published data only\}}

Aller R, Izaola O, Gomez S, Tafur C, Gonzalez G, Berroa E, et al. Effect of silymarin plus vitamin $\mathrm{E}$ in patients with nonalcoholic fatty liver disease. A randomized clinical pilot study. European Review for Medical and Pharmacological Sciences 2015;19(16):3118-24

\section{Amanat 2018 \{published data only\}}

Amanat S, Eftekhari MH, Fararouei M, Bagheri Lankarani K, Massoumi SJ. Genistein supplementation improves insulin resistance and inflammatory state in non-alcoholic fatty liver patients: a randomized, controlled trial. Clinical Nutrition 2018;37(4):1210-5

\section{Amiri 2017 \{published data only\}}

Amiri HL, Agah S, Azar JT, Hosseini S, Shidfar F, Mousavi SN. Effect of daily calcitriol supplementation with and without calcium on disease regression in non-alcoholic fatty liver patients following an energy-restricted diet: randomized, controlled, double-blind trial. Clinical Nutrition 2017;36(6):1490-7.

\section{Amiri-Moghadam 2015 \{published data only\}}

Amiri-Moghadam S, Nematy M, Eghtesadi S, Khalili M, Mojarrad M, Jazayeri S, et al. Effects of L-carnitine supplementation on body composition in patients with nonalcoholic steatohepatitis (NASH). Current Topics in Nutraceutical Research 2015;13(2):71-6.

\section{Amirkhizi 2018 \{published data only\}}

Amirkhizi F, Hamedi-Shahraki S, Hosseinpour-Arjmand S, Ebrahimi-Mameghani M. The effect of alpha-lipoic acid on liver function and metabolic markers in obese patients with non-alcoholic fatty liver disease: a double-blind randomized controlled trial. Iranian Red Crescent Medical Journal 2018;20(3):e65925

* Amirkhizi F, Hamedi-Shahraki S, Hosseinpour-Arjmand S, Vaghef-Mehrabany E, Ebrahimi-Mameghani M. Effects of alphalipoic acid supplementation on oxidative stress status in patients with non-alcoholic fatty liver disease: a randomized, double-blind, placebo-controlled clinical trial. Iranian Red Crescent Medical Journal 2018;20(9):e67615.

Argo 2015 \{published data only\}

Argo CK, Patrie JT, Lackner C, Henry TD, de Lange EE, Weltman AL, et al. Effects of $n-3$ fish oil on metabolic and histological parameters in NASH: a double-blind, randomized, placebo-controlled trial. Journal of Hepatology 2015;62(1):190-7.

\section{Asghari 2018 \{published data only\}}

Asghari S, Rafraf M, Farzin L, Asghari-Jafarabadi M, Ghavami SM, Somi MH. Effects of pharmacologic dose of resveratrol supplementation on oxidative/antioxidative status biomarkers in nonalcoholic fatty liver disease patients: a 
randomized, double-blind, placebo-controlled trial. Advanced Pharmaceutical Bulletin 2018;8(2):307-17.

\section{Asgharian 2016 \{published data only\}}

* Asgharian A, Askari G, Esmailzade A, Feizi A, Mohammadi V. The effect of symbiotic supplementation on liver enzymes, Creactive protein and ultrasound findings in patients with nonalcoholic fatty liver disease: a clinical trial. International Journal of Preventive Medicine 2016;7:59.

Asgharian A, Mohammadi V, Gholi Z, Esmaillzade A, Feizi A, Askari $G$. The effect of synbiotic supplementation on body composition and lipid profile in patients with NAFLD: a randomized, double blind, placebo-controlled clinical trial study. Iranian Red Crescent Medical Journal 2017;19(4):e42902.

\section{Ashraf 2017 \{published data only\}}

Ashraf MS, Jamshed A, Alam SJ, Ahmed KF, Farhana Y. An openlabel randomized clinical study to compare the effects of a nutritional supplement versus vitamin $\mathrm{E}$ on fibroscan score in nonalcoholic steatohepatitis patients. Journal of Hepatology 2017;66(1 Suppl 1):S426.

\section{Askari 2014 \{published data only\}}

Askari F, Rashidkhani B, Hekmatdoost A. Cinnamon may have therapeutic benefits on lipid profile, liver enzymes, insulin resistance, and high-sensitivity C-reactive protein in nonalcoholic fatty liver disease patients. Nutrition Research 2014;34(2):143-8.

\section{Babaei 2020 \{published data only\}}

Babaei A, Taghavi SA, Mohammadi A, Mahdiyar MA, Iranpour P, Ejtehadi $F$, et al. Comparison of the efficacy of oral fenugreek seeds hydroalcoholic extract versus placebo in nonalcoholic fatty liver disease; a randomized, triple-blind controlled pilot clinical trial. Indian Journal of Pharmacology 2020;52(2):86-93.

\section{Bae 2015 \{published data only\}}

Bae JC, Lee WY, Yoon KH, Park JY, Son HS, Han KA, et al. Improvement of nonalcoholic fatty liver disease with carnitineorotate complex in type 2 diabetes (corona): a randomized controlled trial. Diabetes Care 2015;38(7):1245-52.

\section{Bahrami 2020 \{published data only\}}

Bahrami M, Cheraghpour M, Jafarirad S, Alavinejad P, Asadi F, Hekmatdoost A, et al. The effect of melatonin on treatment of patients with non-alcoholic fatty liver disease: a randomized double blind clinical trial. Complementary Therapies in Medicine 2020;52:102452.

\section{Bakhshimoghaddam 2018 \{published data only\}}

Bakhshimoghaddam F, Shateri K, Sina M, Hashemian M, Alizadeh M. Daily consumption of synbiotic yogurt decreases liver steatosis in patients with nonalcoholic fatty liver disease: a randomized controlled clinical trial. Journal of Nutrition 2018;148(8):1276-84.

\section{Barbakadze 2020 \{published data only\}}

Barbakadze GG, Khachidze TG, Sulaberidze GT, Burnadze KG, Jebashvili MI. Role of vitamin E and vitamin C for the treatment of nonalcoholic steatohepatitis in non-diabetic patients. Hepatology International 2020;14:S312-3.

\section{Barchetta 2016 \{published data only\}}

* Barchetta I, Del Ben M, Angelico F, Di Martino M, Fraioli A, La Torre $G$, et al. No effects of oral vitamin D supplementation on non-alcoholic fatty liver disease in patients with type 2 diabetes: a randomized, double-blind, placebo-controlled trial. $B M C$ Medicine 2016;14:92.

EUCTR 2011-003010-17-IT. Randomized double-blind intervention trial to assess the efficacy of vitamin D3 supplementation vs placebo in reducing hepatic steatoinflammation and cardio-metabolic risk profile in patients affected by type 2 diabetes and non-alcoholic fatty liver disease (NAFLD/NASH). www.clinicaltrialsregister.eu/ctr-search/search? query=2011-003010-17 (first received 7 July 2011).

\section{Basu 2012 \{published data only\}}

* Basu P, James Shah N, Farhat S. Effect of vitamin E and alfa lipoic acid (ALA) in non-alcoholic fatty liver disease: a randomised placebo control open label prospective clinical trial: VAIN trial. Gut 2012;61:A204.

Basu PP, Krishnaswamy N, Nair T, Shah NJ, Farhat S. Effect of vitamin $E$ and alfa lipoic acid (ALA) in non alcoholic fatty liver disease: a randomized placebo control open label prospective clinical trial - VAIN trial. American Journal of Gastroenterology 2011;106:S136-7.

Basu PP, Krishnaswamy N, Nair TJ, Shah NJ, Farhat S. Effect of vitamin $E$ and alfa lipoic acid (ALA) in non alcoholic fatty liver disease: a randomized placebo control open label prospective clinical trial - VAIN trial. Hepatology (Baltimore, Md) 2011;54(4 Suppl):1145a.

\section{Basu 2013 \{published data only\}}

* Basu P, Mittimanj K, Shah NJ, Siriki R, Rahaman K, Atluri N, et al. Curcumin, anti-oxidant, and pioglitazone therapy with inclusion of vitamin $\mathrm{E}$ in non-alcoholic fatty liver disease - a randomized open label placebo controlled clinical prospective trial (captive). Journal of Clinical and Experimental Hepatology 2013;3(1 Suppl 1):S26-7

Basu P, Shah NJ, Farhat S, Siriki R, Mittimanj K, Atluri S, et al. Curcumin, anti-oxidant, and pioglitazone therapy with inclusion of vitamin $\mathrm{E}$ in non alcoholic fatty liver disease - a randomized open label placebo controlled clinical prospective trial (captive). Gut 2013;62:A23.

\section{Behrouz 2017 \{published data only\}}

Behrouz V, Aryaeian N, Zahedi MJ, Jazayeri S. Effects of probiotic and prebiotic supplementation on metabolic parameters, liver aminotransferases, and systemic inflammation in nonalcoholic fatty liver disease: a randomized clinical trial. Journal of Food Science 2020;85(10):3611-7.

* Behrouz V, Jazayeri S, Aryaeian N, Zahedi MJ, Hosseini F. Effects of probiotic and prebiotic supplementation on leptin, adiponectin, and glycemic parameters in non-alcoholic fatty liver disease: a randomized clinical trial. Middle East Journal of Digestive Diseases 2017;9(3):150-7. 
Bomhof 2018 \{published data only\}

Bomhof MR, Parnell JA, Ramay HR, Crotty P, Rioux KP, Probert CS, et al. Histological improvement of non-alcoholic steatohepatitis with a prebiotic: a pilot clinical trial. European Journal of Nutrition 2018;19:19.

Bonfrate 2015 \{published data only\}

Bonfrate L, Grattagliano I, Portincasa P. The efficacy of Eurosil 85-vit. E complex on metabolic profile in adults with non alcoholic fatty liver disease (NAFLD). A double-blind randomized placebo-controlled clinical study. European Journal of Clinical Investigation 2015;45:15.

\section{Boonyagard 2016 \{published data only\}}

Boonyagard S, Techathuvanan K. Impact of vitamin D replacement on liver enzymes in non-alcoholic fatty liver disease patients. Hepatology (Baltimore, Md) 2016;63(1 Suppl 1):542a.

\section{Boonyagard 2020 \{published data only\}}

Boonyagard S, Techathuvanan K. Impact of vitamin D replacement on liver enzymes in non-alcoholic fatty liver disease patients: a randomized, double-blind, placebocontrolled trial. Chotmaihet Thangphaet [Journal of the Medical Association of Thailand] 2020;103(12 Suppl 8):A105-12.

\section{Boyraz 2015 \{published data only\}}

Boyraz M, Pirgon O, Dundar B, Cekmez F, Hatipoglu N. Longterm treatment with $n-3$ polyunsaturated fatty acids as a monotherapy in children with nonalcoholic fatty liver disease. Journal of Clinical Research in Pediatric Endocrinology 2015;7(2):121-7.

\section{Bril 2019 \{published data only\}}

* Bril F, Biernacki DM, Kalavalapalli S, Lomonaco R, Subbarayan SK, Lai J, et al. Role of vitamin E for nonalcoholic steatohepatitis in patients with type 2 diabetes: a randomized controlled trial. Diabetes Care 2019;42(8):1481-8.

Bril F, Biernacki DM, Lomonaco R, Kalavalapalli S, Subbarayan SK, Lai J, et al. Role of vitamin E for the treatment of nonalcoholic steatohepatitis (NASH) in patients with $\mathrm{t} 2 \mathrm{dm}-\mathrm{a}$ randomized, controlled trial. Diabetes 2018;67(Suppl 1):A328-9.

\section{Byrne 2014 \{published data only\}}

Byrne CD, Scorletti E, Bhatia L, McCormick K, Clough G, Nash K, et al. Benefits of docosahexanoic acid tissue enrichment in nonalcoholic fatty liver disease: results from a randomised, double-blind, placebo-controlled study testing the effects of omega-3 fatty acid treatment. Diabetes 2014;63:a468.

\section{Cai 2020 \{published data only\}}

Cai GS, Su H, Zhang J. Protective effect of probiotics in patients with non-alcoholic fatty liver disease. Medicine 2020;99(32):e21464.

\section{Celinski 2014 \{published data only\}}

Celinski K, Konturek PC, Slomka M, Cichoz-Lach H, Brzozowski T, Konturek SJ, et al. Effects of treatment with melatonin and tryptophan on liver enzymes, parameters of fat metabolism and plasma levels of cytokines in patients with non-alcoholic fatty liver disease - 14 months follow-up. Journal of Physiology and Pharmacology 2014;65(1):75-82.

Cerletti 2020 \{published data only\}

Cerletti C, Colucci M, Storto M, Semeraro F, Ammollo CT, Incampo F, et al. Randomised trial of chronic supplementation with a nutraceutical mixture in subjects with non-alcoholic fatty liver disease. British Journal of Nutrition 2020;123(2):190-7.

\section{Chachay 2014 \{published data only\}}

Chachay VS, Macdonald GA, Martin JH, Whitehead JP, O'MooreSullivan TM, Lee P, et al. Resveratrol does not benefit patients with nonalcoholic fatty liver disease. Clinical Gastroenterology and Hepatology 2014;12(12):2092-103.e1-6.

\section{Chan 2017 \{published data only\}}

Chan WK, Mustapha NRN, Mahadeva S. Silymarin for the treatment of non-alcoholic steatohepatitis: interim analysis of a randomized, double-blind, placebo-controlled trial. Journal of Gastroenterology and Hepatology 2015;30:324-5.

Chan WK, Mustapha NRN, Mahadeva S. Silymarin improves fibrosis in non-alcoholic steatohepatitis. Journal of Hepatology 2016;64(2 Suppl 1):S225-6.

* Chan WK, Nik Mustapha NR, Mahadeva S. A randomized trial of silymarin for the treatment of nonalcoholic steatohepatitis. Clinical Gastroenterology and Hepatology 2017;15(12):1940-9.e8.

Chan WK, Nik Mustapha NR, Mahadeva S. Silymarin for the treatment of non-alcoholic steatohepatitis: interim analysis of a randomized, double-blind, placebo-controlled trial. Journal of Hepatology 2015;62:S269.

\section{Chande 2006 \{published data only\}}

Chande N, Laidlaw M, Adams P, Marotta P. Yo Jyo Hen Shi Ko (YHK) improves transaminases in nonalcoholic steatohepatitis (NASH): a randomized pilot study. Digestive Diseases and Sciences 2006;51(7):1183-9.

\section{Chen 2008 \{published data only\}}

Chen R, Guo Q, Zhu WJ, Xie Q, Wang H, Cai W. Therapeutic efficacy of omega-3 polyunsaturated fatty acid capsule in treatment of patients with non-alcoholic fatty liver disease. World Chinese Journal of Digestology 2008;16(18):2002-6.

\section{Chen 2015a \{published data only\}}

Chen SH, Zhao XL, Ran L, Wan J, Wang XF, Qin Y, et al. Resveratrol improves insulin resistance, glucose and lipid metabolism in patients with non-alcoholic fatty liver disease: a randomized controlled trial. Digestive and Liver Disease 2015;47(3):226-32.

\section{Chen 2015b \{published data only\}}

Chen SH, Zhao XL, Wan J, Ran L, Qin Y, Wang XF, et al. Dihydromyricetin improves glucose and lipid metabolism and exerts anti-inflammatory effects in nonalcoholic fatty liver disease: a randomized controlled trial. Pharmacological Research 2015;99:74-81. 


\section{Cheraghpour 2019 \{published data only\}}

Cheraghpour M, Imani H, Ommi S, Alavian SM, KarimiShahrbabak E, Hedayati M, et al. Hesperidin improves hepatic steatosis, hepatic enzymes, and metabolic and inflammatory parameters in patients with nonalcoholic fatty liver disease: a randomized, placebo-controlled, double-blind clinical trial. Phytotherapy Research 2019;33(8):2118-25.

\section{Chiou 2021 \{published data only\}}

Chiou YL, Chyau CC, Li TJ, Kuo CF, Kang YY, Chen CC, et al. Hepatoprotective effect of Antrodia cinnamomea mycelium in patients with nonalcoholic steatohepatitis: a randomized, double-blind, placebo-controlled trial. Journal of the American College of Nutrition 2021;40(4):349-57.

\section{Chongsrisawat 2017 \{published data only\}}

Chongsrisawat V, Poparn H, Chatrproedprai S, Sornsiri K, Treeprasertsuk S. Effect of pre/probiotic mixture in children with nonalcoholic fatty liver disease: a randomized controlled trial. European Journal of Pediatrics 2017;176(11):1486.

\section{Chou 2006 \{published data only\}}

Chou SC, Chen KW, Hwang JS, Lu WT, Chu YY, Lin JD, et al. The add-on effects of Gynostemma pentaphyllum on nonalcoholic fatty liver disease. Alternative Therapies in Health and Medicine 2006;12(3):34-9.

\section{Climax 2020 \{published data only\}}

Climax J, Newsome PN, Hamza M, Weissbach M, Coughlan D, Sattar N, et al. Effects of epeleuton, a novel synthetic secondgeneration n-3 fatty acid, on non-alcoholic fatty liver disease, triglycerides, glycemic control, and cardiometabolic and inflammatory markers. Journal of the American Heart Association 2020;9(16):e016334.

\section{Dabbaghmanesh 2018 \{published data only\}}

Dabbaghmanesh MH, Danafar F, Eshraghian A, Omrani GR. Vitamin $D$ supplementation for the treatment of nonalcoholic fatty liver disease: a randomized double blind placebo controlled trial. Diabetes and Metabolic Syndrome 2018;12(4):513-7.

\section{Dallio 2020 \{published data only\}}

Dallio M, Masarone M, Gravina AG, Di Somma A, Romeo M, Tuccillo $C$, et al. Evaluation of the effect derived from silybin with vitamin $\mathrm{D}$ and vitamin $\mathrm{E}$ administration on clinical, metabolic, endothelial dysfunction, oxidative stress parameters and serological worsening markers in non-alcoholic fatty liver disease patients. Digestive and Liver Disease 2020;52(Suppl 2):S165.

\section{Daneshi-Maskooni 2018 \{published data only\}}

Daneshi-Maskooni M, Keshavarz SA, Qorbani M, Mansouri S, Alavian SM, Badri-Fariman M, et al. Green cardamom increases sirtuin-1 and reduces inflammation in overweight or obese patients with non-alcoholic fatty liver disease: a double-blind randomized placebo-controlled clinical trial. Nutrition and Metabolism 2018;15:63.

\section{Dasarathy 2015 \{published data only\}}

* Dasarathy S, Dasarathy J, Khiyami A, Yerian L, Hawkins C, Sargent R, et al. Double-blind randomized placebo-controlled clinical trial of omega 3 fatty acids for the treatment of diabetic patients with nonalcoholic steatohepatitis. Journal of Clinical Gastroenterology 2015;49(2):137-44.

Dasarathy S, Dasarathy J, Khiyami A, Yerian LM, Sargent R, Hawkins CA, et al. Randomized controlled trial of omega 3 fatty acids in the treatment of non-alcoholic steatohepatitis in type 2 diabetes mellitus. Hepatology (Baltimore, Md) 2013;58(4 Suppl 1):518a.

\section{Della Corte 2012 \{published data only\}}

Della Corte C, Alterio A, Papadatou B, Sartorelli MR, Comparcola D, Nobili V. Beneficial effect of dietary supplementation with glucomannan in children with NAFLD: preliminary results. Digestive and Liver Disease 2012;44:S289.

\section{Della Corte 2016 \{published data only\}}

Della Corte C, Carpino G, De Vito R, De Stefanis C, Alisi A, Cianfarani $S$, et al. Docosahexanoic acid plus vitamin D treatment improves features of NALFD in children with serum vitamin D deficiency: results from a single centre trial. PLOS One 2016;11(12):e0168216.

\section{Deng 2005 \{published data only\}}

Deng YQ, Fan XF, Li JP. The state of insulin resistance in patients with nonalcoholic fatty liver and the intervention with gankangyin. Chinese Journal of Integrative Medicine 2005;11(2):117-22.

\section{Dufour 2006 \{published data only\}}

Balmer ML, Siegrist K, Zimmermann A, Dufour JF. Effects of ursodeoxycholic acid in combination with vitamin $E$ on adipokines and apoptosis in patients with nonalcoholic steatohepatitis. Liver International 2009;29(8):1184-8.

* Dufour JF, Oneta CM, Gonvers JJ, Bihl F, Cerny A, Cereda JM, et al. Randomized placebo-controlled trial of ursodeoxycholic acid with vitamin $\mathrm{E}$ in nonalcoholic steatohepatitis. Clinical Gastroenterology and Hepatology 2006;4(12):1537-43.

\section{Duseja 2019 \{published data only\}}

* Duseja A, Acharya SK, Mehta M, Chhabra S, Shalimar, Rana S, et al. High potency multistrain probiotic improves liver histology in non-alcoholic fatty liver disease (NAFLD): a randomised, double-blind, proof of concept study. BMJ Open Gastroenterology 2019;6:e000315.

Duseja AK, Acharya SK, Mehta M, Chhabra S, Shalimar, Rana S, et al. Probiotic VSL\#3 improves liver histology in patients with nonalcoholic fatty liver disease - a proof of concept study. Hepatology (Baltimore, Md) 2016;64(1 Suppl 1):596a.

\section{Ebrahimi-Mameghani 2016 \{published data only\}}

Ebrahimi-Mameghani M, Jamali H, Mahdavi R, Kakaei F, Abedi R, Kabir-Mamdooh B. Conjugated linoleic acid improves glycemic response, lipid profile, and oxidative stress in obese patients with non-alcoholic fatty liver disease: a randomized controlled clinical trial. Croatian Medical Journal 2016;57(4):331-42. 
Ebrahimi-Mameghani 2017 \{published data only\}

Ebrahimi-Mameghani M, Sadeghi Z, Abbasalizad Farhangi M, Vaghef-Mehrabany E, Aliashrafi S. Glucose homeostasis, insulin resistance and inflammatory biomarkers in patients with non-alcoholic fatty liver disease: beneficial effects of supplementation with microalgae chlorella vulgaris: a doubleblind placebo-controlled randomized clinical trial. Clinical Nutrition 2017;36(4):1001-6.

\section{Eghtesadi 2016 \{published data only\}}

Eghtesadi S, Amiri Moghadam S, Nemati M, Khalili M, Mojarrad M, Jazayeri S, et al. Effects of L-carnitine supplementation on inflammatory factors and malondialdehyde in patients with nonalcoholic steatohepatitis (NASH). Obesity Facts 2016;9:184.

\section{Ekhlasi 2016 \{published data only\}}

* Ekhlasi G, Kolahdouz Mohammadi R, Agah S, Zarrati M, Hosseini AF, Arabshahi SS, et al. Do symbiotic and vitamin E supplementation have favorite effects in nonalcoholic fatty liver disease? A randomized, double-blind, placebo-controlled trial. Journal of Research in Medical Sciences 2016;21:106.

Ekhlasi G, Zarrati M, Agah S, Hosseini AF, Hosseini S, Shidfar S, et al. Effects of symbiotic and vitamin E supplementation on blood pressure, nitric oxide and inflammatory factors in nonalcoholic fatty liver disease. Excli Journal 2017;16:278-90.

\section{Eriksson 2018 \{published data only\}}

Eriksson JW, Lundkvist P, Jansson PA, Johansson L, Kvarnstrom M, Moris L, et al. Effects of dapagliflozin and n-3 carboxylic acids on non-alcoholic fatty liver disease in people with type 2 diabetes: a double-blind randomised placebocontrolled study. Diabetologia 2018;61(9):1923-34.

\section{Eslamparast 2014 \{published data only\}}

Eslamparast T, Poustchi H, Zamani F, Sharafkhah M, Malekzadeh R, Hekmatdoost A. Synbiotic supplementation in nonalcoholic fatty liver disease: a randomized, double-blind, placebo-controlled pilot study. American Journal of Clinical Nutrition 2014;99(3):535-42.

\section{EUCTR 2008-008275-34-GB \{published data only\}}

EUCTR 2008-008275-34-GB. The effect of omega-3 fatty acids on non-alcoholic fatty liver disease. www.clinicaltrialsregister.eu/ ctr-search/trial/2008-008275-34/GB (first received 6 July 2009).

\section{EUCTR 2009-017080-41-GB \{published data only\}}

EUCTR 2009-017080-41-GB. A randomised, partially-blind, placebo-controlled, pilot, dose-ranging study to assess the effect of cannabidiol (CBD) on liver fat levels in subjects with fatty liver disease. www.clinicaltrialsregister.eu/ctr-search/ search?query=2009-017080-41 (first received 17 March 2010).

\section{Fabbrini 2010 \{published data only\}}

Fabbrini E, Mohammed BS, Korenblat KM, Magkos F, McCrea J, Patterson BW, et al. Effect of fenofibrate and niacin on intrahepatic triglyceride content, very low-density lipoprotein kinetics, and insulin action in obese subjects with nonalcoholic fatty liver disease. Journal of Clinical Endocrinology and Metabolism 2010;95(6):2727-35.

\section{Faghihzadeh 2015 \{published data only\}}

Faghihzadeh F, Adibi P, Hekmatdoost A. The effects of resveratrol supplementation on cardiovascular risk factors in patients with non-alcoholic fatty liver disease: a randomised, double-blind, placebo-controlled study. British Journal of Nutrition 2015;114(5):796-803.

\section{Famouri 2017a \{published data only\}}

Famouri F, Shariat Z, Hashemipour M, Keikha M, Kelishadi R. Effects of probiotics on nonalcoholic fatty liver disease in obese children and adolescents. Journal of Pediatric Gastroenterology and Nutrition 2017;64(3):413-7.

\section{Farhangi 2014 \{published data only\}}

Farhangi MA, Alipour B, Jafarvand E, Khoshbaten M. Oral coenzyme q10 supplementation in patients with nonalcoholic fatty liver disease: effects on serum vaspin, chemerin, pentraxin 3 , insulin resistance and oxidative stress. Archives of Medical Research 2014;45(7):589-95.

\section{Farsi 2016 \{published data only\}}

Farsi F, Mohammadshahi M, Alavinejad P, Rezazadeh A, Zarei M, Engali KA. Functions of coenzyme $q 10$ supplementation on liver enzymes, markers of systemic inflammation, and adipokines in patients affected by nonalcoholic fatty liver disease: a doubleblind, placebo-controlled, randomized clinical trial. Journal of the American College of Nutrition 2016;35(4):346-53.

\section{Farzin 2020 \{published data only\}}

Farzin L, Asghari S, Rafraf M, Asghari-Jafarabadi M, Shirmohammadi M. No beneficial effects of resveratrol supplementation on atherogenic risk factors in patients with nonalcoholic fatty liver disease. International Journal for Vitamin \& Nutrition Research 2020;90(3-4):279-89.

\section{Fathi 2020 \{published data only\}}

* Fathi M, Alavinejad P, Haidari Z, Amani R. The effect of zinc supplementation on steatosis severity and liver function enzymes in overweight/obese patients with mild to moderate non-alcoholic fatty liver following calorie-restricted diet: a double-blind, randomized placebo-controlled trial. Biological Trace Element Research 2020;197(2):394-404.

Fathi M, Alavinejad P, Haidari Z, Amani R. The effects of zinc supplementation on metabolic profile and oxidative stress in overweight/obese patients with non-alcoholic fatty liver disease: a randomized, double-blind, placebo-controlled trial. Journal of Trace Elements in Medicine \& Biology 2020;62:126635.

\section{Fernandez-Travieso 2020 \{published data only\}}

Fernandez-Travieso JC, Rodriguez-Perez I, Ruenes-Domech C, IIInait-Ferrer J, Fernandez-Dorta L, Mendoza-Castano S. Benefits of the therapy with abexol in patients with non-alcoholic fatty liver disease. Gastroenterology Research 2020;13(2):73-80.

\section{Ferolla 2016 \{published data only\}}

Ferolla SM, Couto CA, Armiliato GN, Silva LC, Lima EC, Lisboa QC, et al. Improvement in hepatic steatosis in nonalcoholic steatohepatitis in response to synbiotic supplementation. Hepatology (Baltimore, Md) 2015;62:1280a. 
* Ferolla SM, Couto CA, Costa-Silva L, Armiliato GN, Pereira CA, Martins FS, et al. Beneficial effect of synbiotic supplementation on hepatic steatosis and anthropometric parameters but not on gut permeability in a population with nonalcoholic steatohepatitis. Nutrients 2016;8(7):28.

\section{Ferro 2020 \{published data only\}}

Ferro Y, Montalcini T, Mazza E, Foti D, Angotti E, Gliozzi M, et al. Randomized clinical trial: Bergamot citrus and wild cardoon reduce liver steatosis and body weight in non-diabetic individuals aged over 50 years. Frontiers in Endocrinology 2020;11:494.

\section{Foroughi 2014 \{published data only\}}

Foroughi M, Maghsoudi Z, Ghiasvand R, Iraj B, Askari G. Effect of vitamin $D$ supplementation on C-reactive protein in patients with nonalcoholic fatty liver. International Journal of Preventive Medicine 2014;5(8):969-75.

\section{Gavrilescu 2017 \{published data only\}}

Gavrilescu O, Savin A, Chiosa AM, Dorobaf A, Mihai C. Effects of prebiotics on non-alcoholic fatty liver disease. Journal of Gastrointestinal and Liver Diseases 2017;26(Suppl 3):10-1.

\section{Geier 2018 \{published data only\}}

Geier A, Eichinger M, Stirnimann G, Semela D, Tay F, Seifert B, et al. Treatment of non-alcoholic steatohepatitis patients with vitamin D: a double-blinded, randomized, placebocontrolled pilot study. Scandinavian Journal of Gastroenterology 2018;53(9):1114-20.

\section{Ghaffari 2018 \{published data only\}}

Ghaffari A, Rafraf M, Navekar R, Asghari-Jafarabadi M. Effects of turmeric and chicory seed supplementation on antioxidant and inflammatory biomarkers in patients with non-alcoholic fatty liver disease (NAFLD). Advances in Integrative Medicine 2018;5(3):89-95.

\section{Ghergherehchi 2013 \{published data only\}}

Ghergherehchi R, Hazhir N, Gharehbaghi MM. Lifestyle intervention and vitamin $\mathrm{E}$ therapy in obese children with nonalcoholic fatty liver disease. Journal of Comprehensive Pediatrics 2013;4(1):62-5.

\section{Gianturco 2013 \{published data only\}}

Gianturco V, Troisi G, Bellomo A, Bernardini S, D'Ottavio E, Formosa V, et al. Impact of combined therapy with alpha-lipoic and ursodeoxycholic acid on nonalcoholic fatty liver disease: double-blind, randomized clinical trial of efficacy and safety. Hepatology International 2013;7(2):570-6.

\section{Gomez 2009 \{published data only\}}

Vilar Gomez E, Rodriguez De Miranda A, Gra Oramas B, Arus Soler E, Llanio Navarro R, Calzadilla Bertot L, et al. Clinical trial: a nutritional supplement viusid, in combination with diet and exercise, in patients with nonalcoholic fatty liver disease. Alimentary Pharmacology \& Therapeutics 2009;30(10):999-1009.

\section{Gonciarz 2012 \{published data only\}}

Gonciarz M, Gonciarz Z, Bielanski W, Mularczyk A, Konturek PC, Brzozowski T, et al. The effects of long-term melatonin treatment on plasma liver enzymes levels and plasma concentrations of lipids and melatonin in patients with nonalcoholic steatohepatitis: a pilot study. Journal of Physiology and Pharmacology 2012;63(1):35-40.

Guo 2016 \{published data only\}

Guo W, Gao M-s, Li W, Peng C, Xie H-y, Tan Y. Effects of metformin combined with enteric bifid-triple viable capsule on nonalcoholic fatty liver disease. Chinese Journal of New Drugs 2016;25(4):439-42.

\section{Harrison 2003 \{published data only\}}

Harrison SA, Torgerson S, Hayashi P, Ward J, Schenker S. Vitamin $\mathrm{E}$ and vitamin $\mathrm{C}$ treatment improves fibrosis in patients with nonalcoholic steatohepatitis. American Journal of Gastroenterology 2003;98(11):2485-90.

\section{Hashemi 2009 \{published data only\}}

Hashemi SJ, Hajiani E, Sardabi EH. A placebo-controlled trial of silymarin in patients with nonalcoholic fatty liver disease. Hepatitis Monthly 2009;9(4):265-70.

\section{Heeboll 2016 \{published data only\}}

Heeboll S, Kreuzfeldt M, Hamilton-Dutoit S, Poulsen MK, Stodkilde-Jorgensen $\mathrm{H}$, Moller HJ, et al. Placebo-controlled, randomised clinical trial: high-dose resveratrol treatment for non-alcoholic fatty liver disease. Scandinavian Journal of Gastroenterology 2016;51(4):456-63.

\section{Hong 2016 \{published data only\}}

Hong M, Lee YH, Kim S, Suk KT, Bang CS, Yoon JH, et al. Antiinflammatory and antifatigue effect of Korean red ginseng in patients with nonalcoholic fatty liver disease. Journal of Ginseng Research 2016;40(3):203-10.

\section{Hong 2021 \{published data only\}}

Hong JT, Lee MJ, Yoon SJ, Shin SP, Bang CS, Baik GH, et al. Effect of Korea red ginseng on nonalcoholic fatty liver disease: an association of gut microbiota with liver function. Journal of Ginseng Research 2021;45(2):316-24.

\section{Hormoznejad 2020 \{published data only\}}

Hormoznejad R, Mohammad Shahi M, Rahim F, Helli B, Alavinejad P, Sharhani A. Combined cranberry supplementation and weight loss diet in non-alcoholic fatty liver disease: a double-blind placebo-controlled randomized clinical trial. International Journal of Food Sciences \& Nutrition 2020;71(8):991-1000.

\section{Hoseini 2020 \{published data only\}}

Hoseini Z, Behpoor N, Hoseini R. Co-treatment with vitamin D supplementation and aerobic training in elderly women with vitamin D deficiency and NAFLD: a single-blind controlled trial. Hepatitis Monthly 2020;20(2):e96437.

\section{Hosseinabadi 2020 \{published data only\}}

* Hosseinabadi S, Rafraf M, Asghari S, Asghari-Jafarabadi M, Vojouhi S. Effect of green coffee extract supplementation on serum adiponectin concentration and lipid profile in patients with non-alcoholic fatty liver disease: a randomized, controlled trial. Complementary Therapies in Medicine 2020;49:102290. 
Hosseinabadi S, Rafraf M, Mahmoodzadeh A, AsghariJafarabadi M, Asghari S. Effects of green coffee extract supplementation on glycemic indexes, leptin, and obesity values in patients with non-alcoholic fatty liver disease. Journal of Herbal Medicine 2020;22:100340.

\section{Hosseini 2018 \{published data only\}}

Hosseini SM, Aliashrafi S, Ebrahimi-Mameghani M. The effect of a single intramuscular injection of cholecalciferol on the serum levels of vitamin D, adiponectin, insulin resistance, and liver function in women with non-alcoholic fatty liver disease (NAFLD): a randomized, controlled clinical trial. Iranian Red Crescent Medical Journal 2018;20(10):e60746.

\section{Hussain 2017 \{published data only\}}

Hussain M, Habib Ur R, Akhtar L. Therapeutic benefits of green tea extract on various parameters in non-alcoholic fatty liver disease patients. Pakistan Journal of Medical Sciences 2017;33(4):931-6.

\section{Illnait 2013 \{published data only\}}

Illnait J, Rodriguez I, Mendoza S, Fernandez Y, Mas R, Miranda M, et al. Effects of $d-002$, a mixture of high molecular weight beeswax alcohols, on patients with nonalcoholic fatty liver disease. Korean Journal of Internal Medicine 2013;28(4):439-48.

\section{Izadi 2021 \{published data only\}}

Izadi F, Farrokhzad A, Tamizifar B, Tarrahi MJ, Entezari MH. Effect of sour tea supplementation on liver enzymes, lipid profile, blood pressure, and antioxidant status in patients with non-alcoholic fatty liver disease: a double-blind randomized controlled clinical trial. Phytotherapy Research 2021;35(1):477-85.

\section{Jameshorani 2017 \{published data only\}}

Jameshorani M, Rafiee E, Nayshaburi H, Kamali K. Treatment effects of probiotic in non alcoholic fatty liver disease. Journal of Zanjan University of Medical Sciences and Health Services 2017;25(109):23-35.

\section{Janczyk 2015 \{published data only\}}

Janczyk W, Lebensztejn D, Wierzbicka-Rucinska A, Mazur A, Neuhoff-Murawska J, Matusik P, et al. Omega-3 fatty acids therapy in children with nonalcoholic fatty liver disease: a randomized controlled trial. Journal of Pediatrics 2015;166(6):1358-63.e1-3.

\section{Javadi 2017 \{published data only\}}

Javadi L, Ghavami M, Khoshbaten M, Safaiyan A, Barzegari A, Gargari BP. The effect of probiotic and/or prebiotic on liver function tests in patients with nonalcoholic fatty liver disease: a double blind randomized clinical trial. Iranian Red Crescent Medical Journal 2017;19(4):e46017.

\section{Javanmardi 2018 \{published data only\}}

Javanmardi MA, Mohammad Shahi M, Seyedian SS, Haghighizadeh MH. Effects of phytosterol supplementation on serum levels of lipid profiles, liver enzymes, inflammatory markers, adiponectin, and leptin in patients affected by nonalcoholic fatty liver disease: a double-blind, placebocontrolled, randomized clinical trial. Journal of the American
College of Nutrition 2018 May 16 [Epub ahead of print]. [DOI: 10.1080/07315724.2018.1466739]

\section{Jazayeri-Tehrani 2019 \{published data only\}}

Jazayeri-Tehrani SA, Rezayat SM, Mansouri S, Qorbani M, Alavian SM, Daneshi-Maskooni M, et al. Efficacy of nanocurcumin supplementation on insulin resistance, lipids, inflammatory factors and nesfatin among obese patients with non-alcoholic fatty liver disease (NAFLD): a trial protocol. BMJ Open 2017;7(7):e016914.

* Jazayeri-Tehrani SA, Rezayat SM, Mansouri S, Qorbani M, Alavian SM, Daneshi-Maskooni M, et al. Nano-curcumin improves glucose indices, lipids, inflammation, and nesfatin in overweight and obese patients with non-alcoholic fatty liver disease (NAFLD): a double-blind randomized placebocontrolled clinical trial. Nutrition \& Metabolism 2019;16:8.

\section{Jeong 2017 \{published data only\}}

Jeong JY, Sohn JH, Baek YH, Cho YK, Kim Y, Kim H. New botanical drug, $\mathrm{hl}$ tablet, reduces hepatic fat as measured by magnetic resonance spectroscopy in patients with nonalcoholic fatty liver disease: a placebo-controlled, randomized, phase II trial. World Journal of Gastroenterology 2017;23(32):5977-85.

Kanoni 2021 \{published data only\}

* Kanoni S, Kumar S, Amerikanou C, Kurth MJ, Stathopoulou MG, Bourgeois S, et al. Nutrigenetic interactions might modulate the antioxidant and anti-inflammatory status in mastiha-supplemented patients with NAFLD. Frontiers in Immunology 2021;12:683028.

NCT03135873. Mastiha treatment for obese with NAFLD diagnosis. clinicaltrials.gov/ct2/show/NCT03135873 (first posted 1 May 2017).

\section{Kazemi 2020 \{published data only\}}

Kazemi S, Shidfar F, Ehsani S, Adibi P, Janani L, Eslami O. The effects of sumac (Rhus coriaria l) powder supplementation in patients with non-alcoholic fatty liver disease: a randomized controlled trial. Complementary Therapies in Clinical Practice 2020;41:101259.

\section{Khoshbaten 2010b \{published data only\}}

Khoshbaten M, Aliasgarzadeh A, Masnadi K, Tarzamani MK, Farhang S, Babaei $\mathrm{H}$, et al. $\mathrm{N}$-acetylcysteine improves liver function in patients with non-alcoholic fatty liver disease. Hepatitis Monthly 2010;10(1):12-6.

\section{Khutsishvili 2020 \{published data only\}}

Khutsishvili N. Prebiotics' plus probiotics' effect on the patients with nonalcoholic fatty liver disease. Hepatology International 2020;14:S329-30.

\section{Kobyliak 2017 \{published data only\}}

Kobyliak N, Abenavoli L, Falalyeyeva T, Mykhalchyshyn G, Boccuto L, Kononenko L, et al. Beneficial effects of probiotic combination with omega-3 fatty acids in NAFLD: a randomized clinical study. Minerva Medica 2018;13:13. 
Kobyliak 2018 \{published data only\}

* Kobyliak N, Abenavoli L, Mykhalchyshyn G, Kononenko L, Boccuto L, Kyriienko D, et al. A multi-strain probiotic reduces the fatty liver index, cytokines and aminotransferase levels in NAFLD patients: evidence from a randomized clinical trial. Journal of Gastrointestinal and Liver Diseases 2018;27(1):41-9.

Kobyliak N, Bosak N, Falalyeyeva T, Beregova T, Bodnar P. Effect of a probiotic on fatty liver index and liver stiffness in NAFLD patients: randomized clinical trial. Journal of Hepatology 2017;66(1 Suppl 1):S426-7.

\section{Kooshki 2020 \{published data only\}}

Kooshki F, Moradi F, Karimi A, Niazkar HR, Khoshbaten M, Maleki V, et al. Chromium picolinate balances the metabolic and clinical markers in nonalcoholic fatty liver disease: a randomized, double-blind, placebo-controlled trial. European Journal of Gastroenterology \& Hepatology 2020;14:14.

\section{Kugelmas 2003 \{published data only\}}

Kugelmas M, Hill DB, Vivian B, Marsano L, McClain CJ. Cytokines and NASH: a pilot study of the effects of lifestyle modification and vitamin E. Hepatology (Baltimore, Md) 2003;38(2):413-9.

\section{Lavine 2011 \{published data only\}}

Bell LN, Molleston JP, Lavine JE, Schwimmer JB, Murray KF, Rosenthal $P$, et al. Changes in adipose tissue insulin resistance correlate with changes in alt and lobular inflammation in children enrolled in the tonic trial. Hepatology (Baltimore, Md) 2011;54:711a.

* Lavine JE, Schwimmer JB, Van Natta ML, Molleston JP, Murray KF, Rosenthal P, et al. Effect of vitamin E or metformin for treatment of nonalcoholic fatty liver disease in children and adolescents: the tonic randomized controlled trial. JAMA 2011;305(16):1659-68.

\section{Lewis 2018 \{published data only\}}

Lewis JE, Atlas SE, Higuera OL, Fiallo A, Rasul A, Farooqi A, et al. Corrigendum to "The Effect of a Hydrolyzed Polysaccharide Dietary Supplement on Biomarkers in Adults with Nonalcoholic Fatty Liver Disease". Evidence-Based Complementary and Alternative Medicine 2020;2020:9575878.

* Lewis JE, Atlas SE, Higuera OL, Fiallo A, Rasul A, Farooqi A, et al. The effect of a hydrolyzed polysaccharide dietary supplement on biomarkers in adults with nonalcoholic fatty liver disease. Evidence-Based Complementary and Alternative Medicine 2018;2018:1751583.

\section{Li 2010 \{published data only\}}

Li L, Zhang XJ, Lan Y, Xu L, Zhang XZ, Wang HH. Treatment of non-alcoholic fatty liver disease by qianggan capsule ((sic)). Chinese Journal of Integrative Medicine 2010;16(1):23-7.

\section{Li 2016 \{published data only\}}

Li YH, Yang LH, Sha KH, Liu TG, Zhang LG, Liu XX. Efficacy of poly-unsaturated fatty acid therapy on patients with nonalcoholic steatohepatitis. World Journal of Gastroenterology 2016;22(22):7008-13.

\section{Loguercio 2012 \{published data only\}}

Loguercio C, Andreone P, Brisc C, Brisc MC, Bugianesi E, Chiaramonte $\mathrm{M}$, et al. Silybin combined with phosphatidylcholine and vitamin $\mathrm{E}$ in patients with nonalcoholic fatty liver disease: a randomized controlled trial. Free Radical Biology and Medicine 2012;52(9):1658-65.

\section{Magosso 2013 \{published data only\}}

Magosso E, Ansari MA, Gopalan Y, Shuaib IL, Wong JW, Khan NA, et al. Tocotrienols for normalisation of hepatic echogenic response in nonalcoholic fatty liver: a randomised placebocontrolled clinical trial. Nutrition Journal 2013;12(1):166.

\section{Malaguarnera 2010 \{published data only\}}

Malaguarnera M, Gargante MP, Russo C, Antic T, Vacante M, Malaguarnera M, et al. L-carnitine supplementation to diet: a new tool in treatment of nonalcoholic steatohepatitis - a randomized and controlled clinical trial. American Journal of Gastroenterology 2010;105(6):1338-45.

\section{Malaguarnera 2012 \{published data only\}}

Malaguarnera M, Vacante M, Antic T, Giordano M, Chisari G, Acquaviva R, et al. Bifidobacterium longum with fructooligosaccharides in patients with non alcoholic steatohepatitis. Digestive Diseases and Sciences 2012;57(2):545-53.

\section{Mansour 2020 \{published data only\}}

Mansour A, Mohajeri-Tehrani MR, Karimi S, Sanginabadi M, Poustchi $\mathrm{H}$, Enayati $\mathrm{S}$, et al. Short term effects of coffee components consumption on gut microbiota in patients with non-alcoholic fatty liver and diabetes: a pilot randomized placebo-controlled, clinical trial. Excli Journal 2020;19:241-50.

\section{Manzhalii 2017 \{published data only\}}

Manzhalii E, Virchenko O, Falalyeyeva T, Beregova T, Stremmel W. Treatment efficacy of a probiotic preparation for non-alcoholic steatohepatitis: a pilot trial. Journal of Digestive Diseases 2017;18(12):698-703.

\section{Martinez-Rodriguez 2014 \{published data only\}}

Martínez-Rodríguez Leonardo A, Rojas Serrano J, Torre A. Siliphos selenium methionine alpha lipoic acid for nonalcoholic fatty liver disease: results of a pilot study. Journal of Clinical \& Experimental Pharmacology 2014;5(1):1-5.

\section{Miglio 2000 \{published data only\}}

Miglio F, Rovati LC, Santoro A, Setnikar I. Efficacy and safety of oral betaine glucuronate in non-alcoholic steatohepatitis - a double-blind, randomized, parallel-group, placebo-controlled prospective clinical study. Arzneimittel-Forschung-Drug Research 2000;50(8):722-7.

\section{Mofidi 2017 \{published data only\}}

Mofidi F, Poustchi H, Yari Z, Nourinayyer B, Merat S, Sharafkhah M, et al. Synbiotic supplementation in lean patients with non-alcoholic fatty liver disease: a pilot, randomised, double-blind, placebo-controlled, clinical trial. British Journal of Nutrition 2017;117(5):662-8. 
Moradi 2020 \{published data only\}

* Moradi B, Rahmati-Ahmadabad S, Farzanegi P, Helalizadeh M, Azarbayjani MA. Effects of non-linear resistance training and curcumin supplementation on the liver biochemical markers levels and structure in older women with non-alcoholic fatty liver disease. Journal of Bodywork and Movement Therapies 2020;24(3):154-60

Moradi F, Kooshki F, Nokhostin F, Khoshbaten M, Bazyar H, Pourghassem Gargari B. A pilot study of the effects of chromium picolinate supplementation on serum fetuin-a, metabolic and inflammatory factors in patients with nonalcoholic fatty liver disease: a double-blind, placebo-controlled trial. Journal of Trace Elements in Medicine and Biology 2021;63:126659.

\section{Morvaridzadeh 2021 \{published data only\}}

Morvaridzadeh M, Nachvak SM, Mohammadi R, Moradi S, Mostafai R, Pizarro AB, et al. Probiotic yogurt fortified with vitamin $D$ can improve glycemic status in non-alcoholic fatty liver disease patients: a randomized clinical trial. Clinical Nutrition Research 2021;10(1):36-47.

\section{Nabavi 2016 \{published data only\}}

Nabavi S, Rafraf M, Somi MH, Homayouni-Rad A, AsghariJafarabadi M. Effects of probiotic yogurt consumption on metabolic factors in individuals with nonalcoholic fatty liver disease. Journal of Dairy Science 2014;97(12):7386-93.

* Nabavi S, Rafraf M, Somi MH, Homayouni-Rad A, AsghariJafarabadi M. Probiotic yogurt improves body mass index and fasting insulin levels without affecting serum leptin and adiponectin levels in non-alcoholic fatty liver disease (NAFLD). Journal of Functional Foods 2015;18:684-91.

Nabavi S, Rafraf M, Somi MH, Homayouni-Rad A, AsghariJafarabadi M. The effects of probiotic yogurt on metabolic factors in nonalcoholic fatty liver disease. Scientific Journal of Kurdistan University of Medical Sciences 2016;20(6):12-25.

Rafraf M, Nabavi S, Somi MH, Homayouni-Rad A, AsghariJafarabadi M. Effect of probiotic yogurt on enzymes and ecogenicity of liver in individuals with non alcoholic fatty liver disease: a double-blind randomized controlled clinical trial. Journal of Zanjan University of Medical Sciences and Health Services 2015;23(99):25-38.

Rafraf M, Nabavi S, Somi MH, Homayouni-Rad A, AsghariJafarabadi Rad M. The effect of probiotic and conventional yogurt consumption on anthropometric parameters in individuals with non-alcoholic fatty liver disease. Journal of Babol University of Medical Sciences 2014;16(9):55-62.

\section{Naganuma 2016 \{published data only\}}

Naganuma A, Ogawa Y, Hoshino T, Hatanaka T, Tanaka H, Inagawa M, et al. Effect of the medical walking and leucine enriched amino acid containing food for female nonalcoholic fatty liver disease: randomized controlled trial. Clinical Nutrition 2016;35:S62-3.

\section{Navekar 2017 \{published data only\}}

Navekar R, Rafraf M, Ghaffari A, Asghari-Jafarabadi M, Khoshbaten M. Turmeric supplementation improves serum glucose indices and leptin levels in patients with nonalcoholic fatty liver diseases. Journal of the American College of Nutrition 2017;36(4):261-7.

\section{NCT00816465 \{published data only\}}

NCT00816465. Safety and efficacy of Hoodia gordonii for treatment of non-alcoholic fatty liver disease (NAFLD). clinicaltrials.gov/ct2/show/NCT00816465 (first posted 1 January 2009).

\section{NCT00845845 \{published data only\}}

NCT00845845. Fish oil and diet for the treatment of nonalcoholic steatohepatitis (NASH). clinicaltrials.gov/ct2/show/ NCT00845845 (first posted 18 February 2009).

\section{NCT00941642 \{published data only\}}

NCT00941642. Placebo controlled study using lovaza as treatment for non-alcoholic fatty liver disease. clinicaltrials.gov/ ct2/show/NCT00941642 (first posted 17 July 2009).

NCT00977730 \{published data only\}

NCT00977730. The effect of protandim on non-alcoholic steatohepatitis. clinicaltrials.gov/ct2/show/NCT00977730 (first posted 16 September 2009).

\section{NCT01083992 \{published data only\}}

NCT01083992. Role of vitagliptin and vitamin D in the treatment of non-alcoholic fatty liver disease (NAFLD). clinicaltrials.gov/ ct2/show/NCT01083992 (first posted 10 March 2010).

NCT01623024 \{published data only\}

NCT01623024. Effect on liver histology of vitamin D in patients with non-alcoholic steatohepatitis. clinicaltrials.gov/ct2/show/ NCT01623024 (first posted 19 June 2012).

NCT02690792 \{published data only\}

NCT02690792. Effect of vitamin E on non-alcoholic fatty liver disease. clinicaltrials.gov/ct2/show/NCT02690792 (first posted 24 February 2016).

\section{NCT04411862 \{published data only\}}

NCT04411862. Efficacy of phosphatidylcholine in NAFLD. clinicaltrials.gov/show/NCT04411862 (first posted 2 June 2020).

\section{Nelson 2009 \{published data only\}}

Nelson JE, Maliken BD, Saunders C, Stevenson J, Richards T, Kowdley KV. A randomized, masked, controlled study of omega-3 polyunsaturated fatty acid vs monounsaturated fatty acid diet supplementation for the treatment of nonalcoholic fatty liver disease. Gastroenterology 2009;136(5):A847-8.

\section{Nobili 2006 \{published data only\}}

Mosca A, Crudele A, Smeriglio A, Braghini MR, Panera N, Comparcola D, et al. Antioxidant activity of hydroxytyrosol and vitamin $E$ reduces systemic inflammation in children with paediatric NAFLD. Digestive \& Liver Disease 2020;12:12.

Mosca A, Crudele A, Tozzi G, Alterio T, Smeriglio A, Trombetta D, et al. The anti-inflammatory effects of hydroxytyrosol and vitamin E on paediatric NAFLD. Digestive and Liver Disease 2020;52(Suppl 1):e42-e3. 
* Nobili V, Manco M, Devito R, Ciampalini P, Piemonte F, Marcellini M. Effect of vitamin E on aminotransferase levels and insulin resistance in children with non-alcoholic fatty liver disease. Alimentary Pharmacology \& Therapeutics 2006;24(11-12):1553-61.

Nobili V, Manco M, Devito R, Di Ciomm V, Comparcola D, Sartorelh MP, et al. Lifestyle intervention and antioxidant therapy in children with nonalcoholic fatty liver disease: a randomized, controlled trial. Hepatology (Baltimore, Md) 2008;48(1):119-28.

Nobili V, Manco M, DeVito R, PietroBattista A, Comparcola D, Sartorelli MR, et al. Lifestyle intervention and antioxidants in children with nonalcoholic fatty liver disease: a randomized, controlled trial. Gastroenterology 2008;134(4):A781.

\section{Nobili 2013 \{published data only\}}

* Nobili V, Alisi A, Della Corte C, Rise P, Galli C, Agostoni C, et al. Docosahexaenoic acid for the treatment of fatty liver: randomised controlled trial in children. Nutrition Metabolism and Cardiovascular Diseases 2013;23(11):1066-70.

Nobili V, Bedogni G, Alisi A, Pietrobattista A, Rise P, Galli C, et al. Docosahexaenoic acid supplementation decreases liver fat content in children with non-alcoholic fatty liver disease: double-blind randomised controlled clinical trial. Archives of Disease in Childhood 2011;96(4):350-3.

\section{Nogueira 2016 \{published data only\}}

Nogueira MA, Oliveira CP, Alves VAF, Stefano JT, Rodrigues LSD, Torrinhas RS, et al. Omega-3 polyunsaturated fatty acids in treating non-alcoholic steatohepatitis: a randomized, double-blind, placebo-controlled trial. Clinical Nutrition 2016;35(3):578-86

\section{Orang 2020 \{published data only\}}

Orang Z, Mohsenpour MA, Mozaffari-Khosravi H. Effect of omega-3 fatty acid supplementation on inflammatory markers and insulin resistance indices in patient with type 2 diabetes and nonalcoholic fatty liver: a randomized double-blind clinical trial. Obesity Medicine 2020;19:100278.

\section{Orr 2015 \{published data only\}}

Orr DW, Murphy R. Prebiotic supplementation with inulin following metronidazole therapy achieves greater alt reduction in non-alcoholic fatty liver disease (NAFLD): a randomised double-blind placebo controlled trial. Hepatology (Baltimore, Md) 2015;62:1268a-9a.

\section{Oscarsson 2018 \{published data only\}}

Oscarsson J, Onnerhag K, Riserus U, Sunden M, Johansson L, Jansson PA, et al. Effects of free omega-3 carboxylic acids and fenofibrate on liver fat content in patients with hypertriglyceridemia and non-alcoholic fatty liver disease: a double-blind, randomized, placebo-controlled study. Journal of Clinical Lipidology 2018;10:10.

\section{Pacifico 2015 \{published data only\}}

Pacifico L, Bonci E, Di Martino M, Versacci P, Andreoli G, Silvestri LM, et al. A double-blind, placebo-controlled randomized trial to evaluate the efficacy of docosahexaenoic acid supplementation on hepatic fat and associated cardiovascular risk factors in overweight children with nonalcoholic fatty liver disease. Nutrition Metabolism and Cardiovascular Diseases 2015;25(8):734-41.

\section{Palamaru 2017 \{published data only\}}

Palamaru AL, Dranga M, Ungureanu I, Cucos A, Prelipcean CC. Vitamin $E$ and non-alcoholic fatty liver disease. Journal of Gastrointestinal and Liver Diseases 2017;26(Suppl 3):28.

Panahi 2012 \{published data only\}

Panahi Y, Ghamarchehreh ME, Beiraghdar F, Zare R, Jalalian HR, Sahebkar A. Investigation of the effects of Chlorella vulgaris supplementation in patients with non-alcoholic fatty liver disease: a randomized clinical trial. Hepato-Gastroenterology 2012;59(119):2099-103.

\section{Panahi 2016 \{published data only\}}

* Panahi Y, Kianpour P, Mohtashami R, Jafari R, SimentalMendia LE, Sahebkar A. Curcumin lowers serum lipids and uric acid in subjects with nonalcoholic fatty liver disease: a randomized controlled trial. Journal of Cardiovascular Pharmacology 2016;68(3):223-9.

Panahi Y, Kianpour P, Mohtashami R, Jafari R, SimentalMendia LE, Sahebkar A. Efficacy and safety of phytosomal curcumin in non-alcoholic fatty liver disease: a randomized controlled trial. Drug Research 2017;67(4):244-51.

\section{Parsi 2020 \{published data only\}}

Parsi A, Torkashvand M, Hajiani E, Rahimlou M, Sadeghi N. The effects of Crocus sativus extract on serum lipid profile and liver enzymes in patients with non-alcoholic fatty liver disease: a randomized placebo-controlled study. Obesity Medicine 2020;17:100165.

\section{Pasdar 2020 \{published data only\}}

Pasdar Y, Oubari F, Zarif MN, Abbasi M, Pourmahmoudi A, Hosseinikia M. Effects of quercetin supplementation on hematological parameters in non-alcoholic fatty liver disease: a randomized, double-blind, placebo-controlled pilot study. Clinical Nutrition Research 2020;9(1):11-9.

\section{Pervez 2018 \{published data only\}}

Pervez MA, Khan DA, ljaz A, Khan S. Effects of delta-tocotrienol supplementation on liver enzymes, inflammation, oxidative stress and hepatic steatosis in patients with nonalcoholic fatty liver disease. Turkish Journal of Gastroenterology 2018;29(2):170-6.

\section{Pervez 2020 \{published data only\}}

Pervez MA, Khan DA, Slehria AUR, ljaz A. Delta-tocotrienol supplementation improves biochemical markers of hepatocellular injury and steatosis in patients with nonalcoholic fatty liver disease: a randomized, placebo-controlled trial. Complementary Therapies in Medicine 2020;52:102494.

Pezeshki 2016 \{published data only\}

Pezeshki A, Safi S, Feizi A, Askari G, Karami F. The effect of green tea extract supplementation on liver enzymes in patients 
with nonalcoholic fatty liver disease. International Journal of Preventive Medicine 2016;7:28.

\section{Poparn 2020 \{published data only\}}

Poparn H, Chatrproedprai S, Treeprasertsuk S, Sonsiri K, Chongsrisawat V. Effect of synbiotic supplementation in children with nonalcoholic fatty liver disease: a randomized controlled trial. Chotmaihet Thangphaet [Journal of the Medical Association of Thailand] 2020;103(12):S99-104.

Poulos 2021 \{published data only\}

Poulos JE, Kalogerinis PT, Milanov V, Kalogerinis CT, Poulos EJ. The effects of vitamin E, silymarin and carnitine on the metabolic abnormalities associated with nonalcoholic liver disease. Journal of Dietary Supplements 2021 Jan 25 [Online ahead of print]. [DOI: 10.1080/19390211.2021.1874587]

\section{Pour 2020 \{published data only\}}

Pour FK, Aryaeian N, Mokhtare M, Mirnasrollahi Parsa RS, Jannani L, Agah S, et al. The effect of saffron supplementation on some inflammatory and oxidative markers, leptin, adiponectin, and body composition in patients with nonalcoholic fatty liver disease: a double-blind randomized clinical trial. Phytotherapy Research 2020;34(12):3367-78.

\section{Qin 2015 \{published data only\}}

Qin Y, Zhou Y, Chen SH, Zhao XL, Ran L, Zeng XL, et al. Fish oil supplements lower serum lipids and glucose in correlation with a reduction in plasma fibroblast growth factor 21 and prostaglandin e2 in nonalcoholic fatty liver disease associated with hyperlipidemia: a randomized clinical trial. PLOS One 2015;10(7):e0133496.

\section{Rafie 2020 \{published data only\}}

Rafie R, Hosseini SA, Hajiani E, Malehi AS, Mard SA. Effect of ginger powder supplementation in patients with non-alcoholic fatty liver disease: a randomized clinical trial. Clinical and Experimental Gastroenterology 2020;13:35-45.

\section{Rahimlou 2016 \{published data only\}}

Rahimlou M, Yari Z, Hekmatdoost A, Alavian SM, Keshavarz SA. Ginger supplementation in nonalcoholic fatty liver disease: a randomized, double-blind, placebo-controlled pilot study. Hepatitis Monthly 2016;16(1):e34897.

\section{Rahmani 2016 \{published data only\}}

Rahmani S, Asgary S, Askari G, Keshvari M, Hatamipour M, Feizi A, et al. Treatment of non-alcoholic fatty liver disease with curcumin: a randomized placebo-controlled trial. Phytotherapy Research 2016;30(9):1540-8.

\section{Ruan 2010 \{published data only\}}

Ruan JW, Yang J. Efficacy of silibin capsules in the treatment of metabolism syndrome-related fatty liver disease: an analysis of 30 cases. World Chinese Journal of Digestology 2010;18(29):3151-4

\section{Sadrkabir 2020 \{published data only\}}

Sadrkabir M, Jahed S, Sadeghi Z, Isazadeh K. The effect of gerilact on non-alcoholic fatty liver disease. Journal of Kerman University of Medical Sciences 2020;27(1):82-90.
Sakpal 2017 \{published data only\}

Sakpal M, Mehta M, Duseja A, Bhadada S, Das A, Dhiman RK, et al. Vitamin D supplementation in patients with nonalcoholic fatty liver disease - a randomized controlled trial. Indian Journal of Gastroenterology 2015;34(1 Suppl 1):A106.

* Sakpal M, Satsangi S, Mehta M, Duseja A, Bhadada S, Das A, et al. Vitamin D supplementation in patients with nonalcoholic fatty liver disease: a randomized controlled trial. JGH Open 2017;1(2):62?7.

\section{Sangouni 2020 \{published data only\}}

* Sangouni AA, Azar MR, Alizadeh M. Effect of garlic powder supplementation on hepatic steatosis, liver enzymes and lipid profile in patients with non-alcoholic fatty liver disease: a double-blind randomised controlled clinical trial. British Journal of Nutrition 2020;124(4):450-6.

Sangouni AA, Mohammad Hosseini Azar MR, Alizadeh M. Effects of garlic powder supplementation on insulin resistance, oxidative stress, and body composition in patients with nonalcoholic fatty liver disease: a randomized controlled clinical trial. Complementary Therapies in Medicine 2020;51:102428.

\section{Sanyal 2010 \{published data only\}}

Bell LN, Wang J, Muralidharan S, Chalasani S, Fullenkamp AM, Wilson LA, et al. Relationship between adipose tissue insulin resistance and liver histology in nonalcoholic steatohepatitis: a pioglitazone versus vitamin E versus placebo for the treatment of nondiabetic patients with nonalcoholic steatohepatitis trial follow-up study. Hepatology (Baltimore, Md) 2012;56(4):1311-8.

Chalasani NP, Sanyal AJ, Kowdley KV, Robuck PR, Hoofnagle J, Kleiner DE, et al. Pioglitazone versus vitamin E versus placebo for the treatment of non-diabetic patients with non-alcoholic steatohepatitis: Pivens trial design. Contemporary Clinical Trials 2009;30(1):88-96.

Hoofnagle JH, Van Natta ML, Kleiner DE, Clark JM, Kowdley KV, Loomba R, et al. Vitamin $E$ and changes in serum alanine aminotransferase levels in patients with non-alcoholic steatohepatitis. Alimentary Pharmacology \& Therapeutics 2013;38(2):134-43.

* Sanyal AJ, Chalasani N, Kowdley KV, McCullough A, Diehl AM, Bass NM, et al. Pioglitazone, vitamin E, or placebo for nonalcoholic steatohepatitis. New England Journal of Medicine 2010;362(18):1675-85.

\section{Sanyal 2014 \{published data only\}}

Sanyal AJ, Abdelmalek MF, Suzuki A, Cummings OW, Chojkier M. No significant effects of ethyl-eicosapentanoic acid on histologic features of nonalcoholic steatohepatitis in a phase 2 trial. Gastroenterology 2014;147(2):377-84.e1.

\section{Saxena 2013 \{published data only\}}

Saxena A, Kaushik N, Vikram NK, Pandey RM, Misra A, Bhushan SM. Double-blind randomized placebo controlled clinical trial of herbal compound livomap (ma-579) in patients with non alcoholic fatty liver disease (NAFLD). Journal of Ayurveda and Integrative Medicine 2013;4:63. 


\section{Sayari 2018 \{published data only\}}

Sayari S, Neishaboori H, Jameshorani M. Combined effects of synbiotic and sitagliptin versus sitagliptin alone in patients with nonalcoholic fatty liver disease. Clinical and Molecular Hepatology 2018;24(3):331-8.

\section{Schattenberg 2017 \{published data only\}}

Schattenberg JM, Schlegel L, Nier A, Huber Y, Galle PR, Bergheim I. Dietary counselling focusing only on fructose consumption improves hepatic inflammation in patients with NAFLD-A prospective, controlled, randomized open-label study (Nuces NASH). Hepatology (Baltimore, Md) 2017;66(Suppl 1):1106a-7a.

\section{Scorletti 2014 \{published data only\}}

Bhatia LS, Scorletti E, Shojaee-Moradie F, Umpleby M, Clough GF, Curzen NP, et al. High-dose n-3 fatty acid treatment in non-alcoholic fatty liver disease is independently associated with reduced hepatic steatosis and improved hepatic insulin sensitivity. Diabetic Medicine 2014;31:53.

Scorletti E, Bhatia B, McCormick KG, Clough GF, Nash K, Hodson $L$, et al. Potential benefits of purified long chain omega-3 fatty acid treatment in non-alcoholic fatty liver disease (NAFLD): a potential treatment for early NAFLD in metabolic syndrome and type 2 diabetes? Results from the Welcome study. Diabetic Medicine 2014;31:1.

* Scorletti E, Bhatia L, McCormick KG, Clough GF, Nash K, Hodson L, et al. Effects of purified eicosapentaenoic and docosahexaenoic acids in nonalcoholic fatty liver disease: results from the Welcome study. Hepatology (Baltimore, Md) 2014;60(4):1211-21.

\section{Scorletti 2020 \{published data only\}}

* Scorletti E, Afolabi PR, Calder PC, Byrne CD. Investigation of the effects of synbiotic treatment on gut microbiota composition in patients with non-alcoholic fatty liver disease and type 2 diabetes. Diabetic Medicine 2020;37(Suppl 1):55.

Scorletti E, Afolabi PR, Miles EA, Smith DE, Almehmadi A, Alshathry $A$, et al. Design and rationale of the Insyte study: a randomised, placebo controlled study to test the efficacy of a synbiotic on liver fat, disease biomarkers and intestinal microbiota in non-alcoholic fatty liver disease. Contemporary Clinical Trials 2018;71:113-23.

Scorletti E, Afolabi PR, Miles EA, Smith DE, Almehmadi A, Alshathry A, et al. Synbiotics alter fecal microbiomes, but not liver fat or fibrosis, in a randomized trial of patients with nonalcoholic fatty liver disease. Gastroenterology 2020;158(6):1597-610.e7.

\section{Sepideh 2016 \{published data only\}}

Sepideh A, Karim P, Hossein A, Leila R, Hamdollah M, Mohammad EG, et al. Effects of multistrain probiotic supplementation on glycemic and inflammatory indices in patients with nonalcoholic fatty liver disease: a double-blind randomized clinical trial. Journal of the American College of Nutrition 2016;35(6):500-5.

\section{Shahmohammadi 2017 \{published data only\}}

Shahmohammadi HA, Hosseini SA, Hajiani E, Malehi AS, Alipour M. Effects of green coffee bean extract supplementation on patients with non-alcoholic fatty liver disease: a randomized clinical trial. Hepatitis Monthly 2017;17(4):e12299.

Sharifi 2014 \{published data only\}

Sharifi N, Amani R, Hajiani E, Cheraghian B. Does vitamin D improve liver enzymes, oxidative stress, and inflammatory biomarkers in adults with non-alcoholic fatty liver disease? A randomized clinical trial. Endocrine 2014;47(1):70-80.

Shavakhi 2013 \{published data only\}

Shavakhi A, Minakari M, Firouzian H, Assali R, Hekmatdoost A, Ferns $\mathrm{G}$. Effect of a probiotic and metformin on liver aminotransferases in non-alcoholic steatohepatitis: a double blind randomized clinical trial. International Journal of Preventive Medicine 2013;4(5):531-7.

Soleimani 2020 \{published data only\}

Soleimani D, Paknahad Z, Askari G, Iraj B, Feizi A. Effect of garlic powder consumption on body composition in patients with nonalcoholic fatty liver disease: a randomized, doubleblind, placebo-controlled trial. Advanced Biomedical Research 2016;5:2.

* Soleimani D, Paknahad Z, Rouhani MH. Therapeutic effects of garlic on hepatic steatosis in nonalcoholic fatty liver disease patients: a randomized clinical trial. Diabetes, Metabolic Syndrome and Obesity Targets and Therapy 2020;13:2389-97.

\section{Soleimani 2021 \{published data only\}}

Soleimani D, Rezaie M, Rajabzadeh F, Gholizadeh Navashenaq J, Abbaspour M, Miryan M, et al. Protective effects of propolis on hepatic steatosis and fibrosis among patients with nonalcoholic fatty liver disease (NAFLD) evaluated by real-time twodimensional shear wave elastography: a randomized clinical trial. Phytotherapy Research 2021;35(3):1669-79.

Solhi 2014 \{published data only\}

Solhi H, Ghahremani R, Kazemifar AM, Yazdi ZH. Silymarin in treatment of non-alcoholic steatohepatitis: a randomized clinical trial. Caspian Journal of Internal Medicine 2014;5(1):9-12.

\section{Somi 2014 \{published data only\}}

Somi MH, Fatahi E, Panahi J, Havasian MR, Judaki A. Data from a randomized and controlled trial of l-carnitine prescription for the treatment for non- alcoholic fatty liver disease. Bioinformation 2014;10(9):575-9.

\section{Song 2020 \{published data only\}}

Song LH, Zhao XG, Ouyang PL, Guan Q, Yang L, Peng FJ, et al. Combined effect of $\mathrm{n}-3$ fatty acids and phytosterol esters on alleviating hepatic steatosis in non-alcoholic fatty liver disease subjects: a double-blind placebo-controlled clinical trial. British Journal of Nutrition 2020;123(10):1148-58.

Spadaro 2008 \{published data only\}

Spadaro L, Magliocco O, Spampinato D, Piro S, Oliveri C, Alagona $\mathrm{C}$, et al. Effects of $\mathrm{n}-3$ polyunsaturated fatty acids in 
subjects with nonalcoholic fatty liver disease. Digestive and Liver Disease 2008;40(3):194-9.

\section{Tabatabaee 2017 \{published data only\}}

Tabatabaee SM, Alavian SM, Ghalichi L, Miryounesi SM, Mousavizadeh K, Jazayeri S, et al. Green tea in non-alcoholic fatty liver disease: a double blind randomized clinical trial. Hepatitis Monthly 2017;17(12):e14993.

\section{Taghvaei 2018 \{published data only\}}

Taghvaei T, Akha O, Mouodi M, Fakheri HT, Kashi Z, Maleki I, et al. Effects of vitamin D supplementation on patients with nonalcoholic fatty liver disease (NAFLD). Acta Medica Mediterranea 2018;34(2):415-22.

\section{Tan 2011 \{published data only\}}

Tan HH, Low ASC, Lim KH, Wan WK, Goh BB, Wang YT, et al. A randomized, unblinded pilot trial of essential phospholipids in Chinese subjects with nonalcoholic fatty liver disease (NASH). Journal of Gastroenterology and Hepatology 2011;26:162.

\section{Tobin 2018 \{published data only\}}

Tobin D, Brevik-Andersen M, Qin Y, Innes JK, Calder PC. Evaluation of a high concentrate omega- 3 for correcting the omega-3 fatty acid nutritional deficiency in non-alcoholic fatty liver disease (condin). Nutrients 2018;10(8):20.

\section{Tutunchi 2020 \{published data only\}}

* Tutunchi H, Naeini F, Saghafi-Asl M, Farrin N, Monshikarimi A, Ostadrahimi A. Effects of oleoylethanolamide supplementation on atherogenic indices and hematological parameters in patients with nonalcoholic fatty liver disease: a clinical trial. Health Promotion Perspectives 2020;10(4):373-82.

Tutunchi H, Ostadrahimi A, Saghafi-Asl M, HosseinzadehAttar MJ, Shakeri A, Asghari-Jafarabadi M, et al.

Oleoylethanolamide supplementation in obese patients newly diagnosed with non-alcoholic fatty liver disease: effects on metabolic parameters, anthropometric indices, and expression of ppar-alpha, ucp1, and ucp2 genes. Pharmacological Research 2020;156:104770.

Tutunchi H, Ostadrahimi A, Saghafi-Asl M, Roshanravan N, Shakeri-Bavil A, Asghari-Jafarabadi M, et al. Expression of NFkappaB, IL-6, and IL-10 genes, body composition, and hepatic fibrosis in obese patients with NAFLD-combined effects of oleoylethanolamide supplementation and calorie restriction: a triple-blind randomized controlled clinical trial. Journal of Cellular Physiology 2021;236(1):417-26.

\section{Uygun 2000 \{published data only\}}

Uygun A, Kadayifci A, Bagci S, Erdil A, Deveci S, Saka M, et al. L-carnitine therapy in non-alcoholic steatohepatitis. Turkish Journal of Gastroenterology 2000;11(3):196-201.

\section{Vajro 2011 \{published data only\}}

Vajro P, Mandato C, Licenziati MR, Franzese A, Vitale DF, Lenta $S$, et al. Effects of Lactobacillus rhamnosus strain GG in pediatric obesity-related liver disease. Journal of Pediatric Gastroenterology and Nutrition 2011;52(6):740-3.
Wang 2008 \{published data only\}

Wang CL, Liang L, Fu JF, Zou CC, Hong F, Xue JZ, et al. Effect of lifestyle intervention on non-alcoholic fatty liver disease in Chinese obese children. World Journal of Gastroenterology 2008;14(10):1598-602.

\section{Wang 2017 \{published data only\}}

Wang X, Yao N. Effect of herb formula xiao-zhi-hua-xian-tang against nonalcoholic steatohepatitis with advanced fibrosis: a preliminary clinical study. Hepatology International 2017;11(1 Suppl 1):S939-40.

\section{Wang 2018 \{published data only\}}

* Wang W, Shi LP, Shi L, Xu L. [Efficacy of probiotics on the treatment of non-alcoholic fatty liver disease]. Chinese Journal of Internal Medicine 2018;57(2):101-6.

Wang W. Clinical observation on the treatment of nonalcoholic fatty liver with probiotics. United European Gastroenterology Journal 2017;5(5 Suppl 1):A163-4.

\section{Wong 2013a \{published data only\}}

Wong VW, Won GL, Chim AM, Chu WC, Yeung DK, Li KC, et al. Treatment of nonalcoholic steatohepatitis with probiotics. A proof-of-concept study. Annals of Hepatology 2013;12(2):256-62.

\section{Wong 2013b \{published data only\}}

Wong VWS, Wong GLH, Chan AWH, Chu WCW, Choi PCL, Chim AML, et al. Treatment of non-alcoholic steatohepatitis with Phyllanthus urinaria: a randomized trial. Journal of Gastroenterology and Hepatology 2013;28(1):57-62.

Yan 2015 \{published data only\}

Yan H, Xia M, Chang X, Bian H, Lin H, Zhang L, et al. Berberine vs. pioglitazone for treatment of nonalcoholic fatty liver disease and its associated impaired glucose metabolism. Diabetes 2014;63:A513-4.

Yan H, Xia M, Chang X, Bian H, Xu Q, Gao X, et al. Pioglitazone versus berberine for treatment of non-alcoholic fatty liver disease patients with impaired glucose regulation or type 2 diabetes mellitus. Diabetologia 2012;55:S36.

Yan H, Xia M, Chang X, Bian H, Zhang L, Lin H, et al. Berberine increases serum osteocalcin levels and improves early phase of insulin secretion in non-alcoholic fatty liver disease patients with impaired glucose metabolism. Diabetologia 2014;57(1 Suppl 1):S351.

* Yan HM, Xia MF, Wang Y, Chang XX, Yao XZ, Rao SX, et al. Efficacy of berberine in patients with non-alcoholic fatty liver disease. PLOS One 2015;10(8):e0134172.

\section{Yari 2016 \{published data only\}}

Yari Z, Rahimlou M, Eslamparast T, Ebrahimi-Daryani N, Poustchi H, Hekmatdoost A. Flaxseed supplementation in nonalcoholic fatty liver disease: a pilot randomized, open labeled, controlled study. International Journal of Food Sciences and Nutrition 2016;67(4):461-9. 
Yari 2020 \{published data only\}

Yari Z, Cheraghpour M, Alavian SM, Hedayati M, Eini-Zinab H, Hekmatdoost A. The efficacy of flaxseed and hesperidin on non-alcoholic fatty liver disease: an open-labeled randomized controlled trial. European Journal of Clinical Nutrition 2021;75(1):99-111.

\section{Youshari 2017 \{published data only\}}

Youshari N, Mehrangiz, Mameghani E. The effect of oral $\beta$-glucan supplement on liver echogenicity and enzymes in non-alcoholic fatty liver disease treating with hypocaloric diet and vitamin $\mathrm{E}$. Iranian Journal of Basic Medical Sciences 2017;20(Supp 1):82-3.

\section{Zamani 2018 \{published data only\}}

Zamani N, Shams M, Nimrouzi M, Zarshenas MM, Abolhasani Foroughi A, Fallahzadeh Abarghooei E, et al. The effects of Zataria multiflora boiss (Shirazi thyme) on nonalcoholic fatty liver disease and insulin resistance: a randomized double-blind placebo-controlled clinical trial. Complementary Therapies in Medicine 2018;41:118-23.

\section{Zanko 2020 \{published data only\}}

Mikolasevic I, Domislovic V, Trkulja V, Krznaric, Skenderevic N, Mijic $A$, et al. The efficacy of vitamin $D$ supplementation on NAFLD: a randomized, double-blind, placebo-controlled 12- month trial on 311 patients. Journal of Hepatology 2020;73(Suppl 1):S447-8.

* Zanko VL, Domislovic V, Trkulja V, Krznaric-Zrnic I, TurkWensveen T, Krznaric Z, et al. Vitamin D for treatment of nonalcoholic fatty liver disease detected by transient elastography: a randomized, double-blind, placebo-controlled trial. Diabetes Obesity \& Metabolism 2020;22(11):2097-106.

\section{Zhang 2015 \{published data only\}}

Zhang PW, Chen FX, Li D, Ling WH, Guo HH. A CONSORTcompliant, randomized, double-blind, placebo-controlled pilot trial of purified anthrocyanin in patients with nonalcoholic fatty liver disease. Medicine 2015;94(20):e758.

\section{Zhu 2008 \{published data only\}}

Zhu FS, Liu S, Chen XM, Huang ZG, Zhang DW. Effects of n-3 polyunsaturated fatty acids from seal oils on nonalcoholic fatty liver disease associated with hyperlipidemia. World Journal of Gastroenterology 2008;14(41):6395-400.

\section{Zohrer 2017 \{published data only\}}

Zohrer E, Alisi A, Jahnel J, Mosca A, Della Corte C, Crudele A, et al. Efficacy of docosahexaenoic acid-choline-vitamin $\mathrm{E}$ in paediatric NASH: a randomized controlled clinical trial. Applied Physiology, Nutrition, and Metabolism 2017;42(9):948-54.

\section{References to studies excluded from this review}

\section{Abenavoli 2017 \{published data only\}}

* Abenavoli L, Greco M, Milic N, Accattato F, Foti D, Gulletta E, et al. Effect of Mediterranean diet and antioxidant formulation in non-alcoholic fatty liver disease: a randomized study. Nutrients 2017;9(8):12
Abenavoli L, Milic N, Larussa T, Suraci E, Imeneo M, Medic M, et al. Efficacy of Mediterranean diet and antioxidants in overweight patients with non-alcoholic fatty liver disease. Digestive and Liver Disease 2016;48:e130-1.

\section{Akcam 2011 \{published data only\}}

Akcam M, Boyaci A, Pirgon O, Kaya S, Uysal S, Dundar BN. Therapeutic effect of metformin and vitamin $E$ versus prescriptive diet in obese adolescents with fatty liver. International Journal for Vitamin and Nutrition Research 2011;81(6):398-406.

Basu 2014 \{published data only\}

* Basu P, Shah NJ, Aloysius M. Berberine with alfa lipoic acid (ALA) in non alcoholic steato-hepatitis (NASH). A randomized double blinded placebo control trial. A clinical pilot - the Banish Trial. Hepatology (Baltimore, Md) 2014;60:624a.

Basu P, Shah NJ, Aloysius MM. Berberine with alfa lipoic acid (ALA) in non alcoholic steatohepatitis (NASH). A randomized double blinded placebo control trial. A clinical pilot - the Banish Trial. Journal of the International Hepato Pancreato Biliary Association 2015;17:43.

Basu PP, Aloysius MM, Shah NJ, Brown RS Jr. Berberine with alfa lipoic acid (ALA) in non alcoholic steatohepatitis (NASH). A randomized double blinded placebo control trial. A clinical pilot: the Banish Trial. Hepatology International 2014;8(1 Suppl 1):S10.

Chambers 2018 \{published data only\}

Chambers ES, Byrne CS, Rugyendo A, Morrison DJ, Preston T, Tedford C, et al. The effects of dietary supplementation with inulin and inulin-propionate ester on hepatic steatosis in adults with non-alcoholic fatty liver disease. Diabetes, Obesity and Metabolism 2018;11:11.

\section{Chang 2014 \{published data only\}}

Chang HC, Peng CH, Yeh DM, Kao ES, Wang CJ. Hibiscus sabdariffa extract inhibits obesity and fat accumulation, and improves liver steatosis in humans. Food and Function 2014;5(4):734-9.

\section{Dela Cruz 2012 \{published data only\}}

Dela Cruz R, Mappala HT. The efficacy of ursodeoxycholic acid, probiotics vs. diet and exercise in the treatment of NAFLD: an open-labelled prospective randomized trial. Journal of Gastroenterology and Hepatology 2012;27:223.

\section{Ersoz 2005 \{published data only\}}

Ersoz G, Gunsar F, Karasu Z, Akay S, Batur Y, Akarca US. Management of fatty liver disease with vitamin $E$ and $C$ compared to ursodeoxycholic acid treatment. Turkish Journal of Gastroenterology 2005;16(3):124-8.

\section{Famouri 2017b \{published data only\}}

Famouri F, Salehi MM, Rostampour N, Hashemi E, Shahsanaee A. The effect of silymarin on non-alcoholic fatty liver disease of children. Journal of Herbmed Pharmacology 2017;6(1):16-20. 
Guo 2014 \{published data only\}

Guo H, Zhong R, Liu Y, Jiang X, Tang X, Li Z, et al. Effects of bayberry juice on inflammatory and apoptotic markers in young adults with features of non-alcoholic fatty liver disease. Nutrition 2014;30(2):198-203.

\section{Hajiaghamohammadi 2012 \{published data only\}}

Hajiaghamohammadi AA, Ziaee A, Oveisi S, Masroor H. Effects of metformin, pioglitazone, and silymarin treatment on nonalcoholic fatty liver disease: a randomized controlled pilot study. Hepatitis Monthly 2012;12(8):e6099.

Han 2014 \{published data only\}

Han Y, Shi JP, Ma AL, Xu Y, Ding XD, Fan JG. Randomized, vitamin E-controlled trial of bicyclol plus metformin in non-alcoholic fatty liver disease patients with impaired fasting glucose. Clinical Drug Investigation 2014;34(1):1-7.

\section{Khoshbaten 2010a \{published data only\}}

Khoshbaten M, Aliasgarzadeh A, Masnadi K, Farhang S, Tarzamani MK, Babaei H, et al. Grape seed extract to improve liver function in patients with nonalcoholic fatty liver change. Saudi Journal of Gastroenterology 2010;16(3):194-7.

\section{Mahmoudi 2020 \{published data only\}}

Mahmoudi L, Asadi S, Al-Mousavi Z, Niknam R. A randomized controlled clinical trial comparing calcitriol versus cholecalciferol supplementation to reduce insulin resistance in patients with non-alcoholic fatty liver disease. Clinical Nutrition 2020 Dec 8 [Online ahead of print]. [10.1016/j.clnu.2020.11.037]

\section{NCT00820651 \{published data only\}}

NCT00820651. Efficacy and safety of diamel in patients with nonalcoholic steatohepatitis. clinicaltrials.gov/ct2/show/ NCT00820651 (first posted 12 January 2009).

NCT04281121 \{published data only\}

NCT04281121. Omega 3 supplementation in children with non alcoholic fatty liver. clinicaltrials.gov/show/NCT04281121 (first posted 24 February 2020).

\section{Petyaev 2018 \{published data only\}}

Petyaev IM, Dovgalevsky PY, Chalyk NE, Klochkov VA, Kyle NH, Bashmakov YK. Reduction of liver span and parameters of inflammation in nonalcoholic fatty liver disease patients treated with lycosome formulation of phosphatidylcholine: a preliminary report. International Journal of Chronic Diseases Print 2018;2018:4549614.

\section{Podszun 2020 \{published data only\}}

Podszun MC, Alawad AS, Lingala S, Morris N, Huang WA, Yang S, et al. Vitamin E treatment in NAFLD patients demonstrates that oxidative stress drives steatosis through upregulation of denovo lipogenesis. Redox Biology 2020;37:101710.

\section{Saarinen 2011 \{published data only\}}

Saarinen M, Kotronen A, Yamaki G, Matsuzaki S, Mitsuya N, Tiihonen K. Effects of low dose betaine in subjects with mild fatty liver. Annals of Nutrition and Metabolism 2011;58:112.

\section{Semiserin 2016 \{published data only\}}

Semiserin VA, Karakozov AG, Malkuta MA, Zolotareva LA Levchenko OB, Kalyagin IE, et al. [Evaluation of the efficiency of hepatoprotective monotherapy using succinic acid and methionine for nonalcoholic fatty liver disease at the stage of steatohepatitis]. Terapevticheskii Arkhiv 2016;88(2):58-63.

Singhal 2015 \{published data only\}

Singhal P, Nesari T, Gupta GS. Efficacy of herbomineral compounds and pathya (ayurvedic dietary regime and physical exercise) in the management of yakrt roga (non-alcoholic fatty liver disease). Ancient Science of Life 2015;34(4):216-22.

\section{Zhang 2008 \{published data only\}}

Zhang SJ, Chen ZX, Jiang KP, Cheng YH, Gu YL. The effect of quyuhuatantongluo decoction on the non-alcoholic steatohepatitis. Complementary Therapies in Medicine 2008;16(4):192-8.

\section{References to ongoing studies}

ChiCTR2000034740 \{published data only\}

ChiCTR2000034740. To explore the protective effect of Lycium barbarum polysaccharide combined with exercise intervention on nonalcoholic fatty liver disease based on gut microbiota: a randomized controlled trial. www.chictr.org.cn/ hvshowproject.aspx?id=43624 (date of approval by ethics committee 25 July 2019).

\section{ChicTR2000035899 \{published data only\}}

ChiCTR2000035899. Efficacy and safety of caffeic acid in nonalcoholic fatty liver disease. www.chictr.org.cn/ hvshowproject.aspx?id=49625 (date of approval by ethics committee 14 August 2020).

\section{CTRI/2020/05/025322 \{published data only\}}

CTRI/2020/05/025322. Effect of herbal blend in non-alcoholic subjects with elevated fatty liver index. ctri.nic.in/Clinicaltrials/pmaindet2.php? trialid $=41930 \&$ EncHid=\&userName $=$ CTRI/2020/05/025322 (first registered 22 May 2020)

\section{CTRI/2020/07/026362 \{published data only\}}

CTRI/2020/07/026362. To evaluate the efficacy of sarvakalpa-ghan-vati in management of non-alcoholic liver disorder. ctri.nic.in/Clinicaltrials/pmaindet2.php? trialid=44069\&EncHid=\&userName=2020/07/026362 (first registered 4 July 2020).

Han 2020 \{published data only\}

Han K, Kwon O, Park HJ, Jung SY, Yang C, Son CG. Effect of daesiho-tang on obesity with non-alcoholic fatty liver disease: a study protocol for a randomised, double-blind, placebocontrolled pilot trial. Trials 2020;21(1):128.

IRCT20131125015536N \{published data only\} IRCT20131125015536N. Effects of naringenin supplement on non-alcoholic fatty liver disease. www.irct.ir/trial/49713 (first registered 6 August 2020). 
IRCT20191009045043N \{published data only\}

IRCT20191009045043N. Investigation the effect of the sumac powder consumption on patients with non-alcoholic fatty liver. www.irct.ir/trial/43090 (first registered 20 January 2020).

\section{IRCT20200304046692N \{published data only\}}

IRCT20200304046692N. Evaluating the effect of spirulina in non-alcoholic fatty liver patient. www.irct.ir/trial/46330 (first registered 11 March 2020).

\section{IRCT20200531047614N \{published data only\}}

IRCT20200531047614N. Zinc supplementation in treatment of obese children and adolescents with nonalcoholic fatty liver disease. www.irct.ir/trial/48543 (first registered 19 June 2020).

\section{Lambert 2015 \{published data only\}}

Lambert JE, Parnell JA, Eksteen B, Raman M, Bomhof MR, Rioux KP, et al. Gut microbiota manipulation with prebiotics in patients with non-alcoholic fatty liver disease: a randomized controlled trial protocol. BMC Gastroenterology 2015;15:169.

\section{NCT02289235 \{published data only\}}

NCT02289235. The effects of ginger on nonalcoholic fatty liver disease. clinicaltrials.gov/ct2/show/NCT02289235 (first posted 13 November 2014).

\section{NCT02642172 \{published data only\}}

NCT02642172. Prebiotics in patients with non-alcoholic liver disease. clinicaltrials.gov/ct2/show/NCT02642172 (first posted 30 December 2015)

\section{NCT02647294 \{published data only\}}

NCT02647294. Polyunsaturated fatty acids in patients with NAFLD. clinicaltrials.gov/ct2/show/NCT02647294 (first posted 6 January 2016)

\section{NCT02764047 \{published data only\}}

NCT02764047. Probiotics in the treatment of NAFLD. clinicaltrials.gov/ct2/show/NCT02764047 (first posted 6 May 2016)

\section{NCT03439917 \{published data only\}}

NCT03439917. Effects of carnitine supplementation on liver and muscle. clinicaltrials.gov/ct2/show/NCT03439917 (first posted 20 February 2018).

\section{NCT03467282 \{published data only\}}

NCT03467282. Probiotics in NASH Patients - PROBILIVER TRIAL (NASH). clinicaltrials.gov/ct2/show/NCT03467282 (first posted 15 March 2018).

\section{NCT04175392 \{published data only\}}

NCT04175392. Effect of probiotics in non-alcoholic fatty liver disease and steatohepatitis. clinicaltrials.gov/ct2/show/ NCT04175392 (first posted 25 November 2019).

\section{NCT04193982 \{published data only\}}

NCT04193982. An investigator initiated prospective, four arms randomized comparative study of efficacy and safety of saroglitazar, vitamin $\mathrm{E}$ and life style modification in patients with nonalcoholic fatty liver disease (NAFLD)/ non- alcoholic steatohepatitis (NASH). clinicaltrials.gov/ct2/show/ NCT04193982 (first posted 11 December 2019).

NCT04198805 \{published data only\}

NCT04198805. Vitamin E and DHA-EE on NAFLD - randomized, double-blind, placebo-controlled, parallel-group clinical trial (PUVENAFLD). clinicaltrials.gov/ct2/show/NCT04198805 (first posted 13 December 2019).

NCT04330326 \{published data only\}

NCT04330326. Metabolic cofactor supplementation in obese patients with non-alcoholic fatty liver disease. clinicaltrials.gov/ show/NCT04330326 (first posted 1 April 2020).

NCT04475276 \{published data only\}

NCT04475276. Effect of alpha lipoic acid on non-alcoholic fatty liver diseases. clinicaltrials.gov/show/NCT04475276 (first posted 17 July 2020).

\section{NCT04555434 \{published data only\}}

None of the outcomes of interest for this review will be reported in this trial. Effects of probiotics (P pentosaceus, I lactis or I helveticus) in NASH. clinicaltrials.gov/show/NCT04555434 (first posted 18 September 2020).

NCT04671186 \{published data only\}

NCT04671186. Role of probiotics in treatment of pediatric NAFLD patients by assessing with fibroscan. clinicaltrials.gov/ show/NCT04671186 (first posted 17 December 2020).

\section{NCT04704063 \{published data only\}}

NCT04704063. A randomized, double-blind, placebo-controlled study to assess the efficacy and safety of Tocovid Suprabio 200mg in non-alcoholic fatty liver (NAFL). clinicaltrials.gov/ct2/ show/NCT04704063 (first posted 11 January 2021).

\section{NCT04718051 \{published data only\}}

NCT04718051. S.P.Pro liver powder is used to improve liver and metabolic indexes in people with non-alcoholic fatty liver disease. clinicaltrials.gov/ct2/show/NCT04718051 (first posted 22 January 2021).

\section{Wicklow 2015 \{published data only\}}

Wicklow B, Wittmeier K, Gw TJ, McGavock J, Robert M, Duhamel T, et al. Proposed trial: safety and efficacy of resveratrol for the treatment of non-alcoholic fatty liver disease (NAFLD) and associated insulin resistance in adolescents who are overweight or obese adolescents - rationale and protocol. Biochemistry and Cell Biology 2015;93(5):522-30.

\section{Zang 2018 \{published data only\}}

Zang S, Chen J, Song Y, Bai L, Chen J, Chi X, et al. Haptoglobin genotype and vitamin $\mathrm{E}$ versus placebo for the treatment of nondiabetic patients with nonalcoholic steatohepatitis in China: a multicenter, randomized, placebo-controlled trial design. Advances in Therapy 2018;35(2):218-31. 


\section{Additional references}

\section{Abdelmalek 2007}

Abdelmalek MF, Diehl AM. Nonalcoholic fatty liver disease as a complication of insulin resistance. Medical Clinics of North America 2007;91(6):1125-49, ix.

\section{Abenavoli 2013}

Abenavoli L, Bellentani S. Milk thistle to treat non-alcoholic fatty liver disease: dream or reality? Expert Review of Gastroenterology \& Hepatology 2013;7(8):677-9.

\section{Abenavoli 2015}

Abenavoli L, Greco M, Nazionale I, Peta V, Milic N, Accattato F, et al. Effects of Mediterranean diet supplemented with silybinvitamin E-phospholipid complex in overweight patients with non-alcoholic fatty liver disease. Expert Review of Gastroenterology \& Hepatology 2015;9(4):519-27.

\section{Adams 2005}

Adams LA, Lymp JF, St Sauver J, Sanderson SO, Lindor KD, Feldstein $\mathrm{A}$, et al. The natural history of nonalcoholic fatty liver disease: a population-based cohort study. Gastroenterology 2005;129(1):113-21.

\section{Adorini 2012}

Adorini L, Pruzanski M, Shapiro D. Farnesoid X receptor targeting to treat nonalcoholic steatohepatitis. Drug Discovery Today 2012;17(17-18):988-97.

\section{Alberti 2009}

Alberti KG, Eckel RH, Grundy SM, Zimmet PZ, Cleeman JI, Donato KA, et al. Harmonizing the metabolic syndrome: a joint interim statement of the International Diabetes Federation Task Force on Epidemiology and Prevention; National Heart, Lung, and Blood Institute; American Heart Association; World Heart Federation; International Atherosclerosis Society; and International Association for the Study of Obesity. Circulation 2009;120(16):1640-5.

\section{Al-Muzafar 2017}

Al-Muzafar HM, Amin KA. Probiotic mixture improves fatty liver disease by virtue of its action on lipid profiles, leptin, and inflammatory biomarkers. BMC Complementary and Alternative Medicine 2017;17(1):43.

\section{Angulo 2002}

Angulo P. Nonalcoholic fatty liver disease. New England Journal of Medicine 2002;346(16):1221-31.

\section{Angulo 2015}

Angulo P, Kleiner DE, Dam-Larsen S, Adams LA, Bjornsson ES, Charatcharoenwitthaya $P$, et al. Liver fibrosis, but no other histologic features, is associated with long-term outcomes of patients with nonalcoholic fatty liver disease. Gastroenterology 2015;149(2):389-97.e10.

\section{Anstee 2012}

Anstee QM, Day CP. S-adenosylmethionine (SAMe) therapy in liver disease: a review of current evidence and clinical utility. Journal of Hepatology 2012;57(5):1097-109.

\section{Ballestri 2016}

Ballestri S, Zona S, Targher G, Romagnoli D, Baldelli E, Nascimbeni F, et al. Nonalcoholic fatty liver disease is associated with an almost twofold increased risk of incident type 2 diabetes and metabolic syndrome. Evidence from a systematic review and meta-analysis. Journal of Gastroenterology and Hepatology 2016;31(5):936-44.

\section{Barritt 2017}

Barritt AS 4th, Gitlin N, Klein S, Lok AS, Loomba R, Malahias L, et al. Design and rationale for a real-world observational cohort of patients with nonalcoholic fatty liver disease: the TARGET-NASH study. Contemporary Clinical Trials 2017;61:33-8.

\section{Bedogni 2005}

Bedogni G, Miglioli L, Masutti F, Tiribelli C, Marchesini G, Bellentani S. Prevalence of and risk factors for nonalcoholic fatty liver disease: the Dionysos Nutrition and Liver Study. Hepatology (Baltimore, Md) 2005;42(1):44-52.

\section{Bedogni 2007}

Bedogni G, Miglioli L, Masutti F, Castiglione A, Croce LS, Tiribelli $C$, et al. Incidence and natural course of fatty liver in the general population: the Dionysos Study. Hepatology (Baltimore, Md) 2007;46(5):1387-91.

\section{Bernsmeier 2015}

Bernsmeier C, Weisskopf DM, Pflueger MO, Mosimann J, Campana B, Terracciano L, et al. Sleep disruption and daytime sleepiness correlating with disease severity and insulin resistance in non-alcoholic fatty liver disease: a comparison with healthy controls. PLOS One 2015;10(11):e0143293.

\section{Best 2018}

Best LMJ, Freeman S, Sutton AJ, Hawkins N, Tsochatzis E, Gurusamy KS. Treatment for hepatorenal syndrome in people with decompensated liver cirrhosis: a network meta-analysis. Cochrane Database of Systematic Reviews 2018, Issue 9. Art. No: CD013103. [DOI: 10.1002/14651858.CD013103]

\section{Buzzetti 2016}

Buzzetti E, Pinzani M, Tsochatzis EA. The multiple-hit pathogenesis of non-alcoholic fatty liver disease (NAFLD). Metabolism: Clinical and Experimental 2016;65(8):1038-48.

\section{Buzzetti 2021}

Buzzetti E, Linden A, Best LMJ, Madden AM, Roberts D, Chase TJ G, et al. Lifestyle modifications for nonalcohol-related fatty liver disease: a network meta-analysis. Cochrane Database of Systematic Reviews 2021, Issue 3. Art. No: CD013156. [DOI: 10.1002/14651858.CD013156.pub2]

\section{Cerovic 2013}

Cerovic I, Mladenovic D, Jesic R, Naumovic T, Brankovic M, Vucevic D, et al. Alcoholic liver disease/nonalcoholic fatty liver disease index: distinguishing alcoholic from nonalcoholic fatty liver disease. European Journal of Gastroenterology \& Hepatology 2013;25(8):899-904. 


\section{Chaimani 2012}

Chaimani A, Salanti G. Using network meta-analysis to evaluate the existence of small-study effects in a network of interventions. Research Synthesis Methods 2012;3(2):161-76.

\section{Chaimani 2013}

Chaimani A, Higgins JP, Mavridis D, Spyridonos P, Salanti G. Graphical tools for network meta-analysis in STATA. PLOS One 2013;8(10):e76654.

\section{Chalasani 2012}

Chalasani N, Younossi Z, Lavine JE, Diehl AM, Brunt EM, Cusi K, et al. The diagnosis and management of non-alcoholic fatty liver disease: practice guideline by the American Association for the Study of Liver Diseases, American College of Gastroenterology, and the American Gastroenterological Association. Hepatology (Baltimore, Md) 2012;55(6):2005-23.

\section{Chan 2013}

Chan AW, Tetzlaff JM, Altman DG, Laupacis A, Gøtzsche PC, Krleža-Jerić K, et al. SPIRIT 2013 statement: defining standard protocol items for clinical trials. Annals of Internal Medicine 2013;158(3):200-7.

\section{Cholankeril 2017}

Cholankeril G, Wong RJ, Hu M, Perumpail RB, Yoo ER, Puri P, et al. Liver transplantation for nonalcoholic steatohepatitis in the US: temporal trends and outcomes. Digestive Diseases and Sciences 2017;62(10):2915-22.

\section{Clearfield 2021}

Clearfield E, Miller V, Nadglowski J, Barradas K, Al Naber J, Sanyal AJ, et al. coreNASH: multi-stakeholder consensus on core outcomes for decision making about nonalcoholic steatohepatitis treatment. Hepatology Communications 2021;5(5):774-85.

\section{Cowie 2017}

Cowie MR, Blomster JI, Curtis LH, Duclaux S, Ford I, Fritz F, et al. Electronic health records to facilitate clinical research. Clinical Research in Cardiology 2017;106(1):1-9.

\section{Dam-Larsen 2005}

Dam-Larsen S, Franzmann MB, Christoffersen P, Larsen K, Becker U, Bendtsen F. Histological characteristics and prognosis in patients with fatty liver. Scandinavian Journal of Gastroenterology 2005;40(4):460-7.

\section{Dassanayake 2009}

Dassanayake AS, Kasturiratne A, Rajindrajith S, Kalubowila U, Chakrawarthi S, De Silva AP, et al. Prevalence and risk factors for non-alcoholic fatty liver disease among adults in an urban Sri Lankan population. Journal of Gastroenterology and Hepatology 2009;24(7):1284-8

\section{Del Giovane 2013}

Del Giovane C, Vacchi L, Mavridis D, Filippini G, Salanti G. Network meta-analysis models to account for variability in treatment definitions: application to dose effects. Statistics in Medicine 2013;32(1):25-39.

\section{Dias 2010}

Dias S, Welton NJ, Caldwell DM, Ades AE. Checking consistency in mixed treatment comparison meta-analysis. Statistics in Medicine 2010;29(7-8):932-44.

\section{Dias 2012a}

Dias S, Sutton AJ, Welton NJ, Ades AE. NICE DSU technical support document 3: heterogeneity: subgroups, metaregression, bias and bias-adjustment, September 2011 (last updated April 2012). nicedsu.org.uk/wp-content/ uploads/2016/03/TSD3-Heterogeneity.final-report.08.05.12.pdf (accessed 26 February 2021).

\section{Dias 2012b}

Dias S, Welton NJ, Sutton AJ, Ades AE. NICE DSU technical support document 1: introduction to evidence synthesis for decision making, April 2011 (last updated April 2012). silo.tips/download/nice-dsu-technical-support-document-1introduction-to-evidence-synthesis-for-dec (accessed 26 February 2021).

\section{Dias 2014}

Dias S, Welton NJ, Sutton AJ, Caldwell DM, Lu G, Ades AE. NICE DSU technical support document 4: inconsistency in networks of evidence based on randomised controlled trials, May 2011 (last updated April 2014). nicedsu.org.uk/wp-content/ uploads/2016/03/TSD4-Inconsistency.final_.15April2014.pdf (accessed 26 February 2021).

\section{Dias 2016}

Dias S, Welton NJ, Sutton AJ, Ades AE. NICE DSU technical support document 2: a generalised linear modelling framework for pairwise and network meta-analysis of randomised controlled trials, August 2011 (last updated September 2016). www.ncbi.nlm.nih.gov/pubmedhealth/PMH0088912/pdf/ PubMedHealth_PMH0088912.pdf (accessed 26 February 2021).

\section{Edgeworth 1887}

Edgeworth FY. On observations relating to several quantities. Hermathena 1887;6(13):285-79.

\section{Ekstedt 2015}

Ekstedt M, Hagstrom H, Nasr P, Fredrikson M, Stal P, Kechagias $\mathrm{S}$, et al. Fibrosis stage is the strongest predictor for disease-specific mortality in NAFLD after up to 33 years of follow-up. Hepatology (Baltimore, Md) 2015;61(5):1547-54.

\section{Eslam 2019}

Eslam M, Sanyal AJ, George J. Toward more accurate nomenclature for fatty liver diseases. Gastroenterology 2019;157(3):590-3.

\section{EuroQol 2018}

EuroQol. EQ-5D Instruments | About EQ-5D, 2018. euroqol.org/ eq-5d-instruments/ (accessed 26 February 2021).

\section{Fleischman 2014}

Fleischman MW, Budoff M, Zeb I, Li D, Foster T. NAFLD prevalence differs among Hispanic subgroups: the multi-ethnic study of atherosclerosis. World Journal of Gastroenterology 2014;20(17):4987-93. 


\section{Gurusamy 2019}

Gurusamy K, Walmsley M, Davidson BR, Frier C, Fuller B, Madden A, et al. Top research priorities in liver and gallbladder disorders in the United Kingdom. BMJ Open 2019;9(3):e025045.

\section{Guyatt 2011}

Guyatt G, Oxman AD, Akl EA, Kunz R, Vist G, Brozek J, et al. GRADE guidelines: 1. Introduction - GRADE evidence profiles and summary of findings tables. Journal of Clinical Epidemiology 2011;64(4):383-94.

\section{Hardy 2020}

Hardy T, Wonders K, Younes R, Aithal GP, Aller R, Allison M, et al. The European NAFLD Registry: a real-world longitudinal cohort study of nonalcoholic fatty liver disease. Contemporary Clinical Trials 2020;98:106175.

\section{Hernaez 2011}

Hernaez R, Lazo M, Bonekamp S, Kamel I, Brancati FL, Guallar E, et al. Diagnostic accuracy and reliability of ultrasonography for the detection of fatty liver: a meta-analysis. Hepatology (Baltimore, Md) 2011;54(3):1082-90.

\section{Higgins 2003}

Higgins JT, Thompson SG, Deeks JJ, Altman DG. Measuring inconsistency in meta-analyses. BMJ (Clinical research edition) 2003;327(7414):557-60.

\section{Higgins 2011a}

Higgins JT, Altman DG, Sterne JA (editors). Chapter 8. Assessing risk of bias in included studies. In: Higgins JT, Green S (editors). Cochrane Handbook for Systematic Reviews of Interventions Version 5.1.0 (updated March 2011). The Cochrane Collaboration, 2011. Available from www.handbook.cochrane.org.

\section{Higgins 2011b}

Higgins JT, Deeks JJ, Altman DG (editors). Chapter 16. Special topics in statistics. In: Higgins JT, Green S (editors), Cochrane Handbook for Systematic Reviews of Interventions Version 5.1.0 (updated March 2011). The Cochrane Collaboration, 2011. Available from www.handbook.cochrane.org..

\section{Higgins 2012}

Higgins JT, Jackson D, Barrett JK, Lu G, Ades AE, White IR. Consistency and inconsistency in network meta-analysis: concepts and models for multi-arm studies. Research Synthesis Methods 2012;3(2):98-110.

\section{Houghton 2017}

Houghton D, Thoma C, Hallsworth K, Cassidy S, Hardy T, Burt AD, et al. Exercise reduces liver lipids and visceral adiposity in patients with nonalcoholic steatohepatitis in a randomized controlled trial. Clinical Gastroenterology and Hepatology 2017;15(1):96-102.e3.

\section{Hutton 2015}

Hutton B, Salanti G, Caldwell DM, Chaimani A, Schmid CH, Cameron $\mathrm{C}$, et al. The PRISMA extension statement for reporting of systematic reviews incorporating network meta-analyses of health care interventions: checklist and explanations. Annals of Internal Medicine 2015;162(11):777-84.

\section{ICH-GCP 1997}

International Conference on Harmonisation Expert Working Group. International Conference on Harmonisation of Technical Requirements for Registration of Pharmaceuticals for Human Use. ICH Harmonised Tripartite Guideline. Guideline for Good Clinical Practice CFR \& ICH Guidelines. Vol. 1. Philadelphia (PA): Barnett International/PAREXEL, 1997.

\section{Jackson 2014}

Jackson D, Barrett JK, Rice S, White IR, Higgins JT. A designby-treatment interaction model for network meta-analysis with random inconsistency effects. Statistics in Medicine 2014;33(21):3639-54.

\section{James 2015}

James S, Rao SV, Granger CB. Registry-based randomized clinical trials - a new clinical trial paradigm. Nature Reviews. Cardiology 2015;12(5):312-6.

\section{Kjaergard 2001}

Kjaergard LL, Villumsen J, Gluud C. Reported methodologic quality and discrepancies between large and small randomized trials in meta-analyses. Annals of Internal Medicine 2001;135(11):982-9.

\section{Koehler 2012}

Koehler EM, Schouten JN, Hansen BE, van Rooij FJ, Hofman A, Stricker $\mathrm{BH}$, et al. Prevalence and risk factors of non-alcoholic fatty liver disease in the elderly: results from the Rotterdam study. Journal of Hepatology 2012;57(6):1305-11.

\section{Lazo 2013}

Lazo M, Hernaez R, Eberhardt MS, Bonekamp S, Kamel I, Guallar E, et al. Prevalence of nonalcoholic fatty liver disease in the United States: the Third National Health and Nutrition Examination Survey, 1988-1994. American Journal of Epidemiology 2013;178(1):38-45.

\section{Li 2014}

Li Z, Xue J, Chen P, Chen L, Yan S, Liu L. Prevalence of nonalcoholic fatty liver disease in mainland of China: a metaanalysis of published studies. Journal of Gastroenterology and Hepatology 2014;29(1):42-51.

\section{Li 2015}

Li YH, Yang LH, Sha KH, Liu TG, Zhang LG, Liu XX. Efficacy of poly-unsaturated fatty acid therapy on patients with nonalcoholic steatohepatitis. World Journal of Gastroenterology 2015;21(22):7008-13.

\section{Lombardi 2017}

Lombardi R, Onali S, Thorburn D, Davidson BR, Gurusamy KS, Tsochatzis E. Pharmacological interventions for non-alcohol related fatty liver disease (NAFLD). Cochrane Database of Systematic Reviews 2017, Issue 3. Art. No: CD011640. [DOI: 10.1002/14651858.CD011640.pub2] 


\section{Lonardo 2015}

Lonardo A, Bellentani S, Argo CK, Ballestri S, Byrne CD, Caldwell SH, et al. Epidemiological modifiers of non-alcoholic fatty liver disease: focus on high-risk groups. Digestive and Liver Disease 2015;47(12):997-1006.

\section{Lu 2006}

Lu G, Ades AE. Assessing evidence inconsistency in mixed treatment comparisons. Journal of the American Statistical Association 2006;101(474):447-59.

\section{Mann 2018}

Mann JP, Vreugdenhil A, Socha P, Janczyk W, Baumann U, Rajwal S, et al. European Paediatric Non-Alcoholic Fatty Liver Disease registry (EU-PNAFLD): design and rationale. Contemporary Clinical Trials 2018;75:67-71.

\section{Mills 2012}

Mills EJ, loannidis JP, Thorlund K, Schünemann HJ, Puhan MA, Guyatt $\mathrm{GH}$. How to use an article reporting a multiple treatment comparison meta-analysis. JAMA 2012;308(12):1246-53.

\section{Moher 1998}

Moher D, Pham B, Jones A, Cook DJ, Jadad AR, Moher M, et al. Does quality of reports of randomised trials affect estimates of intervention efficacy reported in meta-analyses? Lancet 1998;352(9128):609-13.

\section{Nabavi 2014}

Nabavi S, Rafraf M, Somi MH, Homayouni-Rad A, AsghariJafarabadi M. Effects of probiotic yogurt consumption on metabolic factors in individuals with nonalcoholic fatty liver disease. Journal of Dairy Science 2014;97(12):7386-93.

\section{NCBI 2018}

NCBI. Fatty liver. www.ncbi.nlm.nih.gov/mesh/68005234 (accessed 26 February 2021).

\section{Newell 1992}

Newell DJ. Intention-to-treat analysis: implications for quantitative and qualitative research. International Journal of Epidemiology 1992;21(5):837-41.

\section{Nishioji 2015}

Nishioji K, Sumida Y, Kamaguchi M, Mochizuki N, Kobayashi M, Nishimura T, et al. Prevalence of and risk factors for nonalcoholic fatty liver disease in a non-obese Japanese population, 2011-2012. Journal of Gastroenterology 2015;50(1):95-108.

\section{OmniPage 18.0 [Computer program]}

OmniPage 18.0. Nuance, 2011.

\section{Ong 2008}

Ong JP, Pitts A, Younossi ZM. Increased overall mortality and liver-related mortality in non-alcoholic fatty liver disease. Journal of Hepatology 2008;49(4):608-12.

\section{Onnerhag 2014}

Onnerhag K, Nilsson PM, Lindgren S. Increased risk of cirrhosis and hepatocellular cancer during long-term follow-up of patients with biopsy-proven NAFLD. Scandinavian Journal of Gastroenterology 2014;49(9):1111-8.

\section{OpenBUGS 3.2.3 [Computer program]}

Members of OpenBUGS project management group OpenBUGS. Members of OpenBUGS project management group, 2014.

\section{Optum 2018}

Optum. Patient-reported Outcomes | What We Do | SF Health Surveys | SF-36v2 Health Survey, 2018. www.qualitymetric.com/ health-surveys/the-sf-36v2-health-survey/ (accessed 26 February 2021).

\section{Park 2006}

Park SH, Jeon WK, Kim SH, Kim HJ, Park DI, Cho YK, et al. Prevalence and risk factors of non-alcoholic fatty liver disease among Korean adults. Journal of Gastroenterology and Hepatology 2006;21(1 Pt 1):138-43.

\section{Paschos 2012}

Paschos P, Tziomalos K. Nonalcoholic fatty liver disease and the renin-angiotensin system: implications for treatment. World Journal of Hepatology 2012;4(12):327-31.

\section{Piscaglia 2016}

Piscaglia F, Svegliati-Baroni G, Barchetti A, Pecorelli A, Marinelli S, Tiribelli C, et al. Clinical patterns of hepatocellular carcinoma in nonalcoholic fatty liver disease: a multicenter prospective study. Hepatology (Baltimore, Md) 2016;63(3):827-38

\section{Puhan 2014}

Puhan MA, Schünemann HJ, Murad MH, Li T, BrignardelloPetersen R, Singh JA, et al. A GRADE Working Group approach for rating the quality of treatment effect estimates from network meta-analysis. BMJ (Clinical research edition) 2014;349:g5630.

\section{Reeves 2018}

Reeves D, Howells K, Sidaway M, Blakemore A, Hann M, Panagioti $\mathrm{M}$, et al. The cohort multiple randomized controlled trial design was found to be highly susceptible to low statistical power and internal validity biases. Journal of Clinical Epidemiology 2018;95:111-9.

\section{Relton 2010}

Relton C, Torgerson D, O'Cathain A, Nicholl J. Rethinking pragmatic randomised controlled trials: introducing the "cohort multiple randomised controlled trial" design. BMJ (Clinical research edition) 2010;340:C1066. [PMID: 20304934]

\section{Riley 2007}

Riley P, O'Donohue J, Crook M. A growing burden: the pathogenesis, investigation and management of nonalcoholic fatty liver disease. Journal of Clinical Pathology 2007;60(12):1384-91.

\section{Rinella 2015}

Rinella ME. Nonalcoholic fatty liver disease: a systematic review. JAMA 2015;313(22):2263-73. 


\section{Salanti 2011}

Salanti G, Ades AE, loannidis JP. Graphical methods and numerical summaries for presenting results from multipletreatment meta-analysis: an overview and tutorial. Journal of Clinical Epidemiology 2011;64(2):163-71.

\section{Salanti 2012}

Salanti G. Indirect and mixed-treatment comparison, network, or multiple-treatments meta-analysis: many names, many benefits, many concerns for the next generation evidence synthesis tool. Research Synthesis Methods 2012;3(2):80-97.

\section{Savović 2012a}

Savović J, Jones HE, Altman DG, Harris RJ, Jüni P, Pildal J, et al. Influence of reported study design characteristics on intervention effect estimates from randomized controlled trials: combined analysis of meta-epidemiological studies. Health Technology Assessment 2012;16(35):1-82.

\section{Savović 2012b}

Savović J, Jones HE, Altman DG, Harris RJ, Jüni P, Pildal J, et al. Influence of reported study design characteristics on intervention effect estimates from randomized controlled trials. Annals of Internal Medicine 2012;157(6):429-38.

\section{Savović 2018}

Savović J, Turner RM, Mawdsley D, Jones HE, Beynon R, Higgins JT, et al. Association between risk-of-bias assessments and results of randomized trials in Cochrane Reviews: the ROBES Meta-Epidemiologic Study. American Journal of Epidemiology 2018;187(5):1113-22.

\section{Schulz 1995}

Schulz KF, Chalmers I, Hayes RJ, Altman DG. Empirical evidence of bias. Dimensions of methodological quality associated with estimates of treatment effects in controlled trials. JAMA 1995;273(5):408-12.

\section{Schulz 2010}

Schulz KF, Altman DG, Moher D, CONSORT Group. CONSORT 2010 statement: updated guidelines for reporting parallel group randomized trials. Annals of Internal Medicine 2010;152(11):726-32.

\section{Schünemann 2011}

Schünemann HJ, Oxman AD, Vist GE, Higgins JT, Deeks JJ, Glasziou P, Guyatt GH. Chapter 12. Interpreting results and drawing conclusions. In: Higgins JT, Green S (editors), Cochrane Handbook for Systematic Reviews of Interventions Version 5.1.0 (updated March 2011). The Cochrane Collaboration, 2011. Available from www.handbook.cochrane.org.

\section{Severini 1993}

Severini TA. Bayesian interval estimates which are also confidence intervals. Journal of the Royal Statistical Society. Series B (Methodological) 1993;55(2):533-40.

\section{Shen 2014}

Shen H, Shahzad G, Jawairia M, Bostick RM, Mustacchia P. Association between aspirin use and the prevalence of nonalcoholic fatty liver disease: a cross-sectional study from
The Third National Health and Nutrition Examination Survey. Alimentary Pharmacology \& Therapeutics 2014;40(9):1066-73.

\section{Shojaee-Moradie 2016}

Shojaee-Moradie F, Cuthbertson DJ, Barrett M, Jackson NC, Herring $\mathrm{R}$, Thomas EL, et al. Exercise training reduces liver fat and increases rates of VLDL clearance but not VLDL production in NAFLD. Journal of Clinical Endocrinology and Metabolism 2016;101(11):4219-28.

\section{Soderberg 2010}

Soderberg C, Stal P, Askling J, Glaumann H, Lindberg G, Marmur J, et al. Decreased survival of subjects with elevated liver function tests during a 28-year follow-up. Hepatology (Baltimore, Md) 2010;51(2):595-602.

\section{Sookoian 2011}

Sookoian S, Pirola CJ. Meta-analysis of the influence of I148M variant of patatin-like phospholipase domain containing 3 gene (PNPLA3) on the susceptibility and histological severity of nonalcoholic fatty liver disease. Hepatology (Baltimore, Md) 2011;53(6):1883-94.

\section{Stata/SE 15.1 [Computer program]}

Stata/SE. Version 15.1. Texas, USA: StataCorp LLC, 2016. www.stata.com/.

\section{Tilg 2010}

Tilg H, Moschen AR. Evolution of inflammation in nonalcoholic fatty liver disease: the multiple parallel hits hypothesis. Hepatology (Baltimore, Md) 2010;52(5):1836-46.

\section{van Valkenhoef 2012}

van Valkenhoef G, Lu G, de Brock B, Hillege H, Ades AE, Welton NJ. Automating network meta-analysis. Research Synthesis Methods 2012;3(4):285-99.

\section{Wang 2016}

Wang J, Li P, Jiang Z, Yang Q, Mi Y, Liu Y, et al. Diagnostic value of alcoholic liver disease (ALD)/nonalcoholic fatty liver disease (NAFLD) index combined with gamma-glutamyl transferase in differentiating ALD and NAFLD. Korean Journal of Internal Medicine 2016;31(3):479-87.

\section{White 2012}

White DL, Kanwal F, El-Serag HB. Association between nonalcoholic fatty liver disease and risk for hepatocellular cancer, based on systematic review. Clinical Gastroenterology and Hepatology 2012;10(12):1342-59.e2.

\section{Wood 2008}

Wood L, Egger M, Gluud LL, Schulz KF, Juni P, Altman DG, et al. Empirical evidence of bias in treatment effect estimates in controlled trials with different interventions and outcomes: meta-epidemiological study. BMJ (Clinical research edition) 2008;336(7644):601-5

\section{Yepes-Nunez 2019}

Yepes-Nunez JJ, Li SA, Guyatt G, Jack SM, Brozek JL, Beyene J, et al. Development of the summary of findings table for network meta-analysis. Journal of Clinical Epidemiology 2019;115:1-13. 


\section{Young-Afat 2016}

Young-Afat DA, Verkooijen HA, van Gils CH, van der Velden JM, Burbach JP, Elias SG, et al. Brief report: staged-informed consent in the cohort multiple randomized controlled trial design. Epidemiology (Cambridge, Mass) 2016;27(3):389-92. [PMID: 27035689]

\section{Zhang 2016}

Zhang HJ, He J, Pan LL, Ma ZM, Han CK, Chen CS, et al. Effects of moderate and vigorous exercise on nonalcoholic fatty liver disease: a randomized clinical trial. JAMA Internal Medicine 2016;176(8):1074-82.

CHARACTERISTICS OF STUDIES

Characteristics of included studies [ordered by study ID]

\section{References to other published versions of this review Gurusamy 2018}

Gurusamy KS, Tsochatzis E, Madden AM. Nutritional supplementation for non-alcohol-related fatty liver disease: a network meta-analysis. Cochrane Database of Systematic Reviews 2018, Issue 10. Art. No: CD013157. [DOI: 10.1002/14651858.CD013157]

* Indicates the major publication for the study

Abdelmalek 2009

\section{Study characteristics}

\begin{tabular}{ll}
\hline Methods & Randomised clinical trial \\
\hline Participants & Country: USA \\
& Period of recruitment: 2003 to 2005 \\
& Number randomised: 55 \\
& Post-randomisation dropouts: 20 (36.4\%) \\
& Revised sample size: 35 \\
& Reasons for post-randomisation dropouts: lack of compliance, did not receive treatment \\
& Average age, years: 47 \\
& Females: $35(100.0 \%)$ \\
& NASH: 35 (100.0\%) \\
& Diabetes mellitus: 18 (51.4\%) \\
Inclusion criteria: 1. Age 18 to 70.2 . Histological features of NASH. 3. Elevated ALT or AST $\geq 1.5 \times$ times \\
normal on $\geq 2$ different occasions within 6 months of enrolment. 4. Ethanol consumption $<20 \mathrm{~g} / \mathrm{d}$ for \\
females and $<30 \mathrm{~g} / \mathrm{d}$ for males \\
Exclusion criteria: 1 . Pregnant women, lactating women. 2 . ALT/AST $>5 \times$ normal. 3 . Abnormal total \\
bilirubin or albumin, prolonged PT, or platelets less than lower limit of normal. 4 . Creatinine $\geq 1.5$ mg/ \\
dL. 5. Creatine kinase $\geq 3 \times$ normal. 6 . Drugs known to cause hepatosteatosis or therapy with drugs that \\
may have potential benefit in treatment of NAFLD \\
Method for diagnosis of NAFLD: liver biopsy
\end{tabular}

$\begin{array}{ll}\text { Interventions } & \text { Group 1: amino acids }(\mathrm{n}=17) \\ & \text { Further details: betaine } 20 \mathrm{~g} \text { for } 12 \text { months } \\ & \text { Group 2: no active intervention }(\mathrm{n}=18) \\ & \text { Further details: placebo }\end{array}$

$\begin{array}{ll}\text { Outcomes } & \text { Outcomes reported: fibrosis score, NAFLD activity score } \\ & \text { Follow-up, months: } 12\end{array}$

Follow-up, months: 12

$\begin{array}{ll}\text { Notes } & \text { Source of funding: not stated } \\ \text { Trial name/Trial registry number: not stated } \\ \text { Attempts were made to contact study authors in December } 2020\end{array}$

\section{Risk of bias}

\begin{tabular}{lll}
\hline Bias & Authors' judgement & Support for judgement \\
\hline $\begin{array}{l}\text { Random sequence genera- } \\
\text { tion (selection bias) }\end{array}$ & Unclear risk & Comment: this information was not available \\
\hline \hline
\end{tabular}


Abdelmalek 2009 (Continued)

$\begin{aligned} & \text { Allocation concealment } \\ & \text { (selection bias) }\end{aligned} \quad$ Unclear risk Comment: this information was not available

Blinding of participants Low risk Quote: "double-blind, placebo-controlled trial"
and personnel (perfor-
mance bias)
All outcomes

\begin{tabular}{lll}
\hline $\begin{array}{l}\text { Blinding of outcome as- } \\
\text { sessment (detection bias) } \\
\text { All outcomes }\end{array}$ & Low risk & Quote: "double-blind, placebo-controlled trial" \\
\hline $\begin{array}{l}\text { Incomplete outcome data } \\
\text { (attrition bias) } \\
\text { All outcomes }\end{array}$ & High risk & $\begin{array}{l}\text { Comment: participants were excluded from analysis for reasons likely to be re- } \\
\text { lated to intervention and outcomes }\end{array}$ \\
\hline $\begin{array}{l}\text { Selective reporting (re- } \\
\text { porting bias) }\end{array}$ & High risk & $\begin{array}{l}\text { Comment: no previously published protocol was available; adverse events, } \\
\text { mortality, fatty liver resolution were not reported }\end{array}$ \\
\hline Other bias & Low risk & Comment: no other bias noted \\
\hline
\end{tabular}

Abhari 2020

\section{Study characteristics}

\begin{tabular}{|c|c|}
\hline Methods & Randomised clinical trial \\
\hline Participants & $\begin{array}{l}\text { Country: Iran } \\
\text { Period of recruitment: not stated } \\
\text { Number randomised: } 53 \\
\text { Post-randomisation dropouts: } 8 \text { (15.1\%) } \\
\text { Revised sample size: } 45 \\
\text { Reasons for post-randomisation dropouts: did not follow protocol } \\
\text { Average age, years: } 47 \\
\text { Females: } 20 \text { (44.4\%) } \\
\text { NASH: not stated } \\
\text { Diabetes mellitus: } 0(0.0 \%) \\
\text { Inclusion criteria: } 1.18 \text { to } 75 \text { years of age. } 2 \text {. Presence of steatosis on FibroScan (controlled attenuation } \\
\text { parameter (CAP) > } 270 \mathrm{~dB} / \mathrm{m}) \text {. } 3 \text {. High concentration of liver enzyme ALT > 1.5 upper limit of normal. } \\
\text { 4. No history of alcohol consumption. } 5 \text {. No history of disease such as diabetes or severe hepatic dis- } \\
\text { ease (hepatitis B, C) or cirrhosis (according to gastroenterologist diagnosis). } 6 \text {. Absence of antimicrobial } \\
\text { medication within previous } 3 \text { months. } 7 \text {. No history of weight loss or bariatric surgery in recent years } \\
\text { Exclusion criteria: } 1 \text {. Pregnancy or lactation. } 2 \text {. Unexpected adverse effects. 3. Supplement intolerance } \\
\text { Method for diagnosis of NAFLD: not stated }\end{array}$ \\
\hline Interventions & $\begin{array}{l}\text { Group 1: prebiotics/probiotics/synbiotics }(\mathrm{n}=23 \text { ) } \\
\text { Further details: patients in the synbiotic group received } 1 \text { synbiotic capsule containing } 10^{9} \text { spores of } B \\
\text { coagulans (GBI-30) plus } 0.4 \mathrm{~g} \text { inulin per day } \\
\text { Group 2: no active intervention ( } \mathrm{n}=22) \\
\text { Further details: patients in the placebo group received } 1 \text { capsule of placebo (maltodextrin), which ap- } \\
\text { parently was similar to the synbiotic capsule in terms of size, colour, and shape of capsules }\end{array}$ \\
\hline Outcomes & $\begin{array}{l}\text { Outcomes reported: mortality at maximal follow-up, serious adverse events (number of people), any } \\
\text { adverse events (number of people), liver transplantation at maximal follow-up, decompensation (num- } \\
\text { ber of people), cirrhosis (number of people), resolution of fatty liver disease, hepatocellular carcinoma } \\
\text { Follow-up, months: } 3\end{array}$ \\
\hline
\end{tabular}


Abhari 2020 (Continued)

Notes

Source of funding (quote): "the study was supported by Shahid Beheshti University of Medical Science"

Trial name/Trial registry number: IRCT20100524004010N23

Attempts were made to contact study authors in April 2021

\section{Risk of bias}

\begin{tabular}{lll}
\hline Bias & Authors' judgement & Support for judgement \\
\hline $\begin{array}{l}\text { Random sequence genera- } \\
\text { tion (selection bias) }\end{array}$ & Low risk & Quote: "computer-generated by a statistician" \\
\hline $\begin{array}{l}\text { Allocation concealment } \\
\text { (selection bias) }\end{array}$ & Low risk & $\begin{array}{l}\text { Quote: "subjects and investigators were blind to the treatment assignment un- } \\
\text { til the end of the study...placebo" } \\
\text { Comment: both allocation concealment and blinding were achieved with use } \\
\text { of placebo }\end{array}$ \\
\hline
\end{tabular}

\begin{tabular}{|c|c|c|}
\hline $\begin{array}{l}\text { Blinding of participants } \\
\text { and personnel (perfor- } \\
\text { mance bias) } \\
\text { All outcomes }\end{array}$ & Low risk & $\begin{array}{l}\text { Quote: "subjects and investigators were blind to the treatment assignment un- } \\
\text { til the end of the study... placebo" }\end{array}$ \\
\hline $\begin{array}{l}\text { Blinding of outcome as- } \\
\text { sessment (detection bias) } \\
\text { All outcomes }\end{array}$ & Low risk & $\begin{array}{l}\text { Quote: "subjects and investigators were blind to the treatment assignment un- } \\
\text { til the end of the study...placebo" }\end{array}$ \\
\hline $\begin{array}{l}\text { Incomplete outcome data } \\
\text { (attrition bias) } \\
\text { All outcomes }\end{array}$ & High risk & $\begin{array}{l}\text { Comment: there were post-randomisation dropouts on the basis of not follow- } \\
\text { ing the protocol. This was probably related to intervention and to outcomes }\end{array}$ \\
\hline $\begin{array}{l}\text { Selective reporting (re- } \\
\text { porting bias) }\end{array}$ & Low risk & $\begin{array}{l}\text { Comment: adverse events and either mortality or fatty liver resolution or both } \\
\text { were reported }\end{array}$ \\
\hline Other bias & Low risk & Comment: no other bias noted \\
\hline
\end{tabular}

Afsharinasab 2020

\section{Study characteristics}

\begin{tabular}{ll}
\hline Methods & Randomised clinical trial \\
\hline Participants & Country: Iran \\
& Period of recruitment: not stated \\
Number randomised: 42 \\
Post-randomisation dropouts: not stated \\
Revised sample size: 42 \\
Average age, years: 34 \\
Females: 13 (31.0\%) \\
NASH: not stated \\
Diabetes mellitus: 0 (0.0\%) \\
Inclusion criteria: 1 . Elevated levels of aspartate aminotransferase (AST), alanine aminotransferase \\
(ALT). 2 . Age range 20 to 45 years \\
Exclusion criteria: 1. Patients using alcohol or daily Berberis. 2. Patients having allergy to Berberis and \\
its compounds. 3 . Patients with diabetes, high blood pressure, perceptual disorders, nephrotic syn- \\
drome, uremia ischaemic heart disease, chronic liver disease such as hepatitis. 4 . Pregnancy or lacta- \\
tion \\
Method for diagnosis of NAFLD: ultrasound
\end{tabular}


Afsharinasab 2020 (Continued)

Interventions Group 1: other supplements $(\mathrm{n}=21)$

Further details: experimental group received a gelatin capsule containing $750 \mathrm{mg}$ hydroalcoholic extract of Berberis integerrima every 12 hours for 2 months. All patients in this study received metformin and vitamin $\mathrm{E}$ as standard treatment during the study

Group 2: no active intervention $(n=21)$

Further details: control group received a gelatin capsule containing $750 \mathrm{mg}$ cellulose every 12 hours for

2 months. All patients in this study received metformin and vitamin $\mathrm{E}$ as standard treatment during the study

\begin{tabular}{ll}
\hline Outcomes & No outcomes of interest were reported \\
\hline Notes & Source of funding (quote): "this work was supported by the RUMS by the grant number P/31/1/2806" \\
& Trial name/Trial registry number: not stated \\
& Attempts were made to contact study authors in April 2021
\end{tabular}

\section{Risk of bias}

\begin{tabular}{lll}
\hline Bias & Authors' judgement & Support for judgement \\
\hline $\begin{array}{l}\text { Random sequence genera- } \\
\text { tion (selection bias) }\end{array}$ & Unclear risk & Comment: this information was not available \\
\hline $\begin{array}{l}\text { Allocation concealment } \\
\text { (selection bias) }\end{array}$ & Unclear risk & Comment: this information was not available \\
\hline $\begin{array}{l}\text { Blinding of participants } \\
\begin{array}{l}\text { and personnel (perfor- } \\
\text { mance bias) }\end{array}\end{array}$ & Low risk & Quote: "double blind...placebo" \\
All outcomes & \\
\hline
\end{tabular}

\begin{tabular}{|c|c|c|}
\hline $\begin{array}{l}\text { Blinding of outcome as- } \\
\text { sessment (detection bias) } \\
\text { All outcomes }\end{array}$ & Low risk & Quote: "double blind...placebo" \\
\hline $\begin{array}{l}\text { Incomplete outcome data } \\
\text { (attrition bias) } \\
\text { All outcomes }\end{array}$ & Low risk & Comment: there were no post-randomisation dropouts \\
\hline $\begin{array}{l}\text { Selective reporting (re- } \\
\text { porting bias) }\end{array}$ & High risk & $\begin{array}{l}\text { Comment: no previously published protocol was available; adverse events, } \\
\text { mortality, fatty liver resolution were not reported }\end{array}$ \\
\hline Other bias & Low risk & Comment: no other bias noted \\
\hline
\end{tabular}

Afzali 2020

\section{Study characteristics}

\begin{tabular}{ll}
\hline Methods & Randomised clinical trial \\
\hline Participants & Country: Iran \\
& Period of recruitment: 2018 to 2019 \\
& Number randomised: 120 \\
& Post-randomisation dropouts: 3 (2.5\%) \\
& Revised sample size: 117 \\
& Reasons for post-randomisation dropouts: side effects and unusual values \\
& Average age, years: 47
\end{tabular}


Afzali 2020 (Continued)

Females: 55 (47.0\%)

NASH: not stated

Diabetes mellitus: not stated

Inclusion criteria: 1. Age between 18 and 70 years. 2. Primary diagnosis of NAFLD. 3. Increased levels of liver enzymes ( 2 or $3 \times$ higher than normal)

Exclusion criteria: 1. Patients with liver disease, including Wilson's disease, haemochromatosis, alcoholic fatty liver disease, autoimmune liver disease, or cirrhosis. 2. Pregnant and lactating women Method for diagnosis of NAFLD: ultrasound

Group 1: vitamin E plus other antioxidants plus other supplements $(\mathrm{n}=60)$
Further details: the Beta vulgaris group received vitamin E pearl $(300 \mathrm{IU} /$ twice daily), Livergol tablet
$(140 \mathrm{mg} / \mathrm{d})$, and Beta vulgaris capsule $(400 \mathrm{mg} / \mathrm{d})$ for 6 months
Group 2: vitamin E plus other antioxidants $(\mathrm{n}=57)$
Further details: the placebo group received the same dosages of vitamin E pearl and Livergol tablet,
but placebo capsules instead of Beta vulgaris capsules for the same amount of time

\begin{tabular}{|c|c|}
\hline Outcomes & $\begin{array}{l}\text { Outcomes reported: mortality, liver transplantation, decompensated cirrhosis } \\
\text { Follow-up, months: } 6\end{array}$ \\
\hline Notes & $\begin{array}{l}\text { Source of funding (quote): "this study was supported by a teaching and research scholarship from the } \\
\text { Faculty of Pharmacy of Tehran University of Medical Sciences" } \\
\text { Trial name/Trial registry number: IRCT20121017011145N20 } \\
\text { Attempts were made to contact study authors in April } 2021\end{array}$ \\
\hline
\end{tabular}

\section{Risk of bias}

\begin{tabular}{|c|c|c|}
\hline Bias & Authors' judgement & Support for judgement \\
\hline $\begin{array}{l}\text { Random sequence genera- } \\
\text { tion (selection bias) }\end{array}$ & Low risk & Quote: "the sequence of groups was drawn up by coin tossing" \\
\hline $\begin{array}{l}\text { Allocation concealment } \\
\text { (selection bias) }\end{array}$ & Unclear risk & Comment: this information was not available \\
\hline $\begin{array}{l}\text { Blinding of participants } \\
\text { and personnel (perfor- } \\
\text { mance bias) } \\
\text { All outcomes }\end{array}$ & Low risk & Quote: "double blind...placebo" \\
\hline $\begin{array}{l}\text { Blinding of outcome as- } \\
\text { sessment (detection bias) } \\
\text { All outcomes }\end{array}$ & Low risk & Quote: "double blind...placebo" \\
\hline $\begin{array}{l}\text { Incomplete outcome data } \\
\text { (attrition bias) } \\
\text { All outcomes }\end{array}$ & High risk & $\begin{array}{l}\text { Comment: there were post-randomisation dropouts on the basis of adverse } \\
\text { events and usual values; these were probably related to the intervention and } \\
\text { to outcomes }\end{array}$ \\
\hline $\begin{array}{l}\text { Selective reporting (re- } \\
\text { porting bias) }\end{array}$ & High risk & $\begin{array}{l}\text { Comment: no previously published protocol was available; adverse events } \\
\text { were not reported adequately }\end{array}$ \\
\hline Other bias & Low risk & Comment: no other bias noted \\
\hline
\end{tabular}


Ahn 2018 (Continued)

\begin{tabular}{ll} 
Methods & Randomised clinical trial \\
\hline Participants & Country: South Korea \\
& Period of recruitment: not stated \\
& Number randomised: 68 \\
& Post-randomisation dropouts: not stated \\
& Revised sample size: 68 \\
& Average age, years: not stated \\
& Females: not stated \\
& NASH: not stated \\
& Diabetes mellitus: not stated \\
Inclusion and exclusion criteria: not stated & Method for diagnosis of NAFLD: not stated \\
\hline Interventions & Group 1: prebiotics/probiotics/synbiotics ( $\mathrm{n}=$ not stated) \\
& Further details: probiotics mixture consists of 6 kinds of probiotics (Lactobacillus acidophilus, Lacto- \\
& bacillus rhamnosus, Lactobacillus paracasei, Pediococcus pentosaceus, Bifidobacterium lactis, Bifidobac- \\
& terium breve) for 12 weeks \\
& Group 2: no active intervention ( $\mathrm{n}=$ not stated) \\
& Further details: placebo \\
\hline No outcomes of interest were reported \\
\hline Outcomes & Source of funding: not stated \\
Trial name/Trial registry number: not stated \\
Attempts were made to contact study authors in December 2020
\end{tabular}

\section{Risk of bias}

\begin{tabular}{|c|c|c|}
\hline Bias & Authors' judgement & Support for judgement \\
\hline $\begin{array}{l}\text { Random sequence genera- } \\
\text { tion (selection bias) }\end{array}$ & Unclear risk & Comment: this information was not available \\
\hline $\begin{array}{l}\text { Allocation concealment } \\
\text { (selection bias) }\end{array}$ & Unclear risk & Comment: this information was not available \\
\hline $\begin{array}{l}\text { Blinding of participants } \\
\text { and personnel (perfor- } \\
\text { mance bias) } \\
\text { All outcomes }\end{array}$ & Low risk & Quote: "double-blind, placebo-controlled trial" \\
\hline $\begin{array}{l}\text { Blinding of outcome as- } \\
\text { sessment (detection bias) } \\
\text { All outcomes }\end{array}$ & Low risk & Quote: "double-blind, placebo-controlled trial" \\
\hline $\begin{array}{l}\text { Incomplete outcome data } \\
\text { (attrition bias) } \\
\text { All outcomes }\end{array}$ & Unclear risk & Comment: this information was not available \\
\hline $\begin{array}{l}\text { Selective reporting (re- } \\
\text { porting bias) }\end{array}$ & High risk & $\begin{array}{l}\text { Comment: no previously published protocol was available; adverse events, } \\
\text { mortality, fatty liver resolution were not reported }\end{array}$ \\
\hline Other bias & Low risk & Comment: no other bias noted \\
\hline
\end{tabular}


Akbarzadeh 2015

\section{Study characteristics}

\begin{tabular}{|c|c|}
\hline Methods & Randomised clinical trial \\
\hline Participants & $\begin{array}{l}\text { Country: Iran } \\
\text { Period of recruitment: } 2015 \\
\text { Number randomised: } 80 \\
\text { Post-randomisation dropouts: } 5 \text { (6.3\%) } \\
\text { Revised sample size: } 75 \\
\text { Reasons for post-randomisation dropouts: surgery, disliked the intervention, personal reasons } \\
\text { Average age, years: } 45 \\
\text { Females: } 40 \text { (53.3\%) } \\
\text { NASH: not stated } \\
\text { Diabetes mellitus: not stated } \\
\text { Inclusion criteria: } 1 \text {. NAFLD diagnosed by physical examination and/or ALT > } 40 \text { and/or elastometry > } 4 \\
\text { kPa in FibroScan } \\
\text { Exclusion criteria: } 1 \text {. Alcohol. 2. Pregnancy/Lactation. 3. Weight loss diet over previous } 3 \text { months. } 4 \text {. Ma- } \\
\text { lignancy. } 5 \text {. Medication affecting liver enzymes. } 6 \text {. Multi-vitamin supplements, omega } 3 \text { supplements in } \\
\text { last } 3 \text { months. } 5 . \text { CLD except NAFLD } \\
\text { Method for diagnosis of NAFLD: ultrasound plus/minus elastography plus/minus transaminases }\end{array}$ \\
\hline Interventions & $\begin{array}{l}\text { Group 1: prebiotics/probiotics/synbiotics }(\mathrm{n}=38) \\
\text { Further details: psyllium } 10 \mathrm{~g} \text { daily for } 10 \text { weeks } \\
\text { Group 2: no active intervention ( } \mathrm{n}=37) \\
\text { Further details: placebo } \\
\text { Additional details: both groups received lifestyle interventions }\end{array}$ \\
\hline Outcomes & No outcomes of interest were reported \\
\hline Notes & $\begin{array}{l}\text { Source of funding (quote): "the present study was supported by Isfahan University of Medical Sciences, } \\
\text { Isfahan, Iran" } \\
\text { Trial name/Trial registry number: not stated } \\
\text { Attempts were made to contact study authors in December } 2020\end{array}$ \\
\hline
\end{tabular}

\section{Risk of bias}

\begin{tabular}{lll}
\hline Bias & Authors' judgement & Support for judgement \\
\hline $\begin{array}{l}\text { Random sequence genera- } \\
\text { tion (selection bias) }\end{array}$ & Unclear risk & Comment: this information was not available \\
\hline $\begin{array}{l}\text { Allocation concealment } \\
\text { (selection bias) }\end{array}$ & Unclear risk & Comment: this information was not available \\
\hline $\begin{array}{l}\text { Blinding of participants } \\
\text { and personnel (perfor- } \\
\text { mance bias) }\end{array}$ & Low risk & Quote: "placebo-controlled, double-blind, randomized clinical trial" \\
All outcomes & & \\
\hline
\end{tabular}

\begin{tabular}{lll}
\hline $\begin{array}{l}\text { Blinding of outcome as- } \\
\text { sessment (detection bias) } \\
\text { All outcomes }\end{array}$ & Low risk & Quote: "placebo-controlled, double-blind, randomized clinical trial" \\
\hline $\begin{array}{l}\text { Incomplete outcome data } \\
\text { (attrition bias) } \\
\text { All outcomes }\end{array}$ & Unclear risk & $\begin{array}{l}\text { Comment: participants were excluded from analysis for reasons that may be } \\
\text { related to the intervention and to outcomes }\end{array}$ \\
\hline
\end{tabular}


Akbarzadeh 2015 (Continued)

\begin{tabular}{|c|c|c|}
\hline $\begin{array}{l}\text { Selective reporting (re- } \\
\text { porting bias) }\end{array}$ & High risk & $\begin{array}{l}\text { Comment: no previously published protocol was available; adverse events, } \\
\text { mortality, fatty liver resolution were not reported }\end{array}$ \\
\hline
\end{tabular}

Other bias Low risk Comment: no other bias noted

Aliashrafi 2014

\section{Study characteristics}

\begin{tabular}{|c|c|}
\hline Methods & Randomised clinical trial \\
\hline Participants & $\begin{array}{l}\text { Country: Iran } \\
\text { Period of recruitment: } 2011 \text { to } 2012 \\
\text { Number randomised: } 60 \\
\text { Post-randomisation dropouts: } 5 \text { (8.3\%) } \\
\text { Revised sample size: } 55 \\
\text { Reasons for post-randomisation dropouts: discontinuation (3), pregnancy (1), travel (1) } \\
\text { Average age, years: } 37 \\
\text { Females: } 25 \text { (45.5\%) } \\
\text { NASH: not stated } \\
\text { Diabetes mellitus: not stated } \\
\text { Inclusion criteria: } 1 \text {. Fatty liver on USS. 2. Aged } 20 \text { to } 50.3 \text {. BMI } \geq 30 \\
\text { Exclusion criteria: } 1 \text {. Other liver diseases. } 2 \text {. Those taking the following medications - hepatotoxic or } \\
\text { lipid-lowering agent, metformin, antihypertensive, contraceptive, oestrogen } \\
\text { Method for diagnosis of NAFLD: ultrasound plus transaminases }\end{array}$ \\
\hline Interventions & $\begin{array}{l}\text { Group 1: vitamin E plus other supplements }(n=29) \\
\text { Further details: four } 300-\mathrm{mg} \text { tablets of Chlorella vulgaris per day for } 8 \text { weeks plus vitamin } \mathrm{E} 400 \mathrm{mg} / \mathrm{d} \\
\text { Group 2: vitamin } E(n=26) \\
\text { Further details: four placebos per day for } 8 \text { weeks, similar to Chlorella vulgaris tablets in colour and size, } \\
\text { plus vitamin E } 400 \mathrm{mg} / \mathrm{d}\end{array}$ \\
\hline Outcomes & No outcomes of interest were reported \\
\hline Notes & $\begin{array}{l}\text { Source of funding (quote): "the authors would like to thanks Iranians Green Future Co. (Tehran, Iran) for } \\
\text { providing C vulgaris tablets" } \\
\text { Trial name/Trial registry number: IRCT201202233320N7 } \\
\text { Attempts were made to contact study authors in December } 2020\end{array}$ \\
\hline
\end{tabular}

\section{Risk of bias}

\begin{tabular}{|c|c|c|}
\hline Bias & Authors' judgement & Support for judgement \\
\hline $\begin{array}{l}\text { Random sequence genera- } \\
\text { tion (selection bias) }\end{array}$ & Low risk & $\begin{array}{l}\text { Quote: "patients were randomly allocated using a computer-generated ran- } \\
\text { dom sequence into two groups" }\end{array}$ \\
\hline $\begin{array}{l}\text { Allocation concealment } \\
\text { (selection bias) }\end{array}$ & Unclear risk & Comment: this information was not available \\
\hline $\begin{array}{l}\text { Blinding of participants } \\
\text { and personnel (perfor- } \\
\text { mance bias) } \\
\text { All outcomes }\end{array}$ & Low risk & $\begin{array}{l}\text { Quote: "double-blind randomized placebo-controlled clinical trial. The place- } \\
\text { bo tablets were similar to C vulgaris tablets in color and size" }\end{array}$ \\
\hline $\begin{array}{l}\text { Blinding of outcome as- } \\
\text { sessment (detection bias) }\end{array}$ & Low risk & Quote: "double-blind randomized placebo-controlled clinical trial" \\
\hline
\end{tabular}


Aliashrafi 2014 (Continued)

All outcomes

\begin{tabular}{lll}
$\begin{array}{l}\text { Incomplete outcome data } \\
\text { (attrition bias) } \\
\text { All outcomes }\end{array}$ & Unclear risk & $\begin{array}{l}\text { Comment: there were post-randomisation dropouts, but it is not clear whether } \\
\text { these were related to the intervention or to outcomes }\end{array}$ \\
\hline $\begin{array}{l}\text { Selective reporting (re- } \\
\text { porting bias) }\end{array}$ & High risk & $\begin{array}{l}\text { Comment: a published protocol was available; recruitment had commenced } \\
\text { before the protocol was published, and several pre-defined primary outcomes } \\
\text { were not reported }\end{array}$
\end{tabular}

Other bias Low risk Comment: no other bias noted

Alisi 2014

\section{Study characteristics}

\begin{tabular}{|c|c|c|}
\hline Methods & \multicolumn{2}{|c|}{ Randomised clinical trial } \\
\hline Participants & \multicolumn{2}{|c|}{$\begin{array}{l}\text { Country: Italy } \\
\text { Period of recruitment: } 2012 \text { to } 2013 \\
\text { Number randomised: } 48 \\
\text { Post-randomisation dropouts: } 4 \text { (8.3\%) } \\
\text { Revised sample size: } 44 \\
\text { Reasons for post-randomisation dropouts: lost to follow-up } \\
\text { Average age, years: } 11 \\
\text { Females: } 20 \text { (45.5\%) } \\
\text { NASH: } 44 \text { (100.0\%) } \\
\text { Diabetes mellitus: not stated } \\
\text { Inclusion criteria: } 1 \text {. Obese children with NAFLD } \\
\text { Exclusion criteria: } 1 \text {. Liver disease due to known other cause. } 2 \text {. Use of NSAIDs, antibiotics, probiotics, } \\
\text { or anti-secretory drugs capable of causing achlorhydria within } 2 \text { months preceding enrolment } \\
\text { Method for diagnosis of NAFLD: clinical examination plus transaminases plus liver biopsy }\end{array}$} \\
\hline Interventions & \multicolumn{2}{|c|}{$\begin{array}{l}\text { Group 2: no active intervention }(n=22) \\
\text { Further details: placebo }\end{array}$} \\
\hline Outcomes & \multicolumn{2}{|c|}{ No outcomes of interest were reported } \\
\hline Notes & \multicolumn{2}{|c|}{$\begin{array}{l}\text { Source of funding (quote): "this study was funded by the Italian Ministry of Health (Fondi di Ricerca Cor- } \\
\text { rente and 5*1000) to Prof. Valerio Nobili. Prof. Anania is supported by US Public Health Service Grant } \\
\text { DK062092 and Departments of Veterans' Affairs Grant BX001746" } \\
\text { Trial name/Trial registry number: NCT01650025 } \\
\text { Attempts were made to contact study authors in December } 2020\end{array}$} \\
\hline \multicolumn{3}{|l|}{ Risk of bias } \\
\hline Bias & Authors' judgement & Support for judgement \\
\hline $\begin{array}{l}\text { Random sequence genera- } \\
\text { tion (selection bias) }\end{array}$ & Low risk & $\begin{array}{l}\text { Quote: "a statistician blinded to participants' clinical data, and who did not } \\
\text { perform the final analysis, generated the allocation sequence and randomly } \\
\text { assigned participants to the VSL\#3 or placebo group" }\end{array}$ \\
\hline
\end{tabular}


Alisi 2014 (Continued)

Allocation concealment Low risk Quote: "a statistician blinded to participants' clinical data, and who did not (selection bias) perform the final analysis, generated the allocation sequence and randomly assigned participants to the VSL\#3 or placebo group"

$\begin{array}{ll}\begin{array}{l}\text { Blinding of participants } \\ \text { and personnel (perfor- }\end{array} & \text { Low risk }\end{array}$
mance bias)

All outcomes

Blinding of outcome as- Low risk sessment (detection bias)

All outcomes

Quote: "parallel-arm double-blind RCT...only the statistician had access to the treatment codes"
Incomplete outcome data Unclear risk Comment: participants were excluded from analysis for reasons that may be (attrition bias) related to the intervention and to outcomes

All outcomes

\begin{tabular}{|c|c|c|}
\hline $\begin{array}{l}\text { Selective reporting (re- } \\
\text { porting bias) }\end{array}$ & High risk & $\begin{array}{l}\text { Comment: no previously published protocol was available; adverse events, } \\
\text { mortality, fatty liver resolution were not reported }\end{array}$ \\
\hline
\end{tabular}

Other bias Low risk Comment: no other bias noted

Aller 2011

\section{Study characteristics}

\begin{tabular}{|c|c|}
\hline Methods & Randomised clinical trial \\
\hline Participants & $\begin{array}{l}\text { Country: Spain } \\
\text { Period of recruitment: not stated } \\
\text { Number randomised: } 30 \\
\text { Post-randomisation dropouts: } 2(6.7 \%) \\
\text { Revised sample size: } 28 \\
\text { Reasons for post-randomisation dropouts: lost to follow-up } \\
\text { Average age, years: } 47 \\
\text { Females: } 8 \text { ( } 28.6 \%) \\
\text { NASH: not stated } \\
\text { Diabetes mellitus: } 0 \text { (0.0\%) } \\
\text { Inclusion criteria: } 1 \text {. NAFLD } \\
\text { Exclusion criteria: } 1 \text {. Hep B/C, CMV, EBV. 2. Alcohol consumption. 3. Diabetes mellitus, impaired glucose } \\
\text { tolerance. } 4 \text {. Use of blood pressure-lowering medication or statins. 5. Hereditary defects. 6. Non-or- } \\
\text { gan-specific autoantibodies } \\
\text { Method for diagnosis of NAFLD: liver biopsy }\end{array}$ \\
\hline Interventions & $\begin{array}{l}\text { Group 1: prebiotics/probiotics/synbiotics }(n=14) \\
\text { Further details: } 500 \text { million Lactobacillus bulgaricus and Streptococcus thermophilus organisms for } 3 \\
\text { months } \\
\text { Group 2: no active intervention }(n=14) \\
\text { Further details: placebo }\end{array}$ \\
\hline Outcomes & No outcomes of interest were reported \\
\hline Notes & $\begin{array}{l}\text { Source of funding: not stated } \\
\text { Trial name/Trial registry number: not stated } \\
\text { Attempts were made to contact study authors in December } 2020\end{array}$ \\
\hline
\end{tabular}


Aller 2011 (Continued)

Risk of bias

\begin{tabular}{|c|c|c|}
\hline Bias & Authors' judgement & Support for judgement \\
\hline $\begin{array}{l}\text { Random sequence genera- } \\
\text { tion (selection bias) }\end{array}$ & Low risk & Quote: "table of numbers" \\
\hline $\begin{array}{l}\text { Allocation concealment } \\
\text { (selection bias) }\end{array}$ & Unclear risk & Comment: this information was not available \\
\hline $\begin{array}{l}\text { Blinding of participants } \\
\text { and personnel (perfor- } \\
\text { mance bias) } \\
\text { All outcomes }\end{array}$ & Low risk & Quote: "double-blind randomized clinical trial" \\
\hline $\begin{array}{l}\text { Blinding of outcome as- } \\
\text { sessment (detection bias) } \\
\text { All outcomes }\end{array}$ & Low risk & Quote: "double-blind randomized clinical trial" \\
\hline $\begin{array}{l}\text { Incomplete outcome data } \\
\text { (attrition bias) } \\
\text { All outcomes }\end{array}$ & Unclear risk & $\begin{array}{l}\text { Comment: participants were excluded from analysis for reasons that may be } \\
\text { related to the intervention and to outcomes }\end{array}$ \\
\hline $\begin{array}{l}\text { Selective reporting (re- } \\
\text { porting bias) }\end{array}$ & High risk & $\begin{array}{l}\text { Comment: no previously published protocol was available; adverse events, } \\
\text { mortality, fatty liver resolution were not reported }\end{array}$ \\
\hline Other bias & Low risk & Comment: no other bias noted \\
\hline
\end{tabular}

Aller 2015

\section{Study characteristics}

\begin{tabular}{|c|c|}
\hline Methods & Randomised clinical trial \\
\hline Participants & $\begin{array}{l}\text { Country: Spain } \\
\text { Period of recruitment: not stated } \\
\text { Number randomised: } 36 \\
\text { Post-randomisation dropouts: } 0(0.0 \%) \\
\text { Revised sample size: } 36 \\
\text { Average age, years: } 47 \\
\text { Females: } 14(38.9 \%) \\
\text { NASH: not stated } \\
\text { Diabetes mellitus: } 0 \text { (0.0\%) } \\
\text { Inclusion criteria: } 1 . \text { NAFLD } \\
\text { Exclusion criteria: } 1 \text {. Hep B/C, CMV, EBV. } 2 \text {. Alcohol consumption. 3. Diabetes mellitus, impaired glucose } \\
\text { tolerance. } 4 \text {. Use of blood pressure-lowering medication or statins. 5. Hereditary defects. } 6 \text {. Non-or- } \\
\text { gan-specific autoantibodies } \\
\text { Method for diagnosis of NAFLD: liver biopsy }\end{array}$ \\
\hline Interventions & $\begin{array}{l}\text { Group 1: vitamin E plus other antioxidants }(n=18) \\
\text { Further details: silymarin plus vitamin } E(\text { Eurosil85 } \\
\text { Group 2: Mo no active intervention }(n=18) \\
\text { Further details: no treatment } \\
\text { Additional details: both groups received lifestyle modification intervention }\end{array}$ \\
\hline
\end{tabular}


Aller 2015 (Continued)

Outcomes
Outcomes reported: mortality at maximal follow-up, serious adverse events (number of people), any adverse events (number of people), fibrosis score

Follow-up, months: 3

$\begin{array}{ll}\text { Notes } & \text { Source of funding: not stated } \\ & \text { Trial name/Trial registry number: not stated } \\ & \text { Attempts were made to contact study authors in December } 2020\end{array}$

\section{Risk of bias}

\begin{tabular}{|c|c|c|}
\hline Bias & Authors' judgement & Support for judgement \\
\hline $\begin{array}{l}\text { Random sequence genera- } \\
\text { tion (selection bias) }\end{array}$ & Low risk & Quote: "all patients were randomized (table of numbers)" \\
\hline $\begin{array}{l}\text { Allocation concealment } \\
\text { (selection bias) }\end{array}$ & Unclear risk & Comment: this information was not available \\
\hline $\begin{array}{l}\text { Blinding of participants } \\
\text { and personnel (perfor- } \\
\text { mance bias) } \\
\text { All outcomes }\end{array}$ & Unclear risk & Comment: this information was not available \\
\hline $\begin{array}{l}\text { Blinding of outcome as- } \\
\text { sessment (detection bias) } \\
\text { All outcomes }\end{array}$ & Unclear risk & Comment: this information was not available \\
\hline $\begin{array}{l}\text { Incomplete outcome data } \\
\text { (attrition bias) } \\
\text { All outcomes }\end{array}$ & Low risk & Comment: there were no post-randomisation dropouts \\
\hline $\begin{array}{l}\text { Selective reporting (re- } \\
\text { porting bias) }\end{array}$ & Low risk & $\begin{array}{l}\text { Comment: no previously published protocol was available; adverse events and } \\
\text { either mortality or fatty liver resolution or both were reported }\end{array}$ \\
\hline Other bias & Low risk & Comment: no other bias noted \\
\hline
\end{tabular}

Amanat 2018

\section{Study characteristics}

\begin{tabular}{ll}
\hline Methods & Randomised clinical trial \\
\hline Participants & Country: Iran \\
& Period of recruitment: not stated \\
& Number randomised: 82 \\
& Post-randomisation dropouts: 4 (4.9\%) \\
& Revised sample size: 78 \\
& Reasons for post-randomisation dropouts: missing samples \\
& Average age, years: 44 \\
& Females: 21 (26.9\%) \\
& NASH: not stated \\
& Diabetes mellitus: 0 (0.0\%) \\
Inclusion criteria: 1.18 to 69 years of age with steatosis grade $\geq 2$ by USS \\
Exclusion criteria: 1 . Viral hepatitis, cirrhosis, or other CLD. $2.5 \times$ increase in ALT. 3. Regular alcohol con- \\
sumption. 4 . Pregnancy. 5 . Parenteral nutrition. 6 . Lipid-lowering drugs or antidiabetic drugs \\
Method for diagnosis of NAFLD: ultrasound
\end{tabular}


Amanat 2018 (Continued)

$\begin{array}{ll}\text { Interventions } & \text { Group 1: oestrogen }(n=37) \\ & \text { Further details: } 250 \text { mg genistein in capsules once a day for } 8 \text { weeks } \\ & \text { Group 2: no active intervention }(n=41) \\ & \text { Further details: placebo }\end{array}$

\begin{tabular}{|c|c|}
\hline Outcomes & $\begin{array}{l}\text { Outcomes reported: serious adverse events (number of people), any adverse events (number of peo- } \\
\text { ple) } \\
\text { Follow-up, months: } 2\end{array}$ \\
\hline Notes & $\begin{array}{l}\text { Source of funding (quote): "the trial was supported by a research funding (no. 94-7516) from SUMS, Iran } \\
\text { (SUMS = Shiraz University of Medical Sciences)" } \\
\text { Trial name/Trial registry number: IRCT20131213240NS } \\
\text { Attempts were made to contact study authors in December } 2020\end{array}$ \\
\hline
\end{tabular}

\section{Risk of bias}

\begin{tabular}{lll}
\hline Bias & Authors' judgement & Support for judgement \\
\hline $\begin{array}{l}\text { Random sequence genera- } \\
\text { tion (selection bias) }\end{array}$ & Low risk & $\begin{array}{l}\text { Quote: "participants were randomly assigned to either genistein or placebo } \\
\text { groups by a computer-generated random sequence" }\end{array}$ \\
\hline $\begin{array}{l}\text { Allocation concealment } \\
\text { (selection bias) }\end{array}$ & Low risk & $\begin{array}{l}\text { Quote: "eight-week double-blinded randomized controlled trial...participants, } \\
\text { investigators and laboratory technicians were unaware of the participant's } \\
\text { group allocation" }\end{array}$ \\
$\begin{array}{l}\text { Comment: both allocation concealment and blinding were achieved with use } \\
\text { of a placebo }\end{array}$
\end{tabular}

$\begin{array}{ll}\begin{array}{l}\text { Blinding of participants } \\ \text { and personnel (perfor- }\end{array} & \text { Low risk } \\ \begin{array}{l}\text { mance bias) } \\ \text { All outcomes }\end{array} & \begin{array}{l}\text { Quote: "eight-week double-blinded randomized controlled trial...participants, } \\ \text { investigators and laboratory technicians were unaware of the participant's } \\ \text { group allocation" }\end{array}\end{array}$

\begin{tabular}{|c|c|c|}
\hline $\begin{array}{l}\text { Blinding of outcome as- } \\
\text { sessment (detection bias) } \\
\text { All outcomes }\end{array}$ & Low risk & $\begin{array}{l}\text { Quote: "eight-week double-blinded randomized controlled trial...participants, } \\
\text { investigators and laboratory technicians were unaware of the participant's } \\
\text { group allocation" }\end{array}$ \\
\hline $\begin{array}{l}\text { Incomplete outcome data } \\
\text { (attrition bias) } \\
\text { All outcomes }\end{array}$ & Unclear risk & $\begin{array}{l}\text { Comment: participants were excluded from analysis for reasons that may be } \\
\text { related to the intervention and to outcomes }\end{array}$ \\
\hline $\begin{array}{l}\text { Selective reporting (re- } \\
\text { porting bias) }\end{array}$ & High risk & $\begin{array}{l}\text { Comment: no previously published protocol was available; neither mortality } \\
\text { nor fatty liver resolution was reported }\end{array}$ \\
\hline Other bias & Low risk & Comment: no other bias noted \\
\hline
\end{tabular}

Amiri 2017

\section{Study characteristics}

\begin{tabular}{ll}
\hline Methods & Randomised clinical trial \\
\hline Participants & Country: Iran \\
& Period of recruitment: 2015 to 2016 \\
& Number randomised: 120 \\
& Post-randomisation dropouts: $10(8.3 \%)$ \\
&
\end{tabular}


Amiri 2017 (Continued)

Revised sample size: 110

Reasons for post-randomisation dropouts: lost to follow-up, non-compliant, discontinued intervention

Average age, years: 42

Females: $28(25.5 \%)$

NASH: not stated

Diabetes mellitus: not stated

Inclusion criteria: 1. NAFLD via USS. 2. BMI<35. 3. Age 18 to 65. 4. Serum $25(\mathrm{OH}) \mathrm{D}$ level $<15 \mathrm{ng} / \mathrm{mL} .5$. Iranian

Exclusion criteria: 1. BMI < 25. 2. Lactating/pregnant. 3. Diagnosis of chronic disease including inflammatory disease, heart failure, liver failure, renal failure, cancer, acute myocardial infarction, diabetes, stroke, or serious injury. 4. Use of hepatotoxic drugs or multi-vitamin or antioxidant supplements within 3 months. 5. Smoking or alcohol consumption. 6. Malabsorption disease. 7. Hereditary liver disease. 8. Athlete

Method for diagnosis of NAFLD: ultrasound

\begin{tabular}{|c|c|}
\hline Interventions & $\begin{array}{l}\text { Group 1: vitamin } D(n=74) \\
\text { Further details: } 25 \text { mcg calcitriol ( } 37 \text { patients also received calcium carbonate } 500 \mathrm{mg} \text {, which was deter- } \\
\text { mined at random) for } 12 \text { weeks } \\
\text { Group 2: no active intervention }(n=36) \\
\text { Further details: placebo }\end{array}$ \\
\hline Outcomes & No outcomes of interest were reported \\
\hline Notes & $\begin{array}{l}\text { Source of funding (quote): "this research did not receive any specific grant from funding agencies in the } \\
\text { public, commercial, or not-for-profit sectors" } \\
\text { Trial name/Trial registry number: IRC T201408312709N29 } \\
\text { Attempts were made to contact study authors in December } 2020\end{array}$ \\
\hline
\end{tabular}

\section{Risk of bias}

\begin{tabular}{|c|c|c|}
\hline Bias & Authors' judgement & Support for judgement \\
\hline $\begin{array}{l}\text { Random sequence genera- } \\
\text { tion (selection bias) }\end{array}$ & Low risk & $\begin{array}{l}\text { Quote: "eligible participants were randomly assigned by using a comput- } \\
\text { er-generated random-numbers method by the project coordinator" }\end{array}$ \\
\hline $\begin{array}{l}\text { Allocation concealment } \\
\text { (selection bias) }\end{array}$ & Low risk & $\begin{array}{l}\text { Quote: "eligible participants were randomly assigned by using a comput- } \\
\text { er-generated random-numbers method by the project coordinator" }\end{array}$ \\
\hline $\begin{array}{l}\text { Blinding of participants } \\
\text { and personnel (perfor- } \\
\text { mance bias) } \\
\text { All outcomes }\end{array}$ & Low risk & $\begin{array}{l}\text { Quote: "randomized, controlled, double-blind trial...products were adminis- } \\
\text { tered by a blinded researcher assistant to blinded patients" }\end{array}$ \\
\hline $\begin{array}{l}\text { Blinding of outcome as- } \\
\text { sessment (detection bias) } \\
\text { All outcomes }\end{array}$ & Low risk & $\begin{array}{l}\text { Quote: "randomized, controlled, double-blind trial...products were adminis- } \\
\text { tered by a blinded researcher assistant to blinded patients" }\end{array}$ \\
\hline $\begin{array}{l}\text { Incomplete outcome data } \\
\text { (attrition bias) } \\
\text { All outcomes }\end{array}$ & Unclear risk & $\begin{array}{l}\text { Comment: participants were excluded from analysis for reasons that may be } \\
\text { related to the intervention and to outcomes }\end{array}$ \\
\hline $\begin{array}{l}\text { Selective reporting (re- } \\
\text { porting bias) }\end{array}$ & High risk & $\begin{array}{l}\text { Comment: no previously published protocol was available; adverse events, } \\
\text { mortality, fatty liver resolution were not reported }\end{array}$ \\
\hline Other bias & Low risk & Comment: no other bias noted \\
\hline
\end{tabular}


Amiri-Moghadam 2015

\section{Study characteristics}

\begin{tabular}{|c|c|}
\hline Methods & Randomised clinical trial \\
\hline Participants & $\begin{array}{l}\text { Country: Iran } \\
\text { Period of recruitment: } 2013 \text { to } 2014 \\
\text { Number randomised: } 72 \\
\text { Post-randomisation dropouts: } 4 \text { (5.6\%) } \\
\text { Revised sample size: } 68 \\
\text { Reasons for post-randomisation dropouts: withdrew from the study } \\
\text { Average age, years: } 43 \\
\text { Females: not stated } \\
\text { NASH: } 68 \text { (100.0\%) } \\
\text { Diabetes mellitus: not stated } \\
\text { Inclusion criteria: } 1 \text {. ALT >3 times upper limit of normal and ultrasonography outcomes for diagnosis of } \\
\text { NASH. } 2.18 \text { to } 65 \text { years old. 3. BMI > } 25 \\
\text { Exclusion criteria: } 1 \text {. Smoking. } 2 \text {. Pregnancy. 3. Use of insulin, blood lipid-lowering agents, or steroid } \\
\text { drugs. } 3 \text {. Hemochromatosis. } 4 \text {. Wilson's disease. } 5 \text {. Consumption of supplement in previous month. } 6 . \\
\text { Use of high doses of oestrogen. } 7 \text {. Cushing's disease. } 8 \text {. Hyperthyroidism. 9. Total parenteral nutrition } \\
\text { in past } 6 \text { months } \\
\text { Method for diagnosis of NAFLD: ultrasound plus transaminases }\end{array}$ \\
\hline Interventions & $\begin{array}{l}\text { Group 1: amino acids ( } \mathrm{n}=36) \\
\text { Further details: } \mathrm{L} \text {-carnitine } 2000 \mathrm{mg} / \mathrm{d} \text { for } 12 \text { weeks } \\
\text { Group 2: no active intervention }(\mathrm{n}=32 \text { ) } \\
\text { Further details: placebo } \\
\text { Additional details: both groups received lifestyle advice }\end{array}$ \\
\hline Outcomes & No outcomes of interest were reported \\
\hline Notes & $\begin{array}{l}\text { Source of funding (quote): "the study was supported by a grant (no. 19463) from Iran University of Med- } \\
\text { ical Sciences, Tehran, Iran" } \\
\text { Trial name/Trial registry number: not stated } \\
\text { Attempts were made to contact study authors in December } 2020\end{array}$ \\
\hline
\end{tabular}

\section{Risk of bias}

\begin{tabular}{lll}
\hline Bias & Authors' judgement & Support for judgement \\
\hline $\begin{array}{l}\text { Random sequence genera- } \\
\text { tion (selection bias) }\end{array}$ & Unclear risk & Comment: this information was not available \\
\hline $\begin{array}{l}\text { Allocation concealment } \\
\text { (selection bias) }\end{array}$ & Low risk & $\begin{array}{l}\text { Quote: "neither the researchers nor the patients were informed about the allo- } \\
\text { cated group during the study" } \\
\text { Comment: both allocation concealment and blinding were achieved with use } \\
\text { of placebo }\end{array}$ \\
\hline
\end{tabular}

\begin{tabular}{|c|c|c|}
\hline $\begin{array}{l}\text { Blinding of participants } \\
\text { and personnel (perfor- } \\
\text { mance bias) } \\
\text { All outcomes }\end{array}$ & Low risk & Quote: "randomised double-blind placebo-controlled clinical trial" \\
\hline $\begin{array}{l}\text { Blinding of outcome as- } \\
\text { sessment (detection bias) } \\
\text { All outcomes }\end{array}$ & Low risk & Quote: "randomised double-blind placebo-controlled clinical trial" \\
\hline $\begin{array}{l}\text { Incomplete outcome data } \\
\text { (attrition bias) }\end{array}$ & Unclear risk & $\begin{array}{l}\text { Comment: participants were excluded from analysis for reasons that may be } \\
\text { related to the intervention and to outcomes }\end{array}$ \\
\hline
\end{tabular}

Nutritional supplementation for nonalcohol-related fatty liver disease: a network meta-analysis (Review) 
Amiri-Moghadam 2015 (Continued)

All outcomes

\begin{tabular}{|c|c|c|}
\hline $\begin{array}{l}\text { Selective reporting (re- } \\
\text { porting bias) }\end{array}$ & High risk & $\begin{array}{l}\text { Comment: no previously published protocol was available; adverse events, } \\
\text { mortality, fatty liver resolution were not reported }\end{array}$ \\
\hline
\end{tabular}

Other bias Low risk Comment: no other bias noted

Amirkhizi 2018

\section{Study characteristics}

\begin{tabular}{|c|c|}
\hline Methods & Randomised clinical trial \\
\hline Participants & $\begin{array}{l}\text { Country: Iran } \\
\text { Period of recruitment: not stated } \\
\text { Number randomised: } 50 \\
\text { Post-randomisation dropouts: } 5(10.0 \%) \\
\text { Revised sample size: } 45 \\
\text { Reasons for post-randomisation dropouts: discontinued treatment or withdrawal } \\
\text { Average age, years: } 40 \\
\text { Females: } 22 \text { (48.9\%) } \\
\text { NASH: not stated } \\
\text { Diabetes mellitus: } 0 \text { (0.0\%) } \\
\text { Inclusion criteria: } 1 \text {. Hepatic steatosis on USS. 2. BMI } 30 \text { to } 40.3 \text {. Age } 20 \text { to } 50 \text { years } \\
\text { Exclusion criteria: } 1 \text {. Pregnancy/Lactation. } 2 \text {. Hormone therapy or use of oral contraceptive pill. } 3 \text {. } \\
\text { Chemotherapy in previous year. } 4 \text {. Cardiovascular disease, hypertension, diabetes mellitus, thyroid dis- } \\
\text { orders, or kidney dysfunction. } 5 \text {. Viral hepatitis, cirrhosis, autoimmune hepatitis, or other hepatic dis- } \\
\text { ease. } 6 \text {. Consumption of antioxidant supplements. } 7 \text {. Use of lipid-lowering or antihypertensive medica- } \\
\text { tion. } 8 \text {. Smoking. } 9 \text {. Calorie-restricted diet } \\
\text { Method for diagnosis of NAFLD: ultrasound }\end{array}$ \\
\hline
\end{tabular}

\begin{tabular}{ll}
\hline Interventions & Group 1: vitamin E plus other antioxidants $(n=23)$ \\
& Further details: vitamin E $400 \mathrm{mg}$ plus $1200 \mathrm{mg}$ alpha-lipoic acid per day for 12 weeks \\
& Group 2: vitamin $\mathrm{E}(\mathrm{n}=22)$ \\
& Further details: vitamin E $400 \mathrm{mg}$ per day for 12 weeks
\end{tabular}

\begin{tabular}{ll}
\hline Outcomes & No outcomes of interest were reported \\
\hline Notes & Source of funding (quote): "we thank the Research Vice-Chancellor and Nutrition Research Center of \\
& Tabriz University of Medical Sciences, Tabriz, Iran, for the financial support" \\
& Trial name/Trial registry number: IRCT201511143320N12 \\
& Attempts were made to contact study authors in December 2020
\end{tabular}

\section{Risk of bias}

\begin{tabular}{lll}
\hline Bias & Authors' judgement & Support for judgement \\
\hline $\begin{array}{l}\text { Random sequence genera- } \\
\text { tion (selection bias) }\end{array}$ & Low risk & Quote: "random allocation software (RAS)" \\
\hline $\begin{array}{l}\text { Allocation concealment } \\
\text { (selection bias) }\end{array}$ & Low risk & $\begin{array}{l}\text { Quote: "once the random sequences were generated by the software, they } \\
\text { were kept in a secure location and managed by a third party, who had no in- } \\
\text { volvement in the study" }\end{array}$ \\
\hline $\begin{array}{l}\text { Blinding of participants } \\
\begin{array}{l}\text { and personnel (perfor- } \\
\text { mance bias) }\end{array}\end{array}$ & Low risk & Quote: "double-blind placebo-controlled randomized clinical trial" \\
\hline
\end{tabular}


Amirkhizi 2018 (Continued)

All outcomes

Blinding of outcome as-
sessment (detection bias) $\quad$ Low risk $\quad$ Quote: "double-blind placebo-controlled randomized clinical trial"

sessment (detection bias)

All outcomes

\begin{tabular}{|c|c|c|}
\hline $\begin{array}{l}\text { Incomplete outcome data } \\
\text { (attrition bias) }\end{array}$ & Unclear risk & $\begin{array}{l}\text { Comment: participants were excluded from analysis for reasons that may be } \\
\text { related to the intervention and to outcomes }\end{array}$ \\
\hline
\end{tabular}

All outcomes related to the intervention and to outcomes

Selective reporting (re- High risk
porting bias)

porting bias)

Comment: no previously published protocol was available; adverse events, mortality, fatty liver resolution were not reported

\begin{tabular}{ll}
\hline Other bias $\quad$ Low risk $\quad$ Comment: no other bias noted \\
\hline
\end{tabular}

Argo 2015

\section{Study characteristics}

\begin{tabular}{|c|c|}
\hline Methods & Randomised clinical trial \\
\hline Participants & $\begin{array}{l}\text { Country: USA } \\
\text { Period of recruitment: } 2007 \text { to } 2010 \\
\text { Number randomised: } 41 \\
\text { Post-randomisation dropouts: } 7 \text { (17.1\%) } \\
\text { Revised sample size: } 34 \\
\text { Reasons for post-randomisation dropouts: cirrhosis (1), relocation (1), transportation difficulties (5) } \\
\text { Average age, years: } 47 \\
\text { Females: } 21(61.8 \%) \\
\text { NASH: } 34 \text { (100.0\%) } \\
\text { Diabetes mellitus: } 11 \text { (32.4\%) } \\
\text { Inclusion criteria: } 1 \text {. Biopsies demonstrating steatohepatitis. } 2 \text {. Ethanol }<30 \mathrm{~g} / \mathrm{d} \text { for males and }<20 \mathrm{~g} / \mathrm{d} \\
\text { females } \\
\text { Exclusion criteria: } 1 \text {. Cirrhosis. } 2 \text {. Secondary forms of steatohepatitis. } 3 \text {. Treatment with thiazolidine- } \\
\text { diones. } 4 \text {. Viral hepatitis or autoimmune metabolic liver disease } \\
\text { Method for diagnosis of NAFLD: liver biopsy }\end{array}$ \\
\hline Interventions & $\begin{array}{l}\text { Group 1: PUFA }(n=17) \\
\text { Further details: } n-3 \text { fish oil } 3000 \mathrm{mg} / \mathrm{d} \text { for } 1 \text { year } \\
\text { Group 2: no active intervention }(n=17) \\
\text { Further details: placebo }\end{array}$ \\
\hline Outcomes & No outcomes of interest were reported \\
\hline Notes & $\begin{array}{l}\text { Source of funding (quote): "study medication and identical appearing placebo was provid- } \\
\text { ed at no charge by Nordic Natural. RBC phospholipid profile was performed by Metametrix } \\
\text { (www.metametrix.com)" } \\
\text { Trial name/Trial registry number: NCT00681408 } \\
\text { Attempts were made to contact study authors in December } 2020\end{array}$ \\
\hline
\end{tabular}

\section{Risk of bias}

\begin{tabular}{lll}
\hline Bias & Authors' judgement & Support for judgement \\
\hline $\begin{array}{l}\text { Random sequence genera- } \\
\text { tion (selection bias) }\end{array}$ & Low risk & $\begin{array}{l}\text { Quote: "an independent biostatistician generated the randomization list } \\
\text { which was confidentially forwarded to the investigational pharmacy" }\end{array}$ \\
\hline
\end{tabular}


Argo 2015 (Continued)

$\begin{aligned} & \text { Allocation concealment } \\ & \text { (selection bias) }\end{aligned} \quad$ Low risk
which was confidentially forwarded to the investigational pharmacy"
(selection bias)

Low risk

Quote: "double-blind, randomized, placebo-controlled trial"

Blinding of participants and personnel (performance bias)

All outcomes

Blinding of outcome as- Low risk Quote: "double-blind, randomized, placebo-controlled trial"
sessment (detection bias)

All outcomes

\begin{tabular}{lll}
\hline $\begin{array}{l}\text { Incomplete outcome data } \\
\text { (attrition bias) } \\
\text { All outcomes }\end{array}$ & Unclear risk & $\begin{array}{l}\text { Comment: participants were excluded from analysis for reasons that may be } \\
\text { related to the intervention and to outcomes }\end{array}$ \\
\hline $\begin{array}{l}\text { Selective reporting (re- } \\
\text { porting bias) }\end{array}$ & High risk & $\begin{array}{l}\text { Comment: no previously published protocol was available; adverse events, } \\
\text { mortality, fatty liver resolution were not reported }\end{array}$ \\
\hline Other bias & Low risk & Comment: no other bias noted \\
\hline
\end{tabular}

\section{Asghari 2018}

\section{Study characteristics}

\begin{tabular}{|c|c|}
\hline Methods & Randomised clinical trial \\
\hline Participants & $\begin{array}{l}\text { Country: Iran } \\
\text { Period of recruitment: not stated } \\
\text { Number randomised: } 60 \\
\text { Post-randomisation dropouts: } 0(0.0 \%) \\
\text { Revised sample size: } 60 \\
\text { Average age, years: } 39 \\
\text { Females: } 20 \text { (33.3\%) } \\
\text { NASH: not stated } \\
\text { Diabetes mellitus: } 0 \text { (0.0\%) } \\
\text { Inclusion criteria: } 1.20 \text { - to } 60 \text {-year-olds. 2. BMI } 25 \text { to } 35.3 \text {. NAFLD } \\
\text { Exclusion criteria: } 1 \text {. Pregnant/lactating women. } 2 \text {. Postmenopausal women. 3. Professional athletes. } 4 \\
\text { Smoking. } 5 \text {. Alcohol consumption. } 6 \text {. Inherited liver disorders. 7. Liver disease, cardiovascular disease, } \\
\text { kidney disease, gastrointestinal disease, diabetes mellitus, thyroid dysfunction, or malignancy. } 8 \text {. Use } \\
\text { of hepatotoxic drugs, steroids, or hormonal drugs } \\
\text { Method for diagnosis of NAFLD: ultrasound }\end{array}$ \\
\hline Interventions & $\begin{array}{l}\text { Group 1: other supplements }(n=30) \\
\text { Further details: resveratrol } 600 \mathrm{mg} \text { pure trans-resveratrol capsules for } 12 \text { weeks } \\
\text { Group 2: no active intervention }(n=30) \\
\text { Further details: placebo capsules } \\
\text { Additional details: another group not relevant to this review was excluded }\end{array}$ \\
\hline Outcomes & $\begin{array}{l}\text { Outcomes reported: serious adverse events (number of people), any adverse events (number of peo- } \\
\text { ple), resolution of fatty liver disease } \\
\text { Follow-up, months: } 3\end{array}$ \\
\hline Notes & $\begin{array}{l}\text { Source of funding (quote): "we thank the Research Vice-Chancellor and Nutrition Research Center of } \\
\text { Tabriz University of Medical Sciences, Tabriz, Iran, for the financial support" } \\
\text { Trial name/Trial registry number: IRCT201511233664N16 }\end{array}$ \\
\hline
\end{tabular}


Asghari 2018 (Continued)

Attempts were made to contact study authors in December 2020

\section{Risk of bias}

\begin{tabular}{|c|c|c|}
\hline Bias & Authors' judgement & Support for judgement \\
\hline $\begin{array}{l}\text { Random sequence genera- } \\
\text { tion (selection bias) }\end{array}$ & Low risk & $\begin{array}{l}\text { Quote: "random allocation software was used for generating a random se- } \\
\text { quence, by the study statistician" }\end{array}$ \\
\hline $\begin{array}{l}\text { Allocation concealment } \\
\text { (selection bias) }\end{array}$ & Low risk & $\begin{array}{l}\text { Quote: "an independent person not involved in the study process prepared } \\
\text { both resveratrol and placebo bottles, and labeled them as A or B" }\end{array}$ \\
\hline $\begin{array}{l}\text { Blinding of participants } \\
\text { and personnel (perfor- } \\
\text { mance bias) } \\
\text { All outcomes }\end{array}$ & Low risk & Quote: "randomized, double-blind, placebo-controlled clinical trial" \\
\hline $\begin{array}{l}\text { Blinding of outcome as- } \\
\text { sessment (detection bias) } \\
\text { All outcomes }\end{array}$ & Low risk & Quote: "randomized, double-blind, placebo-controlled clinical trial" \\
\hline $\begin{array}{l}\text { Incomplete outcome data } \\
\text { (attrition bias) } \\
\text { All outcomes }\end{array}$ & Low risk & Comment: an intention-treat analysis was performed \\
\hline $\begin{array}{l}\text { Selective reporting (re- } \\
\text { porting bias) }\end{array}$ & Low risk & $\begin{array}{l}\text { Comment: no previously published protocol was available; adverse events and } \\
\text { either mortality or fatty liver resolution or both were reported }\end{array}$ \\
\hline Other bias & Low risk & Comment: no other bias noted \\
\hline
\end{tabular}

Asgharian 2016

\section{Study characteristics}

\begin{tabular}{ll}
\hline Methods & Randomised clinical trial \\
\hline Participants & Country: Iran \\
& Period of recruitment: 2014 \\
& Number randomised: 80 \\
& Post-randomisation dropouts: 6 (7.5\%) \\
& Revised sample size: 74 \\
& Reasons for post-randomisation dropouts: lost to follow up/poor compliance (4), unwilling (2) \\
& Average age, years: 47 \\
& Females: 55 (74.3\%) \\
& NASH: not stated \\
& Diabetes mellitus: not stated \\
Inclusion criteria: 1.18 to 60 years of age. 2. NAFLD. & Exclusion criteria: 1. Other liver disease. 2. Inflammatory bowel disease. 3. Malignancy. 4. Pregnancy \\
& or lactation. 5. Use of corticosteroids, amiodarone, tamoxifen, cyclines, perhexiline, methotrexate, hy- \\
dralazine, laxatives, or oral contraceptives; use of vitamin-mineral, antioxidant, or omega-3 supple- \\
ment \\
Method for diagnosis of NAFLD: ultrasound \\
Group 1: prebiotics/probiotics/synbiotics ( $\mathrm{n}=38$ ) \\
Further details: 500 -mg capsule (Familact, produced by Zisttakhmir Company) containing 7 species of \\
probiotic bacteria (Lactobacillus casei, Lactobacillus acidophilus, Lactobacillus rhamnosus, Lactobacil-
\end{tabular}


Asgharian 2016 (Continued)

lus bulgaricus, Bifidobacterium breve, Bifidobacterium longum, Streptococcus thermophilus) and fructo-oligosaccharides. Capsule ingested once daily for 8 weeks

Group 2: no active intervention $(n=36)$

Further details: placebo capsule (containing $120 \mathrm{mg}$ starch) similar in shape and appearance to symbiotic capsule. Capsule ingested once daily for 8 weeks

\begin{tabular}{ll}
\hline Outcomes & $\begin{array}{l}\text { Outcomes reported: resolution of fatty liver disease } \\
\text { Follow-up, months: } 2\end{array}$ \\
\hline Notes & $\begin{array}{l}\text { Source of funding (quote): "financial support and sponsorship: Food Security Research Centre, School } \\
\text { of Nutrition and Food Science, Isfahan University of Medical sciences, Isfahan, Iran" } \\
\text { Trial name/Trial registry number: IRCT2013122811763N15 } \\
\text { Attempts were made to contact study authors in December } 2020\end{array}$ \\
&
\end{tabular}

\section{Risk of bias}

\begin{tabular}{|c|c|c|}
\hline Bias & Authors' judgement & Support for judgement \\
\hline $\begin{array}{l}\text { Random sequence genera- } \\
\text { tion (selection bias) }\end{array}$ & Low risk & $\begin{array}{l}\text { Quote: "participants were randomly allocated to two numerically equal groups } \\
\text { from a double-blind, } 80 \text {-person list, using a table of random digits" }\end{array}$ \\
\hline $\begin{array}{l}\text { Allocation concealment } \\
\text { (selection bias) }\end{array}$ & Low risk & $\begin{array}{l}\text { Quote: "the supplements and placebo tablets will be coded as A andB in sim- } \\
\text { ilar packets by a person that will be unaware from goals of the study (quote } \\
\text { from protocol)" }\end{array}$ \\
\hline $\begin{array}{l}\text { Blinding of participants } \\
\text { and personnel (perfor- } \\
\text { mance bias) } \\
\text { All outcomes }\end{array}$ & Low risk & $\begin{array}{l}\text { Quote: "randomized, double-blind, placebo-controlled clinical tri- } \\
\text { al" }\end{array}$ \\
\hline $\begin{array}{l}\text { Blinding of outcome as- } \\
\text { sessment (detection bias) } \\
\text { All outcomes }\end{array}$ & Low risk & $\begin{array}{l}\text { Quote: "randomized, double-blind, placebo-controlled clinical tri- } \\
\text { al" }\end{array}$ \\
\hline $\begin{array}{l}\text { Incomplete outcome data } \\
\text { (attrition bias) } \\
\text { All outcomes }\end{array}$ & Unclear risk & $\begin{array}{l}\text { Comment: there were post-randomisation dropouts, but it is not clear whether } \\
\text { these were related to the intervention or to outcomes }\end{array}$ \\
\hline $\begin{array}{l}\text { Selective reporting (re- } \\
\text { porting bias) }\end{array}$ & Low risk & $\begin{array}{l}\text { Comment: all pre-defined outcomes in the protocol published before recruit- } \\
\text { ment were reported }\end{array}$ \\
\hline Other bias & Low risk & Comment: no other bias noted \\
\hline
\end{tabular}

Ashraf 2017

\section{Study characteristics}

\begin{tabular}{ll}
\hline Methods & Randomised clinical trial \\
\hline Participants & Country: Bangladesh \\
& Period of recruitment: not stated \\
& Number randomised: 52 \\
& Post-randomisation dropouts: not stated \\
& Revised sample size: 52 \\
& Average age, years: 46 \\
& Females: $18(34.6 \%)$ \\
NASH: $52(100.0 \%)$
\end{tabular}


Ashraf 2017 (Continued)

Diabetes mellitus: 0 (0.0\%)

Inclusion criteria: 1 . Patients with fibrotic NASH

Exclusion criteria: 1. Diabetes. 2. Cirrhosis

Method for diagnosis of NAFLD: elastography

\begin{tabular}{ll}
\hline Interventions & Group 1: vitamin C plus other antioxidants ( $\mathrm{n}=25)$ \\
& Further details: Viusid (Catalysis Laboratory, Madrid, Spain) is a nutritional supplement that contains \\
& activated glycyrrhizic acid, ascorbic acid, folic acid, and zinc. Patients were given Viusid 3 sachets daily \\
& for 3 months \\
& Group 2: vitamin E $(\mathrm{n}=27)$ \\
& Further details: vitamin E 800 IU daily for 3 months \\
& Additional details: both groups received lifestyle intervention \\
\hline Outcomes & No outcomes of interest were reported \\
\hline \multirow{2}{*}{ Notes } & Source of funding: not stated \\
& Trial name/Trial registry number: not stated \\
Attempts were made to contact study authors in December 2020
\end{tabular}

\section{Risk of bias}

\begin{tabular}{lll}
\hline Bias & Authors' judgement & Support for judgement \\
\hline $\begin{array}{l}\text { Random sequence genera- } \\
\text { tion (selection bias) }\end{array}$ & Unclear risk & Comment: this information was not available \\
\hline $\begin{array}{l}\text { Allocation concealment } \\
\text { (selection bias) }\end{array}$ & Unclear risk & Comment: this information was not available \\
\hline $\begin{array}{l}\text { Blinding of participants } \\
\text { and personnel (perfor- } \\
\text { mance bias) }\end{array}$ & High risk & Quote: "open-label" \\
All outcomes & & \\
\hline
\end{tabular}

\begin{tabular}{lll}
\hline $\begin{array}{l}\text { Blinding of outcome as- } \\
\text { sessment (detection bias) } \\
\text { All outcomes }\end{array}$ & High risk & Quote: "open-label" \\
\hline $\begin{array}{l}\text { Incomplete outcome data } \\
\text { (attrition bias) } \\
\text { All outcomes }\end{array}$ & Unclear risk & Comment: this information was not available \\
\hline $\begin{array}{l}\text { Selective reporting (re- } \\
\text { porting bias) }\end{array}$ & High risk & $\begin{array}{l}\text { Comment: no previously published protocol was available; adverse events, } \\
\text { mortality, fatty liver resolution were not reported }\end{array}$ \\
\hline Other bias & Low risk & Comment: no other bias noted \\
\hline
\end{tabular}

\section{Askari 2014}

\section{Study characteristics}

\begin{tabular}{ll}
\hline Methods & Randomised clinical trial \\
\hline Participants & Country: Iran \\
& Period of recruitment: not stated \\
& Number randomised: 50
\end{tabular}


Askari 2014 (Continued)

Post-randomisation dropouts: 5 (10.0\%)

Revised sample size: 45

Reasons for post-randomisation dropouts: did not complete study

Average age, years: 45

Females: 24 (53.3\%)

NASH: not stated

Diabetes mellitus: 0 (0.0\%)

Inclusion criteria: 1. NAFLD diagnosis in past 6 months. 2 . 20 to 65 years old. 3. ALT $<60$. 4. Evidence of

fatty liver in USS with score $\geq 2$

Exclusion: 1. No alcohol/drug abuse. 2. No chemotherapy in previous year. 3. No incidence of other acute or chronic liver disease, cirrhosis, biliary disease, autoimmune disease, or cancer. 4. Pregnan$\mathrm{cy} /$ Lactation. 5. Diabetes. 6. Hyperlipidaemia. 7. Hypertension requiring medication. 8. Use of vitamin $\mathrm{E}$ or hepatotoxic drugs in last 6 months

Method for diagnosis of NAFLD: ultrasound

\begin{tabular}{|c|c|}
\hline Interventions & $\begin{array}{l}\text { Group 1: other supplements }(n=23) \\
\text { Further details: cinnamon } 1500 \mathrm{mg} \text { daily for } 12 \text { weeks } \\
\text { Group 2: no active intervention }(\mathrm{n}=22) \\
\text { Further details: placebo } \\
\text { Additional details: both groups received lifestyle advice }\end{array}$ \\
\hline Outcomes & No outcomes of interest were reported \\
\hline Notes & $\begin{array}{l}\text { Source of funding (quote): "this work was financially supported by the National Nutrition and Food } \\
\text { Technology Research Institute" } \\
\text { Trial name/Trial registry number: IRCT201207114010N9 } \\
\text { Attempts were made to contact study authors in December } 2020\end{array}$ \\
\hline
\end{tabular}

\section{Risk of bias}

\begin{tabular}{|c|c|c|}
\hline Bias & Authors' judgement & Support for judgement \\
\hline $\begin{array}{l}\text { Random sequence genera- } \\
\text { tion (selection bias) }\end{array}$ & Unclear risk & Comment: this information was not available \\
\hline $\begin{array}{l}\text { Allocation concealment } \\
\text { (selection bias) }\end{array}$ & Low risk & $\begin{array}{l}\text { Quote: "in order to blind the study, supplement and placebo capsules were } \\
\text { packaged by a third person who had no involvement in the study" }\end{array}$ \\
\hline $\begin{array}{l}\text { Blinding of participants } \\
\text { and personnel (perfor- } \\
\text { mance bias) } \\
\text { All outcomes }\end{array}$ & Low risk & Quote: "randomized double blinded, placebo-controlled trial" \\
\hline $\begin{array}{l}\text { Blinding of outcome as- } \\
\text { sessment (detection bias) } \\
\text { All outcomes }\end{array}$ & Low risk & Quote: "randomized double blinded, placebo-controlled trial" \\
\hline $\begin{array}{l}\text { Incomplete outcome data } \\
\text { (attrition bias) } \\
\text { All outcomes }\end{array}$ & Unclear risk & $\begin{array}{l}\text { Comment: participants were excluded from analysis for reasons that may be } \\
\text { related to the intervention and to outcomes }\end{array}$ \\
\hline $\begin{array}{l}\text { Selective reporting (re- } \\
\text { porting bias) }\end{array}$ & High risk & $\begin{array}{l}\text { Comment: no previously published protocol was available; adverse events, } \\
\text { mortality, fatty liver resolution were not reported }\end{array}$ \\
\hline Other bias & Low risk & Comment: no other bias noted \\
\hline
\end{tabular}


Babaei 2020

\section{Study characteristics}

\begin{tabular}{|c|c|}
\hline Methods & Randomised clinical trial \\
\hline Participants & 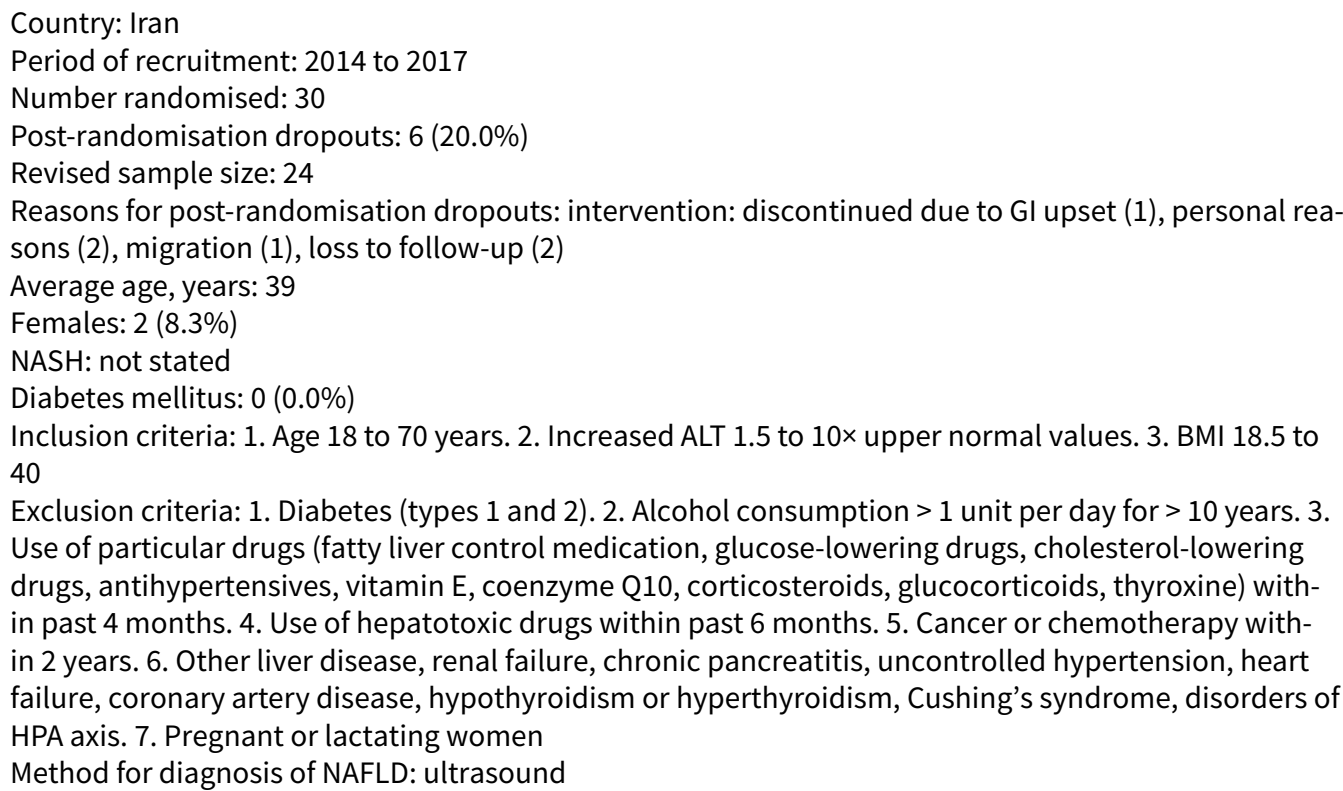 \\
\hline
\end{tabular}

Group 1: other supplements $(\mathrm{n}=13)$
Further details: $1 \mathrm{~g}$ hydroalcoholic extract of Fenugreek (Trigonella foenum-graecum) seed capsules
daily for 3 months
Group 2: no active intervention $(\mathrm{n}=11)$
Further details: placebo (rice flour) capsules daily for 3 months. Placebo capsules were the same as
Fenugreek placebo in colour, package, shape, and size
Additional details: both groups received lifestyle intervention

\begin{tabular}{ll}
\hline Outcomes & No outcomes of interest were reported \\
\hline Notes & $\begin{array}{l}\text { Source of funding (quote): "this article was supported financially by the Vice Chancellor for Research of } \\
\text { Shiraz University of Medical Sciences (grant number: 92-01-21-6352)" } \\
\text { Trial name/Trial registry number: NCT02303314; IRCT2013102015083N1 } \\
\text { Attempts were made to contact study authors in December 2020 }\end{array}$ \\
\hline
\end{tabular}

\section{Risk of bias}

\begin{tabular}{lll}
\hline Bias & Authors' judgement & Support for judgement \\
\hline $\begin{array}{l}\text { Random sequence genera- } \\
\text { tion (selection bias) }\end{array}$ & Low risk & $\begin{array}{l}\text { Quote: "randomization was done by blocked randomization method. A com- } \\
\text { puter random number generator generated the sequence of permuted blocks" }\end{array}$ \\
\hline $\begin{array}{l}\text { Allocation concealment } \\
\text { (selection bias) }\end{array}$ & Low risk & $\begin{array}{l}\text { Quote: "randomized, placebo-controlled, pilot, triple-blind (participants, in- } \\
\text { vestigator, and outcomes assessor)" } \\
\text { Comment: although the precise method was not reported, allocation was } \\
\text { probably concealed by use of a placebo }\end{array}$ \\
\hline $\begin{array}{l}\text { Blinding of participants } \\
\text { and personnel (perfor- } \\
\text { mance bias) } \\
\begin{array}{l}\text { All outcomes } \\
\hline\end{array}\end{array}$ & $\begin{array}{l}\text { Low risk } \\
\text { variables and hepatologist who perform FibroScan), and the statistician who } \\
\text { analyzed the data were all blinded to treatment allocation until the statistical } \\
\text { analysis was complete...placebo" }\end{array}$ \\
\hline
\end{tabular}


Babaei 2020 (Continued)

Blinding of outcome as- Low risk Quote: "participants, investigators (the nurse who measures anthropometric sessment (detection bias) variables and hepatologist who perform FibroScan), and the statistician who All outcomes analyzed the data were all blinded to treatment allocation until the statistical analysis was complete... placebo"

\section{Incomplete outcome data Unclear risk} (attrition bias)

Comment: there were post-randomisation dropouts, but it is not clear whether All outcomes these were related to the intervention or to outcomes

Selective reporting (re- High risk
porting bias)
porting bias)

Comment: a published protocol was available, but it is not clear whether recruitment had commenced before the protocol was published; adverse events, mortality, fatty liver resolution were not reported

Other bias Unclear risk Comment: there were baseline differences in important prognostic factors

Bae 2015

\section{Study characteristics}

\begin{tabular}{|c|c|}
\hline Methods & Randomised clinical trial \\
\hline Participants & $\begin{array}{l}\text { Country: South Korea } \\
\text { Period of recruitment: } 2011 \text { to } 2012 \\
\text { Number randomised: } 78 \\
\text { Post-randomisation dropouts: } 0 \text { (0.0\%) } \\
\text { Revised sample size: } 78 \\
\text { Average age, years: } 51 \\
\text { Females: } 24(30.8 \%) \\
\text { NASH: not stated } \\
\text { Diabetes mellitus: } 78(100.0 \%) \\
\text { Inclusion criteria: } 1.20 \text { to } 70 \text { years of age. } 2 \text {. Known diabetes with previous type } 2 \text { diabetes diagnosis } \geq 3 \\
\text { months before screening. } 3 \text {. Glycated haemoglobin }>6.4 \% \text { or fasting plasma glucose } 130 \text { to } 300 \mathrm{mg} / \mathrm{dL} \\
\text { at screening. } 4 \text {. ALT } 50 \text { to } 350 \text { IU/L at screening } \\
\text { Exclusion criteria: } 1 \text {. Alcohol > } 30 \text { g/d in men, }>20 \text { g/d in women. } 2 \text {. Viral hepatitis, cirrhosis, platelets } \\
<150,000 / m^{3} \text {, or other liver disease. } 3 \text {. Thiazolidinediones for treatment of diabetes or anti-obesi- } \\
\text { ty drug within } 1 \text { month before screening. } 4 \text {. History of malignancy or severe heart disease. } 5 \text {. Pregnan- } \\
\text { cy/Lactation } \\
\text { Method for diagnosis of NAFLD: CT scan }\end{array}$ \\
\hline
\end{tabular}

\begin{tabular}{ll}
\hline Interventions & Group 1: amino acids $(n=39)$ \\
& Further details: carnitine-orotate complex (824 mg, 3 times daily) for 12 weeks \\
& Group 2: no active intervention $(n=39)$ \\
& Further details: placebo
\end{tabular}

Outcomes Outcomes reported: mortality at maximal follow-up, serious adverse events (number of people), serious adverse events (number of events), any adverse events (number of people), any adverse events (number of events)

Follow-up, months: 3

\begin{tabular}{ll}
\hline Notes & $\begin{array}{l}\text { Source of funding (quote): "this study was funded by Celltrion Pharm (Seoul, Korea)" } \\
\text { Trial name/Trial registry number: KCT0000505 } \\
\text { Attempts were made to contact study authors in December } 2020\end{array}$ \\
\hline Risk of bias & Authors' judgement Support for judgement \\
\hline Bias & Suld \\
\hline \hline
\end{tabular}


Bae 2015 (Continued)

Random sequence genera- Low risk tion (selection bias)
Quote: "the randomization sequence was produced by an independent clinical research organization (Medical Excellence, Seoul, Korea) and was computer-generated and stratified by sites with block sizes of four"

\begin{tabular}{|c|c|c|}
\hline $\begin{array}{l}\text { Allocation concealment } \\
\text { (selection bias) }\end{array}$ & Low risk & $\begin{array}{l}\text { Quote: "allocation concealment was implemented by use of sequentially num- } \\
\text { bered, opaque, and sealed envelopes" }\end{array}$ \\
\hline
\end{tabular}

Low risk

Blinding of participants

Quote: "randomized, controlled, double-blind trial...all patients and investigaand personnel (perfortors were masked to the treatment assignment"

mance bias)

All outcomes

Blinding of outcome as- Low risk

sessment (detection bias)

All outcomes

Incomplete outcome data Low risk

(attrition bias)

All outcomes

\begin{tabular}{|c|c|c|}
\hline $\begin{array}{l}\text { Selective reporting (re- } \\
\text { porting bias) }\end{array}$ & Low risk & $\begin{array}{l}\text { Comment: no previously published protocol was available; adverse events and } \\
\text { either mortality or fatty liver resolution or both were reported }\end{array}$ \\
\hline
\end{tabular}

Other bias Low risk Comment: no other bias noted

Bahrami 2020

\section{Study characteristics}

\begin{tabular}{|c|c|}
\hline Methods & Randomised clinical trial \\
\hline Participants & $\begin{array}{l}\text { Country: Iran } \\
\text { Period of recruitment: not stated } \\
\text { Number randomised: } 50 \\
\text { Post-randomisation dropouts: } 5(10.0 \%) \\
\text { Revised sample size: } 45 \\
\text { Reasons for post-randomisation dropouts: discontinued, travel } \\
\text { Average age, years: } 40 \\
\text { Females: } 14 \text { (31.1\%) } \\
\text { NASH: not stated } \\
\text { Diabetes mellitus: not stated } \\
\text { Inclusion criteria: } 1 . \text { Age } \geq 18.2 \text {. Evidence of non-alcoholic fatty liver by ultrasound (steatosis score } \geq 1 \text { ) } \\
\text { and serum levels of alanine aminotransferase > } 30 \text { U/L for men and > } 19 \text { U/L for women } \\
\text { Exclusion criteria: } 1 . \text { Autoimmune disorders. } 2 \text {. Liver and biliary tract disease. 3. Cardiovascular dis- } \\
\text { ease. } 4 \text {. Diabetes mellitus. } 5 \text {. Renal disease. } 6 \text {. Metabolic disease. } 7 \text {. Malignancy. } 8 \text {. Hypothyroidism. } \\
\text { 9. Cushing's syndrome. } 10 \text {. Alcohol abuse and use of hepatotoxic drugs such as methotrexate, tamox- } \\
\text { ifen, amiodarone, and corticosteroids. } 11 \text {. Pregnancy or lactation. } 12 \text {. Night shift workers. 13. History of } \\
\text { bariatric surgery during the last year } \\
\text { Method for diagnosis of NAFLD: ultrasound }\end{array}$ \\
\hline
\end{tabular}

Interventions

Group 1: other supplements $(n=24)$

Further details: $6 \mathrm{mg}$ melatonin daily, 1 hour before bedtime (each tablet contains $3 \mathrm{mg}$ melatonin), for

12 weeks

Group 2: no active intervention $(n=21)$

Further details: participants in the placebo group received matching placebo (with same shape and colour of melatonin tablets) at the same time. Starch was the main ingredient in the placebo 
Bahrami 2020 (Continued)

Outcomes Outcomes reported: resolution of fatty liver disease

Follow-up, months: 2.8

Notes

Source of funding (quote): "Hyperlipidemia Research Center, Ahvaz Jundishapur University of Medical

Sciences (grant number: HLRC-9503)"

Trial name/Trial registry number: IRCT2016061516123N8

Attempts were made to contact study authors in April 2021

\section{Risk of bias}

\begin{tabular}{|c|c|c|}
\hline Bias & Authors' judgement & Support for judgement \\
\hline $\begin{array}{l}\text { Random sequence genera- } \\
\text { tion (selection bias) }\end{array}$ & Unclear risk & Comment: this information was not available \\
\hline $\begin{array}{l}\text { Allocation concealment } \\
\text { (selection bias) }\end{array}$ & Unclear risk & $\begin{array}{l}\text { Quote: "sealed envelopes were used to allocate patients to treatment or place- } \\
\text { bo groups" } \\
\text { Comment: further details were not available }\end{array}$ \\
\hline $\begin{array}{l}\text { Blinding of participants } \\
\text { and personnel (perfor- } \\
\text { mance bias) } \\
\text { All outcomes }\end{array}$ & Low risk & Quote: "double blind...placebo" \\
\hline $\begin{array}{l}\text { Blinding of outcome as- } \\
\text { sessment (detection bias) } \\
\text { All outcomes }\end{array}$ & Low risk & Quote: "double blind...placebo" \\
\hline $\begin{array}{l}\text { Incomplete outcome data } \\
\text { (attrition bias) } \\
\text { All outcomes }\end{array}$ & Unclear risk & $\begin{array}{l}\text { Comment: there were post-randomisation dropouts related to discontinuation } \\
\text { of treatment; it is not clear if this was related to the intervention and to out- } \\
\text { comes }\end{array}$ \\
\hline $\begin{array}{l}\text { Selective reporting (re- } \\
\text { porting bias) }\end{array}$ & High risk & Comment: outcomes specified in pre-published protocol were not reported \\
\hline Other bias & Low risk & Comment: no other bias noted \\
\hline
\end{tabular}

Bakhshimoghaddam 2018

\section{Study characteristics}

\begin{tabular}{ll}
\hline Methods & Randomised clinical trial \\
\hline Participants & Country: Iran \\
& Period of recruitment: 2016 to 2017 \\
& Number randomised: 68 \\
& Post-randomisation dropouts: 8 (11.8\%) \\
& Revised sample size: 60 \\
& Reasons for post-randomisation dropouts: lost-to follow-up, pregnant, taking excluded medication \\
& Average age, years: 40 \\
& Females: 52 (86.7\%) \\
& NASH: not stated \\
& Diabetes mellitus: 0 (0.0\%) \\
Inclusion criteria: 1. Grade 1 to 3 fatty liver. $2 . \geq 18$ years old \\
Exclusion criteria: 1. Alcohol history $>10 \mathrm{~g} / \mathrm{d}$ for women, $\geq 20 \mathrm{~g} / \mathrm{d}$ for men. 2 . Chronic viral hepatitis, au- \\
toimmune hepatitis, PBC, Wilson's disease, other liver disease. 3 . Diabetes. 4 . Impaired renal function.
\end{tabular}


Bakhshimoghaddam 2018 (Continued)

5. Drugs affecting glucose and lipid metabolism and medications known to increase risk of NAFLD. 6.

Preganancy/Lactation

Method for diagnosis of NAFLD: ultrasound

$\begin{array}{ll}\text { Interventions } & \text { Group 1: prebiotics/probiotics/synbiotics }(\mathrm{n}=32) \\ & \text { Further details: } 300 \mathrm{~g} \text { synbiotic yogurt (Bifidobacterium animalis for 24 weeks) } \\ & \text { Group 2: no active intervention }(\mathrm{n}=28) \\ & \text { Further details: no active intervention } \\ & \text { Additional details: both groups received lifestyle advice; another group not relevant to this review was } \\ & \text { excluded }\end{array}$

Outcomes Outcomes reported: mortality at maximal follow-up, serious adverse events (number of people), liver transplantation at maximal follow-up, decompensation (number of people), cirrhosis (number of people), resolution of fatty liver disease, hepatocellular carcinoma

Follow-up, months: 6

Notes
Azarbaijan Pegah Dairy Company (Urmia, Iran) supplied the synbiotic and conventional yogurts (author
replies)"
Trial name/Trial registry number: IRCT2017020932417N2
Attempts were made to contact study authors in December 2020

\section{Risk of bias}

\begin{tabular}{lll}
\hline Bias & Authors' judgement & Support for judgement \\
\hline $\begin{array}{l}\text { Random sequence genera- } \\
\text { tion (selection bias) }\end{array}$ & Low risk & Quote: "randomization lists were computer-generated by a statistician" \\
\hline $\begin{array}{l}\text { Allocation concealment } \\
\text { (selection bias) }\end{array}$ & Low risk & $\begin{array}{l}\text { Quote: "sequentially numbered, opaque, and sealed envelopes were used to } \\
\text { conceal allocation" } \\
\text { Comment: information from study author's email reply }\end{array}$
\end{tabular}

Blinding of participants High risk Quote: "open-label"
and personnel (perfor-
mance bias)
All outcomes

\begin{tabular}{lll}
\hline $\begin{array}{l}\text { Blinding of outcome as- } \\
\text { sessment (detection bias) } \\
\text { All outcomes }\end{array}$ & Unclear risk & $\begin{array}{l}\text { Quote: "the radiologist, laboratory staff, and statistician were blinded to the in- } \\
\text { tervention assignment until the end of the study" } \\
\text { Comment: it is not clear whether healthcare professionals assessing adverse } \\
\text { events were blinded }\end{array}$ \\
\hline $\begin{array}{l}\text { Incomplete outcome data } \\
\begin{array}{l}\text { (attrition bias) } \\
\text { All outcomes }\end{array}\end{array}$ & Unclear risk & $\begin{array}{l}\text { Comment: participants were excluded from analysis for reasons that may be } \\
\text { related to the intervention and to outcomes }\end{array}$ \\
\hline $\begin{array}{l}\text { Selective reporting (re- } \\
\text { porting bias) }\end{array}$ & Low risk & $\begin{array}{l}\text { Comment: no previously published protocol was available; adverse events and } \\
\text { either mortality or fatty liver resolution or both were reported }\end{array}$ \\
\hline \begin{tabular}{l} 
Other bias \\
\hline
\end{tabular} & Low risk & Comment: no other bias noted \\
\hline
\end{tabular}

\section{Study characteristics}


Barbakadze 2020 (Continued)

\begin{tabular}{|c|c|}
\hline Methods & Randomised clinical trial \\
\hline Participants & $\begin{array}{l}\text { Country: Georgia } \\
\text { Period of recruitment: not stated } \\
\text { Number randomised: } 72 \\
\text { Post-randomisation dropouts: not stated } \\
\text { Revised sample size: } 72 \\
\text { Average age, years: not stated } \\
\text { Females: not stated } \\
\text { NASH: } 72 \text { (100.0\%) } \\
\text { Diabetes mellitus: not stated } \\
\text { Inclusion criteria: } 1 \text {. Elevated aminotransferase levels. 2. Drinking < } 40 \mathrm{~g} \text { alcohol/week. 3. NASH diagno- } \\
\text { sis } \\
\text { Exclusion criteria: } 1 \text {. Drinking } \geq 40 \text { g alcohol/week } \\
\text { Method for diagnosis of NAFLD: not stated }\end{array}$ \\
\hline Interventions & $\begin{array}{l}\text { Group 1: vitamin E plus vitamin C }(n=52) \\
\text { Further details: vitamin E } 800 \mathrm{mg} / \mathrm{d} \text { plus vitamin C } 500 \mathrm{mg} / \mathrm{d} \\
\text { Group 2: no active intervention }(\mathrm{n}=20) \\
\text { Further details: did not receive any medical treatment }\end{array}$ \\
\hline Outcomes & No outcomes of interest were reported \\
\hline Notes & $\begin{array}{l}\text { Source of funding: not stated } \\
\text { Trial name/Trial registry number: not stated } \\
\text { Attempts were made to contact study authors in April } 2021\end{array}$ \\
\hline
\end{tabular}

\section{Risk of bias}

\begin{tabular}{|c|c|c|}
\hline Bias & Authors' judgement & Support for judgement \\
\hline $\begin{array}{l}\text { Random sequence genera- } \\
\text { tion (selection bias) }\end{array}$ & Unclear risk & Comment: this information was not available \\
\hline $\begin{array}{l}\text { Allocation concealment } \\
\text { (selection bias) }\end{array}$ & Unclear risk & Comment: this information was not available \\
\hline $\begin{array}{l}\text { Blinding of participants } \\
\text { and personnel (perfor- } \\
\text { mance bias) } \\
\text { All outcomes }\end{array}$ & Unclear risk & Comment: this information was not available \\
\hline $\begin{array}{l}\text { Blinding of outcome as- } \\
\text { sessment (detection bias) } \\
\text { All outcomes }\end{array}$ & Unclear risk & Comment: this information was not available \\
\hline $\begin{array}{l}\text { Incomplete outcome data } \\
\text { (attrition bias) } \\
\text { All outcomes }\end{array}$ & Unclear risk & Comment: this information was not available \\
\hline $\begin{array}{l}\text { Selective reporting (re- } \\
\text { porting bias) }\end{array}$ & High risk & $\begin{array}{l}\text { Comment: no previously published protocol was available; adverse events, } \\
\text { mortality, fatty liver resolution were not reported }\end{array}$ \\
\hline Other bias & Low risk & Comment: no other bias noted \\
\hline
\end{tabular}


Barchetta 2016

\section{Study characteristics}

\begin{tabular}{ll}
\hline Methods & Randomised clinical trial \\
\hline Participants & Country: Italy \\
& Period of recruitment: not stated \\
& Number randomised: 65 \\
& Post-randomisation dropouts: 10 (15.4\%) \\
& Revised sample size: 55 \\
& Reasons for post-randomisation dropouts: discontinued treatment, including for adverse event \\
& Average age, years: 59 \\
& Females: 21 (38.2\%) \\
& NASH: not stated \\
& Diabetes mellitus: 55 (100.0\%) \\
Inclusion criteria: 1.25 to 70 years old. 2. Diagnosis of type 2 diabetes mellitus. 3. Fatty liver on USS and \\
confirmed on MRI in patients with suspected NAFLD (raised transaminase with no other cause of chron- \\
ic liver disease) \\
Exclusion criteria: 1. History of alcohol abuse (> 30 g/d men, > 20 g/d women). 2. Cirrhosis, viral hepati- \\
tis, autoimmune hepatitis, and other causes of liver disease. 3. Advanced renal failure. 4 . Cancer. 5 . Hy- \\
per/Hypoparathyroidism. 6. Previous 6 months' supplementation with vitamin D, calcium, multi-vita- \\
mins, agents affecting bone and calcium/vitamin D metabolism. 7 . UV radiation exposure. 8 . Pregnan- \\
cy/Lactation \\
Method for diagnosis of NAFLD: ultrasound plus MRI plus transaminases
\end{tabular}

\begin{tabular}{ll}
\hline Interventions & $\begin{array}{l}\text { Group 1: vitamin D }(\mathrm{n}=26) \\
\text { Further details: cholecalciferol }(2000 \mathrm{IU} / \mathrm{d}) \text { for } 24 \text { weeks } \\
\text { Group 2: no active intervention }(\mathrm{n}=29) \\
\text { Further details: placebo }\end{array}$ \\
\hline Outcomes & No outcomes of interest were reported \\
\hline Notes & $\begin{array}{l}\text { Source of funding (quote): "this work was funded by research grants from the Sapienza University Ate- } \\
\text { neo Scientific Research (MGC, IB) and the Italian Minister of University and Research (MGC, MGB)" } \\
\text { Trial name/Trial registry number: } 2011-003010-17 \\
\text { Attempts were made to contact study authors in December } 2020\end{array}$ \\
\hline
\end{tabular}

\section{Risk of bias}

\begin{tabular}{lll}
\hline Bias & Authors' judgement & Support for judgement \\
\hline $\begin{array}{l}\text { Random sequence genera- } \\
\text { tion (selection bias) }\end{array}$ & Low risk & $\begin{array}{l}\text { Quote: "randomization was performed by the statistician following acquisition } \\
\text { of participants' informed consent, through a computer-generated and central- } \\
\text { ly administered procedure" }\end{array}$ \\
\hline
\end{tabular}

\begin{tabular}{ll}
\hline $\begin{array}{l}\text { Allocation concealment } \\
\text { (selection bias) }\end{array}$ & $\begin{array}{l}\text { Quote: "randomization was performed by the statistician following acquisition } \\
\text { of participants' informed consent, through a computer-generated and central- } \\
\text { ly administered procedure" }\end{array}$
\end{tabular}

\begin{tabular}{|c|c|c|}
\hline $\begin{array}{l}\text { Blinding of participants } \\
\text { and personnel (perfor- } \\
\text { mance bias) } \\
\text { All outcomes }\end{array}$ & Low risk & Quote: "a randomized, double-blind, placebo-controlled trial" \\
\hline $\begin{array}{l}\text { Blinding of outcome as- } \\
\text { sessment (detection bias) } \\
\text { All outcomes }\end{array}$ & Low risk & Quote: "a randomized, double-blind, placebo-controlled trial" \\
\hline $\begin{array}{l}\text { Incomplete outcome data } \\
\text { (attrition bias) }\end{array}$ & Unclear risk & $\begin{array}{l}\text { Comment: participants were excluded from analysis for reasons that may be } \\
\text { related to the intervention and to outcomes }\end{array}$ \\
\hline
\end{tabular}

Nutritional supplementation for nonalcohol-related fatty liver disease: a network meta-analysis (Review) 
Barchetta 2016 (Continued)

All outcomes

\begin{tabular}{|c|c|c|}
\hline $\begin{array}{l}\text { Selective reporting (re- } \\
\text { porting bias) }\end{array}$ & High risk & $\begin{array}{l}\text { Comment: no previously published protocol was available; adverse events, } \\
\text { mortality, fatty liver resolution were not reported }\end{array}$ \\
\hline
\end{tabular}

Other bias Low risk Comment: no other bias noted

Basu 2012

\section{Study characteristics}

\begin{tabular}{ll}
\hline Methods & Randomised clinical trial \\
\hline Participants & Country: USA \\
Period of recruitment: not stated \\
Number randomised: 155 \\
Post-randomisation dropouts: not stated \\
Revised sample size: 155 \\
Average age, years: not stated \\
Females: not stated \\
NASH: 155 (100.0\%) \\
Diabetes mellitus: 0 (0.0\%) \\
Inclusion criteria: 1 . NAFLD, NASH. 2. BMI $\geq 28$ to $<33$ \\
Exclusion criteria: 1 . Normal antibodies for known liver disease. 2. Diabetes. 3. Viral hepatitis. 4. Hy- \\
po/Hyperthyroidism. 5 . Syndrome with known insulin resistance. 6 . Alcohol $>30 \mathrm{~g} / \mathrm{d}$. 7 . Use of other \\
medication including herbs and supplements \\
Method for diagnosis of NAFLD: not stated
\end{tabular}

Group 1: vitamin E plus other antioxidants ( $n=40)$
Further details: vitamin $E 700$ IU plus alfa lipoic acid 300 mg daily orally for 6 months
Group 2: other antioxidants $(n=40)$
Further details: alfa lipoic acid $300 \mathrm{mg}$ daily orally for 6 months
Group 3: vitamin $E(n=40)$
Further details: vitamin E $700 \mathrm{IU}$ daily orally for 6 months
Group 4: no active intervention ( $\mathrm{n}=35)$
Further details: no intervention

\begin{tabular}{ll}
\hline Outcomes & No outcomes of interest were reported \\
\hline Notes & Source of funding: not stated \\
& Trial name/Trial registry number: not stated \\
& Attempts were made to contact study authors in December 2020 \\
\hline
\end{tabular}

\section{Risk of bias}

\begin{tabular}{lll}
\hline Bias & Authors' judgement & Support for judgement \\
\hline $\begin{array}{l}\text { Random sequence genera- } \\
\text { tion (selection bias) }\end{array}$ & Unclear risk & Comment: this information was not available \\
\hline $\begin{array}{l}\text { Allocation concealment } \\
\text { (selection bias) }\end{array}$ & Unclear risk & Comment: this information was not available \\
\hline $\begin{array}{l}\text { Blinding of participants } \\
\text { and personnel (perfor- } \\
\text { mance bias) }\end{array}$ & High risk & Quote: "open label" \\
\end{tabular}


Basu 2012 (Continued)

All outcomes

Blinding of outcome as- $\quad$ High risk Quote: "open label"
sessment (detection bias)
All outcomes

All outcomes

Incomplete outcome data Unclear risk $\quad$ Comment: this information was not available
(attrition bias)

All outcomes

$\begin{array}{lll}\begin{array}{l}\text { Selective reporting (re- } \\ \text { porting bias) }\end{array} & \text { High risk } & \begin{array}{l}\text { Comment: no previously published protocol was available; adverse events, } \\ \text { mortality, fatty liver resolution were not reported }\end{array}\end{array}$
porting bias) mortality, fatty liver resolution were not reported

\begin{tabular}{ll}
\hline Other bias Low risk Comment: no other bias noted \\
\hline
\end{tabular}

\section{Basu 2013}

\section{Study characteristics}

\begin{tabular}{ll}
\hline Methods & Randomised clinical trial \\
\hline Participants & Country: USA \\
Period of recruitment: not stated & Number randomised: 60 \\
Post-randomisation dropouts: not stated & Revised sample size: 60 \\
Average age, years: not stated & Females: not stated \\
NASH: not stated \\
Diabetes mellitus: not stated \\
Inclusion criteria: 1 . Alcohol < 30 g/d \\
Exclusion criteria: 1 . HIV. 2. Medications causing fatty liver including herbal supplements. 3.Lipodystro- \\
phy. 4 . Overt diabetes mellitus. 5 . Pregnancy. 5 . Hypersensitivity to study medications \\
Method for diagnosis of NAFLD: not stated
\end{tabular}

Interventions

Group 1: vitamin E plus other supplements $(n=20)$

Further details: vitamin E plus curcumin (no further details available, probably for 12 months)

Group 2: vitamin $\mathrm{E}(\mathrm{n}=20)$

Further details: vitamin $\mathrm{E}$ (no further details available, probably for 12 months)

Group 3: other supplements $(n=20)$

Further details: curcumin (no further details available, probably for 12 months)

Additional details: another group not relevant to this review was excluded

\begin{tabular}{ll}
\hline Outcomes & No outcomes of interest were reported \\
\hline Notes & Source of funding: not stated \\
& Trial name/Trial registry number: not stated \\
& Attempts were made to contact study authors in December 2020
\end{tabular}

\section{Risk of bias}

\begin{tabular}{lll}
\hline Bias & Authors' judgement & Support for judgement \\
\hline $\begin{array}{l}\text { Random sequence genera- } \\
\text { tion (selection bias) }\end{array}$ & Unclear risk & Comment: this information was not available \\
\hline
\end{tabular}


Basu 2013 (Continued)

Allocation concealment Unclear risk $\quad$ Comment: this information was not available
(selection bias)

Blinding of participants

High risk

Quote: "open label"

and personnel (perfor-

mance bias)

All outcomes

$\begin{array}{lll}\text { Blinding of outcome as- } & \text { High risk } & \text { Quote: "open label" } \\ \text { sessment (detection bias) } & \end{array}$

detection bias

All outcomes

Incomplete outcome data Unclear risk $\quad$ Comment: this information was not available
(attrition bias)

All outcomes

\begin{tabular}{|c|c|c|}
\hline $\begin{array}{l}\text { Selective reporting (re- } \\
\text { porting bias) }\end{array}$ & High risk & $\begin{array}{l}\text { Comment: no previously published protocol was available; adverse events, } \\
\text { mortality, fatty liver resolution were not reported }\end{array}$ \\
\hline
\end{tabular}

porting bias)

Other bias Low risk Comment: no other bias noted

Behrouz 2017

\section{Study characteristics}

\begin{tabular}{|c|c|}
\hline Methods & Randomised clinical trial \\
\hline Participants & $\begin{array}{l}\text { Country: Iran } \\
\text { Period of recruitment: not stated } \\
\text { Number randomised: } 111 \\
\text { Post-randomisation dropouts: } 22 \text { (19.8\%) } \\
\text { Revised sample size: } 89 \\
\text { Reasons for post-randomisation dropouts: non-compliance (1), lost to follow-up (14), withdrew (1), } \\
\text { travel (3), refused to give blood (2), pregnant (1) } \\
\text { Average age, years: } 38 \\
\text { Females: } 26 \text { ( } 29.2 \%) \\
\text { NASH: not stated } \\
\text { Diabetes mellitus: } 0 \text { (0.0\%) } \\
\text { Inclusion criteria: } 1 . \text { BMI } 25 \text { to } 40.2 .20 \text { to } 60 \text { years old. 3. NAFLD diagnosis based on ALT > 1.5× upper } \\
\text { limit and steatosis on USS > grade } 2 \\
\text { Exclusion criteria: } 1 \text {. Pregnancy/Lactation. 2. Consumption of omega-3 fatty acids and nutritional sup- } \\
\text { plements in previous year. } 3 \text {. Other acute and chronic liver disease, cirrhosis. 4. Coeliac disease. 5. Dia- } \\
\text { betes. } 6 \text {. Hypertension, cardiovascular disease, kidney disease, or lung disease. } 7 \text {. Alcohol abuse. } 8 \text {. An- } \\
\text { tibiotic use over } 1 \text { week during the study. } 9 \text {. Contraceptive pill, corticosteroid, NSAID, another drug. } 9 \text {. } \\
\text { Significant changes in recommended diet and daily physical activity } \\
\text { Method for diagnosis of NAFLD: ultrasound and transaminases }\end{array}$ \\
\hline
\end{tabular}

Interventions Group 1: prebiotics/probiotics/synbiotics ( $\mathrm{n}=59)$

Further details: 1 Webber Naturals capsule (probiotic containing Lactobacillus casei, Lactobacillus rhamnosus, Lactobacillus acidophilus, Bifidobacterium longum, Bifidobacterium breve) or ORAFTI P95

(oligofructose) $16 \mathrm{~g} / \mathrm{d}$ (prebiotic group) for 12 weeks

Group 2: no active intervention $(n=30)$

Further details: placebo

Additional details: both groups received lifestyle advice

Outcomes No outcomes of interest were reported


Behrouz 2017 (Continued)

Notes

Source of funding (quote): "the present study was supported by a grant from Vice Chancellor for Research, Iran University of Medical Sciences, Tehran, Iran (no. 24996)"

Trial name/Trial registry number: IRCT201410052394N13

Attempts were made to contact study authors in December 2020

\section{Risk of bias}

\begin{tabular}{|c|c|c|}
\hline Bias & Authors' judgement & Support for judgement \\
\hline $\begin{array}{l}\text { Random sequence genera- } \\
\text { tion (selection bias) }\end{array}$ & Unclear risk & Comment: this information was not available \\
\hline $\begin{array}{l}\text { Allocation concealment } \\
\text { (selection bias) }\end{array}$ & Unclear risk & Comment: this information was not available \\
\hline $\begin{array}{l}\text { Blinding of participants } \\
\text { and personnel (perfor- } \\
\text { mance bias) } \\
\text { All outcomes }\end{array}$ & Low risk & Quote: "double-blind, placebo-controlled trial" \\
\hline $\begin{array}{l}\text { Blinding of outcome as- } \\
\text { sessment (detection bias) } \\
\text { All outcomes }\end{array}$ & Low risk & Quote: "double-blind, placebo-controlled trial" \\
\hline $\begin{array}{l}\text { Incomplete outcome data } \\
\text { (attrition bias) } \\
\text { All outcomes }\end{array}$ & Unclear risk & $\begin{array}{l}\text { Comment: participants were excluded from analysis for reasons that may be } \\
\text { related to the intervention and to outcomes }\end{array}$ \\
\hline $\begin{array}{l}\text { Selective reporting (re- } \\
\text { porting bias) }\end{array}$ & High risk & $\begin{array}{l}\text { Comment: no previously published protocol was available; adverse events, } \\
\text { mortality, fatty liver resolution were not reported }\end{array}$ \\
\hline Other bias & Low risk & Comment: no other bias noted \\
\hline
\end{tabular}

Bomhof 2018

\section{Study characteristics}

\begin{tabular}{|c|c|}
\hline Methods & Randomised clinical trial \\
\hline Participants & $\begin{array}{l}\text { Country: Canada } \\
\text { Period of recruitment: not stated } \\
\text { Number randomised: } 14 \\
\text { Post-randomisation dropouts: } 1(7.1 \%) \\
\text { Revised sample size: } 13 \\
\text { Reasons for post-randomisation dropouts: inadequate liver biopsy } \\
\text { Average age, years: } 49 \\
\text { Females: } 6 \text { ( } 46.2 \%) \\
\text { NASH: } 13 \text { (100.0\%) } \\
\text { Diabetes mellitus: not stated } \\
\text { Inclusion criteria: } 1 . \text { Liver biopsy-confirmed NASH (NAS } \geq 5) .2 . \geq 18 \text { years old. } 3 \text {. BMI > } 25 \text { (Caucasians), } \\
\geq 23 \text { (Asians). } 4 \text {. Serum ALT } \geq 1.5 \text { upper limit normal. } 5 \text {. No changes to lipid-lowering or diabetic medica- } \\
\text { tion in last } 3 \text { months } \\
\text { Exclusion criteria: } 1 \text {. Alcohol > } 20 \text { g/d women, > } 30 \mathrm{~g} / \mathrm{d} \text { men. } 2 \text {. Alternate aetiology for liver disease. } 3 \text {. } \\
\text { Use of orlistat, liraglutide, prebiotic, probiotic, antibiotic in last } 3 \text { months } \\
\text { Method for diagnosis of NAFLD: ultrasound plus transaminases }\end{array}$ \\
\hline
\end{tabular}


Bomhof 2018 (Continued)

Interventions Group 1: prebiotics/probiotics/synbiotics ( $\mathrm{n}=8)$

Further details: oligofructose (prebiotic $8 \mathrm{~g} / \mathrm{d}$ for 12 weeks followed by $16 \mathrm{~g} / \mathrm{d}$ for 24 weeks)

Group 2: no active intervention $(n=5)$

Further details: placebo

$\begin{array}{ll}\text { Outcomes } & \text { Outcomes reported: NAFLD activity score } \\ & \text { Follow-up, months: } 8\end{array}$

Notes $\quad$ Source of funding (quote): "MRB was supported by Alberta Innovates Health Solutions (AlHS)"

Trial name/Trial registry number: NCT03184376

Attempts were made to contact study authors in December 2020

\section{Risk of bias}

\begin{tabular}{lll}
\hline Bias & Authors' judgement & Support for judgement \\
\hline $\begin{array}{l}\text { Random sequence genera- } \\
\text { tion (selection bias) }\end{array}$ & Unclear risk & Comment: this information was not available \\
\hline $\begin{array}{l}\text { Allocation concealment } \\
\text { (selection bias) }\end{array}$ & Low risk & $\begin{array}{l}\text { Quote: "participants were randomly assigned to one of two groups by a senior } \\
\text { study investigator not involved in recruiting participants" }\end{array}$ \\
\hline $\begin{array}{l}\text { Blinding of participants } \\
\begin{array}{l}\text { and personnel (perfor- } \\
\text { mance bias) } \\
\text { All outcomes }\end{array}\end{array}$ & Unclear risk & $\begin{array}{l}\text { Quote: "placebo-controlled, randomized pilot trial...participants were blinded } \\
\text { to the treatment allocation" } \\
\text { Comment: it is not clear whether investigators were blinded }\end{array}$ \\
\hline
\end{tabular}

\begin{tabular}{lll}
\hline $\begin{array}{l}\text { Blinding of outcome as- } \\
\text { sessment (detection bias) } \\
\text { All outcomes }\end{array}$ & Unclear risk & $\begin{array}{l}\text { Quote: "placebo-controlled, randomized pilot trial...participants were blinded } \\
\text { to the treatment allocation" } \\
\text { Comment: it is not clear whether investigators were blinded }\end{array}$ \\
\hline $\begin{array}{l}\text { Incomplete outcome data } \\
\text { (attrition bias) }\end{array}$ & Low risk & $\begin{array}{l}\text { Comment: } 1 \text { participant was excluded because of inadequate liver biopsy; this } \\
\text { is unlikely to be related to outcomes }\end{array}$ \\
\hline
\end{tabular}

\begin{tabular}{|c|c|c|}
\hline $\begin{array}{l}\text { Selective reporting (re- } \\
\text { porting bias) }\end{array}$ & High risk & $\begin{array}{l}\text { Comment: no previously published protocol was available; adverse events, } \\
\text { mortality, fatty liver resolution were not reported }\end{array}$ \\
\hline
\end{tabular}

Other bias Low risk Comment: no other bias noted

Bonfrate 2015

\section{Study characteristics}

\begin{tabular}{ll}
\hline Methods & Randomised clinical trial \\
\hline Participants & Country: Italy \\
& Period of recruitment: not stated \\
& Number randomised: 40 \\
& Post-randomisation dropouts: not stated \\
& Revised sample size: 40 \\
& Average age, years: not stated \\
& Females: not stated \\
& NASH: not stated \\
Diabetes mellitus: not stated & Inclusion criteria: 1 . NAFLD and metabolic abnormalities
\end{tabular}


Bonfrate 2015 (Continued)

Exclusion criteria: not stated

Method for diagnosis of NAFLD: not stated

\begin{tabular}{ll}
\hline Interventions & Group 1: vitamin E plus other antioxidants ( $\mathrm{n}=$ not stated) \\
& Further details: Eurosil 85 complex (silybin-vit E complex) for 6 months \\
& Group 2: no active intervention ( $\mathrm{n}=$ not stated) \\
& Further details: placebo
\end{tabular}

\begin{tabular}{ll}
\hline Outcomes & No outcomes of interest were reported \\
\hline Notes & Source of funding: not stated \\
& Trial name/Trial registry number: not stated \\
& Attempts were made to contact study authors in December 2020
\end{tabular}

\section{Risk of bias}

\begin{tabular}{|c|c|c|}
\hline Bias & Authors' judgement & Support for judgement \\
\hline $\begin{array}{l}\text { Random sequence genera- } \\
\text { tion (selection bias) }\end{array}$ & Unclear risk & Comment: this information was not available \\
\hline $\begin{array}{l}\text { Allocation concealment } \\
\text { (selection bias) }\end{array}$ & Unclear risk & Comment: this information was not available \\
\hline $\begin{array}{l}\text { Blinding of participants } \\
\text { and personnel (perfor- } \\
\text { mance bias) } \\
\text { All outcomes }\end{array}$ & Low risk & Quote: "double-blind randomized placebo-controlled clinical study" \\
\hline $\begin{array}{l}\text { Blinding of outcome as- } \\
\text { sessment (detection bias) } \\
\text { All outcomes }\end{array}$ & Low risk & Quote: "double-blind randomized placebo-controlled clinical study" \\
\hline $\begin{array}{l}\text { Incomplete outcome data } \\
\text { (attrition bias) } \\
\text { All outcomes }\end{array}$ & Unclear risk & Comment: this information was not available \\
\hline $\begin{array}{l}\text { Selective reporting (re- } \\
\text { porting bias) }\end{array}$ & High risk & $\begin{array}{l}\text { Comment: no previously published protocol was available; adverse events, } \\
\text { mortality, fatty liver resolution were not reported }\end{array}$ \\
\hline Other bias & Low risk & Comment: no other bias noted \\
\hline
\end{tabular}

Boonyagard 2016

\section{Study characteristics}

\begin{tabular}{ll}
\hline Methods & Randomised clinical trial \\
\hline Participants & Country: Thailand \\
& Period of recruitment: 2015 \\
& Number randomised: 60 \\
& Post-randomisation dropouts: not stated \\
& Revised sample size: 60 \\
Average age, years: not stated & Females: not stated \\
NASH: not stated
\end{tabular}


Boonyagard 2016 (Continued)

Diabetes mellitus: not stated

Inclusion criteria: 1. NAFLD and vitamin D deficiency

Exclusion criteria: not stated

Method for diagnosis of NAFLD: not stated

Group 1: vitamin D ( $\mathrm{n}=30)$

Further details: vitamin $D$ for 20 weeks (no further details)

Group 2: no active intervention ( $n=30$ )

Further details: placebo

\begin{tabular}{ll}
\hline Outcomes & No outcomes of interest were reported \\
\hline Notes & Source of funding: not stated \\
& Trial name/Trial registry number: not stated \\
& Attempts were made to contact study authors in December 2020 \\
\hline
\end{tabular}

\section{Risk of bias}

\begin{tabular}{lll}
\hline Bias & Authors' judgement & Support for judgement \\
\hline $\begin{array}{l}\text { Random sequence genera- } \\
\text { tion (selection bias) }\end{array}$ & Unclear risk & Comment: this information was not available \\
\hline $\begin{array}{l}\text { Allocation concealment } \\
\text { (selection bias) }\end{array}$ & Unclear risk & Comment: this information was not available \\
\hline $\begin{array}{l}\text { Blinding of participants } \\
\text { and personnel (perfor- } \\
\text { mance bias) }\end{array}$ & Unclear risk & Comment: this information was not available \\
All outcomes & & \\
\hline
\end{tabular}

$\begin{array}{ll}\text { Blinding of outcome as- } & \text { Unclear risk } \quad \text { Comment: this information was not available } \\ \text { sessment (detection bias) } & \\ \text { All outcomes } & \end{array}$

\begin{tabular}{lll}
$\begin{array}{l}\text { Incomplete outcome data } \\
\text { (attrition bias) } \\
\text { All outcomes }\end{array}$ & Unclear risk & Comment: this information was not available \\
\hline $\begin{array}{l}\text { Selective reporting (re- } \\
\text { porting bias) }\end{array}$ & High risk & $\begin{array}{l}\text { Comment: no previously published protocol was available; adverse events, } \\
\text { mortality, fatty liver resolution were not reported }\end{array}$ \\
\hline Other bias & Low risk & Comment: no other bias noted \\
\hline
\end{tabular}

Boonyagard 2020

\section{Study characteristics}

\begin{tabular}{ll}
\hline Methods & Randomised clinical trial \\
\hline Participants & Country: Thailand \\
& Period of recruitment: 2015 to 2018 \\
& Number randomised: 63 \\
& Post-randomisation dropouts: $3(4.8 \%)$ \\
& Revised sample size: 60 \\
& Reasons for post-randomisation dropouts: did not receive the intervention
\end{tabular}


Boonyagard 2020 (Continued)

Average age, years: 54

Females: $31(51.7 \%)$

NASH: not stated

Diabetes mellitus: 24 (40.0\%)

Inclusion criteria: 1. Diagnosed NAFLD by ultrasonography. 2. Increased levels of alanine transaminase

(ALT). 3. Serum vitamin D level $<30 \mathrm{ng} / \mathrm{mL}$

Exclusion criteria: 1 . Alcohol consumption > 14 drinks/week in women, > 21 drinks/week in men. 2.

Pregnancy and nursing. 3. Known hepatic disease such as hereditary haemochromatosis, Wilson's dis-

ease, and a1-antitrypsin deficiency. 4. History of jejunoileal bypass surgery or gastroplasty. 5. Using to-

tal parenteral nutrition in the past 6 months. 6 . Taking potential hepatotoxic drugs such as high dos-

es of synthetic oestrogens, methotrexate, amiodarone, or chloroquine. 7 . History of hypothyroidism,

Cushing's syndrome, renal failure, or kidney stones. 8. Serum calcium levels $>10.6 \mathrm{mg} / \mathrm{dL}$ and use of vi-

tamin D, vitamin E, and calcium supplements during the last 6 months

Method for diagnosis of NAFLD: ultrasonography and transaminases

\begin{tabular}{ll}
\hline Interventions & Group 1: vitamin D $(n=30)$ \\
& Further details: 40,000 IU vitamin D2 weekly for 5 months \\
& Group 2: no active intervention $(n=30)$ \\
& Further details: placebo
\end{tabular}

\begin{tabular}{ll}
\hline Outcomes & $\begin{array}{l}\text { Outcomes reported: mortality, adverse events, liver transplantation, cirrhosis, decompensated cirrho- } \\
\text { sis, hepatocellular carcinoma } \\
\text { Follow-up, months: } 5\end{array}$ \\
\hline Notes & Source of funding (quote): "we would like to thank Faculty of Medicine and Vajira Hospital, Navamin- \\
& dradhiraj University Research Fund for the funding support" \\
& Trial name/Trial registry number: not stated \\
& Attempts were made to contact study authors in April 2021
\end{tabular}

\section{Risk of bias}

\begin{tabular}{lll}
\hline Bias & Authors' judgement & Support for judgement \\
\hline $\begin{array}{l}\text { Random sequence genera- } \\
\text { tion (selection bias) }\end{array}$ & Low risk & Quote: "computer random number generator" \\
\hline $\begin{array}{l}\text { Allocation concealment } \\
\text { (selection bias) }\end{array}$ & Low risk & $\begin{array}{l}\text { Quote: "randomization and allocation were concealed from the researchers } \\
\text { and participants until the statistical analysis was completed...placebo" } \\
\text { Comment: both allocation concealment and blinding were achieved with use } \\
\text { of placebo }\end{array}$
\end{tabular}

\begin{tabular}{ll}
\hline $\begin{array}{l}\text { Blinding of participants } \\
\text { and personnel (perfor- }\end{array}$ & Low risk \\
manote: "randomization and allocation were concealed from the researchers \\
and participants until the statistical analysis was completed...placebo"
\end{tabular}

mance bias)

All outcomes

\begin{tabular}{|c|c|c|}
\hline $\begin{array}{l}\text { Blinding of outcome as- } \\
\text { sessment (detection bias) }\end{array}$ & Low risk & $\begin{array}{l}\text { Quote: "randomization and allocation were concealed from the researchers } \\
\text { and participants until the statistical analysis was completed...placebo" }\end{array}$ \\
\hline
\end{tabular}

All outcomes

\begin{tabular}{lll}
\hline $\begin{array}{l}\text { Incomplete outcome data } \\
\text { (attrition bias) } \\
\text { All outcomes }\end{array}$ & Unclear risk & $\begin{array}{l}\text { Comment: there were post-randomisation dropouts; it is not clear whether } \\
\text { these could be related to the intervention and to outcomes }\end{array}$ \\
\hline $\begin{array}{l}\text { Selective reporting (re- } \\
\text { porting bias) }\end{array}$ & Low risk & $\begin{array}{l}\text { Comment: no previously published protocol was available; adverse events and } \\
\text { either mortality or fatty liver resolution or both were reported }\end{array}$
\end{tabular}

\begin{tabular}{ll}
\hline Other bias $\quad$ Low risk $\quad$ Comment: no other bias noted \\
\hline
\end{tabular}


Boyraz 2015

\section{Study characteristics}

\begin{tabular}{|c|c|}
\hline Methods & Randomised clinical trial \\
\hline Participants & $\begin{array}{l}\text { Country: Turkey } \\
\text { Period of recruitment: } 2010 \text { to } 2012 \\
\text { Number randomised: } 138 \\
\text { Post-randomisation dropouts: } 30 \text { (21.7\%) } \\
\text { Revised sample size: } 108 \\
\text { Reasons for post-randomisation dropouts: did not complete the protocol } \\
\text { Average age, years: } 14 \\
\text { Females: } 53 \text { ( } 49.1 \%) \\
\text { NASH: not stated } \\
\text { Diabetes mellitus: not stated } \\
\text { Inclusion criteria: } 1 \text {. BMI > } 95 \text { th percentile for age and sex. 2. Persistently elevated serum aminotrans- } \\
\text { ferase levels. 3. Diffusely echogenic liver in imaging studies suggestive of fatty liver } \\
\text { Exclusion criteria: } 1 \text {. Viral hepatitis. 2. Alcohol consumption. 3. History of parenteral nutrition. 4. Use of } \\
\text { drugs known to induce steatosis } \\
\text { Method for diagnosis of NAFLD: ultrasound plus transaminases }\end{array}$ \\
\hline Interventions & $\begin{array}{l}\text { Group 1: PUFA ( } n=56) \\
\text { Further details: Marincap ( } n 3-P U F A) \text { once daily for } 12 \text { months } \\
\text { Group 2: no active intervention ( } n=52 \text { ) } \\
\text { Further details: placebo } \\
\text { Additional details: both groups received lifestyle intervention and advice (scheduled exercise and calo- } \\
\text { rie restriction advice) }\end{array}$ \\
\hline Outcomes & No outcomes of interest were reported \\
\hline Notes & $\begin{array}{l}\text { Source of funding (quote): "we had no source of funding" } \\
\text { Trial name/Trial registry number: not stated } \\
\text { Attempts were made to contact study authors in December } 2020\end{array}$ \\
\hline
\end{tabular}

\section{Risk of bias}

\begin{tabular}{lll}
\hline Bias & Authors' judgement & Support for judgement \\
\hline $\begin{array}{l}\text { Random sequence genera- } \\
\text { tion (selection bias) }\end{array}$ & Unclear risk & Comment: this information was not available \\
\hline $\begin{array}{l}\text { Allocation concealment } \\
\text { (selection bias) }\end{array}$ & Unclear risk & Comment: this information was not available \\
\hline $\begin{array}{l}\text { Blinding of participants } \\
\text { and personnel (perfor- } \\
\text { mance bias) }\end{array}$ & Unclear risk & $\begin{array}{l}\text { Quote: "double-blind...the radiologist was blinded to all clinical and biochem- } \\
\text { ical characteristics of the subjects" } \\
\text { All outcomes }\end{array}$ \\
\hline $\begin{array}{l}\text { Blinding of outcome as- } \\
\text { sessment (detection bias) } \\
\text { All outcomes }\end{array}$ & Low risk & $\begin{array}{l}\text { Quote: "double-blind...the radiologist was blinded to all clinical and biochem- } \\
\text { ical characteristics of the subjects" } \\
\text { Comment: there were no clinical outcomes reported in this trial; therefore, ra- } \\
\text { diologist blinding indicates outcome assessor blinding }\end{array}$ \\
\hline $\begin{array}{l}\text { Incomplete outcome data } \\
\text { (attrition bias) } \\
\text { All outcomes }\end{array}$ & Unclear risk & $\begin{array}{l}\text { Comment: participants were excluded from analysis for reasons that may be } \\
\text { related to the intervention and to outcomes }\end{array}$ \\
\hline
\end{tabular}


Boyraz 2015 (Continued)

\begin{tabular}{|c|c|c|}
\hline $\begin{array}{l}\text { Selective reporting (re- } \\
\text { porting bias) }\end{array}$ & High risk & $\begin{array}{l}\text { Comment: no previously published protocol was available; adverse events, } \\
\text { mortality, fatty liver resolution were not reported }\end{array}$ \\
\hline
\end{tabular}

Other bias Low risk Comment: no other bias noted

Bril 2019

\section{Study characteristics}

\begin{tabular}{|c|c|c|}
\hline Methods & \multicolumn{2}{|c|}{ Randomised clinical trial } \\
\hline Participants & \multicolumn{2}{|c|}{$\begin{array}{l}\text { Country: USA } \\
\text { Period of recruitment: } 2010 \text { to } 2016 \\
\text { Number randomised: } 68 \\
\text { Post-randomisation dropouts: } 0(0.0 \%) \\
\text { Revised sample size: } 68 \\
\text { Average age, years: } 59 \\
\text { Females: } 5 \text { ( } 7.4 \%) \\
\text { NASH: } 68 \text { (100.0\%) } \\
\text { Diabetes mellitus: } 68 \text { (100.0\%) } \\
\text { Inclusion criteria: } 1 \text {. Diagnosis of type } 2 \text { diabetes mellitus. } 2 \text {. Histologically confirmed NASH } \\
\text { Exclusion criteria: } 1 \text {. Other cause of chronic liver disease (e.g. viral hepatitis). 2. Alcohol abuse. 3. Type } \\
1 \text { diabetes mellitus. } 4 \text {. Total parenteral nutrition within past } 6 \text { months. 5. Hepatotoxic drugs. } 6 \text {. Severe } \\
\text { osteoporosis } \\
\text { Method for diagnosis of NAFLD: liver biopsy }\end{array}$} \\
\hline Interventions & \multicolumn{2}{|c|}{$\begin{array}{l}\text { Group 1: vitamin E ( } n=36) \\
\text { Further details: vitamin E } 400 \text { IU orally twice daily for } 18 \text { months } \\
\text { Group 2: no active intervention ( } n=32 \text { ) } \\
\text { Further details: placebo } \\
\text { Additional details: both groups received lifestyle intervention; another group not relevant to this review } \\
\text { was excluded }\end{array}$} \\
\hline Outcomes & \multicolumn{2}{|c|}{$\begin{array}{l}\text { Outcomes reported: mortality at maximal follow-up, serious adverse events (number of people), seri- } \\
\text { ous adverse events (number of events), any adverse events (number of events), fibrosis score } \\
\text { Follow-up, months: } 18\end{array}$} \\
\hline Notes & \multicolumn{2}{|c|}{$\begin{array}{l}\text { Source of funding (quote): "this work was supported by a U.S. Department of Veterans Affairs Merit } \\
\text { Award" } \\
\text { Trial name/Trial registry number: NCT01002547 } \\
\text { Attempts were made to contact study authors in December } 2020\end{array}$} \\
\hline \multicolumn{3}{|l|}{ Risk of bias } \\
\hline Bias & Authors' judgement & Support for judgement \\
\hline $\begin{array}{l}\text { Random sequence genera- } \\
\text { tion (selection bias) }\end{array}$ & Low risk & $\begin{array}{l}\text { Quote: "the computer-generated randomization and patient allocation were } \\
\text { performed by the research pharmacist without any stratification and using a } \\
\text { block factor of } 4 \text {, which was unknown to investigators" }\end{array}$ \\
\hline $\begin{array}{l}\text { Allocation concealment } \\
\text { (selection bias) }\end{array}$ & Low risk & $\begin{array}{l}\text { Quote: "the computer-generated randomization and patient allocation were } \\
\text { performed by the research pharmacist without any stratification and using a } \\
\text { block factor of } 4 \text {, which was unknown to investigators" }\end{array}$ \\
\hline
\end{tabular}


Bril 2019 (Continued)

Blinding of participants Low risk Quote: "randomized, double-blind, placebo-controlled" and personnel (perfor-

mance bias)

All outcomes

Blinding of outcome as-
sessment (detection bias) $\quad$ Low risk Quote: "randomized, double-blind, placebo-controlled"

All outcomes

Incomplete outcome data Low risk Comment: there were no post-randomisation dropouts
(attrition bias)

All outcomes

\begin{tabular}{lll}
\hline $\begin{array}{l}\text { Selective reporting (re- } \\
\text { porting bias) }\end{array}$ & Low risk & $\begin{array}{l}\text { Comment: no previously published protocol was available; adverse events and } \\
\text { either mortality or fatty liver resolution or both were reported }\end{array}$ \\
\hline Other bias & Low risk & Comment: no other bias noted \\
\hline
\end{tabular}

Byrne 2014

\section{Study characteristics}

\begin{tabular}{|c|c|c|}
\hline Methods & \multicolumn{2}{|c|}{ Randomised clinical trial } \\
\hline Participants & \multicolumn{2}{|c|}{$\begin{array}{l}\text { Country: United Kingdom } \\
\text { Period of recruitment: not stated } \\
\text { Number randomised: } 103 \\
\text { Post-randomisation dropouts: not stated } \\
\text { Revised sample size: } 103 \\
\text { Average age, years: not stated } \\
\text { Females: not stated } \\
\text { NASH: not stated } \\
\text { Diabetes mellitus: not stated } \\
\text { Inclusion and exclusion criteria: not stated } \\
\text { Method for diagnosis of NAFLD: not stated }\end{array}$} \\
\hline Interventions & \multicolumn{2}{|c|}{$\begin{array}{l}\text { Group 1: PUFA }(n=51) \\
\text { Further details: Omacor }(4 \mathrm{~g} / \mathrm{d})(\mathrm{n}-3 \text { fatty acid containing eicosapentaenoic acid }(E P A) \text { and docosa- } \\
\text { hexaenoic acid }(D H A)) \text { for } 15 \text { to } 18 \text { months } \\
\text { Group 2: no active intervention ( } n=52) \\
\text { Further details: placebo }\end{array}$} \\
\hline Outcomes & \multicolumn{2}{|c|}{ No outcomes of interest were reported } \\
\hline Notes & \multicolumn{2}{|c|}{$\begin{array}{l}\text { Source of funding (quote): "supported by: NIHR; Diabetes UK" } \\
\text { Trial name/Trial registry number: not stated } \\
\text { Attempts were made to contact study authors in December } 2020\end{array}$} \\
\hline \multicolumn{3}{|l|}{ Risk of bias } \\
\hline Bias & Authors' judgement & Support for judgement \\
\hline $\begin{array}{l}\text { Random sequence genera- } \\
\text { tion (selection bias) }\end{array}$ & Unclear risk & Comment: this information was not available \\
\hline
\end{tabular}


Byrne 2014 (Continued)

Allocation concealment $\quad$ Unclear risk
(selection bias)

Blinding of participants Low risk Quote: "randomised double blind placebo controlled trial"
and personnel (perfor-
mance bias)
All outcomes

\begin{tabular}{lll}
\hline $\begin{array}{l}\text { Blinding of outcome as- } \\
\text { sessment (detection bias) } \\
\text { All outcomes }\end{array}$ & Low risk & Quote: "randomised double blind placebo controlled trial" \\
\hline $\begin{array}{l}\text { Incomplete outcome data } \\
\text { (attrition bias) } \\
\text { All outcomes }\end{array}$ & Unclear risk & Comment: this information was not available \\
\hline $\begin{array}{l}\text { Selective reporting (re- } \\
\text { porting bias) }\end{array}$ & High risk & $\begin{array}{l}\text { Comment: no previously published protocol was available; adverse events, } \\
\text { mortality, fatty liver resolution were not reported }\end{array}$ \\
\hline Other bias & Low risk & Comment: no other bias noted \\
\hline
\end{tabular}

Cai 2020

\section{Study characteristics}

\begin{tabular}{|c|c|}
\hline Methods & Randomised clinical trial \\
\hline Participants & $\begin{array}{l}\text { Country: China } \\
\text { Period of recruitment: } 2017 \text { to } 2019 \\
\text { Number randomised: } 140 \\
\text { Post-randomisation dropouts: not stated } \\
\text { Revised sample size: } 140 \\
\text { Average age, years: } 48 \\
\text { Females: } 55 \text { (39.3\%) } \\
\text { NASH: not stated } \\
\text { Diabetes mellitus: not stated } \\
\text { Inclusion criteria: } 1 \text {. With or without abnormal indexes of liver function. 2. Diffuse fatty liver detected } \\
\text { on ultrasound and NAFLD confirmed by ultrasound-guided biopsy. 3. Aged between } 18 \text { and } 59 \\
\text { Exclusion criteria: } 1 \text {. Other liver disease with definite aetiology, such as viral hepatitis. 2. Liver fibrosis } \\
\text { or cirrhosis. 3. Use of drugs for weight loss, regulating glucose, lipid metabolism, or intestinal flora, and } \\
\text { antibiotics in the past } 3 \text { months. } 4 \text {. Autoimmune disease or other severely chronic co-morbidities. } 5 \text {. } \\
\text { Shedding criteria: could not tolerate diet and exercise therapy; failure to co-operate during interven- } \\
\text { tion treatment as required; occurrence of other disease during intervention or follow-up } \\
\text { Method for diagnosis of NAFLD: ultrasound and biopsy }\end{array}$ \\
\hline Interventions & $\begin{array}{l}\text { Group 1: prebiotics/probiotics/synbiotics }(\mathrm{n}=70) \\
\text { Further details: live combined Bifidobacterium, Lactobacillus, and Enterococcus powder (Bifid Triple Vi- } \\
\text { able) was given orally, } 2 \mathrm{~g} / \mathrm{d} \\
\text { Group 2: no active intervention }(\mathrm{n}=70) \\
\text { Further details: } \text { no treatment } \\
\text { Additional details: both groups received lifestyle modification intervention }\end{array}$ \\
\hline Outcomes & No outcomes of interest were reported \\
\hline Notes & $\begin{array}{l}\text { Source of funding: not stated } \\
\text { Trial name/Trial registry number: not stated }\end{array}$ \\
\hline
\end{tabular}


Cai 2020 (Continued)

Attempts were made to contact study authors in April 2021

\section{Risk of bias}

\begin{tabular}{|c|c|c|}
\hline Bias & Authors' judgement & Support for judgement \\
\hline $\begin{array}{l}\text { Random sequence genera- } \\
\text { tion (selection bias) }\end{array}$ & Low risk & Comment: random numbers table \\
\hline $\begin{array}{l}\text { Allocation concealment } \\
\text { (selection bias) }\end{array}$ & Unclear risk & Comment: this information was not available \\
\hline $\begin{array}{l}\text { Blinding of participants } \\
\text { and personnel (perfor- } \\
\text { mance bias) } \\
\text { All outcomes }\end{array}$ & Unclear risk & Comment: this information was not available \\
\hline $\begin{array}{l}\text { Blinding of outcome as- } \\
\text { sessment (detection bias) } \\
\text { All outcomes }\end{array}$ & Unclear risk & Comment: this information was not available \\
\hline $\begin{array}{l}\text { Incomplete outcome data } \\
\text { (attrition bias) } \\
\text { All outcomes }\end{array}$ & Unclear risk & Comment: this information was not available \\
\hline $\begin{array}{l}\text { Selective reporting (re- } \\
\text { porting bias) }\end{array}$ & High risk & $\begin{array}{l}\text { Comment: no previously published protocol was available; adverse events, } \\
\text { mortality, fatty liver resolution were not reported }\end{array}$ \\
\hline Other bias & Low risk & Comment: no other bias noted \\
\hline
\end{tabular}

Celinski 2014

\section{Study characteristics}

\begin{tabular}{ll}
\hline Methods & Randomised clinical trial \\
\hline Participants & Country: Poland \\
& Period of recruitment: not stated \\
& Number randomised: 74 \\
& Post-randomisation dropouts: not stated \\
& Revised sample size: 74 \\
& Average age, years: 33 \\
& Females: $51(68.9 \%)$ \\
& NASH: 18 (24.3\%) \\
& Diabetes mellitus: 34 (45.9\%) \\
Inclusion and exclusion criteria: not stated & Method for diagnosis of NAFLD: liver biopsy \\
& Group 1: amino acids plus PUFA ( $\mathrm{n}=51$ ) \\
& Further details: tryptophan 1000 mg/d or melatonin $10 \mathrm{mg} / \mathrm{d}$ plus Essentiale forte (major component is \\
& PUFA) 3 tablets/d for 14 months \\
Group 2: PUFA ( $\mathrm{n}=23$ ) & Further details: Essentiale forte (PUFA) 3 tablets/d for 14 months \\
\hline Interventions & No outcomes of interest were reported \\
\hline Outcomes & \\
\hline
\end{tabular}


Celinski 2014 (Continued)

Notes

Source of funding: not stated

Trial name/Trial registry number: not stated

Attempts were made to contact study authors in December 2020

\section{Risk of bias}

\begin{tabular}{|c|c|c|}
\hline Bias & Authors' judgement & Support for judgement \\
\hline $\begin{array}{l}\text { Random sequence genera- } \\
\text { tion (selection bias) }\end{array}$ & Unclear risk & Comment: this information was not available \\
\hline $\begin{array}{l}\text { Allocation concealment } \\
\text { (selection bias) }\end{array}$ & Unclear risk & Comment: this information was not available \\
\hline $\begin{array}{l}\text { Blinding of participants } \\
\text { and personnel (perfor- } \\
\text { mance bias) } \\
\text { All outcomes }\end{array}$ & Unclear risk & Comment: this information was not available \\
\hline $\begin{array}{l}\text { Blinding of outcome as- } \\
\text { sessment (detection bias) } \\
\text { All outcomes }\end{array}$ & Unclear risk & Comment: this information was not available \\
\hline $\begin{array}{l}\text { Incomplete outcome data } \\
\text { (attrition bias) } \\
\text { All outcomes }\end{array}$ & Unclear risk & Comment: this information was not available \\
\hline $\begin{array}{l}\text { Selective reporting (re- } \\
\text { porting bias) }\end{array}$ & High risk & $\begin{array}{l}\text { Comment: no previously published protocol was available; adverse events, } \\
\text { mortality, fatty liver resolution were not reported }\end{array}$ \\
\hline Other bias & Low risk & Comment: no other bias noted \\
\hline
\end{tabular}

Cerletti 2020

\section{Study characteristics}

\begin{tabular}{|c|c|}
\hline Methods & Randomised clinical trial \\
\hline Participants & $\begin{array}{l}\text { Country: Italy } \\
\text { Period of recruitment: } 2015 \text { to } 2016 \\
\text { Number randomised: } 126 \\
\text { Post-randomisation dropouts: } 13 \text { (10.3\%) } \\
\text { Revised sample size: } 113 \\
\text { Reasons for post-randomisation dropouts: bone fracture (1), relocation (1), discontinued supplementa- } \\
\text { tion due to dermatitis (1), discontinued supplementation for faecal colour abnormality (green faeces) } \\
\text { (1), unmotivated personal decision (9) } \\
\text { Average age, years: } 55 \\
\text { Females: } 39 \text { (34.5\%) } \\
\text { NASH: not stated } \\
\text { Diabetes mellitus: } 27 \text { ( } 23.9 \% \text { ) } \\
\text { Inclusion criteria: } 1.18 \text { to } 80 \text { years of age. } 2 \text {. Mild or moderate hepatic steatosis on USS. 3. At least } 1 \text { of } \\
\text { serum ALT/AST/GGT levels higher than normal } \\
\text { Exclusion criteria: } 1 \text {. Alcohol abuse history (> } 210 \text { g/week in men, > } 140 \text { g/week in women). 2. Drugs as- } \\
\text { sociated with hepatic steatosis. 3. Malnutrition. } 4 \text {. Other liver disease. } 5 \text {. Severe renal, cardiac, or respi- } \\
\text { ratory failure. } 6 \text {. Malignancy. } 7 \text {. Intolerance to formulation. } 8 \text {. Pregnancy, lactation } \\
\text { Method for diagnosis of NAFLD: ultrasound plus transaminases }\end{array}$ \\
\hline
\end{tabular}


Cerletti 2020 (Continued)

Interventions

Group 1: control $(n=58)$

Further details: 2 capsules of control mixture once a day for 3 months. Comparator capsules (control) contained formulation excipients and the same amount of choline present in the active mixture (in the form of bitartrate salt)

Group 2: other supplements $(n=55)$

Further details: 2 capsules of nutraceutical mixture once a day for 3 months. Study product was a mixture of active ingredients, formulated as soft gel capsules, each composed of fish oil containing $70 \%$ DHA (250 mg), phosphatidylcholine concentrated in sunflower oil (150 mg), silymarin (75 mg), choline bitartrate (35 mg), curcumin ( $35 \mathrm{mg}$ ), and D- $\alpha$-tocopherol $(10 \mathrm{mg})$, for a total of $830 \mathrm{mg}$. Total content of choline is $21.5 \mathrm{mg}$ per capsule $(43 \mathrm{mg} / \mathrm{d})$

Additional details: both groups received lifestyle advice

Outcomes Outcomes reported: any adverse events (number of people)

Follow-up, months: 3

Notes Source of funding (quote): "the present study was supported by the Italian Ministry of University and Research (MIUR, PON01_01226/1 - Decr. N.I/ Ric 18-1-2010)"

Trial name/Trial registry number: NCT02369536

Attempts were made to contact study authors in April 2021

\section{Risk of bias}

\begin{tabular}{lll}
\hline Bias & Authors' judgement & Support for judgement \\
\hline $\begin{array}{l}\text { Random sequence genera- } \\
\text { tion (selection bias) }\end{array}$ & Low risk & $\begin{array}{l}\text { Quote: "the random allocation sequence to treatment was computer based } \\
\text { per blocks of four or six subjects stratified for each recruiting centre; it was } \\
\text { generated by the principal investigator (PI)'s statistician and forwarded to } \\
\text { each recruitment centre" }\end{array}$ \\
\hline
\end{tabular}

\begin{tabular}{ll}
\hline $\begin{array}{l}\text { Allocation concealment } \\
\text { (selection bias) }\end{array}$ & $\begin{array}{l}\text { Quote: "the random allocation sequence was generated by the principal inves- } \\
\text { tigator (PI)'s statistician and forwarded to each recruitment centre. The doc- } \\
\text { tor responsible for each recruitment centre enrolled participants and assigned } \\
\text { them to interventions, according to the allocation sequence received by the PI } \\
\text { of the trial" }\end{array}$
\end{tabular}

\begin{tabular}{|c|c|c|}
\hline $\begin{array}{l}\text { Blinding of participants } \\
\text { and personnel (perfor- } \\
\text { mance bias) }\end{array}$ & Low risk & $\begin{array}{l}\text { Quote: "double-blind, randomised, multicentre controlled trial... active and } \\
\text { control capsules were identical for organoleptic properties and coded as A and } \\
\text { B by the producer" }\end{array}$ \\
\hline
\end{tabular}

All outcomes

\begin{tabular}{|c|c|c|}
\hline $\begin{array}{l}\text { Blinding of outcome as- } \\
\text { sessment (detection bias) } \\
\text { All outcomes }\end{array}$ & Low risk & $\begin{array}{l}\text { Quote: "double-blind, randomised, multicentre controlled trial... active and } \\
\text { control capsules were identical for organoleptic properties and coded as A and } \\
\text { B by the producer" }\end{array}$ \\
\hline
\end{tabular}

Incomplete outcome data Unclear risk (attrition bias) these were related to the intervention or to outcomes

All outcomes

\begin{tabular}{lll}
\hline $\begin{array}{l}\text { Selective reporting (re- } \\
\text { porting bias) }\end{array}$ & Low risk & $\begin{array}{l}\text { Comment: all pre-defined outcomes in the protocol published before recruit- } \\
\text { ment were reported }\end{array}$ \\
\hline Other bias & Low risk & Comment: no other bias noted \\
\hline
\end{tabular}

\section{Study characteristics}

Nutritional supplementation for nonalcohol-related fatty liver disease: a network meta-analysis (Review)

Copyright @ 2021 The Cochrane Collaboration. Published by John Wiley \& Sons, Ltd. 
Chachay 2014 (Continued)

\begin{tabular}{ll} 
Methods & Randomised clinical trial \\
\hline Participants & Country: Australia \\
Period of recruitment: 2011 to 2012 \\
Number randomised: 20 \\
Post-randomisation dropouts: $0(0.0 \%)$ \\
Revised sample size: 20 \\
Average age, years: 48 \\
Females: 0 (0.0\%) \\
NASH: not stated \\
Diabetes mellitus: not stated \\
Inclusion criteria: 1 . Male. 2. BMI > 25. 3. Waist circumference $>90 \mathrm{~cm}$. 4 . Hepatic steatosis on USS \\
Exclusion: 1 . Any known cause of steatosis (e.g. viral hepatitis). 2 . Alcohol > 40 g/d. 3. Use of steatogenic \\
medication. 4 . Cirrhosis. 5 . Type 2 diabetes mellitus. 6 . History of chronic kidney disease or serious car- \\
diovascular disorder \\
Method for diagnosis of NAFLD: ultrasound
\end{tabular}

\begin{tabular}{ll}
\hline Interventions & Group 1: other supplements $(\mathrm{n}=10)$ \\
& $\begin{array}{l}\text { Further details: resveratrol } 3000 \mathrm{mg} \text { daily for } 8 \text { weeks } \\
\text { Group 2: no active intervention }(\mathrm{n}=10) \\
\text { Further details: placebo }\end{array}$ \\
\hline Outcomes & Outcomes reported: mortality at maximal follow-up \\
& Follow-up, months: 2 \\
\hline Notes & Source of funding: not stated \\
& Trial name/Trial registry number: ACTRN12612001135808 \\
& Attempts were made to contact study authors in December 2020
\end{tabular}

\section{Risk of bias}

\begin{tabular}{lll}
\hline Bias & Authors' judgement & Support for judgement \\
\hline $\begin{array}{l}\text { Random sequence genera- } \\
\text { tion (selection bias) }\end{array}$ & Low risk & Quote: "computer-generated" \\
\hline $\begin{array}{l}\text { Allocation concealment } \\
\text { (selection bias) }\end{array}$ & Low risk & $\begin{array}{l}\text { Quote: "randomized, double-blind, placebo-controlled trial...participants and } \\
\text { investigating staff were blinded to the randomization until completion of re- } \\
\text { sults analyses" }\end{array}$ \\
& $\begin{array}{l}\text { Comment: both allocation concealment and blinding were achieved with use } \\
\text { of placebo }\end{array}$
\end{tabular}

Blinding of participants Low risk Quote: "randomized, double-blind, placebo-controlled trial"
and personnel (perfor-
mance bias)
All outcomes

\begin{tabular}{ll}
\hline Blinding of outcome as- & Low risk $\quad$ Quote: "randomized, double-blind, placebo-controlled trial" \\
sessment (detection bias) &
\end{tabular}

sessment (detection bias)

All outcomes

\begin{tabular}{lll}
\hline $\begin{array}{l}\text { Incomplete outcome data } \\
\text { (attrition bias) } \\
\text { All outcomes }\end{array}$ & Low risk & Comment: there were no post-randomisation dropouts \\
\hline $\begin{array}{l}\text { Selective reporting (re- } \\
\text { porting bias) }\end{array}$ & High risk & $\begin{array}{l}\text { Comment: no previously published protocol was available; adverse events } \\
\text { were not reported adequately }\end{array}$ \\
\hline
\end{tabular}


Chachay 2014 (Continued)

Other bias Low risk Comment: no other bias noted

Chan 2017

\section{Study characteristics}

\begin{tabular}{|c|c|}
\hline Methods & Randomised clinical trial \\
\hline Participants & $\begin{array}{l}\text { Country: Malaysia } \\
\text { Period of recruitment: } 2012 \text { to } 2014 \\
\text { Number randomised: } 99 \\
\text { Post-randomisation dropouts: } 0(0.0 \%) \\
\text { Revised sample size: } 99 \\
\text { Average age, years: } 50 \\
\text { Females: } 53(53.5 \%) \\
\text { NASH: } 99 \text { (100.0\%) } \\
\text { Diabetes mellitus: } 53 \text { (53.5\%) } \\
\text { Inclusion criteria: } 1 .>18 \text { years old. } 2 \text {. Serum alanine aminotransferase (ALT) and/or aspartate amino- } \\
\text { transferase (AST) levels } \geq 40 \text { IU/L } \\
\text { Exclusion criteria: } 1 \text {. Cirrhosis. } 2 \text {. Significant alcohol intake. 3. Medications causing hepatic steatosis. } 4 . \\
\text { Viral hepatitis or other cause of chronic liver disease. } 5 \text {. Use of silymarin or other milk-thistle prepara- } \\
\text { tions, vitamin C, vitamin E, glutathione, alpha-tocopherol, or non-prescribed complementary alterna- } \\
\text { tive medications within the past } 30 \text { days } \\
\text { Method for diagnosis of NAFLD: liver biopsy }\end{array}$ \\
\hline
\end{tabular}

\begin{tabular}{ll}
\hline Interventions & Group 1: other antioxidants $(n=49)$ \\
& Further details: Silymarin $700 \mathrm{mg}, 3$ times daily, for 48 weeks \\
& Group 2: no active intervention $(n=50)$ \\
& Further details: placebo \\
& Additional details: both groups received lifestyle advice
\end{tabular}

Outcomes Outcomes reported: mortality at maximal follow-up, serious adverse events (number of people), any adverse events (number of people), liver transplantation at maximal follow-up, decompensation (number of people), cirrhosis (number of people), resolution of fatty liver disease, hepatocellular carcinoma, fibrosis score, NAFLD activity score

Follow-up, months: 11

Notes

Source of funding (quote): "this study was funded by the University of Malaya Research Grant (Project Number: RG536-13HTM) and Meda Group"

Trial name/Trial registry number: NCT02006498

Attempts were made to contact study authors in December 2020

\section{Risk of bias}

\begin{tabular}{lll}
\hline Bias & Authors' judgement & Support for judgement \\
\hline $\begin{array}{l}\text { Random sequence genera- } \\
\text { tion (selection bias) }\end{array}$ & Low risk & Quote: "a computer-generated table of random numbers was utilized" \\
\hline $\begin{array}{l}\text { Allocation concealment } \\
\text { (selection bias) }\end{array}$ & Low risk & $\begin{array}{l}\text { Quote: "the numbers were kept centrally at this institution's Clinical Investi- } \\
\text { gation Center and released to a research assistant only after a subject was re- } \\
\text { cruited into the study" }\end{array}$ \\
\hline $\begin{array}{l}\text { Blinding of participants } \\
\text { and personnel (perfor- } \\
\text { mance bias) }\end{array}$ & Low risk & Quote: "randomized, double-blind, placebo-controlled study" \\
\hline
\end{tabular}


Chan 2017 (Continued)

All outcomes

Blinding of outcome as-
sessment (detection bias) $\quad$ Low risk Quote: "randomized, double-blind, placebo-controlled study"

sessment (detection bias)

All outcomes

Incomplete outcome data Low risk Comment: there were no post-randomisation dropouts
(attrition bias)

All outcomes

\begin{tabular}{lll}
\hline $\begin{array}{l}\text { Selective reporting (re- } \\
\text { porting bias) }\end{array}$ & Low risk & $\begin{array}{l}\text { Comment: adverse events and either mortality or fatty liver resolution or both } \\
\text { were reported }\end{array}$ \\
\hline Other bias & Low risk & Comment: no other bias noted
\end{tabular}

\section{Chande 2006}

\section{Study characteristics}

\begin{tabular}{|c|c|}
\hline Methods & Randomised clinical trial \\
\hline Participants & $\begin{array}{l}\text { Country: Canada } \\
\text { Period of recruitment: } 2003 \text { to } 2004 \\
\text { Number randomised: } 8 \\
\text { Post-randomisation dropouts: } 0(0.0 \%) \\
\text { Revised sample size: } 8 \\
\text { Average age, years: } 53 \\
\text { Females: } 5(62.5 \%) \\
\text { NASH: } 8 \text { (100.0\%) } \\
\text { Diabetes mellitus: } 4 \text { (50.0\%) } \\
\text { Inclusion criteria: } 1.18 \text { to } 75 \text { years of age. 2. Minimum of } 3 \text { months elevated AST/ALT. 3. < } 20 \text { g alco- } \\
\text { hol/week. } 4 \text {. Biopsy-proven NASH } \\
\text { Exclusion criteria: } 1 \text {. Other liver, gastrointestinal, renal, cardiovascular, neurological, or haematological } \\
\text { disease or psychiatric disorder. 2. Pregnancy, breastfeeding, or lack of birth control. 3. Use of herbal/di- } \\
\text { etary supplements other than multi-vitamin/mineral formulations. 4. Any change in medication within } \\
4 \text { weeks. } 5 \text {. Participation in clinical trial within } 6 \text { weeks } \\
\text { Method for diagnosis of NAFLD: liver biopsy plus transaminases }\end{array}$ \\
\hline Interventions & $\begin{array}{l}\text { Group 1: other supplements }(\mathrm{n}=5) \\
\text { Further details: YHK ( } 500 \mathrm{mg} 3 \text { times daily) for } 8 \text { weeks. Yo Jyo Hen Shi Ko (YHK; Kyotsu Jigyo, Inc., } \\
\text { Japan), which is derived from Panax pseudoginseng, Eucommia ulmoides, Polygonati rhizoma, and Gly- } \\
\text { cyrrhiza glabra } \\
\text { Group 2: no active intervention ( } \mathrm{n}=3 \text { ) } \\
\text { Further details: identical placebo for } 8 \text { weeks }\end{array}$ \\
\hline Outcomes & $\begin{array}{l}\text { Outcomes reported: serious adverse events (number of people), serious adverse events (number of } \\
\text { events), any adverse events (number of events), quality of life (maximal follow-up) } \\
\text { Follow-up, months: } 3\end{array}$ \\
\hline Notes & $\begin{array}{l}\text { Source of funding (quote): "this study was funded by Kyotsu Jigyo, Inc., Japan" } \\
\text { Trial name/Trial registry number: not stated } \\
\text { Attempts were made to contact study authors in December } 2020\end{array}$ \\
\hline
\end{tabular}

\section{Risk of bias}


Chande 2006 (Continued)

\begin{tabular}{lll} 
Bias & Authors' judgement & Support for judgement \\
\hline $\begin{array}{l}\text { Random sequence genera- } \\
\text { tion (selection bias) }\end{array}$ & Unclear risk & Comment: this information was not available \\
\hline $\begin{array}{l}\text { Allocation concealment } \\
\text { (selection bias) }\end{array}$ & Low risk & $\begin{array}{l}\text { Quote: "masking: the YHK or identical placebo was prepackaged and coded } \\
\text { prior to delivery to our research office, to ensure complete double-blinding of } \\
\text { the study coordinator, investigators, and patients. The blinding code was not } \\
\text { broken until all patients had completed the study" }\end{array}$
\end{tabular}

\begin{tabular}{|c|c|c|}
\hline $\begin{array}{l}\text { Blinding of participants } \\
\text { and personnel (perfor- } \\
\text { mance bias) } \\
\text { All outcomes }\end{array}$ & Low risk & Quote: "randomized, double-blind, placebo-controlled trial" \\
\hline $\begin{array}{l}\text { Blinding of outcome as- } \\
\text { sessment (detection bias) } \\
\text { All outcomes }\end{array}$ & Low risk & Quote: "randomized, double-blind, placebo-controlled trial" \\
\hline $\begin{array}{l}\text { Incomplete outcome data } \\
\text { (attrition bias) } \\
\text { All outcomes }\end{array}$ & Low risk & Comment: there were no post-randomisation dropouts \\
\hline $\begin{array}{l}\text { Selective reporting (re- } \\
\text { porting bias) }\end{array}$ & High risk & $\begin{array}{l}\text { Comment: no previously published protocol was available; neither mortality } \\
\text { nor fatty liver resolution was reported }\end{array}$ \\
\hline Other bias & Low risk & Comment: no other bias noted \\
\hline
\end{tabular}

\section{Chen 2008}

\section{Study characteristics}

\begin{tabular}{ll}
\hline Methods & Randomised clinical trial \\
\hline Participants & Country: China \\
& Period of recruitment: not stated \\
& Number randomised: 46 \\
& Post-randomisation dropouts: not stated \\
& Revised sample size: 46 \\
& Average age, years: not stated \\
& Females: not stated \\
& NASH: not stated \\
& Diabetes mellitus: not stated \\
& Inclusion and exclusion criteria: not stated \\
& Method for diagnosis of NAFLD: clinical examination plus transaminases \\
\hline Interventions & Group 1: PUFA ( $\mathrm{n}=30$ ) \\
& Further details: omega-3 polyunsaturated fatty acid capsule (sea seal oil $4 \mathrm{~g} / \mathrm{d}$ or $5 \mathrm{~g} / \mathrm{d}$ decided ran- \\
domly) for 24 weeks \\
Group 2: no active intervention ( $\mathrm{n}=16$ ) \\
Further details: placebo \\
\hline No outcomes of interest were reported \\
\hline Outcomes & Source of funding: not stated \\
& Trial name/trial registry number: not stated \\
\hline
\end{tabular}


Chen 2008 (Continued)

Attempts were made to contact study authors in December 2020

\section{Risk of bias}

\begin{tabular}{|c|c|c|}
\hline Bias & Authors' judgement & Support for judgement \\
\hline $\begin{array}{l}\text { Random sequence genera- } \\
\text { tion (selection bias) }\end{array}$ & Unclear risk & Comment: this information was not available \\
\hline $\begin{array}{l}\text { Allocation concealment } \\
\text { (selection bias) }\end{array}$ & Unclear risk & Comment: this information was not available \\
\hline $\begin{array}{l}\text { Blinding of participants } \\
\text { and personnel (perfor- } \\
\text { mance bias) } \\
\text { All outcomes }\end{array}$ & Low risk & Quote: "randomized, double-blind, placebo-controlled trial" \\
\hline $\begin{array}{l}\text { Blinding of outcome as- } \\
\text { sessment (detection bias) } \\
\text { All outcomes }\end{array}$ & Low risk & Quote: "randomized, double-blind, placebo-controlled trial" \\
\hline $\begin{array}{l}\text { Incomplete outcome data } \\
\text { (attrition bias) } \\
\text { All outcomes }\end{array}$ & Unclear risk & Comment: this information was not available \\
\hline $\begin{array}{l}\text { Selective reporting (re- } \\
\text { porting bias) }\end{array}$ & High risk & $\begin{array}{l}\text { Comment: no previously published protocol was available; adverse events, } \\
\text { mortality, fatty liver resolution were not reported }\end{array}$ \\
\hline Other bias & Low risk & Comment: no other bias noted \\
\hline
\end{tabular}

Chen 2015a

\section{Study characteristics}

\begin{tabular}{ll}
\hline Methods & Randomised clinical trial \\
\hline Participants & Country: China \\
& Period of recruitment: 2012 to 2013 \\
& Number randomised: 60 \\
& Post-randomisation dropouts: 0 (0.0\%) \\
& Revised sample size: 60 \\
& Average age, years: 44 \\
& Females: 18 (30.0\%) \\
& NASH: not stated \\
& Diabetes mellitus: 0 (0.0\%) \\
& Inclusion criteria: 1.20 to 60 years old. 2. 'Bright liver' on USS. 3. BMI 30:30. 4. Fasting blood glucose < \\
& 7.8 \\
& Exclusion criteria: 1. Known aetiology of chronic liver disease. 2. Liver/kidney dysfunction. 3. Malignant \\
tumour. 4. Alcohol $>140$ g/week male, $>70$ g/week female. 5. Any medication over last 6 months alter- \\
ing glucose and lipid metabolism \\
Method for diagnosis of NAFLD: ultrasound
\end{tabular}

Interventions

Group 1: other supplements $(n=30)$

Further details: resveratrol $300 \mathrm{mg}$ twice daily for 3 months

Group 2: no active intervention $(n=30)$

Further details: placebo 
Chen 2015a (Continued)

Outcomes Outcomes reported: mortality at maximal follow-up

Follow-up, months: 3

Notes

Source of funding (quote): "this study was supported by the National Natural Science Foundation of China (No. 30972469; No. 81273059), the Science and Technology Key Project Foundation of Chongqing

(No. CSTC, 2011AB5040), and the National Science-Technology Support Plan Projects Foundation of

China (No. 2012BAI35B02)"

Trial name/Trial registry number: CHICTR-TRC12002378

Attempts were made to contact study authors in December 2020

\section{Risk of bias}

\begin{tabular}{|c|c|c|}
\hline Bias & Authors' judgement & Support for judgement \\
\hline $\begin{array}{l}\text { Random sequence genera- } \\
\text { tion (selection bias) }\end{array}$ & Low risk & $\begin{array}{l}\text { Quote: "an independent investigator determined whether a patient would be } \\
\text { treated with either placebo or resveratrol according to a computer-generated } \\
\text { randomization list" }\end{array}$ \\
\hline $\begin{array}{l}\text { Allocation concealment } \\
\text { (selection bias) }\end{array}$ & Low risk & $\begin{array}{l}\text { Quote: "an independent investigator determined whether a patient would be } \\
\text { treated with either placebo or resveratrol according to a computer-generated } \\
\text { randomization list" }\end{array}$ \\
\hline $\begin{array}{l}\text { Blinding of participants } \\
\text { and personnel (perfor- } \\
\text { mance bias) } \\
\text { All outcomes }\end{array}$ & Low risk & Quote: "double-blind, randomized, placebo-controlled trial" \\
\hline $\begin{array}{l}\text { Blinding of outcome as- } \\
\text { sessment (detection bias) } \\
\text { All outcomes }\end{array}$ & Low risk & Quote: "double-blind, randomized, placebo-controlled trial" \\
\hline $\begin{array}{l}\text { Incomplete outcome data } \\
\text { (attrition bias) } \\
\text { All outcomes }\end{array}$ & Low risk & Comment: there were no post-randomisation dropouts \\
\hline $\begin{array}{l}\text { Selective reporting (re- } \\
\text { porting bias) }\end{array}$ & High risk & $\begin{array}{l}\text { Comment: no previously published protocol was available; adverse events } \\
\text { were not reported adequately }\end{array}$ \\
\hline Other bias & Low risk & Comment: no other bias noted \\
\hline
\end{tabular}

\section{Chen 2015b}

\section{Study characteristics}

\begin{tabular}{ll}
\hline Methods & Randomised clinical trial \\
\hline Participants & Country: China \\
& Period of recruitment: 2012 to 2013 \\
& Number randomised: 60 \\
& Post-randomisation dropouts: $0(0.0 \%)$ \\
& Revised sample size: 60 \\
& Average age, years: 45 \\
& Females: 20 (33.3\%) \\
& NASH: not stated \\
& Diabetes mellitus: $0(0.0 \%)$
\end{tabular}


Inclusion criteria: 1.20 to 60 years. 2. $20<$ BMI < 30. 3. Fasting blood glucose $<7.8$. 4. No weight gain or loss over last 3 months. 5. No medical therapy

Exclusion criteria: 1. Excessive alcohol. 2. Other liver disease. 3. Any medication that would influence glucose or lipid metabolism in the last 6 months. 4. Liver or kidney dysfunction. 5. Malignant tumour Method for diagnosis of NAFLD: ultrasound

\begin{tabular}{|c|c|}
\hline Interventions & $\begin{array}{l}\text { Group 1: other antioxidants }(n=30) \\
\text { Further details: dihydromyricetin }(D H M) 600 \mathrm{mg} / \mathrm{d} \text { for } 12 \text { weeks. DHM capsules also contained pullulan } \\
\text { and maltodextrin } \\
\text { Group 2: no active intervention ( } \mathrm{n}=30) \\
\text { Further details: placebo capsules (containing only pullulan and maltodextrin) daily for } 12 \text { weeks. Place- } \\
\text { bo and DHM capsules were identically packaged } \\
\text { Additional details: both groups received lifestyle advice }\end{array}$ \\
\hline Outcomes & $\begin{array}{l}\text { Outcomes reported: serious adverse events (number of people), any adverse events (number of } \\
\text { events), resolution of fatty liver disease } \\
\text { Follow-up, months: } 3\end{array}$ \\
\hline Notes & $\begin{array}{l}\text { Source of funding (quote): "this study was supported by the National Natural Science Foundation of } \\
\text { China (No. 30972469; No. 81273059), the Science and Technology Key Project Foundation of Chongqing } \\
\text { (No. CSTC, 2011AB5040), and the National Science-technology Support Plan Projects Foundation of } \\
\text { China (No. 2012BAI35B02)" } \\
\text { Trial name/Trial registry number: ChiCTR-TRC-12002377 } \\
\text { Attempts were made to contact study authors in December } 2020\end{array}$ \\
\hline
\end{tabular}

\section{Risk of bias}

\begin{tabular}{lll}
\hline Bias & Authors' judgement & Support for judgement \\
\hline $\begin{array}{l}\text { Random sequence genera- } \\
\text { tion (selection bias) }\end{array}$ & Low risk & $\begin{array}{l}\text { Quote: "an investigator who was not involved in the trial used Excel's random } \\
\text { number generator to generate a number that would determine whether a pa- } \\
\text { tient would be treated with placebo or DHM" }\end{array}$ \\
\hline $\begin{array}{l}\text { Allocation concealment } \\
\text { (selection bias) }\end{array}$ & Low risk & $\begin{array}{l}\text { Quote: "an investigator who was not involved in the trial used Excel's random } \\
\text { number generator to generate a number that would determine whether a pa- } \\
\text { tient would be treated with placebo or DHM. The placebo and DHM capsules } \\
\text { were identically packaged" }\end{array}$ \\
\hline
\end{tabular}

Blinding of participants $\quad$ Low risk $\quad$ Quote: "randomized, double-blind, placebo-controlled trial"
and personnel (perfor-
and personnel (performance bias)

All outcomes

Blinding of outcome as- Low risk Quote: "randomized, double-blind, placebo-controlled trial"
sessment (detection bias)
All outcomes
All outcomes

Incomplete outcome data Low risk Comment: there were no post-randomisation dropouts
(attrition bias)

All outcomes

\begin{tabular}{lll}
\hline $\begin{array}{l}\text { Selective reporting (re- } \\
\text { porting bias) }\end{array}$ & Low risk & $\begin{array}{l}\text { Comment: no previously published protocol was available; adverse events and } \\
\text { either mortality or fatty liver resolution or both were reported }\end{array}$ \\
\hline Other bias & Low risk & Comment: no other bias noted \\
\hline
\end{tabular}


Cheraghpour 2019

\section{Study characteristics}

\begin{tabular}{|c|c|}
\hline Methods & Randomised clinical trial \\
\hline Participants & $\begin{array}{l}\text { Country: Iran } \\
\text { Period of recruitment: } 2017 \text { to } 2018 \\
\text { Number randomised: } 50 \\
\text { Post-randomisation dropouts: } 1(2.0 \%) \\
\text { Revised sample size: } 49 \\
\text { Reasons for post-randomisation dropouts: discontinued study for personal reasons } \\
\text { Average age, years: } 47 \\
\text { Females: } 25(51.0 \%) \\
\text { NASH: not stated } \\
\text { Diabetes mellitus: } 0(0.0 \%) \\
\text { Inclusion criteria: } 1.18 \text { to } 70 \text { years. } 2 \text {. CAP > } 261 \text { ( } \geq \text { grade } 2) \text { on FibroScan } \\
\text { Exclusion criteria: } 1 . \text { Excessive alcohol consumption }(>10 \mathrm{~g} / \mathrm{d}) \text {. } 2 \text {. Liver cirrhosis, renal disease, cardio- } \\
\text { vascular disease, cancer, or diabetes. 3. Use of vitamin E, betaine, pioglitazone, rosiglitazone, pentox- } \\
\text { ifylline, or gemfibrozil. } 4 \text {. Weight loss or bariatric surgery within past } 6 \text { months. 5. Pregnancy or breast- } \\
\text { feeding } \\
\text { Method for diagnosis of NAFLD: elastography }\end{array}$ \\
\hline
\end{tabular}

$\begin{array}{ll}\text { Interventions } & \text { Group 1: other antioxidants }(n=25) \\ & \text { Further details: oral administration of } 2 \text { capsules of Hesperidin (each contains } 500 \mathrm{mg}) \text { for } 12 \text { weeks } \\ & \text { Group 2: no active intervention }(n=24) \\ & \text { Further details: } 2 \text { placebo (starch) capsules for } 12 \text { weeks. Capsules were similar in size and colour to in- } \\ & \text { tervention } \\ & \text { Additional details: both groups received lifestyle advice }\end{array}$

Outcomes Outcomes reported: mortality at maximal follow-up, serious adverse events (number of people), any adverse events (number of people), liver transplantation at maximal follow-up, decompensation (number of people), cirrhosis (number of people), resolution of fatty liver disease, hepatocellular carcinoma Follow-up, months: 3

\begin{tabular}{ll}
\hline Notes & Source of funding (quote): "no funding has been received for this research (author replies)" \\
Trial name/Trial registry number: NCT03377140 \\
Attempts were made to contact study authors in December 2020
\end{tabular}

\section{Risk of bias}

\begin{tabular}{lll} 
Bias & Authors' judgement & Support for judgement \\
\hline $\begin{array}{l}\text { Random sequence genera- } \\
\text { tion (selection bias) }\end{array}$ & Low risk & $\begin{array}{l}\text { Quote: "randomization lists were computer generated by a statistician and giv- } \\
\text { en to the interviewer" }\end{array}$ \\
\hline $\begin{array}{l}\text { Allocation concealment } \\
\text { (selection bias) }\end{array}$ & Low risk & $\begin{array}{l}\text { Quote: "an investigator who had no clinical involvement in the trial numbered } \\
\text { bottles containing supplements and placebos and assigned the participants to } \\
\text { the trial groups in accordance with the randomization list" }\end{array}$
\end{tabular}

\begin{tabular}{|c|c|c|}
\hline $\begin{array}{l}\text { Blinding of participants } \\
\text { and personnel (perfor- } \\
\text { mance bias) } \\
\text { All outcomes }\end{array}$ & Low risk & $\begin{array}{l}\text { Quote: "subjects, investigators, and staff were blind to the treatment assign- } \\
\text { ment until the end of the study... placebo" }\end{array}$ \\
\hline $\begin{array}{l}\text { Blinding of outcome as- } \\
\text { sessment (detection bias) } \\
\text { All outcomes }\end{array}$ & Low risk & $\begin{array}{l}\text { Quote: "subjects, investigators, and staff were blind to the treatment assign- } \\
\text { ment until the end of the study... placebo" }\end{array}$ \\
\hline $\begin{array}{l}\text { Incomplete outcome data } \\
\text { (attrition bias) }\end{array}$ & Unclear risk & $\begin{array}{l}\text { Comment: there were post-randomisation dropouts, but it is not clear whether } \\
\text { these were related to the intervention or to outcomes }\end{array}$ \\
\hline
\end{tabular}


Cheraghpour 2019 (Continued)

All outcomes

\begin{tabular}{|c|c|c|}
\hline $\begin{array}{l}\text { Selective reporting (re- } \\
\text { porting bias) }\end{array}$ & Low risk & $\begin{array}{l}\text { Comment: no previously published protocol was available; adverse events and } \\
\text { either mortality or fatty liver resolution or both were reported }\end{array}$ \\
\hline
\end{tabular}

Other bias Unclear risk Comment: there were baseline differences in important prognostic factors

Chiou 2021

\section{Study characteristics}

\begin{tabular}{|c|c|}
\hline Methods & Randomised clinical trial \\
\hline Participants & $\begin{array}{l}\text { Country: Taiwan } \\
\text { Period of recruitment: } 2015 \text { to } 2016 \\
\text { Number randomised: } 42 \\
\text { Post-randomisation dropouts: } 14 \text { (33.3\%) } \\
\text { Revised sample size: } 28 \\
\text { Reasons for post-randomisation dropouts: loss to follow-up or discontinued intervention } \\
\text { Average age, years: } 46 \\
\text { Females: } 13(46.4 \%) \\
\text { NASH: } 28 \text { (100\%) } \\
\text { Diabetes mellitus: not stated } \\
\text { Inclusion criteria: } 1 \text {. Adult human participants with NASH } \\
\text { Exclusion criteria: } 1 \text {. Patients with other forms of liver disease such as active hepatitis A, active hepati- } \\
\text { tis B, cirrhosis, hepatocellular carcinoma, haemochromatosis, anaemia, and alcoholic liver disease } \\
\text { Method for diagnosis of NAFLD: not stated }\end{array}$ \\
\hline Interventions & $\begin{array}{l}\text { Group 1: other supplements }(n=15) \\
\text { Further details: participants were treated with } 3 \text { capsules per day containing } 420 \text { mg Antrodia cinnamo- } \\
\text { mea Mycelium for } 6 \text { months } \\
\text { Group 2: no active intervention }(n=24) \\
\text { Further details: participants received matching placebo capsules daily for } 6 \text { months } \\
\text { Additional details: both groups received lifestyle modification }\end{array}$ \\
\hline Outcomes & No outcomes of interest were reported \\
\hline Notes & $\begin{array}{l}\text { Source of funding (quote): "the study was supported by the cooperative research projects of } \\
\text { Hungkuang University and Kuang-Tien General Hospital (HK-102147)" } \\
\text { Trial name/Trial registry number: not stated } \\
\text { Attempts were made to contact study authors in April } 2021\end{array}$ \\
\hline
\end{tabular}

\section{Risk of bias}

\begin{tabular}{lll}
\hline Bias & Authors' judgement & Support for judgement \\
\hline $\begin{array}{l}\text { Random sequence genera- } \\
\text { tion (selection bias) }\end{array}$ & Unclear risk & Comment: this information was not available \\
\hline $\begin{array}{l}\text { Allocation concealment } \\
\text { (selection bias) }\end{array}$ & Unclear risk & Comment: this information was not available \\
\hline $\begin{array}{l}\text { Blinding of participants } \\
\text { and personnel (perfor- } \\
\text { mance bias) }\end{array}$ & Low risk & Quote: "double-blind, placebo-controlled' \\
All outcomes & & \\
\hline
\end{tabular}


Chiou 2021 (Continued)
Blinding of outcome as-
Low risk
Quote: "double-blind, placebo-controlled' sessment (detection bias)

All outcomes

\begin{tabular}{lll}
\hline $\begin{array}{l}\text { Incomplete outcome data } \\
\text { (attrition bias) } \\
\text { All outcomes }\end{array}$ & High risk & $\begin{array}{l}\text { Comment: there were post-randomisation dropouts, which probably were re- } \\
\text { lated to intervention and to outcomes }\end{array}$ \\
\hline $\begin{array}{l}\text { Selective reporting (re- } \\
\text { porting bias) }\end{array}$ & High risk & $\begin{array}{l}\text { Comment: a published protocol was not available; adverse events, mortality, } \\
\text { fatty liver resolution were not reported }\end{array}$ \\
\hline Other bias & Low risk & Comment: no other bias noted \\
\hline
\end{tabular}

\section{Chongsrisawat 2017}

\section{Study characteristics}

\begin{tabular}{ll}
\hline Methods & Randomised clinical trial \\
\hline Participants & Country: Thailand \\
& Period of recruitment: 2016 \\
& Number randomised: 37 \\
& Post-randomisation dropouts: not stated \\
& Revised sample size: 37 \\
& Average age, years: not stated \\
& Females: 12 (32.4\%) \\
& NASH: not stated \\
& Diabetes mellitus: not stated \\
Inclusion criteria: 1. Children with NAFLD & Exclusion criteria: not stated \\
& Method for diagnosis of NAFLD: FibroScan \\
& Group 1: prebiotics/probiotics/synbiotics ( $\mathrm{n}=18$ ) \\
& Further details: chicory inulin, Lactobacillus acidophilus, and Bifidobacterium lactis for 16 weeks \\
& Group 2: no active intervention ( $\mathrm{n}=19)$ \\
& Further details: placebo \\
\hline Interventions & No outcomes of interest were reported \\
\hline Outcomes & Source of funding: not stated \\
\hline Notes & Trial name/Trial registry number: not stated \\
& Attempts were made to contact study authors in December 2020 \\
\hline
\end{tabular}

\section{Risk of bias}

Bias Authors' judgement Support for judgement

Random sequence genera- Unclear risk Comment: this information was not available
tion (selection bias)

\begin{tabular}{lll}
\hline $\begin{array}{l}\text { Allocation concealment } \\
\text { (selection bias) }\end{array}$ & Unclear risk & Comment: this information was not available \\
\hline $\begin{array}{l}\text { Blinding of participants } \\
\text { and personnel (perfor- } \\
\text { mance bias) }\end{array}$ & Low risk & Quote: "randomized, double-blind, placebo-controlled trial"
\end{tabular}


Chongsrisawat 2017 (Continued)

All outcomes

Blinding of outcome as- $\quad$ Low risk $\quad$ Quote: "randomized, double-blind, placebo-controlled trial"
sessment (detection bias)

sessment (detection bias)

All outcomes

Incomplete outcome data Unclear risk $\quad$ Comment: this information was not available
(attrition bias)

All outcomes

Selective reporting (re- High risk
porting bias)
porting bias)

Comment: no previously published protocol was available; adverse events, mortality, fatty liver resolution were not reported

\begin{tabular}{ll}
\hline Other bias $\quad$ Low risk $\quad$ Comment: no other bias noted \\
\hline
\end{tabular}

\section{Chou 2006}

\section{Study characteristics}

\begin{tabular}{|c|c|}
\hline Methods & Randomised clinical trial \\
\hline Participants & $\begin{array}{l}\text { Country: Taiwan } \\
\text { Period of recruitment: } 2001 \text { to } 2002 \\
\text { Number randomised: } 60 \\
\text { Post-randomisation dropouts: } 4 \text { (6.7\%) } \\
\text { Revised sample size: } 56 \\
\text { Reasons for post-randomisation dropouts: withdrawal before trial medicine treatment began (i.e. with- } \\
\text { in first } 2 \text { months) because patients could not make scheduled visits } \\
\text { Average age, years: } 43 \\
\text { Females: } 4 \text { (7.1\%) } \\
\text { NASH: not stated } \\
\text { Diabetes mellitus: not stated } \\
\text { Inclusion criteria: } 1.20 \text { years or over. } 2 \text {. Fatty liver on USS. 3. Increased ALT > } 36 \text { or AST > 34 U/L } \\
\text { Exclusion criteria: } 1 \text {. Major cardiovascular disease. 2. Other liver disease. 3. Hypothyroidism. } 4 \text {. } \\
\text { Nephrotic syndrome. } 5 \text {. Cushing's syndrome. } 6 \text {. Use of medications such as diuretics, oestrogen, } \\
\text { steroids, hyperglycaemia requiring oral hypoglycaemic agent or insulin, cancer treatment. } 7 \text {. Pregnan- } \\
\text { cy or lactation. } 8 \text {. Immunosuppression } \\
\text { Method for diagnosis of NAFLD: ultrasound }\end{array}$ \\
\hline Interventions & $\begin{array}{l}\text { Group 1: other supplements }(\mathrm{n}=28) \\
\text { Further details: } 80 \mathrm{~mL} \text { Gynostemma pentaphyllum }(\mathrm{GP}) \text { extraction taken } 3 \text { times daily after meals for } 4 \\
\text { months } \\
\text { Group 2: no active intervention }(\mathrm{n}=28) \\
\text { Further details: placebo capsule with corn starch }(500 \mathrm{mg}) \text { taken } 3 \text { times daily after meals for } 4 \text { months } \\
\text { Additional details: both groups received lifestyle intervention }\end{array}$ \\
\hline Outcomes & $\begin{array}{l}\text { Outcomes reported: serious adverse events (number of people), serious adverse events (number of } \\
\text { events) } \\
\text { Follow-up, months: } 6\end{array}$ \\
\hline Notes & $\begin{array}{l}\text { Source of funding (quote): "the authors would like to thank Chang Gung Memorial Hospital for its finan- } \\
\text { cial support (CMRP 841)" } \\
\text { Trial name/Trial registry number: not stated } \\
\text { Attempts were made to contact study authors in December } 2020\end{array}$ \\
\hline
\end{tabular}

\section{Risk of bias}


Chou 2006 (Continued)

\begin{tabular}{lll} 
Bias & Authors' judgement & Support for judgement \\
\hline $\begin{array}{l}\text { Random sequence genera- } \\
\text { tion (selection bias) }\end{array}$ & Unclear risk & Comment: this information was not available \\
\hline $\begin{array}{l}\text { Allocation concealment } \\
\text { (selection bias) }\end{array}$ & Unclear risk & Comment: this information was not available \\
\hline $\begin{array}{l}\text { Blinding of participants } \\
\text { and personnel (perfor- } \\
\text { mance bias) }\end{array}$ & High risk & $\begin{array}{l}\text { Quote: "a randomized, single-blind, controlled clinical trial. Although the GP } \\
\text { bags and placebo capsules were different in appearance, the research subjects } \\
\text { did not know whether they were taking GP or the placebo" } \\
\text { Comment: only participants were blinded }\end{array}$
\end{tabular}

\section{Blinding of outcome as- High risk} sessment (detection bias) All outcomes
Quote: "a randomized, single-blind, controlled clinical trial. Although the GP bags and placebo capsules were different in appearance, the research subjects did not know whether they were taking GP or the placebo" Comment: only participants were blinded

\begin{tabular}{lll}
\hline $\begin{array}{l}\text { Incomplete outcome data } \\
\text { (attrition bias) } \\
\text { All outcomes }\end{array}$ & Unclear risk & $\begin{array}{l}\text { Comment: there were post-randomisation dropouts, but it is not clear whether } \\
\text { these were related to the intervention or to outcomes }\end{array}$ \\
\hline $\begin{array}{l}\text { Selective reporting (re- } \\
\text { porting bias) }\end{array}$ & High risk & $\begin{array}{l}\text { Comment: no previously published protocol was available; neither mortality } \\
\text { nor fatty liver resolution was reported }\end{array}$ \\
\hline Other bias & Low risk & Comment: no other bias noted \\
\hline
\end{tabular}

\section{Climax 2020}

\section{Study characteristics}

\begin{tabular}{|c|c|}
\hline Methods & Randomised clinical trial \\
\hline Participants & $\begin{array}{l}\text { Country: USA } \\
\text { Period of recruitment: } 2016 \text { to } 2019 \\
\text { Number randomised: } 96 \\
\text { Post-randomisation dropouts: } 3 \text { (3.1\%) } \\
\text { Revised sample size: } 93 \\
\text { Reasons for post-randomisation dropouts: not stated } \\
\text { Average age, years: } 48 \\
\text { Females: } 31 \text { (33.3\%) } \\
\text { NASH: not stated } \\
\text { Diabetes mellitus: } 25 \text { ( } 26.9 \%) \\
\text { Inclusion criteria: } 1 \text {. Age } 18 \text { to } 75 \text { years. } 2 \text {. NAFLD based on the presence of hepatic steatosis on imaging } \\
\text { or histology in the absence of secondary causes. } 3 \text {. BMI between } 25.0 \text { and } 40.0 \mathrm{~kg} / \mathrm{m}^{2} .4 \text {. ALT } \geq 1.5 \text { times } \\
\text { the upper limit of normal and }<5 \text { times the upper limit of normal } \\
\text { Exclusion criteria: } 1 \text {. Weight change }>5 \% \text { in the } 3 \text { months before screening. } 2 \text {. History of gastric bypass } \\
\text { surgery. } 3 \text {. History of or scheduled orthotopic liver transplant. } 4 \text {. Haemoglobin A1C (HbAlc) } \geq 9 \% \text {. } 5 \text {. } \\
\text { Decompensated or severe liver disease. } 6 \text {. Requiring antihyperglycaemic treatment or lipid-lowering } \\
\text { treatment and not on a stable dose for at least } 3 \text { months before screening. } 7 \text {. Use of dietary supple- } \\
\text { ments containing } n-3 \text { or } n-6 \text { fatty acids in the } 4 \text { weeks before baseline } \\
\text { Method for diagnosis of NAFLD: imaging or histology and transaminases }\end{array}$ \\
\hline
\end{tabular}

\footnotetext{
Interventions
}

Group 1: PUFA ( $\mathrm{n}=63)$

Further details: Epeleuton (15-hydroxy eicosapentaenoic acid) $2 \mathrm{~g} / \mathrm{d}$ or $1 \mathrm{~g} / \mathrm{d}$

Group 2: no active intervention $(n=30)$ 
Climax 2020 (Continued)

Further details: placebo (light liquid paraffin) twice daily

Outcomes reported: adverse events (number of people)

Follow-up, months: 4

Notes

Source of funding (quote): "this work was supported by Afimmune. Afimmune was involved in the design and conduct of the trial, collection, analysis, and interpretation of data; and preparation of this manuscript"

Trial name/Trial registry number: NCT02941549

Attempts were made to contact study authors in April 2021

\section{Risk of bias}

\begin{tabular}{|c|c|c|}
\hline Bias & Authors' judgement & Support for judgement \\
\hline $\begin{array}{l}\text { Random sequence genera- } \\
\text { tion (selection bias) }\end{array}$ & Unclear risk & Comment: this information was not available \\
\hline $\begin{array}{l}\text { Allocation concealment } \\
\text { (selection bias) }\end{array}$ & Unclear risk & Comment: this information was not available \\
\hline $\begin{array}{l}\text { Blinding of participants } \\
\text { and personnel (perfor- } \\
\text { mance bias) } \\
\text { All outcomes }\end{array}$ & Low risk & Quote: "double blind...placebo" \\
\hline $\begin{array}{l}\text { Blinding of outcome as- } \\
\text { sessment (detection bias) } \\
\text { All outcomes }\end{array}$ & Low risk & Quote: "double blind...placebo" \\
\hline $\begin{array}{l}\text { Incomplete outcome data } \\
\text { (attrition bias) } \\
\text { All outcomes }\end{array}$ & Unclear risk & $\begin{array}{l}\text { Comment: there were post-randomisation dropouts; it is not clear whether } \\
\text { these could be related to the intervention and to outcomes }\end{array}$ \\
\hline $\begin{array}{l}\text { Selective reporting (re- } \\
\text { porting bias) }\end{array}$ & Low risk & $\begin{array}{l}\text { Comment: all pre-defined outcomes in the protocol published before recruit- } \\
\text { ment were reported }\end{array}$ \\
\hline Other bias & Low risk & Comment: no other bias noted \\
\hline
\end{tabular}

\section{Dabbaghmanesh 2018}

\section{Study characteristics}

\begin{tabular}{ll}
\hline Methods & Randomised clinical trial \\
\hline Participants & Country: Iran \\
& Period of recruitment: 2011 to 2013 \\
& Number randomised: 106 \\
& Post-randomisation dropouts: 15 (14.2\%) \\
& Revised sample size: 91 \\
& Reasons for post-randomisation dropouts: lost-to-follow-up (8), did not follow study protocol or dis- \\
& continued drugs due to side effects (7) \\
& Average age, years: 45 \\
& Females: 54 (59.3\%) \\
& NASH: not stated \\
& Diabetes mellitus: $0(0.0 \%)$ \\
Inclusion criteria: 1.20 to 75 years old. 2 . Hepatic steatosis on USS. 3. Vitamin D $<30 \mathrm{ng} / \mathrm{mL}$
\end{tabular}


Dabbaghmanesh 2018 (Continued)

Exclusion criteria: 1. Cirrhosis. 2. Viral hepatitis. 3. Alcohol $>10 \mathrm{~g} / \mathrm{d}$. 4. Other causes of chronic liver disease. 5. Cancer. 6. Hypercalciuria. 7. Pregnancy/Lactation. 8. Hypersensitivity to D3. 9. Use of oestrogen, tamoxifen, methotrexate, amiodarone, tetracycline, vitamin $\mathrm{D}$, and calcium supplementation in last 6 months

Method for diagnosis of NAFLD: ultrasound

\begin{tabular}{|c|c|}
\hline Interventions & $\begin{array}{l}\text { Group 1: vitamin } D(n=59) \\
\text { Further details: } 50,000 \cup \text { vitamin D3 (cholecalciferol) pearl per week or } 0.25 \mathrm{mg} \text { calcitriol }(1,25 \text { dihy- } \\
\text { droxycholecalciferol) pearl per day for } 12 \text { weeks } \\
\text { Group 2: no active intervention }(n=32) \\
\text { Further details: placebo }\end{array}$ \\
\hline Outcomes & No outcomes of interest were reported \\
\hline Notes & $\begin{array}{l}\text { Source of funding (quote): "this study was supported by a research grant from Shiraz University of Med- } \\
\text { ical Sciences, Shiraz, Iran" } \\
\text { Trial name/Trial registry number: not stated } \\
\text { Attempts were made to contact study authors in December } 2020\end{array}$ \\
\hline
\end{tabular}

\section{Risk of bias}

\begin{tabular}{lll}
\hline Bias & Authors' judgement & Support for judgement \\
\hline $\begin{array}{l}\text { Random sequence genera- } \\
\text { tion (selection bias) }\end{array}$ & Low risk & Quote: "computer based procedure" \\
\hline $\begin{array}{l}\text { Allocation concealment } \\
\text { (selection bias) }\end{array}$ & Low risk & $\begin{array}{l}\text { Quote: "treatments and placebo were provided in identical packages and were } \\
\text { given to the participants by an educated person who was blinded to the drug } \\
\text { and patients" }\end{array}$ \\
\hline $\begin{array}{l}\text { Blinding of participants } \\
\text { and personnel (perfor- } \\
\text { mance bias) } \\
\begin{array}{l}\text { All outcomes } \\
\text { and }\end{array}\end{array}$ & Low risk & Quote: "double blind, randomized, placebo controlled trial" \\
\hline
\end{tabular}

Blinding of outcome as- Low risk Quote: "double blind, randomized, placebo controlled trial"
sessment (detection bias)

All outcomes

\begin{tabular}{lll}
\hline $\begin{array}{l}\text { Incomplete outcome data } \\
\text { (attrition bias) } \\
\text { All outcomes }\end{array}$ & High risk & $\begin{array}{l}\text { Comment: participants were excluded from analysis for reasons that are likely } \\
\text { to be related to the intervention and to outcomes }\end{array}$ \\
\hline $\begin{array}{l}\text { Selective reporting (re- } \\
\text { porting bias) }\end{array}$ & High risk & $\begin{array}{l}\text { Comment: no previously published protocol was available; adverse events, } \\
\text { mortality, fatty liver resolution were not reported }\end{array}$ \\
\hline Other bias & Low risk & Comment: no other bias noted \\
\hline
\end{tabular}

Dallio 2020

\section{Study characteristics}

\begin{tabular}{ll}
\hline Methods & Randomised clinical trial \\
\hline Participants & $\begin{array}{l}\text { Country: Italy } \\
\text { Period of recruitment: not stated }\end{array}$
\end{tabular}


Dallio 2020 (Continued)

Number randomised: 90

Post-randomisation dropouts: not stated

Revised sample size: 90

Average age, years: not stated

Females: not stated

NASH: not stated

Diabetes mellitus: not stated

Inclusion and exclusion criteria: not stated

Method for diagnosis of NAFLD: not stated

$\begin{array}{ll}\text { Interventions } & \text { Group 1: vitamin D plus vitamin E plus other antioxidants }(n=60) \\ & \text { Further details: silybin with vitamin } D \text { and vitamin } E \text { (RealSIL 100D) } \\ \text { Group 2: no active intervention }(n=30) \\ \text { Further details: not treated }\end{array}$

\begin{tabular}{ll}
\hline Outcomes & No outcomes of interest were reported \\
\hline Notes & Source of funding: not stated \\
& Trial name/Trial registry number: not stated \\
& Attempts were made to contact study authors in April 2021 \\
\hline
\end{tabular}

\section{Risk of bias}

\begin{tabular}{|c|c|c|}
\hline Bias & Authors' judgement & Support for judgement \\
\hline $\begin{array}{l}\text { Random sequence genera- } \\
\text { tion (selection bias) }\end{array}$ & Unclear risk & Comment: this information was not available \\
\hline $\begin{array}{l}\text { Allocation concealment } \\
\text { (selection bias) }\end{array}$ & Unclear risk & Comment: this information was not available \\
\hline $\begin{array}{l}\text { Blinding of participants } \\
\text { and personnel (perfor- } \\
\text { mance bias) } \\
\text { All outcomes }\end{array}$ & Unclear risk & Comment: this information was not available \\
\hline $\begin{array}{l}\text { Blinding of outcome as- } \\
\text { sessment (detection bias) } \\
\text { All outcomes }\end{array}$ & Unclear risk & Comment: this information was not available \\
\hline $\begin{array}{l}\text { Incomplete outcome data } \\
\text { (attrition bias) } \\
\text { All outcomes }\end{array}$ & Unclear risk & Comment: this information was not available \\
\hline $\begin{array}{l}\text { Selective reporting (re- } \\
\text { porting bias) }\end{array}$ & High risk & $\begin{array}{l}\text { Comment: no previously published protocol was available; adverse events, } \\
\text { mortality, fatty liver resolution were not reported }\end{array}$ \\
\hline Other bias & Low risk & Comment: no other bias noted \\
\hline
\end{tabular}

Daneshi-Maskooni 2018

\section{Study characteristics}

\begin{tabular}{ll}
\hline Methods & Randomised clinical trial \\
\hline Participants & Country: Iran
\end{tabular}


Daneshi-Maskooni 2018 (Continued)

Period of recruitment: 2016 to 2017

Number randomised: 92

Post-randomisation dropouts: 5 (5.4\%)

Revised sample size: 87

Reasons for post-randomisation dropouts: lost to follow-up, discontinued intervention

Average age, years: 45

Females: 33 (37.9\%)

NASH: not stated

Diabetes mellitus: not stated

Inclusion criteria: 1. Age 30 to 60 years. 2. BMI 25 to 35

Exclusion criteria: 1. Alcohol use in past 12 months. 2. Other liver conditions. 3. Uncontrolled hypertension. 4. Pregnancy or lactation. 5. Professional athlete. 6. Use of ursodeoxycholic acid, probiotics, statins, and antihypertensives. 7. Antioxidant and vitamin supplements within past 3 months. 8 . Weight loss over past 3 months

Method for diagnosis of NAFLD: ultrasound

$\begin{array}{ll}\text { Interventions } & \text { Group 1: other supplements }(\mathrm{n}=43) \\ & \text { Further details: cardamom } 1000 \mathrm{mg} 3 \text { times daily for } 3 \text { months } \\ & \text { Group 2: no active intervention }(\mathrm{n}=44) \\ & \text { Further details: placebo }\end{array}$

\begin{tabular}{ll}
\hline Outcomes & Outcomes reported: any adverse events (number of people) \\
& Follow-up, months: 3
\end{tabular}

Notes Source of funding (quote): "funding was supported by the Tehran University of Medical Sciences (Code: 30123-161-03-94)"

Trial name/Trial registry number: IRCT2015121317254N4

Attempts were made to contact study authors in December 2020

\section{Risk of bias}

\begin{tabular}{|c|c|c|}
\hline Bias & Authors' judgement & Support for judgement \\
\hline $\begin{array}{l}\text { Random sequence genera- } \\
\text { tion (selection bias) }\end{array}$ & Unclear risk & Comment: this information was not available \\
\hline $\begin{array}{l}\text { Allocation concealment } \\
\text { (selection bias) }\end{array}$ & Low risk & $\begin{array}{l}\text { Quote: "both the subjects and investigators were blinded to the intervention } \\
\text { allocation" } \\
\text { Comment: although the precise method was not reported, allocation was } \\
\text { probably concealed by use of a placebo }\end{array}$ \\
\hline $\begin{array}{l}\text { Blinding of participants } \\
\text { and personnel (perfor- } \\
\text { mance bias) } \\
\text { All outcomes }\end{array}$ & Low risk & Quote: "double-blind randomized placebo-controlled clinical trial" \\
\hline $\begin{array}{l}\text { Blinding of outcome as- } \\
\text { sessment (detection bias) } \\
\text { All outcomes }\end{array}$ & Low risk & Quote: "double-blind randomized placebo-controlled clinical trial" \\
\hline $\begin{array}{l}\text { Incomplete outcome data } \\
\text { (attrition bias) } \\
\text { All outcomes }\end{array}$ & Unclear risk & $\begin{array}{l}\text { Comment: participants were excluded from analysis for reasons that may be } \\
\text { related to the intervention and to outcomes }\end{array}$ \\
\hline $\begin{array}{l}\text { Selective reporting (re- } \\
\text { porting bias) }\end{array}$ & High risk & $\begin{array}{l}\text { Comment: a published protocol was available, but recruitment had com- } \\
\text { menced before the protocol was published: neither mortality nor fatty liver } \\
\text { resolution was reported }\end{array}$ \\
\hline Other bias & Low risk & Comment: no other bias noted \\
\hline
\end{tabular}




\section{Study characteristics}

\begin{tabular}{|c|c|}
\hline Methods & Randomised clinical trial \\
\hline Participants & $\begin{array}{l}\text { Country: USA } \\
\text { Period of recruitment: not stated } \\
\text { Number randomised: } 37 \\
\text { Post-randomisation dropouts: } 0(0.0 \%) \\
\text { Revised sample size: } 37 \\
\text { Average age, years: } 51 \\
\text { Females: } 29(78.4 \%) \\
\text { NASH: } 37(100.0 \%) \\
\text { Diabetes mellitus: } 37(100.0 \%) \\
\text { Inclusion criteria: } 1 \text {. Established diagnosis of NASH. 2. NAFLD activity score } \geq 4 \text { on biopsy performed } \\
\text { within } 6 \text { months of entry into the study. 3. Adult patient. } 4 \text {. Diabetic patient with at least moderate con- } \\
\text { trol of blood sugar (HbA1c }<8.5 \%) \text { and stable antidiabetes regimen }>4 \text { months } \\
\text { Exclusion criteria: } 1 \text {. Other liver disease. } 2 \text {. Cirrhosis. 3. Alcohol > } 30 \text { g for males, }>20 \text { g/d for females. } \\
\text { 4. End-stage organ disease associated with diabetes or heart failure NYHA II to IV. Use of fish oil sup- } \\
\text { plement in last } 6 \text { months. } 5 \text {. Use of medication causing steatosis (i.e. vitamin E, thiazolidinedione, S- } \\
\text { adenosyl methionine) } \\
\text { Method for diagnosis of NAFLD: liver biopsy }\end{array}$ \\
\hline
\end{tabular}

Interventions

Group 1: PUFA $(\mathrm{n}=18)$

Further details: $\mathrm{n} 3$ fatty acid containing eicosapentaenoic acid $2160 \mathrm{mg}$ and docosahexaenoic acid

$1440 \mathrm{mg}$ daily for 48 weeks

Group 2: no active intervention $(n=19)$

Further details: placebo

\section{Outcomes}

Outcomes reported: mortality at maximal follow-up, fibrosis score, NAFLD activity score

Follow-up, months: 11

\section{Notes}

Source of funding (quote): "research funding for the study was provided by the National Institutes of Health: U01061732 DK83414, CTSC grant, UL1TR000439. Douglas Laboratories and their Vice President Andrew Hoelpner assisted with providing the PUFA and placebo in a masked manner"

Trial name/Trial registry number: NCT00323414

Attempts were made to contact study authors in December 2020

\section{Risk of bias}

\begin{tabular}{lll}
\hline Bias & Authors' judgement & Support for judgement \\
\hline $\begin{array}{l}\text { Random sequence genera- } \\
\text { tion (selection bias) }\end{array}$ & Low risk & $\begin{array}{l}\text { Quote: "patients were randomized by the sealed envelope technique using a } \\
\text { random numbers table" }\end{array}$ \\
\hline $\begin{array}{l}\text { Allocation concealment } \\
\text { (selection bias) }\end{array}$ & Unclear risk & $\begin{array}{l}\text { Quote: "patients were randomized by the sealed envelope technique using a } \\
\text { random numbers table" } \\
\text { Comment: additional information about sealed envelope technique was not } \\
\text { available }\end{array}$ \\
\hline $\begin{array}{l}\text { Blinding of participants } \\
\begin{array}{l}\text { and personnel (perfor- } \\
\text { mance bias) }\end{array}\end{array}$ & Low risk & Quote: "double blind randomized placebo controlled clinical trial" \\
All outcomes &
\end{tabular}

Blinding of outcome as-
sessment (detection bias) $\quad$ Low risk $\quad$ Quote: "double blind randomized placebo controlled clinical trial"


Dasarathy 2015 (Continued)

All outcomes

\begin{tabular}{lll}
\hline $\begin{array}{l}\text { Incomplete outcome data } \\
\text { (attrition bias) } \\
\text { All outcomes }\end{array}$ & Low risk \\
\hline $\begin{array}{l}\text { Selective reporting (re- } \\
\text { porting bias) }\end{array}$ & High risk & $\begin{array}{l}\text { Comment: } \\
\text { were not reported adequately }\end{array}$ \\
\hline Other bias & Low risk & Comment: no other bias noted \\
\hline
\end{tabular}

Della Corte 2012

\section{Study characteristics}

\begin{tabular}{|c|c|c|}
\hline Methods & \multicolumn{2}{|c|}{ Randomised clinical trial } \\
\hline Participants & \multicolumn{2}{|c|}{$\begin{array}{l}\text { Country: Italy } \\
\text { Period of recruitment: not stated } \\
\text { Number randomised: } 40 \\
\text { Post-randomisation dropouts: not stated } \\
\text { Revised sample size: } 40 \\
\text { Average age, years: } 11 \\
\text { Females: } 17(42.5 \%) \\
\text { NASH: not stated } \\
\text { Diabetes mellitus: not stated } \\
\text { Inclusion and exclusion criteria: not stated } \\
\text { Method for diagnosis of NAFLD: not stated }\end{array}$} \\
\hline Interventions & \multicolumn{2}{|c|}{$\begin{array}{l}\text { Group 1: prebiotics/probiotics/synbiotics ( } \mathrm{n}=\text { not stated) } \\
\text { Further details: glucomannan-enriched biscuits } 5 \mathrm{~g} / \mathrm{d} \text { for } 6 \text { months } \\
\text { Group 2: no active intervention ( } \mathrm{n}=\text { not stated) } \\
\text { Further details: placebo }\end{array}$} \\
\hline Outcomes & \multicolumn{2}{|c|}{ No outcomes of interest were reported } \\
\hline Notes & \multicolumn{2}{|c|}{$\begin{array}{l}\text { Source of funding: not stated } \\
\text { Trial name/Trial registry number: NCT01553500 } \\
\text { Attempts were made to contact study authors in December } 2020\end{array}$} \\
\hline \multicolumn{3}{|l|}{ Risk of bias } \\
\hline Bias & Authors' judgement & Support for judgement \\
\hline $\begin{array}{l}\text { Random sequence genera- } \\
\text { tion (selection bias) }\end{array}$ & Unclear risk & Comment: this information was not available \\
\hline $\begin{array}{l}\text { Allocation concealment } \\
\text { (selection bias) }\end{array}$ & Unclear risk & Comment: this information was not available \\
\hline $\begin{array}{l}\text { Blinding of participants } \\
\text { and personnel (perfor- } \\
\text { mance bias) } \\
\text { All outcomes }\end{array}$ & Low risk & $\begin{array}{l}\text { Quote: "masking: triple (participant, care provider, investigator) (Trial Registry } \\
\text { information)" }\end{array}$ \\
\hline
\end{tabular}


Della Corte 2012 (Continued)

Blinding of outcome as- Low risk Quote: "masking: triple (participant, care provider, investigator) (Trial Registry sessment (detection bias) information)"

All outcomes

\begin{tabular}{lll}
$\begin{array}{l}\text { Incomplete outcome data } \\
\text { (attrition bias) } \\
\text { All outcomes }\end{array}$ & Unclear risk & Comment: this information was not available \\
\hline $\begin{array}{l}\text { Selective reporting (re- } \\
\text { porting bias) }\end{array}$ & High risk & $\begin{array}{l}\text { Comment: no previously published protocol was available; adverse events, } \\
\text { mortality, fatty liver resolution were not reported }\end{array}$
\end{tabular}

Other bias Low risk Comment: no other bias noted

Della Corte 2016

\section{Study characteristics}

\begin{tabular}{|c|c|c|}
\hline Methods & \multicolumn{2}{|c|}{ Randomised clinical trial } \\
\hline Participants & \multicolumn{2}{|c|}{$\begin{array}{l}\text { Country: Italy } \\
\text { Period of recruitment: } 2014 \text { to } 2015 \\
\text { Number randomised: } 43 \\
\text { Post-randomisation dropouts: } 2 \text { (4.7\%) } \\
\text { Revised sample size: } 41 \\
\text { Reasons for post-randomisation dropouts: refused end-of-study biopsy } \\
\text { Average age, years: } 13 \\
\text { Females: } 23(56.1 \%) \\
\text { NASH: } 14 \text { ( } 34.1 \%) \\
\text { Diabetes mellitus: not stated } \\
\text { Inclusion criteria: } 1.4 \text { to } 16 \text { years old. } 2 \text {. Liver biopsy consistent with NAFLD/NASH. 3. Decreased vita- } \\
\text { min D levels < } 20 \mathrm{ng} / \mathrm{mL} \text {. } 4 \text {. ALT < } 10 \times \text { upper limit of normal. } 5 \text {. No lab/clinical signs of liver decompensa- } \\
\text { tion } \\
\text { Exclusion: } 1 . \text { Other causes of liver disease } \\
\text { Method for diagnosis of NAFLD: liver biopsy }\end{array}$} \\
\hline Interventions & \multicolumn{2}{|c|}{$\begin{array}{l}\text { Group 1: PUFA plus vitamin D }(n=18) \\
\text { Further details: } n 3 \text { fatty acid docosahexanoic acid }(D H A)(500 \mathrm{mg}) \text { plus vitamin } D(800 \mathrm{IU}) \text { for } 24 \text { weeks } \\
\text { Group 2: no active intervention }(n=23) \\
\text { Further details: placebo }\end{array}$} \\
\hline Outcomes & \multicolumn{2}{|c|}{ No outcomes of interest were reported } \\
\hline Notes & \multicolumn{2}{|c|}{$\begin{array}{l}\text { Source of funding: not stated } \\
\text { Trial name/Trial registry number: NCT02098317 } \\
\text { Attempts were made to contact study authors in December } 2020\end{array}$} \\
\hline \multicolumn{3}{|l|}{ Risk of bias } \\
\hline Bias & Authors' judgement & Support for judgement \\
\hline $\begin{array}{l}\text { Random sequence genera- } \\
\text { tion (selection bias) }\end{array}$ & Low risk & $\begin{array}{l}\text { Quote: "a computer-generated randomization sequence assigned participants } \\
\text { in a 1:1 ratio to treatment with Vitamin D plus DHA (Treatment arm) or placebo } \\
\text { (Placebo arm) (source: trial registry)" }\end{array}$ \\
\hline
\end{tabular}


Della Corte 2016 (Continued)

Allocation concealment Low risk Quote: "a statistician, who was blinded to participants' clinical data and did (selection bias) not participate in patients' clinical care, generated the allocation sequence and assigned participants to their group (source: trial registry)"
Blinding of participants Low risk and personnel (perfor-

mance bias)

All outcomes
Quote: "randomized, double-blind placebo-controlled trial"

\section{Blinding of outcome as- Low risk sessment (detection bias) \\ All outcomes \\ Quote: "randomized, double-blind placebo-controlled trial"}

\begin{tabular}{|c|c|c|}
\hline $\begin{array}{l}\text { Incomplete outcome data } \\
\text { (attrition bias) }\end{array}$ & Unclear risk & $\begin{array}{l}\text { Comment: participants were excluded from analysis for reasons that may be } \\
\text { related to the intervention and to outcomes }\end{array}$ \\
\hline
\end{tabular}

All outcomes

related to the intervention and to outcomes

\begin{tabular}{|c|c|c|}
\hline $\begin{array}{l}\text { Selective reporting (re- } \\
\text { porting bias) }\end{array}$ & High risk & $\begin{array}{l}\text { Comment: no previously published protocol was available; adverse events, } \\
\text { mortality, fatty liver resolution were not reported }\end{array}$ \\
\hline
\end{tabular}

Other bias Low risk Comment: no other bias noted

Deng 2005

\section{Study characteristics}

\begin{tabular}{|c|c|}
\hline Methods & Randomised clinical trial \\
\hline Participants & $\begin{array}{l}\text { Country: China } \\
\text { Period of recruitment: not stated } \\
\text { Number randomised: } 96 \\
\text { Post-randomisation dropouts: } 0(0.0 \%) \\
\text { Revised sample size: } 96 \\
\text { Average age, years: } 48 \\
\text { Females: } 40 \text { (41.7\%) } \\
\text { NASH: not stated } \\
\text { Diabetes mellitus: } 0(0.0 \%) \\
\text { Inclusion criteria: } 1 \text {. Diagnosis of NAFLD. } 2 \text {. No bibulous history } \\
\text { Exclusion criteria: } 1 \text {. Hypertension, coronary heart disease, diabetes mellitus, other liver disease, or ge- } \\
\text { netic disease. } 2 \text {. Total parenteral nutrition } \\
\text { Method for diagnosis of NAFLD: liver imaging and transaminases }\end{array}$ \\
\hline Interventions & $\begin{array}{l}\text { Group 1: other antioxidants ( } \mathrm{n}=48 \text { ) } \\
\text { Further details: silymarin } 600 \mathrm{mg} \text { daily for } 3 \text { months } \\
\text { Group 2: other supplements ( } \mathrm{n}=48 \text { ) } \\
\text { Further details: gankangyin (GKY) orally administered, } 60 \mathrm{~mL} \text { daily, for } 3 \text { months. GKY is a Chinese } \\
\text { preparation composed of } 8 \text { traditional Chinese drugs, including cudweed } 30 \mathrm{~g} \text {, mung bean } 25 \mathrm{~g} \text {, bu- } \\
\text { pleurum root } 12 \mathrm{~g} \text {, pinellia tuber } 10 \mathrm{~g} \text {, rhubarb root } 3 \mathrm{~g} \text {, laminaria } 20 \mathrm{~g} \text {, grossy privet fruit } 20 \mathrm{~g} \text {, Gynos- } \\
\text { temma pentaphyllum } 30 \mathrm{~g} \text {, with approximately fresh crude drugs } 1.5 \mathrm{~g} \text { per mL }\end{array}$ \\
\hline Outcomes & No outcomes of interest were reported \\
\hline Notes & $\begin{array}{l}\text { Source of funding: not stated } \\
\text { Trial name/Trial registry number: not stated } \\
\text { Attempts were made to contact study authors in December } 2020\end{array}$ \\
\hline
\end{tabular}


Deng 2005 (Continued)

Risk of bias

\begin{tabular}{|c|c|c|}
\hline Bias & Authors' judgement & Support for judgement \\
\hline $\begin{array}{l}\text { Random sequence genera- } \\
\text { tion (selection bias) }\end{array}$ & Low risk & $\begin{array}{l}\text { Quote: "randomized number derived by NDST software developed by Prof. Sun } \\
\text { Rui-yuan" }\end{array}$ \\
\hline $\begin{array}{l}\text { Allocation concealment } \\
\text { (selection bias) }\end{array}$ & Unclear risk & Comment: this information was not available \\
\hline $\begin{array}{l}\text { Blinding of participants } \\
\text { and personnel (perfor- } \\
\text { mance bias) } \\
\text { All outcomes }\end{array}$ & Unclear risk & Comment: this information was not available \\
\hline $\begin{array}{l}\text { Blinding of outcome as- } \\
\text { sessment (detection bias) } \\
\text { All outcomes }\end{array}$ & Unclear risk & Comment: this information was not available \\
\hline $\begin{array}{l}\text { Incomplete outcome data } \\
\text { (attrition bias) } \\
\text { All outcomes }\end{array}$ & Low risk & Comment: there were no post-randomisation dropouts \\
\hline $\begin{array}{l}\text { Selective reporting (re- } \\
\text { porting bias) }\end{array}$ & High risk & $\begin{array}{l}\text { Comment: no previously published protocol was available; adverse events, } \\
\text { mortality, fatty liver resolution were not reported }\end{array}$ \\
\hline Other bias & Low risk & Comment: no other bias noted \\
\hline
\end{tabular}

\section{Dufour 2006}

\section{Study characteristics}

\begin{tabular}{ll}
\hline Methods & Randomised clinical trial \\
\hline Participants & Country: Switzerland \\
& Period of recruitment: 1999 to 2002 \\
& Number randomised: 33 \\
& Post-randomisation dropouts: 12 (36.4\%) \\
& Revised sample size: 21 \\
& Reasons for post-randomisation dropouts: non-compliant, personal reason, lost to follow-up, did not \\
& have follow-up liver biopsy \\
& Average age, years: 46 \\
& Females: 10 (47.6\%) \\
& NASH: 27 (128.6\%) \\
& Diabetes mellitus: 8 (38.1\%) \\
Inclusion criteria: 1.18 to 75 years old. 2. Persistent ALT > 1.5 upper limit of normal for 6 months. 3. Al- \\
cohol < 40 g. 4. Liver biopsy< 6 months before enrolment with macrovesicular steatosis with > 10\% of \\
hepatocytes, hepatocellular injury, and lobular inflammation \\
Exclusion criteria: 1. Other causes of chronic liver disease, decompensation, or cirrhosis. 2. Pregnan- \\
cy/Lactation. 3. Drug inducing NASH. 4. Use of oral anticoagulation. \\
Method for diagnosis of NAFLD: liver biopsy \\
Group 1: vitamin E ( $\mathrm{n}=10)$ \\
Further details: vitamin E 400 IU twice daily for 2 years \\
Group 2: no active intervention ( $\mathrm{n}=11$ ) \\
Further details: placebo \\
\hline Interventions
\end{tabular}


Dufour 2006 (Continued)

Outcomes

Notes
Outcomes reported: fibrosis score, NAFLD activity score

Follow-up, months: 24

Source of funding (quote): "tablets containing vitamin E (natural d-tocopherol) $400 \mathrm{IU}$ and placebo

tablets were provided by Antistress AG (Rapperswil, Switzerland)"

Trial name/Trial registry number: not stated

Attempts were made to contact study authors in December 2020

\section{Risk of bias}

Bias Authors' judgement Support for judgement

Random sequence genera- Low risk tion (selection bias)

Quote: "the pharmacy established before the start of the study a list randomly assigning each patient to..."

Comment: although the precise method of generation of random sequence generation was not reported, the method of allocation concealment suggests that sequence generation was random

Allocation concealment Low risk
(selection bias)

Quote: "the medication was delivered by the pharmacy of the lead center (Inselspital, Bern) and shipped regularly to the patients, depending on their requirements...patients as well as their physicians were blinded to the treatment until completion of the whole study"

Comment: both allocation concealment and blinding were achieved with use of a placebo

\begin{tabular}{ll}
$\begin{array}{l}\text { Blinding of participants } \\
\text { and personnel (perfor- }\end{array}$ & Quote: "the medication was delivered by the pharmacy of the lead center \\
mance bias) & $\begin{array}{l}\text { (Inselspital, Bern) and shipped regularly to the patients, depending on their } \\
\text { requirements...patients as well as their physicians were blinded to the treat- } \\
\text { All outcomes }\end{array}$ \\
\hline
\end{tabular}

Blinding of outcome as- Low risk sessment (detection bias)

All outcomes

Quote: "the medication was delivered by the pharmacy of the lead center (Inselspital, Bern) and shipped regularly to the patients, depending on their requirements... patients as well as their physicians were blinded to the treatment until completion of the whole study"

Incomplete outcome data High risk (attrition bias)

Comment: participants were excluded from analysis for reasons that are likely All outcomes

\begin{tabular}{lll}
\hline $\begin{array}{l}\text { Selective reporting (re- } \\
\text { porting bias) }\end{array}$ & High risk & $\begin{array}{l}\text { Comment: no previously published protocol was available; adverse events, } \\
\text { mortality, fatty liver resolution were not reported }\end{array}$ \\
\hline Other bias & Low risk & Comment: no other bias noted \\
\hline
\end{tabular}

Duseja 2019

\section{Study characteristics}

\begin{tabular}{ll}
\hline Methods & Randomised clinical trial \\
\hline Participants & Country: India \\
& Period of recruitment: not stated \\
& Number randomised: 39 \\
& Post-randomisation dropouts: $24(61.5 \%)$ \\
& Revised sample size: 15
\end{tabular}


Duseja 2019 (Continued)

Reasons for post-randomisation dropouts: did not undergo repeat liver biopsy (15); lost to follow-up

(5); non-compliant (3), pregnant (1)

Average age, years: 36

Females: $11(73.3 \%)$

NASH: not stated

Diabetes mellitus: $0(0.0 \%)$

Inclusion criteria: 1 . Age above 18 years. 2 . Raised AST and ALT at least $1.5 \times$ normal for longer than 3 months. 3.No history of alcohol intake or $<20 \mathrm{~g} / \mathrm{d}$ confirmed by at least 2 family members. 4. Negative viral markers for hepatitis $\mathrm{C}$ virus. 5. Negative autoimmune markers (antinuclear antibodies, anti-smooth muscle antibody, anti-liver kidney macrosomal antibody, anti-mitochondrial antibody, negative Kayser-Fleischer ring with normal ceruloplasmin, and normal iron studies). 6. Liver biopsy consistent with features of NAFLD

Exclusion criteria: 1. Pregnant/lactating. 2. Diabetes mellitus. 3. Liver cirrhosis on imaging or liver biopsy. 4. Use of drugs that can cause NAFLD (e.g. corticosteroids, methotrexate, tamoxifen) Method for diagnosis of NAFLD: liver biopsy

$\begin{array}{ll}\text { Interventions } & \text { Group 1: probiotics }(n=10) \\ & \text { Further details: oral probiotic VSL\#3 (2 capsules, } 3 \text { times daily; } 675 \text { billion CFU/d) for } 1 \text { year } \\ \text { Group 2: no active intervention }(n=5) \\ \text { Further details: placebo } \\ \text { Additional details: both groups received lifestyle modifications. }\end{array}$

Outcomes reported: mortality at maximal follow-up, serious adverse events (number of people), any
adverse events (number of people), cirrhosis (number of people), resolution of fatty liver disease,
NAFLD activity score
Follow-up, months: 12

Notes

Source of funding (quote): "CD Pharma India Private Limited (New Delhi, India) funded the study and supplied the investigational drugs but did not participate in any part of the study"

Trial name/Trial registry number: CTRI/2008/091/000074

Attempts were made to contact study authors in December 2020

\section{Risk of bias}

\begin{tabular}{lll}
\hline Bias & Authors' judgement & Support for judgement \\
\hline $\begin{array}{l}\text { Random sequence genera- } \\
\text { tion (selection bias) }\end{array}$ & Low risk & Quote: "computer generated randomization (source: trial registry)" \\
\hline $\begin{array}{l}\text { Allocation concealment } \\
\text { (selection bias) }\end{array}$ & Low risk & $\begin{array}{l}\text { Quote: "sequentially numbered, sealed, opaque envelopes (source: trial reg- } \\
\text { istry)" }\end{array}$ \\
\hline $\begin{array}{l}\text { Blinding of participants } \\
\text { and personnel (perfor- } \\
\text { mance bias) }\end{array}$ & Low risk & Quote: "prospective, double blind, placebo controlled, randomised" \\
All outcomes & & \\
\hline
\end{tabular}

Blinding of outcome as- Low risk

Quote: "prospective, double blind, placebo controlled, randomised"

sessment (detection bias)

All outcomes

\begin{tabular}{lll}
\hline $\begin{array}{l}\text { Incomplete outcome data } \\
\text { (attrition bias) } \\
\text { All outcomes }\end{array}$ & High risk & $\begin{array}{l}\text { Comment: participants were excluded from analysis for reasons that are likely } \\
\text { to be related to the intervention and to outcomes }\end{array}$ \\
\hline $\begin{array}{l}\text { Selective reporting (re- } \\
\text { porting bias) }\end{array}$ & Low risk & $\begin{array}{l}\text { Comment: no previously published protocol was available; adverse events and } \\
\text { either mortality or fatty liver resolution or both were reported }\end{array}$ \\
\hline Other bias & Low risk & Comment: no other bias noted \\
\hline
\end{tabular}


Ebrahimi-Mameghani 2016

\section{Study characteristics}

\begin{tabular}{ll}
\hline Methods & Randomised clinical trial \\
\hline Participants & Country: Iran \\
& Period of recruitment: 2014 to 2015 \\
& Number randomised: 54 \\
& Post-randomisation dropouts: 16 (29.6\%) \\
& Revised sample size: 38 \\
& Reasons for post-randomisation dropouts: discontinued intervention (8), travelling (3), not stated (5) \\
& Average age, years: 38 \\
& Females: 33 (86.8\%) \\
& NASH: not stated \\
& Diabetes mellitus: not stated \\
Inclusion criteria: 1.20 to 50 years old. 2. BMI 30 to 40. 3. Taking 400 IU vitamin E supplement daily \\
Exclusion criteria: 1. Alcohol consumption. 2. Preganancy/Lactation. 3. Menopause. 4. Athlete. 5 Inflam- \\
matory conditions. 6 . Family history of hyperlipidaemia. 7 . Cardiovascular, lung, renal, or liver disease. \\
8. Other known liver disease. 9. Use of supplements in last 2 months \\
Method for diagnosis of NAFLD: ultrasound
\end{tabular}

\begin{tabular}{ll} 
Interventions & Group 1: PUFA plus vitamin E ( $\mathrm{n}=19)$ \\
& Further details: 3 times $1000 \mathrm{mg}$ softgel of conjugated linoleic acid plus 400 IU vitamin E for 8 weeks \\
& Group 2: vitamin E ( $=19)$ \\
& Further details: vitamin E $400 \mathrm{IU}$ for 8 weeks \\
& Additional details: both groups received weight loss diet \\
\hline Outcomes & No outcomes of interest were reported \\
\hline Notes & Source of funding (quote): "this study was supported by a research grant from Nutrition Research cen- \\
& ter, Tabriz University of Medical Sciences, Iran" \\
& Trial name/Trial registry number: IRCT2014020516491N1 \\
& Attempts were made to contact study authors in December 2020
\end{tabular}

\section{Risk of bias}

\begin{tabular}{|c|c|c|}
\hline Bias & Authors' judgement & Support for judgement \\
\hline $\begin{array}{l}\text { Random sequence genera- } \\
\text { tion (selection bias) }\end{array}$ & Unclear risk & Comment: this information was not available \\
\hline $\begin{array}{l}\text { Allocation concealment } \\
\text { (selection bias) }\end{array}$ & Unclear risk & $\begin{array}{l}\text { Quote: "the person who determined allocation sequence for the study and } \\
\text { those who assigned participants were blinded" } \\
\text { Comment: it is not clear how the random sequence was concealed }\end{array}$ \\
\hline $\begin{array}{l}\text { Blinding of participants } \\
\text { and personnel (perfor- } \\
\text { mance bias) } \\
\text { All outcomes }\end{array}$ & Unclear risk & Comment: this information was not available \\
\hline $\begin{array}{l}\text { Blinding of outcome as- } \\
\text { sessment (detection bias) } \\
\text { All outcomes }\end{array}$ & Unclear risk & Comment: this information was not available \\
\hline $\begin{array}{l}\text { Incomplete outcome data } \\
\text { (attrition bias) } \\
\text { All outcomes }\end{array}$ & Unclear risk & $\begin{array}{l}\text { Comment: participants were excluded from analysis for reasons that may be } \\
\text { related to the intervention and to outcomes }\end{array}$ \\
\hline
\end{tabular}


Ebrahimi-Mameghani 2016 (Continued)

\begin{tabular}{|c|c|c|}
\hline $\begin{array}{l}\text { Selective reporting (re- } \\
\text { porting bias) }\end{array}$ & High risk & $\begin{array}{l}\text { Comment: no previously published protocol was available; adverse events, } \\
\text { mortality, fatty liver resolution were not reported }\end{array}$ \\
\hline
\end{tabular}

Other bias Low risk Comment: no other bias noted

\section{Ebrahimi-Mameghani 2017}

\section{Study characteristics}

\begin{tabular}{|c|c|}
\hline Methods & Randomised clinical trial \\
\hline Participants & $\begin{array}{l}\text { Country: Iran } \\
\text { Period of recruitment: } 2011 \text { to } 2012 \\
\text { Number randomised: } 70 \\
\text { Post-randomisation dropouts: } 15 \text { (21.4\%) } \\
\text { Revised sample size: } 55 \\
\text { Reasons for post-randomisation dropouts: lost-to follow-up, discontinued intervention } \\
\text { Average age, years: } 37 \\
\text { Females: } 25 \text { (45.5\%) } \\
\text { NASH: not stated } \\
\text { Diabetes mellitus: not stated } \\
\text { Inclusion criteria: } 1 \text {. NAFLD. 2. } 20 \text { - to } 50 \text {-year-olds } \\
\text { Exclusion criteria: } 1 \text {. Other causes of liver disease. 2. Use of hepatotoxic drug, antihypertensive medica- } \\
\text { tion, contraceptive, or oestrogen } \\
\text { Method for diagnosis of NAFLD: ultrasound }\end{array}$ \\
\hline Interventions & $\begin{array}{l}\text { Group 1: other supplements }(n=29) \\
\text { Further details: Chlorella vulgaris four } 300-m g \text { tablets for } 8 \text { weeks } \\
\text { Group 2: no active intervention }(n=26) \\
\text { Further details: placebo }\end{array}$ \\
\hline Outcomes & No outcomes of interest were reported \\
\hline Notes & $\begin{array}{l}\text { Source of funding (quote): "also, the authors appreciate Iranians Green Future Co. (Tehran, Iran) for } \\
\text { providing C vulgaris tablets" } \\
\text { Trial name/Trial registry number: IRCT201202233320N7 } \\
\text { Attempts were made to contact study authors in December } 2020\end{array}$ \\
\hline
\end{tabular}

\section{Risk of bias}

\begin{tabular}{|c|c|c|}
\hline Bias & Authors' judgement & Support for judgement \\
\hline $\begin{array}{l}\text { Random sequence genera- } \\
\text { tion (selection bias) }\end{array}$ & Low risk & Quote: "a computer-generated random sequence" \\
\hline \multirow[t]{2}{*}{$\begin{array}{l}\text { Allocation concealment } \\
\text { (selection bias) }\end{array}$} & Low risk & $\begin{array}{l}\text { Quote: "a double-blind placebo-controlled randomized clinical trial...the } \\
\text { study participants, investigators and the laboratory staff were all blinded to } \\
\text { treatment assignment" }\end{array}$ \\
\hline & & $\begin{array}{l}\text { Comment: allocation concealment and blinding were achieved with use of } \\
\text { placebo }\end{array}$ \\
\hline $\begin{array}{l}\text { Blinding of participants } \\
\text { and personnel (perfor- } \\
\text { mance bias) } \\
\text { All outcomes }\end{array}$ & Low risk & Quote: "a double-blind placebo-controlled randomized clinical trial" \\
\hline
\end{tabular}


Ebrahimi-Mameghani 2017 (Continued)
Blinding of outcome as-
Low risk
Quote: "a double-blind placebo-controlled randomized clinical trial" sessment (detection bias)

All outcomes

$\begin{array}{ll}\text { Incomplete outcome data Unclear risk } & \text { Comment: participants were excluded from analysis for reasons that may be } \\ \text { (attrition bias) } & \text { related to the intervention and to outcomes }\end{array}$

All outcomes

related to the intervention and to outcomes

\begin{tabular}{|c|c|c|}
\hline $\begin{array}{l}\text { Selective reporting (re- } \\
\text { porting bias) }\end{array}$ & High risk & $\begin{array}{l}\text { Comment: no previously published protocol was available; adverse events, } \\
\text { mortality, fatty liver resolution were not reported }\end{array}$ \\
\hline
\end{tabular}

Other bias Low risk Comment: no other bias noted

Eghtesadi 2016

\section{Study characteristics}

\begin{tabular}{ll}
\hline Methods & Randomised clinical trial \\
\hline Participants & Country: Iran \\
& Period of recruitment: not stated \\
Number randomised: 68 \\
Post-randomisation dropouts: not stated \\
Revised sample size: 68 \\
Average age, years: not stated \\
Females: not stated \\
NASH: 68 (100.0\%) \\
Diabetes mellitus: not stated \\
Inclusion and exclusion criteria: not stated \\
Method for diagnosis of NAFLD: not stated
\end{tabular}

\begin{tabular}{ll}
\hline Interventions & Group 1: amino acids $(n=36)$ \\
& Further details: $2000 \mathrm{mg}$ L-carnitine supplements for 12 weeks \\
& Group 2: no active intervention $(\mathrm{n}=32)$ \\
& Further details: placebo
\end{tabular}

\begin{tabular}{ll}
\hline Outcomes & No outcomes of interest were reported \\
\hline Notes & Source of funding: not stated \\
& Trial name/Trial registry number: not stated \\
& Attempts were made to contact study authors in December 2020
\end{tabular}

\section{Risk of bias}

\begin{tabular}{lll}
\hline Bias & Authors' judgement & Support for judgement \\
\hline $\begin{array}{l}\text { Random sequence genera- } \\
\text { tion (selection bias) }\end{array}$ & Unclear risk & Comment: this information was not available \\
\hline $\begin{array}{l}\text { Allocation concealment } \\
\text { (selection bias) }\end{array}$ & Unclear risk & Comment: this information was not available \\
\hline $\begin{array}{l}\text { Blinding of participants } \\
\begin{array}{l}\text { and personnel (perfor- } \\
\text { mance bias) }\end{array}\end{array}$ & Low risk & Quote: "randomized double-blind placebo-controlled clinical trial" \\
\end{tabular}


Eghtesadi 2016 (Continued)

All outcomes

$\begin{aligned} & \text { Blinding of outcome as- } \\ & \text { sessment (detection bias) }\end{aligned} \quad$ Low risk $\quad$ Quote: "randomized double-blind placebo-controlled clinical trial"
Alloutcomes

sessment (detection bias)

All outcomes

Incomplete outcome data Unclear risk Comment: this information was not available
(attrition bias)

All outcomes

Selective reporting (re- High risk
porting bias)

porting bias)

Comment: no previously published protocol was available; adverse events, mortality, fatty liver resolution were not reported

\begin{tabular}{ll}
\hline Other bias $\quad$ Low risk $\quad$ Comment: no other bias noted \\
\hline
\end{tabular}

Ekhlasi 2016

\section{Study characteristics}

\begin{tabular}{ll}
\hline Methods & Randomised clinical trial \\
\hline Participants & Country: Iran \\
& Period of recruitment: 2012 to 2013 \\
& Number randomised: 63 \\
& Post-randomisation dropouts: 3 (4.8\%) \\
& Revised sample size: 60 \\
& Reasons for post-randomisation dropouts: did not receive allocated intervention or discontinued treat- \\
& ment \\
Average age, years: not stated \\
Females: 12 (20.0\%) \\
NASH: not stated \\
Diabetes mellitus: 0 (0.0\%) \\
Inclusion criteria: 1. NAFLD plus raised ALT. 2. 25- to 64-year-olds. 3. BMI 25 to 35 \\
Exclusion criteria: 1. Other causes of liver disease. 2. Alcohol consumption. 3. Cancer. 4. Cardiovascu- \\
lar disease. 5 . Diabetes. 6 . Pregnancy/Lactation. 7 . Use of NSAIDs, antibiotics, or probiotic/food supple- \\
ments \\
Method for diagnosis of NAFLD: ultrasound plus transaminases
\end{tabular}

Interventions Group 1: prebiotics/probiotics/synbiotics plus vitamin $\mathrm{E}(\mathrm{n}=15)$

Further details: synbiotics (Protexin; Probiotics International Ltd.; contained Lactobacillus casei, Lactobacillus rhamnosus, Streptococcus thermophilus, Bifidobacterium breve, Lactobacillus acidophilus, Bifidobacterium longum, Lactobacillus bulgaricus, prebiotic (fructo-oligosaccharide), probiotic cultures (magnesium stearate), a vegetable capsule (hydroxypropyl methylcellulose)) twice daily plus vitamin $\mathrm{E}$ 400 IU daily for 8 weeks

Group 2: vitamin $\mathrm{E}(\mathrm{n}=15)$

Further details: vitamin E 400 IU daily plus placebo for 8 weeks

Group 3: prebiotics/probiotics/synbiotics $(n=15)$

Further details: synbiotics (Protexin) twice daily plus placebo for 8 weeks

Group 4: no active intervention $(n=15)$

Further details: placebo

\begin{tabular}{ll}
\hline Outcomes & No outcomes of interest were reported \\
\hline Notes & Source of funding (quote): "this work was supported by the Iran National Science Foundation" \\
& Trial name/Trial registry number: 201111082709 N22 \\
& Attempts were made to contact study authors in December 2020
\end{tabular}


Ekhlasi 2016 (Continued)

Risk of bias

\begin{tabular}{|c|c|c|}
\hline Bias & Authors' judgement & Support for judgement \\
\hline $\begin{array}{l}\text { Random sequence genera- } \\
\text { tion (selection bias) }\end{array}$ & Unclear risk & Comment: this information was not available \\
\hline $\begin{array}{l}\text { Allocation concealment } \\
\text { (selection bias) }\end{array}$ & Unclear risk & Comment: this information was not available \\
\hline $\begin{array}{l}\text { Blinding of participants } \\
\text { and personnel (perfor- } \\
\text { mance bias) } \\
\text { All outcomes }\end{array}$ & Low risk & Quote: "a randomized, double-blind, placebo-controlled trial" \\
\hline $\begin{array}{l}\text { Blinding of outcome as- } \\
\text { sessment (detection bias) } \\
\text { All outcomes }\end{array}$ & Low risk & Quote: "a randomized, double-blind, placebo-controlled trial" \\
\hline $\begin{array}{l}\text { Incomplete outcome data } \\
\text { (attrition bias) } \\
\text { All outcomes }\end{array}$ & Unclear risk & $\begin{array}{l}\text { Comment: participants were excluded from analysis for reasons that may be } \\
\text { related to the intervention and to outcomes }\end{array}$ \\
\hline $\begin{array}{l}\text { Selective reporting (re- } \\
\text { porting bias) }\end{array}$ & High risk & $\begin{array}{l}\text { Comment: no previously published protocol was available; adverse events, } \\
\text { mortality, fatty liver resolution were not reported }\end{array}$ \\
\hline Other bias & Low risk & Comment: no other bias noted \\
\hline
\end{tabular}

\section{Eriksson 2018}

\section{Study characteristics}

\begin{tabular}{|c|c|}
\hline Methods & Randomised clinical trial \\
\hline Participants & $\begin{array}{l}\text { Country: Sweden } \\
\text { Period of recruitment: } 2015 \\
\text { Number randomised: } 84 \\
\text { Post-randomisation dropouts: } 0(0.0 \%) \\
\text { Revised sample size: } 84 \\
\text { Average age, years: } 66 \\
\text { Females: } 25 \text { ( } 29.8 \%) \\
\text { NASH: not stated } \\
\text { Diabetes mellitus: } 84 \text { (100.0\%) } \\
\text { Inclusion criteria: } 1 . \text { Type } 2 \text { diabetes mellitus. } 2.40 \text { - to } 75 \text {-year-olds. 3. Stable dose of metformin or sul- } \\
\text { fonylurea alone or in combination for at least } 3 \text { months. } 4 \text {. Proton density fat fraction (PDFF) }>5.5 \% \text { (as } \\
\text { measured by MRI). } 5 \text {. BMI } 25 \text { to } 40 \\
\text { Exclusion criteria: } 1 \text {. Use of SGLT2is, n-3 fatty acids, insulin, or glucagon-like peptide } 1 \text { receptor agonist. } \\
\text { 2. Other liver disease, or renal disease. 3. Significant alcohol (> } 14 \text { drinks/week) } \\
\text { Method for diagnosis of NAFLD: MRI }\end{array}$ \\
\hline Interventions & $\begin{array}{l}\text { Group 1: PUFA ( } n=42) \\
\text { Further details: } 4 \text { g n3 carboxylic acids (OM-3CA capsules) for } 12 \text { weeks } \\
\text { Group 2: no active intervention ( } n=42) \\
\text { Further details: placebo } \\
\text { Additional details: some participants in both groups also received dapagliflozin, an antidiabetic drug; } \\
\text { this was decided randomly }\end{array}$ \\
\hline
\end{tabular}


Eriksson 2018 (Continued)

Outcomes

Outcomes reported: mortality at maximal follow-up, any adverse events (number of people)

Follow-up, months: 3
Source of funding (quote): "the study was funded by AstraZeneca"

Trial name/Trial registry number: NCT02279407

Attempts were made to contact study authors in December 2020

\section{Risk of bias}

\begin{tabular}{lll}
\hline Bias & Authors' judgement & Support for judgement \\
\hline $\begin{array}{l}\text { Random sequence genera- } \\
\text { tion (selection bias) }\end{array}$ & Low risk & $\begin{array}{l}\text { Quote: "a centralised system, which provided a randomisation code delivered } \\
\text { by an external call centre" }\end{array}$ \\
\hline $\begin{array}{l}\text { Allocation concealment } \\
\text { (selection bias) }\end{array}$ & Low risk & $\begin{array}{l}\text { Quote: "a centralised system, which provided a randomisation code delivered } \\
\text { by an external call centre" }\end{array}$ \\
\hline $\begin{array}{l}\text { Blinding of participants } \\
\text { and personnel (perfor- } \\
\text { mance bias) }\end{array}$ & Low risk & Quote: "a double-blind randomised placebo-controlled study" \\
All outcomes & & \\
\hline
\end{tabular}

Blinding of outcome as- Low risk Quote: "a double-blind randomised placebo-controlled study"

sessment (detection bias)

All outcomes

\begin{tabular}{lll}
\hline $\begin{array}{l}\text { Incomplete outcome data } \\
\text { (attrition bias) } \\
\text { All outcomes }\end{array}$ & Low risk & Comment: there were no post-randomisation dropouts \\
\hline $\begin{array}{l}\text { Selective reporting (re- } \\
\text { porting bias) }\end{array}$ & Low risk & $\begin{array}{l}\text { Comment: no previously published protocol was available; adverse events and } \\
\text { either mortality or fatty liver resolution or both were reported }\end{array}$ \\
\hline Other bias & Low risk & Comment: no other bias noted \\
\hline
\end{tabular}

\section{Eslamparast 2014}

\section{Study characteristics}

\begin{tabular}{ll}
\hline Methods & Randomised clinical trial \\
\hline Participants & Country: Iran \\
& Period of recruitment: 2012 \\
& Number randomised: 52 \\
& Post-randomisation dropouts: $0(0.0 \%)$ \\
& Revised sample size: 52 \\
& Average age, years: 46 \\
& Females: 27 (51.9\%) \\
& NASH: not stated \\
& Diabetes mellitus: 0 (0.0\%) \\
& Inclusion criteria: 1. NAFLD. 2. ALT > 60 \\
& Exclusion criteria: 1. Other causes of chronic liver disease. 2. Alcohol use. 3. Diabetes. 4. Hypothy- \\
roidism that is untreated. 5. Systemic disease. 6 . Pregnancy/Lactation. 7 . Lack of effective birth control \\
in women of childbearing age \\
Method for diagnosis of NAFLD: ultrasound plus transaminases
\end{tabular}


Eslamparast 2014 (Continued)

Interventions
Group 1: prebiotics/probiotics/synbiotics $(n=26)$

Further details: synbiotics (Protexin; Probiotics International Ltd.; contained Lactobacillus casei, Lactobacillus rhamnosus, Streptococcus thermophilus, Bifidobacterium breve, Lactobacillus acidophilus, Bifidobacterium longum, Lactobacillus bulgaricus, prebiotic (fructo-oligosaccharide), probiotic cultures (magnesium stearate), a vegetable capsule (hydroxypropyl methylcellulose)) for 28 weeks Group 2: no active intervention ( $\mathrm{n}=26)$

Further details: placebo

Additional details: both groups received lifestyle modification advice

\begin{tabular}{ll}
\hline Outcomes & $\begin{array}{l}\text { Outcomes reported: fibrosis score } \\
\text { Follow-up, months: } 7\end{array}$ \\
\hline Notes & Source of funding (quote): "Protexin Company, UK, provided the synbiotic supplements, and Nikan Teb \\
& Co provided the FibroScan machine" \\
& Trial name/Trial registry number: NCT01791959 \\
& Attempts were made to contact study authors in December 2020
\end{tabular}

\section{Risk of bias}

\begin{tabular}{lll}
\hline Bias & Authors' judgement & Support for judgement \\
\hline $\begin{array}{l}\text { Random sequence genera- } \\
\text { tion (selection bias) }\end{array}$ & Low risk & Quote: "randomization lists were computer-generated by a statistician" \\
\hline $\begin{array}{l}\text { Allocation concealment } \\
\text { (selection bias) }\end{array}$ & Low risk & $\begin{array}{l}\text { Quote: "a randomized, double-blind, placebo-controlled pilot study...sub- } \\
\text { jects, investigators, and staff were blind to the treatment assignment until the } \\
\text { end of the study" }\end{array}$ \\
$\begin{array}{l}\text { Comment: allocation concealment and blinding were achieved with use of } \\
\text { placebo }\end{array}$
\end{tabular}

Blinding of participants Low risk Quote: "a randomized, double-blind, placebo-controlled pilot study"
and personnel (perfor-
mance bias)
All outcomes

\begin{tabular}{lll}
\hline $\begin{array}{l}\text { Blinding of outcome as- } \\
\text { sessment (detection bias) } \\
\text { All outcomes }\end{array}$ & Low risk & Quote: "a randomized, double-blind, placebo-controlled pilot study" \\
\hline $\begin{array}{l}\text { Incomplete outcome data } \\
\text { (attrition bias) }\end{array}$ & Unclear risk & $\begin{array}{l}\text { Comment: a multiple imputation procedure was performed based on mul- } \\
\text { ti-variate imputation by chained equations; however, values may not be miss- } \\
\text { ing at random, as people were excluded from analysis because of discontinued } \\
\text { intervention or loss to follow-up }\end{array}$ \\
\hline
\end{tabular}

\begin{tabular}{|c|c|c|}
\hline $\begin{array}{l}\text { Selective reporting (re- } \\
\text { porting bias) }\end{array}$ & High risk & $\begin{array}{l}\text { Comment: no previously published protocol was available; adverse events, } \\
\text { mortality, fatty liver resolution were not reported }\end{array}$ \\
\hline
\end{tabular}

\begin{tabular}{ll}
\hline Other bias $\quad$ Low risk $\quad$ Comment: no other bias noted \\
\hline
\end{tabular}

\section{Study characteristics}

Methods Randomised clinical trial


EUCTR 2008-008275-34-GB (Continued)

\begin{tabular}{|c|c|}
\hline Participants & $\begin{array}{l}\text { Country: UK } \\
\text { Period of recruitment: } 2010 \text { to } 2011 \\
\text { Number randomised: } 50 \\
\text { Post-randomisation dropouts: } 1 \text { (2.0\%) } \\
\text { Revised sample size: } 49 \\
\text { Reasons for post-randomisation dropou } \\
\text { Average age, years: } 53 \\
\text { Females: } 23 \text { (46.9\%) } \\
\text { NASH: not stated } \\
\text { Diabetes mellitus: } 16 \text { ( } 32.7 \%) \\
\text { Inclusion criteria: } 1 \text {. Clinical diagnosis of } \\
\text { firmed on ultrasound. } 3 \text {. Abnormal serun } \\
\text { Exclusion criteria: } 1 \text {. Any other establish } \\
\text { class IV). } 3 \text {. Use of medication that could } \\
\text { tiplatelets were permitted). } 5 \text {. Current al } \\
\text { taking fish oil. } 7 \text {. Known allergy to fish oi } \\
\text { Method for diagnosis of NAFLD: clinical p }\end{array}$ \\
\hline Interventions & $\begin{array}{l}\text { Group 1: PUFA }(n=24) \\
\text { Further details: Omacor capsules, } 4 \mathrm{~g} / \mathrm{d} \\
\text { Group 2: no active intervention }(\mathrm{n}=25) \\
\text { Further details: placebo capsules, } 4 \mathrm{~g} / \mathrm{d}\end{array}$ \\
\hline
\end{tabular}

Outcomes

Outcomes reported: mortality at maximal follow-up, serious adverse events (number of people), any adverse events (number of people), liver transplantation at maximal follow-up, decompensation (number of people), cirrhosis (number of people)

Follow-up, months: 9

Notes

Source of funding (quote): "this trial was funded by The University of Edinburgh 'Liver Fund'. No external funding was applied for. No funding or support was received from the pharmaceutical industry" Trial name/Trial registry number: NCT01277237

Attempts were made to contact study authors in April 2021

\section{Risk of bias}

\begin{tabular}{|c|c|c|}
\hline Bias & Authors' judgement & Support for judgement \\
\hline $\begin{array}{l}\text { Random sequence genera- } \\
\text { tion (selection bias) }\end{array}$ & Low risk & $\begin{array}{l}\text { Quote: "the medication was pre-packed and pack numbers were assigned at } \\
\text { random by means of a computer generated list at Tayside Pharmaceuticals" }\end{array}$ \\
\hline $\begin{array}{l}\text { Allocation concealment } \\
\text { (selection bias) }\end{array}$ & Low risk & $\begin{array}{l}\text { Quote: "the study numbers were randomised and allocated by a randomised } \\
\text { list generated by the labelling company (author replies)" }\end{array}$ \\
\hline $\begin{array}{l}\text { Blinding of participants } \\
\text { and personnel (perfor- } \\
\text { mance bias) } \\
\text { All outcomes }\end{array}$ & Low risk & Quote: "participants, radiologist, and investigators were blinded...placebo" \\
\hline $\begin{array}{l}\text { Blinding of outcome as- } \\
\text { sessment (detection bias) } \\
\text { All outcomes }\end{array}$ & Low risk & Quote: "participants, radiologist, and investigators were blinded...placebo" \\
\hline $\begin{array}{l}\text { Incomplete outcome data } \\
\text { (attrition bias) } \\
\text { All outcomes }\end{array}$ & Low risk & $\begin{array}{l}\text { Comment: there was one post-randomisation dropout unrelated to the inter- } \\
\text { vention and to the outcome }\end{array}$ \\
\hline $\begin{array}{l}\text { Selective reporting (re- } \\
\text { porting bias) }\end{array}$ & Low risk & $\begin{array}{l}\text { Comment: all pre-defined outcomes in the protocol published before recruit- } \\
\text { ment were reported }\end{array}$ \\
\hline
\end{tabular}


EUCTR 2008-008275-34-GB (Continued)

Other bias Low risk Comment: no other bias noted

EUCTR 2009-017080-41-GB

\section{Study characteristics}

\begin{tabular}{|c|c|}
\hline Methods & Randomised clinical trial \\
\hline Participants & $\begin{array}{l}\text { Country: UK } \\
\text { Period of recruitment: not stated } \\
\text { Number randomised: } 25 \\
\text { Post-randomisation dropouts: not stated } \\
\text { Revised sample size: } 25 \\
\text { Average age, years: } 46 \\
\text { Females: } 13 \text { (52.0\%) } \\
\text { NASH: not stated } \\
\text { Diabetes mellitus: not stated } \\
\text { Inclusion criteria: } 1 \text {. Aged } 18 \text { years or older. } 2 \text {. Evidence of liver fat content } \geq 5 \% \text { as measured by MRI/ } \\
\text { MRS scanning or biopsy within } 2 \text { months. 3. No changes in levels of exercise or diet for } 4 \text { weeks before } \\
\text { the start of treatment } \\
\text { Exclusion criteria: } 1 \text {. Type } 1 / 2 \text { diabetes. } 2 \text {. Use of recreational cannabis, medicinal cannabis, or cannabi- } \\
\text { noid medications (including Sativex) within } 1 \text { month before study entry. 3. History of alcohol or sub- } \\
\text { stance abuse, or epilepsy or recurrent seizures. } 4 \text {. History of major depression. 5. Cardiac, renal, or he- } \\
\text { patic impairment, or any other significant disease or disorder. } 6 \text {. History of hepatitis B or C. } 7 \text {. Genet- } \\
\text { ic dyslipidaemia. } 8 \text {. Hypersensitivity to cannabinoids or any excipients of the IMP(s). } 9 \text {. Presence of } \\
\text { any metal implants. } 10 \text {. History of claustrophobia. } 11 \text {. Female participants (or partners of male partici- } \\
\text { pants) of childbearing potential not using effective contraception. 12. Pregnant or lactating. 13. Weight } \\
>150 \text { kg } \\
\text { Method for diagnosis of NAFLD: MRI/MRS scanning or liver biopsy }\end{array}$ \\
\hline
\end{tabular}

Interventions Group 1: other supplements $(n=20)$

Further details: Cannabidiol Licaps ${ }^{\circledR}$ Size 00 hard gelatin capsules containing $100 \mathrm{mg}$ of CBD dissolved in vehicle (Gelucire 44/14)

Group 2: no active intervention $(\mathrm{n}=5)$

Further details: placebo

Outcomes

Outcomes reported: serious adverse events (number of people), any adverse events (number of people), any adverse events (number of events)

Follow-up, months: 2

\begin{tabular}{lll}
\hline Notes & $\begin{array}{l}\text { Source of funding: not stated } \\
\text { Trial name/Trial registry number: NCT01284634 } \\
\text { Attempts were made to contact study authors in April } 2021\end{array}$ \\
\hline Risk of bias & Authors' judgement & Support for judgement \\
\hline Bias & Unclear risk & Comment: this information was not available \\
\hline $\begin{array}{l}\text { Random sequence genera- } \\
\text { tion (selection bias) }\end{array}$ & \\
\hline $\begin{array}{l}\text { Allocation concealment } \\
\text { (selection bias) }\end{array}$ & Unclear risk & Comment: this information was not available \\
\hline
\end{tabular}


EUCTR 2009-017080-41-GB (Continued)

\author{
Blinding of participants Low risk Quote: "quadruple blind...placebo" \\ and personnel (perfor- \\ mance bias) \\ All outcomes
}

\begin{tabular}{lll}
\hline $\begin{array}{l}\text { Blinding of outcome as- } \\
\text { sessment (detection bias) } \\
\text { All outcomes }\end{array}$ & Low risk & Quote: "quadruple blind...placebo" \\
\hline $\begin{array}{l}\text { Incomplete outcome data } \\
\text { (attrition bias) } \\
\text { All outcomes }\end{array}$ & Low risk & Comment: intention-to-treat analysis was used \\
\hline $\begin{array}{l}\text { Selective reporting (re- } \\
\text { porting bias) }\end{array}$ & High risk & $\begin{array}{l}\text { Comment: a published protocol was available, but it is not clear whether re- } \\
\text { cruitment had commenced before the protocol was published: neither mortal- } \\
\text { ity nor fatty liver resolution was reported }\end{array}$ \\
\hline Other bias & Low risk & Comment: no other bias noted \\
\hline
\end{tabular}

Fabbrini 2010

\title{
Study characteristics
}

\begin{tabular}{|c|c|}
\hline Methods & Randomised clinical trial \\
\hline Participants & $\begin{array}{l}\text { Country: Italy } \\
\text { Period of recruitment: not stated } \\
\text { Number randomised: } 18 \\
\text { Post-randomisation dropouts: not stated } \\
\text { Revised sample size: } 18 \\
\text { Average age, years: } 42 \\
\text { Females: } 12(66.7 \%) \\
\text { NASH: not stated } \\
\text { Diabetes mellitus: } 0(0.0 \%) \\
\text { Inclusion criteria: } 1 . \text { Obese. } 2 \text {. NAFLD } \\
\text { Exclusion criteria: } 1 \text {. Smokers. } 2 \text {. Alcohol }>20 \mathrm{~g} / \mathrm{d} \text {. 3. Severe hypertriglyceridaemia. 4. Diabetes. 5. Other } \\
\text { causes of chronic liver disease } \\
\text { Method for diagnosis of NAFLD: not stated }\end{array}$ \\
\hline Interventions & $\begin{array}{l}\text { Group 1: amino acids }(n=9) \\
\text { Further details: } \text { nicotinic acid (Niaspan) titrated from } 500 \mathrm{mg} / \text { week to final dose of } 2000 \mathrm{mg} / \text { week dur- } \\
\text { ing first } 3 \text { weeks for } 8 \text { weeks } \\
\text { Group 2: no active intervention }(n=9) \\
\text { Further details: placebo } \\
\text { Additional details: another group not relevant to this review was excluded }\end{array}$ \\
\hline Outcomes & No outcomes of interest were reported \\
\hline Notes & $\begin{array}{l}\text { Source of funding (quote): "kindly provided by Abbott Laboratories. This study was supported by Na- } \\
\text { tional Institutes of Health Grants DK } 37948 \text {, DK } 56341 \text { (to Clinical Nutrition Research Unit), RR024992 (to } \\
\text { Clinical and Translational Science Award), and RR-00954 (to Biomedical Mass Spectrometry Resource)" } \\
\text { Trial name/Trial registry number: not stated } \\
\text { Attempts were made to contact study authors in December } 2020\end{array}$ \\
\hline
\end{tabular}

\section{Risk of bias}


Fabbrini 2010 (Continued)

\begin{tabular}{|c|c|c|}
\hline Bias & Authors' judgement & Support for judgement \\
\hline $\begin{array}{l}\text { Random sequence genera- } \\
\text { tion (selection bias) }\end{array}$ & Unclear risk & Comment: this information was not available \\
\hline $\begin{array}{l}\text { Allocation concealment } \\
\text { (selection bias) }\end{array}$ & Unclear risk & Comment: this information was not available \\
\hline $\begin{array}{l}\text { Blinding of participants } \\
\text { and personnel (perfor- } \\
\text { mance bias) } \\
\text { All outcomes }\end{array}$ & Low risk & $\begin{array}{l}\text { Quote: "subjects were randomly assigned in a double-blind fashion...random- } \\
\text { ized, placebo-controlled trial to evaluate the effect" }\end{array}$ \\
\hline $\begin{array}{l}\text { Blinding of outcome as- } \\
\text { sessment (detection bias) } \\
\text { All outcomes }\end{array}$ & Low risk & $\begin{array}{l}\text { Quote: "subjects were randomly assigned in a double-blind fashion...random- } \\
\text { ized, placebo-controlled trial to evaluate the effect" }\end{array}$ \\
\hline $\begin{array}{l}\text { Incomplete outcome data } \\
\text { (attrition bias) } \\
\text { All outcomes }\end{array}$ & Unclear risk & Comment: this information was not available \\
\hline $\begin{array}{l}\text { Selective reporting (re- } \\
\text { porting bias) }\end{array}$ & High risk & $\begin{array}{l}\text { Comment: no previously published protocol was available; adverse events, } \\
\text { mortality, fatty liver resolution were not reported }\end{array}$ \\
\hline Other bias & Low risk & Comment: no other bias noted \\
\hline
\end{tabular}

Faghihzadeh 2015

\section{Study characteristics}

\begin{tabular}{ll}
\hline Methods & Randomised clinical trial \\
\hline Participants & Country: Iran \\
& Period of recruitment: 2013 to 2014 \\
& Number randomised: 50 \\
& Post-randomisation dropouts: 2 (4.0\%) \\
& Revised sample size: 48 \\
& Reasons for post-randomisation dropouts: discontinued study, had excessive weight loss \\
& Average age, years: 45 \\
& Females: 15 (31.3\%) \\
& NASH: not stated \\
& Diabetes mellitus: 0 (0.0\%) \\
Inclusion criteria: 1. NAFLD. 2. Raised ALT \\
Exclusion criteria: 1 . Viral hepatitis. 2. Alcohol use. 3. Other causes of chronic liver disease. 4. Diabetes. \\
5. Untreated hypothyroidism. 6. Pregnancy/Lactation. 7 . Lack of effective birth control in women of \\
childbearing age \\
Method for diagnosis of NAFLD: ultrasound plus elastography plus transaminases
\end{tabular}

\begin{tabular}{ll}
\hline Interventions & Group 1: other supplements $(n=24)$ \\
& Further details: resveratrol $500 \mathrm{mg}$ daily for 12 weeks \\
& Group 2: no active intervention $(n=24)$ \\
& Further details: placebo \\
& Additional details: both groups received lifestyle advice
\end{tabular}

Outcomes

Outcomes reported: serious adverse events (number of people), resolution of fatty liver disease Follow-up, months: 3 
Faghihzadeh 2015 (Continued)

Notes

Source of funding (quote): "this work was financially supported by the Iran National Science Foundation (A. H., grant number 90008014), and the National Nutrition and Food Technology Research Institute (A. H., grant number 046468)"

Trial name/Trial registry number: IRCT201202014010N7

Attempts were made to contact study authors in December 2020

\section{Risk of bias}

\begin{tabular}{|c|c|c|}
\hline Bias & Authors' judgement & Support for judgement \\
\hline $\begin{array}{l}\text { Random sequence genera- } \\
\text { tion (selection bias) }\end{array}$ & Low risk & $\begin{array}{l}\text { Quote: "stratified randomisation lists were computer generated by a statisti- } \\
\text { cian and given to the investigator, while the supplements were masked as A } \\
\text { product or B product" }\end{array}$ \\
\hline $\begin{array}{l}\text { Allocation concealment } \\
\text { (selection bias) }\end{array}$ & Low risk & $\begin{array}{l}\text { Quote: "stratified randomisation lists were computer generated by a statisti- } \\
\text { cian and given to the investigator, while the supplements were masked as A } \\
\text { product or B product" }\end{array}$ \\
\hline $\begin{array}{l}\text { Blinding of participants } \\
\text { and personnel (perfor- } \\
\text { mance bias) } \\
\text { All outcomes }\end{array}$ & Low risk & Quote: "randomised double-blinded placebo-controlled clinical trial" \\
\hline $\begin{array}{l}\text { Blinding of outcome as- } \\
\text { sessment (detection bias) } \\
\text { All outcomes }\end{array}$ & Low risk & Quote: "randomised double-blinded placebo-controlled clinical trial" \\
\hline $\begin{array}{l}\text { Incomplete outcome data } \\
\text { (attrition bias) } \\
\text { All outcomes }\end{array}$ & Unclear risk & $\begin{array}{l}\text { Comment: participants were excluded from analysis for reasons that may be } \\
\text { related to the intervention and to outcomes }\end{array}$ \\
\hline $\begin{array}{l}\text { Selective reporting (re- } \\
\text { porting bias) }\end{array}$ & Low risk & $\begin{array}{l}\text { Comment: no previously published protocol was available; adverse events and } \\
\text { either mortality or fatty liver resolution or both were reported }\end{array}$ \\
\hline Other bias & Low risk & Comment: no other bias noted \\
\hline
\end{tabular}

Famouri 2017a

\section{Study characteristics}

\begin{tabular}{ll}
\hline Methods & Randomised clinical trial \\
\hline Participants & Country: Iran \\
& Period of recruitment: 2014 \\
& Number randomised: 64 \\
& Post-randomisation dropouts: 0 (0.0\%) \\
& Revised sample size: 64 \\
& Average age, years: 13 \\
& Females: 32 (50.0\%) \\
& NASH: not stated \\
& Diabetes mellitus: not stated \\
& Inclusion criteria: 1.10 to 18 years old. 2. BMI > 85th percentile. 3. NAFLD \\
& Exclusion criteria: 1. Alcohol use. 2. Long-term medication use. 3. Other chronic liver disease \\
& Method for diagnosis of NAFLD: ultrasound \\
& Group 1: prebiotics/probiotics/synbiotics $(\mathrm{n}=32)$ \\
\hline Interventions &
\end{tabular}


Famouri 2017a (Continued)

Further details: probiotic capsule (containing Lactobacillus acidophilus ATCC B3208, Bifidobacterium lactis DSMZ 32269, Bifidobacterium bifidum ATCC SD6576, Lactobacillus rhamnosus DSMZ 21690) for 12 weeks

Group 2: no active intervention $(n=32)$

Further details: placebo

Additional details: both groups received lifestyle advice

Outcomes Outcomes reported: mortality at maximal follow-up, resolution of fatty liver disease

Follow-up, months: 3

Notes Source of funding (quote): "the study was conducted as a part of a thesis, funded by Isfahan University of Medical Sciences"

Trial name/Trial registry number: IRCT2013100414882N1

Attempts were made to contact study authors in December 2020

\section{Risk of bias}

\begin{tabular}{|c|c|c|}
\hline Bias & Authors' judgement & Support for judgement \\
\hline $\begin{array}{l}\text { Random sequence genera- } \\
\text { tion (selection bias) }\end{array}$ & Low risk & Quote: "computer-generated random numbers" \\
\hline $\begin{array}{l}\text { Allocation concealment } \\
\text { (selection bias) }\end{array}$ & Low risk & $\begin{array}{l}\text { Quote: "random allocation of patients to } 2 \text { groups was performed by sequen- } \\
\text { tially numbered containers" }\end{array}$ \\
\hline $\begin{array}{l}\text { Blinding of participants } \\
\text { and personnel (perfor- } \\
\text { mance bias) } \\
\text { All outcomes }\end{array}$ & Low risk & $\begin{array}{l}\text { Quote: "randomized triple-blind trial. An assistant performed randomization, } \\
\text { so the group allocation was blinded for the investigators and participants" }\end{array}$ \\
\hline $\begin{array}{l}\text { Blinding of outcome as- } \\
\text { sessment (detection bias) } \\
\text { All outcomes }\end{array}$ & Low risk & $\begin{array}{l}\text { Quote: "randomized triple-blind trial. An assistant performed randomization, } \\
\text { so the group allocation was blinded for the investigators and participants" }\end{array}$ \\
\hline $\begin{array}{l}\text { Incomplete outcome data } \\
\text { (attrition bias) } \\
\text { All outcomes }\end{array}$ & Low risk & Comment: there were no post-randomisation dropouts \\
\hline $\begin{array}{l}\text { Selective reporting (re- } \\
\text { porting bias) }\end{array}$ & High risk & $\begin{array}{l}\text { Comment: no previously published protocol was available; adverse events } \\
\text { were not reported adequately }\end{array}$ \\
\hline Other bias & Low risk & Comment: no other bias noted \\
\hline
\end{tabular}

Farhangi 2014

\section{Study characteristics}

\begin{tabular}{ll}
\hline Methods & Randomised clinical trial \\
\hline Participants & Country: Iran \\
& Period of recruitment: not stated \\
& Number randomised: 44 \\
& Post-randomisation dropouts: $3(6.8 \%)$ \\
& Revised sample size: 41 \\
& Reasons for post-randomisation dropouts: withdrawal, adverse event, travel \\
& Average age, years: 42 \\
& Females: $10(24.4 \%)$
\end{tabular}


Farhangi 2014 (Continued)

NASH: not stated

Diabetes mellitus: 0 (0.0\%)

Inclusion criteria: 1. NAFLD. 2. Aged 20 to 50 years for women, 20 to 65 years for men

Exclusion criteria: 1 . History of excessive alcohol ( $<30 \mathrm{~g} / \mathrm{d}$ men, $<20 \mathrm{~g} / \mathrm{d}$ women). 2. Cirrhosis, hepatitis B/C, or other chronic liver disease. 3. Diabetes. 4. Gastrointestinal disease. 5. Rheumatoid arthritis, heart failure, or renal disease. 6 . Use of antioxidants and omega 3 supplements

Method for diagnosis of NAFLD: ultrasound

\begin{tabular}{ll}
\hline Interventions & Group 1: other antioxidants $(\mathrm{n}=20)$ \\
& $\begin{array}{l}\text { Further details: coenzyme } \mathrm{Q} 10100 \mathrm{mg} / \mathrm{d} \text { for } 4 \text { weeks } \\
\text { Group 2: no active intervention }(\mathrm{n}=21)\end{array}$ \\
& Further details: placebo \\
\hline Outcomes & No outcomes of interest were reported \\
\hline Notes & $\begin{array}{l}\text { Source of funding (quote): "we thank research undersecretary of Tabriz University of Medical Sciences } \\
\text { for financial support" } \\
\text { Trial name/Trial registry number: IRCT201305254105N12 } \\
\text { Attempts were made to contact study authors in December } 2020\end{array}$
\end{tabular}

\section{Risk of bias}

\begin{tabular}{|c|c|c|}
\hline Bias & Authors' judgement & Support for judgement \\
\hline $\begin{array}{l}\text { Random sequence genera- } \\
\text { tion (selection bias) }\end{array}$ & Unclear risk & Comment: this information was not available \\
\hline $\begin{array}{l}\text { Allocation concealment } \\
\text { (selection bias) }\end{array}$ & Unclear risk & Comment: this information was not available \\
\hline $\begin{array}{l}\text { Blinding of participants } \\
\text { and personnel (perfor- } \\
\text { mance bias) } \\
\text { All outcomes }\end{array}$ & Low risk & Quote: "randomized double-blind placebo controlled trial" \\
\hline $\begin{array}{l}\text { Blinding of outcome as- } \\
\text { sessment (detection bias) } \\
\text { All outcomes }\end{array}$ & Low risk & Quote: "randomized double-blind placebo controlled trial" \\
\hline $\begin{array}{l}\text { Incomplete outcome data } \\
\text { (attrition bias) } \\
\text { All outcomes }\end{array}$ & High risk & $\begin{array}{l}\text { Comment: participants were excluded from analysis for reasons that are likely } \\
\text { to be related to the intervention and to outcomes }\end{array}$ \\
\hline $\begin{array}{l}\text { Selective reporting (re- } \\
\text { porting bias) }\end{array}$ & High risk & $\begin{array}{l}\text { Comment: no previously published protocol was available; adverse events, } \\
\text { mortality, fatty liver resolution were not reported }\end{array}$ \\
\hline Other bias & Low risk & Comment: no other bias noted \\
\hline
\end{tabular}

Farsi 2016

\section{Study characteristics}

\begin{tabular}{ll}
\hline Methods & Randomised clinical trial \\
\hline Participants & $\begin{array}{l}\text { Country: Iran } \\
\text { Period of recruitment: not stated }\end{array}$
\end{tabular}


Farsi 2016 (Continued)

Number randomised: 42

Post-randomisation dropouts: 1 (2.4\%)

Revised sample size: 41

Reasons for post-randomisation dropouts: discontinued intervention

Average age, years: not stated

Females: 13 (31.7\%)

NASH: not stated

Diabetes mellitus: $0(0.0 \%)$

Inclusion criteria: 1. NAFLD. 2. Absence of alcohol consumption

Exclusion criteria: 1. History of chronic liver disease. 2. Renal failure, gastrointestinal disease. 3. Use of

vitamin supplements, anticoagulant medication, hepatotoxic drugs. 4. Alcohol consumption. 5. Dia-

betes

Method for diagnosis of NAFLD: ultrasound plus transaminases

\begin{tabular}{ll}
\hline Interventions & Group 1: other antioxidants $(\mathrm{n}=20)$ \\
& Further details: coenzyme $\mathrm{Q} 10100 \mathrm{mg} / \mathrm{d}$ for 12 weeks \\
& Group 2: no active intervention $(\mathrm{n}=21)$ \\
& Further details: placebo \\
\hline Outcomes & Outcomes reported: resolution of fatty liver disease \\
& Follow-up, months: 3 \\
\hline Notes & Source of funding (quote): "financial support was provided by Ahvaz Jundishapur University of Medical \\
& Sciences" \\
& Trial name/Trial registry number: IRCT2013071313984N1 \\
& Attempts were made to contact study authors in December 2020
\end{tabular}

\section{Risk of bias}

\begin{tabular}{lll}
\hline Bias & Authors' judgement & Support for judgement \\
\hline $\begin{array}{l}\text { Random sequence genera- } \\
\text { tion (selection bias) }\end{array}$ & Unclear risk & Comment: this information was not available \\
\hline $\begin{array}{l}\text { Allocation concealment } \\
\text { (selection bias) }\end{array}$ & Unclear risk & Comment: this information was not available \\
\hline $\begin{array}{l}\text { Blinding of participants } \\
\text { and personnel (perfor- } \\
\text { mance bias) } \\
\text { All outcomes }\end{array}$ & Low risk & Quote: "randomized double-blind placebo controlled trial" \\
\hline
\end{tabular}

\begin{tabular}{|c|c|c|}
\hline $\begin{array}{l}\text { Blinding of outcome as- } \\
\text { sessment (detection bias) } \\
\text { All outcomes }\end{array}$ & Low risk & Quote: "randomized double-blind placebo controlled trial" \\
\hline
\end{tabular}

\begin{tabular}{lll}
\hline $\begin{array}{l}\text { Incomplete outcome data } \\
\text { (attrition bias) } \\
\text { All outcomes }\end{array}$ & Unclear risk & $\begin{array}{l}\text { Comment: participants were excluded from analysis for reasons that may be } \\
\text { related to the intervention and to outcomes }\end{array}$ \\
\hline $\begin{array}{l}\text { Selective reporting (re- } \\
\text { porting bias) }\end{array}$ & High risk & $\begin{array}{l}\text { Comment: no previously published protocol was available; adverse events } \\
\text { were not reported adequately }\end{array}$ \\
\hline Other bias & Low risk & Comment: no other bias noted \\
\hline
\end{tabular}


Farzin 2020

\section{Study characteristics}

\begin{tabular}{|c|c|}
\hline Methods & Randomised clinical trial \\
\hline Participants & $\begin{array}{l}\text { Country: Iran } \\
\text { Period of recruitment: not stated } \\
\text { Number randomised: } 50 \\
\text { Post-randomisation dropouts: not stated } \\
\text { Revised sample size: } 50 \\
\text { Average age, years: not stated } \\
\text { Females: not stated } \\
\text { NASH: not stated } \\
\text { Diabetes mellitus: not stated } \\
\text { Inclusion and exclusion criteria: not stated } \\
\text { Method for diagnosis of NAFLD: not stated }\end{array}$ \\
\hline Interventions & $\begin{array}{l}\text { Group 1: other supplements }(n=25) \\
\text { Further details: resveratrol } 600 \mathrm{mg} \text { daily for } 12 \text { weeks } \\
\text { Group 2: no active intervention }(n=25) \\
\text { Further details: placebo daily for } 12 \text { weeks }\end{array}$ \\
\hline Outcomes & No outcomes of interest were reported \\
\hline Notes & $\begin{array}{l}\text { Source of funding: not stated } \\
\text { Trial name/Trial registry number: IRCT201511233664N16 } \\
\text { Attempts were made to contact study authors in April } 2021\end{array}$ \\
\hline
\end{tabular}

\section{Risk of bias}

Bias Authors' judgement Support for judgement

Random sequence genera- Unclear risk Comment: this information was not available
tion (selection bias)

\begin{tabular}{lll}
\hline $\begin{array}{l}\text { Allocation concealment } \\
\text { (selection bias) }\end{array}$ & Unclear risk & Comment: this information was not available \\
\hline $\begin{array}{l}\text { Blinding of participants } \\
\text { and personnel (perfor- } \\
\text { mance bias) }\end{array}$ & Low risk & Quote: "double blind...placebo" \\
All outcomes & \\
\hline
\end{tabular}

\begin{tabular}{lll}
\hline $\begin{array}{l}\text { Blinding of outcome as- } \\
\text { sessment (detection bias) } \\
\text { All outcomes }\end{array}$ & Low risk & \\
\hline $\begin{array}{l}\text { Incomplete outcome data } \\
\text { (attrition bias) }\end{array}$ & Unclear risk & Comment: this information was not available \\
All outcomes & &
\end{tabular}

\begin{tabular}{lll}
\hline $\begin{array}{l}\text { Selective reporting (re- } \\
\text { porting bias) }\end{array}$ & High risk & $\begin{array}{l}\text { Comment: no previously published protocol was available; adverse events, } \\
\text { mortality, fatty liver resolution were not reported }\end{array}$ \\
\hline Other bias & Low risk & Comment: no other bias noted \\
\hline
\end{tabular}


Fathi 2020

\section{Study characteristics}

\begin{tabular}{|c|c|}
\hline Methods & Randomised clinical trial \\
\hline Participants & $\begin{array}{l}\text { Country: Iran } \\
\text { Period of recruitment: } 2018 \text { to } 2019 \\
\text { Number randomised: } 56 \\
\text { Post-randomisation dropouts: } 6 \text { (10.7\%) } \\
\text { Revised sample size: } 50 \\
\text { Reasons for post-randomisation dropouts: lost to follow-up } \\
\text { Average age, years: } 40 \\
\text { Females: } 18 \text { (36.0\%) } \\
\text { NASH: not stated } \\
\text { Diabetes mellitus: } 0 \text { (0.0\%) } \\
\text { Inclusion criteria: } 1 . \text { NAFLD participants. 2. Overweight or obese (body mass index (BMI) between } 25 \\
\left.\text { and } 35 \text { kg/m }{ }^{2}\right) .3 \text {. Aged } 18 \text { to } 65 \text { years. } 4 \text {. Abdominal obesity (waist circumference (WC) }>80 \text { cm for } \\
\text { women, > } 94 \text { cm for men) } \\
\text { Exclusion criteria: } 1 \text {. Highly physically active (> } 3 \text { hours/week). } 2 \text {. Grade } 3 \text { NAFLD. 3. Smoking. } 4 \text {. Preg- } \\
\text { nant or lactating women. } 5 \text {. Chronic disease such as diabetes, major cardiovascular disease, cancer, } \\
\text { hepatic or kidney dysfunction, hypothyroidism or hyperthyroidism, malabsorption disorders. } 6 \text {. Taking } \\
\text { medication that may interfere with zinc function. } 7 \text {. Not taking more than } 10 \% \text { of zinc supplement. } 8 \text {. } \\
\text { Using dietary supplements or adhering to special diets or heavy physical activity programmes over past } \\
3 \text { months. } 9 \text {. History of regular alcohol drinking over past } 3 \text { months } \\
\text { Method for diagnosis of NAFLD: ultrasound }\end{array}$ \\
\hline Interventions & $\begin{array}{l}\text { Group 1: other supplements ( } \mathrm{n}=25) \\
\text { Further details: } 220 \mathrm{mg} \text { zinc gluconate (as } 30 \mathrm{mg} \text { elemental zinc) supplement per day for } 12 \text { weeks } \\
\text { Group 2: no active intervention }(n=25) \\
\text { Further details: placebo daily for } 12 \text { weeks } \\
\text { Additional details: all participants also followed a weight-loss calorie-restricted diet for } 12 \text { weeks }\end{array}$ \\
\hline Outcomes & $\begin{array}{l}\text { Outcome reported: resolution of fatty liver disease } \\
\text { Follow-up, months: } 3\end{array}$ \\
\hline Notes & $\begin{array}{l}\text { Source of funding (quote): "the study was funded by a research grant from Iran National Science Foun- } \\
\text { dation (INSF) (Grant Number } 97014520) " \\
\text { Trial name/Trial registry number: IRCT 20181005041238N1 } \\
\text { Attempts were made to contact study authors in April } 2021\end{array}$ \\
\hline
\end{tabular}

\section{Risk of bias}

\begin{tabular}{lll}
\hline Bias & Authors' judgement & Support for judgement \\
\hline $\begin{array}{l}\text { Random sequence genera- } \\
\text { tion (selection bias) }\end{array}$ & Low risk & $\begin{array}{l}\text { Quote: "randomization was performed according to the blocked randomiza- } \\
\text { tion schedule with a block size of 4 subjects provided by the computer" }\end{array}$ \\
\hline $\begin{array}{l}\text { Allocation concealment } \\
\text { (selection bias) }\end{array}$ & Low risk & $\begin{array}{l}\text { Quote: "allocation concealment was conducted to decrease the possible bias. } \\
\text { For this purpose, the tablets containers were coded by the producing compa- } \\
\text { ny" } \\
\text { Comment: both allocation concealment and blinding were achieved with use } \\
\text { of placebo }\end{array}$
\end{tabular}

Blinding of participants Low risk Quote: "double blind...placebo"

and personnel (perfor-

mance bias)

All outcomes

\begin{tabular}{ll}
\hline $\begin{array}{l}\text { Blinding of outcome as- } \\
\text { sessment (detection bias) }\end{array} \quad$ Low risk $\quad$ Quote: "double blind...placebo" \\
\hline
\end{tabular}


Fathi 2020 (Continued)

All outcomes

\begin{tabular}{lll}
$\begin{array}{l}\text { Incomplete outcome data } \\
\text { (attrition bias) } \\
\text { All outcomes }\end{array}$ & Unclear risk & $\begin{array}{l}\text { Comment: there were post-randomisation dropouts related to loss to fol- } \\
\text { low-up; it is not clear whether these were related to the intervention and to } \\
\text { outcomes }\end{array}$ \\
\hline $\begin{array}{l}\text { Selective reporting (re- } \\
\text { porting bias) }\end{array}$ & High risk & $\begin{array}{l}\text { Comment: a published protocol was available, but recruitment had com- } \\
\text { menced before the protocol was published; adverse events were not reported } \\
\text { adequately }\end{array}$
\end{tabular}

Other bias Low risk Comment: no other bias noted

Fernandez-Travieso 2020

\section{Study characteristics}

\begin{tabular}{|c|c|c|}
\hline Methods & \multicolumn{2}{|c|}{ Randomised clinical trial } \\
\hline Participants & \multicolumn{2}{|c|}{$\begin{array}{l}\text { Country: Cuba } \\
\text { Period of recruitment: not stated } \\
\text { Number randomised: } 100 \\
\text { Post-randomisation dropouts: not stated } \\
\text { Revised sample size: } 100 \\
\text { Average age, years: } 53 \\
\text { Females: } 54 \text { (54.0\%) } \\
\text { NASH: not stated } \\
\text { Diabetes mellitus: } 22 \text { ( } 22.0 \%) \\
\text { Inclusion criteria: } 1 \text {. Both sexes. 2. Aged between } 25 \text { and } 70 \text { years. 3. History of liver enzyme elevation, } \\
\text { obesity or overweight, diabetes, or dyslipidaemia, or with ultrasound history of liver disease due to } \\
\text { non-alcoholic fat deposition } \\
\text { Exclusion criteria: } 1 \text {. Current alcohol consumption. 2. Hepatitis C and B virus infection. 3. Autoimmune } \\
\text { liver disease, haemochromatosis, hepatotoxicity, human immunodeficiency virus (HIV), secondary } \\
\text { cause of NAFLD, cirrhosis. 4. Pregnant or nursing. } 5 \text {. Uncompensated diabetic patients. 6. Treatment } \\
\text { that could influence liver function } \\
\text { Method for diagnosis of NAFLD: ultrasound }\end{array}$} \\
\hline Interventions & \multicolumn{2}{|c|}{$\begin{array}{l}\text { Group 1: other supplements }(n=50) \\
\text { Further details: Abexol tablets } 100 \mathrm{mg} / \mathrm{d} \text { for } 6 \text { months } \\
\text { Group 2: no active intervention }(n=50) \\
\text { Further details: placebo tablets twice daily for } 6 \text { months }\end{array}$} \\
\hline Outcomes & \multicolumn{2}{|c|}{$\begin{array}{l}\text { Outcomes reported: any adverse events (number of people), resolution of fatty liver disease } \\
\text { Follow-up, months: } 6\end{array}$} \\
\hline Notes & \multicolumn{2}{|c|}{$\begin{array}{l}\text { Source of funding (quote): "this study was support by the National Centre for Scientific Research, as } \\
\text { part of its research-development projects" } \\
\text { Trial name/Trial registry number: not stated } \\
\text { Attempts were made to contact study authors in April } 2021\end{array}$} \\
\hline \multicolumn{3}{|l|}{ Risk of bias } \\
\hline Bias & Authors' judgement & Support for judgement \\
\hline $\begin{array}{l}\text { Random sequence genera- } \\
\text { tion (selection bias) }\end{array}$ & Low risk & $\begin{array}{l}\text { Quote: "randomization was computer generated using blocks and } 1 / 1 \text { ran- } \\
\text { domization" }\end{array}$ \\
\hline
\end{tabular}


Fernandez-Travieso 2020 (Continued)

$\begin{array}{ll}\begin{array}{l}\text { Allocation concealment } \\ \text { (selection bias) }\end{array} & \text { Low risk } \\ & \begin{array}{l}\text { Quote: "identical coded packages...placebo" } \\ \text { of identical coded packages }\end{array}\end{array}$

Blinding of participants Low risk Comment: double-blind...placebo

and personnel (perfor-

mance bias)

All outcomes

\begin{tabular}{lll}
\hline $\begin{array}{l}\text { Blinding of outcome as- } \\
\text { sessment (detection bias) } \\
\text { All outcomes }\end{array}$ & Low risk & Comment: double-blind...placebo \\
\hline $\begin{array}{l}\text { Incomplete outcome data } \\
\text { (attrition bias) }\end{array}$ & Unclear risk & Comment: this information was not available \\
All outcomes & &
\end{tabular}

\begin{tabular}{ll}
\hline $\begin{array}{l}\text { Selective reporting (re- } \\
\text { porting bias) }\end{array}$ & Low risk \\
& $\begin{array}{l}\text { Comment: no previously published protocol was available; adverse events and } \\
\text { either mortality or fatty liver resolution or both were reported }\end{array}$ \\
\hline
\end{tabular}

\begin{tabular}{ll}
\hline Other bias Low risk $\quad$ Comment: no other bias noted \\
\hline
\end{tabular}

Ferolla 2016

\section{Study characteristics}

\begin{tabular}{|c|c|}
\hline Methods & Randomised clinical trial \\
\hline Participants & $\begin{array}{l}\text { Country: Brazil } \\
\text { Period of recruitment: } 2014 \text { to } 2015 \\
\text { Number randomised: } 50 \\
\text { Post-randomisation dropouts: } 0(0.0 \%) \\
\text { Revised sample size: } 50 \\
\text { Average age, years: } 57 \\
\text { Females: } 38(76.0 \%) \\
\text { NASH: } 50(100.0 \%) \\
\text { Diabetes mellitus: not stated } \\
\text { Inclusion criteria: } 1 \text {. NASH confirmed on biopsy (NAS } \geq 3) \\
\text { Exclusion criteria: } 1 \text {. Other causes of liver disease. } 2 \text {. Evidence of decompensated liver disease. 3. Con- } \\
\text { traindication to MRI examination } \\
\text { Method for diagnosis of NAFLD: liver biopsy }\end{array}$ \\
\hline Interventions & $\begin{array}{l}\text { Group 1: prebiotics/probiotics/synbiotics ( } n=27 \text { ) } \\
\text { Further details: } 5 \mathrm{~g} \text { of synbiotic Fiber Mais Flora (Nestlé Health Science), which consisted of } 4 \mathrm{~g} \text { of di- } \\
\text { etary fibre (partially hydrolysed guar gum and inulin) and } 1 \times 10^{8} \text { CFU of } L \text { reuteri, twice daily for } 3 \\
\text { months } \\
\text { Group 2: no active intervention ( } n=23) \\
\text { Further details: no treatment } \\
\text { Additional details: both groups received nutritional advice }\end{array}$ \\
\hline
\end{tabular}

Outcomes

Outcomes reported: mortality at maximal follow-up, serious adverse events (number of people), any adverse events (number of events)

Follow-up, months: 3

Notes

Source of funding: not stated

Trial name/Trial registry number: not stated 
Ferolla 2016 (Continued)

Attempts were made to contact study authors in December 2020

\section{Risk of bias}

\begin{tabular}{|c|c|c|}
\hline Bias & Authors' judgement & Support for judgement \\
\hline $\begin{array}{l}\text { Random sequence genera- } \\
\text { tion (selection bias) }\end{array}$ & Unclear risk & Comment: this information was not available \\
\hline $\begin{array}{l}\text { Allocation concealment } \\
\text { (selection bias) }\end{array}$ & Unclear risk & Comment: this information was not available \\
\hline $\begin{array}{l}\text { Blinding of participants } \\
\text { and personnel (perfor- } \\
\text { mance bias) } \\
\text { All outcomes }\end{array}$ & Unclear risk & Comment: this information was not available \\
\hline $\begin{array}{l}\text { Blinding of outcome as- } \\
\text { sessment (detection bias) } \\
\text { All outcomes }\end{array}$ & Unclear risk & Comment: this information was not available \\
\hline $\begin{array}{l}\text { Incomplete outcome data } \\
\text { (attrition bias) } \\
\text { All outcomes }\end{array}$ & Low risk & Comment: there were no post-randomisation dropouts \\
\hline $\begin{array}{l}\text { Selective reporting (re- } \\
\text { porting bias) }\end{array}$ & Low risk & $\begin{array}{l}\text { Comment: no previously published protocol was available; adverse events and } \\
\text { either mortality or fatty liver resolution or both were reported }\end{array}$ \\
\hline Other bias & Low risk & Comment: no other bias noted \\
\hline
\end{tabular}

Ferro 2020

\section{Study characteristics}

\begin{tabular}{|c|c|}
\hline Methods & Randomised clinical trial \\
\hline Participants & $\begin{array}{l}\text { Country: Italy } \\
\text { Period of recruitment: } 2019 \\
\text { Number randomised: } 102 \\
\text { Post-randomisation dropouts: } 16 \text { (15.7\%) } \\
\text { Revised sample size: } 86 \\
\text { Reasons for post-randomisation dropouts: personal reasons, health reasons, work reasons } \\
\text { Average age, years: } 51 \\
\text { Females: } 33 \text { (38.4\%) } \\
\text { NASH: not stated } \\
\text { Diabetes mellitus: } 0 \text { (0.0\%) } \\
\text { Inclusion criteria: } 1 . \text { Liver steatosis. } 2 \text {. Not taking nutraceuticals, supplements, or functional food } \\
\text { Exclusion criteria: } 1 \text {. Past and current alcohol abuse (> } 20 \text { g of alcohol per day). 2. Chronic hepatitis B } \\
\text { and/or C virus infection. 3. Allergies to cardoon, artichoke, or maize. 4. Triglyceride concentration > } \\
250 \text { mg/dL. } 5 \text {. Diabetes. } 6 \text {. Autoimmune or cholestatic liver disease. 7. Liver cirrhosis. 8. Pregnancy. 9. } \\
\text { Nephrotic syndrome, chronic renal failure. 10. Gastroesophageal reflux. 11. Cancer. 12. Taking amio- } \\
\text { darone, antiretroviral agents, corticosteroids, methotrexate, tamoxifen, or valproate. 13. Recent initia- } \\
\text { tion of lipid-lowering drugs (less than } 6 \text { weeks) } \\
\text { Method for diagnosis of NAFLD: elastography }\end{array}$ \\
\hline
\end{tabular}

Interventions

Group 1: other supplements $(n=45)$ 
Ferro 2020 (Continued)

Further details: Bergamot and wild cardoon nutraceutical capsule $300 \mathrm{mg} / \mathrm{d}$ for 12 weeks (capsule contained a combination product with bergamot polyphenolic fraction (BPF) and wild-type Cynara cardunculus extract $(\mathrm{CyC})$ plus excipients including PUFA and a mixture of bergamot pulp and albedo derivative (registered Patents RM2008A000615, PCT/IB2009/055061, and 102017000040866) (batch number 18R049, expiration date 10/2020))

Group 2: no active intervention $(n=41)$

Further details: placebo capsule daily for 12 weeks (placebo contained maltodextrin plus excipients including PUFA and a mixture of bergamot pulp and albedo derivative (batch number 18R050, expiration date 10/2020))

\begin{tabular}{ll}
\hline Outcomes & No outcomes of interest were reported \\
\hline Notes & Source of funding (quote): "this study was funded by Italian Ministry of University and Research, grant \\
& number: Nutramed Project, PON 03PE000_78_1" \\
& Trial name/Trial registry number: ISRCTN12833814 \\
& Attempts were made to contact study authors in April 2021 \\
& \\
\hline
\end{tabular}

\section{Risk of bias}

\begin{tabular}{|c|c|c|}
\hline Bias & Authors' judgement & Support for judgement \\
\hline $\begin{array}{l}\text { Random sequence genera- } \\
\text { tion (selection bias) }\end{array}$ & Low risk & $\begin{array}{l}\text { Quote: "computer-generated random numbers were used for the simple ran- } \\
\text { domization of subjects" }\end{array}$ \\
\hline $\begin{array}{l}\text { Allocation concealment } \\
\text { (selection bias) }\end{array}$ & Unclear risk & Comment: this information was not available \\
\hline $\begin{array}{l}\text { Blinding of participants } \\
\text { and personnel (perfor- } \\
\text { mance bias) } \\
\text { All outcomes }\end{array}$ & Low risk & Comment: double-blind...placebo \\
\hline $\begin{array}{l}\text { Blinding of outcome as- } \\
\text { sessment (detection bias) } \\
\text { All outcomes }\end{array}$ & Low risk & Comment: double-blind...placebo \\
\hline $\begin{array}{l}\text { Incomplete outcome data } \\
\text { (attrition bias) } \\
\text { All outcomes }\end{array}$ & Unclear risk & $\begin{array}{l}\text { Comment: there were post-randomisation dropouts related to personal rea- } \\
\text { sons, health reasons, and work reasons - it is not clear whether they were re- } \\
\text { lated to the intervention and to outcomes }\end{array}$ \\
\hline $\begin{array}{l}\text { Selective reporting (re- } \\
\text { porting bias) }\end{array}$ & High risk & $\begin{array}{l}\text { Comment: a published protocol was available, but recruitment had com- } \\
\text { menced before the protocol was published; adverse events, mortality, fatty liv- } \\
\text { er resolution were not reported }\end{array}$ \\
\hline Other bias & Low risk & Comment: no other bias noted \\
\hline
\end{tabular}

Foroughi 2014

\section{Study characteristics}

\begin{tabular}{ll}
\hline Methods & Randomised clinical trial \\
\hline Participants & Country: Iran \\
& Period of recruitment: not stated \\
& Number randomised: 60 \\
& Post-randomisation dropouts: $0(0.0 \%)$ \\
& Revised sample size: 60
\end{tabular}


Foroughi 2014 (Continued)

Average age, years: 49

Females: 31 (51.7\%)

NASH: not stated

Diabetes mellitus: not stated

Inclusion criteria: 1. NAFLD on USS

Exclusion criteria: 1. Acute illness. 2. Chronic kidney disease. 3. Hyperparathyroid, hypoparathyroid. 4.

Coronary heart disease. 5. Other chronic liver disease. 6. Pregnancy. 7. Taking drugs affecting ALT in-

cluding metformin

Method for diagnosis of NAFLD: ultrasound

\begin{tabular}{ll}
\hline Interventions & Group 1: vitamin $D(n=30)$ \\
& Further details: vitamin D 50,000 IU weekly for 10 weeks \\
& Group 2: no active intervention $(n=30)$ \\
& Further details: placebo
\end{tabular}

\begin{tabular}{ll}
\hline Outcomes & Outcomes reported: mortality at maximal follow-up \\
& Follow-up, months: 2
\end{tabular}

\begin{tabular}{ll}
\hline Notes & Source of funding (quote): "source of support: nil" \\
& Trial name/Trial registry number: not stated \\
& Attempts were made to contact study authors in December 2020 \\
\hline
\end{tabular}

\section{Risk of bias}

\begin{tabular}{lll}
\hline Bias & Authors' judgement & Support for judgement \\
\hline $\begin{array}{l}\text { Random sequence genera- } \\
\text { tion (selection bias) }\end{array}$ & Unclear risk & Comment: this information was not available \\
\hline $\begin{array}{l}\text { Allocation concealment } \\
\text { (selection bias) }\end{array}$ & Unclear risk & Comment: this information was not available \\
\hline $\begin{array}{l}\text { Blinding of participants } \\
\text { and personnel (perfor- } \\
\text { mance bias) }\end{array}$ & Low risk & Quote: "randomized double-blind placebo-controlled clinical trial" \\
All outcomes & \\
\hline
\end{tabular}

\begin{tabular}{|c|c|c|}
\hline $\begin{array}{l}\text { Blinding of outcome as- } \\
\text { sessment (detection bias) } \\
\text { All outcomes }\end{array}$ & Low risk & Quote: "randomized double-blind placebo-controlled clinical trial" \\
\hline $\begin{array}{l}\text { Incomplete outcome data } \\
\text { (attrition bias) } \\
\text { All outcomes }\end{array}$ & Low risk & Comment: there were no post-randomisation dropouts \\
\hline $\begin{array}{l}\text { Selective reporting (re- } \\
\text { porting bias) }\end{array}$ & High risk & $\begin{array}{l}\text { Comment: no previously published protocol was available; adverse events } \\
\text { were not reported adequately }\end{array}$ \\
\hline Other bias & Low risk & Comment: no other bias noted \\
\hline
\end{tabular}

\section{Gavrilescu 2017}

\section{Study characteristics}

Methods Randomised clinical trial


Gavrilescu 2017 (Continued)

\begin{tabular}{|c|c|}
\hline Participants & $\begin{array}{l}\text { Country: Romania } \\
\text { Period of recruitment: } 2015 \text { to } 2016 \\
\text { Number randomised: } 42 \\
\text { Post-randomisation dropouts: not stated } \\
\text { Revised sample size: } 42 \\
\text { Average age, years: not stated } \\
\text { Females: not stated } \\
\text { NASH: not stated } \\
\text { Diabetes mellitus: not stated } \\
\text { Inclusion and exclusion criteria: not stated } \\
\text { Method for diagnosis of NAFLD: not stated }\end{array}$ \\
\hline Interventions & $\begin{array}{l}\text { Group 1: prebiotics/probiotics/synbiotics ( } n=\text { not stated) } \\
\text { Further details: prebiotic supplements for } 24 \text { weeks } \\
\text { Group 2: no active intervention ( } n=\text { not stated) } \\
\text { Further details: no treatment } \\
\text { Additional details: both groups received lifestyle modification }\end{array}$ \\
\hline Outcomes & No outcomes of interest were reported \\
\hline Notes & $\begin{array}{l}\text { Source of funding: not stated } \\
\text { Trial name/Trial registry number: not stated } \\
\text { Attempts were made to contact study authors in December } 2020\end{array}$ \\
\hline
\end{tabular}

\section{Risk of bias}

\begin{tabular}{lll}
\hline Bias & Authors' judgement & Support for judgement \\
\hline $\begin{array}{l}\text { Random sequence genera- } \\
\text { tion (selection bias) }\end{array}$ & Unclear risk & Comment: this information was not available \\
\hline $\begin{array}{l}\text { Allocation concealment } \\
\text { (selection bias) }\end{array}$ & Unclear risk & Comment: this information was not available \\
\hline $\begin{array}{l}\text { Blinding of participants } \\
\text { and personnel (perfor- } \\
\text { mance bias) }\end{array}$ & Unclear risk & Comment: this information was not available \\
All outcomes & & \\
\hline
\end{tabular}

\begin{tabular}{lll}
\hline $\begin{array}{l}\text { Blinding of outcome as- } \\
\text { sessment (detection bias) } \\
\text { All outcomes }\end{array}$ & Unclear risk & Comment: this information was not available \\
\hline $\begin{array}{l}\text { Incomplete outcome data } \\
\text { (attrition bias) } \\
\text { All outcomes }\end{array}$ & Unclear risk & Comment: this information was not available \\
\hline $\begin{array}{l}\text { Selective reporting (re- } \\
\text { porting bias) }\end{array}$ & High risk & $\begin{array}{l}\text { Comment: no previously published protocol was available; adverse events, } \\
\text { mortality, fatty liver resolution were not reported }\end{array}$ \\
\hline Other bias & Low risk & Comment: no other bias noted \\
\hline
\end{tabular}

\section{Geier 2018}

\section{Study characteristics}

Nutritional supplementation for nonalcohol-related fatty liver disease: a network meta-analysis (Review)

Copyright @ 2021 The Cochrane Collaboration. Published by John Wiley \& Sons, Ltd. 
Geier 2018 (Continued)

\begin{tabular}{|c|c|}
\hline Methods & Randomised clinical trial \\
\hline Participants & $\begin{array}{l}\text { Country: Switzerland } \\
\text { Period of recruitment: not stated } \\
\text { Number randomised: } 20 \\
\text { Post-randomisation dropouts: } 2(10.0 \%) \\
\text { Revised sample size: } 18 \\
\text { Reasons for post-randomisation dropouts: missing values } \\
\text { Average age, years: not stated } \\
\text { Females: not stated } \\
\text { NASH: } 18 \text { (100.0\%) } \\
\text { Diabetes mellitus: not stated } \\
\text { Inclusion criteria: } 1 \text {. Increased alanine aminotransferase (ALT) level ( } \geq 1.2 \text {-fold ULN). } 2 \text {. Histological di- } \\
\text { agnosis of NASH diagnosed according to SAF score obtained within } 18 \text { months preceding entry. } 3 \text {. De- } \\
\text { creased } 25-O H \text { vitamin D level (<30 } \mu \text { g/L). } 4 \text {. Non-excessive alcohol consumption ( }<21 \text { standard drinks } \\
\text { on average per week in males, }<14 \text { standard drinks on average per week in females) } \\
\text { Exclusion criteria: } 1 \text {. Cirrhosis. } 2 \text {. HCV RNA positivity. } 3 \text {. HBs antigen positivity. } 4 \text {. Other liver disease in- } \\
\text { cluding autoimmune hepatitis, hereditary haemochromatosis, alpha-1-antitrypsin deficiency, Wilson's } \\
\text { disease. } 5 \text {. Drug-induced fatty liver disease. } 6 \text {. Serious disease limiting life expectancy. } 7 \text {. Pregnant or } \\
\text { breastfeeding. } 8 \text {. Intention to become pregnant during the course of the study, or childbearing poten- } \\
\text { tial in women who were not using safe contraception } \\
\text { Method for diagnosis of NAFLD: liver biopsy }\end{array}$ \\
\hline
\end{tabular}

\begin{tabular}{ll}
\hline Interventions & Group 1: vitamin $D(n=8)$ \\
& Further details: vitamin D 2100 IU once daily for 48 weeks \\
& Group 2: no active intervention $(n=10)$ \\
& Further details: placebo
\end{tabular}

Outcomes Outcomes reported: serious adverse events (number of people), serious adverse events (number of events), any adverse events (number of events)

Follow-up, months: 11

Notes

Source of funding (quote): "the study medication (vitamin D3 2100 IU daily) and placebo were produced and provided by Antistress AG, Rapperswil-Jona, Switzerland"

Trial name/Trial registry number: NCT01571063

Attempts were made to contact study authors in December 2020

\section{Risk of bias}

\begin{tabular}{lll}
\hline Bias & Authors' judgement & Support for judgement \\
\hline $\begin{array}{l}\text { Random sequence genera- } \\
\text { tion (selection bias) }\end{array}$ & Low risk & $\begin{array}{l}\text { Quote: "randomization on day 0 (stratified for the presence of diabetes, block } \\
\text { size 10, not stratified by center) was performed by the Cantonal Pharmacy } \\
\text { Zurich before starting the } 48 \text { week treatment period" } \\
\text { Comment: although the precise method of generation of random sequence } \\
\text { generation was not reported, the method of allocation concealment suggests } \\
\text { that sequence generation was random }\end{array}$ \\
& \\
&
\end{tabular}

\begin{tabular}{ll}
\hline $\begin{array}{l}\text { Allocation concealment } \\
\text { (selection bias) }\end{array}$ & Quote: "randomization on day 0 (stratified for the presence of diabetes, block \\
& size 10, not stratified by center) was performed by the Cantonal Pharmacy \\
& Zurich before starting the 48 week treatment period"
\end{tabular}

Blinding of participants Low risk Quote: "double-blinded, randomized, placebo-controlled pilot study"
and personnel (perfor-
mance bias)
All outcomes

$\begin{aligned} & \text { Blinding of outcome as- } \\ & \text { sessment (detection bias) }\end{aligned} \quad$ Low risk Quote: "double-blinded, randomized, placebo-controlled pilot study"


Geier 2018 (Continued)

All outcomes

\begin{tabular}{|c|c|c|}
\hline $\begin{array}{l}\text { Incomplete outcome data } \\
\text { (attrition bias) }\end{array}$ & Unclear risk & $\begin{array}{l}\text { Comment: participants were excluded from analysis for reasons that may be } \\
\text { related to the intervention and to outcomes }\end{array}$ \\
\hline
\end{tabular}

All outcomes

related to the intervention and to outcomes

\begin{tabular}{|c|c|c|}
\hline $\begin{array}{l}\text { Selective reporting (re- } \\
\text { porting bias) }\end{array}$ & High risk & $\begin{array}{l}\text { Comment: no previously published protocol was available; neither mortality } \\
\text { nor fatty liver resolution was reported }\end{array}$ \\
\hline
\end{tabular}

Other bias Low risk Comment: no other bias noted

\section{Ghaffari 2018}

\section{Study characteristics}

\begin{tabular}{|c|c|}
\hline Methods & Randomised clinical trial \\
\hline Participants & $\begin{array}{l}\text { Country: Iran } \\
\text { Period of recruitment: not stated } \\
\text { Number randomised: } 92 \\
\text { Post-randomisation dropouts: } 7(7.6 \%) \\
\text { Revised sample size: } 85 \\
\text { Reasons for post-randomisation dropouts: discontinuation of intervention, lack of compliance, trans- } \\
\text { port difficulties } \\
\text { Average age, years: } 41 \\
\text { Females: } 46 \text { (54.1\%) } \\
\text { NASH: not stated } \\
\text { Diabetes mellitus: not stated } \\
\text { Inclusion criteria: } 1 \text {. NAFLD diagnosis confirmed by gastroenterologist } \\
\text { Exclusion criteria: } 1 \text {. Thyroid disorder. } 2 \text {. Cancer. 3. Biliary and kidney stones. 4. Viral hepatitis plus oth- } \\
\text { er hepatic disease. 5. Post-menopausal. } 6 \text {. Pregnant or breastfeeding } \\
\text { Method for diagnosis of NAFLD: ultrasound }\end{array}$ \\
\hline Interventions & $\begin{array}{l}\text { Group 1: other supplements }(n=64) \\
\text { Further details: turmeric }(3 \mathrm{~g} / \mathrm{d}) \text { and/or chicory seeds }(9 \mathrm{~g} / \mathrm{d}) \text { for } 12 \text { weeks } \\
\text { Group 2: no active intervention }(n=21) \\
\text { Further details: placebo }\end{array}$ \\
\hline Outcomes & No outcomes of interest were reported \\
\hline Notes & $\begin{array}{l}\text { Source of funding: not stated } \\
\text { Trial name/Trial registry number: IRCT201406183664N12 } \\
\text { Attempts were made to contact study authors in December } 2020\end{array}$ \\
\hline
\end{tabular}

\section{Risk of bias}

\begin{tabular}{lll}
\hline Bias & Authors' judgement & Support for judgement \\
\hline $\begin{array}{l}\text { Random sequence genera- } \\
\text { tion (selection bias) }\end{array}$ & Unclear risk & Comment: this information was not available \\
\hline $\begin{array}{l}\text { Allocation concealment } \\
\text { (selection bias) }\end{array}$ & Low risk & $\begin{array}{l}\text { Quote: "turmeric capsules and chicory seed powder were delivered in identical } \\
\text { packs for all patients by a coworker who did not involve in project" }\end{array}$ \\
\hline $\begin{array}{l}\text { Blinding of participants } \\
\text { and personnel (perfor- } \\
\text { mance bias) }\end{array}$ & Low risk & Quote: "double-blind, randomized controlled clinical trial...placebo" \\
\hline
\end{tabular}


Ghaffari 2018 (Continued)

All outcomes

Blinding of outcome as-
sessment (detection bias) $\quad$ Low risk $\quad$ Quote: "double-blind, randomized controlled clinical trial...placebo"

sessment (detection bias)

All outcomes

\begin{tabular}{|c|c|c|}
\hline $\begin{array}{l}\text { Incomplete outcome data } \\
\text { (attrition bias) }\end{array}$ & Unclear risk & $\begin{array}{l}\text { Comment: participants were excluded from analysis for reasons that may be } \\
\text { related to the intervention and to outcomes }\end{array}$ \\
\hline
\end{tabular}

All outcomes related to the intervention and to outcomes

Selective reporting (re- High risk
porting bias)
porting bias)
Comment: no previously published protocol was available; adverse events, mortality, fatty liver resolution were not reported

\begin{tabular}{ll}
\hline Other bias $\quad$ Low risk $\quad$ Comment: no other bias noted \\
\hline
\end{tabular}

Ghergherehchi 2013

\section{Study characteristics}

\begin{tabular}{ll}
\hline Methods & Randomised clinical trial \\
\hline Participants & Country: Iran \\
& Period of recruitment: 2008 to 2009 \\
& Number randomised: 33 \\
& Post-randomisation dropouts: not stated \\
& Revised sample size: 33 \\
& Average age, years: 7 \\
& Females: 15 (45.5\%) \\
& NASH: not stated \\
& Diabetes mellitus: not stated \\
Inclusion criteria: 1 . BMI > 97th percentile for age and sex. 2 . ALT/AST $>1.5 \times$ normal. 3. Signs of hepatic \\
steatosis on USS \\
Exclusion criteria: 1 . Other causes of liver disease. 2 . Drugs causing steatosis \\
Method for diagnosis of NAFLD: ultrasound plus transaminases
\end{tabular}

Interventions $\quad$ Group 1: vitamin $\mathrm{E}(\mathrm{n}=17)$

Further details: vitamin $\mathrm{E} 400 \mathrm{mg} / \mathrm{d}$ for 6 months

Group 2: no active intervention $(n=16)$

Further details: placebo

Additional details: both groups underwent lifestyle intervention (exercise and low-calorie diet)

\begin{tabular}{lll}
\hline Outcomes & No outcomes of interest were reported \\
\hline Notes & $\begin{array}{l}\text { Source of funding (quote): "this study is funded by Tabriz University of Medical Sciences" } \\
\text { Trial name/Trial registry number: not stated } \\
\text { Attempts were made to contact study authors in December } 2020\end{array}$ \\
\hline Risk of bias & Authors' judgement & Support for judgement \\
\hline Bias & Low risk & $\begin{array}{l}\text { Quote: "eligible patients were randomized into two groups by a person who } \\
\text { was not aware of the main objective by using software Random List" }\end{array}$ \\
\hline $\begin{array}{l}\text { Random sequence genera- } \\
\text { tion (selection bias) }\end{array}$ & Low risk & $\begin{array}{l}\text { Quote: "eligible patients were randomized into two groups by a person who } \\
\text { was not aware of the main objective by using software Random List" }\end{array}$ \\
\hline $\begin{array}{l}\text { Allocation concealment } \\
\text { (selection bias) }\end{array}$ & &
\end{tabular}


Ghergherehchi 2013 (Continued)
Blinding of participants
Low risk
Quote: "double-blind placebo study" and personnel (perfor- mance bias)
All outcomes

\begin{tabular}{lll}
\hline $\begin{array}{l}\text { Blinding of outcome as- } \\
\text { sessment (detection bias) } \\
\text { All outcomes }\end{array}$ & Low risk & Quote: "double-blind placebo study" \\
\hline $\begin{array}{l}\text { Incomplete outcome data } \\
\text { (attrition bias) } \\
\text { All outcomes }\end{array}$ & Unclear risk & Comment: this information was not available \\
\hline $\begin{array}{l}\text { Selective reporting (re- } \\
\text { porting bias) }\end{array}$ & High risk & $\begin{array}{l}\text { Comment: no previously published protocol was available; adverse events, } \\
\text { mortality, fatty liver resolution were not reported }\end{array}$ \\
\hline Other bias & Low risk & Comment: no other bias noted \\
\hline
\end{tabular}

Gianturco 2013

\section{Study characteristics}

\begin{tabular}{|c|c|}
\hline Methods & Randomised clinical trial \\
\hline Participants & $\begin{array}{l}\text { Country: Italy } \\
\text { Period of recruitment: not stated } \\
\text { Number randomised: } 200 \\
\text { Post-randomisation dropouts: } 4(2.0 \%) \\
\text { Revised sample size: } 196 \\
\text { Reasons for post-randomisation dropouts: onset of diabetes } \\
\text { Average age, years: } 62 \\
\text { Females: } 92(46.9 \%) \\
\text { NASH: not stated } \\
\text { Diabetes mellitus: } 0 \text { (0.0\%) } \\
\text { Inclusion criteria: } 1 \text {. Histologically confirmed NAFLD } \\
\text { Exclusion criteria: } 1 \text {. Hepatitis B/C. 2. Gallstones. 3. Alcohol consumption. 4. Renal failure. 5. Diabetes } \\
\text { Method for diagnosis of NAFLD: liver biopsy }\end{array}$ \\
\hline Interventions & $\begin{array}{l}\text { Group 1: other antioxidants }(\mathrm{n}=104) \\
\text { Further details: alpha-lipoic acid } 400 \mathrm{mg} / \mathrm{d} \text { for } 12 \text { months } \\
\text { Group 2: no active intervention }(\mathrm{n}=92) \\
\text { Further details: placebo } \\
\text { Additional details: both groups received hypocaloric diet. A proportion of patients in each group re- } \\
\text { ceived ursodeoxycholic acid; this was decided randomly }\end{array}$ \\
\hline Outcomes & $\begin{array}{l}\text { Outcomes reported: fibrosis score, NAFLD activity score } \\
\text { Follow-up, months: } 12\end{array}$ \\
\hline Notes & $\begin{array}{l}\text { Source of funding: not stated } \\
\text { Trial name/Trial registry number: not stated } \\
\text { Attempts were made to contact study authors in December } 2020\end{array}$ \\
\hline
\end{tabular}

\section{Risk of bias}


Gomez 2009 (Continued)

Additional details: both groups received hypocaloric diet and exercise

Outcomes reported: mortality at maximal follow-up, fibrosis score, NAFLD activity score

Follow-up, months: 6

$\begin{array}{ll}\text { Notes } & \text { Source of funding (quote): "supported in part by a grant from Catalysis Laboratories, Spain" } \\ & \text { Trial name/Trial registry number: NCT00509418 }\end{array}$

Attempts were made to contact study authors in December 2020

\section{Risk of bias}

\begin{tabular}{lll}
\hline Bias & Authors' judgement & Support for judgement \\
\hline $\begin{array}{l}\text { Random sequence genera- } \\
\text { tion (selection bias) }\end{array}$ & Unclear risk & Comment: this information was not available \\
\hline $\begin{array}{l}\text { Allocation concealment } \\
\text { (selection bias) }\end{array}$ & Low risk & $\begin{array}{l}\text { Quote: "it was performed by a health worker experienced in randomization } \\
\text { techniques who was not involved in the evaluation or treatment of the partici- } \\
\text { pants. The physicians, study coordinators and patients did not have access to } \\
\text { the randomization scheme" }\end{array}$ \\
\hline
\end{tabular}

Blinding of participants Unclear risk Comment: this information was not available

and personnel (perfor-

mance bias)

All outcomes

Blinding of outcome as-
sessment (detection bias) $\quad$ Unclear risk Comment: this information was not available

(detection bias)

All outcomes

\begin{tabular}{lll}
\hline $\begin{array}{l}\text { Incomplete outcome data } \\
\text { (attrition bias) } \\
\text { All outcomes }\end{array}$ & Low risk & Comment: there were no post-randomisation dropouts \\
\hline $\begin{array}{l}\text { Selective reporting (re- } \\
\text { porting bias) }\end{array}$ & High risk & $\begin{array}{l}\text { Comment: no previously published protocol was available; adverse events } \\
\text { were not reported adequately }\end{array}$ \\
\hline Other bias & Low risk & Comment: no other bias noted \\
\hline
\end{tabular}

Gonciarz 2012

\section{Study characteristics}

\begin{tabular}{ll}
\hline Methods & Randomised clinical trial \\
\hline Participants & Country: Poland \\
& Period of recruitment: 2008 to 2010 \\
& Number randomised: 45 \\
& Post-randomisation dropouts: $3(6.7 \%)$ \\
& Revised sample size: 42 \\
& Reasons for post-randomisation dropouts: not stated \\
& Average age, years: 41 \\
& Females: 16 (38.1\%) \\
& NASH: not stated \\
& Diabetes mellitus: not stated \\
Inclusion criteria: 1 . NASH on liver biopsy. 2. Alcohol $<20 \mathrm{~g} / \mathrm{d}$. 3. Elevated aminotransferases \\
Exclusion criteria: 1 . Other causes of liver disease. 2. On supplements containing antioxidants
\end{tabular}




$\begin{array}{ll}\text { Interventions } & \text { Group 1: other antioxidants }(\mathrm{n}=30) \\ & \text { Further details: melatonin } 5 \mathrm{mg} \text { twice daily for } 24 \text { weeks } \\ \text { Group 2: no active intervention }(\mathrm{n}=12) \\ \text { Further details: placebo } \\ \text { Additional details: both groups received lifestyle intervention comprising exercise and diet }\end{array}$

\begin{tabular}{ll}
\hline Outcomes & No outcomes of interest were reported \\
\hline Notes & Source of funding (quote): "this work is supported by grant No K/PBW/000495 from the Polish Ministry \\
& of Science and Higher Education" \\
& Trial name/Trial registry number: not stated \\
& Attempts were made to contact study authors in December 2020 \\
& \\
\hline
\end{tabular}

\section{Risk of bias}

\begin{tabular}{|c|c|c|}
\hline Bias & Authors' judgement & Support for judgement \\
\hline $\begin{array}{l}\text { Random sequence genera- } \\
\text { tion (selection bias) }\end{array}$ & Unclear risk & Comment: this information was not available \\
\hline $\begin{array}{l}\text { Allocation concealment } \\
\text { (selection bias) }\end{array}$ & Unclear risk & Comment: this information was not available \\
\hline $\begin{array}{l}\text { Blinding of participants } \\
\text { and personnel (perfor- } \\
\text { mance bias) } \\
\text { All outcomes }\end{array}$ & Unclear risk & Comment: this information was not available \\
\hline $\begin{array}{l}\text { Blinding of outcome as- } \\
\text { sessment (detection bias) } \\
\text { All outcomes }\end{array}$ & Unclear risk & Comment: this information was not available \\
\hline $\begin{array}{l}\text { Incomplete outcome data } \\
\text { (attrition bias) } \\
\text { All outcomes }\end{array}$ & Unclear risk & $\begin{array}{l}\text { Comment: participants were excluded from analysis for reasons that may be } \\
\text { related to the intervention and to outcomes }\end{array}$ \\
\hline $\begin{array}{l}\text { Selective reporting (re- } \\
\text { porting bias) }\end{array}$ & High risk & $\begin{array}{l}\text { Comment: no previously published protocol was available; adverse events, } \\
\text { mortality, fatty liver resolution were not reported }\end{array}$ \\
\hline Other bias & Low risk & Comment: no other bias noted \\
\hline
\end{tabular}

Guo 2016

\section{Study characteristics}

\begin{tabular}{ll}
\hline Methods & Randomised clinical trial \\
\hline Participants & Country: China \\
& Period of recruitment: 2011 to 2013 \\
& Number randomised: 88 \\
& Post-randomisation dropouts: 4 (4.5\%) \\
& Revised sample size: 84 \\
& Reasons for post-randomisation dropouts: poor compliance/did not complete experimental protocol \\
& (2), diarrhoea/flatulence/nausea (1), lost to follow up (1)
\end{tabular}


Guo 2016 (Continued)

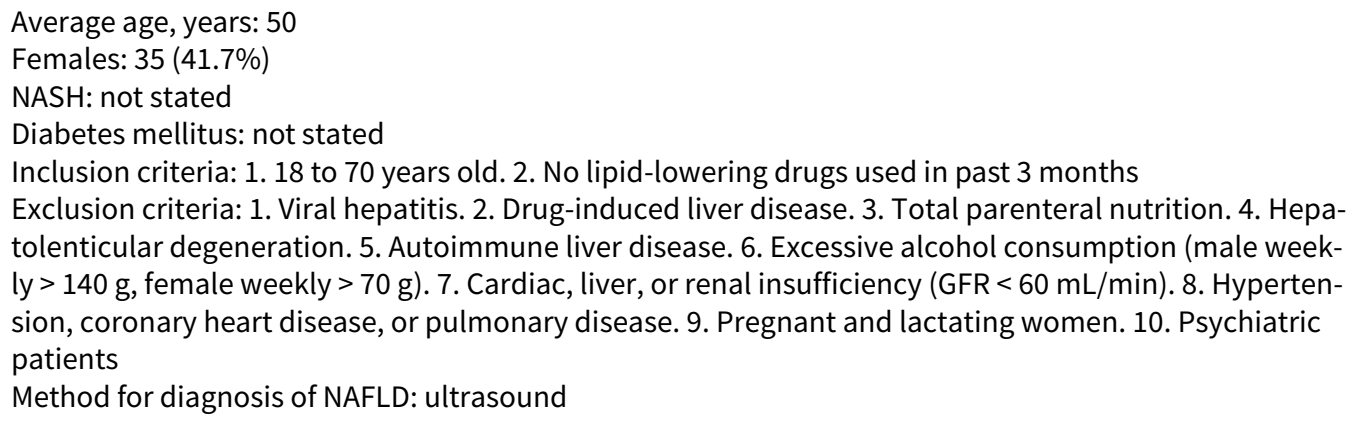

$\begin{array}{ll}\text { Interventions } & \text { Group 1: prebiotics/probiotics/synbiotics }(\mathrm{n}=42) \\ & \text { Further details: routine treatment of diet, exercise, and metformin combined with enteric bifid-triple } \\ & \text { viable capsule (Bifidobacterium: } 1260 \mathrm{mg} \text { per day) for } 8 \text { weeks } \\ & \text { Group 2: no active intervention }(\mathrm{n}=42) \\ & \text { Further details: routine treatment of diet, exercise, and metformin plus dimethicone }(20 \mathrm{mg} / \mathrm{kg} / \mathrm{d}) \mathrm{for} 8 \\ & \text { weeks } \\ & \text { Additional details: both groups received lifestyle advice }\end{array}$

Outcomes Outcomes reported: serious adverse events (number of people), adverse events (number of people)
Follow-up, months: 2

\begin{tabular}{ll}
\hline Notes & Source of funding: not stated \\
& Trial name/Trial registry number: not stated \\
& Attempts were made to contact study authors in December 2020
\end{tabular}

\section{Risk of bias}

\begin{tabular}{lll}
\hline Bias & Authors' judgement & Support for judgement \\
\hline $\begin{array}{l}\text { Random sequence genera- } \\
\text { tion (selection bias) }\end{array}$ & Unclear risk & Comment: this information was not available \\
\hline $\begin{array}{l}\text { Allocation concealment } \\
\text { (selection bias) }\end{array}$ & Unclear risk & Comment: this information was not available \\
\hline $\begin{array}{l}\text { Blinding of participants } \\
\text { and personnel (perfor- } \\
\text { mance bias) } \\
\text { All outcomes }\end{array}$ & Unclear risk & Comment: this information was not available \\
\hline
\end{tabular}

\begin{tabular}{ll} 
Blinding of outcome as- & Unclear risk Comment: this information was not available \\
sessment (detection bias) & \\
All outcomes & \\
\hline
\end{tabular}

\begin{tabular}{|c|c|c|}
\hline $\begin{array}{l}\text { Incomplete outcome data } \\
\text { (attrition bias) } \\
\text { All outcomes }\end{array}$ & Unclear risk & $\begin{array}{l}\text { Comment: there were post-randomisation dropouts, but it is not clear whether } \\
\text { these were related to the intervention or to outcomes }\end{array}$ \\
\hline
\end{tabular}

\begin{tabular}{lll}
\hline $\begin{array}{l}\text { Selective reporting (re- } \\
\text { porting bias) }\end{array}$ & High risk & $\begin{array}{l}\text { Comment: no previously published protocol was available; adverse events, } \\
\text { mortality, fatty liver resolution were not reported }\end{array}$ \\
\hline Other bias & Low risk & Comment: no other bias noted \\
\hline
\end{tabular}


Harrison 2003

\section{Study characteristics}

\begin{tabular}{|c|c|}
\hline Methods & Randomised clinical trial \\
\hline Participants & $\begin{array}{l}\text { Country: USA } \\
\text { Period of recruitment: } 2000 \text { to } 2002 \\
\text { Number randomised: } 49 \\
\text { Post-randomisation dropouts: } 4(8.2 \%) \\
\text { Revised sample size: } 45 \\
\text { Reasons for post-randomisation dropouts: did not want biopsy or moved away } \\
\text { Average age, years: } 51 \\
\text { Females: } 25(55.6 \%) \\
\text { NASH: } 45 \text { (100.0\%) } \\
\text { Diabetes mellitus: } 19 \text { ( } 42.2 \%) \\
\text { Inclusion criteria: } 1 . \text { Clinical and histological diagnosis of NASH. } 2 .>18 \text { years old. 3. Liver biopsy in } \\
\text { past } 6 \text { months for elevated aminotransferases. } 4 \text {. Well-compensated liver disease. } 5 \text {. Hb }>12 \mathrm{~g} / \mathrm{dL} \text { in } \\
\text { women, > } 13 \mathrm{~g} / \mathrm{dL} \text { in men. } 6 \text {. WCC }>3000 \mathrm{~mm}^{3} \text {, neutrophils }>15,000 \text {, platelets }>70,000 \text {, and creatinine }< \\
1.4 \text { mg/dL } \\
\text { Exclusion criteria: } 1 . \text { Other causes of chronic liver disease. } 2 \text {. Drugs causing steatohepatitis (e.g. tamox- } \\
\text { ifen, steroids, amiodarone). 3. Prior surgery such as gastroplasty, jejunoileal or jejunocolic bypass. } 4 \text {. } \\
\text { Decompensated liver disease. } 5 \text {. Pregnancy. } 6 \text {. Total parenteral nutrition in last } 6 \text { months. } 7 \text {. Previous } \\
\text { transplant. } 8 \text {. Alcohol consumption }>10 \mathrm{~g} / \mathrm{d} \\
\text { Method for diagnosis of NAFLD: liver biopsy }\end{array}$ \\
\hline
\end{tabular}

Interventions Group 1: vitamin E plus vitamin C $(n=23)$

Further details: vitamin E 1000 IU plus vitamin C $1000 \mathrm{mg}$ daily for 6 months

Group 2: no active intervention $(n=22)$

Further details: placebo

\begin{tabular}{ll} 
Outcomes & Outcomes reported: fibrosis score \\
& Follow-up, months: 6 \\
\hline Notes & Source of funding: not stated \\
& Trial name/Trial registry number: not stated \\
& Attempts were made to contact study authors in December 2020
\end{tabular}

\section{Risk of bias}

\begin{tabular}{lll}
\hline Bias & Authors' judgement & Support for judgement \\
\hline $\begin{array}{l}\text { Random sequence genera- } \\
\text { tion (selection bias) }\end{array}$ & Low risk & Quote: "computer-generated randomization table" \\
\hline $\begin{array}{l}\text { Allocation concealment } \\
\text { (selection bias) }\end{array}$ & Low risk & $\begin{array}{l}\text { Quote: "this randomization table was kept by the pharmacy where the vita- } \\
\text { mins or placebo were to be obtained by the patient" }\end{array}$ \\
\hline $\begin{array}{l}\text { Blinding of participants } \\
\text { and personnel (perfor- } \\
\text { mance bias) } \\
\text { All outcomes }\end{array}$ & Low risk & Quote: "prospective, double-blind, randomized, placebo-controlled trial" \\
\hline $\begin{array}{l}\text { Blinding of outcome as- } \\
\text { sessment (detection bias) } \\
\text { All outcomes }\end{array}$ & Low risk & \\
\hline $\begin{array}{l}\text { Incomplete outcome data } \\
\text { (attrition bias) } \\
\text { All outcomes }\end{array}$ & Unclear risk & $\begin{array}{l}\text { Quote: "prospective, double-blind, randomized, placebo-controlled trial" } \\
\text { related to the intervention and to outcomes }\end{array}$ \\
\hline
\end{tabular}


Harrison 2003 (Continued)

Selective reporting (re- High risk Comment: no previously published protocol was available; adverse events, porting bias)

mortality, fatty liver resolution were not reported

Other bias Low risk Comment: no other bias noted

Hashemi 2009

\section{Study characteristics}

\begin{tabular}{ll}
\hline Methods & Randomised clinical trial \\
\hline Participants & Country: Iran \\
Period of recruitment: 2007 to 2008 \\
Number randomised: 100 \\
Post-randomisation dropouts: not stated \\
Revised sample size: 100 \\
Average age, years: 39 \\
Females: $43(43.0 \%)$ \\
NASH: 100 (100.0\%) \\
Diabetes mellitus: not stated \\
Inclusion criteria: 1 . Sonographic evidence of fatty liver. 2. ALT > 1.2× normal. 3. Exclusion of other \\
chronic liver disease. 4. Suggestive histological evidence of NASH or strong risk factors such as type 2 \\
diabetes or BMI > 30 \\
Exclusion criteria: 1 . Alcohol > 20 g/d. 2. Drugs causing fatty liver disease. 3. Severe comorbid medical \\
condition \\
Method for diagnosis of NAFLD: ultrasound plus transaminases
\end{tabular}

\begin{tabular}{ll}
\hline Interventions & $\begin{array}{l}\text { Group 1: other antioxidants }(\mathrm{n}=50) \\
\text { Further details: silymarin } 280 \mathrm{mg} \text { for } 24 \text { weeks } \\
\text { Group 2: no active intervention }(\mathrm{n}=50) \\
\text { Further details: placebo }\end{array}$ \\
\hline Outcomes & No outcomes of interest were reported \\
\hline Notes & $\begin{array}{l}\text { Source of funding: not stated } \\
\text { Trial name/Trial registry number: not stated } \\
\text { Attempts were made to contact study authors in December } 2020\end{array}$
\end{tabular}

\section{Risk of bias}

\begin{tabular}{lll}
\hline Bias & Authors' judgement & Support for judgement \\
\hline $\begin{array}{l}\text { Random sequence genera- } \\
\text { tion (selection bias) }\end{array}$ & Unclear risk & Comment: this information was not available \\
\hline $\begin{array}{l}\text { Allocation concealment } \\
\text { (selection bias) }\end{array}$ & Unclear risk & Comment: this information was not available \\
\hline $\begin{array}{l}\text { Blinding of participants } \\
\text { and personnel (perfor- } \\
\text { mance bias) } \\
\text { All outcomes }\end{array}$ & Unclear risk & Comment: this information was not available \\
\hline
\end{tabular}

Blinding of outcome as-
sessment (detection bias) $\quad$ Unclear risk $\quad$ Comment: this information was not available

All outcomes 
Hashemi 2009 (Continued)
Incomplete outcome data
Unclear risk
Comment: this information was not available
(attrition bias)

All outcomes

\begin{tabular}{lll}
\hline $\begin{array}{l}\text { Selective reporting (re- } \\
\text { porting bias) }\end{array}$ & High risk & $\begin{array}{l}\text { Comment: no previously published protocol was available; adverse events, } \\
\text { mortality, fatty liver resolution were not reported }\end{array}$ \\
\hline Other bias & Low risk & Comment: no other bias noted \\
\hline
\end{tabular}

Heeboll 2016

\section{Study characteristics}

\begin{tabular}{|c|c|}
\hline Methods & Randomised clinical trial \\
\hline Participants & $\begin{array}{l}\text { Country: Denmark } \\
\text { Period of recruitment: } 2011 \text { to } 2014 \\
\text { Number randomised: } 28 \\
\text { Post-randomisation dropouts: } 0(0.0 \%) \\
\text { Revised sample size: } 28 \\
\text { Average age, years: not stated } \\
\text { Females: not stated } \\
\text { NASH: not stated } \\
\text { Diabetes mellitus: } 0(0.0 \%) \\
\text { Inclusion criteria: } 1 \text {. BMI } \geq 25 \mathrm{~kg} / \mathrm{m}^{2} \text {. 2. ALT }>70 \text { in men, }>45 \text { in women. } 3 \text {. At least } 1 \text { element of metabol- } \\
\text { ic syndrome } \\
\text { Exclusion criteria: } 1 \text {. Type } 2 \text { diabetes mellitus. } 2 \text {. Severe systemic or malignant disease } \\
\text { Method for diagnosis of NAFLD: ultrasound }\end{array}$ \\
\hline Interventions & $\begin{array}{l}\text { Group 1: other supplements }(n=15) \\
\text { Further details: resveratrol } 1.5 \mathrm{~g} \text { daily for } 6 \text { months } \\
\text { Group 2: no active intervention }(n=13) \\
\text { Further details: placebo }\end{array}$ \\
\hline Outcomes & $\begin{array}{l}\text { Outcomes reported: mortality at maximal follow-up, any adverse events (number of people), any ad- } \\
\text { verse events (number of events), liver transplantation at maximal follow-up, decompensation (number } \\
\text { of people), cirrhosis (number of people), resolution of fatty liver disease, hepatocellular carcinoma } \\
\text { Follow-up, months: } 6\end{array}$ \\
\hline Notes & $\begin{array}{l}\text { Source of funding (quote): "resveratrol was provided by Evolva SA (Basel, Switzerland), free of charge" } \\
\text { Trial name/Trial registry number: NCT01464801 } \\
\text { Attempts were made to contact study authors in December } 2020\end{array}$ \\
\hline
\end{tabular}

\section{Risk of bias}

\begin{tabular}{lll}
\hline Bias & Authors' judgement & Support for judgement \\
\hline $\begin{array}{l}\text { Random sequence genera- } \\
\text { tion (selection bias) }\end{array}$ & Low risk & $\begin{array}{l}\text { Quote: "a block randomization of four was generated (using www.randomiza- } \\
\text { tion.com)" }\end{array}$ \\
\hline $\begin{array}{l}\text { Allocation concealment } \\
\text { (selection bias) }\end{array}$ & Low risk & $\begin{array}{l}\text { Quote: "a block randomization of four was generated (using www.randomiza- } \\
\text { tion.com), by the hospital pharmacist at study start. Study medication was ad- } \\
\text { ministered as tablets in sealed, sequentially numbered containers" }\end{array}$ \\
\hline
\end{tabular}


Heeboll 2016 (Continued)

Blinding of participants Low risk Quote: "prospective, placebo-controlled, randomised and double-blind cliniand personnel (perforcal trial"

mance bias)

All outcomes
Blinding of outcome as- Low risk sessment (detection bias)

All outcomes

\begin{tabular}{lll}
\hline $\begin{array}{l}\text { Incomplete outcome data } \\
\text { (attrition bias) } \\
\text { All outcomes }\end{array}$ & Low risk & Comment: there were no post-randomisation dropouts \\
\hline $\begin{array}{l}\text { Selective reporting (re- } \\
\text { porting bias) }\end{array}$ & Low risk & $\begin{array}{l}\text { Comment: no previously published protocol was available; adverse events and } \\
\text { either mortality or fatty liver resolution or both were reported }\end{array}$ \\
\hline Other bias & Low risk & Comment: no other bias noted \\
\hline
\end{tabular}

Hong 2016

\section{Study characteristics}

\begin{tabular}{|c|c|}
\hline Methods & Randomised clinical trial \\
\hline \multirow[t]{12}{*}{ Participants } & Country: China \\
\hline & Period of recruitment: 2011 to 2012 \\
\hline & Number randomised: 80 \\
\hline & Post-randomisation dropouts: 14 (17.5\%) \\
\hline & Revised sample size: 66 \\
\hline & $\begin{array}{l}\text { Reasons for post-randomisation dropouts: did not complete full therapy (inadequate data, patient re- } \\
\text { fusal) }\end{array}$ \\
\hline & Average age, years: 49 \\
\hline & Females: $10(15.2 \%)$ \\
\hline & NASH: not stated \\
\hline & Diabetes mellitus: not stated \\
\hline & Inclusion criteria: 1 . Fatty liver disease. 2 . AST $\geq 50$ or $A L T \geq 50$ \\
\hline & $\begin{array}{l}\text { Exclusion: 1. Other causes of liver disease (e.g. viral hepatitis, autoimmune hepatitis, haemochromato- } \\
\text { sis). 2. Pancreatitis. 3. Cancer. 4. Drug-induced liver injury. } \\
\text { Method for diagnosis of NAFLD: not stated }\end{array}$ \\
\hline \multirow[t]{5}{*}{ Interventions } & Group 1: other supplements plus other antioxidants $(n=35)$ \\
\hline & $\begin{array}{l}\text { Further details: Korean red ginseng ( } 3000 \text { mg/d) plus Silybum marianum (Legalon) capsule ( } 450 \text { mg/d) } \\
\text { for } 3 \text { weeks }\end{array}$ \\
\hline & Group 2: other antioxidants $(n=31)$ \\
\hline & Further details: Silybum marianum (Legalon) capsule (450 mg/d) plus placebo for 3 weeks \\
\hline & Additional details: both groups received lifestyle intervention \\
\hline Outcomes & No outcomes of interest were reported \\
\hline \multirow[t]{3}{*}{ Notes } & $\begin{array}{l}\text { Source of funding (quote): "this research was supported by a grant from the Korea Society of Ginseng } \\
\text { funded by Korea Ginseng Corporation (Korean Red Ginseng; 2011)" }\end{array}$ \\
\hline & Trial name/Trial registry number: NCT02331589 \\
\hline & Attempts were made to contact study authors in December 2020 \\
\hline
\end{tabular}

\section{Risk of bias}

Nutritional supplementation for nonalcohol-related fatty liver disease: a network meta-analysis (Review) 
Hong 2016 (Continued)

Bias Authors' judgement Support for judgement

Random sequence genera- Low risk Quote: "randomization was performed using a computerized procedure"
tion (selection bias)

Allocation concealment Unclear risk Comment: this information was not available
(selection bias)

\begin{tabular}{|c|c|c|}
\hline $\begin{array}{l}\text { Blinding of participants } \\
\text { and personnel (perfor- }\end{array}$ & Unclear risk & $\begin{array}{l}\text { Quote: "a single-blind, randomized, controlled clinical trial" } \\
\text { Comment: not clear who were blinded }\end{array}$ \\
\hline
\end{tabular}

mance bias)

All outcomes

$\begin{array}{lll}\text { Blinding of outcome as- } & \text { Unclear risk } & \text { Quote: "a single-blind, randomized, controlled clinical trial" } \\ \text { sessment (detection bias) } & & \text { Comment: not clear whether outcome assessors were blinded } \\ \text { All outcomes } & \end{array}$

\begin{tabular}{lll}
\hline $\begin{array}{l}\text { Incomplete outcome data } \\
\text { (attrition bias) } \\
\text { All outcomes }\end{array}$ & Unclear risk & $\begin{array}{l}\text { Comment: participants were excluded from analysis for reasons that may be } \\
\text { related to the intervention and to outcomes }\end{array}$ \\
\hline $\begin{array}{l}\text { Selective reporting (re- } \\
\text { porting bias) }\end{array}$ & High risk & $\begin{array}{l}\text { Comment: no previously published protocol was available; adverse events, } \\
\text { mortality, fatty liver resolution were not reported }\end{array}$ \\
\hline Other bias & Low risk & Comment: no other bias noted \\
\hline
\end{tabular}

Hong 2021

\section{Study characteristics}

\begin{tabular}{|c|c|}
\hline Methods & Randomised clinical trial \\
\hline Participants & $\begin{array}{l}\text { Country: Korea } \\
\text { Period of recruitment: } 2017 \text { to } 2018 \\
\text { Number randomised: } 94 \\
\text { Post-randomisation dropouts: } 7 \text { (7.4\%) } \\
\text { Revised sample size: } 87 \\
\text { Reasons for post-randomisation dropouts: patient refusal, loss to follow-up } \\
\text { Average age, years: } 50 \\
\text { Females: } 35 \text { (40.2\%) } \\
\text { NASH: not stated } \\
\text { Diabetes mellitus: not stated } \\
\text { Inclusion criteria: } 1 \text {. Aspartate aminotransferase (AST) or alanine aminotransferase (ALT) levels } \geq 50 \text { IU/ } \\
\text { L. 2. Fatty liver disease in abdominal ultrasound. } 3 \text {. Older than } 20 \text { years } \\
\text { Exclusion criteria: } 1 \text {. Excessive alcohol drinking (male }>60 \text { g/week, female }>40 \text { g/week). } 2 \text {. Virus-related } \\
\text { hepatitis, alcohol-induced hepatitis, autoimmune disease, haemochromatosis, infiltrative liver disease. } \\
\text { 3. Pancreas problem. } 4 \text {. Drug-related liver injury. } 5 \text {. Cancer } \\
\text { Method for diagnosis of NAFLD: ultrasound and transaminases }\end{array}$ \\
\hline Interventions & $\begin{array}{l}\text { Group 1: other supplements ( } n=43) \\
\text { Further details: Korean red ginseng (KRG, ginsenosides Rg1pRb1pRg3 } 4.5 \mathrm{mg} / \mathrm{g} ; 2000 \mathrm{mg} / \mathrm{d} \text { ) and milk- } \\
\text { thistle dried extracts powder ( } 450 \mathrm{mg} / \mathrm{d}) \text { for } 30 \text { days } \\
\text { Group 2: no active intervention ( } \mathrm{n}=44) \\
\text { Further details: placebo capsules (cellulose) and milk-thistle dried extracts powder ( } 450 \mathrm{mg} / \mathrm{d} \text { ) daily for } \\
30 \text { days. Placebos were of the same size and shape as the KRG capsule powder } \\
\text { Additional details: all patients were educated about diet and exercise according to recommendations }\end{array}$ \\
\hline
\end{tabular}


Hong 2021 (Continued)

Outcomes No outcomes of interest were reported
Source of funding (quote): "this research was supported by Hallym University ResearchFund, the Korea Society of Ginseng funded by Korea Ginseng Corporation (Korea Red Ginseng; 2016), the Basic Science ResearchProgram through the National Research Foundation of Korea (NRF)funded by the Ministry of Education, Science and Technology (NRF-2018M3A9F3020956 and NRF-2019R1I1A3A01060447) and Hallym University Research Fund 2018 (HURF-2018-67)"

Trial name/Trial registry number: NCT03945123

Attempts were made to contact study authors in April 2021

\section{Risk of bias}

\begin{tabular}{|c|c|c|}
\hline Bias & Authors' judgement & Support for judgement \\
\hline $\begin{array}{l}\text { Random sequence genera- } \\
\text { tion (selection bias) }\end{array}$ & Low risk & Quote: "a computerized procedure was used for the randomization" \\
\hline $\begin{array}{l}\text { Allocation concealment } \\
\text { (selection bias) }\end{array}$ & Unclear risk & Comment: this information was not available \\
\hline $\begin{array}{l}\text { Blinding of participants } \\
\text { and personnel (perfor- } \\
\text { mance bias) } \\
\text { All outcomes }\end{array}$ & Unclear risk & $\begin{array}{l}\text { Comment: an identical placebo was used, but there is no mention about blind- } \\
\text { ing }\end{array}$ \\
\hline $\begin{array}{l}\text { Blinding of outcome as- } \\
\text { sessment (detection bias) } \\
\text { All outcomes }\end{array}$ & Unclear risk & $\begin{array}{l}\text { Comment: an identical placebo was used, but there is no mention about blind- } \\
\text { ing }\end{array}$ \\
\hline $\begin{array}{l}\text { Incomplete outcome data } \\
\text { (attrition bias) } \\
\text { All outcomes }\end{array}$ & Unclear risk & $\begin{array}{l}\text { Comment: there were post-randomisation dropouts related to patient refusal, } \\
\text { follow-up loss, and other reasons - it is not clear whether they were related to } \\
\text { the intervention and to outcomes }\end{array}$ \\
\hline $\begin{array}{l}\text { Selective reporting (re- } \\
\text { porting bias) }\end{array}$ & High risk & $\begin{array}{l}\text { Comment: a published protocol was available, but recruitment had com- } \\
\text { menced before the protocol was published; adverse events, mortality, fatty liv- } \\
\text { er resolution were not reported }\end{array}$ \\
\hline Other bias & Low risk & Comment: no other bias noted \\
\hline
\end{tabular}

Hormoznejad 2020

\section{Study characteristics}

\begin{tabular}{ll}
\hline Methods & Randomised clinical trial \\
\hline Participants & Country: Iran \\
& Period of recruitment: not stated \\
& Number randomised: 50 \\
& Post-randomisation dropouts: $9(18.0 \%)$ \\
& Revised sample size: 41 \\
& Reasons for post-randomisation dropouts: not taking tablets regularly, weight-loss diet \\
& Average age, years: 42 \\
& Females: $18(43.9 \%)$ \\
& NASH: not stated \\
& Diabetes mellitus: $0(0.0 \%)$
\end{tabular}


Hormoznejad 2020 (Continued)

Inclusion criteria: 1.18 years of age or older. 2 . BMI $25 \mathrm{~kg} / \mathrm{m}^{2}$ - upper limit wrongly stated in the text. 3 . Confirmed NAFLD (grade of steatosis $\geq 2$ at ultrasonography)

Exclusion criteria: 1 . History of significant alcohol intake (>10 mL/d for women, $20 \mathrm{~mL} / \mathrm{d}$ for men). 2 .

Smoking habits. 3. Other liver disease; other cardiovascular, respiratory, or kidney disorder; malignan-

cy. 4. Diabetes mellitus. 5. Pregnancy or breastfeeding. 6 . Medication in previous 6 months. 7. Supple-

mentation with antioxidants or vitamins. 8. Weight loss over the past 3 months. 9. Metabolism or en-

docrine disorder

Method for diagnosis of NAFLD: ultrasound

Group 1: other supplements $(\mathrm{n}=20)$
Further details: cranberry tablets $(2$ tablets daily) for 12 weeks. Each tablet contained $144 \mathrm{mg}$ of Vac-
cinium macrocarpon extract with $\geq 36 \mathrm{mg}$ proanthrocyanidine (equal to $13 \mathrm{~g}$ dried cranberry fruit);
composition of the remaining $144 \mathrm{mg}$ was unknown
Group 2: no active intervention $(\mathrm{n}=21)$
Further details: placebo tablets twice daily for 12 weeks. Placebo tablets contained $288 \mathrm{mg}$ of starch
and were similar in colour, size, and weight to cranberry tablets
Additional details: all participants were on a hypocaloric diet of 500 to 1000 kcal under estimated ener-
gy requirements

Outcomes Outcomes reported: resolution of fatty liver disease

Follow-up, months: 3

Notes Source of funding (quote): "this work was financially supported by a Vice-Chancellor for Research Af-

fairs of Ahvaz Jundishapur University of Medical Sciences from [grant number NRC-9718]"

Trial name/Trial registry number: IRCT20150124020765N2

Attempts were made to contact study authors in April 2021

\section{Risk of bias}

\begin{tabular}{|c|c|c|}
\hline Bias & Authors' judgement & Support for judgement \\
\hline $\begin{array}{l}\text { Random sequence genera- } \\
\text { tion (selection bias) }\end{array}$ & Low risk & $\begin{array}{l}\text { Quote: "eligible patients were block randomised based on gender and body } \\
\text { mass index (BMI), then using a randomisation number table, they were as- } \\
\text { signed to receive either cranberry or placebo tablets for } 12 \text { weeks" }\end{array}$ \\
\hline $\begin{array}{l}\text { Allocation concealment } \\
\text { (selection bias) }\end{array}$ & Unclear risk & Comment: this information was not available \\
\hline $\begin{array}{l}\text { Blinding of participants } \\
\text { and personnel (perfor- } \\
\text { mance bias) } \\
\text { All outcomes }\end{array}$ & Low risk & Quote: "double blind...placebo" \\
\hline $\begin{array}{l}\text { Blinding of outcome as- } \\
\text { sessment (detection bias) } \\
\text { All outcomes }\end{array}$ & Low risk & Quote: "double blind...placebo" \\
\hline $\begin{array}{l}\text { Incomplete outcome data } \\
\text { (attrition bias) } \\
\text { All outcomes }\end{array}$ & High risk & $\begin{array}{l}\text { Comment: there were post-randomisation dropouts that probably were relat- } \\
\text { ed to the intervention and to outcomes }\end{array}$ \\
\hline $\begin{array}{l}\text { Selective reporting (re- } \\
\text { porting bias) }\end{array}$ & High risk & $\begin{array}{l}\text { Comment: no previously published protocol was available; adverse events } \\
\text { were not reported adequately }\end{array}$ \\
\hline Other bias & Low risk & Comment: no other bias noted \\
\hline
\end{tabular}


Hoseini 2020

\section{Study characteristics}

\begin{tabular}{|c|c|}
\hline Methods & Randomised clinical trial \\
\hline Participants & $\begin{array}{l}\text { Country: Iran } \\
\text { Period of recruitment: not stated } \\
\text { Number randomised: } 40 \\
\text { Post-randomisation dropouts: } 0(0.0 \%) \\
\text { Revised sample size: } 40 \\
\text { Average age, years: } 62 \\
\text { Females: } 40 \text { (100.0\%) } \\
\text { NASH: not stated } \\
\text { Diabetes mellitus: not stated } \\
\text { Inclusion criteria: } 1 \text {. NAFLD } \\
\text { Exclusion criteria: } 1 \text {. Alcohol consumption }>20 \mathrm{~g} / \mathrm{d} \text {. } 2 \text {. Viral and autoimmune hepatitis. 3. Haemochro- } \\
\text { matosis. } 4 \text {. Drug-induced liver disease. } 5 \text {. Excessive weight loss. } 6 \text {. Surgical treatment for obesity. } 7 \text {. } \\
\text { Pursuing physical activity programmes } 6 \text { months before the intervention. } 8 \text {. Wilson's disease. } 9 \text {. Coeliac } \\
\text { disease } \\
\text { Method for diagnosis of NAFLD: not stated }\end{array}$ \\
\hline Interventions & $\begin{array}{l}\text { Group 1: vitamin } D(n=20) \\
\text { Further details: } 50,000 \text { units of vitamin } D \text { supplement once per week at the beginning of the week } \\
\text { Group 2: no active intervention ( } n=20) \\
\text { Further details: received placebo weekly, with the same shape, colour, smell, and taste } \\
\text { Additional details: } 50 \% \text { of participants in each group received aerobic training (factorial trial design) }\end{array}$ \\
\hline Outcomes & No outcomes of interest were reported \\
\hline Notes & $\begin{array}{l}\text { Source of funding (quote): "the authors declare that the research did not receive any financial grants" } \\
\text { Trial name/Trial registry number: IRCT20190423043359N1 } \\
\text { Attempts were made to contact study authors in April } 2021\end{array}$ \\
\hline
\end{tabular}

\section{Risk of bias}

\begin{tabular}{|c|c|c|}
\hline Bias & Authors' judgement & Support for judgement \\
\hline $\begin{array}{l}\text { Random sequence genera- } \\
\text { tion (selection bias) }\end{array}$ & Unclear risk & Comment: this information was not available \\
\hline $\begin{array}{l}\text { Allocation concealment } \\
\text { (selection bias) }\end{array}$ & Unclear risk & Comment: this information was not available \\
\hline $\begin{array}{l}\text { Blinding of participants } \\
\text { and personnel (perfor- } \\
\text { mance bias) } \\
\text { All outcomes }\end{array}$ & Unclear risk & $\begin{array}{l}\text { Quote: "single blind" } \\
\text { Comment: states single-blind, but it is not clear who was blinded }\end{array}$ \\
\hline $\begin{array}{l}\text { Blinding of outcome as- } \\
\text { sessment (detection bias) } \\
\text { All outcomes }\end{array}$ & Unclear risk & $\begin{array}{l}\text { Quote: "single blind" } \\
\text { Comment: states single-blind, but it is not clear who was blinded }\end{array}$ \\
\hline $\begin{array}{l}\text { Incomplete outcome data } \\
\text { (attrition bias) } \\
\text { All outcomes }\end{array}$ & Low risk & Comment: there were no post-randomisation dropouts \\
\hline $\begin{array}{l}\text { Selective reporting (re- } \\
\text { porting bias) }\end{array}$ & High risk & $\begin{array}{l}\text { Comment: no previously published protocol was available; adverse events, } \\
\text { mortality, fatty liver resolution were not reported }\end{array}$ \\
\hline
\end{tabular}


Hoseini 2020 (Continued)

Other bias Low risk Comment: no other bias noted

Hosseinabadi 2020

\section{Study characteristics}

\begin{tabular}{|c|c|}
\hline Methods & Randomised clinical trial \\
\hline Participants & $\begin{array}{l}\text { Country: Iran } \\
\text { Period of recruitment: } 2016 \\
\text { Number randomised: } 48 \\
\text { Post-randomisation dropouts: } 4 \text { (8.3\%) } \\
\text { Revised sample size: } 44 \\
\text { Reasons for post-randomisation dropouts: discontinued intervention } \\
\text { Average age, years: } 41 \\
\text { Females: } 21 \text { ( } 47.7 \%) \\
\text { NASH: not stated } \\
\text { Diabetes mellitus: } 0 \text { (0.0\%) } \\
\text { Inclusion criteria: } 1 . \text { NAFLD. 2. Aged } 20 \text { to } 60 \text { years. 3. Body mass index (BMI) ranging from } 25 \text { to } 35 \mathrm{~kg} / \\
\mathrm{m}^{2} \\
\text { Exclusion criteria: } 1 . \text { Pregnant, breastfeeding, post menopause. 2. Professional athlete. } 3 \text {. Known doc- } \\
\text { umented liver disease (such as hepatitis B, hepatitis C, and biliary disease). 4. Inherited disorder affect- } \\
\text { ing liver (iron and copper storage disease). 5. History of diagnosed cardiovascular, kidney, diabetes, } \\
\text { gastrointestinal, pulmonary, autoimmune disease, thyroid dysfunction, or cancer. } 6 \text {. Recent surgery. } 7 . \\
\text { Use of alcohol and cigarettes, nutritional supplements, or weight-loss diet within past } 3 \text { months. } 8 \text {. Use } \\
\text { of medications such as corticosteroids, hepatotoxic agent, anticoagulant, antidiuretic, or lipid-lower- } \\
\text { ing drug } \\
\text { Method for diagnosis of NAFLD: ultrasound }\end{array}$ \\
\hline
\end{tabular}

Group 1: other supplements $(n=21)$

Further details: green coffee extract group received 2 capsules per day (each contained $200 \mathrm{mg}$ GCE) for 8 weeks. GCE capsules were purchased from Bonyan Salamat Kasra Co., Tehran, Iran, and were provided by the hydro-alcoholic extraction of green coffee beans. They contained $50 \%$ (100 mg) CGA (chlorogenic acids) as the main ingredient and $<2 \%$ caffeine

Group 2: no active intervention $(n=23)$

Further details: placebo capsules contained starch and were similar in taste and appearance to GCE capsules

\begin{tabular}{ll}
\hline Outcomes & Outcomes reported: resolution of fatty liver disease \\
& Follow-up, months: 2 \\
\hline Notes & Source of funding (quote): "Research Vice-Chancellor of Tabriz University of Medical Sciences,Tabriz, \\
& $\begin{array}{l}\text { Iran" } \\
\text { Trial name/Trial registry number: not stated } \\
\text { Attempts were made to contact study authors in April } 2021\end{array}$
\end{tabular}

\section{Risk of bias}

\begin{tabular}{lll}
\hline Bias & Authors' judgement & Support for judgement \\
\hline $\begin{array}{l}\text { Random sequence genera- } \\
\text { tion (selection bias) }\end{array}$ & Low risk & $\begin{array}{l}\text { Quote: "the sequence of the randomization was generated using random allo- } \\
\text { cation software" }\end{array}$ \\
\hline $\begin{array}{l}\text { Allocation concealment } \\
\text { (selection bias) }\end{array}$ & Low risk & $\begin{array}{l}\text { Quote: "the medication boxes were labeled as A and B and the researchers and } \\
\text { participants were blinded to the allocation until the statistical analyses were } \\
\text { completed" }\end{array}$ \\
\hline
\end{tabular}




\begin{tabular}{|c|c|c|}
\hline $\begin{array}{l}\text { Blinding of participants } \\
\text { and personnel (perfor- } \\
\text { mance bias) } \\
\text { All outcomes }\end{array}$ & Low risk & Quote: "double-blind, placebo-controlled" \\
\hline $\begin{array}{l}\text { Blinding of outcome as- } \\
\text { sessment (detection bias) } \\
\text { All outcomes }\end{array}$ & Low risk & Quote: "double-blind, placebo-controlled" \\
\hline $\begin{array}{l}\text { Incomplete outcome data } \\
\text { (attrition bias) } \\
\text { All outcomes }\end{array}$ & Unclear risk & $\begin{array}{l}\text { Comment: there were post-randomisation dropouts because the intervention } \\
\text { was discontinued. It is not clear whether these were related to the intervention } \\
\text { and to the outcome }\end{array}$ \\
\hline $\begin{array}{l}\text { Selective reporting (re- } \\
\text { porting bias) }\end{array}$ & High risk & $\begin{array}{l}\text { Comment: no previously published protocol was available; adverse events } \\
\text { were not reported adequately }\end{array}$ \\
\hline Other bias & Low risk & Comment: no other bias noted \\
\hline
\end{tabular}

\section{Hosseini 2018}

\section{Study characteristics}

\begin{tabular}{|c|c|}
\hline Methods & Randomised clinical trial \\
\hline Participants & $\begin{array}{l}\text { Country: Iran } \\
\text { Period of recruitment: } 2015 \text { to } 2016 \\
\text { Number randomised: } 82 \\
\text { Post-randomisation dropouts: } 7(8.5 \%) \\
\text { Revised sample size: } 75 \\
\text { Reasons for post-randomisation dropouts: lost to follow-up } \\
\text { Average age, years: } 34 \\
\text { Females: } 75 \text { (100.0\%) } \\
\text { NASH: not stated } \\
\text { Diabetes mellitus: } 0(0.0 \%) \\
\text { Inclusion criteria: } 1 \text {. Women. } 2.18 \text { to } 50 \text { years old. } 3 \text {. BMI } 25 \text { to } 40 \mathrm{~kg} / \mathrm{m}^{2} .3 \text {. NAFLD confirmed on USS. } 4 . \\
\text { Vitamin D insufficiency (serum } 25(\mathrm{OH}) \mathrm{D}<30 \mathrm{ng} / \mathrm{mL}) .5 \text {. Not taking any dietary supplements including } \\
\text { calcium and vit D over last } 6 \text { months } \\
\text { Exclusion criteria: } 1 \text {. Renal, hepatic, other endocrine disorder; malignancy. 2. Pregnancy/Lactation. } 3 . \\
\text { Alcohol consumption. } 4 \text {. Menopause condition. } 5 \text {. Receiving medications influencing vit D metabolism } \\
\text { or insulin } \\
\text { Method for diagnosis of NAFLD: ultrasound }\end{array}$ \\
\hline Interventions & $\begin{array}{l}\text { Group 1: vitamin } D(n=37) \\
\text { Further details: a single intramuscular injection of } 600,000 \text { IU of cholecalciferol } \\
\text { Group 2: no active intervention }(n=38) \\
\text { Further details: no treatment }\end{array}$ \\
\hline Outcomes & No outcomes of interest were reported \\
\hline Notes & $\begin{array}{l}\text { Source of funding (quote): "this study was supported by Research Vice Chancellor of Tabriz University } \\
\text { of Medical Sciences" } \\
\text { Trial name/Trial registry number: IRCT201503163320N10 } \\
\text { Attempts were made to contact study authors in December } 2020\end{array}$ \\
\hline
\end{tabular}


Hosseini 2018 (Continued)

Risk of bias

\begin{tabular}{|c|c|c|}
\hline Bias & Authors' judgement & Support for judgement \\
\hline $\begin{array}{l}\text { Random sequence genera- } \\
\text { tion (selection bias) }\end{array}$ & Low risk & Quote: "computer Random Allocation Software, version 1" \\
\hline $\begin{array}{l}\text { Allocation concealment } \\
\text { (selection bias) }\end{array}$ & Unclear risk & Comment: this information was not available \\
\hline $\begin{array}{l}\text { Blinding of participants } \\
\text { and personnel (perfor- } \\
\text { mance bias) } \\
\text { All outcomes }\end{array}$ & Unclear risk & Comment: this information was not available \\
\hline $\begin{array}{l}\text { Blinding of outcome as- } \\
\text { sessment (detection bias) } \\
\text { All outcomes }\end{array}$ & Unclear risk & Comment: this information was not available \\
\hline $\begin{array}{l}\text { Incomplete outcome data } \\
\text { (attrition bias) } \\
\text { All outcomes }\end{array}$ & Unclear risk & $\begin{array}{l}\text { Comment: participants were excluded from analysis for reasons that may be } \\
\text { related to the intervention and to outcomes }\end{array}$ \\
\hline $\begin{array}{l}\text { Selective reporting (re- } \\
\text { porting bias) }\end{array}$ & High risk & $\begin{array}{l}\text { Comment: no previously published protocol was available; adverse events, } \\
\text { mortality, fatty liver resolution were not reported }\end{array}$ \\
\hline Other bias & Low risk & Comment: no other bias noted \\
\hline
\end{tabular}

Hussain 2017

\section{Study characteristics}

\begin{tabular}{|c|c|}
\hline Methods & Randomised clinical trial \\
\hline Participants & $\begin{array}{l}\text { Country: Pakistan } \\
\text { Period of recruitment: } 2016 \\
\text { Number randomised: } 80 \\
\text { Post-randomisation dropouts: } 0(0.0 \%) \\
\text { Revised sample size: } 80 \\
\text { Average age, years: } 27 \\
\text { Females: } 26 \text { ( } 32.5 \%) \\
\text { NASH: not stated } \\
\text { Diabetes mellitus: } 0 \text { ( } 0.0 \%) \\
\text { Inclusion criteria: } 1.20 \text { to } 55 \text { years old. 2. BMI } \geq 27 \text {. 3. Elevated aminotransferases. 4. USS with fatty liver } \\
\text { grading } 1 \text {, 2, or } 3 \\
\text { Exclusion criteria: } 1 \text {. Alcohol and drug abuse. 2. Smoking. 3. Pregnancy/Lactation. 4. Diabetes, 5, Hy- } \\
\text { pothyroid. } 6 \text {. Biliary disease. } 7 \text {. Autoimmune disease. 8. Drug-induced hepatitis. } 9 \text {. Chronic kidney dis- } \\
\text { ease. } 10 \text {. Cardiac disease. } 11 \text {. Decompensated liver disease or other cause of chronic liver disease } \\
\text { Method for diagnosis of NAFLD: ultrasound plus transaminases }\end{array}$ \\
\hline Interventions & $\begin{array}{l}\text { Group 1: other supplements }(n=40) \\
\text { Further details: green tea extract } 500 \text { mg twice daily for } 12 \text { weeks } \\
\text { Group 2: no active intervention }(n=40) \\
\text { Further details: placebo }\end{array}$ \\
\hline
\end{tabular}


Hussain 2017 (Continued)

Follow-up, months: 3

Source of funding (quote): "Grant Support \& Financial Disclosures: none"

Trial name/Trial registry number: not stated

Attempts were made to contact study authors in December 2020

\section{Risk of bias}

\begin{tabular}{|c|c|c|}
\hline Bias & Authors' judgement & Support for judgement \\
\hline $\begin{array}{l}\text { Random sequence genera- } \\
\text { tion (selection bias) }\end{array}$ & Low risk & Quote: "random numbers generated by computer for each subject" \\
\hline $\begin{array}{l}\text { Allocation concealment } \\
\text { (selection bias) }\end{array}$ & Unclear risk & Comment: this information was not available \\
\hline $\begin{array}{l}\text { Blinding of participants } \\
\text { and personnel (perfor- } \\
\text { mance bias) } \\
\text { All outcomes }\end{array}$ & Unclear risk & Comment: this information was not available \\
\hline $\begin{array}{l}\text { Blinding of outcome as- } \\
\text { sessment (detection bias) } \\
\text { All outcomes }\end{array}$ & Unclear risk & $\begin{array}{l}\text { Quote: "the imaging study of fatty liver was done by experienced radiologist } \\
\text { who was blinded to the all data of patients" } \\
\text { Comment: it is not clear whether outcome assessors of remaining outcomes } \\
\text { were blinded }\end{array}$ \\
\hline $\begin{array}{l}\text { Incomplete outcome data } \\
\text { (attrition bias) } \\
\text { All outcomes }\end{array}$ & Low risk & Comment: there were no post-randomisation dropouts \\
\hline $\begin{array}{l}\text { Selective reporting (re- } \\
\text { porting bias) }\end{array}$ & High risk & $\begin{array}{l}\text { Comment: no previously published protocol was available; adverse events } \\
\text { were not reported adequately }\end{array}$ \\
\hline Other bias & Low risk & Comment: no other bias noted \\
\hline
\end{tabular}

Illnait 2013

\section{Study characteristics}

\begin{tabular}{ll}
\hline Methods & Randomised clinical trial \\
\hline Participants & Country: Cuba \\
& Period of recruitment: not stated \\
& Number randomised: 50 \\
& Post-randomisation dropouts: $0(0.0 \%)$ \\
& Revised sample size: 50 \\
Average age, years: 55 & Females: $27(54.0 \%)$ \\
& NASH: not stated \\
& Diabetes mellitus: 7 (14.0\%) \\
& Inclusion criteria: 1.25 to 70 years old. 2. Prior diagnosis of NAFLD and/or persistent increase in liver \\
& enzymes without excessive alcohol ingestion (< 70 g female, 40 g male) \\
& Exclusion criteria: 1. Overuse of alcohol. 2. Other cause of chronic liver disease. 3. Uncontrolled dia- \\
betes. 4 . Pregnancy/Lactation. 5. Lack of effective birth control in women of childbearing age. 6. Unsta- & ble angina, MI, stroke, or any other serious adverse event within 3 months prior to study. 7 . Any other \\
treatment that could influence liver function
\end{tabular}


IIInait 2013 (Continued)

Method for diagnosis of NAFLD: ultrasound

\begin{tabular}{ll}
\hline Interventions & Group 1: other supplements $(n=25)$ \\
& Further details: bees wax D-002 $(100 \mathrm{mg} / \mathrm{d})$ for 24 weeks \\
& Group 2: no active intervention $(n=25)$ \\
& Further details: placebo
\end{tabular}

\begin{tabular}{ll}
\hline Outcomes & Outcomes reported: mortality at maximal follow-up, resolution of fatty liver disease \\
& Follow-up, months: 6
\end{tabular}

\begin{tabular}{ll}
\hline Notes & Source of funding: not stated \\
& Trial name/Trial registry number: not stated \\
& Attempts were made to contact study authors in December 2020 \\
\hline
\end{tabular}

\section{Risk of bias}

\begin{tabular}{|c|c|c|}
\hline Bias & Authors' judgement & Support for judgement \\
\hline $\begin{array}{l}\text { Random sequence genera- } \\
\text { tion (selection bias) }\end{array}$ & Low risk & Quote: "computer-generated" \\
\hline $\begin{array}{l}\text { Allocation concealment } \\
\text { (selection bias) }\end{array}$ & Low risk & Quote: "treatments were given in identical coded packages accordingly" \\
\hline $\begin{array}{l}\text { Blinding of participants } \\
\text { and personnel (perfor- } \\
\text { mance bias) } \\
\text { All outcomes }\end{array}$ & Low risk & Quote: "randomized, double-blind, placebo-controlled trial" \\
\hline $\begin{array}{l}\text { Blinding of outcome as- } \\
\text { sessment (detection bias) } \\
\text { All outcomes }\end{array}$ & Low risk & Quote: "randomized, double-blind, placebo-controlled trial" \\
\hline $\begin{array}{l}\text { Incomplete outcome data } \\
\text { (attrition bias) } \\
\text { All outcomes }\end{array}$ & Low risk & Comment: there were no post-randomisation dropouts \\
\hline $\begin{array}{l}\text { Selective reporting (re- } \\
\text { porting bias) }\end{array}$ & High risk & $\begin{array}{l}\text { Comment: no previously published protocol was available; adverse events } \\
\text { were not reported adequately }\end{array}$ \\
\hline Other bias & Low risk & Comment: no other bias noted \\
\hline
\end{tabular}

Izadi 2021

\section{Study characteristics}

\begin{tabular}{ll}
\hline Methods & Randomised clinical trial \\
\hline Participants & Country: Iran \\
& Period of recruitment: 2018 \\
& Number randomised: 70 \\
& Post-randomisation dropouts: 9 (12.9\%) \\
& Revised sample size: 61 \\
& Reasons for post-randomisation dropouts: personal reasons (7), travel (1), discontinued intervention \\
& $(1)$ \\
& Average age, years: 43
\end{tabular}


Izadi 2021 (Continued)

Females: 25 (41.0\%)

NASH: not stated

Diabetes mellitus: not stated

Inclusion criteria: 1. Diagnosis of NAFLD according to ultrasound (patients with grade 1 to 3 ) and serum alanine aminotransferase (ALT) $>30 \mathrm{IU} / \mathrm{L}$ for men, $>19 \mathrm{IU} / \mathrm{L}$ for women. 2.20 to 55 years of age. 3. Body mass index $(\mathrm{BMI})>25$ and $<40 \mathrm{~kg} / \mathrm{m}^{2}$. 3. Lack of history of alcohol consumption. 4 . No evidence of any other acute or chronic disorder of the liver (hepatitis B, C, etc.), nor biliary disease, autoimmune disease, cancer, or inherited disorders affecting the liver

Exclusion criteria: 1 . Any allergic reaction to sour tea supplement. 2. Use of antioxidant drugs or any other supplements/drugs that could interfere with the study objectives within 3 months. 3 . Weight loss $>10 \%$ during the study period. 4 . Breastfeeding or pregnant. 5. Irregular use of capsules (consuming < $80 \%$ of capsules delivered to participants during the study)

Method for diagnosis of NAFLD: ultrasound and transaminases

$\begin{array}{ll}\text { Interventions } & \text { Group 1: other supplements }(\mathrm{n}=30) \\ & \text { Further details: } 1 \text { capsule of sour tea powder (450-mg capsule containing } \geq 250 \mathrm{mg} \text { of anthocyanin) } \\ \text { daily for } 8 \text { weeks. Hibiscus sabdariffa } L \text { was obtained from a local market in } 2018 \text { and was scientifically } \\ \text { identified by M. Kamalinejad } \\ \text { Group 2: no active intervention }(\mathrm{n}=31) \\ \text { Further details: } 1 \text { placebo capsule (pure microcrystalline cellulose) daily for } 8 \text { weeks. Placebo was indis- } \\ \text { tinguishable in colour, shape, size, and packaging from sour tea capsules } \\ \text { Additional details: all participants were advised to maintain their usual diet and physical activity during } \\ \text { the intervention period }\end{array}$

\begin{tabular}{ll} 
Outcomes & $\begin{array}{l}\text { Outcomes reported: serious adverse events (number of people), serious adverse events (number of } \\
\text { events) } \\
\text { Follow-up, months: } 2\end{array}$ \\
\hline Notes & Source of funding (quote): "the authors would like to thank the Isfahan University of Medical Sciences \\
& for financial support" \\
& Trial name/Trial registry number: IRCT20140208016529N3 \\
& Attempts were made to contact study authors in April 2021
\end{tabular}

\section{Risk of bias}

Bias Authors' judgement Support for judgement

Random sequence genera- Low risk Quote: "randomization lists were computer-generated by a statistician"
tion (selection bias)

\begin{tabular}{lll}
\hline $\begin{array}{l}\text { Allocation concealment } \\
\text { (selection bias) }\end{array}$ & Unclear risk & Comment: this information was not available \\
\hline $\begin{array}{l}\text { Blinding of participants } \\
\text { and personnel (perfor- } \\
\text { mance bias) } \\
\begin{array}{l}\text { All outcomes } \\
\text { Blinding of outcome as- }\end{array}\end{array}$ & Low risk & $\begin{array}{l}\text { Quote: "all participants, the physician, and the laboratory personnel were } \\
\text { blind to the intervention type...placebo" }\end{array}$ \\
$\begin{array}{l}\text { sessment (detection bias) } \\
\text { All outcomes }\end{array}$ & Low risk & $\begin{array}{l}\text { Quote: "all participants, the physician, and the laboratory personnel were } \\
\text { blind to the intervention type...placebo" }\end{array}$ \\
\hline $\begin{array}{l}\text { Incomplete outcome data } \\
\text { (attrition bias) } \\
\text { All outcomes }\end{array}$ & Unclear risk & $\begin{array}{l}\text { Comment: there were post-randomisation dropouts because the intervention } \\
\text { was discontinued. it is not clear whether these were related to the intervention } \\
\text { and to the outcome }\end{array}$ \\
\hline $\begin{array}{l}\text { Selective reporting (re- } \\
\text { porting bias) }\end{array}$ & High risk & $\begin{array}{l}\text { Comment: no previously published protocol was available; neither mortality } \\
\text { nor fatty liver resolution was reported }\end{array}$ \\
\hline
\end{tabular}


Izadi 2021 (Continued)
Other bias
Low risk
Comment: no other bias noted

Jameshorani 2017

\section{Study characteristics}

\begin{tabular}{ll}
\hline Methods & Randomised clinical trial \\
\hline Participants & Country: Iran \\
& Period of recruitment: not stated \\
& Number randomised: 90 \\
& Post-randomisation dropouts: not stated \\
& Revised sample size: 90 \\
Average age, years: not stated & Females: not stated \\
NASH: not stated & Diabetes mellitus: not stated \\
Inclusion and exclusion criteria: not stated \\
Method for diagnosis of NAFLD: not stated
\end{tabular}

Interventions Group 1: prebiotics/probiotics/synbiotics $(n=45)$

Further details: $500 \mathrm{mg} / \mathrm{d}$ Familact (duration not stated)

Group 2: no active intervention $(n=45)$

Further details: no treatment

Additional details: both groups received diet and exercise interventions

\begin{tabular}{ll}
\hline Outcomes & No outcomes of interest were reported \\
\hline Notes & Source of funding: not stated \\
& Trial name/Trial registry number: not stated \\
& Attempts were made to contact study authors in December 2020
\end{tabular}

\section{Risk of bias}

\begin{tabular}{lll}
\hline Bias & Authors' judgement & Support for judgement \\
\hline $\begin{array}{l}\text { Random sequence genera- } \\
\text { tion (selection bias) }\end{array}$ & Unclear risk & Comment: this information was not available \\
\hline $\begin{array}{l}\text { Allocation concealment } \\
\text { (selection bias) }\end{array}$ & Unclear risk & Comment: this information was not available \\
\hline $\begin{array}{l}\text { Blinding of participants } \\
\text { and personnel (perfor- } \\
\text { mance bias) }\end{array}$ & Unclear risk & Comment: this information was not available \\
$\begin{array}{l}\text { All outcomes } \\
\text { Blinding of outcome as- } \\
\text { sessment (detection bias) } \\
\text { All outcomes }\end{array}$ & Unclear risk & Comment: this information was not available \\
\hline $\begin{array}{l}\text { Incomplete outcome data } \\
\text { (attrition bias) } \\
\text { All outcomes }\end{array}$ & Unclear risk & Comment: this information was not available \\
\hline
\end{tabular}


Jameshorani 2017 (Continued)
Selective reporting (re-
High risk
Comment: no previously published protocol was available; adverse events, porting bias)
mortality, fatty liver resolution were not reported

Other bias Low risk Comment: no other bias noted

\section{Janczyk 2015}

\section{Study characteristics}

\begin{tabular}{|c|c|c|}
\hline Methods & \multicolumn{2}{|c|}{ Randomised clinical trial } \\
\hline Participants & \multicolumn{2}{|c|}{$\begin{array}{l}\text { Country: Poland } \\
\text { Period of recruitment: } 2008 \text { to } 2011 \\
\text { Number randomised: } 76 \\
\text { Post-randomisation dropouts: } 12 \text { (15.8\%) } \\
\text { Revised sample size: } 64 \\
\text { Reasons for post-randomisation dropouts: withdrawn consent (1), personal reasons (2), contact lost } \\
\text { (3), withdrawn by parents/patients (6) } \\
\text { Average age, years: } 13 \\
\text { Females: } 11 \text { (17.2\%) } \\
\text { NASH: not stated } \\
\text { Diabetes mellitus: } 0 \text { (0.0\%) } \\
\text { Inclusion criteria: } 1.5 \text { to } 19 \text { years old. } 2 \text {. Overweight or obesity (according to BMI). 3. ALT } \geq 1.3 \times \text { upper } \\
\text { limit of normal. } 4 \text {. Hyperechogenic liver on USS or liver histology consistent with NAFLD/NASH } \\
\text { Exclusion criteria: } 1 \text {. Significant alcohol consumption history. 2. Other cause of chronic liver disease. } 3 . \\
\text { Diabetes. } 4 \text {. Hypothyroidism. } 5 \text {. Vitamin E treatment. } 6 \text {. Use of statins, ursodeoxycholic acid, probiotics, } \\
\text { metformin within } 3 \text { months. } 7 \text {. History of total parenteral nutrition } \\
\text { Method for diagnosis of NAFLD: ultrasound plus transaminases }\end{array}$} \\
\hline Interventions & \multicolumn{2}{|c|}{$\begin{array}{l}\text { Group 1: PUFA }(n=30) \\
\text { Further details: omega-3 fatty acid (docosahexaenoic acid and eicosapentaenoic acid, } 450 \text { to } 1300 \mathrm{mg} / \\
\text { d) for } 24 \text { weeks } \\
\text { Group 2: } \text { no active intervention }(n=34) \\
\text { Further details: placebo }\end{array}$} \\
\hline Outcomes & \multicolumn{2}{|c|}{$\begin{array}{l}\text { Outcomes reported: serious adverse events (number of people), any adverse events (number of peo- } \\
\text { ple), resolution of fatty liver disease } \\
\text { Follow-up, months: } 6\end{array}$} \\
\hline Notes & \multicolumn{2}{|c|}{$\begin{array}{l}\text { Source of funding (quote): "omega-3 and placebo capsules were manufactured and blinded by Ne- } \\
\text { pentes S.A. and Hasco-Lek Polska S.A." } \\
\text { Trial name/Trial registry number: NCT01547910 } \\
\text { Attempts were made to contact study authors in December } 2020\end{array}$} \\
\hline \multicolumn{3}{|l|}{ Risk of bias } \\
\hline Bias & Authors' judgement & Support for judgement \\
\hline $\begin{array}{l}\text { Random sequence genera- } \\
\text { tion (selection bias) }\end{array}$ & Low risk & $\begin{array}{l}\text { Quote: "computer statistical software StatsDirect version 2.7.9 (StatsDirect } \\
\text { Ltd., England, UK); the list of random treatment assignments was generated" }\end{array}$ \\
\hline $\begin{array}{l}\text { Allocation concealment } \\
\text { (selection bias) }\end{array}$ & Low risk & $\begin{array}{l}\text { Quote: "investigators sent randomization requests by fax to the central ran- } \\
\text { domization center (CRC) responsible for the process of randomization" }\end{array}$ \\
\hline
\end{tabular}


Janczyk 2015 (Continued)

Blinding of participants Low risk Quote: "multicenter, randomized, double-blind, placebo-controlled clinical triand personnel (perforal"

mance bias)

All outcomes

\begin{tabular}{|c|c|c|}
\hline $\begin{array}{l}\text { Blinding of outcome as- } \\
\text { sessment (detection bias) } \\
\text { All outcomes }\end{array}$ & Low risk & $\begin{array}{l}\text { Quote: "multicenter, randomized, double-blind, placebo-controlled clinical tri- } \\
\text { al" }\end{array}$ \\
\hline
\end{tabular}

lcomes

Incomplete outcome data Unclear risk

(attrition bias)

Comment: participants were excluded from analysis for reasons that may be

All outcomes related to the intervention and to outcomes

\begin{tabular}{lll}
\hline $\begin{array}{l}\text { Selective reporting (re- } \\
\text { porting bias) }\end{array}$ & Low risk & $\begin{array}{l}\text { Comment: no previously published protocol was available (recruitment was } \\
\text { completed at the time of protocol publication); adverse events and either mor- } \\
\text { tality or fatty liver resolution or both were reported }\end{array}$ \\
\hline Other bias & Low risk & Comment: no other bias noted \\
\hline
\end{tabular}

Javadi 2017

\section{Study characteristics}

\begin{tabular}{ll}
\hline Methods & Randomised clinical trial \\
\hline Participants & Country: Iran \\
& Period of recruitment: 2013 to 2014 \\
& Number randomised: 84 \\
& Post-randomisation dropouts: 9 (10.7\%) \\
& Revised sample size: 75 \\
& Reasons for post-randomisation dropouts: personal reasons, hepatitis, diabetes \\
& Average age, years: 42 \\
& Females: 15 (20.0\%) \\
& NASH: not stated \\
& Diabetes mellitus: not stated \\
Inclusion criteria: 1 . NAFLD. 2.20 to 60 years of age. 3. ALT/AST higher than normal range \\
Exclusion criteria: 1 . Cardiovascular, thyroid, kidney, autoimmune disease. 2. Other cause of chronic \\
liver disease. 3. Use of vitamin supplements. 4. Alcohol. 5. Pregnant/Lactating \\
Method for diagnosis of NAFLD: ultrasound and transaminases
\end{tabular}

Interventions

Group 1: prebiotics/probiotics/synbiotics $(n=56)$

Further details: probiotic capsules (Bifidobacterium longum and Lactobacillus acidophilus: $2 \times 10^{7} \mathrm{CFU} /$

d) and/or prebiotic inulin high performance $10 \mathrm{~g} / \mathrm{d}$ for 3 months

Group 2: no active intervention $(n=19)$

Further details: placebo

\begin{tabular}{ll}
\hline Outcomes & No outcomes of interest were reported \\
\hline Notes & Source of funding (quote): "the study was granted by nutrition research center, Tabriz University of \\
& Medical Sciences, Iran" \\
& Trial name/Trial registry number: IRCT201301223140N6 \\
& Attempts were made to contact study authors in December 2020 \\
\hline
\end{tabular}

\section{Risk of bias}


Javadi 2017 (Continued)

Random sequence genera- Unclear risk Comment: this information was not available tion (selection bias)

$\begin{array}{ll}\begin{array}{l}\text { Allocation concealment } \\ \text { (selection bias) }\end{array} & \begin{array}{l}\text { Quote: "to ensure blinding, the allocation was performed by an investigator } \\ \text { with no clinical involvement in the study, and the main investigator and statis- } \\ \text { tical data analyst, is the same one, remained blinded to the participant group } \\ \text { until the end of the analysis" }\end{array}\end{array}$

Blinding of participants Low risk Quote: "double blind, placebo-control clinical trial"
and personnel (perfor-
mance bias)

\begin{tabular}{|c|c|c|}
\hline $\begin{array}{l}\text { Blinding of outcome as- } \\
\text { sessment (detection bias) } \\
\text { All outcomes }\end{array}$ & Low risk & Quote: "double blind, placebo-control clinical trial" \\
\hline $\begin{array}{l}\text { Incomplete outcome data } \\
\text { (attrition bias) } \\
\text { All outcomes }\end{array}$ & Unclear risk & $\begin{array}{l}\text { Comment: participants were excluded from analysis for reasons that may be } \\
\text { related to the intervention and to outcomes }\end{array}$ \\
\hline $\begin{array}{l}\text { Selective reporting (re- } \\
\text { porting bias) }\end{array}$ & High risk & $\begin{array}{l}\text { Comment: no previously published protocol was available; adverse events, } \\
\text { mortality, fatty liver resolution were not reported }\end{array}$ \\
\hline Other bias & Low risk & Comment: no other bias noted \\
\hline
\end{tabular}

\section{Javanmardi 2018}

\section{Study characteristics}

\begin{tabular}{|c|c|}
\hline Methods & Randomised clinical trial \\
\hline Participants & $\begin{array}{l}\text { Country: Iran } \\
\text { Period of recruitment: not stated } \\
\text { Number randomised: } 42 \\
\text { Post-randomisation dropouts: } 4 \text { (9.5\%) } \\
\text { Revised sample size: } 38 \\
\text { Reasons for post-randomisation dropouts: personal reasons } \\
\text { Average age, years: } 44 \\
\text { Females: } 21(55.3 \%) \\
\text { NASH: not stated } \\
\text { Diabetes mellitus: not stated } \\
\text { Inclusion criteria: } 1 \text {. NAFLD on USS } \\
\text { Exclusion criteria: } 1 \text {. Liver transplant. 2. Smoking. 3. Alcohol consumption. 4. Use of drugs such as } \\
\text { amiodarone, steroids, tamoxifen, prexillin, methotrexate. 5. Rapid weight loss. 6. Heart failure. } 7 \text {. Thy- } \\
\text { roid disease. 8. Renal disease. } 10 \text {. Other aetiology of chronic liver disease } \\
\text { Method for diagnosis of NAFLD: ultrasound }\end{array}$ \\
\hline Interventions & $\begin{array}{l}\text { Group 1: other supplements }(n=19) \\
\text { Further details: } 1.6-\text { g phytosterol supplement daily for } 8 \text { weeks } \\
\text { Group 2: no active intervention }(n=19) \\
\text { Further details: placebo }\end{array}$ \\
\hline Outcomes & No outcomes of interest were reported \\
\hline Notes & Source of funding: not stated \\
\hline
\end{tabular}


Trial name/Trial registry number: IRCT2017011531958N1

Attempts were made to contact study authors in December 2020

\section{Risk of bias}

\begin{tabular}{|c|c|c|}
\hline Bias & Authors' judgement & Support for judgement \\
\hline $\begin{array}{l}\text { Random sequence genera- } \\
\text { tion (selection bias) }\end{array}$ & Unclear risk & Comment: this information was not available \\
\hline $\begin{array}{l}\text { Allocation concealment } \\
\text { (selection bias) }\end{array}$ & Unclear risk & Comment: this information was not available \\
\hline $\begin{array}{l}\text { Blinding of participants } \\
\text { and personnel (perfor- } \\
\text { mance bias) } \\
\text { All outcomes }\end{array}$ & Low risk & Quote: "randomized, double-blind, placebo-controlled clinical trial" \\
\hline $\begin{array}{l}\text { Blinding of outcome as- } \\
\text { sessment (detection bias) } \\
\text { All outcomes }\end{array}$ & Low risk & Quote: "randomized, double-blind, placebo-controlled clinical trial" \\
\hline $\begin{array}{l}\text { Incomplete outcome data } \\
\text { (attrition bias) } \\
\text { All outcomes }\end{array}$ & Unclear risk & $\begin{array}{l}\text { Comment: participants were excluded from analysis for reasons that may be } \\
\text { related to the intervention and to outcomes }\end{array}$ \\
\hline $\begin{array}{l}\text { Selective reporting (re- } \\
\text { porting bias) }\end{array}$ & High risk & $\begin{array}{l}\text { Comment: no previously published protocol was available; adverse events, } \\
\text { mortality, fatty liver resolution were not reported }\end{array}$ \\
\hline Other bias & Low risk & Comment: no other bias noted \\
\hline
\end{tabular}

Jazayeri-Tehrani 2019

\section{Study characteristics}

\begin{tabular}{|c|c|}
\hline Methods & Randomised clinical trial \\
\hline Participants & $\begin{array}{l}\text { Country: Iran } \\
\text { Period of recruitment: not stated } \\
\text { Number randomised: } 84 \\
\text { Post-randomisation dropouts: not stated } \\
\text { Revised sample size: } 84 \\
\text { Average age, years: } 42 \\
\text { Females: } 38 \text { (45.2\%) } \\
\text { NASH: not stated } \\
\text { Diabetes mellitus: not stated } \\
\text { Inclusion criteria: } 1.25 \text { to } 50 \text { years old. 2. BMI } 25 \text { to } 35 \\
\text { Exclusion criteria: } 1 \text {. Alcohol intake during previous year. 2. Other liver condition, secondary NAFLD. } \\
\text { 3. Uncontrolled hypertension. } 4 \text {. Pregnancy, lactation. } 5 \text {. Professional athlete. } 6 \text {. Use of statins, ur- } \\
\text { sodeoxycholic acid, probiotics, antihypertensive, curcumin-interactive drugs, multi-vitamin/miner- } \\
\text { al/antioxidant supplements over past } 3 \text { months. } 7 \text {. Weight loss over past } 3 \text { months } \\
\text { Method for diagnosis of NAFLD: ultrasound }\end{array}$ \\
\hline Interventions & $\begin{array}{l}\text { Group 1: other supplements ( } n=42 \text { ) } \\
\text { Further details: two } 40-\mathrm{mg} \text { capsules/d of nano-curcumin (supplied by Exir-Nano-Sina Company) after } \\
\text { meals for } 3 \text { months } \\
\text { Group 2: no active intervention ( } n=42 \text { ) }\end{array}$ \\
\hline
\end{tabular}


Jazayeri-Tehrani 2019 (Continued)

Further details: two 40-mg capsules/d of placebo (supplied by Exir-Nano-Sina Company) after meals for

3 months. Capsules were similar in shape, size, and colour to nano-curcumin capsules

Additional details: both groups received lifestyle advice

Outcomes

Outcomes reported: mortality at maximal follow-up, serious adverse events (number of people), any adverse events (number of people), liver transplantation at maximal follow-up, decompensation (number of people), cirrhosis (number of people), resolution of fatty liver disease, hepatocellular carcinoma Follow-up, months: 3

Notes

Source of funding (quote): "the trial funding was supported by Tehran University of Medical Sciences (grant no. 31581)"

Trial name/Trial registry number: IRCT2016071915536N3

Attempts were made to contact study authors in December 2020

\section{Risk of bias}

\begin{tabular}{lll}
\hline Bias & Authors' judgement & Support for judgement \\
\hline $\begin{array}{l}\text { Random sequence genera- } \\
\text { tion (selection bias) }\end{array}$ & Low risk & $\begin{array}{l}\text { Quote: "double-blind, randomized, placebo-controlled clinical trial. Subjects } \\
\text { were divided into two equal groups by the block randomization method, car- } \\
\text { ried out by an assistant" } \\
\text { Comment: although the precise method of generation of random sequence } \\
\text { generation was not reported, the method of allocation concealment suggests } \\
\text { that sequence generation was random }\end{array}$ \\
&
\end{tabular}

\begin{tabular}{ll}
\hline $\begin{array}{l}\text { Allocation concealment } \\
\text { (selection bias) }\end{array}$ & Low risk \\
& $\begin{array}{l}\text { Quote: "double-blind, randomized, placebo-controlled clinical trial. Interven- } \\
\text { tion allocation blinding was performed for both participants and investigators } \\
\text { before the beginning, kept during the intervention, and opened after the data } \\
\text { analysis by a field worker" }\end{array}$ \\
\hline
\end{tabular}

Blinding of participants Low risk Quote: "double-blind, randomized, placebo-controlled clinical trial"
and personnel (performance bias)

All outcomes

\begin{tabular}{|c|c|c|}
\hline $\begin{array}{l}\text { Blinding of outcome as- } \\
\text { sessment (detection bias) } \\
\text { All outcomes }\end{array}$ & Low risk & $\begin{array}{l}\text { Quote: "double-blind, randomized, placebo-controlled clinical trial. Interven- } \\
\text { tion allocation blinding was performed for both participants and investigators } \\
\text { before the beginning, kept during the intervention, and opened after the data } \\
\text { analysis by a field worker" }\end{array}$ \\
\hline
\end{tabular}

\begin{tabular}{lll}
\hline $\begin{array}{l}\text { Incomplete outcome data } \\
\text { (attrition bias) } \\
\text { All outcomes }\end{array}$ & Low risk & Comment: an intention-to-treat analysis was performed \\
\hline $\begin{array}{l}\text { Selective reporting (re- } \\
\text { porting bias) }\end{array}$ & Low risk & $\begin{array}{l}\text { Comment: a published protocol was available, but it is not clear whether re- } \\
\text { cruitment had commenced before the protocol was published; adverse events } \\
\text { and either mortality or fatty liver resolution or both were reported }\end{array}$
\end{tabular}

Other bias Unclear risk Comment: there were baseline differences in important prognostic factors

Jeong 2017

\section{Study characteristics}

\begin{tabular}{ll}
\hline Methods & Randomised clinical trial \\
\hline Participants & Country: South Korea \\
\hline
\end{tabular}

Nutritional supplementation for nonalcohol-related fatty liver disease: a network meta-analysis (Review) 
Period of recruitment: 2013 to 2015

Number randomised: 74

Post-randomisation dropouts: 1 (1.4\%)

Revised sample size: 73

Reasons for post-randomisation dropouts: withdrawing during the study (1)

Average age, years: 42

Females: 14 (19.2\%)

NASH: not stated

Diabetes mellitus: not stated

Inclusion criteria: 1 . Aged 19 to 75 years. 2. USS features of NAFLD. 3. AST or ALT above upper normal limits

Exclusion criteria: 1. AST/ALT > 2. 2. Type 1 diabetes. 3. Other liver disease. 4. Excessive alcohol consumption. 5. Use of steatogenic medication within past 3 months. 5. Serious underlying disease. 6.

Bariatric surgery within past 6 months. 7. Contraindication to magnetic resonance spectroscopy. 8.

Pregnancy, breastfeeding. 9. Hypersensitivity to Magnolia officinalis

Method for diagnosis of NAFLD: ultrasound and transaminases

\begin{tabular}{ll}
\hline Interventions & Group 1: other supplements $(\mathrm{n}=45)$ \\
& Further details: high-dose $(400 \mathrm{mg})$ or low-dose $(133.4 \mathrm{mg}) \mathrm{HL}$ tablet daily for 12 weeks. HL tablet is a \\
new botanical drug extracted from Magnolia officinalis $(\mathrm{MO})$ \\
Group 2: no active intervention $(\mathrm{n}=23)$ \\
Further details: placebo daily for 12 weeks
\end{tabular}

Outcomes $\quad$ Outcomes reported: any adverse events (number of people), any adverse events (number of events)

Follow-up, months: 3

$\begin{array}{ll}\text { Notes } & \text { Source of funding: not stated } \\ & \text { Trial name/Trial registry number: NCT02491905 } \\ & \text { Attempts were made to contact study authors in December } 2020\end{array}$

\section{Risk of bias}

\begin{tabular}{|c|c|c|}
\hline Bias & Authors' judgement & Support for judgement \\
\hline $\begin{array}{l}\text { Random sequence genera- } \\
\text { tion (selection bias) }\end{array}$ & Unclear risk & Comment: this information was not available \\
\hline $\begin{array}{l}\text { Allocation concealment } \\
\text { (selection bias) }\end{array}$ & Unclear risk & Comment: this information was not available \\
\hline $\begin{array}{l}\text { Blinding of participants } \\
\text { and personnel (perfor- } \\
\text { mance bias) } \\
\text { All outcomes }\end{array}$ & Low risk & Quote: "double-blind, placebo controlled" \\
\hline $\begin{array}{l}\text { Blinding of outcome as- } \\
\text { sessment (detection bias) } \\
\text { All outcomes }\end{array}$ & Low risk & Quote: "double-blind, placebo controlled" \\
\hline $\begin{array}{l}\text { Incomplete outcome data } \\
\text { (attrition bias) } \\
\text { All outcomes }\end{array}$ & Unclear risk & $\begin{array}{l}\text { Comment: there were post-randomisation dropouts, but it is not clear whether } \\
\text { these were related to the intervention or to outcomes }\end{array}$ \\
\hline $\begin{array}{l}\text { Selective reporting (re- } \\
\text { porting bias) }\end{array}$ & High risk & $\begin{array}{l}\text { Comment: protocol was published after trial had ended; neither mortality nor } \\
\text { fatty liver resolution was reported }\end{array}$ \\
\hline Other bias & Low risk & Comment: no other bias noted \\
\hline
\end{tabular}


Kanoni 2021

\section{Study characteristics}

\begin{tabular}{ll}
\hline Methods & Randomised clinical trial \\
\hline Participants & Country: multi-centric (Europe) \\
& Period of recruitment: 2017 to 2019 \\
& Number randomised: 98 \\
& Post-randomisation dropouts: $11(11.2 \%)$ \\
& Revised sample size: 87 \\
& Reasons for post-randomisation dropouts: lost to follow-up (11) \\
Average age, years: 49 & Females: not stated \\
& NASH: not stated \\
& Diabetes mellitus: not stated \\
& Inclusion criteria: 1. Men and women, aged 18 to 67 years. 2. Body mass index (BMI) $\geq 30 \mathrm{~kg} / \mathrm{m}^{2} .3$. Es- \\
& tablished NAFLD/NASH based on sensitive LiverMultiScan magnetic resonance imaging (MRI) technique \\
& Exclusion criteria: 1. Hepatotoxic medication. 2 . Concomitant liver disease. 3. Decompensated dia- \\
betes mellitus. 4 . Thyroid disease, hypopituitarism, Cushing's syndrome, alcohol abuse or drug addic- \\
tion, systemic disease, pregnancy, lactation, vegan or lacto- and ovo-lactovegetarianism, psychiatric \\
or mental disorder. 5 . Recent loss of body weight or current diet. 6. Any use of antioxidant-phytochemi- \\
cal-rich supplement, prebiotics, or probiotics. 7 . Change in drug treatment, antibiotic treatment during \\
or before screening \\
Method for diagnosis of NAFLD: magnetic resonance imaging
\end{tabular}

\begin{tabular}{ll}
\hline Interventions & Group 1: other antioxidants $(\mathrm{n}=35)$ \\
& $\begin{array}{l}\text { Further details: Mastiha } 0.35 \mathrm{~g} \text { capsules thrice daily for } 6 \text { months } \\
\text { Group 2: no active intervention }(\mathrm{n}=52) \\
\end{array}$ \\
\hline Further details: placebo daily for 6 months \\
\hline Outcomes & Outcomes reported: any adverse events \\
& Follow-up, months: 6 \\
\hline Notes & $\begin{array}{l}\text { Source of funding: European Union's Horizon } 2020 \text { research and innovation programme MAST4HEALTH } \\
\text { Trial name/Trial registry number: NCT03135873 }\end{array}$
\end{tabular}

\section{Risk of bias}

\begin{tabular}{|c|c|c|}
\hline Bias & Authors' judgement & Support for judgement \\
\hline $\begin{array}{l}\text { Random sequence genera- } \\
\text { tion (selection bias) }\end{array}$ & Unclear risk & Comment: this information was not available \\
\hline $\begin{array}{l}\text { Allocation concealment } \\
\text { (selection bias) }\end{array}$ & Unclear risk & Comment: this information was not available \\
\hline $\begin{array}{l}\text { Blinding of participants } \\
\text { and personnel (perfor- } \\
\text { mance bias) } \\
\text { All outcomes }\end{array}$ & Low risk & $\begin{array}{l}\text { Quote: "all participants and researchers were blinded to the treatment alloca- } \\
\text { tion...double-blind, placebo controlled" }\end{array}$ \\
\hline $\begin{array}{l}\text { Blinding of outcome as- } \\
\text { sessment (detection bias) } \\
\text { All outcomes }\end{array}$ & Low risk & $\begin{array}{l}\text { Quote: "all participants and researchers were blinded to the treatment alloca- } \\
\text { tion...double-blind, placebo controlled" }\end{array}$ \\
\hline
\end{tabular}


Kanoni 2021 (Continued)

Incomplete outcome data Unclear risk Comment: there were post-randomisation dropouts, but it is not clear whether (attrition bias)

All outcomes

\begin{tabular}{lll}
\hline $\begin{array}{l}\text { Selective reporting (re- } \\
\text { porting bias) }\end{array}$ & High risk & $\begin{array}{l}\text { Comment: protocol was published after trial had started; neither mortality nor } \\
\text { fatty liver resolution was reported }\end{array}$ \\
\hline Other bias & Low risk & Comment: no other bias noted
\end{tabular}

Kazemi 2020

\section{Study characteristics}

\begin{tabular}{|c|c|}
\hline Methods & Randomised clinical trial \\
\hline Participants & $\begin{array}{l}\text { Country: Iran } \\
\text { Period of recruitment: not stated } \\
\text { Number randomised: } 84 \\
\text { Post-randomisation dropouts: } 0 \text { (0.0\%) } \\
\text { Revised sample size: } 84 \\
\text { Average age, years: } 42 \\
\text { Females: } 46 \text { (54.8\%) } \\
\text { NASH: not stated } \\
\text { Diabetes mellitus: } 0 \text { (0.0\%) } \\
\text { Inclusion criteria: } 1 \text {. Age range } 20 \text { to } 60 \text { years. } 2 \text {. Body mass index (BMI) between } 25 \text { and } 30 \mathrm{~kg} / \mathrm{m}^{2} .3 \text {. } \\
\text { No evidence of secondary causes for steatosis, including history of alcohol use, haemochromatosis, or } \\
\text { Wilson's disease; history of using hepatotoxic drugs (methotrexate, amiodarone, tamoxifen, corticos- } \\
\text { teroids, valproate, and antiviral drugs); history of hepatitis C and known autoimmune disease. 3. Not } \\
\text { affected by chronic or acute liver disorder; coeliac disease; diabetes; cancer; thyroid disorder; cardio- } \\
\text { vascular, renal, or pulmonary disease; inflammatory disease; or autoimmune disease. } 5 \text {. No history of } \\
\text { smoking. } 6 . \text { Not taking any antioxidant supplements over previous } 1 \text { month. } 7 \text {. Not using any medica- } \\
\text { tion including weight-loss, glucose-lowering, and anti-inflammatory drugs. } 8 \text {. Not following weight-loss } \\
\text { diet over previous } 3 \text { months. } 9 \text {. Not pregnant or breastfeeding } \\
\text { Exclusion criteria: } 1 \text {. Intolerance to sumac and/or placebo supplements or reporting any unexpected } \\
\text { adverse effects. } 2 \text {. Weight loss }>10 \% \text { of baseline weight during the trial. 3. Low compliance with supple- } \\
\text { ments, defined as consuming < } 80 \% \text { of supplements by the end of the trial. } 4 \text {. Presence of any illness } \\
\text { that requires special treatment during the study. } 5 \text {. Unwillingness to continue co-operation } \\
\text { Method for diagnosis of NAFLD: ultrasound }\end{array}$ \\
\hline
\end{tabular}

Interventions Group 1: other supplements $(n=40)$

Further details: 1 capsule containing $500 \mathrm{mg}$ sumac powder, 4 times a day (preferably after each meal),

for 12 weeks

Group 2: no active intervention ( $n=40)$

Further details: received equal amounts of placebo capsule containing dextrin for the same period Additional details: all patients in sumac and placebo groups received a 500-kcal deficit diet plan, which comprised $55 \%$ to $65 \%$ of calories from carbohydrate, $20 \%$ to $30 \%$ from fat, and $10 \%$ to $15 \%$ from protein

\begin{tabular}{ll}
\hline Outcomes & No outcomes of interest were reported \\
\hline Notes & Source of funding (quote): "this work was financially supported by Vice Chancellor for Research, Iran \\
& University of Medical Sciences, Tehran, Iran" \\
& Trial name/Trial registry number: IRCT201701162709N39 \\
& Attempts were made to contact study authors in April 2021 \\
\hline
\end{tabular}

\section{Risk of bias}


Kazemi 2020 (Continued)

Bias Authors' judgement Support for judgement

Random sequence genera- Low risk Quote: "performed using software-generated random numbers"

tion (selection bias)

Allocation concealment $\quad$ Unclear risk
(selection bias)

(selection bias)

Blinding of participants Low risk Quote: "double-blind placebo-controlled"
and personnel (perfor-
mance bias)

Blinding of outcome as-
sessment (detection bias) $\quad$ Low risk Quote: "double-blind placebo-controlled"

All outcomes

Incomplete outcome data Low risk Comment: there were no post-randomisation dropouts
(attrition bias)
All outcomes

\begin{tabular}{lll}
\hline $\begin{array}{l}\text { Selective reporting (re- } \\
\text { porting bias) }\end{array}$ & High risk & $\begin{array}{l}\text { Comment: no previously published protocol was available; adverse events, } \\
\text { mortality, fatty liver resolution were not reported }\end{array}$ \\
\hline Other bias & Low risk & Comment: no other bias noted \\
\hline
\end{tabular}

Khoshbaten 2010b

\section{Study characteristics}

\begin{tabular}{ll}
\hline Methods & Randomised clinical trial \\
\hline Participants & Country: Iran \\
Period of recruitment: 2008 \\
Number randomised: 30 \\
Post-randomisation dropouts: not stated \\
Revised sample size: 30 \\
Average age, years: 43 \\
Females: 19 (63.3\%) \\
NASH: not stated \\
Diabetes mellitus: 3 (10\%) \\
Inclusion criteria: 1. Ongoing NAFLD \\
Exclusion criteria: 1 . History of alcohol consumption or use of medications known to precipitate steato- \\
hepatitis, lipid-reducing agents, ursodeoxycholic acid, or vitamin supplements in the 6 months before \\
the study \\
Method for diagnosis of NAFLD: ultrasound or raised transaminases
\end{tabular}

\begin{tabular}{ll}
\hline Interventions & Group 1: other antioxidants $(n=15)$ \\
& Further details: N-acetyl cysteine $600 \mathrm{mg}$ BD for 3 months \\
& Group 2: vitamin C $(n=15)$ \\
& Further details: vitamin C $1000 \mathrm{mg}$ BD for 3 months
\end{tabular}

Outcomes Outcomes reported: any adverse events


Khoshbaten 2010b (Continued)

Follow-up, months: 3

Notes Source of funding: Drug Applied Research Center at Tabriz University of Medical Sciences

Trial name/Trial registry number: not stated

\section{Risk of bias}

\begin{tabular}{|c|c|c|}
\hline Bias & Authors' judgement & Support for judgement \\
\hline $\begin{array}{l}\text { Random sequence genera- } \\
\text { tion (selection bias) }\end{array}$ & Low risk & $\begin{array}{l}\text { Quote: "patients would pick up a ticket from a box containing mixed labels of } \\
\text { two different treatments, twenty from each category" }\end{array}$ \\
\hline $\begin{array}{l}\text { Allocation concealment } \\
\text { (selection bias) }\end{array}$ & Low risk & $\begin{array}{l}\text { Quote: "patients would pick up a ticket from a box containing mixed labels of } \\
\text { two different treatments, twenty from each category" }\end{array}$ \\
\hline \multirow{2}{*}{$\begin{array}{l}\text { Blinding of participants } \\
\text { and personnel (perfor- } \\
\text { mance bias) } \\
\text { All outcomes }\end{array}$} & Unclear risk & $\begin{array}{l}\text { Quote: "liver hemodynamics, the grade of steatosis and the size of the spleen } \\
\text { were measured by the same radiologist, blinded to the treatment method of } \\
\text { the patients" }\end{array}$ \\
\hline & & $\begin{array}{l}\text { Comment: no information on whether participants or healthcare providers } \\
\text { were blinded }\end{array}$ \\
\hline \multirow[t]{2}{*}{$\begin{array}{l}\text { Blinding of outcome as- } \\
\text { sessment (detection bias) } \\
\text { All outcomes }\end{array}$} & Unclear risk & $\begin{array}{l}\text { Quote: "liver hemodynamics, the grade of steatosis and the size of the spleen } \\
\text { were measured by the same radiologist, blinded to the treatment method of } \\
\text { the patients" }\end{array}$ \\
\hline & & $\begin{array}{l}\text { Comment: there was no information on whether outcome assessors for ad- } \\
\text { verse events were blinded }\end{array}$ \\
\hline $\begin{array}{l}\text { Incomplete outcome data } \\
\text { (attrition bias) } \\
\text { All outcomes }\end{array}$ & Unclear risk & Comment: this information was not available \\
\hline $\begin{array}{l}\text { Selective reporting (re- } \\
\text { porting bias) }\end{array}$ & High risk & $\begin{array}{l}\text { Comment: no pre-published protocol was available; neither mortality nor fatty } \\
\text { liver resolution was reported }\end{array}$ \\
\hline Other bias & Low risk & Comment: no other bias noted \\
\hline
\end{tabular}

\section{Khutsishvili 2020}

\section{Study characteristics}

\begin{tabular}{ll}
\hline Methods & Randomised clinical trial \\
\hline Participants & Country: Georgia \\
Period of recruitment: not stated \\
Number randomised: 79 \\
Post-randomisation dropouts: $6(7.6 \%)$ \\
Revised sample size: 73 \\
Reasons for post-randomisation dropouts: not stated \\
Average age, years: not stated \\
Females: not stated \\
NASH: not stated \\
Diabetes mellitus: not stated \\
Inclusion criteria: 1 . NAFLD. 2. Alcohol intake $<30 \mathrm{~g} / \mathrm{d}$ \\
Exclusion criteria: not stated
\end{tabular}


Khutsishvili 2020 (Continued)

Method for diagnosis of NAFLD: not stated

Interventions Group 1: prebiotics/probiotics/synbiotics $(\mathrm{n}=35)$

Further details: mixture of 6 probiotic agents (Bifidobacterium bifidum, Bifidobacterium longum, Lacto-

bacillus fermentum, Lactobacillus plantarum, Lactobacillus acidophilus, E coli M-17) and an auxiliary pre-

biotic component: fructo-oligosaccharide $50 \mathrm{mg}$

Group 2: no active intervention $(n=38)$

Further details: placebo

\begin{tabular}{ll}
\hline Outcomes & No outcomes of interest were reported \\
\hline Notes & Source of funding: not stated \\
& Trial name/Trial registry number: not stated \\
& Attempts were made to contact study authors in April 2021 \\
\hline
\end{tabular}

\section{Risk of bias}

\section{Bias}

Authors' judgement Support for judgement

Random sequence genera- Unclear risk Comment: this information was not available tion (selection bias)

Allocation concealment Unclear risk Comment: this information was not available
(selection bias)

Blinding of participants Unclear risk Comment: this information was not available
and personnel (performance bias)

All outcomes

\begin{tabular}{|c|c|c|}
\hline $\begin{array}{l}\text { Blinding of outcome as- } \\
\text { sessment (detection bias) } \\
\text { All outcomes }\end{array}$ & Unclear risk & Comment: this information was not available \\
\hline
\end{tabular}

\begin{tabular}{|c|c|c|}
\hline $\begin{array}{l}\text { Incomplete outcome data } \\
\text { (attrition bias) }\end{array}$ & Unclear risk & $\begin{array}{l}\text { Comment: there were post-randomisation dropouts; reasons for post-ran- } \\
\text { domisation dropouts were not reported }\end{array}$ \\
\hline
\end{tabular}

\begin{tabular}{lll}
\hline $\begin{array}{l}\text { Selective reporting (re- } \\
\text { porting bias) }\end{array}$ & High risk & $\begin{array}{l}\text { Comment: no previously published protocol was available; adverse events, } \\
\text { mortality, fatty liver resolution were not reported }\end{array}$ \\
\hline Other bias & Low risk & Comment: no other bias noted \\
\hline
\end{tabular}

Kobyliak 2017

\section{Study characteristics}

\begin{tabular}{ll}
\hline Methods & Randomised clinical trial \\
\hline Participants & Country: not stated \\
& Period of recruitment: not stated \\
& Number randomised: 48 \\
& Post-randomisation dropouts: not stated \\
& Revised sample size: 48 \\
& Average age, years: not stated \\
& Females: not stated
\end{tabular}


Kobyliak 2017 (Continued)

NASH: not stated

Diabetes mellitus: 48 (100.0\%)

Inclusion and exclusion criteria: not stated

Method for diagnosis of NAFLD: not stated
Group 1: prebiotics/probiotics/synbiotics plus PUFA ( $\mathrm{n}=$ not stated)

Further details: Symbiter omega (combination of probiotic biomass supplemented with flax and wheat germ oil (250 mg of each)) for 8 weeks

Group 2: no active intervention ( $\mathrm{n}=$ not stated)

Further details: placebo

\begin{tabular}{ll}
\hline Outcomes & No outcomes of interest were reported \\
\hline Notes & Source of funding: not stated \\
& Trial name/Trial registry number: not stated \\
& Attempts were made to contact study authors in December 2020
\end{tabular}

\section{Risk of bias}

\begin{tabular}{|c|c|c|}
\hline Bias & Authors' judgement & Support for judgement \\
\hline $\begin{array}{l}\text { Random sequence genera- } \\
\text { tion (selection bias) }\end{array}$ & Unclear risk & Comment: this information was not available \\
\hline $\begin{array}{l}\text { Allocation concealment } \\
\text { (selection bias) }\end{array}$ & Unclear risk & Comment: this information was not available \\
\hline $\begin{array}{l}\text { Blinding of participants } \\
\text { and personnel (perfor- } \\
\text { mance bias) } \\
\text { All outcomes }\end{array}$ & Low risk & Quote: "we studied, in double-blind single center RCT...placebo" \\
\hline $\begin{array}{l}\text { Blinding of outcome as- } \\
\text { sessment (detection bias) } \\
\text { All outcomes }\end{array}$ & Low risk & Quote: "we studied, in double-blind single center RCT...placebo" \\
\hline $\begin{array}{l}\text { Incomplete outcome data } \\
\text { (attrition bias) } \\
\text { All outcomes }\end{array}$ & Unclear risk & Comment: this information was not available \\
\hline $\begin{array}{l}\text { Selective reporting (re- } \\
\text { porting bias) }\end{array}$ & High risk & $\begin{array}{l}\text { Comment: no previously published protocol was available; adverse events, } \\
\text { mortality, fatty liver resolution were not reported }\end{array}$ \\
\hline Other bias & Low risk & Comment: no other bias noted \\
\hline
\end{tabular}

\section{Kobyliak 2018}

\section{Study characteristics}

\begin{tabular}{ll}
\hline Methods & Randomised clinical trial \\
\hline Participants & Country: Ukraine \\
& Period of recruitment: not stated \\
& Number randomised: 58 \\
& Post-randomisation dropouts: $0(0.0 \%)$ \\
& Revised sample size: 58
\end{tabular}


Kobyliak 2018 (Continued)

Average age, years: 55

Females: not stated

NASH: not stated

Diabetes mellitus: 58 (100.0\%)

Inclusion criteria: 1 . Aged 18 to 65 years. 2. BMI $\geq 25$. 3. Diagnosed with NAFLD. 4. Type 2 diabetes mellitus treated with diet and exercise alone or metformin, sulphonylureas, or insulin. 5 . AST/ALT $\leq 3 \times$ upper limit of normal

Exclusion criteria: 1 . Alcohol $>20 \mathrm{~g} / \mathrm{d}$ in women, $>30 \mathrm{~g} / \mathrm{d}$ in men. 2 . Other cause of chronic liver disease.

3. Prebiotic/probiotic supplement in last 3 months. 4. Uncontrolled cardiovascular or respiratory disease, active malignancy, or chronic infection. 5. Use of vitamin E, omega-3 fatty acid, or medication with evidence for effects on NAFLD. 6. Presence of active infection. 7. Pregnancy/Lactation Method for diagnosis of NAFLD: ultrasound

Group 1: no active intervention $(\mathrm{n}=28)$
Further details: 1 sachet $(10$ grams) of placebo per day for 8 weeks. Placebo sachets were identical with
similar organoleptic characteristics $($ e.g. taste, appearance) to probiotic sachets
Group 2: prebiotics/probiotics $/$ synbiotics $(\mathrm{n}=30)$
Further details: 1 sachet $(10$ grams) of Symbiter (concentrated biomass of 14 probiotic bacteria genera:
Lactobacillus + Lactococcus $\left(6 \times 10^{10} \mathrm{CFU} / \mathrm{g}\right)$, Bifidobacterium $\left(1 \times 10^{10} / \mathrm{g}\right)$,
Propionibacterium $\left(3 \times 10^{10} / \mathrm{g}\right)$, Acetobacter $\left.\left(1 \times 10^{6} / \mathrm{g}\right)\right)$ per day for 8 weeks

Outcomes

Outcomes reported: mortality at maximal follow-up, serious adverse events (number of people), any adverse events (number of people), liver transplantation at maximal follow-up, decompensation (number of people), cirrhosis (number of people), resolution of fatty liver disease, hepatocellular carcinoma Follow-up, months: 2

$\begin{array}{ll}\text { Notes } & \text { Source of funding (quote): "the multiprobiotic "Symbiter" was supplied by the Scientific and Produc- } \\ \text { tion Company "O.D. Prolisok" (Kyiv, Ukraine)" } \\ \text { Trial name/Trial registry number: not stated } \\ \text { Attempts were made to contact study authors in December } 2020\end{array}$

\section{Risk of bias}

\begin{tabular}{lll}
\hline Bias & Authors' judgement & Support for judgement \\
\hline $\begin{array}{l}\text { Random sequence genera- } \\
\text { tion (selection bias) }\end{array}$ & Low risk & Quote: "computer-generated list" \\
\hline $\begin{array}{l}\text { Allocation concealment } \\
\text { (selection bias) }\end{array}$ & Low risk & $\begin{array}{l}\text { Quote: "the study pharmacist gave the sachets to the participants according } \\
\text { to their group assignment and was responsible for the delivery of the blinded } \\
\text { supplements" }\end{array}$ \\
\hline $\begin{array}{l}\text { Blinding of participants } \\
\begin{array}{l}\text { and personnel (perfor- } \\
\text { mance bias) } \\
\text { All outcomes }\end{array}\end{array}$ & Low risk & Quote: "double-blind treatment...placebo" \\
\hline
\end{tabular}

Blinding of outcome as- Low risk Quote: "double-blind treatment...placebo"

sessment (detection bias)

All outcomes

Incomplete outcome data Low risk Comment: there were no post-randomisation dropouts
(attrition bias)
All outcomes

\begin{tabular}{lll}
\hline $\begin{array}{l}\text { Selective reporting (re- } \\
\text { porting bias) }\end{array}$ & Low risk & $\begin{array}{l}\text { Comment: no previously published protocol was available; adverse events and } \\
\text { either mortality or fatty liver resolution or both were reported }\end{array}$ \\
\hline Other bias & Low risk & Comment: no other bias noted \\
\hline
\end{tabular}


Kooshki 2020

\section{Study characteristics}

\begin{tabular}{|c|c|}
\hline Methods & Randomised clinical trial \\
\hline Participants & $\begin{array}{l}\text { Country: Iran } \\
\text { Period of recruitment: } 2018 \text { to } 2019 \\
\text { Number randomised: } 46 \\
\text { Post-randomisation dropouts: } 3 \text { (6.5\%) } \\
\text { Revised sample size: } 43 \\
\text { Reasons for post-randomisation dropouts: refusal of treatment (2), withdrawal (1) } \\
\text { Average age, years: } 39 \\
\text { Females: } 17 \text { (39.5\%) } \\
\text { NASH: not stated } \\
\text { Diabetes mellitus: } 0 \text { (0.0\%) } \\
\text { Inclusion criteria: } 1 \text {. Women aged } 20 \text { to } 50 \text { years (pre-menopause) or men aged } 20 \text { to } 65 \text { years. } 2 \text {. BMI } 25 \\
\text { to } 40 . \\
\text { Exclusion criteria: } 1 \text {. Alcohol consumption. 2. Renal disease. 3. Thyroid disorder. } 4 \text {. Statin consumption. } \\
\text { 5. Diabetes mellitus. } 6 \text {. Hepatitis C and B. } 7 \text {. Hereditary liver disease. 8. Pregnancy, lactation. } 9 \text {. Being in } \\
\text { weight loss programme during past } 12 \text { weeks. 10. Taking dietary supplements such as vitamins, miner- } \\
\text { als, fibre, and omega-3 in the past } 12 \text { weeks. 11. Doing professional sports } \\
\text { Method for diagnosis of NAFLD: ultrasound }\end{array}$ \\
\hline
\end{tabular}

Interventions Group 1: other supplements $(\mathrm{n}=22)$

Further details: $200 \mu \mathrm{g}$ tablets of chromium picolinate 2 times per day after a meal for 12 weeks (tablets produced by 21 st Century HealthCare, Inc., Tempe, Arizona, USA)

Group 2: no active intervention $(n=21)$

Further details: placebo tablets 2 times per day after a meal for 12 weeks (placebo tablets with corn starch prepared in Research Center, Tabriz University of Medical Sciences, Tabriz, Iran, and similar in size and colour to CrPic tablets)

\begin{tabular}{ll}
\hline Outcomes & No outcomes of interest were reported \\
\hline Notes & Source of funding (quote): "also, we would like to thank the Nutrition Research Center, Tabriz Universi- \\
& ty of Medical Sciences, and Tabriz, Iran, for providing our study grant" \\
& Trial name/Trial registry number: IRCT20100123003140N15 \\
& Attempts were made to contact study authors in April 2021
\end{tabular}

\section{Risk of bias}

\begin{tabular}{|c|c|c|}
\hline Bias & Authors' judgement & Support for judgement \\
\hline $\begin{array}{l}\text { Random sequence genera- } \\
\text { tion (selection bias) }\end{array}$ & Low risk & Quote: "using the blocked randomization method through RAS software" \\
\hline $\begin{array}{l}\text { Allocation concealment } \\
\text { (selection bias) }\end{array}$ & Unclear risk & Comment: this information was not available \\
\hline $\begin{array}{l}\text { Blinding of participants } \\
\text { and personnel (perfor- } \\
\text { mance bias) } \\
\text { All outcomes }\end{array}$ & Low risk & Quote: "double-blind, placebo-controlled" \\
\hline $\begin{array}{l}\text { Blinding of outcome as- } \\
\text { sessment (detection bias) } \\
\text { All outcomes }\end{array}$ & Low risk & Quote: "double-blind, placebo-controlled" \\
\hline
\end{tabular}


Kooshki 2020 (Continued)

Incomplete outcome data Unclear risk Comment: there were post-randomisation dropouts because the intervention (attrition bias) was discontinued. It is not clear whether these were related to the intervention All outcomes and to the outcome

\begin{tabular}{|c|c|c|}
\hline $\begin{array}{l}\text { Selective reporting (re- } \\
\text { porting bias) }\end{array}$ & High risk & $\begin{array}{l}\text { Comment: no previously published protocol was available; adverse events, } \\
\text { mortality, fatty liver resolution were not reported }\end{array}$ \\
\hline
\end{tabular}

Other bias Low risk Comment: no other bias noted

Kugelmas 2003

\section{Study characteristics}

\begin{tabular}{|c|c|}
\hline Methods & Randomised clinical trial \\
\hline Participants & $\begin{array}{l}\text { Country: USA } \\
\text { Period of recruitment: not stated } \\
\text { Number randomised: } 16 \\
\text { Post-randomisation dropouts: not stated } \\
\text { Revised sample size: } 16 \\
\text { Average age, years: } 48 \\
\text { Females: } 9 \text { (56.3\%) } \\
\text { NASH: } 16 \text { (100.0\%) } \\
\text { Diabetes mellitus: not stated } \\
\text { Inclusion criteria: } 1 \text {. Aged } 18 \text { to } 65 \text { years. } 2 \text {. Diagnosis of NASH with liver biopsy consistent with NASH. } 3 . \\
\text { No more than } 1 \text { drink/week in past } 6 \text { months and no history of alcohol abuse } \\
\text { Exclusion criteria: } 1 \text {. Other cause of chronic liver disease. 2. Decompensated liver disease. 3. Ongoing } \\
\text { total parenteral nutrition. } 4 \text {. HIV. } 5 \text {. Previous vitamin E replacement in last } 3 \text { months } \\
\text { Method for diagnosis of NAFLD: liver biopsy }\end{array}$ \\
\hline Interventions & $\begin{array}{l}\text { Group 1: vitamin } E(n=9) \\
\text { Further details: } 800 \text { IU of vitamin E daily for } 12 \text { weeks } \\
\text { Group 2: no active intervention }(n=7) \\
\text { Further details: no treatment } \\
\text { Additional details: both groups received dietary intervention }\end{array}$ \\
\hline Outcomes & No outcomes of interest were reported \\
\hline Notes & $\begin{array}{l}\text { Source of funding (quote): "supported by National Institutes of Health grants MO1RR02602, AA00297(to } \\
\text { D.B.H.), AA014185(D.B.H.), AA01762 (to C.J.M.), and AA10496(to C.J.M.); a Kentucky Science and Engi- } \\
\text { neering Foundation grant; and the Department of Veterans Affairs" } \\
\text { Trial name/Trial registry number: not stated } \\
\text { Attempts were made to contact study authors in December } 2020\end{array}$ \\
\hline
\end{tabular}

\section{Risk of bias}

\begin{tabular}{lll}
\hline Bias & Authors' judgement & Support for judgement \\
\hline $\begin{array}{l}\text { Random sequence genera- } \\
\text { tion (selection bias) }\end{array}$ & Unclear risk & Comment: this information was not available \\
\hline $\begin{array}{l}\text { Allocation concealment } \\
\text { (selection bias) }\end{array}$ & Unclear risk & Comment: this information was not available \\
\hline
\end{tabular}


Kugelmas 2003 (Continued)

Blinding of participants High risk Quote: "in a single-blinded fashion (principal investigator was blinded)" and personnel (performance bias)

All outcomes

\begin{tabular}{lll}
\hline Blinding of outcome as- & Unclear risk & Quote: "in a single-blinded fashion (principal investigator was blinded)" \\
sessment (detection bias) & & $\begin{array}{l}\text { Comment: it is not clear whether the principal investigator was also the out- } \\
\text { come assessor }\end{array}$
\end{tabular}

Incomplete outcome data Unclear risk (attrition bias)

Comment: this information was not available

All outcomes

\begin{tabular}{lll}
\hline $\begin{array}{l}\text { Selective reporting (re- } \\
\text { porting bias) }\end{array}$ & High risk & $\begin{array}{l}\text { Comment: no previously published protocol was available; adverse events, } \\
\text { mortality, fatty liver resolution were not reported }\end{array}$ \\
\hline Other bias & Low risk & Comment: no other bias noted \\
\hline
\end{tabular}

Lavine 2011

\section{Study characteristics}

\begin{tabular}{|c|c|}
\hline Methods & Randomised clinical trial \\
\hline \multirow[t]{13}{*}{ Participants } & Country: USA \\
\hline & Period of recruitment: 2005 to 2010 \\
\hline & Number randomised: 116 \\
\hline & Post-randomisation dropouts: 19 (16.4\%) \\
\hline & Revised sample size: 97 \\
\hline & Reasons for post-randomisation dropouts: did not have liver biopsy \\
\hline & Average age, years: 13 \\
\hline & Females: $33(34.0 \%)$ \\
\hline & NASH: $49(50.5 \%)$ \\
\hline & Diabetes mellitus: $0(0.0 \%)$ \\
\hline & $\begin{array}{l}\text { Inclusion criteria: } 1 \text {. Aged } 8 \text { to } 17 \text { years. 2. NAFLD. 3. Perisistently raised ALT. } 4 \text {. Liver biopsy }<6 \text { months } \\
\text { before randomisation }\end{array}$ \\
\hline & $\begin{array}{l}\text { Exclusion criteria: 1. Diabetes. 2. Cirrhosis. 3. Viral hepatitis. 4. Alchol use. 5. Other cause of chronic liver } \\
\text { disease. 6. Pregnancy. 7. Inborn error in metabolism }\end{array}$ \\
\hline & Method for diagnosis of NAFLD: liver biopsy \\
\hline
\end{tabular}

\begin{tabular}{|c|c|}
\hline Interventions & $\begin{array}{l}\text { Group 1: vitamin } \mathrm{E}(\mathrm{n}=50) \\
\text { Further details: vitamin E } 800 \text { IU for } 96 \text { weeks } \\
\text { Group 2: no active intervention ( } \mathrm{n}=47 \text { ) } \\
\text { Further details: placebo } \\
\text { Additional details: another group not relevant to this review was excluded }\end{array}$ \\
\hline Outcomes & $\begin{array}{l}\text { Outcomes reported: serious adverse events (number of people), any adverse events (number of peo- } \\
\text { ple), any adverse events (number of events), fibrosis score, NAFLD activity score } \\
\text { Follow-up, months: } 28\end{array}$ \\
\hline Notes & $\begin{array}{l}\text { Source of funding (quote): "multiple authors received consulting fees from many pharmaceutical in- } \\
\text { dustries" } \\
\text { Trial name/Trial registry number: TONIC TRIAL/00063635 } \\
\text { Attempts were made to contact study authors in December } 2020\end{array}$ \\
\hline
\end{tabular}

\section{Risk of bias}

Nutritional supplementation for nonalcohol-related fatty liver disease: a network meta-analysis (Review) 
Lavine 2011 (Continued)

\begin{tabular}{|c|c|c|}
\hline Bias & Authors' judgement & Support for judgement \\
\hline $\begin{array}{l}\text { Random sequence genera- } \\
\text { tion (selection bias) }\end{array}$ & Unclear risk & Comment: this information was not available \\
\hline $\begin{array}{l}\text { Allocation concealment } \\
\text { (selection bias) }\end{array}$ & Unclear risk & Comment: this information was not available \\
\hline $\begin{array}{l}\text { Blinding of participants } \\
\text { and personnel (perfor- } \\
\text { mance bias) } \\
\text { All outcomes }\end{array}$ & Low risk & $\begin{array}{l}\text { Quote: "randomized, double-blind, double-dummy, placebo-controlled clini- } \\
\text { cal trial" }\end{array}$ \\
\hline $\begin{array}{l}\text { Blinding of outcome as- } \\
\text { sessment (detection bias) } \\
\text { All outcomes }\end{array}$ & Low risk & $\begin{array}{l}\text { Quote: "randomized, double-blind, double-dummy, placebo-controlled clini- } \\
\text { cal trial" }\end{array}$ \\
\hline $\begin{array}{l}\text { Incomplete outcome data } \\
\text { (attrition bias) } \\
\text { All outcomes }\end{array}$ & Unclear risk & $\begin{array}{l}\text { Comment: participants were excluded from analysis for reasons that may be } \\
\text { related to the intervention and to outcomes }\end{array}$ \\
\hline $\begin{array}{l}\text { Selective reporting (re- } \\
\text { porting bias) }\end{array}$ & High risk & $\begin{array}{l}\text { Comment: no previously published protocol was available; neither mortality } \\
\text { nor fatty liver resolution was reported }\end{array}$ \\
\hline Other bias & Low risk & Comment: no other bias noted \\
\hline
\end{tabular}

Lewis 2018

\section{Study characteristics}

\begin{tabular}{|c|c|}
\hline Methods & Randomised clinical trial \\
\hline Participants & $\begin{array}{l}\text { Country: USA } \\
\text { Period of recruitment: } 2016 \text { to } 2017 \\
\text { Number randomised: } 23 \\
\text { Post-randomisation dropouts: } 0 \text { (0.0\%) } \\
\text { Revised sample size: } 23 \\
\text { Average age, years: } 54 \\
\text { Females: not stated } \\
\text { NASH: not stated } \\
\text { Diabetes mellitus: not stated } \\
\text { Inclusion criteria: } 1 \text {. Aged > } 18 \text { years. 2. Confirmed NAFLD. 3. Stable medication } \\
\text { Exclusion criteria: } 1 \text {. Allergy to rice, rice bran, mushrooms, or related food product. 2. Gastrointesti- } \\
\text { nal disorder with uncertain absorption. 3. Use of lipid-lowering agent } 3 \text { months before the study. } 4 \text {. Im- } \\
\text { munomodulator use. } 5 \text {. Active chemotherapy. } 6 \text {. Anaemia. } 7 \text {. Pregnancy/Attempting conception. } 8 \text {. Pre- } \\
\text { vious dietary supplements. } 9 \text {. Use of similar polysaccharide formula within } 2 \text { weeks before the study } \\
\text { Method for diagnosis of NAFLD: not stated }\end{array}$ \\
\hline
\end{tabular}

Interventions Group 1: polysaccharides $(\mathrm{n}=12)$

Further details: rice bran arabinoxylan compound 1 gram/d for 90 days

Group 2: no active intervention $(n=11)$

Further details: placebo

\section{Outcomes}

Outcomes reported: mortality at maximal follow-up, serious adverse events (number of people), any adverse events (number of people), liver transplantation at maximal follow-up, decompensation (number of people), cirrhosis (number of people), resolution of fatty liver disease, hepatocellular carcinoma 
Lewis 2018 (Continued)

Follow-up, months: 3

Notes Source of funding (quote): "John E. Lewis has been paid by Daiwa Pharmaceutical to speak at international conferences and write articles on health and wellness for their website"

Trial name/Trial registry number: NCT02568787

Attempts were made to contact study authors in December 2020

\section{Risk of bias}

\begin{tabular}{lll}
\hline Bias & Authors' judgement & Support for judgement \\
\hline $\begin{array}{l}\text { Random sequence genera- } \\
\text { tion (selection bias) }\end{array}$ & Low risk & $\begin{array}{l}\text { Quote: "using a random permutations table created by the principal investiga- } \\
\text { tor" }\end{array}$ \\
\hline $\begin{array}{l}\text { Allocation concealment } \\
\text { (selection bias) }\end{array}$ & Low risk & $\begin{array}{l}\text { Quote: "all subjects and investigators were blinded to the treatment condition } \\
\text { and remained blinded until after data analysis" } \\
\text { Comment: allocation concealment and blinding were achieved with use of } \\
\text { placebo }\end{array}$ \\
\hline
\end{tabular}

Blinding of participants Low risk and personnel (perfor-

mance bias)

All outcomes

\begin{tabular}{|c|c|c|}
\hline $\begin{array}{l}\text { Blinding of outcome as- } \\
\text { sessment (detection bias) } \\
\text { All outcomes }\end{array}$ & Low risk & $\begin{array}{l}\text { Quote: "all subjects and investigators were blinded to the treatment condition } \\
\text { and remained blinded until after data analysis...placebo" }\end{array}$ \\
\hline
\end{tabular}

\begin{tabular}{lll}
\hline $\begin{array}{l}\text { Incomplete outcome data } \\
\text { (attrition bias) } \\
\text { All outcomes }\end{array}$ & Low risk & Comment: there were no post-randomisation dropouts \\
\hline $\begin{array}{l}\text { Selective reporting (re- } \\
\text { porting bias) }\end{array}$ & Low risk & $\begin{array}{l}\text { Comment: no previously published protocol was available; adverse events and } \\
\text { either mortality or fatty liver resolution or both were reported }\end{array}$ \\
\hline Other bias & Low risk & Comment: no other bias noted \\
\hline
\end{tabular}

Li 2010

\section{Study characteristics}

\begin{tabular}{ll}
\hline Methods & Randomised clinical trial \\
\hline Participants & Country: China \\
& Period of recruitment: 2007 to 2008 \\
& Number randomised: 88 \\
& Post-randomisation dropouts: $0(0.0 \%)$ \\
& Revised sample size: 88 \\
& Average age, years: 46 \\
& Females: 33 (37.5\%) \\
& NASH: not stated \\
& Diabetes mellitus: not stated \\
& Inclusion criteria: 1 . Aged 18 to 65 years. 2. NAFLD \\
& Exclusion criteria: 1 . Other liver disease. 2. Severe dysfunction of heart, liver, kidney; cancer, or other \\
severe disease. 3 . Total parenteral nutrition. 4. Simultaneously receiving drug treatments that could in- \\
fluence trial results
\end{tabular}


Li 2010 (Continued)

Method for diagnosis of NAFLD: CT scan

Interventions Group 1: phospholipid ( $n=43)$

Further details: polyene phosphatidylcholine capsule (PPC) (trade name: Essential, product of SanofiAventis Pharmaceutical Co., Ltd., 1 capsule containing $228 \mathrm{mg}$ of PPC), which was given as 2 capsules (456 mg) each time, 3 times a day, for 6 months

Group 2: other supplements $(n=45)$

Further details: Qianggan Capsule (QGC), a product of Shijiazhuang Dongfang Pharmaceutical Co., Ltd., which was given as 3 capsules in the morning, 3 at noon, and 4 in the evening, with a 1-day pause after every 6 days, for 6 months (QGC consists of 16 Chinese drugs, namely, Radix Astragali, Radix Salviae miltiorrhizae, Radix Angelicae sinensis, Radix Paeoniae alba, Radix Curcumae, Radix Codonopsis, Rhizoma Polygonati, Rhizoma Alismatis, Radix Rehmanniae, Rhizoma Dioscoreae, Fructus Crataegi, Massa Fermentata Medicinalis, Herba Artemisiae scopariae, Radix Gentianae Macrophyllae, Radix Isatidis, Radix Glycyrrhizae)

Outcomes

Outcomes reported: serious adverse events (number of people), serious adverse events (number of events), any adverse events (number of people), resolution of fatty liver disease

Follow-up, months: 6

Notes

Source of funding: not stated

Trial name/Trial registry number: not stated

Attempts were made to contact study authors in December 2020

\section{Risk of bias}

Bias Authors' judgement Support for judgement

\begin{tabular}{|c|c|}
\hline $\begin{array}{l}\text { Random sequence genera- Low risk } \\
\text { tion (selection bias) }\end{array}$ & $\begin{array}{l}\text { Quote: "they were assigned, depending on the randomized digital table, to } \\
\text { two groups" }\end{array}$ \\
\hline
\end{tabular}

\begin{tabular}{lll}
\hline $\begin{array}{l}\text { Allocation concealment } \\
\text { (selection bias) }\end{array}$ & Unclear risk & Comment: this information was not available \\
\hline $\begin{array}{l}\text { Blinding of participants } \\
\text { and personnel (perfor- } \\
\text { mance bias) }\end{array}$ & Unclear risk & Comment: this information was not available \\
All outcomes & &
\end{tabular}

\begin{tabular}{|c|c|c|}
\hline $\begin{array}{l}\text { Blinding of outcome as- } \\
\text { sessment (detection bias) } \\
\text { All outcomes }\end{array}$ & Unclear risk & Comment: this information was not available \\
\hline $\begin{array}{l}\text { Incomplete outcome data } \\
\text { (attrition bias) } \\
\text { All outcomes }\end{array}$ & Low risk & Comment: there were no post-randomisation dropouts \\
\hline $\begin{array}{l}\text { Selective reporting (re- } \\
\text { porting bias) }\end{array}$ & Low risk & $\begin{array}{l}\text { Comment: no previously published protocol was available; adverse events and } \\
\text { either mortality or fatty liver resolution or both were reported }\end{array}$ \\
\hline Other bias & Low risk & Comment: no other bias noted \\
\hline
\end{tabular}

Li 2016

\section{Study characteristics}

Methods Randomised clinical trial


Li 2016 (Continued)

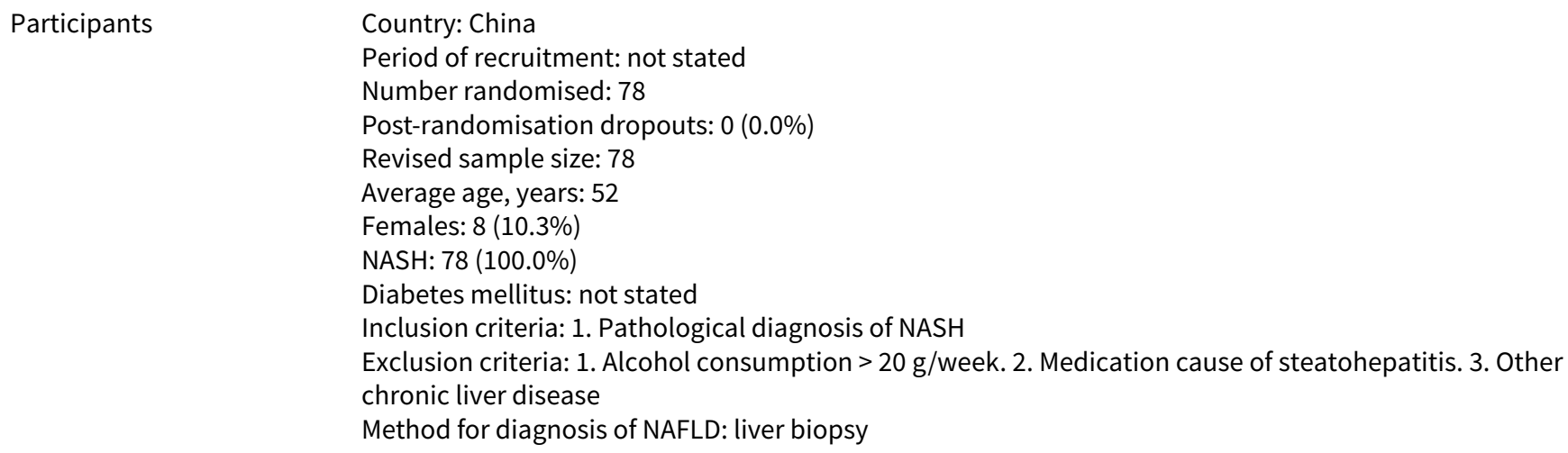

Group 1: PUFA $(\mathrm{n}=39)$

Further details: $50 \mathrm{~mL}$ PUFA with 1:1 ratio of EHA and DHA into daily diet for 6 months

Group 2: no active intervention $(n=39)$

Further details: no treatment

Additional details: both groups received lifestyle modification (exercise) advice

\begin{tabular}{ll}
\hline Outcomes & Outcomes reported: mortality at maximal follow-up, fibrosis score \\
& Follow-up, months: 6 \\
\hline \multirow{2}{*}{ Notes } & Source of funding: not stated \\
& Trial name/Trial registry number: not stated \\
& Attempts were made to contact study authors in December 2020
\end{tabular}

\section{Risk of bias}

\begin{tabular}{lll}
\hline Bias & Authors' judgement & Support for judgement \\
\hline $\begin{array}{l}\text { Random sequence genera- } \\
\text { tion (selection bias) }\end{array}$ & Unclear risk & Comment: this information was not available \\
\hline $\begin{array}{l}\text { Allocation concealment } \\
\text { (selection bias) }\end{array}$ & Unclear risk & Comment: this information was not available \\
\hline $\begin{array}{l}\text { Blinding of participants } \\
\text { and personnel (perfor- } \\
\text { mance bias) }\end{array}$ & High risk & Quote: "not blinded" \\
$\begin{array}{l}\text { All outcomes } \\
\end{array}$ & \\
\hline
\end{tabular}

\begin{tabular}{lll}
\hline $\begin{array}{l}\text { Blinding of outcome as- } \\
\text { sessment (detection bias) } \\
\text { All outcomes }\end{array}$ & High risk & Quote: "not blinded" \\
\hline $\begin{array}{l}\text { Incomplete outcome data } \\
\begin{array}{l}\text { (attrition bias) } \\
\text { All outcomes }\end{array}\end{array}$ & Low risk & Comment: there were no post-randomisation dropouts \\
\hline $\begin{array}{l}\text { Selective reporting (re- } \\
\text { porting bias) }\end{array}$ & High risk & $\begin{array}{l}\text { Comment: no previously published protocol was available; adverse events } \\
\text { were not reported adequately }\end{array}$ \\
\hline Other bias & Low risk & Comment: no other bias noted \\
\hline
\end{tabular}


Loguercio 2012

\section{Study characteristics}

\begin{tabular}{ll}
\hline Methods & Randomised clinical trial \\
\hline Participants & Country: Italy \\
& Period of recruitment: 2005 to 2008 \\
& Number randomised: 179 \\
& Post-randomisation dropouts: 41 (22.9\%) \\
& Revised sample size: 138 \\
& Reasons for post-randomisation dropouts: prematurely withdrawn because of physician's decision (5), \\
patient's decision (21), adverse events (10), other (5) & Average age, years: 42 \\
& Females: 17 (12.3\%) \\
& NASH: not stated \\
& Diabetes mellitus: not stated \\
Inclusion criteria: 1 . Histologically documented liver steatosis or steatohepatitis diagnosed within 12 \\
months. 2 . Aged 18 to 65 years. 3. Persistent increase $\geq 1$ plasma aminotransferase and or ALT and or \\
GGT within 6 months \\
Exclusion criteria: 1 . Pregnancy. 2 . Viral hepatitis (except hep C virus-positive patients with NAFLD with \\
prior HCV treatment failure). 3. Cirrhosis. 4. Other major disease including type 1 diabetes. 5. Daily alco- \\
hol $\geq 20$ g \\
Method for diagnosis of NAFLD: liver biopsy
\end{tabular}

$\begin{array}{ll}\text { Interventions } & \text { Group 1: phospholipids plus vitamin E plus other antioxidants }(\mathrm{n}=69) \\ & \text { Further details: Realsil (RA) (active components: silybin } 94 \mathrm{mg} \text {, phosphatidylcholine } 194 \mathrm{mg} \text {, vitamin } \mathrm{E} \\ & \text { acetate } 50 \% \text { ( } \alpha \text {-tocopherol } 30 \mathrm{mg}) 89.28 \mathrm{mg} \text { ) oral, twice daily, for } 12 \text { months } \\ \text { Group 2: no active intervention }(\mathrm{n}=69) \\ \text { Further details: placebo }\end{array}$

\begin{tabular}{|c|c|}
\hline Outcomes & $\begin{array}{l}\text { Outcomes reported: serious adverse events (number of people), resolution of fatty liver disease, NA } \\
\text { activity score } \\
\text { Follow-up, months: } 12\end{array}$ \\
\hline Notes & $\begin{array}{l}\text { Source of funding (quote): "this study was funded by a grant from the Istituto Biochimico Italiano, } \\
\text { Lorenzini S.p.a., Italy" } \\
\text { Trial name/Trial registry number: not stated } \\
\text { Attempts were made to contact study authors in December } 2020\end{array}$ \\
\hline
\end{tabular}

\section{Risk of bias}

\begin{tabular}{lll}
\hline Bias & Authors' judgement & Support for judgement \\
\hline $\begin{array}{l}\text { Random sequence genera- } \\
\text { tion (selection bias) }\end{array}$ & Low risk & Quote: "computer program" \\
\hline $\begin{array}{l}\text { Allocation concealment } \\
\text { (selection bias) }\end{array}$ & Unclear risk & Comment: this information was not available \\
\hline $\begin{array}{l}\text { Blinding of participants } \\
\text { and personnel (perfor- } \\
\text { mance bias) }\end{array}$ & Low risk & Quote: "multicenter, phase III, double-blind clinical trial...patients and investi- \\
All outcomes & gators were blinded to treatment until trial completion" \\
\hline $\begin{array}{l}\text { Blinding of outcome as- } \\
\text { sessment (detection bias) } \\
\text { All outcomes }\end{array}$ & Low risk & $\begin{array}{l}\text { Quote: "multicenter, phase III, double-blind clinical trial...patients and investi- } \\
\text { gators were blinded to treatment until trial completion" }\end{array}$ \\
\hline $\begin{array}{l}\text { Incomplete outcome data } \\
\text { (attrition bias) }\end{array}$ & Unclear risk & $\begin{array}{l}\text { Comment: participants were excluded from analysis for reasons that are likely } \\
\text { to be related to the intervention and to outcomes }\end{array}$ \\
\hline
\end{tabular}


Loguercio 2012 (Continued)

All outcomes

$\begin{array}{ll}\begin{array}{l}\text { Selective reporting (re- } \\ \text { porting bias) }\end{array} & \text { Low risk } \\ & \begin{array}{l}\text { Comment: no previously published protocol was available; adverse events and } \\ \text { either mortality or fatty liver resolution or both were reported }\end{array}\end{array}$

Other bias Low risk Comment: no other bias noted

Magosso 2013

\section{Study characteristics}

\begin{tabular}{|c|c|}
\hline Methods & Randomised clinical trial \\
\hline Participants & $\begin{array}{l}\text { Country: Malaysia } \\
\text { Period of recruitment: } 2008 \text { to } 2009 \\
\text { Number randomised: } 87 \\
\text { Post-randomisation dropouts: } 0(0.0 \%) \\
\text { Revised sample size: } 87 \\
\text { Average age, years: } 51 \\
\text { Females: } 53(60.9 \%) \\
\text { NASH: not stated } \\
\text { Diabetes mellitus: not stated } \\
\text { Inclusion criteria: } 1 \text {. Aged } \geq 35 \text { years old. } 2 \text {. Mild untreated hypercholesterolaemia (5.2 to } 6.2 \text { for total } \\
\text { cholesterol, } 2.6 \text { to } 4.2 \text { for LDL). } 3 \text {. USS proven NAFLD } \\
\text { Exclusion criteria: } 1 \text {. Alcohol > } 20 \text { g/d and/or history of abuse or excessive intake of alcohol. 2. ALT/AST > } \\
3 x \text { upper limit normal. } 3 \text {. Antihyperlipidaemic medications in last } 3 \text { months. } 4 \text {. Vitamin E intake. 5. Pre- } \\
\text { vious cardiovascular event or hepatitis } \\
\text { Method for diagnosis of NAFLD: ultrasound }\end{array}$ \\
\hline Interventions & $\begin{array}{l}\text { Group 1: vitamin } \mathrm{E}(\mathrm{n}=43) \\
\text { Further details: } 61.5 \mathrm{mg}, 112.8 \mathrm{mg} \text {, and } 25.7 \mathrm{mg} \text { for alpha-, gamma-, and delta-tocotrienol, respectively, } \\
\text { and } 61.1 \mathrm{mg} \text { for alpha-tocopherol (total: } 200 \mathrm{mg} \text { )/ } \mathrm{d} \text { for } 1 \text { year } \\
\text { Group 2: no active intervention ( } \mathrm{n}=44) \\
\text { Further details: placebo }\end{array}$ \\
\hline
\end{tabular}

Outcomes Outcomes reported: mortality at maximal follow-up, serious adverse events (number of people), any adverse events (number of people), resolution of fatty liver disease

Follow-up, months: 12

$\begin{array}{ll}\text { Notes } & \text { Source of funding (quote): "JW Wong, BH Ng \& E Magosso own shares of Hovid" } \\ & \text { Trial name/Trial registry number: NCT00753532 }\end{array}$

Attempts were made to contact study authors in December 2020

\section{Risk of bias}

\begin{tabular}{lll}
\hline Bias & Authors' judgement & Support for judgement \\
\hline $\begin{array}{l}\text { Random sequence genera- } \\
\text { tion (selection bias) }\end{array}$ & Low risk & Quote: "computer generated random allocation sequence" \\
\hline $\begin{array}{l}\text { Allocation concealment } \\
\text { (selection bias) }\end{array}$ & Low risk & $\begin{array}{l}\text { Quote: "the researcher (WJW) who generated the random allocation sequence } \\
\text { and assigned participants was blinded to subjects' clinical data and was inde- } \\
\text { pendent from the persons who enrolled participants" }\end{array}$ \\
\hline $\begin{array}{l}\text { Blinding of participants } \\
\begin{array}{l}\text { and personnel (perfor- } \\
\text { mance bias) }\end{array}\end{array}$ & Unclear risk & $\begin{array}{l}\text { Quote: "researchers and volunteers were blinded to the assigned treatment" } \\
\text { Comment: it is not clear whether participants were blinded }\end{array}$
\end{tabular}


Magosso 2013 (Continued)

All outcomes

Blinding of outcome as- Low risk Quote: "researchers and volunteers were blinded to the assigned treatment"
sessment (detection bias)
All outcomes

All outcomes

Incomplete outcome data Low risk Comment: there were no post-randomisation dropouts

(attrition bias)

All outcomes

\begin{tabular}{lll}
\hline $\begin{array}{l}\text { Selective reporting (re- } \\
\text { porting bias) }\end{array}$ & Low risk & $\begin{array}{l}\text { Comment: no previously published protocol was available; adverse events and } \\
\text { either mortality or fatty liver resolution or both were reported }\end{array}$ \\
\hline Other bias & Low risk & Comment: no other bias noted \\
\hline
\end{tabular}

Malaguarnera 2010

\section{Study characteristics}

\begin{tabular}{|c|c|}
\hline Methods & Randomised clinical trial \\
\hline Participants & $\begin{array}{l}\text { Country: Italy } \\
\text { Period of recruitment: } 2004 \text { to } 2006 \\
\text { Number randomised: } 80 \\
\text { Post-randomisation dropouts: } 6 \text { (7.5\%) } \\
\text { Revised sample size: } 74 \\
\text { Reasons for post-randomisation dropouts: did not receive liver biopsy or had normalisation of ALT } \\
\text { Average age, years: } 48 \\
\text { Females: } 34(45.9 \%) \\
\text { NASH: } 74 \text { (100.0\%) } \\
\text { Diabetes mellitus: not stated } \\
\text { Inclusion criteria: } 1 \text {. NAFLD } \\
\text { Exclusion criteria: } 1 \text {. Other cause of liver disease. } 2 \text {. Significant alcohol consumption }(>10 \mathrm{~g} / \mathrm{d} \text { female, > } \\
20 \text { g/d male). 3. Pregnancy. } 4 \text {. Use of calcium channel blockers, oestrogens, methotrexate, amiodarone, } \\
\text { steroids, chloroquine. } 5 \text {. History of treatment with lipid-lowering agents. } 6 \text {. Hypothyroidism. } 7 \text {. Cush- } \\
\text { ing's syndrome } \\
\text { Method for diagnosis of NAFLD: liver biopsy }\end{array}$ \\
\hline
\end{tabular}

Interventions

Group 1: amino acids $(\mathrm{n}=36)$

Further details: $1 \mathrm{~g}$ carnitine twice daily for 24 weeks

Group 2: no active intervention $(n=38)$

Further details: no treatment

Additional details: both groups received dietary intervention

\begin{tabular}{ll}
\hline Outcomes & Outcomes reported: any adverse events (number of people), fibrosis score, NAFLD activity score \\
Follow-up, months: 6
\end{tabular}

\begin{tabular}{ll}
\hline Notes & $\begin{array}{l}\text { Source of funding (quote): "financial support: none" } \\
\text { Trial name/Trial registry number: not stated } \\
\text { Attempts were made to contact study authors in December } 2020\end{array}$ \\
\hline Risk of bias & Authors' judgement Support for judgement \\
\hline Bias & Sul
\end{tabular}


Malaguarnera 2010 (Continued)

Random sequence genera- Low risk Quote: "computer-generated randomization schedule" tion (selection bias)

\begin{tabular}{|c|c|c|}
\hline $\begin{array}{l}\text { Allocation concealment } \\
\text { (selection bias) }\end{array}$ & Low risk & $\begin{array}{l}\text { Quote: "all drugs and placebos were identical in appearance, and neither in- } \\
\text { vestigators nor patients were informed of the selected agent until the end of } \\
\text { the study phase" }\end{array}$ \\
\hline
\end{tabular}

\begin{tabular}{|c|c|c|}
\hline $\begin{array}{l}\text { Blinding of participants } \\
\text { and personnel (perfor- } \\
\text { mance bias) }\end{array}$ & Low risk & $\begin{array}{l}\text { Quote: "all drugs and placebos were identical in appearance, and neither in- } \\
\text { vestigators nor patients were informed of the selected agent until the end of } \\
\text { the study phase" }\end{array}$ \\
\hline
\end{tabular}

All outcomes

\begin{tabular}{|c|c|c|}
\hline $\begin{array}{l}\text { Blinding of outcome as- } \\
\text { sessment (detection bias) } \\
\text { All outcomes }\end{array}$ & Low risk & $\begin{array}{l}\text { Quote: "all drugs and placebos were identical in appearance, and neither in- } \\
\text { vestigators nor patients were informed of the selected agent until the end of } \\
\text { the study phase" }\end{array}$ \\
\hline
\end{tabular}

$\begin{array}{ll}\begin{array}{l}\text { Incomplete outcome data } \\ \text { (attrition bias) }\end{array} & \text { Unclear risk } \\ \text { Alloutcoment: participants were excluded from analysis for reasons that may be } \\ \text { related to the intervention and to outcomes }\end{array}$

All outcomes related to the intervention and to outcomes

\begin{tabular}{lll}
\hline $\begin{array}{l}\text { Selective reporting (re- } \\
\text { porting bias) }\end{array}$ & High risk & $\begin{array}{l}\text { Comment: no previously published protocol was available; neither mortality } \\
\text { nor fatty liver resolution was reported }\end{array}$ \\
\hline Other bias & Low risk & Comment: no other bias noted \\
\hline
\end{tabular}

Malaguarnera 2012

\section{Study characteristics}

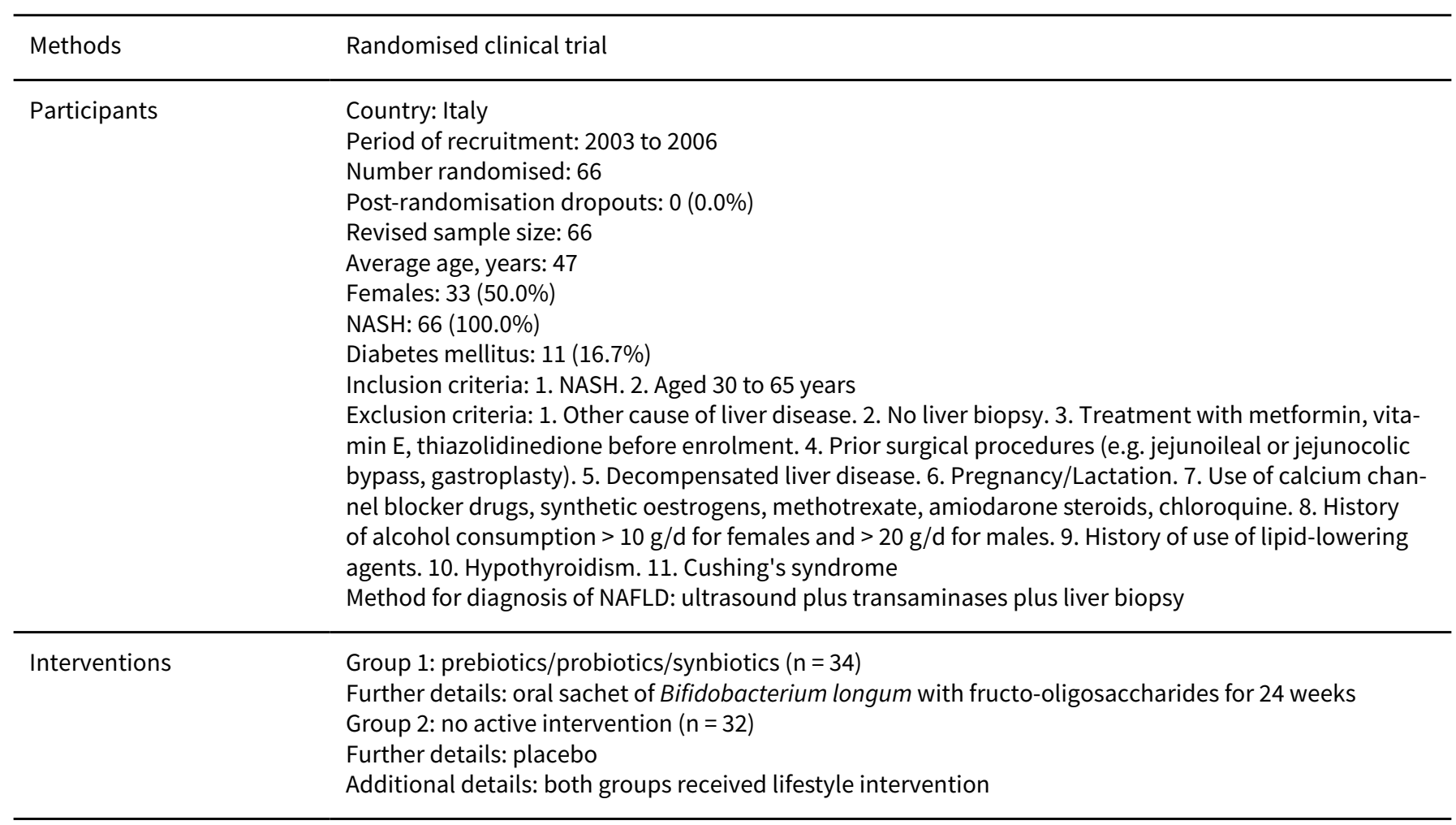


Malaguarnera 2012 (Continued)

Outcomes

Outcomes reported: mortality at maximal follow-up, any adverse events (number of people), fibrosis score, NAFLD activity score

Follow-up, months: 6

$\begin{array}{ll}\text { Notes } & \text { Source of funding (quote): "this study was supported by a grant from the Regional Health Department } \\ & \text { for Sicily (Ric. Fin. 2007)" } \\ & \text { Trial name/Trial registry number: not stated } \\ & \text { Attempts were made to contact study authors in December } 2020\end{array}$

\section{Risk of bias}

\begin{tabular}{|c|c|c|}
\hline Bias & Authors' judgement & Support for judgement \\
\hline $\begin{array}{l}\text { Random sequence genera- } \\
\text { tion (selection bias) }\end{array}$ & Low risk & Quote: "computer-generated" \\
\hline $\begin{array}{l}\text { Allocation concealment } \\
\text { (selection bias) }\end{array}$ & Low risk & $\begin{array}{l}\text { Quote: "neither investigators nor patients were informed of the selected agent } \\
\text { until the end of the study phase" }\end{array}$ \\
\hline & & $\begin{array}{l}\text { Comment: both allocation concealment and blinding were achieved with use } \\
\text { of placebo }\end{array}$ \\
\hline $\begin{array}{l}\text { Blinding of participants } \\
\text { and personnel (perfor- } \\
\text { mance bias) } \\
\text { All outcomes }\end{array}$ & Unclear risk & $\begin{array}{l}\text { Quote: "double-blind treatment...neither investigators nor patients were in- } \\
\text { formed of the selected agent until the end of the study phase" } \\
\text { Comment: it is not clear how this was achieved as a placebo was not used }\end{array}$ \\
\hline $\begin{array}{l}\text { Blinding of outcome as- } \\
\text { sessment (detection bias) } \\
\text { All outcomes }\end{array}$ & Low risk & $\begin{array}{l}\text { Quote: "double-blind treatment...neither investigators nor patients were in- } \\
\text { formed of the selected agent until the end of the study phase" }\end{array}$ \\
\hline $\begin{array}{l}\text { Incomplete outcome data } \\
\text { (attrition bias) } \\
\text { All outcomes }\end{array}$ & Low risk & Comment: there were no post-randomisation dropouts \\
\hline $\begin{array}{l}\text { Selective reporting (re- } \\
\text { porting bias) }\end{array}$ & Low risk & $\begin{array}{l}\text { Comment: no previously published protocol was available; adverse events and } \\
\text { either mortality or fatty liver resolution or both were reported }\end{array}$ \\
\hline Other bias & Low risk & Comment: no other bias noted \\
\hline
\end{tabular}

Mansour 2020

\section{Study characteristics}

\begin{tabular}{ll}
\hline Methods & Randomised clinical trial \\
\hline Participants & Country: Iran \\
& Period of recruitment: not stated \\
& Number randomised: 26 \\
& Post-randomisation dropouts: $0(0.0 \%)$ \\
& Revised sample size: 26 \\
& Average age, years: 45 \\
& Females: 5 (19.2\%) \\
& NASH: not stated \\
& Diabetes mellitus: $26(100.0 \%)$
\end{tabular}


Inclusion criteria: 1. Diabetes type 2. 2. Evidence of hepatic steatosis $\geq$ grade 2 in USS exam and steatosis $\geq$ grade 2 in Fibroscan exam (controlled attenuation parameter (CAP) score $>263$ )

Exclusion criteria: 1. Professional athlete. 2. Taking any medication other than oral diabetes drugs. 3. Viral hepatitis. 4. Alcohol use. 5. Hepatic cirrhosis. 6. Other causes of chronic liver disease. 7. Hypothyroidism. 8. Renal, intestinal, and cardiovascular disorders. 9. Body mass index (BMI) $>35 \mathrm{~kg} / \mathrm{m}^{2}$. 10. On a special diet. 11. Any change in hypoglycaemic medications. 12. Psychiatric disorders impairing patient's ability to provide written informed consent. 13. Pregnancy, lactation Method for diagnosis of NAFLD: ultrasound and elastography

\begin{tabular}{|c|c|}
\hline Interventions & $\begin{array}{l}\text { Group 1: other supplements }(n=20) \\
\text { Further details: } 200 \text { mg caffeine or } 200 \mathrm{mg} \text { chlorogenic acid or both (factorial trial design) for } 12 \text { weeks } \\
\text { Group 2: no active intervention }(n=6) \\
\text { Further details: placebo }\end{array}$ \\
\hline Outcomes & $\begin{array}{l}\text { Outcomes reported: mortality at maximal follow-up, serious adverse events (number of people), any } \\
\text { adverse events (number of people), liver transplantation at maximal follow-up, decompensation (num- } \\
\text { ber of people), cirrhosis (number of people), resolution of fatty liver disease, hepatocellular carcinoma } \\
\text { Follow-up, months: } 3\end{array}$ \\
\hline Notes & $\begin{array}{l}\text { Source of funding (quote): "the study was supported by National Institute for Medical Research Devel- } \\
\text { opment (NIMAD) to AH with grant number of 963356" } \\
\text { Trial name/Trial registry number: NCT02929901 } \\
\text { Attempts were made to contact study authors in April } 2021\end{array}$ \\
\hline
\end{tabular}

\section{Risk of bias}

\begin{tabular}{|c|c|c|}
\hline Bias & Authors' judgement & Support for judgement \\
\hline $\begin{array}{l}\text { Random sequence genera- } \\
\text { tion (selection bias) }\end{array}$ & Low risk & Quote: "randomization lists were computer generated by a statistician" \\
\hline $\begin{array}{l}\text { Allocation concealment } \\
\text { (selection bias) }\end{array}$ & Low risk & $\begin{array}{l}\text { Quote: "the supplements were concealed by the production company and re- } \\
\text { vealed to us after the study results analysed (author replies)" }\end{array}$ \\
\hline $\begin{array}{l}\text { Blinding of participants } \\
\text { and personnel (perfor- } \\
\text { mance bias) } \\
\text { All outcomes }\end{array}$ & Low risk & $\begin{array}{l}\text { Quote: "subjects, investigators, and staff were blinded to the treatment assign- } \\
\text { ment until the end of the study...placebo" }\end{array}$ \\
\hline $\begin{array}{l}\text { Blinding of outcome as- } \\
\text { sessment (detection bias) } \\
\text { All outcomes }\end{array}$ & Low risk & $\begin{array}{l}\text { Quote: "subjects, investigators, and staff were blinded to the treatment assign- } \\
\text { ment until the end of the study...placebo" }\end{array}$ \\
\hline $\begin{array}{l}\text { Incomplete outcome data } \\
\text { (attrition bias) } \\
\text { All outcomes }\end{array}$ & Low risk & Comment: there were no post-randomisation dropouts \\
\hline $\begin{array}{l}\text { Selective reporting (re- } \\
\text { porting bias) }\end{array}$ & Low risk & $\begin{array}{l}\text { Comment: no previously published protocol was available; adverse events and } \\
\text { either mortality or fatty liver resolution or both were reported }\end{array}$ \\
\hline Other bias & Low risk & Comment: no other bias noted \\
\hline
\end{tabular}

\section{Study characteristics}


Manzhalii 2017 (Continued)

\begin{tabular}{ll} 
Methods & Randomised clinical trial \\
\hline Participants & Country: Ukraine \\
& Period of recruitment: not stated \\
Number randomised: 75 \\
Post-randomisation dropouts: $0(0.0 \%)$ \\
Revised sample size: 75 \\
Average age, years: 44 \\
Females: $48(64.0 \%)$ \\
NASH: 75 (100.0\%) \\
Diabetes mellitus: 0 (0.0\%) \\
Inclusion criteria: 1. NASH (based on USS detection of steatosis and elevated GGT $>45$ and ALT > 40 and \\
valid Fibroscan results). 2 . Aged 30 to 60 years \\
Exclusion criteria: 1. Other cause of chronic liver disease. 2 . Alcohol $>40 \mathrm{~g} / \mathrm{d}$ male, $>20 \mathrm{~g} / \mathrm{d}$ female. 3. \\
BMI > 30. 4 . Diabetes. 5 . Hypertriglycerides. 6 . Pregnant/lactating. 7. Severe comorbidities \\
Method for diagnosis of NAFLD: ultrasound plus transaminases
\end{tabular}

Interventions

roup 1: prebiotics/probiotics/synbiotics $(n=38)$

Further details: probiotic cocktail (LBSF; Lactiale; Farmak, Kiev, Ukraine, containing L casei, L rhamnosus, $L$ bulgaris, $B$ longum, and $S$ thermophilus $\left(10^{8}\right.$ bacteria/capsule in total) as well as fructo-oligosaccharides), once daily for 12 weeks

Group 2: no active intervention $(n=37)$

Further details: no treatment

Additional details: both groups received low-calorie diet

\begin{tabular}{ll} 
Outcomes & $\begin{array}{l}\text { Outcomes reported: mortality at maximal follow-up, serious adverse events (number of people), any } \\
\text { adverse events (number of people) } \\
\text { Follow-up, months: } 3\end{array}$ \\
\hline Notes & Source of funding: not stated \\
& Trial name/Trial registry number: not stated \\
& Attempts were made to contact study authors in December 2020
\end{tabular}

\section{Risk of bias}

\begin{tabular}{lll}
\hline Bias & Authors' judgement & Support for judgement \\
\hline $\begin{array}{l}\text { Random sequence genera- } \\
\text { tion (selection bias) }\end{array}$ & Low risk & Quote: "closed envelop drawing" \\
\hline $\begin{array}{l}\text { Allocation concealment } \\
\text { (selection bias) }\end{array}$ & Low risk & Quote: "closed envelop drawing" \\
\hline $\begin{array}{l}\text { Blinding of participants } \\
\begin{array}{l}\text { and personnel (perfor- } \\
\text { mance bias) }\end{array}\end{array}$ & High risk \\
All outcomes & Quote: "open-label" \\
\hline
\end{tabular}

\begin{tabular}{lll}
\hline $\begin{array}{l}\text { Blinding of outcome as- } \\
\text { sessment (detection bias) } \\
\text { All outcomes }\end{array}$ & High risk & Quote: "open-label" \\
\hline $\begin{array}{l}\text { Incomplete outcome data } \\
\text { (attrition bias) } \\
\text { All outcomes }\end{array}$ & Low risk & Comment: there were no post-randomisation dropouts \\
\hline $\begin{array}{l}\text { Selective reporting (re- } \\
\text { porting bias) }\end{array}$ & Low risk & $\begin{array}{l}\text { Comment: no previously published protocol was available; adverse events and } \\
\text { either mortality or fatty liver resolution or both were reported }\end{array}$ \\
\hline
\end{tabular}


Manzhalii 2017 (Continued)

Other bias Low risk Comment: no other bias noted

Martinez-Rodriguez 2014

\section{Study characteristics}

\begin{tabular}{ll}
\hline Methods & Randomised clinical trial \\
\hline Participants & Country: Mexico \\
& Period of recruitment: 2013 to 2014 \\
& Number randomised: 40 \\
& Post-randomisation dropouts: $0(0.0 \%)$ \\
& Revised sample size: 40 \\
& Average age, years: 47 \\
& Females: $26(65.0 \%)$ \\
& NASH: not stated \\
& Diabetes mellitus: 0 (0.0\%) \\
& Inclusion criteria: 1. NAFLD. 2. Aged $\geq 18$ years. 3. Pre-diabetes, metabolic syndrome \\
& Exclusion criteria: 1 . Significant alcohol consumption. 2. Other chronic liver disease. 3. Use of multi-vit- \\
& amins within past 3 months. 4 . Allergy to excipients used in study \\
& Method for diagnosis of NAFLD: ultrasound plus liver biopsy
\end{tabular}

Group 1: other supplements $(\mathrm{n}=20)$
Further details: orally administered selenium 15 mcg-methionine 3 mg-alpha lipoic acid 200 mg (SS-
MAL), every alternate day, for 24 weeks
Group 2: no active intervention $(n=20)$
Further details: no additional supplementation
Additional details: both groups received lifestyle advice and metformin

Outcomes
ple), resolution of fatty liver disease
ple), resolution of fatty liver disease

Follow-up, months: 6

\begin{tabular}{ll}
\hline Notes & Source of funding: not stated \\
& Trial name/Trial registry number: NCT01650181 \\
& Attempts were made to contact study authors in December 2020
\end{tabular}

\section{Risk of bias}

\begin{tabular}{|c|c|c|}
\hline Bias & Authors' judgement & Support for judgement \\
\hline $\begin{array}{l}\text { Random sequence genera- } \\
\text { tion (selection bias) }\end{array}$ & Low risk & $\begin{array}{l}\text { Quote: "we used www.randomization.com in order to assign participants to } \\
\text { each arm" }\end{array}$ \\
\hline $\begin{array}{l}\text { Allocation concealment } \\
\text { (selection bias) }\end{array}$ & Unclear risk & Comment: this information was not available \\
\hline $\begin{array}{l}\text { Blinding of participants } \\
\text { and personnel (perfor- } \\
\text { mance bias) } \\
\text { All outcomes }\end{array}$ & Unclear risk & $\begin{array}{l}\text { Quote: "during data collection researchers did not have access to the random- } \\
\text { ization codes or statistical summaries of follow-up data" } \\
\text { Comment: although study authors state double-blind, the method was not re- } \\
\text { ported, as there is no mention of use of placebo }\end{array}$ \\
\hline $\begin{array}{l}\text { Blinding of outcome as- } \\
\text { sessment (detection bias) } \\
\text { All outcomes }\end{array}$ & Unclear risk & $\begin{array}{l}\text { Quote: "during data collection researchers did not have access to the random- } \\
\text { ization codes or statistical summaries of follow-up data" } \\
\text { Comment: although study authors state double-blind, the method was not re- } \\
\text { ported, as there is no mention of use of placebo }\end{array}$ \\
\hline
\end{tabular}


Martinez-Rodriguez 2014 (Continued)
Incomplete outcome data Low risk
Comment: there were no post-randomisation dropouts
(attrition bias)

All outcomes

\begin{tabular}{lll}
\hline $\begin{array}{l}\text { Selective reporting (re- } \\
\text { porting bias) }\end{array}$ & Low risk & $\begin{array}{l}\text { Comment: a published protocol was available, but it is not clear whether re- } \\
\text { cruitment had commenced before the protocol was published; adverse events } \\
\text { and either mortality or fatty liver resolution or both were reported }\end{array}$ \\
\hline Other bias & Low risk & Comment: no other bias noted \\
\hline
\end{tabular}

Miglio 2000

\section{Study characteristics}

\begin{tabular}{|c|c|c|}
\hline Methods & \multicolumn{2}{|c|}{ Randomised clinical trial } \\
\hline Participants & \multicolumn{2}{|c|}{$\begin{array}{l}\text { Country: Italy } \\
\text { Period of recruitment: not stated } \\
\text { Number randomised: } 191 \\
\text { Post-randomisation dropouts: not stated } \\
\text { Revised sample size: } 191 \\
\text { Average age, years: } 59 \\
\text { Females: } 57(29.8 \%) \\
\text { NASH: not stated } \\
\text { Diabetes mellitus: not stated } \\
\text { Inclusion criteria: } 1 \text {. Aged }>18 \text { years. } 2 \text {. Liver enlargement and hepatic steatosis ascertained on USS } \\
\text { Exclusion criteria: } 1 \text {. History of past/present alcohol abuse. } 2 \text {. Other cause of chronic liver disease } \\
\text { Method for diagnosis of NAFLD: ultrasound }\end{array}$} \\
\hline Interventions & \multicolumn{2}{|c|}{$\begin{array}{l}\text { Group 1: amino acids plus vitamin C ( } \mathrm{n}=96) \\
\text { Further details: Letepar (betaine glucuronate } 150 \mathrm{mg} \text {, diethanolamine glucuronate } 30 \mathrm{mg} \text {, nicoti- } \\
\text { namide ascorbate } 20 \mathrm{mg} \text { ) twice daily for } 8 \text { weeks } \\
\text { Group 2: no active intervention ( } \mathrm{n}=95) \\
\text { Further details: placebo } \\
\text { Additional details: both groups received dietary advice }\end{array}$} \\
\hline Outcomes & \multicolumn{2}{|c|}{$\begin{array}{l}\text { Outcomes reported: serious adverse events (number of people), any adverse events (number of peo- } \\
\text { ple) } \\
\text { Follow-up, months: } 2\end{array}$} \\
\hline Notes & \multicolumn{2}{|c|}{$\begin{array}{l}\text { Source of funding: not stated } \\
\text { Trial name/Trial registry number: not stated } \\
\text { Attempts were made to contact study authors in December } 2020\end{array}$} \\
\hline \multicolumn{3}{|l|}{ Risk of bias } \\
\hline Bias & Authors' judgement & Support for judgement \\
\hline $\begin{array}{l}\text { Random sequence genera- } \\
\text { tion (selection bias) }\end{array}$ & Low risk & Quote: "computer-generated" \\
\hline $\begin{array}{l}\text { Allocation concealment } \\
\text { (selection bias) }\end{array}$ & Low risk & $\begin{array}{l}\text { Quote: "double-blind, placebo-controlled" } \\
\text { Comment: allocation concealment and blinding were achieved with use of } \\
\text { placebo }\end{array}$ \\
\hline
\end{tabular}


Miglio 2000 (Continued)

Blinding of participants Low risk Quote: "double-blind, placebo-controlled" and personnel (performance bias)

All outcomes

\begin{tabular}{lll}
\hline $\begin{array}{l}\text { Blinding of outcome as- } \\
\text { sessment (detection bias) } \\
\text { All outcomes }\end{array}$ & Low risk & Quote: "double-blind, placebo-controlled" \\
\hline $\begin{array}{l}\text { Incomplete outcome data } \\
\text { (attrition bias) }\end{array}$ & Unclear risk & Comment: this information was not available \\
All outcomes & &
\end{tabular}

\begin{tabular}{lll}
\hline $\begin{array}{l}\text { Selective reporting (re- } \\
\text { porting bias) }\end{array}$ & High risk & $\begin{array}{l}\text { Comment: no previously published protocol was available; neither mortality } \\
\text { nor fatty liver resolution was reported }\end{array}$ \\
\hline Other bias & Low risk & Comment: no other bias noted \\
\hline
\end{tabular}

Mofidi 2017

\section{Study characteristics}

\begin{tabular}{|c|c|}
\hline Methods & Randomised clinical trial \\
\hline Participants & $\begin{array}{l}\text { Country: Iran } \\
\text { Period of recruitment: not stated } \\
\text { Number randomised: } 50 \\
\text { Post-randomisation dropouts: } 8(16.0 \%) \\
\text { Revised sample size: } 42 \\
\text { Reasons for post-randomisation dropouts: lost to follow-up, travel } \\
\text { Average age, years: } 45 \\
\text { Females: } 19(45.2 \%) \\
\text { NASH: not stated } \\
\text { Diabetes mellitus: not stated } \\
\text { Inclusion criteria: } 1 \text {. NAFLD. } 2 \text {. Aged > } 18 \text { years. 3. BMI } \leq 25.4 \text {. Lack of history of alcohol consumption } \\
\text { Exclusion criteria: } 1 \text {. Other cause of liver disease. } 2 \text {. Use of antibiotics, probiotic supplements, and/or } \\
\text { hepatotoxic medicine within } 6 \text { months. 3. Pregnant/breastfeeding. } 4 .>10 \% \text { body weight loss during } \\
\text { the study } \\
\text { Method for diagnosis of NAFLD: elastography plus transaminases }\end{array}$ \\
\hline Interventions & $\begin{array}{l}\text { Group 1: prebiotics/probiotics/synbiotics ( } \mathrm{n}=21 \text { ) } \\
\text { Further details: synbiotic supplement (Protexin; Probiotics International Ltd.; containing } 200 \text { million } \\
\text { bacteria of } 7 \text { strains (Lactobacillus casei, Lactobacillus rhamnosus, Streptococcus thermophilus, Bifi- } \\
\text { dobacterium breve, Lactobacillus acidophilus, Bifidobacterium longum, Lactobacillus bulgaricus) and } \\
\text { prebiotic ( } 125 \mathrm{mg} \text { fructo-oligosaccharide) and probiotic cultures (magnesium stearate (source: mineral } \\
\text { and vegetable) and a vegetable capsule (hydroxypropylmethyl cellulose)), twice daily, for } 28 \text { weeks } \\
\text { Group 2: no active intervention ( } \mathrm{n}=21 \text { ) } \\
\text { Further details: placebo } \\
\text { Additional details: both groups received lifestyle advice }\end{array}$ \\
\hline Outcomes & $\begin{array}{l}\text { Outcomes reported: serious adverse events (number of people) } \\
\text { Follow-up, months: } 7\end{array}$ \\
\hline Notes & $\begin{array}{l}\text { Source of funding (quote): "Protexin Company, UK, provided the synbiotics supplements" } \\
\text { Trial name/Trial registry number: NCT02530138 } \\
\text { Attempts were made to contact study authors in December } 2020\end{array}$ \\
\hline
\end{tabular}


Mofidi 2017 (Continued)

Risk of bias

\begin{tabular}{|c|c|c|}
\hline Bias & Authors' judgement & Support for judgement \\
\hline $\begin{array}{l}\text { Random sequence genera- } \\
\text { tion (selection bias) }\end{array}$ & Low risk & Quote: "randomization lists will be computer-generated by a statistician" \\
\hline $\begin{array}{l}\text { Allocation concealment } \\
\text { (selection bias) }\end{array}$ & Low risk & $\begin{array}{l}\text { Quote: "double-blind, placebo-controlled, clinical trial" } \\
\text { Comment: allocation concealment and blinding were achieved with use of } \\
\text { placebo }\end{array}$ \\
\hline $\begin{array}{l}\text { Blinding of participants } \\
\text { and personnel (perfor- } \\
\text { mance bias) } \\
\text { All outcomes }\end{array}$ & Low risk & Quote: "double-blind, placebo-controlled, clinical trial" \\
\hline $\begin{array}{l}\text { Blinding of outcome as- } \\
\text { sessment (detection bias) } \\
\text { All outcomes }\end{array}$ & Low risk & Quote: "double-blind, placebo-controlled, clinical trial" \\
\hline $\begin{array}{l}\text { Incomplete outcome data } \\
\text { (attrition bias) } \\
\text { All outcomes }\end{array}$ & Unclear risk & $\begin{array}{l}\text { Comment: participants were excluded from analysis for reasons that may be } \\
\text { related to the intervention and to outcomes }\end{array}$ \\
\hline $\begin{array}{l}\text { Selective reporting (re- } \\
\text { porting bias) }\end{array}$ & High risk & $\begin{array}{l}\text { Comment: a published protocol was available, but it is not clear whether re- } \\
\text { cruitment had commenced before the protocol was published; neither mortal- } \\
\text { ity nor fatty liver resolution was reported }\end{array}$ \\
\hline Other bias & Low risk & Comment: no other bias noted \\
\hline
\end{tabular}

\section{Moradi 2020}

\section{Study characteristics}

\begin{tabular}{ll}
\hline Methods & Randomised clinical trial \\
\hline Participants & Country: Iran \\
Period of recruitment: not stated \\
Number randomised: 45 \\
Post-randomisation dropouts: $0(0.0 \%)$ \\
Revised sample size: 45 \\
Average age, years: 65 \\
Females: $45(100.0 \%)$ \\
NASH: not stated \\
Diabetes mellitus: not stated \\
Inclusion criteria: 1. Obese. 2. Elderly. 3. Female. 4. Fatty liver disease confirmed by ultrasonography \\
Exclusion criteria: 1 . Addiction to smoking. 2. Alcohol abuse. 3. Regular physical exercise in the last 6 \\
months. 4. Lung disease, kidney disease, cardiovascular disease, liver transplantation, high blood pres- \\
sure, chronic disorder. 5. Special medications such as statins, oestrogen intake, additive effects on in- \\
sulin sensitivity, hepatotoxic medication intake. 6 . Special dietary programme. 7 . Allergy to curcumin. \\
8. A cancer record. 9 . Other supplements \\
Method for diagnosis of NAFLD: ultrasound
\end{tabular}

Interventions

Group 1: other supplements $(n=22)$

Further details: 1 curcumin capsule (curcumin $80 \mathrm{mg}$ as Nanomicelle, produced by Minoo Pharmaceutical Co.) per day for 12 weeks 
Moradi 2020 (Continued)

Group 2: no active intervention ( $n=23$ )

Further details: placebo daily for 12 weeks.

Additional details: $50 \%$ of both groups received lifestyle intervention of non-linear resistance training

(factorial trial design)

\begin{tabular}{ll}
\hline Outcomes & No outcomes of interest were reported \\
\hline Notes & Source of funding (quote): "funding: none" \\
& Trial name/Trial registry number: IRCT20190103042219N1 \\
& Attempts were made to contact study authors in April 2021
\end{tabular}

\section{Risk of bias}

\begin{tabular}{|c|c|c|}
\hline Bias & Authors' judgement & Support for judgement \\
\hline $\begin{array}{l}\text { Random sequence genera- } \\
\text { tion (selection bias) }\end{array}$ & Unclear risk & Comment: this information was not available \\
\hline $\begin{array}{l}\text { Allocation concealment } \\
\text { (selection bias) }\end{array}$ & Low risk & $\begin{array}{l}\text { Quote: "the participants were identified using the codes instead of their actual } \\
\text { names. A third party was asked to classify the participants randomly, using the } \\
\text { (labelled) codes" }\end{array}$ \\
\hline $\begin{array}{l}\text { Blinding of participants } \\
\text { and personnel (perfor- } \\
\text { mance bias) } \\
\text { All outcomes }\end{array}$ & Low risk & Quote: "double-blind...placebo" \\
\hline $\begin{array}{l}\text { Blinding of outcome as- } \\
\text { sessment (detection bias) } \\
\text { All outcomes }\end{array}$ & Low risk & Quote: "double-blind...placebo" \\
\hline $\begin{array}{l}\text { Incomplete outcome data } \\
\text { (attrition bias) } \\
\text { All outcomes }\end{array}$ & Low risk & Comment: there were no post-randomisation dropouts \\
\hline $\begin{array}{l}\text { Selective reporting (re- } \\
\text { porting bias) }\end{array}$ & High risk & $\begin{array}{l}\text { Comment: no previously published protocol was available; adverse events, } \\
\text { mortality, fatty liver resolution were not reported }\end{array}$ \\
\hline Other bias & Low risk & Comment: no other bias noted \\
\hline
\end{tabular}

Morvaridzadeh 2021

\section{Study characteristics}

\begin{tabular}{ll}
\hline Methods & Randomised clinical trial \\
\hline Participants & Country: Iran \\
& Period of recruitment: not stated \\
& Number randomised: 104 \\
& Post-randomisation dropouts: 16 (15.4\%) \\
& Revised sample size: 88 \\
& Reasons for post-randomisation dropouts: lost to follow-up \\
& Average age, years: 40 \\
& Females: 41 (46.6\%) \\
& NASH: not stated \\
& Diabetes mellitus: not stated \\
Inclusion criteria: 1 . NAFLD. 2 . Aged 25 to 55 years. 3. Mild to moderate fatty liver
\end{tabular}


Morvaridzadeh 2021 (Continued)

Exclusion criteria: 1. Any supplements and medications within 6 weeks. 2. Sensitive to yoghurt consumption. 3. Other chronic disease such as chronic heart, lung, kidney problems or cancer Method for diagnosis of NAFLD: ultrasound

Group 1: prebiotics/probiotics/synbiotics plus vitamin $D(n=44)$
Further details: 100 g probiotic yoghurt fortified with vitamin $D$ every day for 12 weeks
Group 2: prebiotics/probiotics/synbiotics $(n=44)$
Further details: 100 g probiotic yoghurt every day for 12 weeks

\begin{tabular}{ll}
\hline Outcomes & No outcomes of interest were reported \\
\hline Notes & Source of funding (quote): "the Research Council approved this study of Kermanshah University of \\
& Medical Sciences" \\
& Trial name/Trial registry number: IRCT20131022015111N3 \\
& Attempts were made to contact study authors in April 2021
\end{tabular}

\section{Risk of bias}

\begin{tabular}{|c|c|c|}
\hline Bias & Authors' judgement & Support for judgement \\
\hline $\begin{array}{l}\text { Random sequence genera- } \\
\text { tion (selection bias) }\end{array}$ & Low risk & Quote: "using random numbers table" \\
\hline $\begin{array}{l}\text { Allocation concealment } \\
\text { (selection bias) }\end{array}$ & Unclear risk & Comment: this information was not available \\
\hline $\begin{array}{l}\text { Blinding of participants } \\
\text { and personnel (perfor- } \\
\text { mance bias) } \\
\text { All outcomes }\end{array}$ & Low risk & Quote: "double-blind...placebo" \\
\hline $\begin{array}{l}\text { Blinding of outcome as- } \\
\text { sessment (detection bias) } \\
\text { All outcomes }\end{array}$ & Low risk & Quote: "double-blind...placebo" \\
\hline $\begin{array}{l}\text { Incomplete outcome data } \\
\text { (attrition bias) } \\
\text { All outcomes }\end{array}$ & Unclear risk & $\begin{array}{l}\text { Comment: there were post-randomisation dropouts because of loss to fol- } \\
\text { low-up; it is not clear whether these were related to the intervention and to } \\
\text { outcomes }\end{array}$ \\
\hline $\begin{array}{l}\text { Selective reporting (re- } \\
\text { porting bias) }\end{array}$ & High risk & $\begin{array}{l}\text { Comment: no previously published protocol was available; adverse events, } \\
\text { mortality, fatty liver resolution were not reported }\end{array}$ \\
\hline Other bias & Low risk & Comment: no other bias noted \\
\hline
\end{tabular}

\section{Nabavi 2016}

\section{Study characteristics}

\begin{tabular}{ll}
\hline Methods & Randomised clinical trial \\
\hline Participants & Country: Iran \\
& Period of recruitment: not stated \\
& Number randomised: 72 \\
& Post-randomisation dropouts: $0(0.0 \%)$ \\
& Revised sample size: 72 \\
& Average age, years: 43
\end{tabular}


Nabavi 2016 (Continued)

Females: 37 (51.4\%)

NASH: not stated

Diabetes mellitus: not stated

Inclusion criteria: 1. NAFLD. 2. Aged 23 to 60 years. 3. BMI 25 to 40

Exclusion criteria: 1 . Kidney disease. 2. Other type of liver disease. 3. Inflammatory intestinal disease.

4. Immunodeficiency disease. 5. Use of tobacco or alcohol. 6. Nutritional supplements in previous

3 weeks. 7. Use of cholesterol-lowering medications, oestrogen, progesterone, or diuretics. 8. Preg-

nant/breastfeeding. 9. Consumption of probiotic yoghurt or any other probiotic products in last 2

months

Method for diagnosis of NAFLD: ultrasound

$\begin{array}{ll}\text { Interventions } & \text { Group 1: prebiotics/probiotics/synbiotics }(n=36) \\ & \text { Further details: } 300 \mathrm{~g} / \mathrm{d} \text { of probiotic yoghurt for } 8 \text { weeks } \\ & \text { Group 2: no active intervention }(\mathrm{n}=36) \\ & \text { Further details: placebo }\end{array}$

Outcomes Outcomes reported: mortality at maximal follow-up, serious adverse events (number of people), any adverse events (number of people)

Follow-up, months: 2

Notes Source of funding (quote): "Pegah Dairy Industries Co. (Tabriz, Iran) for supplying the probiotic and conventional yoghurts"

Trial name/Trial registry number: not stated

Attempts were made to contact study authors in December 2020

\section{Risk of bias}

\begin{tabular}{|c|c|c|}
\hline Bias & Authors' judgement & Support for judgement \\
\hline $\begin{array}{l}\text { Random sequence genera- } \\
\text { tion (selection bias) }\end{array}$ & Low risk & $\begin{array}{l}\text { Quote: "the random sequence was generated by study statistician using ran- } \\
\text { dom allocation software" }\end{array}$ \\
\hline $\begin{array}{l}\text { Allocation concealment } \\
\text { (selection bias) }\end{array}$ & Low risk & $\begin{array}{l}\text { Quote: "probiotic and conventional yogurt containers were identical look- } \\
\text { ing...assignment of groups was covered from the investigators and the sub- } \\
\text { jects" }\end{array}$ \\
\hline $\begin{array}{l}\text { Blinding of participants } \\
\text { and personnel (perfor- } \\
\text { mance bias) } \\
\text { All outcomes }\end{array}$ & Low risk & Quote: "randomized double-blind controlled clinical trial...placebo" \\
\hline $\begin{array}{l}\text { Blinding of outcome as- } \\
\text { sessment (detection bias) } \\
\text { All outcomes }\end{array}$ & Low risk & Quote: "randomized double-blind controlled clinical trial...placebo" \\
\hline $\begin{array}{l}\text { Incomplete outcome data } \\
\text { (attrition bias) } \\
\text { All outcomes }\end{array}$ & Low risk & Comment: there were no post-randomisation dropouts \\
\hline $\begin{array}{l}\text { Selective reporting (re- } \\
\text { porting bias) }\end{array}$ & Low risk & $\begin{array}{l}\text { Comment: no previously published protocol was available; adverse events and } \\
\text { either mortality or fatty liver resolution or both were reported }\end{array}$ \\
\hline Other bias & Low risk & Comment: no other bias noted \\
\hline
\end{tabular}


Naganuma 2016

\section{Study characteristics}

\begin{tabular}{|c|c|}
\hline Methods & Randomised clinical trial \\
\hline Participants & $\begin{array}{l}\text { Country: Japan } \\
\text { Period of recruitment: not stated } \\
\text { Number randomised: } 20 \\
\text { Post-randomisation dropouts: not stated } \\
\text { Revised sample size: } 20 \\
\text { Average age, years: not stated } \\
\text { Females: } 20 \text { (100.0\%) } \\
\text { NASH: not stated } \\
\text { Diabetes mellitus: not stated } \\
\text { Inclusion criteria: } 1 \text {. NAFLD. } 2 \text {. ALT } \geq 40.3 \text {. Female } \\
\text { Exclusion criteria: not stated } \\
\text { Method for diagnosis of NAFLD: not stated }\end{array}$ \\
\hline Outcomes & $\begin{array}{l}\text { Outcomes reported: serious adverse events (number of people) } \\
\text { Follow-up, months: } 3\end{array}$ \\
\hline Notes & $\begin{array}{l}\text { Source of funding: not stated } \\
\text { Trial name/Trial registry number: not stated } \\
\text { Attempts were made to contact study authors in December } 2020\end{array}$ \\
\hline
\end{tabular}

\section{Risk of bias}

Bias Authors' judgement Support for judgement

Random sequence genera- Unclear risk Comment: this information was not available
tion (selection bias)

Allocation concealment Unclear risk Comment: this information was not available
(selection bias)

Blinding of participants Unclear risk Comment: this information was not available
and personnel (perfor-
mance bias)
All outcomes

\begin{tabular}{|c|c|c|}
\hline $\begin{array}{l}\text { Blinding of outcome as- } \\
\text { sessment (detection bias) } \\
\text { All outcomes }\end{array}$ & Unclear risk & Comment: this information was not available \\
\hline $\begin{array}{l}\text { Incomplete outcome data } \\
\text { (attrition bias) } \\
\text { All outcomes }\end{array}$ & Unclear risk & Comment: this information was not available \\
\hline $\begin{array}{l}\text { Selective reporting (re- } \\
\text { porting bias) }\end{array}$ & High risk & $\begin{array}{l}\text { Comment: no previously published protocol was available; neither mortality } \\
\text { nor fatty liver resolution was reported }\end{array}$ \\
\hline Other bias & Low risk & Comment: no other bias noted \\
\hline
\end{tabular}


Navekar 2017

\section{Study characteristics}

\begin{tabular}{ll}
\hline Methods & Randomised clinical trial \\
\hline Participants & Country: Iran \\
& Period of recruitment: not stated \\
& Number randomised: 46 \\
& Post-randomisation dropouts: 4 (8.7\%) \\
& Revised sample size: 42 \\
& Reasons for post-randomisation dropouts: personal reasons \\
& Average age, years: 41 \\
& Females: 24 (57.1\%) \\
& NASH: not stated \\
& Diabetes mellitus: 0 (0.0\%) \\
& Inclusion criteria: 1. Males aged 20 to 60 years, females aged 20 to 50 years. 2. BMI 24.9 to 40. 3. NAFLD \\
& Exclusion: 1 . Diabetes. 2. Alcohol consumption. 3. Liver transplantation. 4. Liver disorders (hepatitis B \\
& or C, liver infection, etc.). 5. Biliary disease or presence of gallstones. 6. Inherited disorders affecting liv- \\
& er (iron storage disease, etc.). 7. Autoimmune disease. 8 . Cancer. 9. Anaemia. 10. Medication (e.g. hypo- \\
& glycaemics, dietary supplements). \\
& Method for diagnosis of NAFLD: ultrasound
\end{tabular}

\begin{tabular}{ll}
\hline Interventions & Group 1: other supplements $(n=21)$ \\
& Further details: turmeric capsules $3 \mathrm{~g} / \mathrm{d}$ for 12 weeks \\
& Group 2: no active intervention $(\mathrm{n}=21)$ \\
& Further details: placebo
\end{tabular}

$\begin{array}{ll}\text { Outcomes } & \text { Outcomes reported: resolution of fatty liver disease } \\ & \text { Follow-up, months: } 3\end{array}$

\begin{tabular}{ll}
\hline Notes & Source of funding (quote): "we thank the Research Vice-Chancellor of Tabriz University of Medical \\
Sciences, Tabriz, Iran, for the financial support" \\
Trial name/Trial registry number: IRCT201406183664N12 \\
Attempts were made to contact study authors in December 2020
\end{tabular}

\section{Risk of bias}

\begin{tabular}{lll}
\hline Bias & Authors' judgement & Support for judgement \\
\hline $\begin{array}{l}\text { Random sequence genera- } \\
\text { tion (selection bias) }\end{array}$ & Unclear risk & Comment: this information was not available \\
\hline $\begin{array}{l}\text { Allocation concealment } \\
\text { (selection bias) }\end{array}$ & Unclear risk & Comment: this information was not available \\
\hline $\begin{array}{l}\text { Blinding of participants } \\
\text { and personnel (perfor- } \\
\text { mance bias) }\end{array}$ & Low risk & Quote: "double-blind randomized controlled clinical trial...placebo" \\
$\begin{array}{l}\text { All outcomes } \\
\text { Blinding of outcome as- } \\
\text { sessment (detection bias) } \\
\text { All outcomes }\end{array}$ & Low risk & \\
\hline $\begin{array}{l}\text { Incomplete outcome data } \\
\text { (attrition bias) } \\
\text { All outcomes }\end{array}$ & Unclear risk & $\begin{array}{l}\text { Comment: participants were excluded from analysis for reasons that may be } \\
\text { related to the intervention and to outcomes }\end{array}$ \\
\hline
\end{tabular}


Navekar 2017 (Continued)

Selective reporting (re- High risk Comment: no previously published protocol was available; adverse events porting bias) were not reported adequately

Other bias

Low risk

Comment: no other bias noted

NCT00816465

\section{Study characteristics}

\begin{tabular}{ll}
\hline Methods & Randomised clinical trial \\
\hline Participants & Country: Israel \\
& Period of recruitment: 2009 to 2010 \\
& Number randomised: 20 \\
& Post-randomisation dropouts: not stated \\
& Revised sample size: 20 \\
& Average age, years: not stated \\
& Females: not stated \\
& NASH: not stated \\
& Diabetes mellitus: not stated \\
& Inclusion criteria: 1 . Aged 18 to 65 years. 2. Biopsy-proven NASH with score $\geq 4$. 3. Altered glucose me- \\
& tabolism, including diabetes (non-treated, or treated with up to 2 drugs (not including insulin) without \\
any change in medication 2 months before enrolment), impaired fasting glucose or impaired glucose \\
tolerance, HbA1C between $5.5 \%$ and $14 \%$ \\
Exclusion criteria: not stated \\
Method for diagnosis of NAFLD: liver biopsy \\
\hline
\end{tabular}

Interventions

Group 1: other supplements ( $\mathrm{n}=$ not stated)

Further details: oral administration of 1 tablet of Hoodia gordoni extract per day

Group 2: no active intervention ( $n=$ not stated)

Further details: oral placebo pill

\begin{tabular}{ll}
\hline Outcomes & No outcomes of interest were reported \\
\hline \multirow{2}{*}{ Notes } & Source of funding: not stated \\
& Trial name/Trial registry number: NCT00816465 \\
& Attempts were made to contact study authors in December 2020
\end{tabular}

\section{Risk of bias}

\begin{tabular}{lll}
\hline Bias & Authors' judgement & Support for judgement \\
\hline $\begin{array}{l}\text { Random sequence genera- } \\
\text { tion (selection bias) }\end{array}$ & Unclear risk & Comment: this information was not available \\
\hline $\begin{array}{l}\text { Allocation concealment } \\
\text { (selection bias) }\end{array}$ & Unclear risk & Comment: this information was not available \\
\hline
\end{tabular}

\begin{tabular}{|c|c|c|}
\hline $\begin{array}{l}\text { Blinding of participants } \\
\text { and personnel (perfor- }\end{array}$ & Low risk & $\begin{array}{l}\text { Quote: "masking: quadruple (participant, care provider, investigator, out- } \\
\text { comes assessor)... placebo" }\end{array}$ \\
\hline
\end{tabular}

mance bias)

All outcomes

\begin{tabular}{|c|c|c|}
\hline $\begin{array}{l}\text { Blinding of outcome as- } \\
\text { sessment (detection bias) }\end{array}$ & Low risk & $\begin{array}{l}\text { Quote: "masking: quadruple (participant, care provider, investigator, out- } \\
\text { comes assessor)...placebo" }\end{array}$ \\
\hline
\end{tabular}




\section{NCT00816465 (Continued)}

Incomplete outcome data Unclear risk Comment: this information was not available (attrition bias)

All outcomes

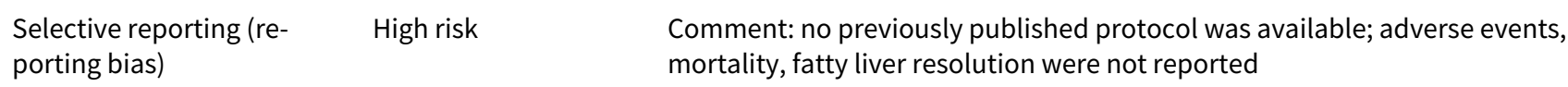

Other bias Low risk Comment: no other bias noted

\section{Study characteristics}

\begin{tabular}{|c|c|}
\hline Methods & Randomised clinical trial \\
\hline Participants & $\begin{array}{l}\text { Country: USA } \\
\text { Period of recruitment: } 2006 \text { to } 2009 \\
\text { Number randomised: } 9 \\
\text { Post-randomisation dropouts: } 0 \text { (0.0\%) } \\
\text { Revised sample size: } 9 \\
\text { Average age, years: not stated } \\
\text { Females: } 4 \text { (44.4\%) } \\
\text { NASH: } 9 \text { (100.0\%) } \\
\text { Diabetes mellitus: not stated } \\
\text { Inclusion criteria: } 1 . \geq 18 \text { years old. } 2 \text {. NASH on liver biopsy within } 6 \text { months. 3. Laboratory parameters } \\
\text { of decompensated liver disease (bilirubin }<2 \text { mg/dL, stable normal albumin, prothrombin time }<3 \text { sec- } \\
\text { onds prolonged). } 4 \text {. Serum creatinine }<1.5 \times \text { upper limit of normal. } 5 \text {. Diabetic patients stable on oral } \\
\text { meds or with }<10 \% \text { change in insulin dose over past } 2 \text { months. } 6 \text {. Normal TSH or FTI. } 7 \text {. Hep C antibody } \\
\text { negative, HBsAg seronegative, ANA }<1: 320 \\
\text { Exclusion criteria: } 1 \text {. Alcohol use exceeding } 10 \text { to } 20 \mathrm{~g} / \mathrm{d} \text { during past } 6 \text { months. 2. Other cause of liv- } \\
\text { er disease, cirrhosis. } 3 \text {. Use of medication associated with NASH within } 6 \text { months. } 5 \text {. Use of NSAIDs, fi- } \\
\text { brates, or warfarin within } 1 \text { month. } 6 \text {. Uncontrolled diabetes, insulin-dependent diabetes. } 7 \text {. History of } \\
\text { small bowel resection. } 8 \text {. Substance abuse within past } 6 \text { months. } 9 \text {. Chemotherapy within } 6 \text { months. } 10 . \\
\text { Taking metformin. } 11 \text {. Unstable thyroid function. } 12 \text {. Pregnancy, breastfeeding. } 13 \text {. Transplant recipi- } \\
\text { ent. } 14 \text {. Use of oral supplements of vitamin E within } 1 \text { month } \\
\text { Method for diagnosis of NAFLD: liver biopsy }\end{array}$ \\
\hline
\end{tabular}

Interventions Group 1: PUFA $(\mathrm{n}=3)$

Further details: participants received $4 \mathrm{mg}$ daily of omega-3-acid ethyl esters (Lovaza) for 24 weeks Group 2: no active intervention $(n=6)$

Further details: participants received daily placebo for 24 weeks

Additional details: both groups received dietary advice

Outcomes Outcomes reported: serious adverse events (number of people), serious adverse events (number of events), adverse events (number of people), adverse events (number of events)

Follow-up, months: 6

Notes Source of funding: not stated

Trial name/Trial registry number: NCT00845845

Attempts were made to contact study authors in December 2020

\section{Risk of bias}

Bias Authors' judgement Support for judgement


NCT00845845 (Continued)

Random sequence genera- Unclear risk Comment: this information was not available tion (selection bias)

Allocation concealment $\quad$ Unclear risk $\quad$ Comment: this information was not available
(selection bias)

Blinding of participants Low risk Quote: "study design - masking: quadruple...placebo"

and personnel (perfor-

mance bias)

All outcomes

\begin{tabular}{lll}
\hline $\begin{array}{l}\text { Blinding of outcome as- } \\
\text { sessment (detection bias) } \\
\text { All outcomes }\end{array}$ & Low risk & Quote: "study design - masking: quadruple...placebo" \\
\hline $\begin{array}{l}\text { Incomplete outcome data } \\
\text { (attrition bias) }\end{array}$ & Low risk & $\begin{array}{l}\text { Comment: although some participants were lost to follow-up, all randomised } \\
\text { participants were included in the analysis }\end{array}$ \\
\hline
\end{tabular}

All outcomes

$\begin{array}{lll}\begin{array}{l}\text { Selective reporting (re- } \\ \text { porting bias) }\end{array} & \text { High risk } & \begin{array}{l}\text { Comment: a published protocol was available, but recruitment had com- } \\ \text { menced before the protocol was published; neither mortality nor fatty liver }\end{array}\end{array}$

$\begin{array}{lll}\begin{array}{l}\text { Selective reporting (re- } \\ \text { porting bias) }\end{array} & \text { High risk } & \begin{array}{l}\text { Comment: a published protocol was available, but recruitment had com- } \\ \text { menced before the protocol was published; neither mortality nor fatty liver }\end{array}\end{array}$ porting bias) resolution was reported

Other bias Low risk Comment: no other bias noted

NCT00941642

\section{Study characteristics}

\begin{tabular}{|c|c|}
\hline Methods & Randomised clinical trial \\
\hline Participants & $\begin{array}{l}\text { Country: USA } \\
\text { Period of recruitment: not stated } \\
\text { Number randomised: } 40 \\
\text { Post-randomisation dropouts: not stated } \\
\text { Revised sample size: } 40 \\
\text { Average age, years: not stated } \\
\text { Females: not stated } \\
\text { NASH: not stated } \\
\text { Diabetes mellitus: not stated } \\
\text { Inclusion criteria: } 1 \text {. Aged > } 18 \text { years. 2. Abnormal liver enzymes 3. NAFLD or NASH on biopsy } \\
\text { Exclusion criteria: not stated } \\
\text { Method for diagnosis of NAFLD: liver biopsy }\end{array}$ \\
\hline Interventions & $\begin{array}{l}\text { Group 1: no active intervention ( } \mathrm{n}=\text { not stated) } \\
\text { Further details: placebo } \\
\text { Group 2: PUFA ( } \mathrm{n}=\text { not stated) } \\
\text { Further details: Lovaza (fish oil supplement) } 4 \mathrm{~g} \text { daily for minimum } 48 \text { weeks }\end{array}$ \\
\hline Outcomes & No outcomes of interest were reported \\
\hline Notes & $\begin{array}{l}\text { Source of funding: not stated } \\
\text { Trial name/Trial registry number: NCT00941642 } \\
\text { Attempts were made to contact study authors in December } 2020\end{array}$ \\
\hline
\end{tabular}

\section{Risk of bias}

Nutritional supplementation for nonalcohol-related fatty liver disease: a network meta-analysis (Review) 
NCT00941642 (Continued)

\section{Bias Authors' judgement Support for judgement}

Random sequence genera- Unclear risk Comment: this information was not available

tion (selection bias)

Allocation concealment $\quad$ Unclear risk Comment: this information was not available
(selection bias)

(selection bias)

Blinding of participants $\quad$ High risk Quote: "masking: single (participant)"
and personnel (perfor-
mance bias)
All outcomes

\begin{tabular}{|c|c|c|}
\hline $\begin{array}{l}\text { Blinding of outcome as- } \\
\text { sessment (detection bias) } \\
\text { All outcomes }\end{array}$ & High risk & Quote: "masking: single (participant)" \\
\hline $\begin{array}{l}\text { Incomplete outcome data } \\
\text { (attrition bias) } \\
\text { All outcomes }\end{array}$ & Unclear risk & Comment: this information was not available \\
\hline $\begin{array}{l}\text { Selective reporting (re- } \\
\text { porting bias) }\end{array}$ & High risk & $\begin{array}{l}\text { Comment: no previously published protocol was available; adverse events, } \\
\text { mortality, fatty liver resolution were not reported }\end{array}$ \\
\hline Other bias & Low risk & Comment: no other bias noted \\
\hline
\end{tabular}

NCT00977730

\section{Study characteristics}

\begin{tabular}{|c|c|}
\hline Methods & Randomised clinical trial \\
\hline Participants & 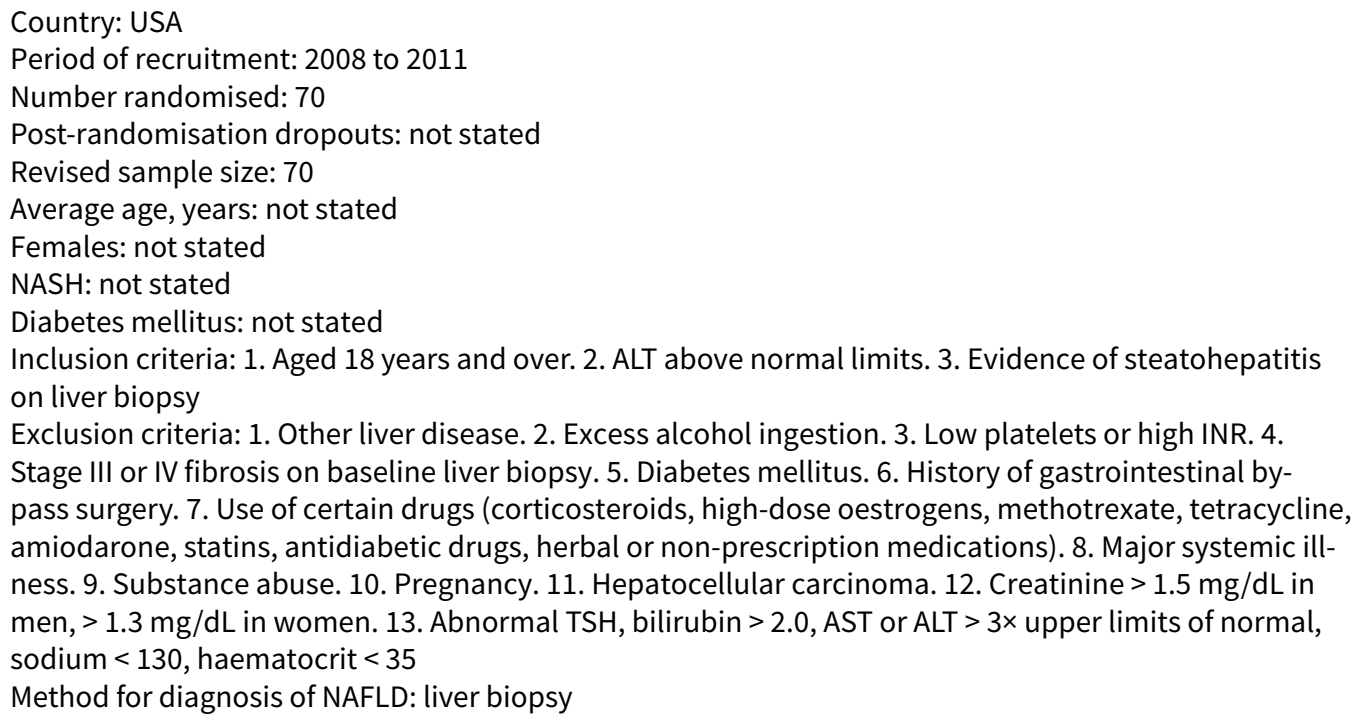 \\
\hline
\end{tabular}

Interventions

Group 1: other antioxidants plus other supplements ( $\mathrm{n}=$ not stated)

Further details: $1 \times 675$-mg capsule Protandim per day for 12 months. Protandim is a nutritional supplement composed of the following 5 botanical extracts: Bacopa Moniera extract, Milk Thistle extract, Ashwagandha powder, Green tea, and Turmeric extract 
NCT00977730 (Continued)

Group 2: no active intervention ( $\mathrm{n}=$ not stated)

Further details: 1 sugar pill per day

\begin{tabular}{ll}
\hline Outcomes & No outcomes of interest were reported \\
\hline Notes & Source of funding: not stated \\
& Trial name/Trial registry number: NCT00977730 \\
& Attempts were made to contact study authors in December 2020
\end{tabular}

\section{Risk of bias}

\begin{tabular}{lll}
\hline Bias & Authors' judgement & Support for judgement \\
\hline $\begin{array}{l}\text { Random sequence genera- } \\
\text { tion (selection bias) }\end{array}$ & Unclear risk & Comment: this information was not available \\
\hline $\begin{array}{l}\text { Allocation concealment } \\
\text { (selection bias) }\end{array}$ & Unclear risk & Comment: this information was not available \\
\hline $\begin{array}{l}\text { Blinding of participants } \\
\begin{array}{l}\text { and personnel (perfor- } \\
\text { mance bias) }\end{array}\end{array}$ & Low risk & $\begin{array}{l}\text { Quote: "masking: triple (participant, investigator, outcomes assessor)...place- } \\
\text { bo" }\end{array}$ \\
All outcomes & & \\
\hline
\end{tabular}

\begin{tabular}{|c|c|c|}
\hline $\begin{array}{l}\text { Blinding of outcome as- } \\
\text { sessment (detection bias) }\end{array}$ & Low risk & $\begin{array}{l}\text { Quote: "masking: triple (participant, investigator, outcomes assessor)...place- } \\
\text { bo" }\end{array}$ \\
\hline
\end{tabular}

\begin{tabular}{lll}
\hline $\begin{array}{l}\text { Incomplete outcome data } \\
\text { (attrition bias) } \\
\text { All outcomes }\end{array}$ & Unclear risk & Comment: this information was not available \\
\hline $\begin{array}{l}\text { Selective reporting (re- } \\
\text { porting bias) }\end{array}$ & High risk & $\begin{array}{l}\text { Comment: no previously published protocol was available; adverse events, } \\
\text { mortality, fatty liver resolution were not reported }\end{array}$ \\
\hline Other bias & Low risk & Comment: no other bias noted \\
\hline
\end{tabular}

\section{Study characteristics}

\begin{tabular}{ll}
\hline Methods & Randomised clinical trial \\
\hline Participants & Country: Israel \\
Period of recruitment: not stated \\
Number randomised: not stated \\
Post-randomisation dropouts: not stated \\
Revised sample size: 0 \\
Average age, years: not stated \\
Females: not stated \\
NASH: not stated \\
Diabetes mellitus: not stated \\
Inclusion criteria: 1 . Aged 18 to 65 years. 2. Men and women with NAFLD per USS. 3. Increased ALT level. \\
4. Hepatomegaly. 5 . Liver biopsy within 2 years \\
Exclusion criteria: 1. Other liver disease (HBV, HCV). 2. Hepatocellular carcinoma. 3. Decompensated liv- \\
er disease. 4. Use of steroids
\end{tabular}


NCT01083992 (Continued)

Method for diagnosis of NAFLD: liver biopsy plus transaminases plus ultrasound

$\begin{array}{ll}\text { Interventions } & \text { Group 1: no active intervention ( } \mathrm{n}=\text { not stated) } \\ & \text { Further details: no intervention } \\ & \text { Group 2: vitamin D ( } \mathrm{n}=\text { not stated) } \\ & \text { Further details: vitamin D (no further details) } \\ & \text { Additional details: both groups received vitagliptin }\end{array}$

\begin{tabular}{ll}
\hline Outcomes & No outcomes of interest were reported \\
\hline Notes & Source of funding: not stated \\
& Trial name/Trial registry number: not stated \\
& Attempts were made to contact study authors in December 2020
\end{tabular}

\section{Risk of bias}

\begin{tabular}{|c|c|c|}
\hline Bias & Authors' judgement & Support for judgement \\
\hline $\begin{array}{l}\text { Random sequence genera- } \\
\text { tion (selection bias) }\end{array}$ & Unclear risk & Comment: this information was not available \\
\hline $\begin{array}{l}\text { Allocation concealment } \\
\text { (selection bias) }\end{array}$ & Unclear risk & Comment: this information was not available \\
\hline $\begin{array}{l}\text { Blinding of participants } \\
\text { and personnel (perfor- } \\
\text { mance bias) } \\
\text { All outcomes }\end{array}$ & High risk & Quote: "open label" \\
\hline $\begin{array}{l}\text { Blinding of outcome as- } \\
\text { sessment (detection bias) } \\
\text { All outcomes }\end{array}$ & High risk & Quote: "open label" \\
\hline $\begin{array}{l}\text { Incomplete outcome data } \\
\text { (attrition bias) } \\
\text { All outcomes }\end{array}$ & Unclear risk & Comment: this information was not available \\
\hline $\begin{array}{l}\text { Selective reporting (re- } \\
\text { porting bias) }\end{array}$ & High risk & $\begin{array}{l}\text { Comment: no previously published protocol was available; adverse events, } \\
\text { mortality, fatty liver resolution were not reported }\end{array}$ \\
\hline Other bias & Low risk & Comment: no other bias noted \\
\hline
\end{tabular}

NCT01623024

\section{Study characteristics}

\begin{tabular}{ll}
\hline Methods & Randomised clinical trial \\
\hline Participants & Country: Italy \\
& Period of recruitment: not stated \\
& Number randomised: 200 \\
& Post-randomisation dropouts: not stated \\
& Revised sample size: 200 \\
& Average age, years: not stated \\
& Females: not stated \\
& NASH: 200 (100.0\%)
\end{tabular}


NCT01623024 (Continued)

Diabetes mellitus: not stated

Inclusion criteria: 1 . Aged > 18 years. 2. Histological diagnosis of possible or definitive NASH according

to Kleiner score within 6 months

Exclusion criteria: not stated

Method for diagnosis of NAFLD: liver biopsy

\begin{tabular}{ll}
\hline Interventions & Group 1: no active intervention ( $\mathrm{n}=$ not stated) \\
& Further details: only lifestyle advice \\
& Group 2: vitamin $\mathrm{D}(\mathrm{n}=\mathrm{not}$ stated) \\
& Further details: vitamin $\mathrm{D} 20,000 \mathrm{IU} /$ week for 96 weeks \\
& Additional details: both groups received lifestyle advice
\end{tabular}

\begin{tabular}{ll}
\hline Outcomes & No outcomes of interest were reported \\
\hline Notes & Source of funding: not stated \\
& Trial name/Trial registry number: NCT01623024 \\
& Attempts were made to contact study authors in December 2020
\end{tabular}

\section{Risk of bias}

\begin{tabular}{|c|c|c|}
\hline Bias & Authors' judgement & Support for judgement \\
\hline $\begin{array}{l}\text { Random sequence genera- } \\
\text { tion (selection bias) }\end{array}$ & Unclear risk & Comment: this information was not available \\
\hline $\begin{array}{l}\text { Allocation concealment } \\
\text { (selection bias) }\end{array}$ & Unclear risk & Comment: this information was not available \\
\hline $\begin{array}{l}\text { Blinding of participants } \\
\text { and personnel (perfor- } \\
\text { mance bias) } \\
\text { All outcomes }\end{array}$ & High risk & Quote: "open label" \\
\hline $\begin{array}{l}\text { Blinding of outcome as- } \\
\text { sessment (detection bias) } \\
\text { All outcomes }\end{array}$ & High risk & Quote: "open label" \\
\hline $\begin{array}{l}\text { Incomplete outcome data } \\
\text { (attrition bias) } \\
\text { All outcomes }\end{array}$ & Unclear risk & Comment: this information was not available \\
\hline $\begin{array}{l}\text { Selective reporting (re- } \\
\text { porting bias) }\end{array}$ & High risk & $\begin{array}{l}\text { Comment: no previously published protocol was available; adverse events, } \\
\text { mortality, fatty liver resolution were not reported }\end{array}$ \\
\hline Other bias & Low risk & Comment: no other bias noted \\
\hline
\end{tabular}

NCT02690792

\section{Study characteristics}

\begin{tabular}{ll}
\hline Methods & Randomised clinical trial \\
\hline Participants & Country: USA \\
& Period of recruitment: 2009 to 2016 \\
& Number randomised: 70 \\
& Post-randomisation dropouts: not stated
\end{tabular}


NCT02690792 (Continued)

Revised sample size: 70

Average age, years: not stated

Females: not stated

NASH: not stated

Diabetes mellitus: not stated

Inclusion and exclusion criteria: not stated

Method for diagnosis of NAFLD: not stated

\begin{tabular}{ll}
\hline Interventions & Group 1: vitamin E ( $=$ not stated) \\
& Further details: vitamin E 200 IU/capsule; 2 capsules each mornin \\
& months \\
& Group 2: no active intervention ( $\mathrm{n}=$ not stated) \\
& Further details: placebo \\
\hline Outcomes & No outcomes of interest were reported \\
\hline Notes & Source of funding: not stated \\
& $\begin{array}{l}\text { Trial name/Trial registry number: NCT02690792 } \\
\text { Attempts were made to contact study authors in December } 2020\end{array}$
\end{tabular}

\section{Risk of bias}

\begin{tabular}{|c|c|c|}
\hline Bias & Authors' judgement & Support for judgement \\
\hline $\begin{array}{l}\text { Random sequence genera- } \\
\text { tion (selection bias) }\end{array}$ & Unclear risk & Comment: this information was not available \\
\hline $\begin{array}{l}\text { Allocation concealment } \\
\text { (selection bias) }\end{array}$ & Unclear risk & Comment: this information was not available \\
\hline $\begin{array}{l}\text { Blinding of participants } \\
\text { and personnel (perfor- } \\
\text { mance bias) } \\
\text { All outcomes }\end{array}$ & Low risk & $\begin{array}{l}\text { Quote: "masking: quadruple (participant, care provider, investigator, out- } \\
\text { comes assessor)...placebo" }\end{array}$ \\
\hline $\begin{array}{l}\text { Blinding of outcome as- } \\
\text { sessment (detection bias) } \\
\text { All outcomes }\end{array}$ & Low risk & $\begin{array}{l}\text { Quote: "masking: quadruple (participant, care provider, investigator, out- } \\
\text { comes assessor)...placebo" }\end{array}$ \\
\hline $\begin{array}{l}\text { Incomplete outcome data } \\
\text { (attrition bias) } \\
\text { All outcomes }\end{array}$ & Unclear risk & Comment: this information was not available \\
\hline $\begin{array}{l}\text { Selective reporting (re- } \\
\text { porting bias) }\end{array}$ & High risk & $\begin{array}{l}\text { Comment: no previously published protocol was available; adverse events, } \\
\text { mortality, fatty liver resolution were not reported }\end{array}$ \\
\hline Other bias & Low risk & Comment: no other bias noted \\
\hline
\end{tabular}

NCT04411862

\section{Study characteristics}

\begin{tabular}{ll}
\hline Methods & Randomised clinical trial \\
\hline Participants & $\begin{array}{l}\text { Country: Egypt } \\
\text { Period of recruitment: } 2016 \text { to } 2019\end{array}$ \\
\hline
\end{tabular}


NCT04411862 (Continued)

Number randomised: 100

Post-randomisation dropouts: not stated

Revised sample size: 100

Average age, years: not stated

Females: not stated

NASH: not stated

Diabetes mellitus: not stated

Inclusion criteria: 1. Fatty liver upon USS/CT/MRI. 2. Increased alanine aminotransferase (ALT). 3. Homeostasis model assessment-insulin resistance (HOMA IR) score $>3$. 4. Presence of liver steatosis or stiffness measured by transient elastography. 5. $\geq 1$ of the following metabolic comorbidities: hypertension, type 2 diabetes mellitus, overweight/obesity $\left(\mathrm{BMI}>27 \mathrm{~kg} / \mathrm{m}^{2}\right)$, serum cholesterol $>200 \mathrm{mg} / \mathrm{dL}$ Exclusion criteria: 1. Evidence of alcoholic or chronic liver disease. 2. Hepatocellular carcinoma. 3. Autoimmune hepatitis. 4. End-stage liver disease. 5. Treatment with other hepatoprotectants. 6. Other concomitant EPL within 30 days. 7. Pregnancy or lactation Method for diagnosis of NAFLD: ultrasound, or CT, or MRI

Interventions

Group 1: phospholipids $(\mathrm{n}=50)$

Further details: dietary supplement: $2.1 \mathrm{~g}$ phosphatidylcholine daily

Group 2: no active intervention $(n=50)$

Further details: no additional dietary supplement

Additional details: both groups received lifestyle behavioural modification and health education by a clinical pharmacist

\begin{tabular}{ll}
\hline Outcomes & No outcomes of interest were reported \\
\hline Notes & Source of funding: not stated \\
& Trial name/Trial registry number: NCT04411862 \\
& Attempts were made to contact study authors in April 2021
\end{tabular}

\section{Risk of bias}

\begin{tabular}{|c|c|c|}
\hline Bias & Authors' judgement & Support for judgement \\
\hline $\begin{array}{l}\text { Random sequence genera- } \\
\text { tion (selection bias) }\end{array}$ & Unclear risk & Comment: this information was not available \\
\hline $\begin{array}{l}\text { Allocation concealment } \\
\text { (selection bias) }\end{array}$ & Unclear risk & Comment: this information was not available \\
\hline $\begin{array}{l}\text { Blinding of participants } \\
\text { and personnel (perfor- } \\
\text { mance bias) } \\
\text { All outcomes }\end{array}$ & High risk & Quote: "open label" \\
\hline $\begin{array}{l}\text { Blinding of outcome as- } \\
\text { sessment (detection bias) } \\
\text { All outcomes }\end{array}$ & High risk & Quote: "open label" \\
\hline $\begin{array}{l}\text { Incomplete outcome data } \\
\text { (attrition bias) } \\
\text { All outcomes }\end{array}$ & Unclear risk & Comment: this information was not available \\
\hline $\begin{array}{l}\text { Selective reporting (re- } \\
\text { porting bias) }\end{array}$ & High risk & $\begin{array}{l}\text { Comment: no previously published protocol was available; adverse events, } \\
\text { mortality, fatty liver resolution were not reported }\end{array}$ \\
\hline Other bias & Low risk & Comment: no other bias noted \\
\hline
\end{tabular}


Nelson 2009

\section{Study characteristics}

\begin{tabular}{|c|c|}
\hline Methods & Randomised clinical trial \\
\hline Participants & $\begin{array}{l}\text { Country: not stated } \\
\text { Period of recruitment: not stated } \\
\text { Number randomised: } 15 \\
\text { Post-randomisation dropouts: not stated } \\
\text { Revised sample size: } 15 \\
\text { Average age, years: not stated } \\
\text { Females: not stated } \\
\text { NASH: not stated } \\
\text { Diabetes mellitus: not stated } \\
\text { Inclusion and exclusion criteria: not stated } \\
\text { Method for diagnosis of NAFLD: not stated }\end{array}$ \\
\hline Interventions & $\begin{array}{l}\text { Group 1: MUFA ( } n=\text { not stated) } \\
\text { Further details: monounsaturated fatty acids (MUFA; i.e. safflower oil) for } 8 \text { weeks } \\
\text { Group 2: PUFA ( } n=\text { not stated) } \\
\text { Further details: omega-3 polyunsaturated fatty acids (PUFA; i.e. fish oil) for } 8 \text { weeks }\end{array}$ \\
\hline Outcomes & No outcomes of interest were reported \\
\hline Notes & $\begin{array}{l}\text { Source of funding: not stated } \\
\text { Trial name/Trial registry number: not stated } \\
\text { Attempts were made to contact study authors in December } 2020\end{array}$ \\
\hline
\end{tabular}

\section{Risk of bias}

\begin{tabular}{|c|c|c|}
\hline Bias & Authors' judgement & Support for judgement \\
\hline $\begin{array}{l}\text { Random sequence genera- } \\
\text { tion (selection bias) }\end{array}$ & Unclear risk & Comment: this information was not available \\
\hline $\begin{array}{l}\text { Allocation concealment } \\
\text { (selection bias) }\end{array}$ & Unclear risk & Comment: this information was not available \\
\hline $\begin{array}{l}\text { Blinding of participants } \\
\text { and personnel (perfor- } \\
\text { mance bias) } \\
\text { All outcomes }\end{array}$ & Unclear risk & Comment: this information was not available \\
\hline $\begin{array}{l}\text { Blinding of outcome as- } \\
\text { sessment (detection bias) } \\
\text { All outcomes }\end{array}$ & Unclear risk & Comment: this information was not available \\
\hline $\begin{array}{l}\text { Incomplete outcome data } \\
\text { (attrition bias) } \\
\text { All outcomes }\end{array}$ & Unclear risk & Comment: this information was not available \\
\hline $\begin{array}{l}\text { Selective reporting (re- } \\
\text { porting bias) }\end{array}$ & High risk & $\begin{array}{l}\text { Comment: no previously published protocol was available; adverse events, } \\
\text { mortality, fatty liver resolution were not reported }\end{array}$ \\
\hline Other bias & Low risk & Comment: no other bias noted \\
\hline
\end{tabular}


Nobili 2006

\section{Study characteristics}

\begin{tabular}{|c|c|}
\hline Methods & Randomised clinical trial \\
\hline Participants & $\begin{array}{l}\text { Country: Italy } \\
\text { Period of recruitment: } 2003 \text { to } 2005 \\
\text { Number randomised: } 90 \\
\text { Post-randomisation dropouts: } 2 \text { (2.2\%) } \\
\text { Revised sample size: } 88 \\
\text { Reasons for post-randomisation dropouts: lost to follow-up } \\
\text { Average age, years: } 12 \\
\text { Females: } 60 \text { (68.2\%) } \\
\text { NASH: not stated } \\
\text { Diabetes mellitus: not stated } \\
\text { Inclusion criteria: } 1 \text {. Otherwise healthy biopsy-proven NAFLD children. 2. Aged } 3 \text { to } 18 \text { years } \\
\text { Exclusion criteria: } 1 \text {. Hepatic virus infection. } 2 \text {. Alcohol consumption. 3. Parenteral nutrition. } 4 \text {. Steato- } \\
\text { sis-inducing drugs. } 5 \text {. Other known chronic liver disease } \\
\text { Method for diagnosis of NAFLD: liver biopsy }\end{array}$ \\
\hline Interventions & $\begin{array}{l}\text { Group 1: vitamin E plus vitamin C }(n=45) \\
\text { Further details: alpha-tocopherol } 600 \mathrm{IU} / \mathrm{d} \text { plus ascorbic acid } 500 \mathrm{mg} / \mathrm{d} \text { for } 12 \text { months } \\
\text { Group 2: no active intervention }(\mathrm{n}=43) \\
\text { Further details: placebo } \\
\text { Additional details: both groups received balanced calorie diet and physical exercise }\end{array}$ \\
\hline Outcomes & $\begin{array}{l}\text { Outcomes reported: serious adverse events (number of people), any adverse events (number of peo- } \\
\text { ple), resolution of fatty liver disease } \\
\text { Follow-up, months: } 12\end{array}$ \\
\hline Notes & $\begin{array}{l}\text { Source of funding: not stated } \\
\text { Trial name/Trial registry number: not stated } \\
\text { Attempts were made to contact study authors in December } 2020\end{array}$ \\
\hline
\end{tabular}

\section{Risk of bias}

\begin{tabular}{|c|c|c|}
\hline Bias & Authors' judgement & Support for judgement \\
\hline $\begin{array}{l}\text { Random sequence genera- } \\
\text { tion (selection bias) }\end{array}$ & Unclear risk & Comment: this information was not available \\
\hline $\begin{array}{l}\text { Allocation concealment } \\
\text { (selection bias) }\end{array}$ & Low risk & $\begin{array}{l}\text { Quote: "double-blind placebo study" } \\
\text { Comment: allocation concealment and blinding were achieved with use of } \\
\text { placebo }\end{array}$ \\
\hline $\begin{array}{l}\text { Blinding of participants } \\
\text { and personnel (perfor- } \\
\text { mance bias) } \\
\text { All outcomes }\end{array}$ & Low risk & Quote: "double-blind placebo study" \\
\hline $\begin{array}{l}\text { Blinding of outcome as- } \\
\text { sessment (detection bias) } \\
\text { All outcomes }\end{array}$ & Low risk & Quote: "double-blind placebo study" \\
\hline $\begin{array}{l}\text { Incomplete outcome data } \\
\text { (attrition bias) } \\
\text { All outcomes }\end{array}$ & Unclear risk & $\begin{array}{l}\text { Comment: participants were excluded from analysis for reasons that may be } \\
\text { related to the intervention and to outcomes }\end{array}$ \\
\hline
\end{tabular}


Nobili 2006 (Continued)

Selective reporting (re- Low risk Comment: no previously published protocol was available; adverse events and porting bias)

Other bias Low risk Comment: no other bias noted

Nobili 2013

\section{Study characteristics}

\begin{tabular}{|c|c|c|}
\hline Methods & \multicolumn{2}{|c|}{ Randomised clinical trial } \\
\hline Participants & \multicolumn{2}{|c|}{$\begin{array}{l}\text { Country: Italy } \\
\text { Period of recruitment: not stated } \\
\text { Number randomised: } 60 \\
\text { Post-randomisation dropouts: } 0(0.0 \%) \\
\text { Revised sample size: } 60 \\
\text { Average age, years: } 12 \\
\text { Females: } 35(58.3 \%) \\
\text { NASH: not stated } \\
\text { Diabetes mellitus: not stated } \\
\text { Inclusion criteria: } 1 \text {. Aged < } 18 \text { years. 2. Persistently elevated ALT } \geq 40 \text {. 3. Diffusely hyperechogenic liver. } \\
4 \text {. Liver biopsy consistent with NAFLD } \\
\text { Exclusion criteria: } 1 \text {. Viral liver disease. } 2 \text {. Autoimmune liver disease. 3. Wilson's disease. 4. } a \text {-1-antit- } \\
\text { rypsin deficiency. } 5 \text {. Coeliac disease. } 6 \text {. Alcohol consumption. } 7 \text {. Parenteral nutrition. 8. Use of drugs } \\
\text { known to induce fatty liver. } 9 \text {. Previous use of N3-LCPUFA } \\
\text { Method for diagnosis of NAFLD: ultrasound plus liver biopsy }\end{array}$} \\
\hline Interventions & \multicolumn{2}{|c|}{$\begin{array}{l}\text { Group 1: PUFA ( } \mathrm{n}=40) \\
\text { Further details: DHA supplementation }(250 \mathrm{mg} / \mathrm{d} \text { and } 500 \mathrm{mg} / \mathrm{d}) \text { for } 24 \text { months } \\
\text { Group 2: no active intervention }(\mathrm{n}=20) \\
\text { Further details: placebo } \\
\text { Additional details: both groups received lifestyle advice }\end{array}$} \\
\hline Outcomes & \multicolumn{2}{|c|}{$\begin{array}{l}\text { Outcomes reported: mortality at maximal follow-up, resolution of fatty liver disease } \\
\text { Follow-up, months: } 24\end{array}$} \\
\hline Notes & \multicolumn{2}{|c|}{$\begin{array}{l}\text { Source of funding (quote): "the study was supported by the 'Bambino Gesù' Children Hospital" } \\
\text { Trial name/Trial registry number: NCT0885313 } \\
\text { Attempts were made to contact study authors in December } 2020\end{array}$} \\
\hline \multicolumn{3}{|l|}{ Risk of bias } \\
\hline Bias & Authors' judgement & Support for judgement \\
\hline $\begin{array}{l}\text { Random sequence genera- } \\
\text { tion (selection bias) }\end{array}$ & Low risk & Quote: "computer-generated randomization list" \\
\hline $\begin{array}{l}\text { Allocation concealment } \\
\text { (selection bias) }\end{array}$ & Low risk & $\begin{array}{l}\text { Quote: "a statistician, who did not perform the final analysis, generated the al- } \\
\text { location sequence and assigned participants to the treatment groups" }\end{array}$ \\
\hline $\begin{array}{l}\text { Blinding of participants } \\
\text { and personnel (perfor- } \\
\text { mance bias) } \\
\text { All outcomes }\end{array}$ & Low risk & $\begin{array}{l}\text { Quote: "participants, investigators and outcome assessors were blinded to the } \\
\text { treatment for all the duration of the study...placebo" }\end{array}$ \\
\hline
\end{tabular}


Nobili 2013 (Continued)

Blinding of outcome as- Low risk Quote: "participants, investigators and outcome assessors were blinded to the sessment (detection bias) treatment for all the duration of the study...placebo"

All outcomes

Incomplete outcome data Low risk Comment: there were no post-randomisation dropouts
(attrition bias)

(attrition bias)

All outcomes

$\begin{array}{lll}\begin{array}{l}\text { Selective reporting (re- } \\ \text { porting bias) }\end{array} & \text { High risk } & \begin{array}{l}\text { Comment: no previously published protocol was available; adverse events } \\ \text { were not reported adequately }\end{array}\end{array}$

Other bias Low risk Comment: no other bias noted

\section{Nogueira 2016}

\section{Study characteristics}

\begin{tabular}{ll}
\hline Methods & Randomised clinical trial \\
\hline Participants & Country: Brazil \\
& Period of recruitment: not stated \\
& Number randomised: 60 \\
& Post-randomisation dropouts: 10 (16.7\%) \\
& Revised sample size: 50 \\
& Reasons for post-randomisation dropouts: lost to follow-up \\
& Average age, years: 53 \\
& Females: 49 (98.0\%) \\
& NASH: 60 (120.0\%) \\
& Diabetes mellitus: not stated \\
& Inclusion criteria: 1. Proven histological diagnosis of NASH \\
& Exclusion criteria: 1 . Other chronic liver disease. 2. Substance abuse. 3. Use of hepatotoxic drugs. 4. Al- \\
& lergy or food intolerance \\
& Method for diagnosis of NAFLD: liver biopsy \\
\hline
\end{tabular}

\begin{tabular}{ll}
\hline Interventions & Group 1: PUFA ( $n=27)$ \\
& Further details: 3 capsules of $0.315 \mathrm{~g}$ omega-3 PUFAs (64\% alphalinolenic (ALA), 16\% eicosapentaenoic \\
& (EPA), and 21\% docosahexaenoic (DHA) acids) per day for 6 months \\
& Group 2: no active intervention $(\mathrm{n}=23)$ \\
& Further details: placebo
\end{tabular}

\begin{tabular}{ll}
\hline Outcomes & $\begin{array}{l}\text { Outcomes reported: fibrosis score, NAFLD activity score } \\
\text { Follow-up, months: } 6\end{array}$ \\
\hline Notes & Source of funding (quote): "omega-3 and placebo capsules were provided by Amway (USA)" \\
& Trial name/Trial registry number: ID01992809 \\
& Attempts were made to contact study authors in December 2020 \\
\hline
\end{tabular}

\section{Risk of bias}

Bias Authors' judgement Support for judgement

Random sequence genera- Low risk Quote: "generated by computer"
tion (selection bias)


Nogueira 2016 (Continued)

Allocation concealment Low risk Quote: "this independent dietician, investigators and clinical staff remained (selection bias) blinded to each study participant's assignment until the end of the statistical analysis phase of the trial"

Comment: both allocation concealment and blinding were achieved with use of placebo

\begin{tabular}{|c|c|c|}
\hline $\begin{array}{l}\text { Blinding of participants } \\
\text { and personnel (perfor- } \\
\text { mance bias) } \\
\text { All outcomes }\end{array}$ & Low risk & Quote: "double-blind, placebo-controlled trial" \\
\hline $\begin{array}{l}\text { Blinding of outcome as- } \\
\text { sessment (detection bias) } \\
\text { All outcomes }\end{array}$ & Low risk & Quote: "double-blind, placebo-controlled trial" \\
\hline $\begin{array}{l}\text { Incomplete outcome data } \\
\text { (attrition bias) } \\
\text { All outcomes }\end{array}$ & Unclear risk & $\begin{array}{l}\text { Comment: participants were excluded from analysis for reasons that may be } \\
\text { related to the intervention and to outcomes }\end{array}$ \\
\hline $\begin{array}{l}\text { Selective reporting (re- } \\
\text { porting bias) }\end{array}$ & High risk & $\begin{array}{l}\text { Comment: no previously published protocol was available; adverse events, } \\
\text { mortality, fatty liver resolution were not reported }\end{array}$ \\
\hline Other bias & Low risk & Comment: no other bias noted \\
\hline
\end{tabular}

\section{Orang 2020}

\section{Study characteristics}

\begin{tabular}{|c|c|}
\hline Methods & Randomised clinical trial \\
\hline Participants & $\begin{array}{l}\text { Country: Iran } \\
\text { Period of recruitment: not stated } \\
\text { Number randomised: } 48 \\
\text { Post-randomisation dropouts: } 4 \text { ( } 8.3 \%) \\
\text { Revised sample size: } 44 \\
\text { Reasons for post-randomisation dropouts: lost to follow-up (2), discontinued intervention (2) } \\
\text { Average age, years: } 48 \\
\text { Females: } 31 \text { (70.5\%) } \\
\text { NASH: not stated } \\
\text { Diabetes mellitus: } 44 \text { (100.0\%) } \\
\text { Inclusion criteria: } 1 \text {. Adults. } 2 \text {. Type } 2 \text { diabetes mellitus. 3. NAFLD } \\
\text { Exclusion criteria: } 1 \text {. Any kind of malignancy. 2. Renal, heart, thyroid, or haemorrhagic disease. 3. Use of } \\
\text { insulin or omega-3 supplement in previous } 3 \text { months. } 4 \text {. Use of alcohol. 5. Diagnosis of hepatitis B or C } \\
\text { Method for diagnosis of NAFLD: not stated }\end{array}$ \\
\hline Interventions & $\begin{array}{l}\text { Group 1: PUFA ( } n=22) \\
\text { Further details: omega-3 consumed as } 2 \text { capsules per day, each containing } 1000 \text { mg of omega-3, for } 12 \\
\text { weeks } \\
\text { Group 2: no active intervention ( } n=22 \text { ) } \\
\text { Further details: } 2 \text { capsules of placebo (edible paraffin oil) per day for } 12 \text { weeks }\end{array}$ \\
\hline Outcomes & No outcomes of interest were reported \\
\hline Notes & $\begin{array}{l}\text { Source of funding (quote): "Nutrition and Food Security Research Center of Shahid Sadoughi University } \\
\text { of Medical Sciences funded this research. The corresponding author, Mozaffari-hassan, is an academ- }\end{array}$ \\
\hline
\end{tabular}


Orang 2020 (Continued)

ic member of Shahid Sadoughi University of Medical Sciences, and the others were master science students. Authors of the presents study have no conflict of interests to declare"

Trial name/Trial registry number: IRCT2017103030489N2

Attempts were made to contact study authors in April 2021

\section{Risk of bias}

\begin{tabular}{lll}
\hline Bias & Authors' judgement & Support for judgement \\
\hline $\begin{array}{l}\text { Random sequence genera- } \\
\text { tion (selection bias) }\end{array}$ & Low risk & Quote: "using computer generated random number" \\
\hline $\begin{array}{l}\text { Allocation concealment } \\
\text { (selection bias) }\end{array}$ & Low risk & $\begin{array}{l}\text { Quote: "random allocation remains blind until the beginning of study and, in } \\
\text { order to blinding both participants and personnel for the type of capsules, the } \\
\text { factory made both omega-3 and placebo capsules with a same appearances } \\
\text { and packed in similar package, named as A and B, and was not revealed after } \\
\text { the analyzing data" } \\
\text { Comment: both allocation concealment and blinding were achieved with use } \\
\text { of placebo }\end{array}$ \\
\hline
\end{tabular}

Blinding of participants Low risk Quote: "double-blind...placebo"
and personnel (perfor-
mance bias)
All outcomes

\begin{tabular}{lll}
\hline $\begin{array}{l}\text { Blinding of outcome as- } \\
\text { sessment (detection bias) } \\
\text { All outcomes }\end{array}$ & Low risk & \\
\hline $\begin{array}{l}\text { Incomplete outcome data } \\
\text { (attrition bias) }\end{array}$ & Unclear risk & $\begin{array}{l}\text { Comment: there were post-randomisation dropouts because the intervention } \\
\text { was discontinued. it is not clear whether these were related to the intervention } \\
\text { and to outcomes }\end{array}$
\end{tabular}

\begin{tabular}{lll}
\hline $\begin{array}{l}\text { Selective reporting (re- } \\
\text { porting bias) }\end{array}$ & High risk & $\begin{array}{l}\text { Comment: no previously published protocol was available; adverse events, } \\
\text { mortality, fatty liver resolution were not reported }\end{array}$ \\
\hline Other bias & Low risk & Comment: no other bias noted \\
\hline
\end{tabular}

Orr 2015

\section{Study characteristics}

\begin{tabular}{ll}
\hline Methods & Randomised clinical trial \\
\hline Participants & Country: New Zealand \\
& Period of recruitment: not stated \\
& Number randomised: not stated \\
& Post-randomisation dropouts: not stated \\
& Revised sample size: 0 \\
& Average age, years: 50 \\
& Females: not stated \\
& NASH: not stated \\
& Diabetes mellitus: not stated \\
Inclusion and exclusion criteria: not stated \\
Method for diagnosis of NAFLD: not stated \\
Group 1: prebiotics/probiotics/synbiotics $(\mathrm{n}=$ not stated) \\
\hline Interventions
\end{tabular}


Orr 2015 (Continued)

Further details: inulin (4 g) sachets twice daily for 12 weeks

Group 2: no active intervention ( $\mathrm{n}=$ not stated)

Further details: placebo

Additional details: another group not relevant to this review was excluded

\begin{tabular}{ll}
\hline Outcomes & No outcomes of interest were reported \\
\hline Notes & Source of funding: not stated \\
& Trial name/Trial registry number: ANZCTR-12613001002774 \\
& Attempts were made to contact study authors in December 2020 \\
\hline
\end{tabular}

\section{Risk of bias}

\begin{tabular}{|c|c|c|}
\hline Bias & Authors' judgement & Support for judgement \\
\hline $\begin{array}{l}\text { Random sequence genera- } \\
\text { tion (selection bias) }\end{array}$ & Unclear risk & Comment: this information was not available \\
\hline $\begin{array}{l}\text { Allocation concealment } \\
\text { (selection bias) }\end{array}$ & Unclear risk & Comment: this information was not available \\
\hline $\begin{array}{l}\text { Blinding of participants } \\
\text { and personnel (perfor- } \\
\text { mance bias) } \\
\text { All outcomes }\end{array}$ & Low risk & Quote: "double-blind, placebo controlled" \\
\hline $\begin{array}{l}\text { Blinding of outcome as- } \\
\text { sessment (detection bias) } \\
\text { All outcomes }\end{array}$ & Low risk & Quote: "double-blind, placebo controlled" \\
\hline $\begin{array}{l}\text { Incomplete outcome data } \\
\text { (attrition bias) } \\
\text { All outcomes }\end{array}$ & Unclear risk & Comment: this information was not available \\
\hline $\begin{array}{l}\text { Selective reporting (re- } \\
\text { porting bias) }\end{array}$ & High risk & $\begin{array}{l}\text { Comment: no previously published protocol was available; adverse events, } \\
\text { mortality, fatty liver resolution were not reported }\end{array}$ \\
\hline Other bias & Low risk & Comment: no other bias noted \\
\hline
\end{tabular}

Oscarsson 2018

\section{Study characteristics}

\begin{tabular}{ll}
\hline Methods & Randomised clinical trial \\
\hline Participants & Country: Sweden \\
& Period of recruitment: not stated \\
& Number randomised: 51 \\
& Post-randomisation dropouts: 5 (9.8\%) \\
& Revised sample size: 46 \\
& Reasons for post-randomisation dropouts: adverse events (3); patients withdrew due to going abroad \\
& (2) \\
& Average age, years: 60 \\
& Females: 21 (45.7\%) \\
& NASH: not stated \\
& Diabetes mellitus: 0 (0.0\%) \\
Inclusion criteria: 1. Aged 40 to 75 years. 2. BMI 25 to 40.3 . Serum TG > 1.7. 4 . Liver PDFF > 5.5\%
\end{tabular}


Exclusion criteria: 1 . History of hepatic disease. 2. Inability to undergo MRI scanning. 3. Significant alcohol intake

Method for diagnosis of NAFLD: liver MRI

\begin{tabular}{ll}
\hline Interventions & Group 1: PUFA $(\mathrm{n}=23)$ \\
& Further details: $4 \mathrm{~g}$ omega 3 fatty acid (Epanova; EPA (500 to $600 \mathrm{mg} / \mathrm{g}), \mathrm{DHA}$ (150 to $250 \mathrm{mg} / \mathrm{g})$, and do- \\
cosapentaenoic acid $(10$ to $80 \mathrm{mg} / \mathrm{g})$ ) for 12 weeks \\
Group 2: no active intervention $(\mathrm{n}=23)$ \\
Further details: placebo \\
Additional details: another group not relevant to this review was excluded \\
\hline
\end{tabular}

Outcomes Outcomes reported: mortality at maximal follow-up, any adverse events (number of people), liver transplantation at maximal follow-up, decompensation (number of people), cirrhosis (number of people), hepatocellular carcinoma

Follow-up, months: 3

\begin{tabular}{ll}
\hline Notes & Source of funding (quote): "the EFFECT I study was funded by AstraZeneca" \\
& Trial name/Trial registry number: EFFECT 1 \\
& Attempts were made to contact study authors in December 2020
\end{tabular}

\section{Risk of bias}

\begin{tabular}{|c|c|c|}
\hline Bias & Authors' judgement & Support for judgement \\
\hline $\begin{array}{l}\text { Random sequence genera- } \\
\text { tion (selection bias) }\end{array}$ & Low risk & Quote: "computer-generated numbers" \\
\hline $\begin{array}{l}\text { Allocation concealment } \\
\text { (selection bias) }\end{array}$ & Low risk & Quote: "centralized randomization system" \\
\hline $\begin{array}{l}\text { Blinding of participants } \\
\text { and personnel (perfor- } \\
\text { mance bias) } \\
\text { All outcomes }\end{array}$ & Low risk & Quote: "double-blind, randomized, placebo-controlled study" \\
\hline $\begin{array}{l}\text { Blinding of outcome as- } \\
\text { sessment (detection bias) } \\
\text { All outcomes }\end{array}$ & Low risk & Quote: "double-blind, randomized, placebo-controlled study" \\
\hline $\begin{array}{l}\text { Incomplete outcome data } \\
\text { (attrition bias) } \\
\text { All outcomes }\end{array}$ & Unclear risk & $\begin{array}{l}\text { Comment: the participant flow was not clear (i.e. it is not clear whether partici- } \\
\text { pants who were withdrawn were included for the outcomes) }\end{array}$ \\
\hline $\begin{array}{l}\text { Selective reporting (re- } \\
\text { porting bias) }\end{array}$ & Low risk & $\begin{array}{l}\text { Comment: no previously published protocol was available; adverse events and } \\
\text { either mortality or fatty liver resolution or both were reported }\end{array}$ \\
\hline Other bias & Low risk & Comment: no other bias noted \\
\hline
\end{tabular}

Pacifico 2015

\section{Study characteristics}

\begin{tabular}{ll}
\hline Methods & Randomised clinical trial \\
\hline Participants & $\begin{array}{l}\text { Country: Italy } \\
\text { Period of recruitment: } 2012 \text { to } 2014\end{array}$ \\
\hline
\end{tabular}


Pacifico 2015 (Continued)

Number randomised: 58

Post-randomisation dropouts: 7 (12.1\%)

Revised sample size: 51

Reasons for post-randomisation dropouts: lost to follow-up, MRI not available

Average age, years: 11

Females: $21(41.2 \%)$

NASH: 33 (64.7\%)

Diabetes mellitus: 0 (0.0\%)

Inclusion criteria: 1 . Aged < 18 years. 2. BMI > 85th percentile. 3. Persistently elevated ALT. 4. MRI-diagnosed NAFLD

Exclusion criteria: 1 . Hepatic viral infection and other secondary cause of steatosis. 2. Smoking. 3. Renal disease. 4. Alcohol intake. 5. Total parenteral nutrition. 6. Hepatotoxic medications. 7. Type 1 or 2 diabetes

Method for diagnosis of NAFLD: liver biopsy plus MRI

$\begin{array}{ll}\text { Interventions } & \text { Group 1: PUFA }(n=25) \\ & \text { Further details: docosahexaenoic acid (DHA) supplementation } 250 \mathrm{mg} / \mathrm{d} \text { for } 6 \text { months } \\ & \text { Group 2: no active intervention }(n=26) \\ & \text { Further details: placebo }\end{array}$

\begin{tabular}{ll}
\hline Outcomes & No outcomes of interest were reported \\
\hline Notes & Source of funding (quote): "this study was funded by the Sapienza University of Rome (Progetti di Ricer- \\
& ca Universitaria 2011e2012)" \\
& Trial name/Trial registry number: not stated \\
& Attempts were made to contact study authors in December 2020
\end{tabular}

\section{Risk of bias}

\begin{tabular}{lll}
\hline Bias & Authors' judgement & Support for judgement \\
\hline $\begin{array}{l}\text { Random sequence genera- } \\
\text { tion (selection bias) }\end{array}$ & Low risk & $\begin{array}{l}\text { Quote: "a randomization list (in a 1:1 ratio to treatment with DHA or place- } \\
\text { bo) was generated by an independent statistician who was blinded to partici- } \\
\text { pants' clinical data and did not perform the final analysis" }\end{array}$ \\
\hline $\begin{array}{l}\text { Allocation concealment } \\
\text { (selection bias) }\end{array}$ & Unclear risk & $\begin{array}{l}\text { Quote: "all participants and research staff were blind to the group assignment" } \\
\text { Comment: allocation concealment and blinding were achieved with use of } \\
\text { placebo }\end{array}$ \\
\hline
\end{tabular}

Blinding of participants Low risk Quote: "double-blind, placebo-controlled randomized trial"
and personnel (performance bias)

All outcomes

\begin{tabular}{|c|c|c|}
\hline $\begin{array}{l}\text { Blinding of outcome as- } \\
\text { sessment (detection bias) } \\
\text { All outcomes }\end{array}$ & Low risk & Quote: "double-blind, placebo-controlled randomized trial" \\
\hline $\begin{array}{l}\text { Incomplete outcome data } \\
\text { (attrition bias) } \\
\text { All outcomes }\end{array}$ & Unclear risk & $\begin{array}{l}\text { Comment: participants were excluded from analysis for reasons that may be } \\
\text { related to the intervention and to outcomes }\end{array}$ \\
\hline $\begin{array}{l}\text { Selective reporting (re- } \\
\text { porting bias) }\end{array}$ & High risk & $\begin{array}{l}\text { Comment: no previously published protocol was available; adverse events, } \\
\text { mortality, fatty liver resolution were not reported }\end{array}$ \\
\hline Other bias & Low risk & Comment: no other bias noted \\
\hline
\end{tabular}


Palamaru 2017

\section{Study characteristics}

\begin{tabular}{|c|c|}
\hline Methods & Randomised clinical trial \\
\hline Participants & $\begin{array}{l}\text { Country: Romania } \\
\text { Period of recruitment: } 2016 \text { to } 2017 \\
\text { Number randomised: } 40 \\
\text { Post-randomisation dropouts: not stated } \\
\text { Revised sample size: } 40 \\
\text { Average age, years: not stated } \\
\text { Females: not stated } \\
\text { NASH: not stated } \\
\text { Diabetes mellitus: not stated } \\
\text { Inclusion and exclusion criteria: not stated } \\
\text { Method for diagnosis of NAFLD: not stated }\end{array}$ \\
\hline Interventions & $\begin{array}{l}\text { Group 1: vitamin } E(n=20) \\
\text { Further details: vitamin E supplements } \\
\text { Group 2: no active intervention ( } n=20) \\
\text { Further details: no additional supplements } \\
\text { Additional details: both groups received lifestyle intervention (i.e. diet and exercise) }\end{array}$ \\
\hline Outcomes & No outcomes of interest were reported \\
\hline Notes & $\begin{array}{l}\text { Source of funding: not stated } \\
\text { Trial name/Trial registry number: not stated } \\
\text { Attempts were made to contact study authors in December } 2020\end{array}$ \\
\hline
\end{tabular}

\section{Risk of bias}

\begin{tabular}{|c|c|c|}
\hline Bias & Authors' judgement & Support for judgement \\
\hline $\begin{array}{l}\text { Random sequence genera- } \\
\text { tion (selection bias) }\end{array}$ & Unclear risk & Comment: this information was not available \\
\hline $\begin{array}{l}\text { Allocation concealment } \\
\text { (selection bias) }\end{array}$ & Unclear risk & Comment: this information was not available \\
\hline $\begin{array}{l}\text { Blinding of participants } \\
\text { and personnel (perfor- } \\
\text { mance bias) } \\
\text { All outcomes }\end{array}$ & Unclear risk & Comment: this information was not available \\
\hline $\begin{array}{l}\text { Blinding of outcome as- } \\
\text { sessment (detection bias) } \\
\text { All outcomes }\end{array}$ & Unclear risk & Comment: this information was not available \\
\hline $\begin{array}{l}\text { Incomplete outcome data } \\
\text { (attrition bias) } \\
\text { All outcomes }\end{array}$ & Unclear risk & Comment: this information was not available \\
\hline $\begin{array}{l}\text { Selective reporting (re- } \\
\text { porting bias) }\end{array}$ & High risk & $\begin{array}{l}\text { Comment: no previously published protocol was available; adverse events, } \\
\text { mortality, fatty liver resolution were not reported }\end{array}$ \\
\hline Other bias & Low risk & Comment: no other bias noted \\
\hline
\end{tabular}




\section{Study characteristics}

\begin{tabular}{ll}
\hline Methods & Randomised clinical trial \\
\hline Participants & Country: Iran \\
& Period of recruitment: 2009 \\
& Number randomised: 76 \\
& Post-randomisation dropouts: 22 (28.9\%) \\
& Revised sample size: 54 \\
& Reasons for post-randomisation dropouts: not stated \\
& Average age, years: 49 \\
& Females: not stated \\
& NASH: not stated \\
& Diabetes mellitus: 11 (20.4\%) \\
Inclusion criteria: 1. Sonographic evidence of fatty liver disease \\
Exclusion criteria: 1 . Alcohol intake. 2. Cirrhosis and other forms of chronic liver disease. 3. Hepatitis \\
(autoimmune, viral, iatrogenic). 4. Surgery \\
Method for diagnosis of NAFLD: ultrasound
\end{tabular}

Interventions

Group 1: vitamin E plus other supplements $(n=21)$

Further details: Chlorella vulgaris extract (Algomed: $1200 \mathrm{mg} / \mathrm{d})$ plus vitamin $\mathrm{E}(200 \mathrm{mg} / \mathrm{d})$ for 3 months

Group 2: vitamin $\mathrm{E}(\mathrm{n}=33)$

Further details: vitamin E $(200 \mathrm{mg} / \mathrm{d})$ for 3 months

Additional details: both groups received metformin

\begin{tabular}{ll}
\hline Outcomes & No outcomes of interest were reported \\
\hline Notes & Source of funding (quote): "this work was financially supported by a grant from the Baqiyatallah Uni- \\
& versity of Medical Sciences (Tehran, Iran)" \\
& Trial name/Trial registry number: not stated \\
& Attempts were made to contact study authors in December 2020
\end{tabular}

\section{Risk of bias}

\begin{tabular}{lll}
\hline Bias & Authors' judgement & Support for judgement \\
\hline $\begin{array}{l}\text { Random sequence genera- } \\
\text { tion (selection bias) }\end{array}$ & Unclear risk & Comment: this information was not available \\
\hline $\begin{array}{l}\text { Allocation concealment } \\
\text { (selection bias) }\end{array}$ & Unclear risk & Comment: this information was not available \\
\hline $\begin{array}{l}\text { Blinding of participants } \\
\text { and personnel (perfor- } \\
\text { mance bias) }\end{array}$ & High risk & Quote: "open-label" \\
All outcomes & \\
\hline
\end{tabular}

\begin{tabular}{lll}
$\begin{array}{l}\text { Blinding of outcome as- } \\
\text { sessment (detection bias) } \\
\text { All outcomes }\end{array}$ & High risk & Quote: "open-label" \\
\hline $\begin{array}{l}\text { Incomplete outcome data } \\
\text { (attrition bias) } \\
\text { All outcomes }\end{array}$ & Unclear risk & $\begin{array}{l}\text { Comment: participants were excluded from analysis for reasons that may be } \\
\text { related to the intervention and to outcomes }\end{array}$ \\
\hline
\end{tabular}


Panahi 2012 (Continued)

\begin{tabular}{|c|c|c|}
\hline $\begin{array}{l}\text { Selective reporting (re- } \\
\text { porting bias) }\end{array}$ & High risk & $\begin{array}{l}\text { Comment: no previously published protocol was available; adverse events, } \\
\text { mortality, fatty liver resolution were not reported }\end{array}$ \\
\hline
\end{tabular}

Other bias Low risk Comment: no other bias noted

Panahi 2016

\section{Study characteristics}

\begin{tabular}{|c|c|c|}
\hline Methods & \multicolumn{2}{|c|}{ Randomised clinical trial } \\
\hline Participants & \multicolumn{2}{|c|}{$\begin{array}{l}\text { Country: Iran } \\
\text { Period of recruitment: not stated } \\
\text { Number randomised: } 102 \\
\text { Post-randomisation dropouts: } 15(14.7 \%) \\
\text { Revised sample size: } 87 \\
\text { Reasons for post-randomisation dropouts: discontinued intervention because of perception of lack of } \\
\text { effect } \\
\text { Average age, years: } 46 \\
\text { Females: } 36(41.4 \%) \\
\text { NASH: not stated } \\
\text { Diabetes mellitus: } 21 \text { ( } 24.1 \%) \\
\text { Inclusion criteria: } 1 \text {. NAFLD } \\
\text { Exclusion criteria: } 1 \text {. Pregnancy/breastfeeding. 2. Alcohol consumption. 3. Smoking. 4. Hepatotoxic } \\
\text { drugs. } 5 \text {. Hepatitis. } 6 \text {. Coronary, renal, pulmonary, and thyroid disease } \\
\text { Method for diagnosis of NAFLD: ultrasound }\end{array}$} \\
\hline Interventions & \multicolumn{2}{|c|}{$\begin{array}{l}\text { Group 1: other supplements }(n=44) \\
\text { Further details: curcumin ( } 1000 \mathrm{mg} / \mathrm{d} \text { in } 2 \text { divided doses) for } 8 \text { weeks } \\
\text { Group 2: no active intervention ( } \mathrm{n}=43) \\
\text { Further details: no treatment } \\
\text { Additional details: both groups received dietary and lifestyle advice }\end{array}$} \\
\hline Outcomes & \multicolumn{2}{|c|}{ No outcomes of interest were reported } \\
\hline Notes & \multicolumn{2}{|c|}{$\begin{array}{l}\text { Source of funding (quote): "the authors are grateful for the supports provided by the Baqiyatallah Uni- } \\
\text { versity of Medical Sciences (Tehran, Iran) and Indena SpA (Milan, Italy)" } \\
\text { Trial name/Trial registry number: IRCT2015122525641N2 } \\
\text { Attempts were made to contact study authors in December } 2020\end{array}$} \\
\hline \multicolumn{3}{|l|}{ Risk of bias } \\
\hline Bias & Authors' judgement & Support for judgement \\
\hline $\begin{array}{l}\text { Random sequence genera- } \\
\text { tion (selection bias) }\end{array}$ & Unclear risk & Comment: this information was not available \\
\hline $\begin{array}{l}\text { Allocation concealment } \\
\text { (selection bias) }\end{array}$ & Unclear risk & Comment: this information was not available \\
\hline $\begin{array}{l}\text { Blinding of participants } \\
\text { and personnel (perfor- } \\
\text { mance bias) } \\
\text { All outcomes }\end{array}$ & Unclear risk & Comment: this information was not available \\
\hline
\end{tabular}


Panahi 2016 (Continued)

Blinding of outcome as- Unclear risk Comment: this information was not available sessment (detection bias)

All outcomes

$\begin{array}{ll}\text { Incomplete outcome data Unclear risk } & \text { Comment: participants were excluded from analysis for reasons that may be } \\ \text { (attrition bias) } & \text { related to the intervention and to outcomes }\end{array}$

All outcomes

related to the intervention and to outcomes

\begin{tabular}{|c|c|c|}
\hline $\begin{array}{l}\text { Selective reporting (re- } \\
\text { porting bias) }\end{array}$ & High risk & $\begin{array}{l}\text { Comment: no previously published protocol was available; adverse events, } \\
\text { mortality, fatty liver resolution were not reported }\end{array}$ \\
\hline
\end{tabular}

Other bias Low risk Comment: no other bias noted

Parsi 2020

\section{Study characteristics}

\begin{tabular}{|c|c|}
\hline Methods & Randomised clinical trial \\
\hline Participants & $\begin{array}{l}\text { Country: Iran } \\
\text { Period of recruitment: not stated } \\
\text { Number randomised: } 60 \\
\text { Post-randomisation dropouts: not stated } \\
\text { Revised sample size: } 60 \\
\text { Average age, years: } 35 \\
\text { Females: } 27 \text { ( } 45.0 \%) \\
\text { NASH: not stated } \\
\text { Diabetes mellitus: not stated } \\
\text { Inclusion criteria: } 1 \text {. Liver ultrasonography (steatosis score } \geq 1 \text { ). } 2 \text {. Serum levels of ALT > } 19 \mathrm{U} / \mathrm{L} \text { for } \\
\text { women, } 30 \text { U/L for men. } 3 \text {. Aged } 20 \text { to } 60 \text { years. } 4 \text {. BMI ranging from } 24.9 \text { to } 40 \mathrm{~kg} / \mathrm{m}^{2} \\
\text { Exclusion criteria: } 1 \text {. Other cause of fatty liver such as alcohol consumption. } 2 \text {. Use of drugs that may } \\
\text { have potential benefit for treatment of NAFLD such as vitamin E, metformin, pentoxifylline, and rosigli- } \\
\text { tazone. } 3 \text {. Pregnant and lactating woman. } 4 \text {. Renal, liver, heart, pituitary, thyroid, or psychiatric disor- } \\
\text { der. } 5 \text {. Weight loss > } 10 \% \text { of initial body weight during the intervention. } 6 \text {. Taking a probiotic, multi-vita- } \\
\text { min-mineral, or antioxidant supplement during past } 3 \text { months. } 7 \text {. Not taking more than } 10 \% \text { of supple- } \\
\text { ments at any follow-up visit } \\
\text { Method for diagnosis of NAFLD: ultrasound and transaminases }\end{array}$ \\
\hline
\end{tabular}

\begin{tabular}{ll}
\hline Interventions & $\begin{array}{l}\text { Group 1: other supplements }(\mathrm{n}=30) \\
\text { Further details: saffron (containing } 15 \mathrm{mg} \text { Crocin) supplement daily for } 8 \text { weeks } \\
\text { Group 2: no active intervention }(\mathrm{n}=30) \\
\text { Further details: Identical-appearing placebo capsules (starch) once daily for } 8 \text { weeks }\end{array}$ \\
\hline Outcomes & No outcomes of interest were reported \\
\hline Notes & Source of funding (quote): "this work was financially supported by Alimentary Tract Research Center, \\
& $\begin{array}{l}\text { Imam Khomeini Hospital, Ahvaz Jundishapur University of Medical Sciences, Ahvaz, Iran (Grant Num- } \\
\text { ber: RDC-9612)" } \\
\text { Trial name/Trial registry number: IRCT20180513039634N } \\
\text { Attempts were made to contact study authors in April 2021 }\end{array}$
\end{tabular}

\section{Risk of bias}

\section{Bias}

Authors' judgement Support for judgement 
Parsi 2020 (Continued)

Random sequence genera- Unclear risk Comment: this information was not available tion (selection bias)

Allocation concealment Unclear risk
(selection bias)

Blinding of participants Low risk Quote: "double-blind...placebo"

and personnel (perfor-

mance bias)

All outcomes

Blinding of outcome as-
sessment (detection bias) $\quad$ Low risk $\quad$ Quote: "double-blind...placebo"

All outcomes

Incomplete outcome data Low risk Comment: there were no post-randomisation dropouts
(attrition bias)

All outcomes

\begin{tabular}{lll}
\hline $\begin{array}{l}\text { Selective reporting (re- } \\
\text { porting bias) }\end{array}$ & High risk & $\begin{array}{l}\text { Comment: no previously published protocol was available; adverse events, } \\
\text { mortality, fatty liver resolution were not reported }\end{array}$ \\
\hline Other bias & Low risk & Comment: no other bias noted \\
\hline
\end{tabular}

\section{Pasdar 2020}

\section{Study characteristics}

\begin{tabular}{|c|c|}
\hline Methods & Randomised clinical trial \\
\hline Participants & $\begin{array}{l}\text { Country: Iran } \\
\text { Period of recruitment: } 2017 \\
\text { Number randomised: } 90 \\
\text { Post-randomisation dropouts: } 12 \text { (13.3\%) } \\
\text { Revised sample size: } 78 \\
\text { Reasons for post-randomisation dropouts: unwillingness to continue (7), non-adherence (3), travel (2) } \\
\text { Average age, years: } 45 \\
\text { Females: } 50 \text { (64.1\%) } \\
\text { NASH: not stated } \\
\text { Diabetes mellitus: not stated } \\
\text { Inclusion criteria: } 1.18 \text { to } 65 \text { years old. } 2 \text {. NAFLD as approved by ultrasonography. 3. Body mass index } \\
\text { between } 25 \text { and } 40 \text { kg/m }{ }^{2} \\
\text { Exclusion criteria: } 1 \text {. History of alcohol abuse. 2. Acute or chronic disease such as liver disorder (hepati- } \\
\text { tis B and C), Wilson's disease, cirrhosis, autoimmune disease, and thyroid disorder. 3. Previous expo- } \\
\text { sure to pesticides and insecticides. } 4 \text {. Taking steatogenic medications, which potentially interact with } \\
\text { quercetin (anticoagulant agents, inhibitors of CYP3A4, etc.) } \\
\text { Method for diagnosis of NAFLD: ultrasound }\end{array}$ \\
\hline Interventions & $\begin{array}{l}\text { Group 1: other supplements }(n=39) \\
\text { Further details: quercetin (Sigma Q4951 } \geq 95 \% \text { purity; Sigma-Aldrich, St. Louis, Missouri, USA); each pa- } \\
\text { tient received } 250-m g \text { capsules twice daily } \\
\text { Group 2: no active intervention ( } n=39 \text { ) } \\
\text { Further details: placebo (Avicel } \mathrm{PH} 101 \text {; Sigma-Aldrich) produced at same size, colour, and smell as } \\
\text { quercetin capsules. Each patient received } 250-\mathrm{mg} \text { capsules twice daily }\end{array}$ \\
\hline Outcomes & No outcomes of interest were reported \\
\hline
\end{tabular}


Pasdar 2020 (Continued)

$\begin{array}{ll}\text { Notes } & \text { Source of funding: not stated } \\ & \text { Trial name/Trial registry number: IRCT2016060628299N1 } \\ & \text { Attempts were made to contact study authors in April } 2021\end{array}$

\section{Risk of bias}

\begin{tabular}{|c|c|c|}
\hline Bias & Authors' judgement & Support for judgement \\
\hline $\begin{array}{l}\text { Random sequence genera- } \\
\text { tion (selection bias) }\end{array}$ & Low risk & $\begin{array}{l}\text { Quote: "random assignment was performed using computer-generated ran- } \\
\text { dom numbers" }\end{array}$ \\
\hline $\begin{array}{l}\text { Allocation concealment } \\
\text { (selection bias) }\end{array}$ & Unclear risk & Comment: this information was not available \\
\hline $\begin{array}{l}\text { Blinding of participants } \\
\text { and personnel (perfor- } \\
\text { mance bias) } \\
\text { All outcomes }\end{array}$ & Low risk & Quote: "double-blind...placebo" \\
\hline $\begin{array}{l}\text { Blinding of outcome as- } \\
\text { sessment (detection bias) } \\
\text { All outcomes }\end{array}$ & Low risk & Quote: "double-blind...placebo" \\
\hline $\begin{array}{l}\text { Incomplete outcome data } \\
\text { (attrition bias) } \\
\text { All outcomes }\end{array}$ & High risk & $\begin{array}{l}\text { Comment: there were post-randomisation dropouts, some of which seem to } \\
\text { be related to the intervention }\end{array}$ \\
\hline $\begin{array}{l}\text { Selective reporting (re- } \\
\text { porting bias) }\end{array}$ & High risk & $\begin{array}{l}\text { Comment: no previously published protocol was available; adverse events, } \\
\text { mortality, fatty liver resolution were not reported }\end{array}$ \\
\hline Other bias & Low risk & Comment: no other bias noted \\
\hline
\end{tabular}

Pervez 2018

\section{Study characteristics}

\begin{tabular}{ll}
\hline Methods & Randomised clinical trial \\
\hline Participants & Country: Pakistan \\
& Period of recruitment: 2015 to 2016 \\
& Number randomised: 71 \\
& Post-randomisation dropouts: 7 (9.9\%) \\
& Revised sample size: 64 \\
& Reasons for post-randomisation dropouts: withdrew consent, lost to follow-up \\
& Average age, years: 44 \\
& Females: 35 (54.7\%) \\
& NASH: not stated \\
& Diabetes mellitus: not stated \\
& Inclusion criteria: 1 . NAFLD \\
& Exclusion criteria: 1 . Chronic hepatitis B/C. 2. Alcoholic liver disease. 3. Autoimmune disease. 4. Hepa- \\
& totoxic medication \\
& Method for diagnosis of NAFLD: ultrasound \\
& Group 1: vitamin $\mathrm{E}(\mathrm{n}=31)$ \\
& Further details: oral delta-tocotrienol 300 mg twice daily for 12 weeks \\
& Group 2: no active intervention $(\mathrm{n}=33)$ \\
\hline Interventions &
\end{tabular}


Pervez 2018 (Continued)

Further details: placebo

Outcomes Outcomes reported: serious adverse events (number of people), any adverse events (number of peo-

ple), resolution of fatty liver disease

Follow-up, months: 3
Source of funding (quote): "this study is funded by the Armed Forces Institute of Pathology, Rawalpindi and Higher Education Commission, Government of Pakistan, Islamabad"

Trial name/Trial registry number: not stated

Attempts were made to contact study authors in December 2020

\section{Risk of bias}

\begin{tabular}{|c|c|c|}
\hline Bias & Authors' judgement & Support for judgement \\
\hline $\begin{array}{l}\text { Random sequence genera- } \\
\text { tion (selection bias) }\end{array}$ & Unclear risk & Comment: this information was not available \\
\hline $\begin{array}{l}\text { Allocation concealment } \\
\text { (selection bias) }\end{array}$ & Low risk & $\begin{array}{l}\text { Quote: "the same opaque capsules containing either } \delta \text {-tocotrienol or placebo } \\
\text { (sucrose) were administered to the patients by a research assistant blinded to } \\
\text { the contents of the capsules" }\end{array}$ \\
\hline $\begin{array}{l}\text { Blinding of participants } \\
\text { and personnel (perfor- } \\
\text { mance bias) } \\
\text { All outcomes }\end{array}$ & Low risk & Quote: "randomized, double-blind, placebo-controlled pilot study" \\
\hline $\begin{array}{l}\text { Blinding of outcome as- } \\
\text { sessment (detection bias) } \\
\text { All outcomes }\end{array}$ & Low risk & Quote: "randomized, double-blind, placebo-controlled pilot study" \\
\hline $\begin{array}{l}\text { Incomplete outcome data } \\
\text { (attrition bias) } \\
\text { All outcomes }\end{array}$ & Unclear risk & $\begin{array}{l}\text { Comment: participants were excluded from analysis for reasons that may be } \\
\text { related to the intervention and to outcomes }\end{array}$ \\
\hline $\begin{array}{l}\text { Selective reporting (re- } \\
\text { porting bias) }\end{array}$ & Low risk & $\begin{array}{l}\text { Comment: no previously published protocol was available; adverse events and } \\
\text { either mortality or fatty liver resolution or both were reported }\end{array}$ \\
\hline Other bias & Low risk & Comment: no other bias noted \\
\hline
\end{tabular}

Pervez 2020

\section{Study characteristics}

\begin{tabular}{ll}
\hline Methods & Randomised clinical trial \\
\hline Participants & Country: Pakistan \\
& Period of recruitment: 2015 to 2016 \\
& Number randomised: 71 \\
& Post-randomisation dropouts: $0(0.0 \%)$ \\
& Revised sample size: 71 \\
& Average age, years: 44 \\
& Females: 37 (52.1\%) \\
& NASH: not stated \\
& Diabetes mellitus: not stated
\end{tabular}


Pervez 2020 (Continued)

Inclusion criteria: 1. Aged 20 to 70 years. 2. Ultrasound-proven fatty liver, fatty liver index (FLI) $\geq 6018$, and mild to moderate persistent elevation of alanine transaminase (ALT) and aspartate transaminase (AST) (i.e. not greater than 4 times the upper limit of $42 \mathrm{IU} / \mathrm{L}$ and $37 \mathrm{IU} / \mathrm{L}$, respectively)

Exclusion criteria: 1. Viral hepatitis B and C. 2. Alcoholic liver disease. 3. Autoimmune hepatitis, haemochromatosis, Wilson's disease. 4. Malignancy. 5. Cardiovascular, respiratory, and kidney disorders. 6. Pregnancy or lactation. 7. History of using hepatotoxic and lipid-lowering drugs and herbal or vitamin supplements. 8 . History of average alcohol consumption $>30 \mathrm{~g} / \mathrm{d}$ in men, $>20 \mathrm{~g} / \mathrm{d}$ in women, in past 3 months

Method for diagnosis of NAFLD: ultrasound

\begin{tabular}{ll}
\hline Interventions & Group 1: vitamin $\mathrm{E}(\mathrm{n}=35)$ \\
& Further details: tocotrienol group received two 300-mg tocotrienol capsules per day. Tocotrienol cap- \\
& sules were manufactured by American River Nutrition, Inc., Hadley, MA. USA. Tocotrienol was extracted \\
& from annatto bean; each capsule contained $90 \% \delta$-tocotrienol and $10 \% \mathrm{\gamma}$ - tocotrienol \\
& Group 2: no active intervention $(\mathrm{n}=36)$ \\
& Further details: placebo group received two $50-\mathrm{mg}$ sucrose capsules per day. Placebo and tocotrienol \\
& capsules were identical in size, colour, and consistency to ensure blinding of patients \\
\hline Outcomes & Outcomes reported: resolution of fatty liver disease \\
& Follow-up, months: 6 \\
\hline Notes & Source of funding (quote): "this work was financially supported by Higher EducationCommission, Gov- \\
& ernment of Pakistan Islamabad" \\
Trial name/Trial registry number: SLCTR/2015/023 \\
Attempts were made to contact study authors in April 2021
\end{tabular}

\section{Risk of bias}

\begin{tabular}{|c|c|c|}
\hline Bias & Authors' judgement & Support for judgement \\
\hline $\begin{array}{l}\text { Random sequence genera- } \\
\text { tion (selection bias) }\end{array}$ & Unclear risk & $\begin{array}{l}\text { Quote: "randomized to receive } \delta \text {-tocotrienol or placebo through the simple } \\
\text { randomization technique by a person not involved in the study" } \\
\text { Comment: further details were not available }\end{array}$ \\
\hline $\begin{array}{l}\text { Allocation concealment } \\
\text { (selection bias) }\end{array}$ & Low risk & $\begin{array}{l}\text { Quote: "randomized to receive } \delta \text {-tocotrienol or placebo through the simple } \\
\text { randomization technique by a person not involved in the study" }\end{array}$ \\
\hline $\begin{array}{l}\text { Blinding of participants } \\
\text { and personnel (perfor- } \\
\text { mance bias) } \\
\text { All outcomes }\end{array}$ & Low risk & $\begin{array}{l}\text { Quote: "both the patients and investigators were blinded to treatment alloca- } \\
\text { tion...placebo" }\end{array}$ \\
\hline $\begin{array}{l}\text { Blinding of outcome as- } \\
\text { sessment (detection bias) } \\
\text { All outcomes }\end{array}$ & Low risk & $\begin{array}{l}\text { Quote: "both the patients and investigators were blinded to treatment alloca- } \\
\text { tion...placebo" }\end{array}$ \\
\hline $\begin{array}{l}\text { Incomplete outcome data } \\
\text { (attrition bias) } \\
\text { All outcomes }\end{array}$ & Low risk & Comment: an intention-to-treat analysis was used \\
\hline $\begin{array}{l}\text { Selective reporting (re- } \\
\text { porting bias) }\end{array}$ & High risk & $\begin{array}{l}\text { Comment: no previously published protocol was available; adverse events } \\
\text { were not reported adequately }\end{array}$ \\
\hline Other bias & Low risk & Comment: no other bias noted \\
\hline
\end{tabular}


Pezeshki 2016

\section{Study characteristics}

\begin{tabular}{|c|c|}
\hline Methods & Randomised clinical trial \\
\hline Participants & $\begin{array}{l}\text { Country: Iran } \\
\text { Period of recruitment: } 2013 \text { to } 2014 \\
\text { Number randomised: } 80 \\
\text { Post-randomisation dropouts: } 9 \text { (11.3\%) } \\
\text { Revised sample size: } 71 \\
\text { Reasons for post-randomisation dropouts: discontinued treatment } \\
\text { Average age, years: not stated } \\
\text { Females: } 39 \text { (54.9\%) } \\
\text { NASH: not stated } \\
\text { Diabetes mellitus: not stated } \\
\text { Inclusion criteria: } 1 \text {. Aged } 20 \text { to } 50 \text { years. } 2 \text {. BMI } \geq 30.3 \text {. NAFLD } \\
\text { Exclusion criteria: } 1 \text {. Other liver disease (Wilson's, autoimmune, haemochromatosis, viral infection). } 2 . \\
\text { Alcoholic liver disease. } 3 \text {. Hepatotoxic medication } \\
\text { Method for diagnosis of NAFLD: ultrasound plus transaminases }\end{array}$ \\
\hline Interventions & $\begin{array}{l}\text { Group 1: other supplements }(n=35) \\
\text { Further details: green tea extract }(G T E) 500 \mathrm{mg} / \mathrm{d} \text { for } 90 \text { days } \\
\text { Group 2: no active intervention }(n=36) \\
\text { Further details: placebo }\end{array}$ \\
\hline Outcomes & No outcomes of interest were reported \\
\hline Notes & $\begin{array}{l}\text { Source of funding: not stated } \\
\text { Trial name/Trial registry number: Protexin } \\
\text { Attempts were made to contact study authors in December } 2020\end{array}$ \\
\hline
\end{tabular}

\section{Risk of bias}

Bias Authors' judgement Support for judgement

Random sequence genera- Low risk Quote: "a random number between 0.0 and 0.99 was generated by the comtion (selection bias) $\quad$ puter for each subject"

Allocation concealment Low risk Quote: "by a research assistant blinded to the contents in the capsules"
(selection bias)

Blinding of participants Low risk Quote: "double-blind, placebo-controlled, randomized clinical trial"
and personnel (performance bias)

All outcomes

\begin{tabular}{|c|c|c|}
\hline $\begin{array}{l}\text { Blinding of outcome as- } \\
\text { sessment (detection bias) } \\
\text { All outcomes }\end{array}$ & Low risk & Quote: "double-blind, placebo-controlled, randomized clinical trial" \\
\hline $\begin{array}{l}\text { Incomplete outcome data } \\
\text { (attrition bias) } \\
\text { All outcomes }\end{array}$ & Unclear risk & $\begin{array}{l}\text { Comment: participants were excluded from analysis for reasons that may be } \\
\text { related to the intervention and to outcomes }\end{array}$ \\
\hline $\begin{array}{l}\text { Selective reporting (re- } \\
\text { porting bias) }\end{array}$ & High risk & $\begin{array}{l}\text { Comment: no previously published protocol was available; adverse events, } \\
\text { mortality, fatty liver resolution were not reported }\end{array}$ \\
\hline Other bias & Low risk & Comment: no other bias noted \\
\hline
\end{tabular}




\section{Study characteristics}

\begin{tabular}{|c|c|}
\hline Methods & Randomised clinical trial \\
\hline Participants & $\begin{array}{l}\text { Country: Thailand } \\
\text { Period of recruitment: } 2016 \\
\text { Number randomised: } 37 \\
\text { Post-randomisation dropouts: } 0(0 \%) \\
\text { Revised sample size: } 37 \\
\text { Average age (years): } 12 \\
\text { Females: } 25 \text { (67.6\%) } \\
\text { NASH: not stated } \\
\text { Diabetes mellitus: not stated } \\
\text { Inclusion criteria: } 1 \text {. Children. 2. NAFLD defined by CAP value }>225 \mathrm{~dB} / \mathrm{m} \text { by Fibroscan } \\
\text { Exclusion criteria: } 1 \text {. Children with metabolic liver disease. } 2 \text {. Viral hepatitis. 3. Wilson's disease. 4. Au- } \\
\text { toimmune hepatitis. } 5 \text {. Hepatotoxic drug exposure. } 6 \text {. Alcohol consumption } \\
\text { Method for diagnosis of NAFLD: FibroScan }\end{array}$ \\
\hline Interventions & $\begin{array}{l}\text { Group 1: prebiotics/probiotics/synbiotics }(n=18) \\
\text { Further details: a powder mixture containing } 2.24 \text { grams chicory inulin, } 1.5 \times 10^{9} \text { colony-forming units } \\
\text { of Lactobacillus acidophilus and Bifidobacterium lactis in each sachet for } 16 \text { weeks } \\
\text { Group 2: no active intervention }(n=19) \\
\text { Further details: indistinguishable placebo for } 16 \text { weeks }\end{array}$ \\
\hline Outcomes & $\begin{array}{l}\text { Outcomes reported: mortality, serious adverse events (number of people), any adverse events (number } \\
\text { of people), liver transplantation, cirrhosis, decompensated cirrhosis, hepatocellular carcinoma } \\
\text { Follow-up, months: } 3\end{array}$ \\
\hline Notes & $\begin{array}{l}\text { Source of funding (quote): "this study was supported by the Ratchadaphiseksomphot Fund, Faculty of } \\
\text { Medicine, Chulalongkorn University (RA59/030)" } \\
\text { Trial name/Trial registry number: TCTR20170128001 } \\
\text { Attempts were made to contact study authors in April } 2021\end{array}$ \\
\hline
\end{tabular}

\section{Risk of bias}

\begin{tabular}{lll}
\hline Bias & Authors' judgement & Support for judgement \\
\hline $\begin{array}{l}\text { Random sequence genera- } \\
\text { tion (selection bias) }\end{array}$ & Low risk & $\begin{array}{l}\text { Quote: "by generating random allocation sequence using computer soft- } \\
\text { ware" (author replies) }\end{array}$ \\
\hline $\begin{array}{l}\text { Allocation concealment } \\
\text { (selection bias) }\end{array}$ & Low risk & $\begin{array}{l}\text { Quote: "the company allocated synbiotics and placebo, which were packed } \\
\text { in indistinguishable sachets to the recruiter (concealed allocation)" (author } \\
\text { replies) }\end{array}$
\end{tabular}

Blinding of participants Low risk Quote: "double-blind...placebo"

and personnel (perfor-

mance bias)

All outcomes

Blinding of outcome as- Low risk Quote: "double-blind...placebo"
sessment (detection bias)
All outcomes

Incomplete outcome data Low risk Comment: there were no post-randomisation dropouts
(attrition bias)
All outcomes


Poparn 2020 (Continued)

Selective reporting (re- Low risk Comment: no previously published protocol was available; adverse events and porting bias)

Other bias Low risk Comment: no other bias noted

Poulos 2021

\section{Study characteristics}

\begin{tabular}{|c|c|}
\hline Methods & Randomised clinical trial \\
\hline Participants & $\begin{array}{l}\text { Country: USA } \\
\text { Period of recruitment: not stated } \\
\text { Number randomised: } 27 \\
\text { Post-randomisation dropouts: } 2 \text { (7.4\%) } \\
\text { Revised sample size: } 25 \\
\text { Reasons for post-randomisation dropouts: lost to follow-up } \\
\text { Average age, years: } 56 \\
\text { Females: } 19 \text { (76.0\%) } \\
\text { NASH: not stated } \\
\text { Diabetes mellitus: not stated } \\
\text { Inclusion criteria. } 1 . \geq 18 \text { years of age. } 2 \text {. Clinical or radiological evidence of NAFLD established by liver } \\
\text { biopsy or radiographic studies revealing hepatic steatosis. 3. Metabolic syndrome. } 4 \text {. Elevated AST/ALT } \\
\text { in the absence of any other metabolic, viral, or autoimmune aetiology. } 5 \text {. Negative urine pregnancy test } \\
\text { (for females of childbearing potential) } \\
\text { Exclusion criteria: } 1 \text {. History of having received any investigational drug within } 3 \text { months. } 2 \text {. History of a } \\
\text { medical condition associated with chronic liver disease other than NAFLD. 3. Baseline increased risk for } \\
\text { anaemia or anaemia would be medically problematic. } 4 \text {. History of severe psychiatric disease, includ- } \\
\text { ing psychosis and/or severe depression, characterised by a suicide attempt, hospitalisation for psy- } \\
\text { chiatric disease, or a period of disability due to psychiatric disease. } 5 \text {. Positive test at screening for he- } \\
\text { patitis A, B, C, or HIV/AIDS. } 6 \text {. Pregnant or breastfeeding. } 7 \text {. Type } 1 \text { or } 2 \text { diabetes with HbA1C }>8.5 \% \text { at } \\
\text { screening. } 8 \text {. History or other evidence of chronic pulmonary disease associated with functional limita- } \\
\text { tion. } 9 \text {. Uncontrolled seizure disorder. } 10 \text {. Poorly controlled thyroid function. } 11 \text {. Bleeding disorder, or } \\
\text { anticoagulant use. } 12 \text {. Poorly controlled hypertension. 13. Evidence of active or suspected cancer, or } \\
\text { history of malignancy within last } 2 \text { years, except those with basal cell carcinoma that has been excised } \\
\text { and cured } \\
\text { Method for diagnosis of NAFLD: liver biopsy or radiographic studies plus transaminases }\end{array}$ \\
\hline
\end{tabular}

Group 1: amino acids plus vitamin E plus other antioxidants $(n=14)$

Further details: drug compound consisted of a pill containing vitamin E $200 \mathrm{IU}$, silymarin $750 \mathrm{mg}$, and $L$ carnitine 1 gram (EuroMED USA, Inc.). Silymarin was $80 \%$ silybin

Group 2: no active intervention $(n=11)$

Further details: placebo consisted of rice flour containing 32 calories per dose and 0.77 net carbs/g

\begin{tabular}{ll}
\hline Outcomes & No outcomes of interest were reported \\
\hline Notes & Source of funding (quote): "Biovil Corporation" \\
& Trial name/Trial registry number: NCT01511523 \\
& Attempts were made to contact study authors in April 2021 \\
\hline
\end{tabular}

\section{Risk of bias}

Bias Authors' judgement Support for judgement

Random sequence genera- Unclear risk Comment: this information was not available tion (selection bias) 
Poulos 2021 (Continued)

Allocation concealment Unclear risk Comment: this information was not available
(selection bias)

Blinding of participants Unclear risk Comment: this information was not available
and personnel (perfor-
mance bias)
All outcomes

\begin{tabular}{lll}
\hline $\begin{array}{l}\text { Blinding of outcome as- } \\
\text { sessment (detection bias) } \\
\text { All outcomes }\end{array}$ & Unclear risk & Comment: this information was not available \\
\hline $\begin{array}{l}\text { Incomplete outcome data } \\
\text { (attrition bias) } \\
\text { All outcomes }\end{array}$ & Unclear risk & $\begin{array}{l}\text { Comment: there were post-randomisation dropouts because of loss to fol- } \\
\text { low-up. it is not clear whether these were related to the intervention and to } \\
\text { outcomes }\end{array}$ \\
\hline $\begin{array}{l}\text { Selective reporting (re- } \\
\text { porting bias) }\end{array}$ & High risk & $\begin{array}{l}\text { Comment: no previously published protocol was available; adverse events, } \\
\text { mortality, fatty liver resolution were not reported }\end{array}$ \\
\hline Other bias & Low risk & Comment: no other bias noted \\
\hline
\end{tabular}

Pour 2020

\section{Study characteristics}

\begin{tabular}{|c|c|}
\hline Methods & Randomised clinical trial \\
\hline Participants & $\begin{array}{l}\text { Country: Iran } \\
\text { Period of recruitment: } 2016 \text { to } 2017 \\
\text { Number randomised: } 76 \\
\text { Post-randomisation dropouts: not stated } \\
\text { Revised sample size: } 76 \\
\text { Average age, years: } 43 \\
\text { Females: } 33 \text { (43.4\%) } \\
\text { NASH: not stated } \\
\text { Diabetes mellitus: not stated } \\
\text { Inclusion criteria: } 1 \text {. NAFLD } \\
\text { Exclusion criteria: } 1 \text {. Chronic liver disease such as viral hepatitis. 2. Diabetes mellitus. 3. Infection. } 4 . \\
\text { Cancer. } 5 \text {. Autoimmune disease. } 6 \text {. Inherited disorder affecting the liver condition (storage disorders of } \\
\text { iron, copper, and others). } 7 \text {. Acute cardiovascular, respiratory, and kidney disorders. 8. Hypertension. } 9 . \\
\text { Hypothyroidism. } 10 \text {. Any disorder that affects weight (hyperprolactinaemia, Cushing's syndrome). } 11 . \\
\text { Pregnant or lactating women. } 12 \text {. Use of hepatotoxic medication. 13. Alcohol use. 14. Taking any med- } \\
\text { ication or on strict diet to lose weight in the past } 3 \text { months. 15. Taking lipid-lowering drugs or antidia- } \\
\text { betic drugs, vitamins, or any antioxidant supplements. 16. Therapy with approved medicine that may } \\
\text { have potential benefit for treatment of NAFLD (i.e. vitamin E, betaine, pioglitazone, milk thistle, thiazo- } \\
\text { lidinedione, anti-TNF- } a \text {, UDCA, SAM-E). } 17 \text {. Consuming < } 80 \% \text { of tablets at any follow-up visit } \\
\text { Method for diagnosis of NAFLD: ultrasound }\end{array}$ \\
\hline
\end{tabular}

Interventions Group 1: other supplements $(\mathrm{n}=38)$

Further details: 1 tablet containing $100 \mathrm{mg}$ saffron per day

Group 2: no active intervention $(n=38)$

Further details: placebo - similar shape and size tablets containing 100 mg maltodextrin

Additional details: both groups were given a healthy diet and physical activity advice

Outcomes

Outcomes reported: serious adverse events (number of people), any adverse events (number of people) 
Pour 2020 (Continued)

Follow-up, months: 3

$\begin{array}{ll}\text { Notes } & \text { Source of funding (quote): "this work was financially supported by the Iran University of Medical } \\ \text { Sciences (IUMS)" } & \\ \text { Trial name/Trial registry number: IRCT number: } 201705309472 \text { N13 } \\ \text { Attempts were made to contact study authors in April } 2021\end{array}$

\section{Risk of bias}

\begin{tabular}{|c|c|c|}
\hline Bias & Authors' judgement & Support for judgement \\
\hline $\begin{array}{l}\text { Random sequence genera- } \\
\text { tion (selection bias) }\end{array}$ & Low risk & $\begin{array}{l}\text { Quote: "the participants were randomly assigned into two groups by a com- } \\
\text { puter-generated random sequence" }\end{array}$ \\
\hline $\begin{array}{l}\text { Allocation concealment } \\
\text { (selection bias) }\end{array}$ & Low risk & $\begin{array}{l}\text { Quote: "a statistical advisor who did not involved in the study encoded unique } \\
\text { codes on the identical boxes, which was generated by the software" }\end{array}$ \\
\hline $\begin{array}{l}\text { Blinding of participants } \\
\text { and personnel (perfor- } \\
\text { mance bias) } \\
\text { All outcomes }\end{array}$ & Low risk & $\begin{array}{l}\text { Quote: "randomization and assignment of the participants into the groups (al- } \\
\text { location) were hidden from both the researchers and the patients until the fi- } \\
\text { nal analyses were completed...placebo" }\end{array}$ \\
\hline $\begin{array}{l}\text { Blinding of outcome as- } \\
\text { sessment (detection bias) } \\
\text { All outcomes }\end{array}$ & Low risk & $\begin{array}{l}\text { Quote: "randomization and assignment of the participants into the groups (al- } \\
\text { location) were hidden from both the researchers and the patients until the fi- } \\
\text { nal analyses were completed...placebo" }\end{array}$ \\
\hline $\begin{array}{l}\text { Incomplete outcome data } \\
\text { (attrition bias) } \\
\text { All outcomes }\end{array}$ & Low risk & Comment: an intention-to-treat analysis was performed \\
\hline $\begin{array}{l}\text { Selective reporting (re- } \\
\text { porting bias) }\end{array}$ & High risk & $\begin{array}{l}\text { Comment: no previously published protocol was available; neither mortality } \\
\text { nor fatty liver resolution was reported }\end{array}$ \\
\hline Other bias & Low risk & Comment: no other bias noted \\
\hline
\end{tabular}

Qin 2015

\section{Study characteristics}

\begin{tabular}{|c|c|}
\hline Methods & Randomised clinical trial \\
\hline Participants & $\begin{array}{l}\text { Country: China } \\
\text { Period of recruitment: } 2012 \text { to } 2013 \\
\text { Number randomised: } 80 \\
\text { Post-randomisation dropouts: } 10(12.5 \%) \\
\text { Revised sample size: } 70 \\
\text { Reasons for post-randomisation dropouts: loss to follow-up } \\
\text { Average age, years: } 45 \\
\text { Females: } 19 \text { (27.1\%) } \\
\text { NASH: not stated } \\
\text { Diabetes mellitus: not stated } \\
\text { Inclusion criteria: } 1 \text {. Steady BMI between } 20 \text { and } 30 \text { over past } 3 \text { months. } 2 \text {. No excessive alcohol con- } \\
\text { sumption } \\
\text { Exclusion criteria: } 1 . \text { Viral hepatitis, autoimmune hepatitis, or other liver disease. 2. Use of medication } \\
\text { or dietary supplement over past } 6 \text { months that could influence NAFLD. 3. Gastrointestinal disease, se- } \\
\text { vere chronic disease, kidney dysfunction, or malignant tumour. 4. Acute or chronic infectious disease } \\
\text { 5. Any surgical procedure }\end{array}$ \\
\hline
\end{tabular}


Qin 2015 (Continued)

Method for diagnosis of NAFLD: ultrasound

$\begin{array}{ll}\text { Interventions } & \text { Group 1: PUFA plus vitamin E }(\mathrm{n}=36) \\ & \text { Further details: fish oil ( } 182 \mathrm{mg} \text { EPA and } 129 \mathrm{mg} \mathrm{DHA} \text {, in addition to vitamin E) } 4 \mathrm{~g} / \mathrm{d} \text { for } 3 \mathrm{months} \\ & \text { Group 2: no active intervention }(\mathrm{n}=34) \\ & \text { Further details: placebo } \\ & \text { Additional details: both groups received lifestyle advice }\end{array}$

Outcomes Outcomes reported: serious adverse events (number of people), any adverse events (number of people)

Follow-up, months: 3

$\begin{array}{ll}\text { Notes } & \text { Source of funding: not stated } \\ \text { Trial name/Trial registry number: ChiCTR-TRC12002380 } & \\ \text { Attempts were made to contact study authors in December } 2020\end{array}$

\section{Risk of bias}

\begin{tabular}{|c|c|c|}
\hline Bias & Authors' judgement & Support for judgement \\
\hline $\begin{array}{l}\text { Random sequence genera- } \\
\text { tion (selection bias) }\end{array}$ & Low risk & $\begin{array}{l}\text { Quote: "the randomized sequence was produced by a randomization protocol } \\
\text { using the IBM SPSS Statistics } 19.0 \text { (IBM, Japan) system" }\end{array}$ \\
\hline $\begin{array}{l}\text { Allocation concealment } \\
\text { (selection bias) }\end{array}$ & Low risk & $\begin{array}{l}\text { Quote: "the information of randomization was sealed until the end of the } \\
\text { study" } \\
\text { Comment: allocation concealment and blinding were achieved with use of } \\
\text { placebo }\end{array}$ \\
\hline $\begin{array}{l}\text { Blinding of participants } \\
\text { and personnel (perfor- } \\
\text { mance bias) } \\
\text { All outcomes }\end{array}$ & Low risk & Quote: "double-blind, randomized clinical trial...placebo" \\
\hline $\begin{array}{l}\text { Blinding of outcome as- } \\
\text { sessment (detection bias) } \\
\text { All outcomes }\end{array}$ & Low risk & Quote: "double-blind, randomized clinical trial...placebo" \\
\hline $\begin{array}{l}\text { Incomplete outcome data } \\
\text { (attrition bias) } \\
\text { All outcomes }\end{array}$ & Unclear risk & $\begin{array}{l}\text { Comment: participants were excluded from analysis for reasons that may be } \\
\text { related to the intervention and to outcomes }\end{array}$ \\
\hline $\begin{array}{l}\text { Selective reporting (re- } \\
\text { porting bias) }\end{array}$ & High risk & $\begin{array}{l}\text { Comment: no previously published protocol was available; neither mortality } \\
\text { nor fatty liver resolution was reported }\end{array}$ \\
\hline Other bias & Low risk & Comment: no other bias noted \\
\hline
\end{tabular}

Rafie 2020

\section{Study characteristics}

\begin{tabular}{ll}
\hline Methods & Randomised clinical trial \\
\hline Participants & Country: Iran \\
& Period of recruitment: not stated \\
& Number randomised: 50 \\
& Post-randomisation dropouts: $4(8.0 \%)$
\end{tabular}


Rafie 2020 (Continued)

Revised sample size: 46

Reasons for post-randomisation dropouts: discontinued intervention (2), received supplementary (2)

Average age, years: 49

Females: 26 (56.5\%)

NASH: not stated

Diabetes mellitus: not stated

Inclusion criteria: 1. Aged 20 to 70 years. 2. $24.9<\mathrm{BMI}<35.19$. 3. High levels of liver enzymes $(>30 \mathrm{U} / \mathrm{L}$ in men, $>19 \mathrm{U} / \mathrm{L}$ in women)

Exclusion criteria: 1. Alcohol $>20$ g per day. 2. Taking drugs with liver toxicity such as calcium channel blockers, methotrexate, NSAIDs, oestrogens, progesterones, immunosuppressants, diuretics, and corticosteroids. 3. Liver disorder other than NAFLD (e.g. hereditary haemochromatosis, Wilson's disease, cirrhosis, hepatitis C, hepatitis B, autoimmune hepatitis). 4. Diabetes and other metabolic disease. 5. History of disease such as Cushing's syndrome, hypothyroidism, heart failure, renal failure, and renal stones. 6 . Weight loss medication. 7. Any supplement in last 6 months. 8 . History of gastric bypass surgery. 9. Severe weight loss during last 6 months. 10. Receiving hormone therapy Method for diagnosis of NAFLD: ultrasound plus transaminases

Group 1: other supplements $(\mathrm{n}=23)$
Further details: $500 \mathrm{mg}$ ginger rhizome powder capsules; 3 capsules daily
Group 2: no active intervention $(\mathrm{n}=23)$
Further details: 3 capsules $500 \mathrm{mg}$ of placebo that were apparently similar to the ginger supplement.
Placebo containing wheat flour was prepared in the same form and colour as a ginger supplement at
Pharmacy Faculty Lab of Ahvaz Jundishapur University of Medical Sciences
Additional details: patients in both groups were advised to receive a diet with energy balanced, accord-
ing to guidelines published by the North American Association, and all patients were asked to exercise
at least 3 times a week for 30 minutes a day

Outcomes Outcomes reported: any adverse events (number of people)

Follow-up, months: 3

Notes

Source of funding (quote): "this study was supported by the Vice Chancellor for research affairs of Ahvaz Jundishapur University of Medical Sciences"

Trial name/Trial registry number: IRCT2016042827652N1

Attempts were made to contact study authors in April 2021

\section{Risk of bias}

\begin{tabular}{lll}
\hline Bias & Authors' judgement & Support for judgement \\
\hline $\begin{array}{l}\text { Random sequence genera- } \\
\text { tion (selection bias) }\end{array}$ & Unclear risk & Comment: this information was not available \\
\hline $\begin{array}{l}\text { Allocation concealment } \\
\text { (selection bias) }\end{array}$ & Low risk & $\begin{array}{l}\text { Quote: "before the beginning of the study, the cans containing the capsules } \\
\text { were coded by a person other than the researcher, in the form of B and A, to } \\
\text { ensure that the researchers did not know the type of capsules received by } \\
\text { each group (given the double-blindness of the study)" }\end{array}$
\end{tabular}

Blinding of participants Low risk Quote: "double-blind randomized clinical trial...placebo"
and personnel (perfor-
mance bias)
All outcomes

Blinding of outcome as- Low risk Quote: "double-blind randomized clinical trial...placebo"
sessment (detection bias)
All outcomes

Incomplete outcome data Unclear risk (attrition bias)

Comment: there were post-randomisation dropouts; it is not clear whether

All outcomes these were related to the intervention and to outcomes 
Rafie 2020 (Continued)

\begin{tabular}{|c|c|c|}
\hline $\begin{array}{l}\text { Selective reporting (re- } \\
\text { porting bias) }\end{array}$ & High risk & $\begin{array}{l}\text { Comment: no previously published protocol was available; neither mortality } \\
\text { nor fatty liver resolution was reported }\end{array}$ \\
\hline
\end{tabular}

Other bias Low risk Comment: no other bias noted

Rahimlou 2016

\section{Study characteristics}

\begin{tabular}{|c|c|c|}
\hline Methods & \multicolumn{2}{|c|}{ Randomised clinical trial } \\
\hline Participants & \multicolumn{2}{|c|}{$\begin{array}{l}\text { Country: Iran } \\
\text { Period of recruitment: not stated } \\
\text { Number randomised: } 50 \\
\text { Post-randomisation dropouts: } 6 \text { (12.0\%) } \\
\text { Revised sample size: } 44 \\
\text { Reasons for post-randomisation dropouts: willingness to continue, immigration } \\
\text { Average age, years: } 45 \\
\text { Females: } 24 \text { (54.5\%) } \\
\text { NASH: not stated } \\
\text { Diabetes mellitus: } 0 \text { (0.0\%) } \\
\text { Inclusion criteria: } 1 \text {. Presence of steatosis on Fibroscan } \\
\text { Exclusion criteria: } 1 \text {. Chronic liver disease. 2. Diabetes mellitus. 3. Cancer. 4. Inherited disorder of liver. } \\
\text { 5. Untreated hypothyroidism. } 6 \text {. Alcohol use.7. Weight loss surgery. 8. Pregnancy/Lactation } \\
\text { Method for diagnosis of NAFLD: elastography plus transaminases }\end{array}$} \\
\hline Interventions & \multicolumn{2}{|c|}{$\begin{array}{l}\text { Group 1: other supplements }(n=23) \\
\text { Further details: ginger supplement } 2 \mathrm{~g} / \mathrm{d} \text { for } 12 \text { weeks } \\
\text { Group 2: no active intervention }(\mathrm{n}=21) \\
\text { Further details: placebo } \\
\text { Additional details: both groups received lifestyle advice }\end{array}$} \\
\hline Outcomes & \multicolumn{2}{|c|}{$\begin{array}{l}\text { Outcomes reported: serious adverse events (number of people) } \\
\text { Follow-up, months: } 3\end{array}$} \\
\hline Notes & \multicolumn{2}{|c|}{$\begin{array}{l}\text { Source of funding (quote): "we thank green plants of life pharmaceutics Co. for providing ginger and } \\
\text { placebo capsules and all participants that contributed to this study" } \\
\text { Trial name/Trial registry number: NCY02535195 } \\
\text { Attempts were made to contact study authors in December } 2020\end{array}$} \\
\hline \multicolumn{3}{|l|}{ Risk of bias } \\
\hline Bias & Authors' judgement & Support for judgement \\
\hline $\begin{array}{l}\text { Random sequence genera- } \\
\text { tion (selection bias) }\end{array}$ & Low risk & Quote: "computer-generated" \\
\hline $\begin{array}{l}\text { Allocation concealment } \\
\text { (selection bias) }\end{array}$ & Low risk & $\begin{array}{l}\text { Quote: "statistician and participants, project managers and employees at the } \\
\text { clinic were completely unaware (blind) about the intervention and control } \\
\text { groups... identical placebos" } \\
\text { Comment: both allocation concealment and blinding were achieved with use } \\
\text { of placebo }\end{array}$ \\
\hline
\end{tabular}


Rahimlou 2016 (Continued)

Blinding of participants Low risk Quote: "randomized, double-blind, placebo-controlled clinical trial" and personnel (performance bias)

All outcomes

\begin{tabular}{lll}
\hline $\begin{array}{l}\text { Blinding of outcome as- } \\
\text { sessment (detection bias) } \\
\text { All outcomes }\end{array}$ & Low risk & Quote: "randomized, double-blind, placebo-controlled clinical trial" \\
\hline $\begin{array}{l}\text { Incomplete outcome data } \\
\text { (attrition bias) } \\
\text { All outcomes }\end{array}$ & Unclear risk & $\begin{array}{l}\text { Comment: participants were excluded from analysis for reasons that may be } \\
\text { related to the intervention and to outcomes }\end{array}$ \\
\hline $\begin{array}{l}\text { Selective reporting (re- } \\
\text { porting bias) }\end{array}$ & High risk & $\begin{array}{l}\text { Comment: no previously published protocol was available; neither mortality } \\
\text { nor fatty liver resolution was reported }\end{array}$ \\
\hline Other bias & Low risk & Comment: no other bias noted \\
\hline
\end{tabular}

Rahmani 2016

\section{Study characteristics}

\begin{tabular}{|c|c|}
\hline Methods & Randomised clinical trial \\
\hline Participants & $\begin{array}{l}\text { Country: Iran } \\
\text { Period of recruitment: not stated } \\
\text { Number randomised: } 80 \\
\text { Post-randomisation dropouts: } 3(3.8 \%) \\
\text { Revised sample size: } 77 \\
\text { Reasons for post-randomisation dropouts: adverse events } \\
\text { Average age, years: } 48 \\
\text { Females: } 42 \text { (54.5\%) } \\
\text { NASH: not stated } \\
\text { Diabetes mellitus: not stated } \\
\text { Inclusion: criteria: } 1 \text {. Diagnosis of NAFLD according to liver ultrasound } \\
\text { Exclusion holiday: } 1 \text {. Pregnancy/breastfeeding. 2. NAFLD secondary to alcohol. 3. Smoking. 4. Hepato- } \\
\text { toxic medication. 5. Hepatitis } \\
\text { Method for diagnosis of NAFLD: ultrasound }\end{array}$ \\
\hline Interventions & $\begin{array}{l}\text { Group 1: other supplements }(n=37) \\
\text { Further details: curcumin }(500 \mathrm{mg} / \mathrm{d} \text { equivalent to } 70 \mathrm{mg} \text { curcumin) for } 8 \text { weeks } \\
\text { Group 2: no active intervention }(n=40) \\
\text { Further details: placebo }\end{array}$ \\
\hline Outcomes & $\begin{array}{l}\text { Outcomes reported: resolution of fatty liver disease } \\
\text { Follow-up, months: } 2\end{array}$ \\
\hline Notes & $\begin{array}{l}\text { Source of funding (quote): "this study was financially supported by the Isfahan University of Medical } \\
\text { Sciences (Isfahan, Iran). The financial support provided by the Iran National Science Foundation (INSF) } \\
\text { is also gratefully acknowledged" } \\
\text { Trial name/Trial registry number: IRCT2014110511763N18 } \\
\text { Attempts were made to contact study authors in December } 2020\end{array}$ \\
\hline
\end{tabular}

\section{Risk of bias}


Rahmani 2016 (Continued)
Random sequence genera-
Unclear risk
Comment: this information was not available tion (selection bias)

Allocation concealment Unclear risk Comment: this information was not available
(selection bias)

Blinding of participants Low risk Quote: "randomized double-blind placebo-controlled trial"
and personnel (perfor-
mance bias)
All outcomes

Blinding of outcome as- Low risk $\quad$ Quote: "randomized double-blind placebo-controlled trial"
sessment (detection bias)

All outcomes

\begin{tabular}{ll}
\hline $\begin{array}{l}\text { Incomplete outcome data } \\
\text { (attrition bias) }\end{array}$ & Unclear risk \\
All outcomes & $\begin{array}{l}\text { Comment: participants were excluded from analysis for reasons which may be } \\
\text { related to the intervention and the outcomes }\end{array}$
\end{tabular}

\begin{tabular}{ll}
\hline $\begin{array}{l}\text { Selective reporting (re- } \\
\text { porting bias) }\end{array}$ & High risk
\end{tabular}$\quad \begin{aligned} & \text { Comment: no previously published protocol was available: adverse events } \\
& \text { were not reported adequately }\end{aligned}$

\begin{tabular}{ll}
\hline Other bias Low risk $\quad$ Comment: no other bias noted \\
\hline
\end{tabular}

\section{Ruan 2010}

\section{Study characteristics}

\begin{tabular}{|c|c|}
\hline Methods & Randomised clinical trial \\
\hline Participants & $\begin{array}{l}\text { Country: china } \\
\text { Period of recruitment: } 2008 \text { to } 2009 \\
\text { Number randomised: } 60 \\
\text { Post-randomisation dropouts: not stated } \\
\text { Revised sample size: } 60 \\
\text { Average age, years: not stated } \\
\text { Females: } 12(20.0 \%) \\
\text { NASH: not stated } \\
\text { Diabetes mellitus: not stated } \\
\text { Inclusion and exclusion criteria: not stated } \\
\text { Method for diagnosis of NAFLD: not stated }\end{array}$ \\
\hline Interventions & $\begin{array}{l}\text { Group 1: other antioxidants }(n=30) \\
\text { Further details: silymarin } 70 \mathrm{mg} 3 \text { times a day for } 24 \text { weeks } \\
\text { Group 2: no active intervention }(n=30) \\
\text { Further details: no treatment } \\
\text { Additional details: both groups received lifestyle intervention }\end{array}$ \\
\hline Outcomes & $\begin{array}{l}\text { Outcomes reported: resolution of fatty liver disease } \\
\text { Follow-up, months: } 6\end{array}$ \\
\hline Notes & $\begin{array}{l}\text { Source of funding: not stated } \\
\text { Trial name/Trial registry number: not stated } \\
\text { Attempts were made to contact study authors in December } 2020\end{array}$ \\
\hline
\end{tabular}

\section{Risk of bias}

Nutritional supplementation for nonalcohol-related fatty liver disease: a network meta-analysis (Review) 
Ruan 2010 (Continued)

Bias Authors' judgement Support for judgement

Random sequence genera- Unclear risk Comment: this information was not available

tion (selection bias)

Allocation concealment $\quad$ Unclear risk Comment: this information was not available
(selection bias)

Blinding of participants High risk $\quad$ Quote: "open, randomized, controlled clinical study"
and personnel (perfor-
mance bias)
All outcomes

$\begin{array}{lll}\text { Blinding of outcome as- } & \text { High risk } & \text { Quote: "open, randomized, controlled clinical study" } \\ \text { sessment (detection bias) } & \end{array}$

All outcomes

\begin{tabular}{lll}
\hline $\begin{array}{l}\text { Incomplete outcome data } \\
\text { (attrition bias) } \\
\text { All outcomes }\end{array}$ & Unclear risk & Comment: this information was not available \\
\hline $\begin{array}{l}\text { Selective reporting (re- } \\
\text { porting bias) }\end{array}$ & High risk & $\begin{array}{l}\text { Comment: no previously published protocol was available; adverse events } \\
\text { were not reported adequately }\end{array}$ \\
\hline Other bias & Low risk & Comment: no other bias noted \\
\hline
\end{tabular}

Sadrkabir 2020

\section{Study characteristics}

\begin{tabular}{|c|c|}
\hline Methods & Randomised clinical trial \\
\hline Participants & $\begin{array}{l}\text { Country: Iran } \\
\text { Period of recruitment: not stated } \\
\text { Number randomised: } 67 \\
\text { Post-randomisation dropouts: } 6 \text { (9.0\%) } \\
\text { Revised sample size: } 61 \\
\text { Reasons for post-randomisation dropouts: none referred to receive treatment (3), cut of treatment (3) } \\
\text { Average age, years: } 44 \\
\text { Females: } 21 \text { (34.4\%) } \\
\text { NASH: not stated } \\
\text { Diabetes mellitus: not stated } \\
\text { Inclusion criteria: } 1 \text {. Aged > } 18 \text { years. } 2 \text {. Newly diagnosed NAFLD, grade } 2 \text { and } 3 \text { fatty liver, treatment } \\
\text { naïve } \\
\text { Exclusion criteria: } 1 \text {. Digestive disease. } 2 \text {. Diabetes. } 3 \text {. Rheumatoid arthritis, other rheumatological dis- } \\
\text { ease treated with immunosuppressive drugs, } 4 \text {. Cholestatic liver disease, advanced liver disease. } 5 \text {. } \\
\text { Heart failure, thyroid and kidney diseases. } 6 \text {. Any cause of chronic liver disease other than NAFLD, such } \\
\text { as positive test for hepatitis B, hepatitis C, and autoimmune hepatitis. } 7 \text {. History of cancer and drug } \\
\text { treatment. } 8 \text {. Antibiotic use in the past } 2 \text { weeks. } 9 \text {. Use of vitamin supplemental antioxidant, fibre, and } \\
\text { omega-3 in } 3 \text { weeks before and during study, pregnancy or lactation, contraceptive use, liver transplan- } \\
\text { tation, and alcohol consumption in the } 3 \text { months before the study } \\
\text { Method for diagnosis of NAFLD: ultrasound }\end{array}$ \\
\hline
\end{tabular}

Interventions

Group 1: prebiotics/probiotics/synbiotics $(n=33)$ 
Sadrkabir 2020 (Continued)

Further details: 2 capsules of $500 \mathrm{mg}$ of GeriLact for 60 days. GeriLact was used in this study as a symbiotic product in capsule form (lactobacilli, cassia, acidophilous, langburoum, bifidobacterial, and streptococcus along with prebiotics (fructolucosaccharide)

Group 2: no active intervention $(n=28)$

Further details: placebo for 60 days

Additional details: diet and exercise recommendations for both groups

\begin{tabular}{ll}
\hline Outcomes & No outcomes of interest were reported \\
\hline Notes & Source of funding (quote): "funder: vice chancellor for research, Ardabil University of Medical Sciences" \\
& Trial name/Trial registry number: IRCT2017102537007N1 \\
& Attempts were made to contact study authors in April 2021
\end{tabular}

\section{Risk of bias}

\begin{tabular}{|c|c|c|}
\hline Bias & Authors' judgement & Support for judgement \\
\hline $\begin{array}{l}\text { Random sequence genera- } \\
\text { tion (selection bias) }\end{array}$ & Unclear risk & Comment: this information was not available \\
\hline $\begin{array}{l}\text { Allocation concealment } \\
\text { (selection bias) }\end{array}$ & Unclear risk & Comment: this information was not available \\
\hline $\begin{array}{l}\text { Blinding of participants } \\
\text { and personnel (perfor- } \\
\text { mance bias) } \\
\text { All outcomes }\end{array}$ & Unclear risk & Comment: placebo was used, but there is no mention about blinding \\
\hline $\begin{array}{l}\text { Blinding of outcome as- } \\
\text { sessment (detection bias) } \\
\text { All outcomes }\end{array}$ & Unclear risk & Comment: placebo was used, but there is no mention about blinding \\
\hline $\begin{array}{l}\text { Incomplete outcome data } \\
\text { (attrition bias) } \\
\text { All outcomes }\end{array}$ & Unclear risk & $\begin{array}{l}\text { Comment: there were post-randomisation dropouts; it is not clear whether } \\
\text { these were related to the intervention and to outcomes }\end{array}$ \\
\hline $\begin{array}{l}\text { Selective reporting (re- } \\
\text { porting bias) }\end{array}$ & High risk & $\begin{array}{l}\text { Comment: no previously published protocol was available; adverse events, } \\
\text { mortality, fatty liver resolution were not reported }\end{array}$ \\
\hline Other bias & Low risk & Comment: no other bias noted \\
\hline
\end{tabular}

\section{Sakpal 2017}

\section{Study characteristics}

\begin{tabular}{ll}
\hline Methods & Randomised clinical trial \\
\hline Participants & Country: India \\
& Period of recruitment: not stated \\
Number randomised: 81 \\
Post-randomisation dropouts: $0(0.0 \%)$ \\
Revised sample size: 81 \\
Average age, years: 38 \\
Females: 26 (32.1\%) \\
NASH: not stated \\
Diabetes mellitus: 13 (16.0\%) \\
Inclusion criteria: 1 . Aged over 12 years. 2. Ultrasound showing features of steatosis \\
\hline
\end{tabular}


Sakpal 2017 (Continued)

Exclusion criteria: 1. Pregnancy. 2. Drug intake likely to cause NAFLD. 3. Extensive small bowel resec-

tion

Method for diagnosis of NAFLD: ultrasound

\begin{tabular}{ll}
\hline Interventions & Group 1: vitamin $D(n=51)$ \\
& Further details: a single intramuscular injection of 600,000 IU of cholecalciferol \\
& Group 2: no active intervention $(n=30)$ \\
& Further details: no treatment \\
& Additional details: both groups received lifestyle modifications
\end{tabular}

Outcomes Outcomes reported: mortality at maximal follow-up

Follow-up, months: 6

\begin{tabular}{ll}
\hline Notes & Source of funding: not stated \\
& Trial name/Trial registry number: not stated \\
& Attempts were made to contact study authors in December 2020
\end{tabular}

\section{Risk of bias}

\begin{tabular}{|c|c|c|}
\hline Bias & Authors' judgement & Support for judgement \\
\hline $\begin{array}{l}\text { Random sequence genera- } \\
\text { tion (selection bias) }\end{array}$ & Unclear risk & Comment: this information was not available \\
\hline $\begin{array}{l}\text { Allocation concealment } \\
\text { (selection bias) }\end{array}$ & Unclear risk & Comment: this information was not available \\
\hline $\begin{array}{l}\text { Blinding of participants } \\
\text { and personnel (perfor- } \\
\text { mance bias) } \\
\text { All outcomes }\end{array}$ & Unclear risk & Comment: this information was not available \\
\hline $\begin{array}{l}\text { Blinding of outcome as- } \\
\text { sessment (detection bias) } \\
\text { All outcomes }\end{array}$ & Unclear risk & Comment: this information was not available \\
\hline $\begin{array}{l}\text { Incomplete outcome data } \\
\text { (attrition bias) } \\
\text { All outcomes }\end{array}$ & Low risk & Comment: there were no post-randomisation dropouts \\
\hline $\begin{array}{l}\text { Selective reporting (re- } \\
\text { porting bias) }\end{array}$ & High risk & $\begin{array}{l}\text { Comment: no previously published protocol was available; adverse events } \\
\text { were not reported adequately }\end{array}$ \\
\hline Other bias & Low risk & Comment: no other bias noted \\
\hline
\end{tabular}

\section{Sangouni 2020}

\section{Study characteristics}

\begin{tabular}{ll}
\hline Methods & Randomised clinical trial \\
\hline Participants & Country: Iran \\
& Period of recruitment: 2018 \\
& Number randomised: 90 \\
& Post-randomisation dropouts: $2(2.2 \%)$ \\
& Revised sample size: 88
\end{tabular}


Reasons for post-randomisation dropouts: undergoing surgery (1), lost to follow-up (1)

Average age, years: 45

Females: 31 (35.2\%)

NASH: not stated

Diabetes mellitus: not stated

Inclusion criteria: 1 . Patients with grade 1 to 3 fatty liver. 2. Aged $\geq 18$ years

Exclusion criteria: 1 . History of alcohol abuse (average daily alcohol consumption $\geq 10 \mathrm{~g}$ for women, $\geq$

$20 \mathrm{~g}$ for men). 2. Viral hepatitis. 3. Liver cancer, other liver disease. 4. Diabetes mellitus. 5. Untreated hypothyroidism. 6. Mental disease. 7. Kidney disease. 8. Pregnancy, lactation. 9. Low blood pressure, taking blood pressure-lowering medications. 10. Allergic to garlic. 11. Unwilling to continue the study. Method for diagnosis of NAFLD: ultrasound

Group 1: other supplements $(\mathrm{n}=45)$
Further details: $400 \mathrm{mg}$ garlic powder tablets (each coated tablet contained $1.5 \mathrm{mg}$ allicin, approxi-
mately $2 \mathrm{~g}$ of fresh garlic) 4 times daily. Garlic and placebo tablets were manufactured at Amin Pharma-
ceutical Company
Group 2: no active intervention $(\mathrm{n}=43)$
Further details: placebo tablets (each $400-\mathrm{mg}$ coated tablet contained starch) 4 times daily. Placebo
and garlic powder tablets had similar appearance
Additional details: the usual treatment for all patients consisted of prescribing milk thistle tablets and
recommending weight loss without offering any method

Outcomes Outcomes reported: serious adverse events (number of people)

Follow-up, months: 3

Source of funding (quote): "we would like to express our gratitude towards the Urmia University of
Medical Sciences, for the facilities and financial support"
Trial name/Trial registry number: IRCT20170206032417N4
Attempts were made to contact study authors in April 2021

\section{Risk of bias}

\begin{tabular}{lll}
\hline Bias & Authors' judgement & Support for judgement \\
\hline $\begin{array}{l}\text { Random sequence genera- } \\
\text { tion (selection bias) }\end{array}$ & Low risk & Quote: "randomisation lists were computer-generated by a statistician" \\
\hline $\begin{array}{l}\text { Allocation concealment } \\
\text { (selection bias) }\end{array}$ & Low risk & $\begin{array}{l}\text { Quote: "patients, researcher, laboratory staff and statistician were blinded to } \\
\text { the study groups until the end of the study...placebo" } \\
\text { Comment: both allocation concealment and blinding were achieved with use } \\
\text { of placebo }\end{array}$ \\
\hline
\end{tabular}

\begin{tabular}{|c|c|c|}
\hline $\begin{array}{l}\text { Blinding of participants } \\
\text { and personnel (perfor- }\end{array}$ & Low risk & $\begin{array}{l}\text { Quote: "patients, researcher, laboratory staff and statistician were blinded to } \\
\text { the study groups until the end of the study...placebo" }\end{array}$ \\
\hline
\end{tabular}
mance bias)

All outcomes

Blinding of outcome as- Low risk sessment (detection bias) All outcomes
Quote: "patients, researcher, laboratory staff and statistician were blinded to the study groups until the end of the study...placebo"

$\begin{array}{ll}\begin{array}{l}\text { Incomplete outcome data } \\ \text { (attrition bias) }\end{array} & \begin{array}{l}\text { Comment: there were post-randomisation dropouts; it is not clear whether } \\ \text { these were related to the intervention and to outcomes }\end{array}\end{array}$

All outcomes these were related to the intervention and to outcomes

\begin{tabular}{|c|c|c|}
\hline $\begin{array}{l}\text { Selective reporting (re- } \\
\text { porting bias) }\end{array}$ & High risk & $\begin{array}{l}\text { Comment: no previously published protocol was available; neither mortality } \\
\text { nor fatty liver resolution was reported }\end{array}$ \\
\hline
\end{tabular}

\begin{tabular}{ll}
\hline Other bias Low risk Comment: no other bias noted \\
\hline \hline
\end{tabular}


Sanyal 2010

\section{Study characteristics}

\begin{tabular}{ll}
\hline Methods & Randomised clinical trial \\
\hline Participants & Country: USA \\
& Period of recruitment: 2005 to 2007 \\
& Number randomised: 167 \\
& Post-randomisation dropouts: $0(0.0 \%)$ \\
& Revised sample size: 167 \\
& Average age, years: 46 \\
& Females: $148(88.6 \%)$ \\
& NASH: 167 (100.0\%) \\
& Diabetes mellitus: 0 (0.0\%) \\
& Inclusion criteria: 1. Definitive or possible steatohepatitis (histologically) \\
& Exclusion criteria: 1 . Alcohol consumption $(>30$ g/d for men, $>20$ g/d for women for at least 3 consec- \\
& utive months over past 5 years. 2. Cirrhosis 3. Hepatitis C or other liver disease. 4. Heart failure (NYHA \\
& Class II to IV). 5 . Drugs causing steatohepatitis \\
& Method for diagnosis of NAFLD: liver biopsy
\end{tabular}

\begin{tabular}{ll}
\hline Interventions & Group 1: vitamin E $(n=84)$ \\
& Further details: vitamin E 800 IU daily for 96 weeks \\
& Group 2: no active intervention $(n=83)$ \\
& Further details: placebo \\
& Additional details: another group not relevant to this review was excluded
\end{tabular}

$\begin{array}{ll}\text { Outcomes } & \text { Outcomes reported: mortality at maximal follow-up, serious adverse events (number of people), any } \\ \text { adverse events (number of events), decompensation (number of events), cirrhosis (number of people), } \\ \text { resolution of fatty liver disease, fibrosis score, NAFLD activity score } \\ \text { Follow-up, months: } 28\end{array}$

Notes Source of funding (quote): "several authors received consulting fees"

Trial name/Trial registry number: NCT00063622

Attempts were made to contact study authors in December 2020

\section{Risk of bias}

\begin{tabular}{lll}
\hline Bias & Authors' judgement & Support for judgement \\
\hline $\begin{array}{l}\text { Random sequence genera- } \\
\text { tion (selection bias) }\end{array}$ & Low risk & $\begin{array}{l}\text { Quote: "the randomization plan was prepared and administered centrally by } \\
\text { the Data Coordinating Center" }\end{array}$ \\
\hline $\begin{array}{l}\text { Allocation concealment } \\
\text { (selection bias) }\end{array}$ & Low risk & $\begin{array}{l}\text { Quote: "the randomization plan was prepared and administered centrally by } \\
\text { the Data Coordinating Center" }\end{array}$ \\
\hline $\begin{array}{l}\text { Blinding of participants } \\
\text { and personnel (perfor- } \\
\text { mance bias) }\end{array}$ & Low risk & $\begin{array}{l}\text { Quote: "multi-center, randomized, placebo-controlled, double-masked, dou- } \\
\text { ble-dummy clinical trial" }\end{array}$ \\
\hline $\begin{array}{l}\text { Blinding of outcome as- } \\
\text { sessment (detection bias) } \\
\text { All outcomes }\end{array}$ & Low risk & $\begin{array}{l}\text { Quote: "multi-center, randomized, placebo-controlled, double-masked, dou- } \\
\text { ble-dummy clinical trial" }\end{array}$ \\
\hline $\begin{array}{l}\text { lncomplete outcome data } \\
\text { (attrition bias) } \\
\text { All outcomes }\end{array}$ & Low risk & Comment: there were no post-randomisation dropouts \\
\hline
\end{tabular}


Sanyal 2010 (Continued)

Selective reporting (re- Low risk Comment: the protocol was published after recruitment was completed; adporting bias) verse events and either mortality or fatty liver resolution or both were reported

Other bias Low risk Comment: no other bias noted

Sanyal 2014

\section{Study characteristics}

\begin{tabular}{|c|c|c|}
\hline Methods & \multicolumn{2}{|c|}{ Randomised clinical trial } \\
\hline Participants & \multicolumn{2}{|c|}{$\begin{array}{l}\text { Country: USA } \\
\text { Period of recruitment: } 2011 \text { to } 2012 \\
\text { Number randomised: } 243 \\
\text { Post-randomisation dropouts: } 0 \text { (0.0\%) } \\
\text { Revised sample size: } 243 \\
\text { Average age, years: } 49 \\
\text { Females: } 148(60.9 \%) \\
\text { NASH: } 243(100.0 \%) \\
\text { Diabetes mellitus: } 85 \text { (35.0\%) } \\
\text { Inclusion criteria: } 1 \text {. Biopsy-confirmed NASH } \\
\text { Exclusion criteria: } 1 \text {. Cirrhosis. 2. Decompensated liver disease with ascites. 3. Encephalopathy or } \\
\text { variceal haemorrhage. } 4 \text {. ALT > } 300 \text { IU/L. } 5 \text {. Pregnancy or lactation. } 6 \text {. Serum creatinine }>2 \text { mg/dL. } 7 \text {. } \\
\text { Symptomatic coronary, peripheral, or neurovascular disease. 8. Symptomatic heart failure (NYHA Class } \\
\text { Il or higher). } 9 \text {. Prolonged QTC. } 9 \text {. Respiratory disease requiring oxygen therapy. 10. History of cerebral } \\
\text { or retinal haemorrhage or known bleeding disorder } \\
\text { Method for diagnosis of NAFLD: liver biopsy }\end{array}$} \\
\hline Interventions & \multicolumn{2}{|c|}{$\begin{array}{l}\text { Group 1: PUFA ( } n=168) \\
\text { Further details: ethyl-eicosapentaenoic acid } 1800 \mathrm{mg} \text { or } 2700 \mathrm{mg} \text { (randomly chosen) for } 12 \text { months } \\
\text { Group 2: no active intervention ( } \mathrm{n}=75) \\
\text { Further details: placebo }\end{array}$} \\
\hline Outcomes & \multicolumn{2}{|c|}{$\begin{array}{l}\text { Outcomes reported: mortality at maximal follow-up, serious adverse events (number of people), any } \\
\text { adverse events (number of events), fibrosis score, NAFLD activity score } \\
\text { Follow-up, months: } 12\end{array}$} \\
\hline Notes & \multicolumn{2}{|c|}{$\begin{array}{l}\text { Source of funding: not stated } \\
\text { Trial name/Trial registry number: NCT01154985 } \\
\text { Attempts were made to contact study authors in December } 2020\end{array}$} \\
\hline \multicolumn{3}{|l|}{ Risk of bias } \\
\hline Bias & Authors' judgement & Support for judgement \\
\hline $\begin{array}{l}\text { Random sequence genera- } \\
\text { tion (selection bias) }\end{array}$ & Low risk & $\begin{array}{l}\text { Quote: "interactive voice-response system" } \\
\text { Comment: although the precise method of random sequence generation was } \\
\text { not reported, the method of allocation concealment suggests that the se- } \\
\text { quence generation was random }\end{array}$ \\
\hline $\begin{array}{l}\text { Allocation concealment } \\
\text { (selection bias) }\end{array}$ & Low risk & Quote: "interactive voice-response system" \\
\hline
\end{tabular}


Sanyal 2014 (Continued)

Blinding of participants Low risk Quote: "prospective, double-blind, randomized, placebo-controlled trial" and personnel (performance bias)

All outcomes

\begin{tabular}{lll}
$\begin{array}{l}\text { Blinding of outcome as- } \\
\text { sessment (detection bias) } \\
\text { All outcomes }\end{array}$ & Low risk & Quote: "prospective, double-blind, randomized, placebo-c \\
\hline $\begin{array}{l}\text { Incomplete outcome data } \\
\text { (attrition bias) }\end{array}$ & Low risk & Comment: there were no post-randomisation dropouts \\
Alloutcomes &
\end{tabular}

All outcomes

$\begin{array}{lll}\begin{array}{l}\text { Selective reporting (re- } \\ \text { porting bias) }\end{array} & \text { Low risk } & \begin{array}{l}\text { Comment: no previously published protocol was available; adverse events and } \\ \text { either mortality or fatty liver resolution or both were reported }\end{array}\end{array}$

Other bias Low risk Comment: no other bias noted

Saxena 2013

\section{Study characteristics}

\begin{tabular}{|c|c|}
\hline Methods & Randomised clinical trial \\
\hline Participants & $\begin{array}{l}\text { Country: India } \\
\text { Period of recruitment: not stated } \\
\text { Number randomised: } 58 \\
\text { Post-randomisation dropouts: } 8(13.8 \%) \\
\text { Revised sample size: } 50 \\
\text { Reasons for post-randomisation dropouts: reasons not stated and breakdown of dropouts in each } \\
\text { group not given } \\
\text { Average age, years: not stated } \\
\text { Females: not stated } \\
\text { NASH: not stated } \\
\text { Diabetes mellitus: not stated } \\
\text { Inclusion criteria: } 1 \text {. NAFLD on USS with/without persistent elevation of serum ALT or AST. 2. Negative } \\
\text { viral markers } \\
\text { Exclusion criteria: not stated } \\
\text { Method for diagnosis of NAFLD: ultrasound }\end{array}$ \\
\hline
\end{tabular}

$\begin{array}{ll}\text { Interventions } & \text { Group 1: other supplements }(\mathrm{n}=26) \\ & \text { Further details: patients received an herbal compound MA 579 (Livomap), } 2 \text { tablets twice daily for } 4 \\ \text { months } & \\ \text { Group 2: no active intervention }(\mathrm{n}=24) \\ \text { Further details: patients received placebo, } 2 \text { tablets twice daily for } 4 \text { months } \\ \text { Additional details: both groups received lifestyle advice }\end{array}$

Outcomes

Outcomes reported: serious adverse events (number of people), serious adverse events (number of events), any adverse events (number of people), any adverse events (number of events)

Follow-up, months: 4

$\begin{array}{ll}\text { Notes } & \text { Source of funding: not stated } \\ \text { Trial name/Trial registry number: not stated } \\ \text { Attempts were made to contact study authors in December } 2020\end{array}$

\section{Risk of bias}

Nutritional supplementation for nonalcohol-related fatty liver disease: a network meta-analysis (Review) 
Saxena 2013 (Continued)

\section{Bias Authors' judgement Support for judgement}

Random sequence genera- Unclear risk Comment: this information was not available

tion (selection bias)

Allocation concealment Unclear risk Comment: this information was not available
(selection bias)

Blinding of participants Low risk Quote: "double-blind randomized placebo controlled clinical trial"
and personnel (perfor-
mance bias)
All outcomes

\begin{tabular}{lll}
\hline $\begin{array}{l}\text { Blinding of outcome as- } \\
\text { sessment (detection bias) } \\
\text { All outcomes }\end{array}$ & Quw risk & \\
\hline $\begin{array}{l}\text { Incomplete outcome data } \\
\text { (attrition bias) }\end{array}$ & Unclear risk & $\begin{array}{l}\text { Comment: there were post-randomisation dropouts, but it is not clear whether } \\
\text { these were related to the intervention or to outcomes }\end{array}$ \\
\hline
\end{tabular}

\begin{tabular}{lll}
\hline $\begin{array}{l}\text { Selective reporting (re- } \\
\text { porting bias) }\end{array}$ & High risk & $\begin{array}{l}\text { Comment: no previously published protocol was available; neither mortality } \\
\text { nor fatty liver resolution was reported }\end{array}$ \\
\hline Other bias & Low risk & Comment: no other bias noted \\
\hline
\end{tabular}

Sayari 2018

\section{Study characteristics}

\begin{tabular}{|c|c|}
\hline Methods & Randomised clinical trial \\
\hline Participants & $\begin{array}{l}\text { Country: Iran } \\
\text { Period of recruitment: } 2015 \text { to } 2017 \\
\text { Number randomised: } 140 \\
\text { Post-randomisation dropouts: } 2 \text { (1.4\%) } \\
\text { Revised sample size: } 138 \\
\text { Reasons for post-randomisation dropouts: loss to follow-up (1), refusal to continue (1) } \\
\text { Average age, years: } 43 \\
\text { Females: } 55 \text { (39.9\%) } \\
\text { NASH: not stated } \\
\text { Diabetes mellitus: not stated } \\
\text { Inclusion criteria: } 1 \text {. Aged } 18 \text { to } 60 \text { years. 2. BMI from } 25 \text { to } 29.9 .3 \text {. Impaired fasting blood glucose and/ } \\
\text { or impaired oral glucose tolerance test. } 4 \text {. New cases of NAFLD } \\
\text { Exclusion criteria: } 1 \text {. Other liver disease. 2. Alcohol consumption > } 10 \mathrm{~g} / \mathrm{d} \text { in women, > } 20 \mathrm{~g} / \mathrm{d} \text { in men. } \\
\text { 3. Presence of kidney disease, thyroid disorder, immunodeficiency disease, heart failure, on choles- } \\
\text { terol-lowering medication. } 4 \text {. Pregnancy, breastfeeding } \\
\text { Method for diagnosis of NAFLD: ultrasound plus transaminases }\end{array}$ \\
\hline Interventions & $\begin{array}{l}\text { Group 1: prebiotics/probiotics/synbiotics ( } \mathrm{n}=70 \text { ) } \\
\text { Further details: synbiotic ( } 500 \mathrm{mg} \text { ) once daily for } 16 \text { weeks. Each synbiotic capsule (Familakt) con- } \\
\text { tained } 109 \text { colony-forming units (CFUs) of } 7 \text { strains of friendly bacteria (Lactobacillus casei, Lactobacil- } \\
\text { lus rhamnosus, Lactobacillus acidophilus, Lactobacillus bulgaricus, Bifidobacterium breve, Bifidobacteri- } \\
\text { um longum, Streptococcus thermophilus) and prebiotic (fructo-oligosaccharide) and probiotic cultures } \\
\text { (magnesium stearate (source: mineral and vegetable), and a vegetable capsule (hydroxypropyl methyl- } \\
\text { cellulose)) }\end{array}$ \\
\hline
\end{tabular}


Sayari 2018 (Continued)

Group 2: no active intervention $(\mathrm{n}=68)$

Further details: placebo (maltodextrin) once daily for 16 weeks

Additional details: both groups received lifestyle advice and sitagliptin

\begin{tabular}{ll}
\hline Outcomes & No outcomes of interest were reported \\
\hline Notes & Source of funding: not stated \\
& Trial name/Trial registry number: ZMDRC approval number: A-12-500-13 \\
& Attempts were made to contact study authors in December 2020
\end{tabular}

\section{Risk of bias}

\begin{tabular}{lll}
\hline Bias & Authors' judgement & Support for judgement \\
\hline $\begin{array}{l}\text { Random sequence genera- } \\
\text { tion (selection bias) }\end{array}$ & Unclear risk & Comment: this information was not available \\
\hline $\begin{array}{l}\text { Allocation concealment } \\
\text { (selection bias) }\end{array}$ & Unclear risk & Comment: this information was not available \\
\hline $\begin{array}{l}\text { Blinding of participants } \\
\text { and personnel (perfor- } \\
\text { mance bias) }\end{array}$ & Low risk & Quote: "randomized, double blind trial...placebo" \\
All outcomes & \\
\hline
\end{tabular}

\begin{tabular}{lll}
$\begin{array}{l}\text { Blinding of outcome as- } \\
\text { sessment (detection bias) } \\
\text { All outcomes }\end{array}$ & Low risk & Quote: "randomized, double blind trial...placebo" \\
\hline $\begin{array}{l}\text { Incomplete outcome data } \\
\text { (attrition bias) } \\
\text { All outcomes }\end{array}$ & Unclear risk & $\begin{array}{l}\text { Comment: there were post-randomisation dropouts, but it is not clear whether } \\
\text { these were related to the intervention or to outcomes }\end{array}$ \\
\hline $\begin{array}{l}\text { Selective reporting (re- } \\
\text { porting bias) }\end{array}$ & High risk & $\begin{array}{l}\text { Comment: no previously published protocol was available; adverse events, } \\
\text { mortality, fatty liver resolution were not reported }\end{array}$ \\
\hline Other bias & Low risk & Comment: no other bias noted \\
\hline
\end{tabular}

Schattenberg 2017

\section{Study characteristics}

\begin{tabular}{ll}
\hline Methods & Randomised clinical trial \\
\hline Participants & Country: Germany \\
& Period of recruitment: not stated \\
& Number randomised: 29 \\
& Post-randomisation dropouts: $0(0.0 \%)$ \\
& Revised sample size: 29 \\
& Average age, years: 48 \\
& Females: $19(65.5 \%)$ \\
& NASH: 29 (100.0\%) \\
& Diabetes mellitus: not stated \\
& Inclusion criteria: 1 . Histologically confirmed NASH or a combination of M30 levels above 200 U/L and \\
& hepatic steatosis on ultrasound. 2. Aged 18 to 75 years \\
& Method for diagnosis of NAFLD: ultrasound plus M30 (biomarker of liver injury) or liver biopsy \\
\hline
\end{tabular}


Schattenberg 2017 (Continued)

$\begin{array}{ll}\text { Interventions } & \text { Group 1: no active intervention }(n=14) \\ & \text { Further details: no additional supplementation } \\ & \text { Group 2: prebiotics/probiotics/synbiotics }(n=15) \\ & \text { Further details: Lactobacillus caseiShirota plus } 2.1 \text { g soluble fibre twice daily (LCS) for } 12 \text { weeks } \\ & \text { Additional details: both groups received dietary advice }\end{array}$

\begin{tabular}{ll}
\hline Outcomes & $\begin{array}{l}\text { Outcomes reported: mortality at maximal follow-up, serious adverse events (number of people), any } \\
\text { adverse events (number of people), liver transplantation at maximal follow-up, decompensation (num- } \\
\text { ber of people), cirrhosis (number of people) } \\
\text { Follow-up, months: } 3\end{array}$ \\
\hline Notes & $\begin{array}{l}\text { Source of funding (quote): "this study was partly funded by H2020 under grant no. } 634413 \text { for the EPoS } \\
\text { projects and by Yakult Europe" } \\
\text { Trial name/Trial registry number: NCT02366052, NUCES NASH } \\
\text { Attempts were made to contact study authors in December } 2020\end{array}$
\end{tabular}

\section{Risk of bias}

\begin{tabular}{|c|c|c|}
\hline Bias & Authors' judgement & Support for judgement \\
\hline $\begin{array}{l}\text { Random sequence genera- } \\
\text { tion (selection bias) }\end{array}$ & Unclear risk & Comment: this information was not available \\
\hline $\begin{array}{l}\text { Allocation concealment } \\
\text { (selection bias) }\end{array}$ & Unclear risk & Comment: this information was not available \\
\hline $\begin{array}{l}\text { Blinding of participants } \\
\text { and personnel (perfor- } \\
\text { mance bias) } \\
\text { All outcomes }\end{array}$ & High risk & $\begin{array}{l}\text { Comment: because of the nature of another intervention (which was excluded } \\
\text { from this review), it is not possible to blind participants }\end{array}$ \\
\hline $\begin{array}{l}\text { Blinding of outcome as- } \\
\text { sessment (detection bias) } \\
\text { All outcomes }\end{array}$ & High risk & $\begin{array}{l}\text { Quote: "blinded lab analysis (author replies)" } \\
\text { Comment: assessors of clinical outcomes were not blinded }\end{array}$ \\
\hline $\begin{array}{l}\text { Incomplete outcome data } \\
\text { (attrition bias) } \\
\text { All outcomes }\end{array}$ & Low risk & Comment: there were no post-randomisation dropouts \\
\hline $\begin{array}{l}\text { Selective reporting (re- } \\
\text { porting bias) }\end{array}$ & Low risk & $\begin{array}{l}\text { Comment: no previously published protocol was available; adverse events and } \\
\text { either mortality or fatty liver resolution or both were reported }\end{array}$ \\
\hline Other bias & Low risk & Comment: no other bias noted \\
\hline
\end{tabular}

\section{Scorletti 2014}

\section{Study characteristics}

\begin{tabular}{ll}
\hline Methods & Randomised clinical trial \\
\hline Participants & Country: United Kingdom \\
& Period of recruitment: 2010 to 2011 \\
& Number randomised: 103 \\
& Post-randomisation dropouts: $0(0.0 \%)$ \\
& Revised sample size: 103 \\
& Average age, years: 51
\end{tabular}


Scorletti 2014 (Continued)

Females: 43 (41.7\%)

NASH: not stated

Diabetes mellitus: $10(9.7 \%)$

Inclusion criteria: 1 . Aged $>18$ years. 2. Recent histological diagnosis of NAFLD or steatosis diagnosed by ultrasound/CT/MRI in context of diabetes and/or metabolic syndrome

Exclusion criteria: 1 . Other cause of liver disease (e.g. viral hepatitis, Wilson's disease). 2. Alcohol consumption (> 35 units/week for women, $>50$ units/week for men). 3. Decompensated acute or chronic liver disease. 4. Cirrhosis. 5. Pregnancy or breastfeeding. 6. Hypersensitivity to Omacar or soya Method for diagnosis of NAFLD: liver biopsy or USS/CT/MRI

\begin{tabular}{|c|c|}
\hline Interventions & $\begin{array}{l}\text { Group 1: PUFA ( } n=51 \text { ) } \\
\text { Further details: } 4 \mathrm{~g} / \mathrm{d} \text { purified long chain omega-3 fatty acids (Omacor; DHA plus EPA) for } 15 \text { to } 18 \\
\text { months } \\
\text { Group 2: no active intervention }(n=52) \\
\text { Further details: placebo }\end{array}$ \\
\hline Outcomes & $\begin{array}{l}\text { Outcomes reported: mortality at maximal follow-up } \\
\text { Follow-up, months: } 15 \text { to } 18\end{array}$ \\
\hline Notes & $\begin{array}{l}\text { Source of funding (quote): "Omacor and placebo were provided by Pronova Biopharma through Abbott } \\
\text { Laboratories, Southampton, UK" } \\
\text { Trial name/Trial registry number: NCT00760513 } \\
\text { Attempts were made to contact study authors in December } 2020\end{array}$ \\
\hline
\end{tabular}

\section{Risk of bias}

\begin{tabular}{|c|c|c|}
\hline Bias & Authors' judgement & Support for judgement \\
\hline $\begin{array}{l}\text { Random sequence genera- } \\
\text { tion (selection bias) }\end{array}$ & Low risk & Quote: "computerized block randomisation" \\
\hline $\begin{array}{l}\text { Allocation concealment } \\
\text { (selection bias) }\end{array}$ & Low risk & $\begin{array}{l}\text { Quote: "patients were randomised according to standardized procedures } \\
\text { (computerized block randomisation) by a research pharmacist" }\end{array}$ \\
\hline $\begin{array}{l}\text { Blinding of participants } \\
\text { and personnel (perfor- } \\
\text { mance bias) } \\
\text { All outcomes }\end{array}$ & Low risk & Quote: "randomised double blind placebo controlled trial" \\
\hline $\begin{array}{l}\text { Blinding of outcome as- } \\
\text { sessment (detection bias) } \\
\text { All outcomes }\end{array}$ & Low risk & Quote: "randomised double blind placebo controlled trial" \\
\hline $\begin{array}{l}\text { Incomplete outcome data } \\
\text { (attrition bias) } \\
\text { All outcomes }\end{array}$ & Low risk & Comment: there were no post-randomisation dropouts \\
\hline $\begin{array}{l}\text { Selective reporting (re- } \\
\text { porting bias) }\end{array}$ & High risk & $\begin{array}{l}\text { Comment: no previously published protocol was available; adverse events } \\
\text { were not reported adequately }\end{array}$ \\
\hline Other bias & Low risk & Comment: no other bias noted \\
\hline
\end{tabular}

\section{Study characteristics}


Scorletti 2020 (Continued)

\begin{tabular}{ll} 
Methods & Randomised clinical trial \\
\hline Participants & Country: UK \\
& Period of recruitment: not stated \\
& Number randomised: 104 \\
& Post-randomisation dropouts: $15(14.4 \%)$ \\
& Revised sample size: 89 \\
& Reasons for post-randomisation dropouts: claustrophobia (5), family circumstances (1), personal cir- \\
& cumstances (2), relocation (2), non-compliance (2), taken over 3 courses of antibiotics (3) \\
& Average age, years: 51 \\
& Females: 31 (34.8\%) \\
& NASH: not stated \\
& Diabetes mellitus: 33 (37.1\%) \\
Inclusion criteria: 1 . Diagnosis of liver fat on normal clinical grounds with either histologic confirmation \\
of NAFLD or imaging evidence of liver fat with exclusion of other liver conditions causing liver fat accu- \\
mulation. 2. Alcohol consumption of < 14 units/week for women and < 21 units/week for men \\
Exclusion criteria: 1 . Abdominal surgery. 2 . Three or more courses of broad-spectrum antibiotics in the \\
previous year that may change gut microbiota. 3. Consumption of probiotic foods or supplements with- \\
in the 2 months preceding enrolment \\
Method for diagnosis of NAFLD: liver biopsy or imaging evidence of liver fat
\end{tabular}

Group 1: prebiotics/probiotics/synbiotics $(\mathrm{n}=45)$
Further details: synbiotic treatment, which consisted of fructo-oligosaccharides $4 \mathrm{~g}$ twice a day (2 sa-
chets a day, stirred into a cold drink) plus Bifidobacterium animalis subspecies lactis BB-12 at a mini-
mum of 10 billion colony-forming units/day (1 capsule a day) for 10 to 14 months
Group 2: no active intervention $(n=44)$
Further details: placebo, which consisted of $4 \mathrm{~g}$ twice a day of maltodextrin (1 capsule a day plus 2 sa-
chets a day, stirred into a cold drink) for 10 to 14 months

Outcomes Outcomes reported: mortality, liver transplantation, cirrhosis, decompensated cirrhosis, hepatocellular carcinoma, fibrosis score

Follow-up, months: 12

Notes

Source of funding (quote): "the synbiotic and placebo were provided at no cost by Chr. Hansen Holding. Chr. Hansen had no input into any aspect of study design or conduct of the trial. Furthermore, Chr. Hansen will have no input into data analysis or subsequent reporting of the trial results. The INSYTE trial was funded by the National Institute for Heath Research Southampton Biomedical Research Centre and JKS is Funded by the Wellcome Trust (Grant Number 206453/Z/17/Z)"

Trial name/Trial registry number: NCT01680640

Attempts were made to contact study authors in April 2021

\section{Risk of bias}

\begin{tabular}{lll}
\hline Bias & Authors' judgement & Support for judgement \\
\hline $\begin{array}{l}\text { Random sequence genera- } \\
\text { tion (selection bias) }\end{array}$ & Low risk & $\begin{array}{l}\text { Quote: "according to a list generated by the BRC Statistics and Data Manage- } \\
\text { ment group" (author replies) }\end{array}$ \\
\hline $\begin{array}{l}\text { Allocation concealment } \\
\text { (selection bias) }\end{array}$ & Low risk & $\begin{array}{l}\text { Quote: "by a person from within University Hospital Southampton or the Uni- } \\
\text { versity of Southampton who was not connected to the INSYTE study" (author } \\
\text { replies) }\end{array}$ \\
\hline $\begin{array}{l}\text { Blinding of participants } \\
\text { and personnel (perfor- } \\
\text { mance bias) } \\
\begin{array}{l}\text { All outcomes } \\
\text { Blinding of outcome as- } \\
\text { sessment (detection bias) }\end{array}\end{array}$ & Low risk & Quote: "double-blind phase 2 trial...placebo" \\
\hline
\end{tabular}


Scorletti 2020 (Continued)

All outcomes

\begin{tabular}{lll}
$\begin{array}{l}\text { Incomplete outcome data } \\
\text { (attrition bias) } \\
\text { All outcomes }\end{array}$ & High risk & $\begin{array}{l}\text { Comment: there were post-randomisation dropouts - differentially more in the } \\
\text { intervention group; some are likely to be related to the intervention }\end{array}$ \\
\hline $\begin{array}{l}\text { Selective reporting (re- } \\
\text { porting bias) }\end{array}$ & High risk & $\begin{array}{l}\text { Comment: no previously published protocol was available; adverse events } \\
\text { were not reported adequately }\end{array}$ \\
\hline Other bias & Low risk & Comment: no other bias noted \\
\hline
\end{tabular}

Sepideh 2016

\section{Study characteristics}

\begin{tabular}{|c|c|c|}
\hline Methods & \multicolumn{2}{|c|}{ Randomised clinical trial } \\
\hline Participants & \multicolumn{2}{|c|}{$\begin{array}{l}\text { Country: Iran } \\
\text { Period of recruitment: } 2013 \\
\text { Number randomised: } 50 \\
\text { Post-randomisation dropouts: } 8 \text { (16.0\%) } \\
\text { Revised sample size: } 42 \\
\text { Reasons for post-randomisation dropouts: withdrew } \\
\text { Average age, years: } 45 \\
\text { Females: } 14 \text { (33.3\%) } \\
\text { NASH: not stated } \\
\text { Diabetes mellitus: not stated } \\
\text { Inclusion criteria: } 1 \text {. Aged } 18 \text { to } 65 \text { years. 2. NAFLD } \\
\text { Exclusion: } 1 \text {. Other cause of liver disease (e.g. Wilson's disease, viral hepatitis, autoimmune disease). } 2 . \\
\text { Pregnancy or lactation. 3. Cardiovascular or kidney disease, haemochromatosis, or immunodeficiency. } \\
\text { 4. Antibiotics, probiotics, NSAIDs, or medicinal plant use in previous } 2 \text { months } \\
\text { Method for diagnosis of NAFLD: ultrasound }\end{array}$} \\
\hline Interventions & \multicolumn{2}{|c|}{$\begin{array}{l}\text { Group 1: prebiotics/probiotics/synbiotics }(n=21) \\
\text { Further details: } 2 \text { capsules/d probiotic (Lactocare) for } 8 \text { weeks } \\
\text { Group 2: no active intervention }(n=21) \\
\text { Further details: placebo }\end{array}$} \\
\hline Outcomes & \multicolumn{2}{|c|}{$\begin{array}{l}\text { Outcomes reported: serious adverse events (number of people) } \\
\text { Follow-up, months: } 2\end{array}$} \\
\hline Notes & \multicolumn{2}{|c|}{$\begin{array}{l}\text { Source of funding: not stated } \\
\text { Trial name/Trial registry number: IRCT2012122911920N1 } \\
\text { Attempts were made to contact study authors in December } 2020\end{array}$} \\
\hline \multicolumn{3}{|l|}{ Risk of bias } \\
\hline Bias & Authors' judgement & Support for judgement \\
\hline $\begin{array}{l}\text { Random sequence genera- } \\
\text { tion (selection bias) }\end{array}$ & Unclear risk & Comment: this information was not available \\
\hline $\begin{array}{l}\text { Allocation concealment } \\
\text { (selection bias) }\end{array}$ & Low risk & $\begin{array}{l}\text { Quote: "the placebo and probiotic were packaged in identical sealed boxes, } \\
\text { identified by a code number only" }\end{array}$ \\
\hline
\end{tabular}


Sepideh 2016 (Continued)

Blinding of participants Low risk Quote: "double-blind randomized clinical trial...placebo" and personnel (performance bias)

All outcomes

\begin{tabular}{lll}
\hline $\begin{array}{l}\text { Blinding of outcome as- } \\
\text { sessment (detection bias) } \\
\text { All outcomes }\end{array}$ & Low risk & Quote: "double-blind randomized clinical trial...placebo" \\
\hline $\begin{array}{l}\text { Incomplete outcome data } \\
\text { (attrition bias) } \\
\text { All outcomes }\end{array}$ & Unclear risk & $\begin{array}{l}\text { Comment: participants were excluded from analysis for reasons that may be } \\
\text { related to the intervention and to outcomes }\end{array}$ \\
\hline $\begin{array}{l}\text { Selective reporting (re- } \\
\text { porting bias) }\end{array}$ & High risk & $\begin{array}{l}\text { Comment: no previously published protocol was available; neither mortality } \\
\text { nor fatty liver resolution was reported }\end{array}$ \\
\hline \begin{tabular}{l} 
Other bias \\
\hline
\end{tabular} & Low risk & Comment: no other bias noted \\
\hline
\end{tabular}

Shahmohammadi 2017

\section{Study characteristics}

\begin{tabular}{|c|c|}
\hline Methods & Randomised clinical trial \\
\hline Participants & $\begin{array}{l}\text { Country: Iran } \\
\text { Period of recruitment: not stated } \\
\text { Number randomised: } 44 \\
\text { Post-randomisation dropouts: } 0(0.0 \%) \\
\text { Revised sample size: } 44 \\
\text { Average age, years: } 43 \\
\text { Females: } 22(50.0 \%) \\
\text { NASH: not stated } \\
\text { Diabetes mellitus: } 0(0.0 \%) \\
\text { Inclusion criteria: } 1 . \text { Age > } 20 \text { years. 2. NAFLD } \\
\text { Exclusion criteria: } 1 . \text { Hepatotoxic drugs. 2. Other forms of liver disease (e.g. viral hepatitis). 3. Metabolic } \\
\text { disease (e.g. diabetes). } 4 \text {. Pregnancy or lactation. 5. HRT. } 6 \text {. Other supplements in previous } 6 \text { months. } 7 . \\
\text { Bariatric surgery } \\
\text { Method for diagnosis of NAFLD: ultrasound }\end{array}$ \\
\hline
\end{tabular}

\begin{tabular}{|c|c|}
\hline Interventions & $\begin{array}{l}\text { Group 1: other supplements }(\mathrm{n}=22) \\
\text { Further details: green coffee bean extract } 1 \mathrm{~g} / \mathrm{d} \text { for } 8 \text { weeks } \\
\text { Group 2: no active intervention }(\mathrm{n}=22) \\
\text { Further details: placebo } \\
\text { Additional details: both groups received lifestyle advice }\end{array}$ \\
\hline Outcomes & $\begin{array}{l}\text { Outcomes reported: mortality at maximal follow-up } \\
\text { Follow-up, months: } 2\end{array}$ \\
\hline Notes & $\begin{array}{l}\text { Source of funding (quote): "this work was financially supported by Vice-Chancellor for Research Affairs } \\
\text { of Ahvaz Jundishapur University of Medical Sciences" } \\
\text { Trial name/Trial registry number: IRCT2016030626941N1 } \\
\text { Attempts were made to contact study authors in December } 2020\end{array}$ \\
\hline
\end{tabular}

\section{Risk of bias}


Shahmohammadi 2017 (Continued)

Random sequence genera- Low risk $\quad$ Quote: "computer-generated"
tion (selection bias)

Allocation concealment Low risk
(selection bias)

Quote: "a person, who was not aware of the nature of the trial, packed the sup(selection bias) plements and placebo capsules in numbered bottles based on the list. The other person who was not aware of random sequences allocated the patients to the numbered bottles"

Blinding of participants Low risk Quote: "parallel, double-blind, placebo-controlled clinical trial"
and personnel (perfor-
mance bias)
All outcomes

Blinding of outcome as-
$\begin{aligned} & \text { sessment (detection bias) } \\ & \text { All outcomes }\end{aligned}$

\begin{tabular}{lll}
\hline $\begin{array}{l}\text { Incomplete outcome data } \\
\text { (attrition bias) } \\
\text { All outcomes }\end{array}$ & Low risk & Comment: there were no post-randomisation dropouts \\
\hline $\begin{array}{l}\text { Selective reporting (re- } \\
\text { porting bias) }\end{array}$ & High risk & $\begin{array}{l}\text { Comment: no previously published protocol was available; adverse events } \\
\text { were not reported adequately }\end{array}$ \\
\hline Other bias & Low risk & Comment: no other bias noted \\
\hline
\end{tabular}

\section{Sharifi 2014}

\section{Study characteristics}

\begin{tabular}{|c|c|}
\hline Methods & Randomised clinical trial \\
\hline Participants & $\begin{array}{l}\text { Country: Iran } \\
\text { Period of recruitment: } 2012 \text { to } 2013 \\
\text { Number randomised: } 60 \\
\text { Post-randomisation dropouts: } 7 \text { (11.7\%) } \\
\text { Revised sample size: } 53 \\
\text { Reasons for post-randomisation dropouts: discontinued intervention, personal reasons } \\
\text { Average age, years: } 42 \\
\text { Females: } 27 \text { (50.9\%) } \\
\text { NASH: not stated } \\
\text { Diabetes mellitus: not stated } \\
\text { Inclusion criteria: } 1 \text {. Aged } 18 \text { to } 70 \text { years. } 2 \text {. Serum ALT > } 19 \text { U/L for women, } 30 \mathrm{U} / \mathrm{L} \text { for men } \\
\text { Exclusion criteria: } 1 \text {. Alcohol consumption > } 20 \mathrm{~g} / \mathrm{d} \text {. } 2 \text {. Pregnancy or lactation. 3. Other forms of hepat- } \\
\text { ic disease (e.g. haemochromatosis). } 4 \text {. History of bypass surgery. } 5 \text {. Total parenteral nutrition in past } 6 \\
\text { months. } 6 \text {. Hepatotoxic drugs. } 7 \text {. Intake of vitamin D/E or calcium in past } 6 \text { months } \\
\text { Method for diagnosis of NAFLD: ultrasound and transaminases }\end{array}$ \\
\hline Interventions & $\begin{array}{l}\text { Group 1: vitamin } D(n=27) \\
\text { Further details: } 50,000 \text { IU vitamin D3 (D-Vitin) every } 14 \text { days for } 4 \text { months } \\
\text { Group 2: no active intervention ( } n=26) \\
\text { Further details: placebo }\end{array}$ \\
\hline Outcomes & $\begin{array}{l}\text { Outcomes reported: resolution of fatty liver disease } \\
\text { Follow-up, months: } 4\end{array}$ \\
\hline
\end{tabular}


Sharifi 2014 (Continued)

Notes
Source of funding (quote): "this work was financially supported by a Grant (No. RDC-9105) from ViceChancellor for Research Affairs of Jundishapur University of Medical Sciences and approved by the Research Institute for Infectious Diseases of the Digestive System, Jundishapur University of Medical Sciences, Ahvaz, Iran"

Trial name/Trial registry number: IRCT2012071810333N1

Attempts were made to contact study authors in December 2020

\section{Risk of bias}

\section{Bias}

Authors' judgement Support for judgement

Random sequence genera- Low risk
tion (selection bias)

$\begin{array}{ll}\begin{array}{l}\text { Allocation concealment } \\ \text { (selection bias) }\end{array} & \begin{array}{l}\text { Quote: "an investigator with no clinical involvement in the trial packed the } \\ \text { supplements and placebos in numbered bottles based on the random list. The } \\ \text { other person, who was not involved in the trial and not aware of random se- } \\ \text { quences, assigned the patients to the numbered bottles of pearls" }\end{array}\end{array}$

Blinding of participants Low risk Quote: "parallel, double-blind, placebo-controlled study"
and personnel (perfor-
mance bias)
All outcomes

Blinding of outcome as-
sessment (detection bias) $\quad$ Low risk $\quad$ Quote: "parallel, double-blind, placebo-controlled study"

All outcomes

\begin{tabular}{ll}
\hline $\begin{array}{l}\text { Incomplete outcome data } \\
\text { (attrition bias) }\end{array}$ & Unclear risk \\
All outcomes & $\begin{array}{l}\text { Comment: participants were excluded from analysis for reasons that may be } \\
\text { related to the intervention and to outcomes }\end{array}$
\end{tabular}

\begin{tabular}{|c|c|c|}
\hline $\begin{array}{l}\text { Selective reporting (re- } \\
\text { porting bias) }\end{array}$ & High risk & $\begin{array}{l}\text { Comment: no previously published protocol was available; adverse events } \\
\text { were not reported adequately }\end{array}$ \\
\hline
\end{tabular}

\begin{tabular}{ll}
\hline Other bias Low risk $\quad$ Comment: no other bias noted \\
\hline
\end{tabular}

\section{Shavakhi 2013}

\section{Study characteristics}

\begin{tabular}{ll}
\hline Methods & Randomised clinical trial \\
\hline Participants & Country: Iran \\
& Period of recruitment: 2010 to 2012 \\
& Number randomised: 70 \\
& Post-randomisation dropouts: 7 (10.0\%) \\
& Revised sample size: 63 \\
& Reasons for post-randomisation dropouts: loss to follow-up, personal reasons \\
& Average age, years: 40 \\
& Females: 31 (49.2\%) \\
& NASH: 63 (100.0\%) \\
& Diabetes mellitus: not stated \\
& Inclusion criteria: 1 . Aged 18 to 75 years. 2. NAFLD
\end{tabular}


Exclusion criteria: 1. Other causes of liver disease (e.g. autoimmune hepatitis). 2. Insulin-dependent diabetes mellitus. 3. Pregnancy or lactation. 4. Impaired renal function. 5. Heart failure. 6. Hepatocellular carcinoma. 7. Hepatotoxic drugs

Method for diagnosis of NAFLD: liver biopsy

\begin{tabular}{|c|c|}
\hline Interventions & $\begin{array}{l}\text { Group 1: no active intervention ( } \mathrm{n}=32 \text { ) } \\
\text { Further details: placebo tablets (similar in shape and appearance to Protexin) } \\
\text { Group 2: prebiotics/probiotics/synbiotics ( } \mathrm{n}=31 \text { ) } \\
\text { Further details: probiotic (Protexin; made by Science and Nature in Balance Co., UK; contained Lac- } \\
\text { tobacillus acidophilus } 1 \times 10^{8} \mathrm{CFUs,} \mathrm{Lactobacillus} \mathrm{casei5} \times 10^{8} \mathrm{CFUs,} \text { Lactobacillus rhamnosus } 7.5 \times 10^{7} \\
\text { CFUs, Lactobacillus bulgaricus } 1.5 \times 10^{8} \mathrm{CFUs} \text {, Bifidobacterium breve } 5 \times 10^{7} \mathrm{CFUs,} \mathrm{Bifidobacterium} \\
\text { longum } 2.5 \times 10^{7} \mathrm{CFUs} \text {, Streptococcus thermophilus } 5 \times 10^{7} \mathrm{CFUs} \text {, fructo-oligosaccharides } 350 \mathrm{mg} \text { ), } 2 \\
\text { tablets per day for } 6 \text { months } \\
\text { Additional details: both groups received metformin }\end{array}$ \\
\hline
\end{tabular}

Outcomes

Outcomes reported: mortality at maximal follow-up, resolution of fatty liver disease

Follow-up, months: 6

\section{Notes}

Source of funding (quote): "source of support: Isfahan University of Medical Science Vice Chancellery for Research"

Trial name/Trial registry number: not stated

Attempts were made to contact study authors in December 2020

\section{Risk of bias}

\begin{tabular}{|c|c|c|}
\hline Bias & Authors' judgement & Support for judgement \\
\hline $\begin{array}{l}\text { Random sequence genera- } \\
\text { tion (selection bias) }\end{array}$ & Unclear risk & Comment: this information was not available \\
\hline $\begin{array}{l}\text { Allocation concealment } \\
\text { (selection bias) }\end{array}$ & Unclear risk & Comment: this information was not available \\
\hline $\begin{array}{l}\text { Blinding of participants } \\
\text { and personnel (perfor- } \\
\text { mance bias) } \\
\text { All outcomes }\end{array}$ & Low risk & Quote: "randomized double-blind clinical trial...placebo" \\
\hline $\begin{array}{l}\text { Blinding of outcome as- } \\
\text { sessment (detection bias) } \\
\text { All outcomes }\end{array}$ & Low risk & Quote: "randomized double-blind clinical trial...placebo" \\
\hline $\begin{array}{l}\text { Incomplete outcome data } \\
\text { (attrition bias) } \\
\text { All outcomes }\end{array}$ & Unclear risk & $\begin{array}{l}\text { Comment: participants were excluded from analysis for reasons that may be } \\
\text { related to the intervention and to outcomes }\end{array}$ \\
\hline $\begin{array}{l}\text { Selective reporting (re- } \\
\text { porting bias) }\end{array}$ & High risk & $\begin{array}{l}\text { Comment: no previously published protocol was available; adverse events } \\
\text { were not reported adequately }\end{array}$ \\
\hline Other bias & Low risk & Comment: no other bias noted \\
\hline
\end{tabular}

Soleimani 2020

\section{Study characteristics}

Methods Randomised clinical trial


Soleimani 2020 (Continued)

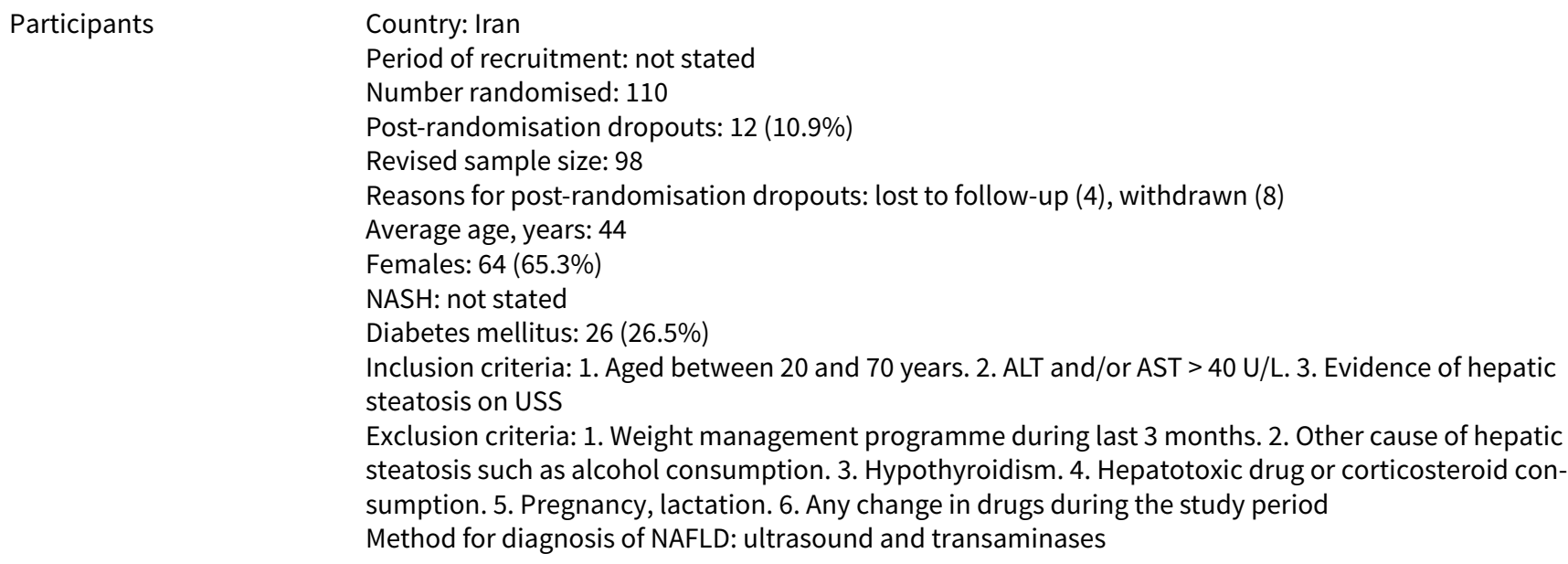

Outcomes

Outcomes reported: serious adverse events (number of people), any adverse events (number of people)

Follow-up, months: 4 fahan, Iran. The funder is not involved in the study design, data analysis and interpretation, or writing of the manuscript"

Trial name/Trial registry number: IRCT2014110819853N1

Attempts were made to contact study authors in April 2021

\section{Risk of bias}

\begin{tabular}{lll}
\hline Bias & Authors' judgement & Support for judgement \\
\hline $\begin{array}{l}\text { Random sequence genera- } \\
\text { tion (selection bias) }\end{array}$ & Unclear risk & Comment: this information was not available \\
\hline $\begin{array}{l}\text { Allocation concealment } \\
\text { (selection bias) }\end{array}$ & Low risk & $\begin{array}{l}\text { Quote: "the treatment assignment was concealed from the patients, investiga- } \\
\text { tors, staff, radiologist, and hepatologist throughout the study...placebo" } \\
\text { Comment: both allocation concealment and blinding were achieved with use } \\
\text { of placebo }\end{array}$ \\
\hline
\end{tabular}

$\begin{array}{ll}\begin{array}{l}\text { Blinding of participants } \\ \text { and personnel (perfor- }\end{array} & \text { Low risk } \\ \begin{array}{l}\text { mance bias) } \\ \text { All outcomes }\end{array} & \text { tors, staff, radiologist, and hepatologist throughout the study...placebo" }\end{array}$

\begin{tabular}{|c|c|c|}
\hline $\begin{array}{l}\text { Blinding of outcome as- } \\
\text { sessment (detection bias) }\end{array}$ & Low risk & $\begin{array}{l}\text { Quote: "the treatment assignment was concealed from the patients, investiga- } \\
\text { tors, staff, radiologist, and hepatologist throughout the study...placebo" }\end{array}$ \\
\hline
\end{tabular}

\begin{tabular}{l}
$\begin{array}{l}\text { Incomplete outcome data Unclear risk } \\
\text { (attrition bias) } \\
\text { All outcomes }\end{array}$ \\
\hline
\end{tabular}


Soleimani 2020 (Continued)

Selective reporting (re- High risk Comment: no previously published protocol was available; neither mortality porting bias) nor fatty liver resolution was reported

Other bias

Low risk

Comment: no other bias noted

Soleimani 2021

\section{Study characteristics}

\begin{tabular}{|c|c|}
\hline Methods & Randomised clinical trial \\
\hline Participants & $\begin{array}{l}\text { Country: Iran } \\
\text { Period of recruitment: } 2018 \\
\text { Number randomised: } 54 \\
\text { Post-randomisation dropouts: } 0 \text { (0.0\%) } \\
\text { Revised sample size: } 54 \\
\text { Average age, years: } 42 \\
\text { Females: } 16 \text { ( } 29.6 \%) \\
\text { NASH: not stated } \\
\text { Diabetes mellitus: } 3 \text { (5.6\%) } \\
\text { Inclusion criteria: } 1 \text {. Aged } 18 \text { to } 60 \text { years. 2. New diagnosis of hepatic steatosis with elastography tech- } \\
\text { nique } \\
\text { Exclusion criteria: } 1 \text {. History of alcohol abuse. 2. Presence of secondary cause of hepatic steatosis (such } \\
\text { as hepatitis B and C virus, autoimmune hepatitis, haemochromatosis, coeliac disease, hypopituitarism, } \\
\text { hypothyroidism, Wilson's disease, abetalipoproteinaemia, or corticosteroid medication). 3. Confound- } \\
\text { ing concomitant drug use (vitamin E, thiazolidinediones, glucagon-like peptide-1 analogs, exogenous } \\
\text { insulin, or ursodeoxycholic acid). } 4 \text {. Pregnancy, lactation. } 5 \text {. Aadverse reactions to honey bee products. } \\
6 \text {. Poor compliance with trial treatment (< } 80 \% \text { were withdrawn from the trial during the follow-up peri- } \\
\text { od) } \\
\text { Method for diagnosis of NAFLD: elastography }\end{array}$ \\
\hline Interventions & $\begin{array}{l}\text { Group 1: other supplements }(\mathrm{n}=27) \\
\text { Further details: patients in the propolis group received poplar propolis tablet ( } 450 \mathrm{mg} \text { containing } 250 \\
\mathrm{mg} \text { of freeze-dried ethanolic extract of Iranian propolis and } 200 \mathrm{mg} \text { of microcrystalline cellulose) twice } \\
\text { daily for } 4 \text { months. Each propolis tablet contains } 90 \mathrm{mg} \text { gallic acid equivalent and } 67 \mathrm{mg} \text { flavonoids } \\
\text { Group 2: no active intervention ( } \mathrm{n}=27) \\
\text { Further details: propolis-like placebo tablet ( } 442 \mathrm{mg} \text { of microcrystalline cellulose and } 8 \mathrm{mg} \text { of various } \\
\text { artificial food dyes) for } 4 \text { months } \\
\text { Additional details: all patients were advised to follow an energy-restricted diet }(\sim 250 \mathrm{kcal}) \text { containing } \\
30 \% \text { calories from fat, } 52 \% \text { from carbohydrate, and } 18 \% \text { from protein, along with } 150 \text { minutes/week of } \\
\text { moderate-intensity exercise such as walking and cycling }\end{array}$ \\
\hline Outcomes & $\begin{array}{l}\text { Outcomes reported: mortality at maximal follow-up, serious adverse events (number of people), any } \\
\text { adverse events (number of events), liver transplantation at maximal follow-up, decompensation (num- } \\
\text { ber of people), cirrhosis (number of people), hepatocellular carcinoma } \\
\text { Follow-up, months: } 4\end{array}$ \\
\hline Notes & $\begin{array}{l}\text { Source of funding (quote): "financial support was provided by Mashhad University of Medical Sciences, } \\
\text { Mashhad, Iran. The funder was not involved in the study design, data analysis and interpretation, or } \\
\text { writing of the manuscript" } \\
\text { Trial name/Trial registry number: IRCT20180824040857N1 } \\
\text { Attempts were made to contact study authors in April } 2021\end{array}$ \\
\hline
\end{tabular}

\section{Risk of bias}

\section{Bias}

Authors' judgement Support for judgement

Nutritional supplementation for nonalcohol-related fatty liver disease: a network meta-analysis (Review) 
Soleimani 2021 (Continued)

Random sequence genera- Low risk Quote: "computer-generated randomization sequence" tion (selection bias)

$\begin{array}{ll}\begin{array}{l}\text { Allocation concealment } \\ \text { (selection bias) }\end{array} & \text { Low risk } \\ & \begin{array}{l}\text { Quote: "the allocation sequence was concealed through consecutively num- } \\ \text { bered, opaque, sealed envelopes" }\end{array}\end{array}$

\begin{tabular}{|c|c|c|}
\hline $\begin{array}{l}\text { Blinding of participants } \\
\text { and personnel (perfor- } \\
\text { mance bias) } \\
\text { All outcomes }\end{array}$ & Low risk & $\begin{array}{l}\text { Quote: "except for the study pharmacist, who provided a randomization list } \\
\text { and sequentially numbered drug containers according to it, other investi- } \\
\text { gators and all patients were blinded from the study-group assignment. The } \\
\text { placebo tablet was identical in terms of shape, color, size, odor, and weight to } \\
\text { the propolis tablet. All tablets were dispensed in similar containers" }\end{array}$ \\
\hline
\end{tabular}

\begin{tabular}{|c|c|c|}
\hline $\begin{array}{l}\text { Blinding of outcome as- } \\
\text { sessment (detection bias) } \\
\text { All outcomes }\end{array}$ & Low risk & $\begin{array}{l}\text { Quote: "except for the study pharmacist, who provided a randomization list } \\
\text { and sequentially numbered drug containers according to it, other investiga- } \\
\text { tors and all patients were blinded from the study-group assignment" }\end{array}$ \\
\hline
\end{tabular}

Incomplete outcome data Low risk Comment: an intention-to-treat analysis was performed

(attrition bias)

All outcomes

\begin{tabular}{|c|c|c|}
\hline $\begin{array}{l}\text { Selective reporting (re- } \\
\text { porting bias) }\end{array}$ & Low risk & $\begin{array}{l}\text { Comment: no previously published protocol was available; adverse events and } \\
\text { either mortality or fatty liver resolution or both were reported }\end{array}$ \\
\hline
\end{tabular}

Other bias Low risk Comment: no other bias noted

Solhi 2014

\section{Study characteristics}

\begin{tabular}{|c|c|}
\hline Methods & Randomised clinical trial \\
\hline Participants & $\begin{array}{l}\text { Country: Iran } \\
\text { Period of recruitment: not stated } \\
\text { Number randomised: } 80 \\
\text { Post-randomisation dropouts: } 16(20.0 \%) \\
\text { Revised sample size: } 64 \\
\text { Reasons for post-randomisation dropouts: did not participate in follow-up } \\
\text { Average age, years: } 27 \\
\text { Females: } 16(25.0 \%) \\
\text { NASH: } 64(100.0 \%) \\
\text { Diabetes mellitus: } 0 \text { ( } 0.0 \%) \\
\text { Inclusion criteria: } 1 \text {. NASH confirmed on ultrasound. 2. Persistent elevation in ALT/AST > } 1.2 \times \text { ULN in } \\
\text { previous } 6 \text { months } \\
\text { Exclusion criteria: } 1 \text {. Other liver disease (e.g. autoimmune hepatitis). 2. Diabetes. 3. Severe cardiac, re- } \\
\text { nal, or pulmonary disease. } 4 \text {. Pregnancy. } 5 \text {. Alcohol consumption }>20 \mathrm{~g} \text { daily or substance abuse. } 6 \text {. He- } \\
\text { patotoxic drugs } \\
\text { Method for diagnosis of NAFLD: ultrasound plus transaminases }\end{array}$ \\
\hline Interventions & $\begin{array}{l}\text { Group 1: other antioxidants }(n=33) \\
\text { Further details: silymarin (Livergol) } 210 \mathrm{mg} / \mathrm{d} \text { orally for } 8 \text { weeks } \\
\text { Group 2: no active intervention }(\mathrm{n}=31) \\
\text { Further details: placebo } \\
\text { Additional details: both groups received lifestyle advice }\end{array}$ \\
\hline Outcomes & No outcomes of interest were reported \\
\hline
\end{tabular}


Solhi 2014 (Continued)

Notes
Source of funding (quote): "the project has been performed under financial support of research department of Arak's university of medical sciences, Arak, Iran"

Trial name/Trial registry number: IRCT201202159018N1

Attempts were made to contact study authors in December 2020

\section{Risk of bias}

\begin{tabular}{|c|c|c|}
\hline Bias & Authors' judgement & Support for judgement \\
\hline $\begin{array}{l}\text { Random sequence genera- } \\
\text { tion (selection bias) }\end{array}$ & Unclear risk & Comment: this information was not available \\
\hline $\begin{array}{l}\text { Allocation concealment } \\
\text { (selection bias) }\end{array}$ & Unclear risk & Comment: this information was not available \\
\hline $\begin{array}{l}\text { Blinding of participants } \\
\text { and personnel (perfor- } \\
\text { mance bias) } \\
\text { All outcomes }\end{array}$ & Unclear risk & Comment: this information was not available \\
\hline $\begin{array}{l}\text { Blinding of outcome as- } \\
\text { sessment (detection bias) } \\
\text { All outcomes }\end{array}$ & Unclear risk & Comment: this information was not available \\
\hline $\begin{array}{l}\text { Incomplete outcome data } \\
\text { (attrition bias) } \\
\text { All outcomes }\end{array}$ & Unclear risk & $\begin{array}{l}\text { Comment: participants were excluded from analysis for reasons that may be } \\
\text { related to the intervention and to outcomes }\end{array}$ \\
\hline $\begin{array}{l}\text { Selective reporting (re- } \\
\text { porting bias) }\end{array}$ & High risk & $\begin{array}{l}\text { Comment: no previously published protocol was available; adverse events, } \\
\text { mortality, fatty liver resolution were not reported }\end{array}$ \\
\hline Other bias & Low risk & Comment: no other bias noted \\
\hline
\end{tabular}

Somi 2014

\section{Study characteristics}

\begin{tabular}{ll}
\hline Methods & Randomised clinical trial \\
\hline Participants & Country: Iran \\
& Period of recruitment: 2012 to 2014 \\
& Number randomised: 80 \\
& Post-randomisation dropouts: 0 (0.0\%) \\
& Revised sample size: 80 \\
& Average age, years: 41 \\
& Females: 14 (17.5\%) \\
& NASH: not stated \\
& Diabetes mellitus: 0 (0.0\%) \\
& Inclusion criteria: 1. NAFLD on ultrasound \\
& Exclusion criteria: 1. Diabetic patients. 2. Other cause of raised liver enzymes (e.g. Wilson's disease). 3. \\
& Hepatotoxic drugs. 4 . Excessive alcohol consumption \\
& Method for diagnosis of NAFLD: ultrasound \\
& Group 1: amino acids ( $\mathrm{n}=40)$ \\
& Further details: L-carnitine 500 mg twice daily for 24 weeks \\
& Group 2: no active intervention ( $\mathrm{n}=40)$ \\
\hline Interventions &
\end{tabular}


Somi 2014 (Continued)

Further details: no treatment

Outcomes Outcomes reported: mortality at maximal follow-up, resolution of fatty liver disease

Follow-up, months: 6

$\begin{array}{ll}\text { Notes } & \text { Source of funding: not stated } \\ \text { Trial name/Trial registry number: N11RCT201102235893 } \\ \text { Attempts were made to contact study authors in December } 2020\end{array}$

\section{Risk of bias}

\section{Bias}

Authors' judgement Support for judgement

Random sequence genera- Unclear risk Comment: this information was not available

tion (selection bias)

Allocation concealment $\quad$ Unclear risk
(selection bias)

\begin{tabular}{lll}
\hline $\begin{array}{l}\text { Blinding of participants } \\
\text { and personnel (perfor- } \\
\text { mance bias) }\end{array}$ & Unclear risk & Comment: this information was not available \\
All outcomes & \\
\hline $\begin{array}{l}\text { Blinding of outcome as- } \\
\text { sessment (detection bias) } \\
\text { All outcomes }\end{array}$ & Low risk & $\begin{array}{l}\text { Quote: "sonography of patients in each group was done by radiologist who } \\
\text { was not aware of biochemical liver tests, with Siemens G40 and pruvconex, } \\
\text { and 3.46 MHz frequency after } 8 \text { hours fasting" } \\
\text { Comment: no clinical outcomes were reported in this trial; therefore, radiolo- } \\
\text { gist blinding indicates outcome assessor blinding }\end{array}$ \\
\hline
\end{tabular}

\begin{tabular}{lll}
\hline $\begin{array}{l}\text { Incomplete outcome data } \\
\text { (attrition bias) } \\
\text { All outcomes }\end{array}$ & Low risk & Comment: there were no post-randomisation dropouts \\
\hline $\begin{array}{l}\text { Selective reporting (re- } \\
\text { porting bias) }\end{array}$ & High risk & $\begin{array}{l}\text { Comment: no previously published protocol was available; adverse events } \\
\text { were not reported adequately }\end{array}$ \\
\hline Other bias & Low risk & Comment: no other bias noted \\
\hline
\end{tabular}

\section{Song 2020}

\section{Study characteristics}

\begin{tabular}{ll}
\hline Methods & Randomised clinical trial \\
\hline Participants & Country: China \\
& Period of recruitment: 2018 \\
& Number randomised: 96 \\
& Post-randomisation dropouts: 21 (21.9\%) \\
& Revised sample size: 75 \\
& Reasons for post-randomisation dropouts: withdrawal from study because of business trips, poor com- \\
& pliance \\
& Average age, years: 46 \\
& Females: 8 (10.7\%) \\
& NASH: not stated \\
& Diabetes mellitus: not stated
\end{tabular}


Song 2020 (Continued)

Inclusion criteria: 1. Adult participants (aged 30 to 67 years). 2. Average liver:spleen attenuation ratio $\leq 1.2$ or liver attenuation $\leq 52$ Hounsfield units, as determined by non-enhanced computerised tomography. 3. Dyslipidaemia (TC $\geq 5.2 \mathrm{mmol} / \mathrm{L}$, LDL cholesterol $\geq 3.36 \mathrm{mmol} / \mathrm{L}$, or TAG $\geq 1.7 \mathrm{mmol} / \mathrm{L}$ ). 4. Overweight $\left(\mathrm{BMI} \geq 24 \mathrm{~kg} / \mathrm{m}^{2}\right)$

Exclusion criteria: 1. Pregnancy. 2. Cardiovascular disease, cancer, disability, diabetes mellitus. 3. Excessive alcohol consumption ( $\geq 30 \mathrm{~g} / \mathrm{d}$ for men, $\geq 20 \mathrm{~g} / \mathrm{d}$ for women). 4. Hepatitis B or C or other liver disease. 5. Use of hypoglycaemic or lipid-regulating drugs (statins, fibrates) or other drugs that may impact glucose and lipid metabolism. 6. Intolerable adverse events from soya milk products. 8. Disease that impacts the participant's metabolism. 9. Hyperthyroidism, mental disorder, or disease associated with serious dysfunction of the heart, liver, or kidney

Method for diagnosis of NAFLD: CT scan plus abnormal serum lipid profile or high BMI

Interventions

Group 1: other supplements $(\mathrm{n}=16)$

Further details: phytosterol esters (PS)-enriched soyamilk powder containing $3.3 \mathrm{~g}$ of PS (Vegapure 67 WDP, equivalent to $2.5 \mathrm{~g}$ phytosterol in free form) and placebo capsules (vegetable oil blend: $30 \%$ palmitic acid, $40 \%$ oleic acid, and $20 \%$ linoleic acid obtained by blending rapeseed, sunflower and palm oils). Intervention lasted for 12 weeks

Group 2: PUFA plus other supplements $(n=17)$

Further details: PS-enriched soyamilk powder containing $3.3 \mathrm{~g}$ of PS plus fish oil capsules containing highly concentrated EPA and DHA (450 mg EPA plus $1500 \mathrm{mg}$ DHA). Intervention lasted for 12 weeks Group 3: PUFA ( $n=21)$

Further details: fish oil capsules containing highly concentrated EPA and DHA (PronovaPure 150:500TG, $450 \mathrm{mg}$ EPA $p 1500 \mathrm{mg}$ DHA) and placebo soyamilk powder. Intervention lasted for 12 weeks

Group 4: no active intervention $(n=21)$

Further details: placebo soyamilk and placebo capsule for 12 weeks

Additional details: no dietary intervention management was performed in the interest of achieving high compliance and isolating the effects of supplements used in the trial. Participants were not encouraged to specifically modify their lifestyles (including dietary habits) but were instructed to refrain from consuming supplements or other products claimed to reduce blood cholesterol

\begin{tabular}{ll} 
Outcomes & $\begin{array}{l}\text { Outcomes reported: serious adverse events (number of people), serious adverse events (number of } \\
\text { events), any adverse events (number of people), any adverse events (number of events) } \\
\text { Follow-up, months: } 3\end{array}$ \\
\hline Notes & $\begin{array}{l}\text { Source of funding (quote): "the authors gratefully acknowledge the kindly provider of intervention and } \\
\text { placebo products by company of Yanling Natural Hygiene Sdn. Bhd" } \\
\text { Trial name/Trial registry number: ChiCTR1800014419 } \\
\text { Attempts were made to contact study authors in April } 2021\end{array}$
\end{tabular}

\section{Risk of bias}

\begin{tabular}{|c|c|c|}
\hline Bias & Authors' judgement & Support for judgement \\
\hline $\begin{array}{l}\text { Random sequence genera- } \\
\text { tion (selection bias) }\end{array}$ & Low risk & $\begin{array}{l}\text { Quote: "randomisation was conducted using computer-generated random } \\
\text { numbers by a trained staff member at the physical examination centre" }\end{array}$ \\
\hline $\begin{array}{l}\text { Allocation concealment } \\
\text { (selection bias) }\end{array}$ & Low risk & $\begin{array}{l}\text { Quote: "all participants, care providers and outcome assessors were blinded to } \\
\text { the treatment allocations...placebo" } \\
\text { Comment: both allocation concealment and blinding were achieved with use } \\
\text { of placebo }\end{array}$ \\
\hline $\begin{array}{l}\text { Blinding of participants } \\
\text { and personnel (perfor- } \\
\text { mance bias) } \\
\text { All outcomes }\end{array}$ & Low risk & $\begin{array}{l}\text { Quote: "all participants, care providers and outcome assessors were blinded to } \\
\text { the treatment allocations...placebo" }\end{array}$ \\
\hline $\begin{array}{l}\text { Blinding of outcome as- } \\
\text { sessment (detection bias) } \\
\text { All outcomes }\end{array}$ & Low risk & $\begin{array}{l}\text { Quote: "all participants, care providers and outcome assessors were blinded to } \\
\text { the treatment allocations...placebo" }\end{array}$ \\
\hline
\end{tabular}


Song 2020 (Continued)

Incomplete outcome data Unclear risk Comment: there were post-randomisation dropouts; it is not clear whether (attrition bias)

All outcomes

\begin{tabular}{|c|c|c|}
\hline $\begin{array}{l}\text { Selective reporting (re- } \\
\text { porting bias) }\end{array}$ & High risk & $\begin{array}{l}\text { Comment: no previously published protocol was available; neither mortality } \\
\text { nor fatty liver resolution was reported }\end{array}$ \\
\hline
\end{tabular}

Other bias Low risk Comment: no other bias noted

Spadaro 2008

\section{Study characteristics}

\begin{tabular}{|c|c|}
\hline Methods & Randomised clinical trial \\
\hline Participants & $\begin{array}{l}\text { Country: Italy } \\
\text { Period of recruitment: not stated } \\
\text { Number randomised: } 40 \\
\text { Post-randomisation dropouts: } 4(10.0 \%) \\
\text { Revised sample size: } 36 \\
\text { Reasons for post-randomisation dropouts: loss to follow-up, non-compliance } \\
\text { Average age, years: } 51 \\
\text { Females: } 17 \text { ( } 47.2 \%) \\
\text { NASH: not stated } \\
\text { Diabetes mellitus: not stated } \\
\text { Inclusion criteria: } 1 \text {. NAFLD } \\
\text { Exclusion criteria: } 1 \text {. Previous PUFA therapy within } 3 \text { months. 2. Inflammatory disease (e.g. IBD, autoim- } \\
\text { mune disease). } 3 \text {. Malignancy. } 4 \text {. Pregnancy } \\
\text { Method for diagnosis of NAFLD: ultrasound plus transaminases }\end{array}$ \\
\hline Interventions & $\begin{array}{l}\text { Group 1: PUFA }(n=18) \\
\text { Further details: polyunsaturated fatty acid } 2 \mathrm{~g} / \mathrm{d} \text { for } 6 \text { months } \\
\text { Group 2: no active intervention }(n=18) \\
\text { Further details: no treatment } \\
\text { Additional details: both groups received modified diet }\end{array}$ \\
\hline Outcomes & $\begin{array}{l}\text { Outcomes reported: resolution of fatty liver disease } \\
\text { Follow-up, months: } 6\end{array}$ \\
\hline Notes & $\begin{array}{l}\text { Source of funding: not stated } \\
\text { Trial name/Trial registry number: not stated } \\
\text { Attempts were made to contact study authors in December } 2020\end{array}$ \\
\hline
\end{tabular}

\section{Risk of bias}

\begin{tabular}{lll}
\hline Bias & Authors' judgement & Support for judgement \\
\hline $\begin{array}{l}\text { Random sequence genera- } \\
\text { tion (selection bias) }\end{array}$ & Low risk & Quote: "random sampling numbers" \\
\hline $\begin{array}{l}\text { Allocation concealment } \\
\text { (selection bias) }\end{array}$ & Unclear risk & Comment: this information was not available \\
\hline $\begin{array}{l}\text { Blinding of participants } \\
\text { and personnel (perfor- } \\
\text { mance bias) }\end{array}$ & Unclear risk & Comment: this information was not available \\
\hline
\end{tabular}


Spadaro 2008 (Continued)

All outcomes

Blinding of outcome as-
sessment (detection bias) $\quad$ Unclear risk Comment: this information was not available
sessment (detection bias) All outcomes

\begin{tabular}{|c|c|c|}
\hline ncomplete outcome data & Unclear risk & $\begin{array}{l}\text { Comment: participants were excluded from analysis for reasons that may be } \\
\text { related to the intervention and to outcomes }\end{array}$ \\
\hline
\end{tabular}

All outcomes

\begin{tabular}{|c|c|c|}
\hline $\begin{array}{l}\text { Selective reporting (re- } \\
\text { porting bias) }\end{array}$ & High risk & $\begin{array}{l}\text { Comment: no previously published protocol was available; adverse events } \\
\text { were not reported adequately }\end{array}$ \\
\hline
\end{tabular}

\begin{tabular}{ll}
\hline Other bias Low risk $\quad$ Comment: no other bias noted \\
\hline
\end{tabular}

Tabatabaee 2017

\section{Study characteristics}

\begin{tabular}{|c|c|c|}
\hline Methods & \multicolumn{2}{|c|}{ Randomised clinical trial } \\
\hline Participants & \multicolumn{2}{|c|}{$\begin{array}{l}\text { Country: Iran } \\
\text { Period of recruitment: not stated } \\
\text { Number randomised: } 67 \\
\text { Post-randomisation dropouts: } 22(32.8 \%) \\
\text { Revised sample size: } 45 \\
\text { Reasons for post-randomisation dropouts: did not receive allocated intervention, lost to follow-up, dis- } \\
\text { continued intervention } \\
\text { Average age, years: } 40 \\
\text { Females: } 40 \text { ( } 88.9 \%) \\
\text { NASH: not stated } \\
\text { Diabetes mellitus: } 14 \text { (31.1\%) } \\
\text { Inclusion criteria: } 1 \text {. NAFLD } \\
\text { Exclusion criteria: } 1 \text {. Iron deficiency anaemia. 2. Green tea allergy. 3. Alcohol consumption (> } 20 \text { g daily). } \\
\text { 4. Other liver disorder (e.g. autoimmune hepatitis). 5. Pregnancy or lactation } \\
\text { Method for diagnosis of NAFLD: ultrasound, elastography, or liver biopsy }\end{array}$} \\
\hline Interventions & \multicolumn{2}{|c|}{$\begin{array}{l}\text { Group 1: other supplements }(n=21) \\
\text { Further details: green tea } 550 \mathrm{mg} \text { daily for } 3 \text { months } \\
\text { Group 2: no active intervention }(\mathrm{n}=24) \\
\text { Further details: placebo } \\
\text { Additional details: both groups received lifestyle advice }\end{array}$} \\
\hline Outcomes & \multicolumn{2}{|c|}{ No outcomes of interest were reported } \\
\hline Notes & \multicolumn{2}{|c|}{$\begin{array}{l}\text { Source of funding: not stated } \\
\text { Trial name/Trial registry number: IRCT201404332365N8 } \\
\text { Attempts were made to contact study authors in December } 2020\end{array}$} \\
\hline \multicolumn{3}{|l|}{ Risk of bias } \\
\hline Bias & Authors' judgement & Support for judgement \\
\hline $\begin{array}{l}\text { Random sequence genera- } \\
\text { tion (selection bias) }\end{array}$ & Low risk & Quote: "list already generated using a random number sequence" \\
\hline
\end{tabular}


Tabatabaee 2017 (Continued)

$\begin{array}{ll}\begin{array}{l}\text { Allocation concealment } \\ \text { (selection bias) }\end{array} & \text { Low risk } \\ \end{array}$

Blinding of participants Low risk Quote: "double blind clinical trial...placebo"

and personnel (perfor-

mance bias)

All outcomes

Blinding of outcome as-
sessment (detection bias) $\quad$ Low risk $\quad$ Quote: "double blind clinical trial...placebo"

sessment (detection bias)

All outcomes

\begin{tabular}{|c|c|c|}
\hline $\begin{array}{l}\text { Incomplete outcome data } \\
\text { (attrition bias) }\end{array}$ & Unclear risk & $\begin{array}{l}\text { Comment: participants were excluded from analysis for reasons that may be } \\
\text { related to the intervention and to outcomes }\end{array}$ \\
\hline
\end{tabular}

All outcomes

Selective reporting (re- High risk Comment: no previously published protocol was available; adverse events, porting bias) mortality, fatty liver resolution were not reported

\begin{tabular}{ll}
\hline Other bias Low risk Comment: no other bias noted \\
\hline
\end{tabular}

\section{Taghvaei 2018}

\section{Study characteristics}

\begin{tabular}{|c|c|}
\hline Methods & Randomised clinical trial \\
\hline Participants & $\begin{array}{l}\text { Country: Iran } \\
\text { Period of recruitment: not stated } \\
\text { Number randomised: } 40 \\
\text { Post-randomisation dropouts: } 0(0.0 \%) \\
\text { Revised sample size: } 40 \\
\text { Average age, years: } 43 \\
\text { Females: } 20 \text { (50.0\%) } \\
\text { NASH: not stated } \\
\text { Diabetes mellitus: } 0 \text { (0.0\%) } \\
\text { Inclusion criteria: } 1 \text {. NAFLD } \\
\text { Exclusion criteria: } 1 \text {. Liver cirrhosis. 2. Pregnancy or lactation. 3. Alcohol consumption. 4. Drug abuse. } \\
\text { 5. Use of vitamins in past } 6 \text { months. 7. Diabetes mellitus. 8. Chronic kidney disease. 9. Hypercalcaemia. } \\
\text { 10. End-stage heart and lung disease. 11. Use of hepatotoxic medication } \\
\text { Method for diagnosis of NAFLD: elastography plus transaminases }\end{array}$ \\
\hline Interventions & $\begin{array}{l}\text { Group 1: vitamin } D(n=20) \\
\text { Further details: } 50,000 \text { IU vitamin D3 weekly for } 12 \text { weeks } \\
\text { Group 2: no active intervention }(n=20) \\
\text { Further details: no further details }\end{array}$ \\
\hline Outcomes & $\begin{array}{l}\text { Outcomes reported: mortality at maximal follow-up } \\
\text { Follow-up, months: } 6\end{array}$ \\
\hline Notes & $\begin{array}{l}\text { Source of funding: not stated } \\
\text { Trial name/Trial registry number: IRCT2015102624725N1 } \\
\text { Attempts were made to contact study authors in December } 2020\end{array}$ \\
\hline
\end{tabular}

\section{Risk of bias}


Taghvaei 2018 (Continued)

\begin{tabular}{|c|c|c|}
\hline Bias & Authors' judgement & Support for judgement \\
\hline $\begin{array}{l}\text { Random sequence genera- } \\
\text { tion (selection bias) }\end{array}$ & Low risk & Quote: "random number table" \\
\hline $\begin{array}{l}\text { Allocation concealment } \\
\text { (selection bias) }\end{array}$ & Unclear risk & Comment: this information was not available \\
\hline $\begin{array}{l}\text { Blinding of participants } \\
\text { and personnel (perfor- } \\
\text { mance bias) } \\
\text { All outcomes }\end{array}$ & Unclear risk & $\begin{array}{l}\text { Quote: "randomized, double-blind, parallel-group, clinical trial" } \\
\text { Comment: no placebo was used; therefore, it is not clear how blinding was } \\
\text { achieved }\end{array}$ \\
\hline $\begin{array}{l}\text { Blinding of outcome as- } \\
\text { sessment (detection bias) } \\
\text { All outcomes }\end{array}$ & Unclear risk & $\begin{array}{l}\text { Quote: "randomized, double-blind, parallel-group, clinical trial" } \\
\text { Comment: no placebo was used; therefore, it is not clear how blinding was } \\
\text { achieved }\end{array}$ \\
\hline $\begin{array}{l}\text { Incomplete outcome data } \\
\text { (attrition bias) } \\
\text { All outcomes }\end{array}$ & Low risk & Comment: there were no post-randomisation dropouts \\
\hline $\begin{array}{l}\text { Selective reporting (re- } \\
\text { porting bias) }\end{array}$ & High risk & $\begin{array}{l}\text { Comment: no previously published protocol was available; adverse events } \\
\text { were not reported adequately }\end{array}$ \\
\hline Other bias & Low risk & Comment: no other bias noted \\
\hline
\end{tabular}

Tan 2011

\section{Study characteristics}

\begin{tabular}{ll}
\hline Methods & Randomised clinical trial \\
\hline Participants & Country: China \\
& Period of recruitment: not stated \\
& Number randomised: 15 \\
& Post-randomisation dropouts: not stated \\
& Revised sample size: 15 \\
& Average age, years: 46 \\
& Females: 1 (6.7\%) \\
& NASH: 15 (100.0\%) \\
& Diabetes mellitus: not stated \\
& Inclusion criteria: 1 . Biopsy-proven NASH \\
& Exclusion criteria: not stated \\
& Method for diagnosis of NAFLD: liver biopsy \\
\end{tabular}

Interventions Group 1: phospholipids $(\mathrm{n}=10)$

Further details: essential phospholipids $1800 \mathrm{mg} / \mathrm{d}$ for 6 months

Group 2: no active intervention $(n=5)$

Further details: no treatment

Additional details: both groups received lifestyle intervention

\begin{tabular}{ll}
\hline Outcomes & No outcomes of interest were reported \\
\hline Notes & Source of funding: not stated \\
& Trial name/Trial registry number: not stated \\
& Attempts were made to contact study authors in December 2020
\end{tabular}


Tan 2011 (Continued)

Risk of bias

\begin{tabular}{|c|c|c|}
\hline Bias & Authors' judgement & Support for judgement \\
\hline $\begin{array}{l}\text { Random sequence genera- } \\
\text { tion (selection bias) }\end{array}$ & Unclear risk & Comment: this information was not available \\
\hline $\begin{array}{l}\text { Allocation concealment } \\
\text { (selection bias) }\end{array}$ & Unclear risk & Comment: this information was not available \\
\hline $\begin{array}{l}\text { Blinding of participants } \\
\text { and personnel (perfor- } \\
\text { mance bias) } \\
\text { All outcomes }\end{array}$ & Unclear risk & Comment: this information was not available \\
\hline $\begin{array}{l}\text { Blinding of outcome as- } \\
\text { sessment (detection bias) } \\
\text { All outcomes }\end{array}$ & Unclear risk & Comment: this information was not available \\
\hline $\begin{array}{l}\text { Incomplete outcome data } \\
\text { (attrition bias) } \\
\text { All outcomes }\end{array}$ & Unclear risk & Comment: this information was not available \\
\hline $\begin{array}{l}\text { Selective reporting (re- } \\
\text { porting bias) }\end{array}$ & High risk & $\begin{array}{l}\text { Comment: no previously published protocol was available; adverse events, } \\
\text { mortality, fatty liver resolution were not reported }\end{array}$ \\
\hline Other bias & Low risk & Comment: no other bias noted \\
\hline
\end{tabular}

Tobin 2018

\section{Study characteristics}

\begin{tabular}{|c|c|}
\hline Methods & Randomised clinical trial \\
\hline Participants & 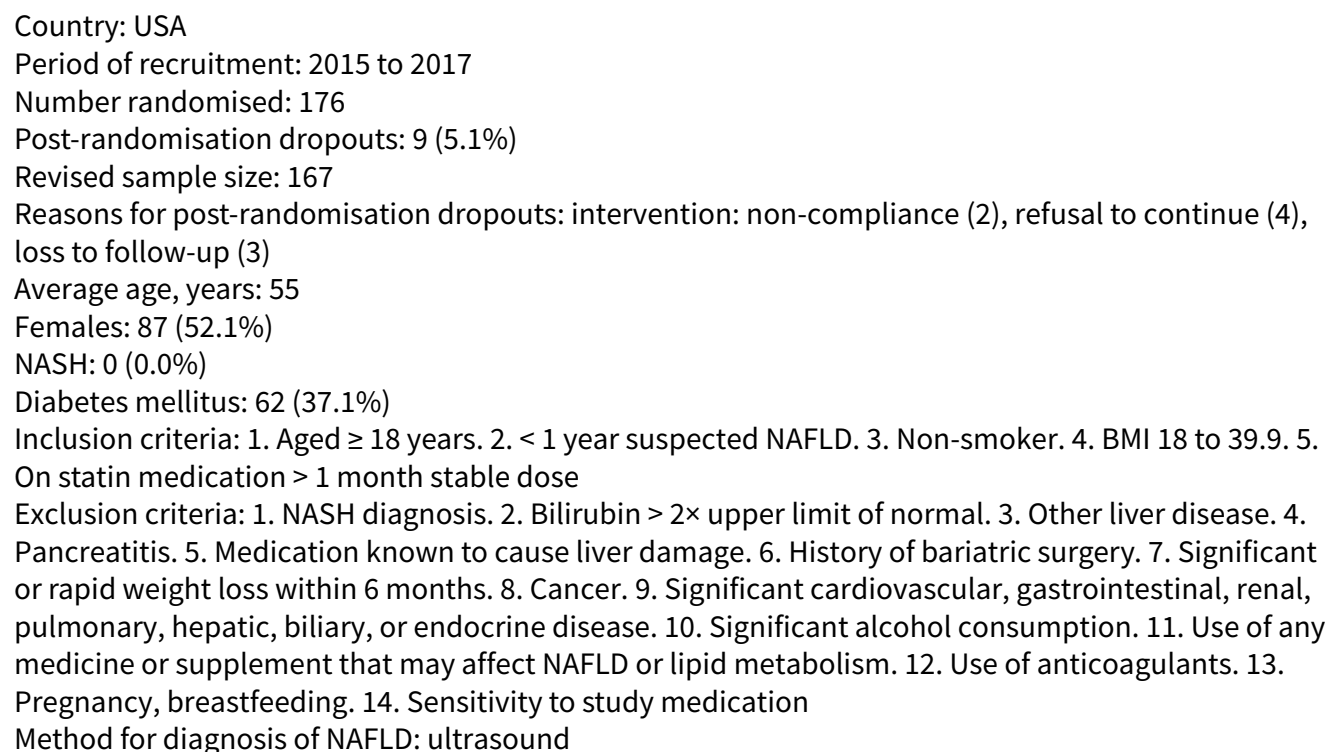 \\
\hline
\end{tabular}


Tobin 2018 (Continued)

Further details: 3 placebo (olive oil) capsules per day for 24 weeks. Placebo capsules were identical in size and appearance to MF4637 and contained $1 \mathrm{~g}$ of olive oil

Group 2: PUFA ( $n=81$ )

Further details: omega-3 concentrate MF4637, 3 capsules daily, for 24 weeks. Omega-3 fatty acid medical food (MF4637; BASF AS, Lysaker, Norway) was provided as soft gel capsules, with each 1-gram capsule containing marine-sourced EPA and DHA as ethyl esters (460 mg and $380 \mathrm{mg}$, respectively) Additional details: both groups received lifestyle advice

\begin{tabular}{ll}
\hline Outcomes & No outcomes of interest were reported \\
\hline Notes & Source of funding (quote): "funding: this study was funded by BASF AS. Conflicts of Interest: D.T. and \\
& Y.Q. are employees of BASF AS; M.B.A. is a former employee of BASF AS; P.C.C. is an advisor to BASF AS" \\
& Trial name/Trial registry number: NCT02923804 \\
& Attempts were made to contact study authors in December 2020 \\
\hline
\end{tabular}

\section{Risk of bias}

\begin{tabular}{lll}
\hline Bias & Authors' judgement & Support for judgement \\
\hline $\begin{array}{l}\text { Random sequence genera- } \\
\text { tion (selection bias) }\end{array}$ & Low risk & $\begin{array}{l}\text { Quote: "randomization numbers corresponding to predetermined interven- } \\
\text { tion were assigned in a sequential manner to each subject via an Interactive } \\
\text { Voice/Web Response System" }\end{array}$ \\
& &
\end{tabular}

\begin{tabular}{ll}
\hline $\begin{array}{l}\text { Allocation concealment } \\
\text { (selection bias) }\end{array}$ & Low risk \\
& $\begin{array}{l}\text { Quote: "each participant was centrally randomized. Randomization numbers } \\
\text { corresponding to predetermined intervention were assigned in a sequential } \\
\text { manner to each subject via an Interactive Voice/Web Response System" }\end{array}$
\end{tabular}

\begin{tabular}{|c|c|c|}
\hline $\begin{array}{l}\text { Blinding of participants } \\
\text { and personnel (perfor- }\end{array}$ & Low risk & $\begin{array}{l}\text { Quote: "the investigational products were administered in a double-blinded } \\
\text { fashion...placebo" }\end{array}$ \\
\hline
\end{tabular}
mance bias)

All outcomes

\begin{tabular}{lll}
\hline $\begin{array}{l}\text { Blinding of outcome as- } \\
\text { sessment (detection bias) } \\
\text { All outcomes }\end{array}$ & Low risk & $\begin{array}{l}\text { Quote: "the investigational products were administered in a double-blinded } \\
\text { fashion...placebo" }\end{array}$ \\
\hline $\begin{array}{l}\text { Incomplete outcome data } \\
\text { (attrition bias) } \\
\text { All outcomes }\end{array}$ & Unclear risk & $\begin{array}{l}\text { Comment: there were post-randomisation dropouts, but it is not clear whether } \\
\text { these were related to the intervention or to outcomes }\end{array}$ \\
\hline $\begin{array}{l}\text { Selective reporting (re- } \\
\text { porting bias) }\end{array}$ & High risk & $\begin{array}{l}\text { Comment: a published protocol was available, but recruitment had com- } \\
\text { menced before the protocol was published; adverse events, mortality, fatty liv- } \\
\text { er resolution were not reported }\end{array}$
\end{tabular}

Other bias Unclear risk Comment: there were baseline differences in important prognostic factors

Tutunchi 2020

\section{Study characteristics}

\begin{tabular}{ll}
\hline Methods & Randomised clinical trial \\
\hline Participants & Country: Iran \\
& Period of recruitment: 2019 \\
& Number randomised: 76 \\
& Post-randomisation dropouts: $0(0.0 \%)$ \\
& Revised sample size: 76 \\
\hline
\end{tabular}


Tutunchi 2020 (Continued)

Average age, years: 41

Females: $36(47.4 \%)$

NASH: not stated

Diabetes mellitus: not stated

Inclusion criteria: 1. Obese. 2. Newly diagnosed NAFLD. 3. Body mass index (BMI) 30 to $40 \mathrm{~kg} / \mathrm{m}^{2} .4$.

Aged 21 to 59 years

Exclusion criteria: 1. Liver disease such as hepatitis, cirrhosis, biliary disorder, inherited disorder affecting the liver. 2. Diabetes, hypertension, cardiovascular disorder, kidney dysfunction, thyroid problem, gastrointestinal disorder, pulmonary and autoimmune disease, malignancy, recent surgery. 3. Alcoholic, smoker, tobacco consumer. 4. Pregnancy or lactation. 5. Weight-loss programme within 3 months prior to the study. 6 . Use of lipid-lowering drugs, weight loss drugs, corticosteroids, hepatotoxic drugs, anticoagulants, antidiuretics, multi-vitamins, minerals, and any dietary supplements during past 3 months

Method for diagnosis of NAFLD: ultrasound

Group 1: other supplements $(n=38)$
Further details: 2 capsules of oleoylethanolamide per day for 12 weeks. OEA capsules contained 125 mg
OEA
Group 2: no active intervention $(n=38)$
Further details: 2 capsules of placebo per day for 12 weeks. Placebo capsules contained 125 mg starch
and were similar in appearance, size, and colour to OEA capsules
Additional details: all patients were given weight loss diet and physical activity recommendations

$\begin{array}{ll}\text { Outcomes } & \text { Outcomes reported: serious adverse events (number of people), serious adverse events (number of } \\ \text { events), resolution of fatty liver disease }\end{array}$
Follow-up, months: 3

Notes

Source of funding (quote): "the study was financially supported by the Nutrition Research Center of Tabriz University of Medical Sciences, and Iran National Science Foundation (INSF)"

Trial name/Trial registry number: IRCT20110530006652N2

Attempts were made to contact study authors in April 2021

\section{Risk of bias}

\begin{tabular}{|c|c|c|}
\hline Bias & Authors' judgement & Support for judgement \\
\hline $\begin{array}{l}\text { Random sequence genera- } \\
\text { tion (selection bias) }\end{array}$ & Low risk & $\begin{array}{l}\text { Quote: "eligible patients were randomized to receive either OEA or placebo for } \\
12 \text { weeks based on the random block procedure developed by random alloca- } \\
\text { tion software" }\end{array}$ \\
\hline $\begin{array}{l}\text { Allocation concealment } \\
\text { (selection bias) }\end{array}$ & Low risk & $\begin{array}{l}\text { Quote: "the random sequence was administered by an independent third in- } \\
\text { vestigator who was not aware of the study clinical process until the outcome } \\
\text { data collection was completed" }\end{array}$ \\
\hline $\begin{array}{l}\text { Blinding of participants } \\
\text { and personnel (perfor- } \\
\text { mance bias) } \\
\text { All outcomes }\end{array}$ & Low risk & $\begin{array}{l}\text { Quote: "both OEA and placebo capsules were labeled as A or B, and the inves- } \\
\text { tigators, participants, and the statistician were blinded to the drug allocation } \\
\text { until the end of the analysis" }\end{array}$ \\
\hline $\begin{array}{l}\text { Blinding of outcome as- } \\
\text { sessment (detection bias) } \\
\text { All outcomes }\end{array}$ & Low risk & $\begin{array}{l}\text { Quote: "both OEA and placebo capsules were labeled as A or B, and the inves- } \\
\text { tigators, participants, and the statistician were blinded to the drug allocation } \\
\text { until the end of the analysis" }\end{array}$ \\
\hline $\begin{array}{l}\text { Incomplete outcome data } \\
\text { (attrition bias) } \\
\text { All outcomes }\end{array}$ & Low risk & Comment: an intention-to-treat analysis was performed \\
\hline $\begin{array}{l}\text { Selective reporting (re- } \\
\text { porting bias) }\end{array}$ & Low risk & $\begin{array}{l}\text { Comment: no previously published protocol was available; adverse events and } \\
\text { either mortality or fatty liver resolution or both were reported }\end{array}$ \\
\hline
\end{tabular}


Tutunchi 2020 (Continued)

Other bias Low risk Comment: no other bias noted

Uygun 2000

\section{Study characteristics}

\begin{tabular}{|c|c|}
\hline Methods & Randomised clinical trial \\
\hline Participants & $\begin{array}{l}\text { Country: Turkey } \\
\text { Period of recruitment: not stated } \\
\text { Number randomised: } 133 \\
\text { Post-randomisation dropouts: } 32(24.1 \%) \\
\text { Revised sample size: } 101 \\
\text { Reasons for post-randomisation dropouts: did not complete the protocol } \\
\text { Average age, years: not stated } \\
\text { Females: } 29(28.7 \%) \\
\text { NASH: } 101(100.0 \%) \\
\text { Diabetes mellitus: not stated } \\
\text { Inclusion criteria: } 1 \text {. Elevated ALT levels. 2. NASH. 3. No other liver disease } \\
\text { Exclusion criteria: not stated } \\
\text { Method for diagnosis of NAFLD: liver biopsy }\end{array}$ \\
\hline Interventions & $\begin{array}{l}\text { Group 1: amino acids ( } \mathrm{n}=78 \text { ) } \\
\text { Further details: carnitine } 1 \text { to } 3 \mathrm{~g} / \mathrm{d} \text { for } 6 \text { months } \\
\text { Group 2: no active intervention ( } \mathrm{n}=23 \text { ) } \\
\text { Further details: no treatment } \\
\text { Additional details: both groups received lifestyle intervention }\end{array}$ \\
\hline Outcomes & No outcomes of interest were reported \\
\hline Notes & $\begin{array}{l}\text { Source of funding: not stated } \\
\text { Trial name/Trial registry number: not stated } \\
\text { Attempts were made to contact study authors in December } 2020\end{array}$ \\
\hline
\end{tabular}

\section{Risk of bias}

\begin{tabular}{lll}
\hline Bias & Authors' judgement & Support for judgement \\
\hline $\begin{array}{l}\text { Random sequence genera- } \\
\text { tion (selection bias) }\end{array}$ & Unclear risk & Comment: this information was not available \\
\hline $\begin{array}{l}\text { Allocation concealment } \\
\text { (selection bias) }\end{array}$ & Unclear risk & Comment: this information was not available \\
\hline $\begin{array}{l}\text { Blinding of participants } \\
\text { and personnel (perfor- } \\
\text { mance bias) }\end{array}$ & Unclear risk & Comment: this information was not available \\
All outcomes & \\
\hline
\end{tabular}

\begin{tabular}{ll} 
Blinding of outcome as- & Unclear risk \\
sessment (detection bias) & \\
All outcomes & \\
\hline
\end{tabular}

$\begin{array}{ll}\begin{array}{l}\text { Incomplete outcome data } \\ \text { (attrition bias) }\end{array} & \text { Unclear risk } \\ \text { All outcomes } & \begin{array}{l}\text { Comment: participants were excluded from analysis for reasons that may be } \\ \text { related to the intervention and to outcomes }\end{array}\end{array}$


Uygun 2000 (Continued)

\begin{tabular}{|c|c|c|}
\hline $\begin{array}{l}\text { Selective reporting (re- } \\
\text { porting bias) }\end{array}$ & High risk & $\begin{array}{l}\text { Comment: no previously published protocol was available; adverse events, } \\
\text { mortality, fatty liver resolution were not reported }\end{array}$ \\
\hline
\end{tabular}

Other bias Low risk Comment: no other bias noted

Vajro 2011

\section{Study characteristics}

\begin{tabular}{|c|c|c|}
\hline Methods & \multicolumn{2}{|c|}{ Randomised clinical trial } \\
\hline Participants & \multicolumn{2}{|c|}{$\begin{array}{l}\text { Country: Italy } \\
\text { Period of recruitment: not stated } \\
\text { Number randomised: } 20 \\
\text { Post-randomisation dropouts: } 0(0.0 \%) \\
\text { Revised sample size: } 20 \\
\text { Average age, years: } 11 \\
\text { Females: } 2(10.0 \%) \\
\text { NASH: not stated } \\
\text { Diabetes mellitus: } 0(0.0 \%) \\
\text { Inclusion criteria: } 1 \text {. BMI > 95th percentile for age and sex. } 2 \text {. NAFLD } \\
\text { Exclusion criteria: } 1 . \text { Other cause of transaminitis (e.g. viral hepatitis, alcohol abuse, drug toxicity). } 2 . \\
\text { Antibiotic use } \\
\text { Method for diagnosis of NAFLD: elastography plus transaminases }\end{array}$} \\
\hline Interventions & \multicolumn{2}{|c|}{$\begin{array}{l}\text { Group 1: prebiotics/probiotics/synbiotics }(n=10) \\
\text { Further details: probiotic Lactobacillus rhamnosus strain GG (12 billion CFUs/d) for } 8 \text { weeks } \\
\text { Group 2: no active intervention ( } n=10) \\
\text { Further details: placebo }\end{array}$} \\
\hline Outcomes & \multicolumn{2}{|c|}{$\begin{array}{l}\text { Outcomes reported: mortality at maximal follow-up, serious adverse events (number of people), any } \\
\text { adverse events (number of people) } \\
\text { Follow-up, months: } 2\end{array}$} \\
\hline Notes & \multicolumn{2}{|c|}{$\begin{array}{l}\text { Source of funding (quote): "this work was partly funded by the Italian Ministry of University and Re- } \\
\text { search (MIUR) PRIN 2005" } \\
\text { Trial name/Trial registry number: not stated } \\
\text { Attempts were made to contact study authors in December } 2020\end{array}$} \\
\hline \multicolumn{3}{|l|}{ Risk of bias } \\
\hline Bias & Authors' judgement & Support for judgement \\
\hline $\begin{array}{l}\text { Random sequence genera- } \\
\text { tion (selection bias) }\end{array}$ & Unclear risk & Comment: this information was not available \\
\hline $\begin{array}{l}\text { Allocation concealment } \\
\text { (selection bias) }\end{array}$ & Unclear risk & $\begin{array}{l}\text { Quote: "sealed envelopes" } \\
\text { Comment: further information was not available }\end{array}$ \\
\hline $\begin{array}{l}\text { Blinding of participants } \\
\text { and personnel (perfor- } \\
\text { mance bias) } \\
\text { All outcomes }\end{array}$ & Low risk & Quote: "double-blind, placebo-controlled pilot study" \\
\hline
\end{tabular}


Vajro 2011 (Continued)

Blinding of outcome as- Low risk Quote: "double-blind, placebo-controlled pilot study" sessment (detection bias)

All outcomes

\begin{tabular}{lll}
$\begin{array}{l}\text { Incomplete outcome data } \\
\text { (attrition bias) } \\
\text { All outcomes }\end{array}$ & Low risk & Comment: there were no post-randomisation dropouts \\
\hline $\begin{array}{l}\text { Selective reporting (re- } \\
\text { porting bias) }\end{array}$ & Low risk & $\begin{array}{l}\text { Comment: no previously published protocol was available; adverse events and } \\
\text { either mortality or fatty liver resolution or both were reported }\end{array}$
\end{tabular}

Other bias Low risk Comment: no other bias noted

Wang 2008

\section{Study characteristics}

\begin{tabular}{|c|c|c|}
\hline Methods & \multicolumn{2}{|c|}{ Randomised clinical trial } \\
\hline Participants & \multicolumn{2}{|c|}{$\begin{array}{l}\text { Country: China } \\
\text { Period of recruitment: not stated } \\
\text { Number randomised: } 57 \\
\text { Post-randomisation dropouts: } 0(0.0 \%) \\
\text { Revised sample size: } 57 \\
\text { Average age, years: } 14 \\
\text { Females: } 18(31.6 \%) \\
\text { NASH: } 57(100.0 \%) \\
\text { Diabetes mellitus: not stated } \\
\text { Inclusion criteria: } 1 \text {. Aged } 10 \text { to } 17 \text { years. 2. Obesity. 3. NAFLD } \\
\text { Exclusion criteria: } 1 \text {. History of alcohol intake. } 2 \text {. Positive markers for other liver disease (e.g. hepatitis) } \\
\text { Method for diagnosis of NAFLD: not stated }\end{array}$} \\
\hline Interventions & \multicolumn{2}{|c|}{$\begin{array}{l}\text { Group 1: vitamin } E(n=19) \\
\text { Further details: vitamin E } 100 \mathrm{mg} / \mathrm{d} \text { for } 1 \text { month } \\
\text { Group 2: no active intervention }(\mathrm{n}=38) \\
\text { Further details: no treatment } \\
\text { Additional details: another group not relevant to this review was excluded }\end{array}$} \\
\hline Outcomes & \multicolumn{2}{|c|}{$\begin{array}{l}\text { Outcomes reported: mortality at maximal follow-up } \\
\text { Follow-up, months: not stated }\end{array}$} \\
\hline Notes & \multicolumn{2}{|c|}{$\begin{array}{l}\text { Source of funding: not stated } \\
\text { Trial name/Trial registry number: not stated } \\
\text { Attempts were made to contact study authors in December } 2020\end{array}$} \\
\hline \multicolumn{3}{|l|}{ Risk of bias } \\
\hline Bias & Authors' judgement & Support for judgement \\
\hline $\begin{array}{l}\text { Random sequence genera- } \\
\text { tion (selection bias) }\end{array}$ & Unclear risk & Comment: this information was not available \\
\hline $\begin{array}{l}\text { Allocation concealment } \\
\text { (selection bias) }\end{array}$ & Unclear risk & Comment: this information was not available \\
\hline
\end{tabular}


Wang 2008 (Continued)

Blinding of participants Unclear risk Comment: this information was not available and personnel (perfor-

mance bias)

All outcomes

\section{Blinding of outcome as- Unclear risk sessment (detection bias)}

All outcomes
Quote: "all patients underwent an ultrasonographic study of the liver performed by one operator who was blinded to the groups"

Comment: not clear whether remaining outcomes were measured by blinded outcome assessors

Comment: there were no post-randomisation dropouts

Incomplete outcome data Low risk

(attrition bias)

All outcomes

\begin{tabular}{|c|c|c|}
\hline $\begin{array}{l}\text { Selective reporting (re- } \\
\text { porting bias) }\end{array}$ & High risk & $\begin{array}{l}\text { Comment: no previously published protocol was available; adverse events } \\
\text { were not reported adequately }\end{array}$ \\
\hline
\end{tabular}

Other bias Low risk Comment: no other bias noted

Wang 2017

\section{Study characteristics}

\begin{tabular}{ll}
\hline Methods & Randomised clinical trial \\
\hline Participants & Country: China \\
& Period of recruitment: not stated \\
& Number randomised: 36 \\
& Post-randomisation dropouts: not stated \\
& Revised sample size: 36 \\
& Average age, years: 41 \\
& Females: $13(36.1 \%)$ \\
& NASH: 36 (100.0\%) \\
& Diabetes mellitus: 0 (0.0\%) \\
Inclusion criteria: 1. NASH with advanced fibrosis \\
Exclusion criteria: 1. Other liver disease. 2. Diabetes. 3. Pregnancy. 4. Breastfeeding \\
Method for diagnosis of NAFLD: elastography
\end{tabular}

Interventions Group 1: other supplements $(\mathrm{n}=24)$

Further details: Xiao-Zhi-Hua-Xian-Tang (XZHXT), a traditional Chinese medicine herbal formulation

(Lotus Leaf, Semen Coicis) 3 times daily

Group 2: no active intervention $(n=12)$

Further details: no supplementation

Additional details: both groups received lifestyle intervention

\begin{tabular}{ll}
\hline Outcomes & No outcomes of interest were reported \\
\hline Notes & Source of funding: not stated \\
& Trial name/Trial registry number: not stated \\
& Attempts were made to contact study authors in December 2020
\end{tabular}

\section{Risk of bias}

Bias Authors' judgement Support for judgement


Wang 2017 (Continued)

Random sequence genera- Unclear risk Comment: this information was not available tion (selection bias)

Allocation concealment Unclear risk Comment: this information was not available
(selection bias)

Blinding of participants Unclear risk Comment: this information was not available
and personnel (perfor-
mance bias)
All outcomes

\begin{tabular}{|c|c|c|}
\hline $\begin{array}{l}\text { Blinding of outcome as- } \\
\text { sessment (detection bias) }\end{array}$ & Unclear risk & Comment: this information was not available \\
\hline
\end{tabular}

All outcomes

Incomplete outcome data Unclear risk Comment: this information was not available
(attrition bias)

All outcomes

\begin{tabular}{lll}
\hline $\begin{array}{l}\text { Selective reporting (re- } \\
\text { porting bias) }\end{array}$ & High risk & $\begin{array}{l}\text { Comment: no previously published protocol was available; adverse events, } \\
\text { mortality, fatty liver resolution were not reported }\end{array}$ \\
\hline Other bias & Low risk & Comment: no other bias noted \\
\hline
\end{tabular}

\section{Wang 2018}

\section{Study characteristics}

\begin{tabular}{|c|c|}
\hline Methods & Randomised clinical trial \\
\hline \multirow[t]{11}{*}{ Participants } & Country: China \\
\hline & Period of recruitment: 2010 to 2015 \\
\hline & Number randomised: 200 \\
\hline & Post-randomisation dropouts: not stated \\
\hline & Revised sample size: 200 \\
\hline & Average age, years: not stated \\
\hline & Females: not stated \\
\hline & NASH: not stated \\
\hline & Diabetes mellitus: not stated \\
\hline & Inclusion and exclusion criteria: not stated \\
\hline & Method for diagnosis of NAFLD: not stated \\
\hline \multirow[t]{4}{*}{ Interventions } & Group 1: phospholipids $(n=50)$ \\
\hline & Further details: polyene phosphatidylcholine $456 \mathrm{mg} 3$ times daily for 1 month \\
\hline & Group 2: prebiotics/probiotics/synbiotics $(n=150)$ \\
\hline & $\begin{array}{l}\text { Further details: } 2 \text { live combined Bifidobacterium, Lactobacillus, and Enterococcus powder or } 2 \text { live com- } \\
\text { bined Bacillus subtilis and Enterococcus or } 4 \text { live combined bacteria for } 1 \text { month }\end{array}$ \\
\hline Outcomes & No outcomes of interest were reported \\
\hline \multirow[t]{3}{*}{ Notes } & Source of funding: not stated \\
\hline & Trial name/Trial registry number: not stated \\
\hline & Attempts were made to contact study authors in December 2020 \\
\hline
\end{tabular}

\section{Risk of bias}


Wang 2018 (Continued)

\begin{tabular}{lll} 
Bias & Authors' judgement & Support for judgement \\
\hline $\begin{array}{l}\text { Random sequence genera- } \\
\text { tion (selection bias) }\end{array}$ & Low risk & Quote: "random number table" \\
\hline $\begin{array}{l}\text { Allocation concealment } \\
\text { (selection bias) }\end{array}$ & Unclear risk & Comment: this information was not available \\
\hline $\begin{array}{l}\text { Blinding of participants } \\
\text { and personnel (perfor- } \\
\text { mance bias) }\end{array}$ & Unclear risk & Comment: this information was not available \\
All outcomes & & \\
\hline
\end{tabular}

\begin{tabular}{|c|c|c|}
\hline $\begin{array}{l}\text { Blinding of outcome as- } \\
\text { sessment (detection bias) } \\
\text { All outcomes }\end{array}$ & Unclear risk & Comment: this information was not available \\
\hline $\begin{array}{l}\text { Incomplete outcome data } \\
\text { (attrition bias) } \\
\text { All outcomes }\end{array}$ & Unclear risk & Comment: this information was not available \\
\hline $\begin{array}{l}\text { Selective reporting (re- } \\
\text { porting bias) }\end{array}$ & High risk & $\begin{array}{l}\text { Comment: no previously published protocol was available; adverse events, } \\
\text { mortality, fatty liver resolution were not reported }\end{array}$ \\
\hline Other bias & Low risk & Comment: no other bias noted \\
\hline
\end{tabular}

Wong 2013a

\section{Study characteristics}

\begin{tabular}{|c|c|}
\hline Methods & Randomised clinical trial \\
\hline Participants & $\begin{array}{l}\text { Country: China } \\
\text { Period of recruitment: } 2009 \\
\text { Number randomised: } 20 \\
\text { Post-randomisation dropouts: } 0(0.0 \%) \\
\text { Revised sample size: } 20 \\
\text { Average age, years: } 49 \\
\text { Females: } 7(35.0 \%) \\
\text { NASH: } 20 \text { (100.0\%) } \\
\text { Diabetes mellitus: } 7 \text { (35.0\%) } \\
\text { Inclusion criteria: } 1 \text {. NAFLD } \\
\text { Exclusion criteria: } 1 \text {. Viral hepatitis. 2. Liver decompensation. 3. ALT > 10x upper limit of normal. 4. Ma- } \\
\text { lignancy. } 5 \text {. Use of steroids or methotrexate in past } 6 \text { months } \\
\text { Method for diagnosis of NAFLD: liver biopsy plus transaminases }\end{array}$ \\
\hline Interventions & $\begin{array}{l}\text { Group 1: prebiotics/probiotics/synbiotics }(\mathrm{n}=10) \\
\text { Further details: Lepicol probiotic formula } 1 \text { sachet twice daily for } 6 \text { months. Lepicol probiotic formula } \\
\text { contained Lactobacillus plantarum, Lactobacillus deslbrueckii, Lactobacillus acidophilus, Lactobacillus } \\
\text { rhamnosus, and Bifidobacterium bifidum } \\
\text { Group 2: no active intervention }(\mathrm{n}=10) \\
\text { Further details: no treatment }\end{array}$ \\
\hline Outcomes & $\begin{array}{l}\text { Outcomes reported: mortality at maximal follow-up, serious adverse events (number of people), any } \\
\text { adverse events (number of people) } \\
\text { Follow-up, months: } 6\end{array}$ \\
\hline
\end{tabular}


Wong 2013a (Continued)

Notes
Source of funding (quote): "the work described in this paper was partially supported by the direct grant of The Chinese University of Hong Kong (Ref 2010.1.042)"

Trial name/Trial registry number: NCT00870012

Attempts were made to contact study authors in December 2020

\section{Risk of bias}

\begin{tabular}{|c|c|c|}
\hline Bias & Authors' judgement & Support for judgement \\
\hline $\begin{array}{l}\text { Random sequence genera- } \\
\text { tion (selection bias) }\end{array}$ & Low risk & Quote: "computer-generated list" \\
\hline $\begin{array}{l}\text { Allocation concealment } \\
\text { (selection bias) }\end{array}$ & Unclear risk & $\begin{array}{l}\text { Quote: "sealed envelopes" } \\
\text { Comment: further information was not available }\end{array}$ \\
\hline $\begin{array}{l}\text { Blinding of participants } \\
\text { and personnel (perfor- } \\
\text { mance bias) } \\
\text { All outcomes }\end{array}$ & Unclear risk & Comment: this information was not available \\
\hline $\begin{array}{l}\text { Blinding of outcome as- } \\
\text { sessment (detection bias) } \\
\text { All outcomes }\end{array}$ & Unclear risk & Comment: this information was not available \\
\hline $\begin{array}{l}\text { Incomplete outcome data } \\
\text { (attrition bias) } \\
\text { All outcomes }\end{array}$ & Low risk & Comment: there were no post-randomisation dropouts \\
\hline $\begin{array}{l}\text { Selective reporting (re- } \\
\text { porting bias) }\end{array}$ & Low risk & $\begin{array}{l}\text { Comment: no previously published protocol was available; adverse events and } \\
\text { either mortality or fatty liver resolution or both were reported }\end{array}$ \\
\hline Other bias & Low risk & Comment: no other bias noted \\
\hline
\end{tabular}

Wong 2013b

\section{Study characteristics}

\begin{tabular}{ll}
\hline Methods & Randomised clinical trial \\
\hline Participants & Country: China \\
& Period of recruitment: 2010 to 2011 \\
& Number randomised: 60 \\
& Post-randomisation dropouts: not stated \\
& Revised sample size: 60 \\
& Average age, years: 51 \\
& Females: $27(45.0 \%)$ \\
& NASH: 60 (100.0\%) \\
& Diabetes mellitus: 23 (38.3\%) \\
& Inclusion criteria: 1 . Histology-proven NASH. 2. Aged 18 to 70 years \\
& Exclusion criteria: 1. Other liver disease. 2 Significant alcohol consumption. 3. Liver decompensation. 4. \\
& Type 1 diabetes. 5 . Malignancy \\
Method for diagnosis of NAFLD: liver biopsy
\end{tabular}

Interventions

Group 1: other supplements $(n=40)$ 
Wong 2013b (Continued)

Further details: Phyllanthus urinaria $1 \mathrm{~g}$ ( 2 tablets) 3 times daily for 24 weeks. Each Phyllanthus tablet contained $400 \mathrm{mg}$ of Phyllanthus urinaria together with inactive ingredients of microcrystalline cellulose, hydroxypropylmethylcelllose, and magnesium stearate

Group 2: no active intervention $(n=20)$

Further details: Phyllanthus-like placebo, 2 tablets, 3 times daily, for 24 weeks

Additional details: both groups received lifestyle advice

Outcomes Outcomes reported: serious adverse events (number of people), any adverse events (number of people), resolution of fatty liver disease, fibrosis score, NAFLD activity score

Follow-up, months: 6

Notes Source of funding (quote): "the Phyllanthus tablets and placebo were provided by Hepaguard Company Limited, Hong Kong"

Trial name/Trial registry number: NCT01210989

Attempts were made to contact study authors in December 2020

\section{Risk of bias}

\begin{tabular}{|c|c|c|}
\hline Bias & Authors' judgement & Support for judgement \\
\hline $\begin{array}{l}\text { Random sequence genera- } \\
\text { tion (selection bias) }\end{array}$ & Low risk & $\begin{array}{l}\text { Quote: "randomization was carried out through the use of a computer-gener- } \\
\text { ated list of random numbers in blocks of 6" }\end{array}$ \\
\hline $\begin{array}{l}\text { Allocation concealment } \\
\text { (selection bias) }\end{array}$ & Low risk & $\begin{array}{l}\text { Quote: "study medications were stored in consecutively numbered, sealed } \\
\text { bottles and the preparation was done at a separate office" }\end{array}$ \\
\hline $\begin{array}{l}\text { Blinding of participants } \\
\text { and personnel (perfor- } \\
\text { mance bias) } \\
\text { All outcomes }\end{array}$ & Low risk & $\begin{array}{l}\text { Quote: "the hepatologists, pathologists, nurses and patients were all blinded } \\
\text { to the treatment assignment...placebo" }\end{array}$ \\
\hline $\begin{array}{l}\text { Blinding of outcome as- } \\
\text { sessment (detection bias) } \\
\text { All outcomes }\end{array}$ & Low risk & $\begin{array}{l}\text { Quote: "the hepatologists, pathologists, nurses and patients were all blinded } \\
\text { to the treatment assignment...placebo" }\end{array}$ \\
\hline $\begin{array}{l}\text { Incomplete outcome data } \\
\text { (attrition bias) } \\
\text { All outcomes }\end{array}$ & Low risk & Comment: an intention-treat analysis was performed \\
\hline $\begin{array}{l}\text { Selective reporting (re- } \\
\text { porting bias) }\end{array}$ & Low risk & $\begin{array}{l}\text { Comment: a published protocol was available, but recruitment had com- } \\
\text { menced before the protocol was published; adverse events and either mortali- } \\
\text { ty or fatty liver resolution or both were reported }\end{array}$ \\
\hline Other bias & Low risk & Comment: no other bias noted \\
\hline
\end{tabular}

Yan 2015

\section{Study characteristics}

\begin{tabular}{ll}
\hline Methods & Randomised clinical trial \\
\hline Participants & Country: China \\
& Period of recruitment: 2008 to 2011 \\
& Number randomised: 124 \\
& Post-randomisation dropouts: 16 (12.9\%) \\
& Revised sample size: 108 \\
& Reasons for post-randomisation dropouts: discontinued intervention, lost to follow-up \\
\hline
\end{tabular}


Yan 2015 (Continued)

Average age, years: 52

Females: 54 (50.0\%)

NASH: not stated

Diabetes mellitus: not stated

Inclusion criteria: 1. NAFLD

Exclusion criteria: 1. Hepatitis B/C. 2. Hepatotoxic drugs. 3. Alcohol consumption (>10 g/d for women, >

$20 \mathrm{~g} / \mathrm{d}$ for men)

Method for diagnosis of NAFLD: magnetic resonance spectroscopy

\begin{tabular}{|c|c|}
\hline Interventions & $\begin{array}{l}\text { Group 1: other supplements }(n=55) \\
\text { Further details: berberine } 0.5 \mathrm{mg}, 3 \text { times daily for } 16 \text { weeks } \\
\text { Group 2: no active intervention }(n=53) \\
\text { Further details: no treatment } \\
\text { Additional details: both groups received lifestyle intervention; another group not relevant to this review } \\
\text { was excluded }\end{array}$ \\
\hline Outcomes & $\begin{array}{l}\text { Outcomes reported: serious adverse events (number of people), any adverse events (number of events) } \\
\text { Follow-up, months: } 4\end{array}$ \\
\hline Notes & $\begin{array}{l}\text { Source of funding (quote): "this work was supported by grants from the Major State Basic Research De- } \\
\text { velopment Program of China (2012CB524906 to Gao X.; http// www.973.gov.cn/Default_3.aspx), Nation- } \\
\text { al Natural Science Foundation of China ( } 81270933 \text { to Gao X.), Major State Basic Research Development } \\
\text { Program of China ( } 2011 \text { CB504004 to Gao X.), the Science and Technology Commission of Shanghai Mu- } \\
\text { nicipality" } \\
\text { Trial name/Trial registry number: NCT00633282 } \\
\text { Attempts were made to contact study authors in December } 2020\end{array}$ \\
\hline
\end{tabular}

\section{Risk of bias}

\begin{tabular}{lll}
\hline Bias & Authors' judgement & Support for judgement \\
\hline $\begin{array}{l}\text { Random sequence genera- } \\
\text { tion (selection bias) }\end{array}$ & Low risk & Quote: "computer-generated" \\
\hline $\begin{array}{l}\text { Allocation concealment } \\
\text { (selection bias) }\end{array}$ & Unclear risk & Comment: this information was not available \\
\hline $\begin{array}{l}\text { Blinding of participants } \\
\text { and personnel (perfor- } \\
\text { mance bias) }\end{array}$ & High risk & Quote: "open-label" \\
All outcomes & \\
\hline
\end{tabular}

Blinding of outcome as- High risk Quote: "open-label"

sessment (detection bias)

All outcomes

\begin{tabular}{lll}
\hline $\begin{array}{l}\text { Incomplete outcome data } \\
\text { (attrition bias) } \\
\text { All outcomes }\end{array}$ & Unclear risk & $\begin{array}{l}\text { Comment: participants were excluded from analysis for reasons that may be } \\
\text { related to the intervention and to outcomes }\end{array}$ \\
\hline $\begin{array}{l}\text { Selective reporting (re- } \\
\text { porting bias) }\end{array}$ & High risk & $\begin{array}{l}\text { Comment: no previously published protocol was available; neither mortality } \\
\text { nor fatty liver resolution was reported }\end{array}$ \\
\hline Other bias & Low risk & Comment: no other bias noted \\
\hline
\end{tabular}


Yari 2016

\section{Study characteristics}

\begin{tabular}{|c|c|}
\hline Methods & Randomised clinical trial \\
\hline Participants & $\begin{array}{l}\text { Country: Iran } \\
\text { Period of recruitment: not stated } \\
\text { Number randomised: } 50 \\
\text { Post-randomisation dropouts: } 0(0.0 \%) \\
\text { Revised sample size: } 50 \\
\text { Average age, years: } 45 \\
\text { Females: } 23(46.0 \%) \\
\text { NASH: not stated } \\
\text { Diabetes mellitus: } 0 \text { (0.0\%) } \\
\text { Inclusion criteria: } 1 . \text { Aged } 18 \text { to } 70 \text { years. 2. NAFLD } \\
\text { Exclusion criteria: } 1 \text {. Allergy to nuts, flaxseed, or sesame seeds. 2. Excessive alcohol use. 3. Cardiovas- } \\
\text { cular disease. } 4 \text {. Cancer. } 5 \text {. Diabetes mellitus. } 6 \text {. Therapy that may benefit NAFLD (e.g. vitamin E). } 7 . \\
\text { Weight loss in past } 6 \text { months. } 8 \text {. Pregnancy or lactation } \\
\text { Method for diagnosis of NAFLD: elastography }\end{array}$ \\
\hline Interventions & $\begin{array}{l}\text { Group 1: PUFA }(n=25) \\
\text { Further details: flaxseed (milled) } 30 \mathrm{~g} / \mathrm{d} \text { for } 12 \text { weeks } \\
\text { Group 2: no active intervention }(\mathrm{n}=25) \\
\text { Further details: no treatment } \\
\text { Additional details: both groups received lifestyle modification }\end{array}$ \\
\hline Outcomes & $\begin{array}{l}\text { Outcomes reported: mortality at maximal follow-up } \\
\text { Follow-up, months: } 3\end{array}$ \\
\hline Notes & $\begin{array}{l}\text { Source of funding (quote): "this study was supported by a grant from the National Nutrition and Food } \\
\text { Technology Research Institute of the ShahidBeheshti University and the Digestive Disease Research } \\
\text { Center of the Shariati Hospital" } \\
\text { Trial name/Trial registry number: NCT02395900 } \\
\text { Attempts were made to contact study authors in December } 2020\end{array}$ \\
\hline
\end{tabular}

\section{Risk of bias}

\begin{tabular}{lll}
\hline Bias & Authors' judgement & Support for judgement \\
\hline $\begin{array}{l}\text { Random sequence genera- } \\
\text { tion (selection bias) }\end{array}$ & Unclear risk & Comment: this information was not available \\
\hline $\begin{array}{l}\text { Allocation concealment } \\
\text { (selection bias) }\end{array}$ & Unclear risk & Comment: this information was not available \\
\hline $\begin{array}{l}\text { Blinding of participants } \\
\begin{array}{l}\text { and personnel (perfor- } \\
\text { mance bias) }\end{array}\end{array}$ & High risk & Quote: "open labeled" \\
All outcomes & & \\
\hline
\end{tabular}

\begin{tabular}{|c|c|c|}
\hline $\begin{array}{l}\text { Blinding of outcome as- } \\
\text { sessment (detection bias) } \\
\text { All outcomes }\end{array}$ & High risk & Quote: "open labeled" \\
\hline
\end{tabular}

Incomplete outcome data Low risk Comment: there were no post-randomisation dropouts
(attrition bias)

(attrition bias)
All outcomes 
Yari 2016 (Continued)

\begin{tabular}{|c|c|c|}
\hline $\begin{array}{l}\text { Selective reporting (re- } \\
\text { porting bias) }\end{array}$ & High risk & $\begin{array}{l}\text { Comment: no previously published protocol was available; adverse events } \\
\text { were not reported adequately }\end{array}$ \\
\hline
\end{tabular}

Other bias Low risk Comment: no other bias noted

Yari 2020

\section{Study characteristics}

\begin{tabular}{ll}
\hline Methods & Randomised clinical trial \\
\hline Participants & Country: Iran \\
& Period of recruitment: 2018 to 2019 \\
& Number randomised: 100 \\
& Post-randomisation dropouts: $8(8.0 \%)$ \\
& Revised sample size: 92 \\
& Reasons for post-randomisation dropouts: loss to follow-up \\
& Average age, years: 45 \\
& Females: 44 (47.8\%) \\
& NASH: not stated \\
& Diabetes mellitus: not stated \\
& Inclusion criteria: 1 . Aged 18 to 70 years. 2. BMI between 25 and $40.3 .>37 \%$ hepatic fat content (CAP $\geq$ \\
& 260, grade $\geq 2$ ) \\
& Exclusions criteria: 1 . Alcohol consumption $>10$ g/d (women), $>20$ g/d (men). 2. Other liver disease. \\
& 3. Athlete. 4 . Pregnancy, lactation. 5 . Taking hypoglycaemic, lipid-regulating, anti-inflammatory med- \\
& ications, drugs, or herbal supplements affecting liver function. 6. Presence of gastrointestinal, cardiac, \\
& renal, pulmonary, autoimmune, thyroid disease; severe metabolic abnormalities. 7 . History of weight \\
& loss surgery or concurrent weight-reduction programme \\
& Method for diagnosis of NAFLD: elastography
\end{tabular}

Group 1: other supplements $(n=24)$
Further details: $30 \mathrm{~g}$ whole brown milled flaxseed powder daily for 12 weeks
Group 2: other antioxidants plus other supplements $(n=25)$
Further details: combination of $1 \mathrm{~g}$ hesperidin and $30 \mathrm{~g}$ flaxseed daily for 12 weeks
Group 3: other antioxidants $(n=22)$
Further details: $1 \mathrm{~g}$ hesperidin supplementation daily for 12 weeks
Group 4: no active intervention $(n=21)$
Further details: control reference group had not undertaken any intervention
Additional details: both groups received lifestyle advice

Outcomes Outcomes reported: mortality at maximal follow-up, serious adverse events (number of people), any adverse events (number of people), liver transplantation at maximal follow-up, decompensation (number of people), cirrhosis (number of people), resolution of fatty liver disease, hepatocellular carcinoma Follow-up, months: 3

\begin{tabular}{|c|c|c|}
\hline Notes & $\begin{array}{l}\text { Source of funding (qu } \\
\text { Trial name/Trial regist } \\
\text { Attempts were made } t\end{array}$ & $\begin{array}{l}\text { e): "funded by the University (author replies)" } \\
\text { / number: NCT03734510 } \\
\text { contact study authors in December } 2020\end{array}$ \\
\hline \multicolumn{3}{|l|}{ Risk of bias } \\
\hline Bias & Authors' judgement & Support for judgement \\
\hline $\begin{array}{l}\text { Random sequence genera- } \\
\text { tion (selection bias) }\end{array}$ & Low risk & $\begin{array}{l}\text { Quote: "the random allocations were carried out using a random numbers ta- } \\
\text { ble" }\end{array}$ \\
\hline
\end{tabular}


Yari 2020 (Continued)

\begin{tabular}{l}
$\begin{array}{l}\text { Allocation concealment Low risk } \\
\text { (selection bias) }\end{array} \quad$ Quote: "it was concealed by a third person" (author replies) \\
\hline
\end{tabular}

(selection bias)

Blinding of participants

High risk

Quote: "open-label"

and personnel (perfor-

mance bias)

All outcomes

$\begin{array}{lll}\text { Blinding of outcome as- } & \text { High risk } & \text { Quote: "open-label" } \\ \text { sessment (detection bias) } & \end{array}$

sessment (detection bias)

All outcomes

Incomplete outcome data Unclear risk

(attrition bias)

All outcomes

Selective reporting (re- Low risk
porting bias)

porting bias)

Other bias Unclear risk Comment: there were baseline differences in important prognostic factors

Comment: there were post-randomisation dropouts, but it is not clear whether these were related to the intervention or to outcomes

Youshari 2017

\section{Study characteristics}

\begin{tabular}{|c|c|}
\hline Methods & Randomised clinical trial \\
\hline Participants & $\begin{array}{l}\text { Country: Iran } \\
\text { Period of recruitment: not stated } \\
\text { Number randomised: } 41 \\
\text { Post-randomisation dropouts: not stated } \\
\text { Revised sample size: } 41 \\
\text { Average age, years: not stated } \\
\text { Females: not stated } \\
\text { NASH: not stated } \\
\text { Diabetes mellitus: not stated } \\
\text { Inclusion and exclusion criteria: not stated } \\
\text { Method for diagnosis of NAFLD: not stated }\end{array}$ \\
\hline Interventions & $\begin{array}{l}\text { Group 1: vitamin E plus other supplements }(n=20) \\
\text { Further details: } 3 \text { grams of oats beta-glucan daily for } 8 \text { weeks plus vitamin } E \text { (no further details) } \\
\text { Group 2: vitamin } E(n=21) \\
\text { Further details: } 3 \text { grams of maltodextrin as placebo daily for } 8 \text { weeks plus vitamin } E \text { (no further details) } \\
\text { Additional details: both groups received lifestyle intervention }\end{array}$ \\
\hline Outcomes & No outcomes of interest were reported \\
\hline Notes & $\begin{array}{l}\text { Source of funding: not stated } \\
\text { Trial name/Trial registry number: not stated } \\
\text { Attempts were made to contact study authors in December } 2020\end{array}$ \\
\hline \multicolumn{2}{|l|}{ Risk of bias } \\
\hline Bias & Authors' judgement Support for judgement \\
\hline
\end{tabular}

Nutritional supplementation for nonalcohol-related fatty liver disease: a network meta-analysis (Review) 
Youshari 2017 (Continued)

Random sequence genera- Unclear risk Comment: this information was not available tion (selection bias)

Allocation concealment $\quad$ Unclear risk Comment: this information was not available
(selection bias)

Blinding of participants $\quad$ Low risk
$\begin{aligned} & \text { and personnel (perfor- } \\ & \text { mance }\end{aligned}$

mance bias)

All outcomes

Blinding of outcome as-
sessment (detection bias) $\quad$ Low risk $\quad$ Quote: "randomized, double-blind, parallel, placebo-controlled study"

All outcomes

Incomplete outcome data Unclear risk Comment: this information was not available
(attrition bias)

All outcomes

\begin{tabular}{lll}
\hline $\begin{array}{l}\text { Selective reporting (re- } \\
\text { porting bias) }\end{array}$ & High risk & $\begin{array}{l}\text { Comment: no previously published protocol was available; adverse events, } \\
\text { mortality, fatty liver resolution were not reported }\end{array}$ \\
\hline Other bias & Low risk & Comment: no other bias noted \\
\hline
\end{tabular}

\section{Zamani 2018}

\section{Study characteristics}

\begin{tabular}{|c|c|}
\hline Methods & Randomised clinical trial \\
\hline Participants & $\begin{array}{l}\text { Country: Iran } \\
\text { Period of recruitment: } 2017 \\
\text { Number randomised: } 90 \\
\text { Post-randomisation dropouts: } 5 \text { (5.6\%) } \\
\text { Revised sample size: } 85 \\
\text { Reasons for post-randomisation dropouts: immigration (2), use of some drugs (2), missed follow-up (1) } \\
\text { Average age, years: } 39 \\
\text { Females: } 24 \text { ( } 28.2 \%) \\
\text { NASH: not stated } \\
\text { Diabetes mellitus: } 0 \text { (0.0\%) } \\
\text { Inclusion criteria: } 1 \text {. Aged } 18 \text { to } 65 \text { years. 2. BMI } 18 \text { to } 36 \text {. 3. Fatty liver on USS. 4. ALT } \geq 1.5 \times \text { upper limit of } \\
\text { normal } \\
\text { Exclusion criteria: } 1 \text {. Pregnancy, lactation. } 2 \text {. Alcohol consumption. 3. Diabetes mellitus. } 4 \text {. Sensitivity } \\
\text { to ZM or thyme. } 5 \text {. Other liver disease. } 6 \text {. Malignancy. } 7 \text {. Use of hepatotoxic drugs within past } 6 \text { months. } \\
\text { 8. Use of drugs with effects on biochemical tests of the study within previous } 3 \text { months (e.g. metformin, } \\
\text { vitamin E, oral contraceptive pills, omega-3, statins, glucocorticoids). 8. Hypothyroidism and hyperthy- } \\
\text { roidism. } 9 \text {. Renal insufficiency } \\
\text { Method for diagnosis of NAFLD: ultrasound }\end{array}$ \\
\hline Interventions & $\begin{array}{l}\text { Group 1: other supplements ( } \mathrm{n}=45 \text { ) } \\
\text { Further details: Zataria multiflora Boiss (Lamiaceae) is an herbal plant (ZM, Shirazi thyme). Patients in } \\
\text { the treatment group received } 4 \text { capsules (each containing } 350 \mathrm{mg} Z \mathrm{ZM} \text { ) daily for a period of } 12 \text { weeks } \\
\text { Group 2: no active intervention ( } \mathrm{n}=40 \text { ) } \\
\text { Further details: in the control group, patients received identical placebo (wheat flour) capsules, } 4 \text { cap- } \\
\text { sules daily for a period of } 12 \text { weeks. Each placebo capsule contained } 350 \text { mg wheat flour including } 35 \\
\text { mg of ZM aerial part powder (to improve blindness regarding taste and aroma) }\end{array}$ \\
\hline
\end{tabular}


Zamani 2018 (Continued)

Additional details: both groups received dietary advice

Outcomes Outcomes reported: mortality at maximal follow-up, serious adverse events (number of people), any adverse events (number of people), liver transplantation at maximal follow-up, decompensation (number of people), cirrhosis (number of people), hepatocellular carcinoma

Follow-up, months: 3

$\begin{array}{ll}\text { Notes } & \text { Source of funding (quote): "this study was supported by Shiraz University Medical Sciences (Grant No. } \\ \text { 94-7648) as part of a PhD thesis" } \\ \text { Trial name/Trial registry number: NCT02983669 } \\ \text { Attempts were made to contact study authors in December } 2020\end{array}$

\section{Risk of bias}

\begin{tabular}{lll}
\hline Bias & Authors' judgement & Support for judgement \\
\hline $\begin{array}{l}\text { Random sequence genera- } \\
\text { tion (selection bias) }\end{array}$ & Low risk & $\begin{array}{l}\text { Quote: "patients were randomly allocated into treatment and placebo groups } \\
\text { by block randomization which was generated by a computer as a non-strati- } \\
\text { fied list" }\end{array}$ \\
\hline $\begin{array}{l}\text { Allocation concealment } \\
\text { (selection bias) }\end{array}$ & Low risk & $\begin{array}{l}\text { Quote: "randomized double-blind placebo-controlled clinical trial. Both the } \\
\text { participants and investigators were blind to the intervention and placebo } \\
\text { groups" } \\
\text { Comment: although the precise method was not reported, allocation was } \\
\text { probably concealed by use of placebo }\end{array}$ \\
\hline
\end{tabular}

\begin{tabular}{|c|c|c|}
\hline $\begin{array}{l}\text { Blinding of participants } \\
\text { and personnel (perfor- } \\
\text { mance bias) } \\
\text { All outcomes }\end{array}$ & Low risk & $\begin{array}{l}\text { Quote: "both the participants and investigators were blind to the intervention } \\
\text { and control groups...placebo" }\end{array}$ \\
\hline $\begin{array}{l}\text { Blinding of outcome as- } \\
\text { sessment (detection bias) } \\
\text { All outcomes }\end{array}$ & Low risk & $\begin{array}{l}\text { Quote: "both the participants and investigators were blind to the intervention } \\
\text { and control groups...placebo" }\end{array}$ \\
\hline $\begin{array}{l}\text { Incomplete outcome data } \\
\text { (attrition bias) } \\
\text { All outcomes }\end{array}$ & Unclear risk & $\begin{array}{l}\text { Comment: there were post-randomisation dropouts, but it is not clear whether } \\
\text { these were related to the intervention or to outcomes }\end{array}$ \\
\hline $\begin{array}{l}\text { Selective reporting (re- } \\
\text { porting bias) }\end{array}$ & Low risk & $\begin{array}{l}\text { Comment: all pre-defined outcomes in the protocol published before recruit- } \\
\text { ment were reported }\end{array}$ \\
\hline Other bias & Unclear risk & Comment: there were baseline differences in important prognostic factors \\
\hline
\end{tabular}

Zanko 2020

\section{Study characteristics}

\begin{tabular}{ll}
\hline Methods & Randomised clinical trial \\
\hline Participants & Country: Croatia \\
& Period of recruitment: 2015 to 2019 \\
& Number randomised: 311 \\
& Post-randomisation dropouts: 0 (0.0\%) \\
& Revised sample size: 311 \\
& Average age, years: 65 \\
& Females: 133 (42.8\%) \\
\hline
\end{tabular}


Zanko 2020 (Continued)

NASH: not stated

Diabetes mellitus: $171(55.0 \%)$

Inclusion criteria: 1 . Aged $\geq 18$ years. 2. Irrespective of serum vitamin D levels. 3. NAFLD confirmed by ultrasound and transient elastography (TE) with CAP $\geq 238 \mathrm{~dB} / \mathrm{m}$. 4. Positively excluded other cause of chronic liver disease. 5. Metabolic syndrome

Exclusion criteria: 1 . Significant alcohol consumption (> $30 \mathrm{~g} / \mathrm{d}$ in men, $20 \mathrm{~g} / \mathrm{d}$ in women). 2. Presence of autoimmune, viral, or other metabolic chronic liver disease. 3. Pregnancy. 4. Right-sided heart failure. 5 Malignancy. 6 . Ascites. 7. Jejunoileal bypass or extensive small bowel resection or total parenteral nutrition. 8. Clinical, laboratory, and imaging features of liver cirrhosis. 9. Consumption of drugs that might induce hepatic steatosis (e.g. corticosteroids, high-dose oestrogen, methotrexate, amiodarone) during the 9 pre-study months. 10. Inability of reliable TE measurement (TE failure; including inadequate results) at pre-study screening Method for diagnosis of NAFLD: ultrasound plus transient elastography

$\begin{array}{ll}\text { Interventions } & \text { Group 1: vitamin } D(n=201) \\ & \text { Further details: vitamin D3 oral solution (cholecalciferol } 1000 \mathrm{IU} / \mathrm{d} \text {; delivered as } 5 \text { drops, } 200 \mathrm{IU} \text { each) } \\ & \text { for } 12 \text { months } \\ & \text { Group 2: no active intervention }(\mathrm{n}=110) \\ & \text { Further details: matching placebo (containing vehicles: castor oil, purified water, and methylparaben } \\ \text { preservative) for } 12 \text { months } \\ \text { Additional details: dietary and physical activity measures that were in place before the trial; continued } \\ \text { as usual throughout the trial }\end{array}$

\begin{tabular}{ll}
\hline Outcomes & $\begin{array}{l}\text { Outcomes reported: serious adverse events (number of people), serious adverse events (number of } \\
\text { events) } \\
\text { Follow-up, months: } 12\end{array}$ \\
\hline Notes & $\begin{array}{l}\text { Source of funding (quote): "the authors declare that there was no financial support for this study" } \\
\text { Trial name/Trial registry number: NCT04038853 } \\
\text { Attempts were made to contact study authors in April } 2021\end{array}$
\end{tabular}

\section{Risk of bias}

\begin{tabular}{lll}
\hline Bias & Authors' judgement & Support for judgement \\
\hline $\begin{array}{l}\text { Random sequence genera- } \\
\text { tion (selection bias) }\end{array}$ & Low risk & $\begin{array}{l}\text { Quote: "simple randomization was implemented using a random number gen- } \\
\text { erator" }\end{array}$ \\
\hline $\begin{array}{l}\text { Allocation concealment } \\
\text { (selection bias) }\end{array}$ & Low risk & $\begin{array}{l}\text { Quote: "the randomization list was kept in a sealed envelope by a staff mem- } \\
\text { ber not participating in patient recruitment and follow-up. The same person } \\
\text { dispensed the allocated treatments, which were labelled at the hospital phar- } \\
\text { macy to contain only the patient code" }\end{array}$
\end{tabular}

\begin{tabular}{ll}
\hline $\begin{array}{l}\text { Blinding of participants } \\
\text { and personnel (perfor- }\end{array}$ & Low risk \\
$\begin{array}{l}\text { mance bias) } \\
\text { All outcomes }\end{array}$ & $\begin{array}{l}\text { Quote: "double-blind, placebo-controlled trial. At scheduled visits, investiga- } \\
\text { tors were unaware of the assigned treatment and had no information about } \\
\text { the serum vitamin D3 levels" }\end{array}$
\end{tabular}

\begin{tabular}{|c|c|c|}
\hline $\begin{array}{l}\text { Blinding of outcome as- } \\
\text { sessment (detection bias) } \\
\text { All outcomes }\end{array}$ & Low risk & $\begin{array}{l}\text { Quote: "double-blind, placebo-controlled trial. At scheduled visits, investiga- } \\
\text { tors were unaware of the assigned treatment and had no information about } \\
\text { the serum vitamin D3 levels" }\end{array}$ \\
\hline $\begin{array}{l}\text { Incomplete outcome data } \\
\text { (attrition bias) } \\
\text { All outcomes }\end{array}$ & Low risk & Comment: there were no post-randomisation dropouts \\
\hline $\begin{array}{l}\text { Selective reporting (re- } \\
\text { porting bias) }\end{array}$ & High risk & $\begin{array}{l}\text { Comment: no previously published protocol was available; neither mortality } \\
\text { nor fatty liver resolution was reported }\end{array}$ \\
\hline
\end{tabular}


Zanko 2020 (Continued)
Other bias
Low risk
Comment: no other bias noted

Zhang 2015

\section{Study characteristics}

\begin{tabular}{|c|c|}
\hline Methods & Randomised clinical trial \\
\hline Participants & $\begin{array}{l}\text { Country: China } \\
\text { Period of recruitment: } 2013 \\
\text { Number randomised: } 74 \\
\text { Post-randomisation dropouts: } 0(0.0 \%) \\
\text { Revised sample size: } 74 \\
\text { Average age, years: } 46 \\
\text { Females: } 35 \text { ( } 47.3 \%) \\
\text { NASH: not stated } \\
\text { Diabetes mellitus: not stated } \\
\text { Inclusion criteria: } 1 \text {. NAFLD } \\
\text { Exclusion criteria: } 1 \text {. Cirrhosis. } 2 \text {. Viral hepatitis. 3. Cardiovascular disease. 4. Cancer. 5. Excessive alco- } \\
\text { hol consumption. } 6 \text {. Hepatotoxic drugs } \\
\text { Method for diagnosis of NAFLD: ultrasound }\end{array}$ \\
\hline Interventions & $\begin{array}{l}\text { Group 1: other supplements }(n=37) \\
\text { Further details: purified anthocyanin (biolink) } 320 \mathrm{mg} / \mathrm{d} \text { for } 12 \text { weeks } \\
\text { Group 2: no active intervention }(n=37) \\
\text { Further details: placebo }\end{array}$ \\
\hline Outcomes & $\begin{array}{l}\text { Outcomes reported: mortality at maximal follow-up, serious adverse events (number of people), liver } \\
\text { transplantation at maximal follow-up, decompensation (number of people), cirrhosis (number of peo- } \\
\text { ple), hepatocellular carcinoma, fibrosis score } \\
\text { Follow-up, months: } 3\end{array}$ \\
\hline Notes & $\begin{array}{l}\text { Source of funding (quote): "this work was funded by grants from the National Basic Research Program } \\
\text { (973 Program, 2012CB517506), National Natural Science Foundation (81372994, 81172655), and Guang- } \\
\text { dong Industry-University Research Foundation (2013B090600138)" } \\
\text { Trial name/Trial registry number: NCT01940263 } \\
\text { Attempts were made to contact study authors in December } 2020\end{array}$ \\
\hline
\end{tabular}

\section{Risk of bias}

\begin{tabular}{lll}
\hline Bias & Authors' judgement & Support for judgement \\
\hline $\begin{array}{l}\text { Random sequence genera- } \\
\text { tion (selection bias) }\end{array}$ & Low risk & Quote: "computer-generated list of random numbers" \\
\hline $\begin{array}{l}\text { Allocation concealment } \\
\text { (selection bias) }\end{array}$ & Low risk & $\begin{array}{l}\text { Quote: "scheduled participants were consecutively assigned by a medical } \\
\text { technologist, who was unaware of enrolment status, to treatment codes that } \\
\text { corresponded to labels on otherwise identical concealed containers" }\end{array}$ \\
\hline $\begin{array}{l}\text { Blinding of participants } \\
\text { and personnel (perfor- } \\
\text { mance bias) }\end{array}$ & Low risk & Quote: "double-blind, randomized study...placebo" \\
$\begin{array}{l}\text { All outcomes } \\
\begin{array}{l}\text { Blinding of outcome as- } \\
\text { sessment (detection bias) }\end{array}\end{array}$ & Low risk & Quote: "double-blind, randomized study...placebo" \\
\hline
\end{tabular}


Zhang 2015 (Continued)

All outcomes

Incomplete outcome data Low risk Comment: there were no post-randomisation dropouts
(attrition bias)

(attrition bias)

All outcomes

\begin{tabular}{lll}
\hline $\begin{array}{l}\text { Selective reporting (re- } \\
\text { porting bias) }\end{array}$ & Low risk & $\begin{array}{l}\text { Comment: no previously published protocol was available; adverse events and } \\
\text { either mortality or fatty liver resolution or both were reported }\end{array}$ \\
\hline Other bias & Low risk & Comment: no other bias noted \\
\hline
\end{tabular}

\section{Zhu 2008}

\section{Study characteristics}

\begin{tabular}{|c|c|c|}
\hline Methods & \multicolumn{2}{|c|}{ Randomised clinical trial } \\
\hline Participants & \multicolumn{2}{|c|}{$\begin{array}{l}\text { Country: China } \\
\text { Period of recruitment: } 2006 \text { to } 2008 \\
\text { Number randomised: } 144 \\
\text { Post-randomisation dropouts: } 10 \text { (6.9\%) } \\
\text { Revised sample size: } 134 \\
\text { Reasons for post-randomisation dropouts: did not complete protocol } \\
\text { Average age, years: } 45 \\
\text { Females: } 37 \text { ( } 27.6 \%) \\
\text { NASH: not stated } \\
\text { Diabetes mellitus: not stated } \\
\text { Inclusion criteria: } 1 \text {. NAFLD } \\
\text { Exclusion criteria: } 1 \text {. Alcohol excess. 2. Other cause of liver disease (e.g. viral hepatitis). 3. Pregnancy } \\
\text { and breastfeeding. } 4 \text {. Hepatotoxic medication } \\
\text { Method for diagnosis of NAFLD: ultrasound }\end{array}$} \\
\hline Interventions & \multicolumn{2}{|c|}{$\begin{array}{l}\text { Group 1: PUFA ( } n=66) \\
\text { Further details: omega-3 fatty acids, } 2 \mathrm{~g} \text { from seal oils (Shanghai Hengsheng Biology \& Medicine Co. } \\
\text { Ltd., Shanghai, China), } 3 \text { times daily for } 24 \text { weeks } \\
\text { Group 2: no active intervention }(n=68) \\
\text { Further details: placebo }\end{array}$} \\
\hline Outcomes & \multicolumn{2}{|c|}{$\begin{array}{l}\text { Outcomes reported: serious adverse events (number of people), resolution of fatty liver disease } \\
\text { Follow-up, months: } 6\end{array}$} \\
\hline Notes & \multicolumn{2}{|c|}{$\begin{array}{l}\text { Source of funding: not stated } \\
\text { Trial name/Trial registry number: not stated } \\
\text { Attempts were made to contact study authors in December } 2020\end{array}$} \\
\hline \multicolumn{3}{|l|}{ Risk of bias } \\
\hline Bias & Authors' judgement & Support for judgement \\
\hline $\begin{array}{l}\text { Random sequence genera- } \\
\text { tion (selection bias) }\end{array}$ & Unclear risk & Comment: this information was not available \\
\hline $\begin{array}{l}\text { Allocation concealment } \\
\text { (selection bias) }\end{array}$ & Unclear risk & Comment: this information was not available \\
\hline
\end{tabular}


Zhu 2008 (Continued)

Blinding of participants and personnel (perfor-

Unclear risk Comment: this information was not available

All outcomes

\begin{tabular}{|c|c|c|}
\hline $\begin{array}{l}\text { Blinding of outcome as- } \\
\text { sessment (detection bias) }\end{array}$ & Unclear risk & $\begin{array}{l}\text { Quote: "ultrasound scans were performed by a trained operator who was blind } \\
\text { to the treatment of participants" }\end{array}$ \\
\hline All outcomes & & $\begin{array}{l}\text { Comment: it is not clear whether outcome assessors of remaining outcomes } \\
\text { were blinded }\end{array}$ \\
\hline
\end{tabular}

Incomplete outcome data Unclear risk (attrition bias)

Comment: participants were excluded from analysis for reasons that may be

All outcomes related to the intervention and to outcomes

\section{Selective reporting (re- Low risk} porting bias)

Comment: no previously published protocol was available; adverse events and either mortality or fatty liver resolution or both were reported

Other bias Low risk Comment: no other bias noted

Zohrer 2017

\section{Study characteristics}

\begin{tabular}{|c|c|}
\hline Methods & Randomised clinical trial \\
\hline Participants & $\begin{array}{l}\text { Country: Italy } \\
\text { Period of recruitment: not stated } \\
\text { Number randomised: } 43 \\
\text { Post-randomisation dropouts: } 3(7.0 \%) \\
\text { Revised sample size: } 40 \\
\text { Reasons for post-randomisation dropouts: did not complete the study } \\
\text { Average age, years: } 13 \\
\text { Females: } 16(40.0 \%) \\
\text { NASH: } 40 \text { (100.0\%) } \\
\text { Diabetes mellitus: not stated } \\
\text { Inclusion criteria: } 1 \text {. NAFLD } \\
\text { Exclusion criteria: } 1 \text {. Other cause of liver disease (e.g. autoimmune hepatitis). 2. Parenteral nutrition. } 3 . \\
\text { Previous gastrointestinal surgery. } 4 \text {. Use of NSAIDs, antibiotics, probiotics within } 2 \text { months } \\
\text { Method for diagnosis of NAFLD: liver biopsy }\end{array}$ \\
\hline Interventions & $\begin{array}{l}\text { Group 1: phospholipids plus PUFA plus vitamin E }(n=20) \\
\text { Further details: docosahexaenoic acid, choline, and vitamin E (DHA-CHO-VE) (Pro DHA Steatolip Plus) } \\
\text { for } 6 \text { months } \\
\text { Group 2: no active intervention }(n=20) \\
\text { Further details: placebo } \\
\text { Additional details: both groups received lifestyle intervention. }\end{array}$ \\
\hline Outcomes & $\begin{array}{l}\text { Outcomes reported: serious adverse events (number of people), any adverse events (number of events) } \\
\text { Follow-up, months: } 12\end{array}$ \\
\hline Notes & $\begin{array}{l}\text { Source of funding (quote): "we thank DMF Dietetic Metabolic Food (Italy) who provided Pro DHA } \\
\text { Steatolip Plus with verified composition and indistinguishable placebo" } \\
\text { Trial name/Trial registry number: NCT01934777 } \\
\text { Attempts were made to contact study authors in December } 2020\end{array}$ \\
\hline
\end{tabular}

\section{Risk of bias}

Nutritional supplementation for nonalcohol-related fatty liver disease: a network meta-analysis (Review) 
Zohrer 2017 (Continued)

\begin{tabular}{lll} 
Bias & Authors' judgement & Support for judgement \\
\hline $\begin{array}{l}\text { Random sequence genera- } \\
\text { tion (selection bias) }\end{array}$ & Low risk & Quote: "children were randomized by computer" \\
\hline $\begin{array}{l}\text { Allocation concealment } \\
\text { (selection bias) }\end{array}$ & Low risk & $\begin{array}{l}\text { Quote: "children were randomized by computer" } \\
\text { Comment: both allocation concealment and blinding were achieved with use } \\
\text { of placebo }\end{array}$
\end{tabular}

$\begin{array}{ll}\begin{array}{l}\text { Blinding of participants } \\ \text { and personnel (perfor- }\end{array} & \text { Low risk }\end{array}$
assignment...placebo"

All outcomes

\begin{tabular}{lll}
\hline $\begin{array}{l}\text { Blinding of outcome as- } \\
\text { sessment (detection bias) } \\
\text { All outcomes }\end{array}$ & Low risk & $\begin{array}{l}\text { Quote: "patients and investigators were blinded before and after intervention } \\
\text { assignment...placebo" }\end{array}$ \\
\hline $\begin{array}{l}\text { Incomplete outcome data } \\
\text { (attrition bias) } \\
\text { All outcomes }\end{array}$ & Unclear risk & $\begin{array}{l}\text { Comment: participants were excluded from analysis for reasons that may be } \\
\text { related to the intervention and to outcomes }\end{array}$ \\
\hline $\begin{array}{l}\text { Selective reporting (re- } \\
\text { porting bias) }\end{array}$ & High risk & $\begin{array}{l}\text { Quote: "however, we underline that the current primary endpoint differs from } \\
\text { that in the protocol submitted to ClinicalTrials.gov because liver biopsy at } 12 \\
\text { months in the placebo group was not performed for ethical reasons" }\end{array}$ \\
\hline Other bias & Low risk & Comment: no other bias noted \\
\hline
\end{tabular}

ALT: alanine aminotransferase.

AST: aspartate aminotransferase.

BMI: body mass index.

CAP: controlled attenuation parameter.

CLD: chronic lung disease.

CMV: cytomegalovirus.

DHA: docosahexaenoic acid.

EBV: Epstein-Barr virus.

EPA: eicosapentaenoic acid.

HbA1c: glycosylated haemoglobin.

HPA: hypothalamic-pituitary-adrenal.

LFT: liver function test.

MUFA: monounsaturated fatty acid.

NAFLD: non-alcohol-related fatty liver disease.

NASH: non-alcoholic steatohepatitis.

NSAID: non-steroidal anti-inflammatory drug.

NYHA: New York Heart Association.

PDFF: proton density fat fraction.

PT: prothrombin time.

PUFA: polyunsaturated fatty acid.

$\mathrm{RCT}$ : randomised controlled trial.

SAM-E: S-adenosyl methionine.

TG: triglyceride.

TNF: tumour necrosis factor.

TSH: thyroid-stimulating hormone.

UDCA: ursodeoxycholic acid.

USS: ultrasound scan. 
Characteristics of excluded studies [ordered by study ID]

\begin{tabular}{|c|c|}
\hline Study & Reason for exclusion \\
\hline Abenavoli 2017 & $\begin{array}{l}\text { Study authors used systematic sampling method. Although they mention the word "randomisa- } \\
\text { tion", they also state: "the control group (Group C), [which] refused any pharmacological and/or } \\
\text { nutritional treatment, was also studied" (in an earlier report) }\end{array}$ \\
\hline Akcam 2011 & $\begin{array}{l}\text { Comparison of nutritional supplementation with pharmacological intervention or lifestyle inter- } \\
\text { vention }\end{array}$ \\
\hline Basu 2014 & $\begin{array}{l}\text { Interventions were not reported clearly enough to assess whether this trial is eligible for the review } \\
\text { (i.e. it is not clear what intervention and control were) }\end{array}$ \\
\hline Chambers 2018 & Comparison of a polysaccharide with a non-nutritional supplement \\
\hline Chang 2014 & Not clear whether participants had NAFLD \\
\hline Dela Cruz 2012 & $\begin{array}{l}\text { Comparison of nutritional supplementation with pharmacological intervention and lifestyle inter- } \\
\text { vention }\end{array}$ \\
\hline Ersoz 2005 & Comparison of nutritional supplementation with pharmacological intervention \\
\hline Famouri 2017b & Quasi-randomised study (alternation) \\
\hline Guo 2014 & $\begin{array}{l}\text { In this cross-over study, cross-over was at } 4 \text { weeks, with no outcomes available prior to cross-over; } \\
\text { therefore, the RCT will not meet objectives of this Review }\end{array}$ \\
\hline Hajiaghamohammadi 2012 & Comparison of nutritional supplementation with pharmacological intervention \\
\hline Han 2014 & $\begin{array}{l}\text { Only } 1 \text { of the groups received metformin, a pharmacological intervention (i.e. unequal co-interven- } \\
\text { tions) }\end{array}$ \\
\hline Khoshbaten 2010a & Comparison of nutritional supplementation with lifestyle intervention \\
\hline Mahmoudi 2020 & Comparison of variations in same treatment node \\
\hline NCT00820651 & Comparison of nutritional supplementation with lifestyle intervention \\
\hline NCT04281121 & $\begin{array}{l}\text { In this study, which is available only from a trial register, although study authors state that alloca- } \\
\text { tion is random, they also state "single group assignment", and the description of interventions is } \\
\text { identical }\end{array}$ \\
\hline Petyaev 2018 & $\begin{array}{l}\text { Comparison of different formulations of supplement (i.e. comparison of variations in treatment } \\
\text { node) }\end{array}$ \\
\hline Podszun 2020 & Comparison of variations in same treatment node \\
\hline Saarinen 2011 & Not clear whether participants had NAFLD \\
\hline Semiserin 2016 & Not clear whether this was an RCT \\
\hline Singhal 2015 & Although study authors state "randomly divided", they also state "retrospective...study" \\
\hline Zhang 2008 & Comparison with nutritional supplementation with pharmacological intervention \\
\hline
\end{tabular}

NAFLD: non-alcohol-related fatty liver disease. 
Characteristics of ongoing studies [ordered by study ID]

\section{ChicTR2000034740}

\begin{tabular}{ll}
\hline Study name & ChiCTR2000034740 \\
\hline Methods & Randomised clinical trial \\
\hline Participants & People with NAFLD \\
\hline Interventions & Other supplement vs no active intervention (placebo) \\
\hline Outcomes & No outcomes of interest will be measured in this trial \\
\hline Starting date & August 2020 \\
\hline Contact information & Gao Lulu (gaolu755243@163.com) \\
\hline Notes & \\
\hline
\end{tabular}

\section{ChiCTR2000035899}

\begin{tabular}{ll}
\hline Study name & ChiCTR2000035899 \\
\hline Methods & Randomised clinical trial \\
\hline Participants & People with NAFLD \\
\hline Interventions & Other supplement vs no active intervention (placebo) \\
\hline Outcomes & No outcomes of interest will be measured in this trial \\
\hline Starting date & October 2020 \\
\hline Contact information & Chen Yuanwen (chenyuanwen@xinhuamed.com.cn) \\
\hline Notes & \\
\hline
\end{tabular}

\section{CTRI/2020/05/025322}

\begin{tabular}{ll}
\hline Study name & CTRI/2020/05/025322 \\
\hline Methods & Randomised clinical trial \\
\hline Participants & People with NAFLD \\
\hline Interventions & Other supplement vs no active intervention (placebo) \\
\hline Outcomes & No outcomes of interest will be measured in this trial \\
\hline Starting date & June 2020 \\
\hline
\end{tabular}


CTRI/2020/05/025322 (Continued)

Contact information

Mr Krishna Chaitanya (highaspirer@gmail.com)

\section{Notes}

\section{CTRI/2020/07/026362}

\begin{tabular}{ll}
\hline Study name & CTRI/2020/07/026362 \\
\hline Methods & Randomised clinical trial \\
\hline Participants & People with NAFLD \\
\hline Interventions & Other supplement vs no active intervention (placebo) \\
\hline Outcomes & No outcomes of interest will be measured in this trial \\
\hline Starting date & July 2020 \\
\hline Contact information & Dr Gyanendra Datta Shukla (dr.gdshukla@gmail.com) \\
\hline Notes & \\
\hline
\end{tabular}

Han 2020

\begin{tabular}{ll}
\hline Study name & KCT0003554 \\
\hline Methods & Randomised clinical trial \\
\hline Participants & People with NAFLD \\
\hline Interventions & Other supplements vs no active intervention (placebo) \\
\hline Outcomes & Change in liver fat \\
\hline Starting date & March 2019 \\
\hline Contact information & Changsop Yang (yangunja@kiom.re.kr) \\
\hline Notes & \\
\hline
\end{tabular}

IRCT20131125015536N

\begin{tabular}{ll}
\hline Study name & IRCT20131125015536N \\
\hline Methods & Randomised clinical trial \\
\hline Participants & People with NAFLD \\
\hline Interventions & Other supplements vs no active intervention (placebo) \\
\hline
\end{tabular}


IRCT20131125015536N (Continued)

\begin{tabular}{ll} 
Outcomes & No outcomes of interest will be measured in this trial \\
\hline Starting date & October 2020 \\
\hline Contact information & Mohammad Javad Hosseinzadeh (mhosseinzadeh@tums.ac.ir) \\
\hline Notes & \\
\hline
\end{tabular}

\section{IRCT20191009045043N}

\begin{tabular}{ll}
\hline Study name & IRCT20191009045043N \\
\hline Methods & Randomised clinical trial \\
\hline Participants & People with NAFLD \\
\hline Interventions & Other supplements vs no active intervention (placebo) \\
\hline Outcomes & No outcomes of interest will be measured in this trial \\
\hline Starting date & January 2020 \\
\hline Contact information & Mohsen Mohit (mohsen.mohit20@yahoo.com) \\
\hline Notes & \\
\hline
\end{tabular}

\section{IRCT20200304046692N}

\begin{tabular}{ll}
\hline Study name & IRCT20200304046692N \\
\hline Methods & Randomised clinical trial \\
\hline Participants & People with NAFLD \\
\hline Interventions & Other supplements vs no active intervention (placebo) \\
\hline Outcomes & Change in liver fat \\
\hline Starting date & December 2019 \\
\hline Contact information & Reza Barati-Boldaji (reza93barati@gmail.com) \\
\hline Notes & \\
\hline
\end{tabular}

\section{IRCT20200531047614N}

\begin{tabular}{ll} 
Study name & IRCT20200531047614N \\
\hline Methods & Randomised clinical trial
\end{tabular}


IRCT20200531047614N (Continued)

\begin{tabular}{ll} 
Participants & People with NAFLD \\
\hline Interventions & Other supplements vs no active intervention (placebo) \\
\hline Outcomes & Change in liver fat \\
\hline Starting date & May 2020 \\
\hline Contact information & Mohammad Rajabi (rajabi-m@kaums.ac.ir) \\
\hline Notes & \\
\hline
\end{tabular}

\section{Lambert 2015}

\begin{tabular}{ll}
\hline Study name & NCT02568605 \\
\hline Methods & Randomised clinical trial \\
\hline Participants & People with NAFLD \\
\hline Interventions & Prebiotics/Probiotics/Synbiotics vs no active intervention (placebo) \\
\hline Outcomes & Quality of life, change in liver fat \\
\hline Starting date & May 2015 \\
\hline Contact information & Raylene A. Reimer (reimer@ucalgary.ca) \\
\hline Notes & \\
\hline
\end{tabular}

NCT02289235

\begin{tabular}{ll}
\hline Study name & NCT02289235 \\
\hline Methods & Randomised clinical trial \\
\hline Participants & People with NAFLD \\
\hline Interventions & Other supplements (ginger) vs no active intervention (placebo) \\
\hline Outcomes & Change in liver fat \\
\hline Starting date & May 2019 \\
\hline Contact information & Mesbah Shams (Internal Medicine \& Endocrinology, Shiraz University of Medical Sciences) \\
\hline Notes & \\
\hline
\end{tabular}


NCT02642172

\begin{tabular}{|c|c|}
\hline Study name & NCT02642172 \\
\hline Methods & Randomised clinical trial \\
\hline Participants & People with NAFLD \\
\hline Interventions & Prebiotics/Probiotics/Synbiotics vs no active intervention (placebo) \\
\hline Outcomes & Change in liver fat \\
\hline Starting date & January 2016 \\
\hline Contact information & $\begin{array}{l}\text { Yaakov Maor (NCT02642172; \%20PN-837-CTIL,\%20Prebiotics\%20in\%20Patients\%20With\%20Non- } \\
\text { alcoholic\%20Liver\%20Disease" type="EXTERNAL">yaakovma1@clalit.org.il) }\end{array}$ \\
\hline \multicolumn{2}{|l|}{ Notes } \\
\hline \multicolumn{2}{|l|}{ NCT02647294 } \\
\hline Study name & NCT02647294 \\
\hline Methods & Randomised clinical trial \\
\hline Participants & People with NAFLD \\
\hline Interventions & PUFA vs no active intervention (placebo) \\
\hline Outcomes & Change in liver fat, liver fibrosis \\
\hline Starting date & February 2016 \\
\hline Contact information & Radan Bruha (General University Hospital in Prague) \\
\hline Notes & \\
\hline
\end{tabular}

\section{NCT02764047}

\begin{tabular}{ll}
\hline Study name & NCT02764047 \\
\hline Methods & Randomised clinical trial \\
\hline Participants & People with NAFLD \\
\hline Interventions & Prebiotics/Probiotics/Synbiotics vs no active intervention (placebo) \\
\hline Outcomes & Liver fibrosis \\
\hline Starting date & January 2015 \\
\hline Contact information & $\begin{array}{l}\text { Gabriela Z Port (NCT02764047; \%20852.771,\%20Probiotics\%20in\%20the\%20Treatment\%200f } \\
\text { \%20NAFLD" type="EXTERNAL">gabriela.port@hotmail.com) }\end{array}$ \\
\hline
\end{tabular}


NCT02764047 (Continued)

Notes

NCT03439917

\begin{tabular}{ll}
\hline Study name & NCT03439917 \\
\hline Methods & Randomised clinical trial \\
\hline Participants & People with NAFLD \\
\hline Interventions & Amino acids (carnitine) vs no active intervention (placebo) \\
\hline Outcomes & No outcomes of interest for this review will be reported in this trial \\
\hline Starting date & April 2018 \\
\hline Contact information & Guru Aithal (University of Nottingham) \\
\hline
\end{tabular}

Notes

NCT03467282

\begin{tabular}{ll}
\hline Study name & NCT03467282 \\
\hline Methods & Randomised clinical trial \\
\hline Participants & People with NAFLD \\
\hline Interventions & Prebiotics/Probiotics/Synbiotics vs no active intervention (placebo) \\
\hline Outcomes & Liver fibrosis \\
\hline Starting date & November 2017 \\
\hline Contact information & Valesca Dall Alba (Hospital de Clinicas de Porto Alegre) \\
\hline
\end{tabular}

Notes

\section{NCT04175392}

\begin{tabular}{ll}
\hline Study name & PRONE Study \\
\hline Methods & Randomised clinical trial \\
\hline Participants & People with NAFLD and NASH \\
\hline Interventions & Prebiotics/Probiotics/Synbiotics vs no active intervention (placebo) \\
\hline Outcomes & Liver fibrosis \\
\hline
\end{tabular}


NCT04175392 (Continued)

\begin{tabular}{ll} 
Starting date & January 2021 \\
\hline Contact information & Mark Anthony Raphael (mark.raphael@beaumont.org) \\
\hline
\end{tabular}

Notes

NCT04193982

\begin{tabular}{ll}
\hline Study name & NCT04193982 \\
\hline Methods & Randomised clinical trial \\
\hline Participants & People with NAFLD \\
\hline Interventions & Vitamin E vs no active intervention \\
\hline Outcomes & No outcomes of interest for this review will be reported in this trial \\
\hline Starting date & January 2021 \\
\hline Contact information & Mithun Sharma (drmithunsharma@gmail.com) \\
\hline
\end{tabular}

Notes

\section{NCT04198805}

\begin{tabular}{ll}
\hline Study name & PUVENAFLD \\
\hline Methods & Randomised clinical trial \\
\hline Participants & People with NAFLD \\
\hline Interventions & Vitamin E vs PUFA vs Vitamin E plus PUFA vs no active intervention \\
\hline Outcomes & Health-related quality of life \\
\hline Starting date & January 2020 \\
\hline Contact information & Stacey Richardson (stlyrich@iu.edu) \\
\hline Notes & \\
\hline
\end{tabular}

\section{NCT04330326}

\begin{tabular}{ll}
\hline Study name & NCT04330326 \\
\hline Methods & Randomised clinical trial \\
\hline Participants & People with NAFLD \\
\hline
\end{tabular}


NCT04330326 (Continued)

\begin{tabular}{ll} 
Interventions & Other supplement vs no active intervention \\
\hline Outcomes & Change in liver fat \\
\hline Starting date & July 2019 \\
\hline Contact information & Mujdat Zeybel (mzeybel@kuh.ku.edu.tr) \\
\hline Notes &
\end{tabular}

\section{NCT04475276}

\begin{tabular}{ll}
\hline Study name & NCT04475276 \\
\hline Methods & Randomised clinical trial \\
\hline Participants & People with NAFLD \\
\hline Interventions & Other antioxidants vs no active intervention \\
\hline Outcomes & Change in fat \\
\hline Starting date & August 2020 \\
\hline Contact information & Monalisa Jena (drmonalisajena@gmail.com) \\
\hline Notes & \\
\hline
\end{tabular}

\section{NCT04555434}

\begin{tabular}{ll}
\hline Study name & NCT04555434 \\
\hline Methods & Randomised clinical trial \\
\hline Participants & People with NAFLD \\
\hline Interventions & Prebiotics/Probiotics/Synbiotics vs no active intervention (placebo) \\
\hline Outcomes & No outcomes of interest for this review will be reported in this trial \\
\hline Starting date & October 2019 \\
\hline Contact information & Ki Tae Suk (ktsuk@hallym.ac.kr) \\
\hline Notes & \\
\hline
\end{tabular}

\section{NCT04671186}


NCT04671186 (Continued)

\begin{tabular}{ll} 
Methods & Randomised clinical trial \\
\hline Participants & People with NAFLD \\
\hline Interventions & Prebiotics/Probiotics/Synbiotics vs no active intervention \\
\hline Outcomes & Change in liver fat \\
\hline Starting date & September 2020 \\
\hline Contact information & Kanya Ahuja (kahuja3@northwell.edu) \\
\hline Notes &
\end{tabular}

Notes

NCT04704063

\begin{tabular}{ll} 
Study name & NCT04704063 \\
\hline Methods & Randomised clinical trial \\
\hline Participants & People with NAFLD \\
\hline Interventions & Vitamin E vs no active intervention \\
\hline Outcomes & Change in liver fat \\
\hline Starting date & January 2021 \\
\hline Contact information & Siok Yee Chan (sychan@usm.my) \\
\hline Notes & \\
\hline
\end{tabular}

NCT04718051

\begin{tabular}{ll}
\hline Study name & NCT04718051 \\
\hline Methods & Randomised clinical trial \\
\hline Participants & People with NAFLD \\
\hline Interventions & Other supplements vs no active intervention \\
\hline Outcomes & No outcomes of interest for this review will be reported in this trial \\
\hline Starting date & January 2021 \\
\hline Contact information & Ming Shun Wu (vw1017@gmail.com) \\
\hline Notes & \\
\hline
\end{tabular}


Wicklow 2015

\begin{tabular}{ll}
\hline Study name & INSYTE study \\
\hline Methods & Randomised clinical trial \\
\hline Participants & People with NAFLD \\
\hline Interventions & Other supplements (resveratrol) vs no active intervention (placebo) \\
\hline Outcomes & No outcomes of interest for this review will be reported in this trial \\
\hline Starting date & December 2013 \\
\hline Contact information & Brandy Wicklow (bwicklow@hsc.mb.ca) \\
\hline Notes & \\
\hline
\end{tabular}

\section{Zang 2018}

\begin{tabular}{ll}
\hline Study name & NCT02962297 \\
\hline Methods & Randomised clinical trial \\
\hline Participants & People with NASH \\
\hline Interventions & Vitamin E vs no active intervention (placebo) \\
\hline Outcomes & Liver fibrosis, NAFLD activity scores \\
\hline Starting date & December 2016 \\
\hline Contact information & Junping Shi (davidshi0571@126.com) \\
\hline
\end{tabular}

Notes

NAFLD: non-alcohol-related fatty liver disease.

NASH: non-alcoholic steatohepatitis.

PUFA: polyunsaturated fatty acid.

ADDITIONAL TABLES

Table 1. Summary of characteristics of included studies

\section{Features \\ Summary}

\section{Participant characteristics}

Mean or median age in trials ranged from 7 to 66 years in trials that reported this information (Miglio 2000; Harrison 2003; Kugelmas 2003; Deng 2005; Chande 2006; Chou 2006; Dufour 2006; Nobili 2006; Spadaro 2008; Wang 2008; Zhu 2008; Abdelmalek 2009; Gomez 2009; Hashemi 2009; Fabbrini 2010; Khoshbaten 2010b; Li 2010; Malaguarnera 2010; Sanyal 2010; Aller 2011; Lavine 2011; Tan 2011; Vajro 2011; Della Corte 2012; Gonciarz 2012; Loguercio 2012; Malaguarnera 2012; Panahi 2012; Ghergherehchi 2013; Gianturco 2013; Illnait 2013; Magosso 2013; Nobili 2013; Shavakhi 2013; Wong 2013a; Wong 2013b; Aliashrafi 2014; Alisi 2014; Askari 2014; Celinski 2014; Chachay 2014; Eslamparast 2014; Farhangi 2014; Foroughi 2014; Martinez-Rodriguez 2014; Sanyal 2014; Scorletti 2014; Sharifi 2014; Solhi 2014; Somi 2014; Akbarzadeh 2015; Aller 2015; Amiri-Moghadam 2015; 
Table 1. Summary of characteristics of included studies (Continued)

Argo 2015; Bae 2015; Boyraz 2015; Chen 2015a; Chen 2015b; Dasarathy 2015; Faghihzadeh 2015; Janczyk 2015; Orr 2015; Pacifico 2015; Qin 2015; Yan 2015; Zhang 2015; Asgharian 2016; Barchetta 2016; Della Corte 2016; Ebrahimi-Mameghani 2016; Ferolla 2016; Guo 2016; Hong 2016; Li 2016; Nabavi 2016; Nogueira 2016; Panahi 2016; Rahimlou 2016; Rahmani 2016; Sepideh 2016; Yari 2016; Amiri 2017; Ashraf 2017; Behrouz 2017; Chan 2017; Ebrahimi-Mameghani 2017; Famouri 2017a; Hussain 2017; Javadi 2017; Jeong 2017; Manzhalii 2017; Mofidi 2017; Navekar 2017; Sakpal 2017; Schattenberg 2017; Shahmohammadi 2017; Tabatabaee 2017; Wang 2017; Zohrer 2017; Amanat 2018; Amirkhizi 2018; Asghari 2018; Bakhshimoghaddam 2018; Bomhof 2018; Dabbaghmanesh 2018; Daneshi-Maskooni 2018; Eriksson 2018; Ghaffari 2018; Hosseini 2018; Javanmardi 2018; Kobyliak 2018; Lewis 2018; Oscarsson 2018; Pervez 2018; Sayari 2018; Taghvaei 2018; Tobin 2018; Zamani 2018; Bril 2019; Cheraghpour 2019; Duseja 2019; Jazayeri-Tehrani 2019; Abhari 2020; Afsharinasab 2020; Afzali 2020; Babaei 2020; Bahrami 2020; Boonyagard 2020; Cai 2020; Cerletti 2020; Climax 2020; Fathi 2020; Fernandez-Travieso 2020; Ferro 2020; Hormoznejad 2020; Hoseini 2020; Hosseinabadi 2020; Kazemi 2020; Kooshki 2020; Mansour 2020; Moradi 2020; Orang 2020; Parsi 2020; Pasdar 2020; Pervez 2020; Poparn 2020; Pour 2020; Rafie 2020; Sadrkabir 2020; Sangouni 2020; Scorletti 2020; Soleimani 2020; Song 2020; Tutunchi 2020; Yari 2020; Zanko 2020; Chiou 2021; Hong 2021; Izadi 2021; Kanoni 2021; Morvaridzadeh 2021; Poulos 2021; Soleimani 2021; EUCTR 2008-008275-34-GB; EUCTR 2009-017080-41-GB)

In 9 trials, all participants were females (Abdelmalek 2009; Panahi 2012; Amiri-Moghadam 2015; Orr 2015; Hosseini 2018; Kobyliak 2018; Lewis 2018; Hoseini 2020; Moradi 2020). In 1 trial, all participants were males (Chachay 2014). In the remaining 154 trials that reported information on gender of participants, the proportion of females ranged from 6.7\% to 98\% (Miglio 2000; Harrison 2003; Kugelmas 2003; Deng 2005; Chande 2006; Chou 2006; Dufour 2006; Nobili 2006; Spadaro 2008; Wang 2008; Zhu 2008; Gomez 2009; Hashemi 2009; Fabbrini 2010; Li 2010; Khoshbaten 2010b; Malaguarnera 2010; Sanyal 2010; Aller 2011; Lavine 2011; Tan 2011; Vajro 2011; Della Corte 2012; Gonciarz 2012; Loguercio 2012; Malaguarnera 2012; Ghergherehchi 2013; Gianturco 2013; Illnait 2013; Magosso 2013; Nobili 2013; Shavakhi 2013; Wong 2013a; Wong 2013b; Aliashrafi 2014; Alisi 2014; Askari 2014; Celinski 2014; Eslamparast 2014; Farhangi 2014; Foroughi 2014; Martinez-Rodriguez 2014; Sanyal 2014; Scorletti 2014; Sharifi 2014; Solhi 2014; Somi 2014; Akbarzadeh 2015; Aller 2015; Argo 2015; Bae 2015; Boyraz 2015; Chen 2015a; Chen 2015b; Dasarathy 2015; Faghihzadeh 2015; Janczyk 2015; Pacifico 2015; Qin 2015; Yan 2015; Zhang 2015; Asgharian 2016; Barchetta 2016; Della Corte 2016; Ebrahimi-Mameghani 2016; Ferolla 2016; Guo 2016; Hong 2016; Li 2016; Nabavi 2016; Nogueira 2016; Panahi 2016; Rahimlou 2016; Rahmani 2016; Sepideh 2016; Yari 2016; Amiri 2017; Ashraf 2017; Behrouz 2017; Chan 2017; Ebrahimi-Mameghani 2017; Famouri 2017a; Hussain 2017; Javadi 2017; Jeong 2017; Manzhalii 2017; Mofidi 2017; Navekar 2017; Sakpal 2017; Schattenberg 2017; Shahmohammadi 2017; Tabatabaee 2017; Wang 2017; Zohrer 2017; Amanat 2018; Amirkhizi 2018; Asghari 2018; Bakhshimoghaddam 2018; Bomhof 2018; Dabbaghmanesh 2018; Daneshi-Maskooni 2018; Eriksson 2018; Ghaffari 2018; Javanmardi 2018; Oscarsson 2018; Pervez 2018; Sayari 2018; Taghvaei 2018; Tobin 2018; Zamani 2018; Bril 2019; Cheraghpour 2019; Duseja 2019; Jazayeri-Tehrani 2019; Abhari 2020; Afsharinasab 2020; Afzali 2020; Babaei 2020; Bahrami 2020; Boonyagard 2020; Cai 2020; Cerletti 2020; Climax 2020; Fathi 2020; Fernandez-Travieso 2020; Ferro 2020; Hormoznejad 2020; Hosseinabadi 2020; Kazemi 2020; Kooshki 2020; Mansour 2020; Orang 2020; Parsi 2020; Pasdar 2020; Pervez 2020; Poparn 2020; Pour 2020; Rafie 2020; Sadrkabir 2020; Sangouni 2020; Scorletti 2020; Soleimani 2020; Song 2020; Tutunchi 2020; Yari 2020; Zanko 2020; Chiou 2021; Hong 2021; Izadi 2021; Morvaridzadeh 2021; Poulos 2021; Soleimani 2021; EUCTR 2008-008275-34-GB; EUCTR 2009-017080-41-GB)

A total of 44 trials reported the proportion of participants who had NASH: in 1 trial, no participants had NASH (Tobin 2018); in 39 trials, all participants had NASH (Uygun 2000; Harrison 2003; Kugelmas 2003; Chande 2006; Dufour 2006; Wang 2008; Abdelmalek 2009; Hashemi 2009; Malaguarnera 2010; Sanyal 2010; Tan 2011; Basu 2012; Malaguarnera 2012; Shavakhi 2013; Wong 2013a; Wong 2013b; Alisi 2014; Sanyal 2014; Solhi 2014; Amiri-Moghadam 2015; Argo 2015; Dasarathy 2015; Eghtesadi 2016; Ferolla 2016; Li 2016; Nogueira 2016; Ashraf 2017; Chan 2017; Manzhalii 2017; Schattenberg 2017; Wang 2017; Zohrer 2017; Bomhof 2018; Geier 2018; Bril 2019; Barbakadze 2020; Chiou 2021; NCT00845845; NCT01623024); in the remaining 4 trials, the proportion of participants who had NASH ranged from 24.3\% to 64.7\% (Lavine 2011; Celinski 2014; Pacifico 2015; Della Corte 2016)

In all, 90 trials reported the proportion of participants who had diabetes mellitus: in 53 trials, no participants had diabetes mellitus (Deng 2005; Gomez 2009; Fabbrini 2010; Sanyal 2010; Aller 2011; Lavine 2011; Vajro 2011; Basu 2012; Gianturco 2013; Askari 2014; Eslamparast 2014; Farhangi 2014; 
Table 1. Summary of characteristics of included studies (Continued)

Martinez-Rodriguez 2014; Solhi 2014; Somi 2014; Aller 2015; Chen 2015a; Chen 2015b; Faghihzadeh 2015; Janczyk 2015; Pacifico 2015; Ekhlasi 2016; Farsi 2016; Heeboll 2016; Rahimlou 2016; Yari 2016; Ashraf 2017; Behrouz 2017; Hussain 2017; Manzhalii 2017; Navekar 2017; Shahmohammadi 2017; Wang 2017; Amanat 2018; Amirkhizi 2018; Asghari 2018; Bakhshimoghaddam 2018; Dabbaghmanesh 2018; Hosseini 2018; Oscarsson 2018; Taghvaei 2018; Zamani 2018; Cheraghpour 2019; Duseja 2019; Abhari 2020; Afsharinasab 2020; Babaei 2020; Fathi 2020; Ferro 2020; Hormoznejad 2020; Hosseinabadi 2020; Kazemi 2020; Kooshki 2020); in 9 trials, all participants had diabetes mellitus (Bae 2015; Dasarathy 2015; Barchetta 2016; Kobyliak 2017; Eriksson 2018; Kobyliak 2018; Bril 2019; Mansour 2020; Orang 2020); in the remaining 28 trials, the proportion of participants who had diabetes mellitus ranged from 5.6\% to 55.0\% (Harrison 2003; Chande 2006; Dufour 2006; Abdelmalek 2009; Khoshbaten 2010b; Malaguarnera 2012; Panahi 2012; Illnait 2013; Wong 2013a; Wong 2013b; Celinski 2014; Sanyal 2014; Scorletti 2014; Argo 2015; Panahi 2016; Chan 2017; Sakpal 2017; Tabatabaee 2017; Tobin 2018; Boonyagard 2020; Cerletti 2020; Climax 2020; Fernandez-Travieso 2020; Scorletti 2020; Soleimani 2020; Zanko 2020; Soleimani 2021; EUCTR 2008-008275-34-GB)

The method of diagnosis of NAFLD included biopsy, transaminases, and imaging methods including ultrasound, elastography, CT examination, or a combination of these methods. The method of diagnosis used in each study is available in Table 2 and under Characteristics of included studies

\section{Interventions compared}

Interventions compared in these 202 trials included amino acids, amino acids plus PUFA, amino acids plus vitamin C, amino acids plus vitamin E plus other antioxidants, oestrogen, MUFA, other antioxidants, other antioxidants plus other supplements, other supplements, other supplements plus other antioxidants, phospholipids, phospholipids plus PUFA plus vitamin E, phospholipids plus vitamin E plus other antioxidants, polysaccharides, prebiotics/probiotics/synbiotics plus vitamin E, prebiotics/probiotics/synbiotics, prebiotics/probiotics/synbiotics plus PUFA, prebiotics/probiotics/synbiotics plus vitamin D, PUFA, PUFA plus other supplements, PUFA plus vitamin D, PUFA plus vitamin $E$, vitamin $C$, vitamin $C$ plus other antioxidants, vitamin $D$, vitamin $D$ plus vitamin $E$ plus other antioxidants, vitamin E, vitamin E plus other antioxidants, vitamin E plus other antioxidants plus other supplements, vitamin E plus other supplements, vitamin E plus vitamin $C$, and no active intervention

\section{Trials reporting outcomes}

A total of 115 trials (7732 participants) reported 1 or more outcomes for this review (Miglio 2000; Harrison 2003; Chande 2006; Chou 2006; Dufour 2006; Nobili 2006; Spadaro 2008; Wang 2008; Zhu 2008; Abdelmalek 2009; Gomez 2009; Khoshbaten 2010b; Li 2010; Malaguarnera 2010; Ruan 2010; Sanyal 2010; Lavine 2011; Vajro 2011; Loguercio 2012; Malaguarnera 2012; Gianturco 2013; Illnait 2013; Magosso 2013; Nobili 2013; Saxena 2013; Shavakhi 2013; Wong 2013a; Wong 2013b; Chachay 2014; Eslamparast 2014; Foroughi 2014; Martinez-Rodriguez 2014; Sanyal 2014; Scorletti 2014; Sharifi 2014; Somi 2014; Aller 2015; Bae 2015; Chen 2015a; Chen 2015b; Dasarathy 2015; Faghihzadeh 2015; Janczyk 2015; Qin 2015; Yan 2015; Zhang 2015; Asgharian 2016; Farsi 2016; Ferolla 2016; Guo 2016; Heeboll 2016; Li 2016; Nabavi 2016; Naganuma 2016; Nogueira 2016; Rahimlou 2016; Rahmani 2016; Sepideh 2016; Yari 2016; Chan 2017; Famouri 2017a; Hussain 2017; Jeong 2017; Manzhalii 2017; Mofidi 2017; Navekar 2017; Sakpal 2017; Schattenberg 2017; Shahmohammadi 2017; Zohrer 2017; Amanat 2018; Asghari 2018; Bakhshimoghaddam 2018; Bomhof 2018; Daneshi-Maskooni 2018; Eriksson 2018; Geier 2018; Kobyliak 2018; Lewis 2018; Oscarsson 2018; Pervez 2018; Taghvaei 2018; Zamani 2018; Bril 2019; Cheraghpour 2019; Duseja 2019; Jazayeri-Tehrani 2019; Abhari 2020; Afzali 2020; Bahrami 2020; Boonyagard 2020; Cerletti 2020; Climax 2020; Fathi 2020; Fernandez-Travieso 2020; Hormoznejad 2020; Hosseinabadi 2020; Mansour 2020; Pervez 2020; Poparn 2020; Pour 2020; Rafie 2020; Sangouni 2020; Scorletti 2020; Soleimani 2020; Song 2020; Tutunchi 2020; Yari 2020; Zanko 2020; Izadi 2021; Kanoni 2021; Soleimani 2021; EUCTR 2008-008275-34-GB; EUCTR 2009-017080-41-GB; NCT00845845)

\section{Follow-up}

The follow-up period in trials ranged from 1 to 28 months. In 104 trials, follow-up was less than 3 months (Miglio 2000; Kugelmas 2003; Deng 2005; Chande 2006; Nelson 2009; Fabbrini 2010; Khoshbaten 2010b; Aller 2011; Vajro 2011; Panahi 2012; Aliashrafi 2014; Askari 2014; Chachay 2014; Farhangi 2014; Foroughi 2014; Solhi 2014; Aller 2015; Amiri-Moghadam 2015; Bae 2015; Chen 2015a; Chen 2015b; Faghihzadeh 2015; Orr 2015; Qin 2015; Zhang 2015; Asgharian 2016; Ebrahimi-Mameghani 2016; Eghtesadi 2016; Ekhlasi 2016; Farsi 2016; Ferolla 2016; Guo 2016; Hong 2016; Nabavi 2016; Naganuma 2016; Panahi 2016; Pezeshki 2016; Rahimlou 2016; Rahmani 2016; Sepideh 2016; Yari 2016; Amiri 2017; Ashraf 2017; Behrouz 2017; Ebrahimi-Mameghani 2017; Famouri 2017a; Hussain 2017; Javadi 2017; Jeong 2017; Kobyliak 2017; Manzhalii 2017; Navekar 2017; Schattenberg 
Table 1. Summary of characteristics of included studies (Continued)

2017; Shahmohammadi 2017; Tabatabaee 2017; Youshari 2017; Amanat 2018; Amirkhizi 2018; Asghari 2018; Dabbaghmanesh 2018; Daneshi-Maskooni 2018; Eriksson 2018; Ghaffari 2018; Hosseini 2018; Javanmardi 2018; Kobyliak 2018; Lewis 2018; Oscarsson 2018; Pervez 2018; Wang 2018; Zamani 2018; Cheraghpour 2019; Jazayeri-Tehrani 2019; Abhari 2020; Afsharinasab 2020; Babaei 2020; Bahrami 2020; Cai 2020; Cerletti 2020; Fathi 2020; Ferro 2020; Hormoznejad 2020; Hoseini 2020; Hosseinabadi 2020; Kazemi 2020; Kooshki 2020; Mansour 2020; Moradi 2020; Orang 2020; Parsi 2020; Pasdar 2020; Poparn 2020; Pour 2020; Rafie 2020; Sadrkabir 2020; Sangouni 2020; Song 2020; Tutunchi 2020; Yari 2020; Hong 2021; Izadi 2021; Morvaridzadeh 2021; EUCTR 2009-017080-41-GB; NCT00816465); in 92 trials, follow-up was between 3 months and 28 months (Uygun 2000; Harrison 2003; Chou 2006; Dufour 2006; Nobili 2006; Chen 2008; Spadaro 2008; Zhu 2008; Abdelmalek 2009; Gomez 2009; Hashemi 2009; Li 2010; Malaguarnera 2010; Ruan 2010; Sanyal 2010; Lavine 2011; Tan 2011; Basu 2012; Della Corte 2012; Gonciarz 2012; Loguercio 2012; Malaguarnera 2012; Basu 2013; Ghergherehchi 2013; Gianturco 2013; Illnait 2013; Magosso 2013; Nobili 2013; Saxena 2013; Shavakhi 2013; Wong 2013a; Wong 2013b; Alisi 2014; Byrne 2014; Celinski 2014; Eslamparast 2014; Martinez-Rodriguez 2014; Sanyal 2014; Scorletti 2014; Sharifi 2014; Somi 2014; Akbarzadeh 2015; Argo 2015; Bonfrate 2015; Boyraz 2015; Dasarathy 2015; Janczyk 2015; Pacifico 2015; Yan 2015; Barchetta 2016; Boonyagard 2016; Della Corte 2016; Heeboll 2016; Li 2016; Nogueira 2016; Chan 2017; Chongsrisawat 2017; Gavrilescu 2017; Mofidi 2017; Palamaru 2017; Sakpal 2017; Wang 2017; Zohrer 2017; Ahn 2018; Bakhshimoghaddam 2018; Bomhof 2018; Geier 2018; Sayari 2018; Taghvaei 2018; Tobin 2018; Bril 2019; Duseja 2019; Afzali 2020; Barbakadze 2020; Boonyagard 2020; Climax 2020; Dallio 2020; Fernandez-Travieso 2020; Pervez 2020; Scorletti 2020; Soleimani 2020; Zanko 2020; Chiou 2021; Kanoni 2021; Poulos 2021; Soleimani 2021; EUCTR 2008-008275-34-GB; NCT00845845; NCT00977730; NCT01083992; NCT02690792; NCT04411862); follow-up was not reported in 6 trials (Wang 2008; Jameshorani 2017; Farzin 2020; Khutsishvili 2020; NCT00941642; NCT01623024) sults (Chande 2006; Dufour 2006; Gomez 2009; Fabbrini 2010; Sanyal 2010; Lavine 2011; Magosso 2013; Wong 2013b; Aliashrafi 2014; Eslamparast 2014; Scorletti 2014; Argo 2015; Bae 2015; Dasarathy 2015; Janczyk 2015; Heeboll 2016; Hong 2016; Nabavi 2016; Nogueira 2016; Panahi 2016; Rahimlou 2016; Chan 2017; Ebrahimi-Mameghani 2017; Mofidi 2017; Schattenberg 2017; Zohrer 2017; Bomhof 2018; Eriksson 2018; Geier 2018; Kobyliak 2018; Lewis 2018; Oscarsson 2018; Tobin 2018; Duseja 2019; Cerletti 2020; Climax 2020; Scorletti 2020; Song 2020; Hong 2021; Poulos 2021; EUCTR 2009-017080-41-GB); 87 trials were funded by neutral organisations that have no vested interests in results of the study (Kugelmas 2003; Chou 2006; Khoshbaten 2010b; Malaguarnera 2010; Gonciarz 2012; Loguercio 2012; Malaguarnera 2012; Panahi 2012; Ghergherehchi 2013; Nobili 2013; Shavakhi 2013; Alisi 2014; Askari 2014; Byrne 2014; Farhangi 2014; Foroughi 2014; Sharifi 2014; Solhi 2014; Akbarzadeh 2015; Amiri-Moghadam 2015; Boyraz 2015; Chen 2015a; Chen 2015b; Faghihzadeh 2015; Pacifico 2015; Yan 2015; Zhang 2015; Asgharian 2016; Barchetta 2016; Ebrahimi-Mameghani 2016; Ekhlasi 2016; Farsi 2016; Rahmani 2016; Yari 2016; Amiri 2017; Behrouz 2017; Famouri 2017a; Hussain 2017; Javadi 2017; Navekar 2017; Shahmohammadi 2017; Amanat 2018; Amirkhizi 2018; Asghari 2018; Bakhshimoghaddam 2018; Dabbaghmanesh 2018; DaneshiMaskooni 2018; Hosseini 2018; Pervez 2018; Zamani 2018; Bril 2019; Cheraghpour 2019; Jazayeri-Tehrani 2019; Abhari 2020; Afsharinasab 2020; Afzali 2020; Babaei 2020; Bahrami 2020; Boonyagard 2020; Fathi 2020; Fernandez-Travieso 2020; Ferro 2020; Hormoznejad 2020; Hoseini 2020; Hosseinabadi 2020; Kazemi 2020; Kooshki 2020; Mansour 2020; Moradi 2020; Orang 2020; Parsi 2020; Pervez 2020; Poparn 2020; Pour 2020; Rafie 2020; Sadrkabir 2020; Sangouni 2020; Soleimani 2020; Tutunchi 2020; Yari 2020; Zanko 2020; Chiou 2021; Izadi 2021; Kanoni 2021; Morvaridzadeh 2021; Soleimani 2021; EUCTR 2008-008275-34-GB); the source of funding for the remaining 74 trials was unclear (Miglio 2000; Uygun 2000; Harrison 2003; Deng 2005; Nobili 2006; Chen 2008; Spadaro 2008; Wang 2008; Zhu 2008; Abdelmalek 2009; Hashemi 2009; Nelson 2009; Li 2010; Ruan 2010; Aller 2011; Tan 2011; Vajro 2011; Basu 2012; Della Corte 2012; Basu 2013; Gianturco 2013; Illnait 2013; Saxena 2013; Wong 2013a; Celinski 2014; Chachay 2014; Martinez-Rodriguez 2014; Sanyal 2014; Somi 2014; Aller 2015; Bonfrate 2015; Orr 2015; Qin 2015; Boonyagard 2016; Della Corte 2016; Eghtesadi 2016; Ferolla 2016; Guo 2016; Li 2016; Naganuma 2016; Pezeshki 2016; Sepideh 2016; Ashraf 2017; Chongsrisawat 2017; Gavrilescu 2017; Jameshorani 2017; Jeong 2017; Kobyliak 2017; Manzhalii 2017; Palamaru 2017; Sakpal 2017; Tabatabaee 2017; Wang 2017; Youshari 2017; Ahn 2018; Ghaffari 2018; Javanmardi 2018; Sayari 2018; Taghvaei 2018; Wang 2018; Barbakadze 2020; Cai 2020; Dallio 2020; Farzin 2020; Khutsishvili 2020; Pasdar 2020; NCT00816465; NCT00845845; NCT00941642; NCT00977730; NCT01083992; NCT01623024; NCT02690792; NCT04411862) 
MUFA: monounsaturated fatty acid.

NAFLD: non-alcohol-related fatty liver disease.

PUFA: polyunsaturated fatty acid. 


\begin{tabular}{|c|c|c|c|c|c|c|c|c|}
\hline Study name & $\begin{array}{l}\text { Intervention } 1 \text { (number of partici- } \\
\text { pants) vs intervention } 2 \text { (number } \\
\text { of participants) }\end{array}$ & NASH & $\begin{array}{l}\text { Diabetes melli- } \\
\text { tus }\end{array}$ & $\begin{array}{l}\text { Supplementary } \\
\text { lifestyle modifi- } \\
\text { cation }\end{array}$ & $\begin{array}{l}\text { Method of } \\
\text { diagnosis } \\
\text { of NAFLD }\end{array}$ & $\begin{array}{l}\text { Period of } \\
\text { recruit- } \\
\text { ment }\end{array}$ & $\begin{array}{l}\text { Follow-up } \\
\text { in months }\end{array}$ & Risk of bias \\
\hline $\begin{array}{l}\text { Afshari- } \\
\text { nasab } 2020\end{array}$ & $\begin{array}{l}\text { Other supplements }(n=21) \text { vs No ac- } \\
\text { tive intervention }(n=21)\end{array}$ & Not stated & $\begin{array}{l}\text { No participants } \\
\text { had diabetes } \\
\text { mellitus }\end{array}$ & Not stated & Ultrasound & Not stated & 2 & High \\
\hline $\begin{array}{l}\text { Asghari } \\
2018\end{array}$ & $\begin{array}{l}\text { Other supplements }(n=30) \text { vs No ac- } \\
\text { tive intervention }(n=30)\end{array}$ & Not stated & $\begin{array}{l}\text { No participants } \\
\text { had diabetes } \\
\text { mellitus }\end{array}$ & Not stated & Ultrasound & Not stated & 2.8 & Low \\
\hline Askari 2014 & $\begin{array}{l}\text { Other supplements }(n=23) \text { vs No ac- } \\
\text { tive intervention }(n=22)\end{array}$ & Not stated & $\begin{array}{l}\text { No participants } \\
\text { had diabetes } \\
\text { mellitus }\end{array}$ & $\begin{array}{l}\text { All participants } \\
\text { had supplemen- } \\
\text { tary lifestyle } \\
\text { modification }\end{array}$ & Not stated & Not stated & 2.8 & High \\
\hline Babaei 2020 & $\begin{array}{l}\text { Other supplements }(n=13) \text { vs No ac- } \\
\text { tive intervention }(n=11)\end{array}$ & Not stated & $\begin{array}{l}\text { No participants } \\
\text { had diabetes } \\
\text { mellitus }\end{array}$ & $\begin{array}{l}\text { All participants } \\
\text { had supplemen- } \\
\text { tary lifestyle } \\
\text { modification }\end{array}$ & Ultrasound & $2014-2017$ & 3 & High \\
\hline $\begin{array}{l}\text { Bahrami } \\
2020\end{array}$ & $\begin{array}{l}\text { Other supplements }(n=24) \text { vs No ac- } \\
\text { tive intervention }(n=21)\end{array}$ & Not stated & $\begin{array}{l}\text { No participants } \\
\text { had diabetes } \\
\text { mellitus }\end{array}$ & Not stated & Ultrasound & Not stated & 2.8 & High \\
\hline Cerletti 2020 & $\begin{array}{l}\text { Other supplements }(n=55) \text { vs No ac- } \\
\text { tive intervention }(n=58)\end{array}$ & Not stated & $\begin{array}{l}\text { Participants } \\
\text { with and with- } \\
\text { out diabetes } \\
\text { mellitus }\end{array}$ & $\begin{array}{l}\text { All participants } \\
\text { had supplemen- } \\
\text { tary lifestyle } \\
\text { modification }\end{array}$ & $\begin{array}{l}\text { Ultrasound } \\
+ \text { transami- } \\
\text { nases }\end{array}$ & $2015-2016$ & 3 & High \\
\hline $\begin{array}{l}\text { Chachay } \\
2014\end{array}$ & $\begin{array}{l}\text { Other supplements }(n=10) \text { vs No ac- } \\
\text { tive intervention }(n=10)\end{array}$ & Not stated & Not stated & Not stated & Ultrasound & $2011-2012$ & 1.8 & High \\
\hline $\begin{array}{l}\text { Chande } \\
2006\end{array}$ & $\begin{array}{l}\text { Other supplements }(n=5) \text { vs No ac- } \\
\text { tive intervention }(n=3)\end{array}$ & $\begin{array}{l}\text { All partici- } \\
\text { pants had } \\
\text { NASH }\end{array}$ & $\begin{array}{l}\text { Participants } \\
\text { with and with- } \\
\text { out diabetes } \\
\text { mellitus }\end{array}$ & Not stated & $\begin{array}{l}\text { Liver biopsy } \\
+ \text { transami- } \\
\text { nases }\end{array}$ & $2003-2004$ & 2.8 & High \\
\hline Chen 2015a & $\begin{array}{l}\text { Other supplements }(n=30) \text { vs No ac- } \\
\text { tive intervention }(n=30)\end{array}$ & Not stated & $\begin{array}{l}\text { No participants } \\
\text { had diabetes } \\
\text { mellitus }\end{array}$ & Not stated & Ultrasound & $2012-2013$ & 3 & High \\
\hline
\end{tabular}




\begin{tabular}{|c|c|c|c|c|c|c|c|c|}
\hline Chiou 2021 & $\begin{array}{l}\text { Other supplements }(n=15) \text { vs No ac- } \\
\text { tive intervention }(n=13)\end{array}$ & $\begin{array}{l}\text { All partici- } \\
\text { pants had } \\
\text { NASH }\end{array}$ & Not stated & $\begin{array}{l}\text { All participants } \\
\text { had supplemen- } \\
\text { tary lifestyle } \\
\text { modification }\end{array}$ & Not stated & $2015-2016$ & 6 & High \\
\hline Chou 2006 & $\begin{array}{l}\text { Other supplements }(n=28) \text { vs No ac- } \\
\text { tive intervention }(n=28)\end{array}$ & Not stated & Not stated & $\begin{array}{l}\text { All participants } \\
\text { had supplemen- } \\
\text { tary lifestyle } \\
\text { modification }\end{array}$ & Ultrasound & 2001-2002 & 6 & High \\
\hline $\begin{array}{l}\text { Daneshi- } \\
\text { Maskooni } \\
2018\end{array}$ & $\begin{array}{l}\text { Other supplements }(n=43) \text { vs No ac- } \\
\text { tive intervention }(n=44)\end{array}$ & Not stated & Not stated & Not stated & Ultrasound & $2016-2017$ & 3 & High \\
\hline $\begin{array}{l}\text { Ebrahi- } \\
\text { mi-Mameghan } \\
2017\end{array}$ & $\begin{array}{l}\text { Other supplements }(n=29) \text { vs No ac- } \\
\text { i tive intervention }(n=26)\end{array}$ & Not stated & Not stated & Not stated & Ultrasound & 2011-2012 & 1.8 & High \\
\hline $\begin{array}{l}\text { EUCTR } \\
2009-017080-4 \\
\text { GB }\end{array}$ & $\begin{array}{l}\text { Other supplements }(n=20) \text { vs No ac- } \\
\text { 1tive intervention }(n=5)\end{array}$ & Not stated & Not stated & Not stated & $\begin{array}{l}\text { MRI, MRS, or } \\
\text { biopsy }\end{array}$ & Not stated & 2 & High \\
\hline $\begin{array}{l}\text { Faghi- } \\
\text { hzadeh } 2015\end{array}$ & $\begin{array}{l}\text { Other supplements }(n=24) \text { vs No ac- } \\
\text { tive intervention }(n=24)\end{array}$ & Not stated & $\begin{array}{l}\text { No participants } \\
\text { had diabetes } \\
\text { mellitus }\end{array}$ & $\begin{array}{l}\text { All participants } \\
\text { had supplemen- } \\
\text { tary lifestyle } \\
\text { modification }\end{array}$ & $\begin{array}{l}\text { Ultrasound } \\
+ \text { elastog- } \\
\text { raphy + } \\
\text { transami- } \\
\text { nases }\end{array}$ & $2013-2014$ & 2.8 & High \\
\hline Farzin 2020 & $\begin{array}{l}\text { Other supplements }(n=25) \text { vs No ac- } \\
\text { tive intervention }(n=25)\end{array}$ & Not stated & Not stated & Not stated & Not stated & Not stated & Not stated & High \\
\hline Fathi 2020 & $\begin{array}{l}\text { Other supplements }(n=25) \text { vs No ac- } \\
\text { tive intervention }(n=25)\end{array}$ & Not stated & $\begin{array}{l}\text { No participants } \\
\text { had diabetes } \\
\text { mellitus }\end{array}$ & Not stated & Ultrasound & 2018-2019 & 2.8 & High \\
\hline $\begin{array}{l}\text { Fernan- } \\
\text { dez-Travieso } \\
2020\end{array}$ & $\begin{array}{l}\text { Other supplements }(n=50) \text { vs No ac- } \\
\text { tive intervention }(n=50)\end{array}$ & Not stated & $\begin{array}{l}\text { Participants } \\
\text { with and with- } \\
\text { out diabetes } \\
\text { mellitus }\end{array}$ & Not stated & Ultrasound & Not stated & 6 & High \\
\hline Ferro 2020 & $\begin{array}{l}\text { Other supplements }(n=45) \text { vs No ac- } \\
\text { tive intervention }(n=41)\end{array}$ & Not stated & $\begin{array}{l}\text { No participants } \\
\text { had diabetes } \\
\text { mellitus }\end{array}$ & Not stated & Ultrasound & 2019 & 2.8 & High \\
\hline
\end{tabular}




\begin{tabular}{|c|c|c|c|c|c|c|c|c|}
\hline $\begin{array}{l}\text { Ghaffari } \\
2018\end{array}$ & $\begin{array}{l}\text { Other supplements }(n=64) \text { vs No ac- } \\
\text { tive intervention }(n=21)\end{array}$ & Not stated & Not stated & Not stated & Ultrasound & Not stated & 2.8 & High \\
\hline $\begin{array}{l}\text { Heeboll } \\
2016\end{array}$ & $\begin{array}{l}\text { Other supplements }(n=15) \text { vs No ac- } \\
\text { tive intervention }(n=13)\end{array}$ & $\begin{array}{l}\text { Participants } \\
\text { with and } \\
\text { without } \\
\text { NASH }\end{array}$ & $\begin{array}{l}\text { No participants } \\
\text { had diabetes } \\
\text { mellitus }\end{array}$ & Not stated & Ultrasound & $2011-2014$ & 6 & Low \\
\hline Hong 2021 & $\begin{array}{l}\text { Other supplements }(n=43) \text { vs No ac- } \\
\text { tive intervention }(n=44)\end{array}$ & Not stated & Not stated & Not stated & Ultrasound & $2017-2018$ & 0.9 & High \\
\hline $\begin{array}{l}\text { Hormozne- } \\
\text { jad } 2020\end{array}$ & $\begin{array}{l}\text { Other supplements }(n=20) \text { vs No ac- } \\
\text { tive intervention }(n=21)\end{array}$ & Not stated & $\begin{array}{l}\text { No participants } \\
\text { had diabetes } \\
\text { mellitus }\end{array}$ & Not stated & Ultrasound & Not stated & 2.8 & High \\
\hline $\begin{array}{l}\text { Hosseinaba- } \\
\text { di } 2020\end{array}$ & $\begin{array}{l}\text { Other supplements }(n=21) \text { vs No ac- } \\
\text { tive intervention }(n=23)\end{array}$ & Not stated & $\begin{array}{l}\text { No participants } \\
\text { had diabetes } \\
\text { mellitus }\end{array}$ & Not stated & Ultrasound & 2016 & 1.8 & High \\
\hline $\begin{array}{l}\text { Hussain } \\
2017\end{array}$ & $\begin{array}{l}\text { Other supplements }(n=40) \text { vs No ac- } \\
\text { tive intervention }(n=40)\end{array}$ & Not stated & $\begin{array}{l}\text { No participants } \\
\text { had diabetes } \\
\text { mellitus }\end{array}$ & Not stated & $\begin{array}{l}\text { Ultrasound } \\
+ \text { transami- } \\
\text { nases }\end{array}$ & 2016 & 2.8 & High \\
\hline Illnait 2013 & $\begin{array}{l}\text { Other supplements }(n=25) \text { vs No ac- } \\
\text { tive intervention }(n=25)\end{array}$ & Not stated & $\begin{array}{l}\text { Participants } \\
\text { with and with- } \\
\text { out diabetes } \\
\text { mellitus }\end{array}$ & Not stated & Ultrasound & Not stated & 5.5 & High \\
\hline Izadi 2021 & $\begin{array}{l}\text { Other supplements }(n=30) \text { vs No ac- } \\
\text { tive intervention }(n=31)\end{array}$ & Not stated & Not stated & Not stated & Ultrasound & 2018 & 1.8 & High \\
\hline $\begin{array}{l}\text { Javanmardi } \\
2018\end{array}$ & $\begin{array}{l}\text { Other supplements }(n=19) \text { vs No ac- } \\
\text { tive intervention }(n=19)\end{array}$ & Not stated & Not stated & Not stated & Ultrasound & Not stated & 1.8 & High \\
\hline $\begin{array}{l}\text { Jazay- } \\
\text { eri-Tehrani } \\
2019\end{array}$ & $\begin{array}{l}\text { Other supplements }(n=42) \text { vs No ac- } \\
\text { tive intervention }(n=42)\end{array}$ & Not stated & Not stated & $\begin{array}{l}\text { All participants } \\
\text { had supplemen- } \\
\text { tary lifestyle } \\
\text { modification }\end{array}$ & Ultrasound & Not stated & 3 & High \\
\hline Jeong 2017 & $\begin{array}{l}\text { Other supplements }(n=45) \text { vs No ac- } \\
\text { tive intervention }(n=23)\end{array}$ & Not stated & Not stated & Not stated & Ultrasound & $2013-2015$ & 2.8 & High \\
\hline
\end{tabular}




\begin{tabular}{|c|c|c|c|c|c|c|c|c|}
\hline Kazemi 2020 & $\begin{array}{l}\text { Other supplements }(n=40) \text { vs No ac- } \\
\text { tive intervention }(n=40)\end{array}$ & Not stated & $\begin{array}{l}\text { No participants } \\
\text { had diabetes } \\
\text { mellitus }\end{array}$ & Not stated & Ultrasound & Not stated & 2.8 & High \\
\hline $\begin{array}{l}\text { Kooshki } \\
2020\end{array}$ & $\begin{array}{l}\text { Other supplements }(n=22) \text { vs No ac- } \\
\text { tive intervention }(n=21)\end{array}$ & Not stated & $\begin{array}{l}\text { No participants } \\
\text { had diabetes } \\
\text { mellitus }\end{array}$ & Not stated & Ultrasound & $2018-2019$ & 2.8 & High \\
\hline $\begin{array}{l}\text { Mansour } \\
2020\end{array}$ & $\begin{array}{l}\text { Other supplements }(n=20) \text { vs No ac- } \\
\text { tive intervention }(n=6)\end{array}$ & Not stated & $\begin{array}{l}\text { All participants } \\
\text { had diabetes } \\
\text { mellitus only }\end{array}$ & Not stated & Ultrasound & Not stated & 2.8 & Low \\
\hline $\begin{array}{l}\text { Mar- } \\
\text { tinez-Ro- } \\
\text { driguez } \\
2014\end{array}$ & $\begin{array}{l}\text { Other supplements }(n=20) \text { vs No ac- } \\
\text { tive intervention }(n=20)\end{array}$ & Not stated & $\begin{array}{l}\text { No participants } \\
\text { had diabetes } \\
\text { mellitus }\end{array}$ & $\begin{array}{l}\text { All participants } \\
\text { had supplemen- } \\
\text { tary lifestyle } \\
\text { modification }\end{array}$ & $\begin{array}{l}\text { Ultrasound } \\
+ \text { liver biop- } \\
\text { sy }\end{array}$ & $2013-2014$ & 5.5 & High \\
\hline Moradi 2020 & $\begin{array}{l}\text { Other supplements }(n=22) \text { vs No ac- } \\
\text { tive intervention }(n=23)\end{array}$ & Not stated & Not stated & $\begin{array}{l}50 \% \text { of partici- } \\
\text { pants (factorial } \\
\text { trial design) }\end{array}$ & Ultrasound & Not stated & 2.8 & High \\
\hline $\begin{array}{l}\text { Navekar } \\
2017\end{array}$ & $\begin{array}{l}\text { Other supplements }(n=21) \text { vs No ac- } \\
\text { tive intervention }(n=21)\end{array}$ & Not stated & $\begin{array}{l}\text { No participants } \\
\text { had diabetes } \\
\text { mellitus }\end{array}$ & Not stated & Ultrasound & Not stated & 2.8 & High \\
\hline NCT00816465 & $\begin{array}{l}\text { Other supplements }(\mathrm{n}=\text { not stated) } \\
\text { vs No active intervention }(\mathrm{n}=\text { not } \\
\text { stated) }\end{array}$ & $\begin{array}{l}\text { All partici- } \\
\text { pants had } \\
\text { NASH }\end{array}$ & $\begin{array}{l}\text { Participants } \\
\text { with and with- } \\
\text { out diabetes } \\
\text { mellitus }\end{array}$ & Not stated & Liver biopsy & $2009-2010$ & 2 & High \\
\hline Panahi 2016 & $\begin{array}{l}\text { Other supplements }(n=44) \text { vs No ac- } \\
\text { tive intervention }(n=43)\end{array}$ & Not stated & $\begin{array}{l}\text { Participants } \\
\text { with and with- } \\
\text { out diabetes } \\
\text { mellitus }\end{array}$ & $\begin{array}{l}\text { All participants } \\
\text { had supplemen- } \\
\text { tary lifestyle } \\
\text { modification }\end{array}$ & Ultrasound & Not stated & 1.8 & High \\
\hline Parsi 2020 & $\begin{array}{l}\text { Other supplements }(n=30) \text { vs No ac- } \\
\text { tive intervention }(n=30)\end{array}$ & Not stated & Not stated & Not stated & $\begin{array}{l}\text { Ultrasound } \\
\text { and liver en- } \\
\text { zymes }\end{array}$ & Not stated & 1.8 & High \\
\hline Pasdar 2020 & $\begin{array}{l}\text { Other supplements }(n=39) \text { vs No ac- } \\
\text { tive intervention }(n=39)\end{array}$ & Not stated & Not stated & Not stated & Ultrasound & 2017 & 2.8 & High \\
\hline
\end{tabular}




\begin{tabular}{|c|c|c|c|c|c|c|c|c|}
\hline $\begin{array}{l}\text { Pezeshki } \\
2016\end{array}$ & $\begin{array}{l}\text { Other supplements }(n=35) \text { vs No ac- } \\
\text { tive intervention }(n=36)\end{array}$ & Not stated & Not stated & Not stated & $\begin{array}{l}\text { Ultrasound } \\
+ \text { transami- } \\
\text { nases }\end{array}$ & $2013-2014$ & 3 & High \\
\hline $\begin{array}{l}\text { Rahimlou } \\
2016\end{array}$ & $\begin{array}{l}\text { Other supplements }(n=23) \text { vs No ac- } \\
\text { tive intervention }(n=21)\end{array}$ & Not stated & $\begin{array}{l}\text { No participants } \\
\text { had diabetes } \\
\text { mellitus }\end{array}$ & $\begin{array}{l}\text { All participants } \\
\text { had supplemen- } \\
\text { tary lifestyle } \\
\text { modification }\end{array}$ & $\begin{array}{l}\text { Elastog- } \\
\text { raphy + } \\
\text { transami- } \\
\text { nases }\end{array}$ & Not stated & 2.8 & High \\
\hline $\begin{array}{l}\text { Rahmani } \\
2016\end{array}$ & $\begin{array}{l}\text { Other supplements }(n=37) \text { vs No ac- } \\
\text { tive intervention }(n=40)\end{array}$ & Not stated & Not stated & Not stated & Ultrasound & Not stated & 1.8 & High \\
\hline $\begin{array}{l}\text { Sangouni } \\
2020\end{array}$ & $\begin{array}{l}\text { Other supplements }(n=45) \text { vs No ac- } \\
\text { tive intervention }(n=43)\end{array}$ & Not stated & $\begin{array}{l}\text { No participants } \\
\text { had diabetes } \\
\text { mellitus }\end{array}$ & $\begin{array}{l}\text { All participants } \\
\text { had supplemen- } \\
\text { tary lifestyle } \\
\text { modification }\end{array}$ & Ultrasound & 2018 & 2.8 & High \\
\hline Saxena 2013 & $\begin{array}{l}\text { Other supplements }(n=26) \text { vs No ac- } \\
\text { tive intervention }(n=24)\end{array}$ & Not stated & Not stated & $\begin{array}{l}\text { All participants } \\
\text { had supplemen- } \\
\text { tary lifestyle } \\
\text { modification }\end{array}$ & Ultrasound & Not stated & 3.9 & High \\
\hline $\begin{array}{l}\text { Shahmo- } \\
\text { hammadi } \\
2017\end{array}$ & $\begin{array}{l}\text { Other supplements }(n=22) \text { vs No ac- } \\
\text { tive intervention }(n=22)\end{array}$ & Not stated & $\begin{array}{l}\text { No participants } \\
\text { had diabetes } \\
\text { mellitus }\end{array}$ & $\begin{array}{l}\text { All participants } \\
\text { had supplemen- } \\
\text { tary lifestyle } \\
\text { modification }\end{array}$ & Ultrasound & Not stated & 1.8 & High \\
\hline $\begin{array}{l}\text { Soleimani } \\
2020\end{array}$ & $\begin{array}{l}\text { Other supplements }(n=47) \text { vs No ac- } \\
\text { tive intervention }(n=51)\end{array}$ & Not stated & $\begin{array}{l}\text { Participants } \\
\text { with and with- } \\
\text { out diabetes } \\
\text { mellitus }\end{array}$ & Not stated & Ultrasound & Not stated & 3.5 & High \\
\hline $\begin{array}{l}\text { Soleimani } \\
2021\end{array}$ & $\begin{array}{l}\text { Other supplements }(n=27) \text { vs No ac- } \\
\text { tive intervention }(n=27)\end{array}$ & Not stated & $\begin{array}{l}\text { Participants } \\
\text { with and with- } \\
\text { out diabetes } \\
\text { mellitus }\end{array}$ & $\begin{array}{l}\text { All participants } \\
\text { had supplemen- } \\
\text { tary lifestyle } \\
\text { modification }\end{array}$ & $\begin{array}{l}\text { Elastogra- } \\
\text { phy tech- } \\
\text { nique }\end{array}$ & 2018 & 4 & Low \\
\hline Song 2020 & $\begin{array}{l}\text { Other supplements }(n=16) \text { vs No ac- } \\
\text { tive intervention }(n=21)\end{array}$ & Not stated & $\begin{array}{l}\text { No participants } \\
\text { had diabetes } \\
\text { mellitus }\end{array}$ & $\begin{array}{l}\text { All participants } \\
\text { had supplemen- } \\
\text { tary lifestyle } \\
\text { modification }\end{array}$ & $\begin{array}{l}\text { CT scan + } \\
\text { abnormal } \\
\text { serum lipid } \\
\text { profile or } \\
\text { high BMI }\end{array}$ & 2018 & 2.8 & High \\
\hline
\end{tabular}




\begin{tabular}{|c|c|c|c|c|c|c|c|c|}
\hline $\begin{array}{l}\text { Tabatabaee } \\
2017\end{array}$ & $\begin{array}{l}\text { Other supplements }(n=21) \text { vs No ac- } \\
\text { tive intervention }(n=24)\end{array}$ & Not stated & $\begin{array}{l}\text { Participants } \\
\text { with and with- } \\
\text { out diabetes } \\
\text { mellitus }\end{array}$ & $\begin{array}{l}\text { All participants } \\
\text { had supplemen- } \\
\text { tary lifestyle } \\
\text { modification }\end{array}$ & $\begin{array}{l}\text { Ultrasound, } \\
\text { elastogra- } \\
\text { phy, or liver } \\
\text { biopsy }\end{array}$ & Not stated & 3 & High \\
\hline $\begin{array}{l}\text { Tutunchi } \\
2020\end{array}$ & $\begin{array}{l}\text { Other supplements }(n=38) \text { vs No ac- } \\
\text { tive intervention }(n=38)\end{array}$ & Not stated & $\begin{array}{l}\text { No participants } \\
\text { had diabetes } \\
\text { mellitus }\end{array}$ & $\begin{array}{l}\text { All participants } \\
\text { had supplemen- } \\
\text { tary lifestyle } \\
\text { modification }\end{array}$ & Ultrasound & 2019 & 2.8 & Low \\
\hline Wang 2017 & $\begin{array}{l}\text { Other supplements }(n=24) \text { vs No ac- } \\
\text { tive intervention }(n=12)\end{array}$ & $\begin{array}{l}\text { All partici- } \\
\text { pants had } \\
\text { NASH }\end{array}$ & $\begin{array}{l}\text { No participants } \\
\text { had diabetes } \\
\text { mellitus }\end{array}$ & $\begin{array}{l}\text { All participants } \\
\text { had supplemen- } \\
\text { tary lifestyle } \\
\text { modification }\end{array}$ & $\begin{array}{l}\text { Elastogra- } \\
\text { phy }\end{array}$ & Not stated & 5.5 & High \\
\hline Wong 2013b & $\begin{array}{l}\text { Other supplements }(n=40) \text { vs No ac- } \\
\text { tive intervention }(n=20)\end{array}$ & $\begin{array}{l}\text { All partici- } \\
\text { pants had } \\
\text { NASH }\end{array}$ & $\begin{array}{l}\text { Participants } \\
\text { with and with- } \\
\text { out diabetes } \\
\text { mellitus }\end{array}$ & $\begin{array}{l}\text { All participants } \\
\text { had supplemen- } \\
\text { tary lifestyle } \\
\text { modification }\end{array}$ & Liver biopsy & $2010-2011$ & 5.5 & Low \\
\hline Yan 2015 & $\begin{array}{l}\text { Other supplements }(n=55) \text { vs No ac- } \\
\text { tive intervention }(n=53)\end{array}$ & Not stated & Not stated & $\begin{array}{l}\text { All participants } \\
\text { had supplemen- } \\
\text { tary lifestyle } \\
\text { modification }\end{array}$ & MRS & $2008-2011$ & 3.7 & High \\
\hline Yari 2020 & $\begin{array}{l}\text { Other supplements }(n=24) \text { vs No ac- } \\
\text { tive intervention }(n=21)\end{array}$ & Not stated & Not stated & $\begin{array}{l}\text { All participants } \\
\text { had supplemen- } \\
\text { tary lifestyle } \\
\text { modification }\end{array}$ & $\begin{array}{l}\text { Elastogra- } \\
\text { phy }\end{array}$ & $2018-2019$ & 2.8 & High \\
\hline $\begin{array}{l}\text { Zamani } \\
2018\end{array}$ & $\begin{array}{l}\text { Other supplements }(n=45) \text { vs No ac- } \\
\text { tive intervention }(n=40)\end{array}$ & Not stated & $\begin{array}{l}\text { No participants } \\
\text { had diabetes } \\
\text { mellitus }\end{array}$ & $\begin{array}{l}\text { All participants } \\
\text { had supplemen- } \\
\text { tary lifestyle } \\
\text { modification }\end{array}$ & Ultrasound & 2017 & 2.8 & High \\
\hline Zhang 2015 & $\begin{array}{l}\text { Other supplements }(n=37) \text { vs No ac- } \\
\text { tive intervention }(n=37)\end{array}$ & Not stated & Not stated & Not stated & Ultrasound & 2013 & 2.8 & Low \\
\hline Abhari 2020 & $\begin{array}{l}\text { Prebiotics/Probiotics/Synbiotics }(n= \\
23) \text { vs No active intervention }(n=22)\end{array}$ & Not stated & $\begin{array}{l}\text { No participants } \\
\text { had diabetes } \\
\text { mellitus }\end{array}$ & Not stated & Not stated & Not stated & 2.8 & High \\
\hline
\end{tabular}




\begin{tabular}{|c|c|c|c|c|c|c|c|c|}
\hline Ahn 2018 & $\begin{array}{l}\text { Prebiotics/Probiotics/Synbiotics }(n= \\
\text { not stated) vs No active intervention } \\
\text { ( } n=\text { not stated) }\end{array}$ & Not stated & Not stated & Not stated & Not stated & Not stated & 8 & High \\
\hline $\begin{array}{l}\text { Akbarzadeh } \\
2015\end{array}$ & $\begin{array}{l}\text { Prebiotics/Probiotics/Synbiotics }(n= \\
\text { 38) vs No active intervention }(n=37)\end{array}$ & Not stated & Not stated & $\begin{array}{l}\text { All participants } \\
\text { had supplemen- } \\
\text { tary lifestyle } \\
\text { modification }\end{array}$ & $\begin{array}{l}\text { Ultrasound } \\
\pm \text { elastog- } \\
\text { raphy } \pm \\
\text { transami- } \\
\text { nases }\end{array}$ & 2015 & 6.3 & High \\
\hline Alisi 2014 & $\begin{array}{l}\text { Prebiotics/Probiotics/Synbiotics }(n= \\
22) \text { vs No active intervention }(n=22)\end{array}$ & $\begin{array}{l}\text { All partici- } \\
\text { pants had } \\
\text { NASH }\end{array}$ & Not stated & Not stated & $\begin{array}{l}\text { Clinical ex- } \\
\text { amination } \\
+ \text { transami- } \\
\text { nases + liver } \\
\text { biopsy }\end{array}$ & $2012-2013$ & 4 & High \\
\hline Aller 2011 & $\begin{array}{l}\text { Prebiotics/Probiotics/Synbiotics }(n= \\
\text { 14) vs No active intervention }(n=14)\end{array}$ & Not stated & $\begin{array}{l}\text { No participants } \\
\text { had diabetes } \\
\text { mellitus }\end{array}$ & Not stated & Liver biopsy & Not stated & 3 & High \\
\hline Guo 2016 & $\begin{array}{l}\text { Prebiotics/Probiotics/Synbiotics }(n= \\
42) \text { vs No active intervention }(n=42)\end{array}$ & Not stated & Not stated & $\begin{array}{l}\text { All participants } \\
\text { had supplemen- } \\
\text { tary lifestyle } \\
\text { modification }\end{array}$ & Ultrasound & $2011-2013$ & 1.8 & High \\
\hline $\begin{array}{l}\text { Asgharian } \\
2016\end{array}$ & $\begin{array}{l}\text { Prebiotics/Probiotics/Synbiotics }(n= \\
\text { 38) vs No active intervention }(n=36)\end{array}$ & $\begin{array}{l}\text { Participants } \\
\text { with and } \\
\text { without } \\
\text { NASH }\end{array}$ & Not stated & Not stated & Ultrasound & 2014 & 1.8 & High \\
\hline $\begin{array}{l}\text { Bakhshi- } \\
\text { moghad- } \\
\text { dam } 2018\end{array}$ & $\begin{array}{l}\text { Prebiotics/Probiotics/Synbiotics }(n= \\
\text { 32) vs No active intervention }(n=28)\end{array}$ & Not stated & $\begin{array}{l}\text { No participants } \\
\text { had diabetes } \\
\text { mellitus }\end{array}$ & $\begin{array}{l}\text { All participants } \\
\text { had supplemen- } \\
\text { tary lifestyle } \\
\text { modification }\end{array}$ & Ultrasound & $2016-2017$ & 5.5 & High \\
\hline $\begin{array}{l}\text { Behrouz } \\
2017\end{array}$ & $\begin{array}{l}\text { Prebiotics/Probiotics/Synbiotics }(n= \\
\text { 59) vs No active intervention }(n=30)\end{array}$ & Not stated & $\begin{array}{l}\text { No participants } \\
\text { had diabetes } \\
\text { mellitus }\end{array}$ & $\begin{array}{l}\text { All participants } \\
\text { had supplemen- } \\
\text { tary lifestyle } \\
\text { modification }\end{array}$ & $\begin{array}{l}\text { Ultrasound } \\
\text { and ALT }\end{array}$ & Not stated & 2.8 & High \\
\hline $\begin{array}{l}\text { Bomhof } \\
2018\end{array}$ & $\begin{array}{l}\text { Prebiotics/Probiotics/Synbiotics }(n= \\
\text { 8) vs No active intervention }(n=5)\end{array}$ & $\begin{array}{l}\text { All partici- } \\
\text { pants had } \\
\text { NASH }\end{array}$ & $\begin{array}{l}\text { Participants } \\
\text { with and with- }\end{array}$ & Not stated & $\begin{array}{l}\text { Ultrasound } \\
+ \text { transami- } \\
\text { nases }\end{array}$ & Not stated & 8.3 & High \\
\hline
\end{tabular}




\begin{tabular}{|c|c|c|c|c|c|c|c|c|}
\hline & & & $\begin{array}{l}\text { out diabetes } \\
\text { mellitus }\end{array}$ & & & & & \\
\hline Cai 2020 & $\begin{array}{l}\text { Prebiotics/Probiotics/Synbiotics }(n= \\
70) \text { vs No active intervention }(n=70)\end{array}$ & Not stated & Not stated & $\begin{array}{l}\text { All participants } \\
\text { had supplemen- } \\
\text { tary lifestyle } \\
\text { modification }\end{array}$ & $\begin{array}{l}\text { Ultrasound } \\
\text { and biopsy }\end{array}$ & 2017-2019 & 3 & High \\
\hline $\begin{array}{l}\text { Chongsri- } \\
\text { sawat } 2017\end{array}$ & $\begin{array}{l}\text { Prebiotics/Probiotics/Synbiotics }(n= \\
\text { 18) vs No active intervention }(n=19)\end{array}$ & Not stated & Not stated & Not stated & FibroScan & 2016 & 3.7 & High \\
\hline $\begin{array}{l}\text { Della Corte } \\
2012\end{array}$ & $\begin{array}{l}\text { Prebiotics/Probiotics/Synbiotics }(n= \\
\text { not stated) vs No active intervention } \\
\text { ( } n=\text { not stated) }\end{array}$ & Not stated & Not stated & Not stated & Not stated & Not stated & 6 & High \\
\hline Duseja 2019 & $\begin{array}{l}\text { Prebiotics/Probiotics/Synbiotics }(n= \\
\text { 10) vs No active intervention }(n=5)\end{array}$ & Not stated & $\begin{array}{l}\text { No participants } \\
\text { had diabetes } \\
\text { mellitus }\end{array}$ & $\begin{array}{l}\text { All participants } \\
\text { had supplemen- } \\
\text { tary lifestyle } \\
\text { modification }\end{array}$ & Biopsy & Not stated & 12 & High \\
\hline Ekhlasi 2016 & $\begin{array}{l}\text { Prebiotics/Probiotics/Synbiotics }(n= \\
\text { 15) vs No active intervention }(n=15)\end{array}$ & Not stated & $\begin{array}{l}\text { No participants } \\
\text { had diabetes } \\
\text { mellitus }\end{array}$ & Not stated & $\begin{array}{l}\text { Ultrasound } \\
+ \text { transami- } \\
\text { nases }\end{array}$ & $2012-2013$ & 1.8 & High \\
\hline $\begin{array}{l}\text { Eslamparast } \\
2014\end{array}$ & $\begin{array}{l}\text { Prebiotics/Probiotics/Synbiotics }(n= \\
\text { 26) vs No active intervention }(n=26)\end{array}$ & Not stated & $\begin{array}{l}\text { No participants } \\
\text { had diabetes } \\
\text { mellitus }\end{array}$ & $\begin{array}{l}\text { All participants } \\
\text { had supplemen- } \\
\text { tary lifestyle } \\
\text { modification }\end{array}$ & $\begin{array}{l}\text { Ultrasound } \\
+ \text { transami- } \\
\text { nases }\end{array}$ & 2012 & 6.5 & High \\
\hline $\begin{array}{l}\text { Famouri } \\
2017 a\end{array}$ & $\begin{array}{l}\text { Prebiotics/Probiotics/Synbiotics }(n= \\
\text { 32) vs No active intervention }(n=32)\end{array}$ & Not stated & Not stated & $\begin{array}{l}\text { All participants } \\
\text { had supplemen- } \\
\text { tary lifestyle } \\
\text { modification }\end{array}$ & Ultrasound & 2014 & 2.8 & High \\
\hline Ferolla 2016 & $\begin{array}{l}\text { Prebiotics/Probiotics/Synbiotics }(n= \\
\text { 27) vs No active intervention }(n=23)\end{array}$ & $\begin{array}{l}\text { All partici- } \\
\text { pants had } \\
\text { NASH }\end{array}$ & $\begin{array}{l}\text { Participants } \\
\text { with and with- } \\
\text { out diabetes } \\
\text { mellitus }\end{array}$ & $\begin{array}{l}\text { All participants } \\
\text { had supplemen- } \\
\text { tary lifestyle } \\
\text { modification }\end{array}$ & Liver biopsy & $2014-2015$ & 3 & High \\
\hline $\begin{array}{l}\text { Gavrilescu } \\
2017\end{array}$ & $\begin{array}{l}\text { Prebiotics/Probiotics/Synbiotics }(n= \\
\text { not stated) vs No active intervention } \\
\text { ( } n=\text { not stated) }\end{array}$ & Not stated & Not stated & $\begin{array}{l}\text { All participants } \\
\text { had supplemen- } \\
\text { tary lifestyle } \\
\text { modification }\end{array}$ & Not stated & $2015-2016$ & 5.5 & High \\
\hline
\end{tabular}




\begin{tabular}{|c|c|c|c|c|c|c|c|c|}
\hline $\begin{array}{l}\text { Jameshorani } \\
2017\end{array}$ & $\begin{array}{l}\text { Prebiotics/Probiotics/Synbiotics }(n= \\
45) \text { vs No active intervention }(n=45)\end{array}$ & Not stated & Not stated & $\begin{array}{l}\text { All participants } \\
\text { had supplemen- } \\
\text { tary lifestyle } \\
\text { modification }\end{array}$ & Not stated & Not stated & Not stated & High \\
\hline Javadi 2017 & $\begin{array}{l}\text { Prebiotics/Probiotics/Synbiotics }(n= \\
56) \text { vs No active intervention }(n=19)\end{array}$ & Not stated & Not stated & Not stated & Ultrasound & $2013-2014$ & 3 & High \\
\hline $\begin{array}{l}\text { Khutsishvili } \\
2020\end{array}$ & $\begin{array}{l}\text { Prebiotics/Probiotics/Synbiotics }(n= \\
\text { 35) vs No active intervention }(n=38)\end{array}$ & Not stated & Not stated & Not stated & Not stated & Not stated & Not stated & High \\
\hline $\begin{array}{l}\text { Kobyliak } \\
2018\end{array}$ & $\begin{array}{l}\text { Prebiotics/Probiotics/Synbiotics }(n= \\
\text { 30) vs No active intervention }(n=28)\end{array}$ & Not stated & $\begin{array}{l}\text { All participants } \\
\text { had diabetes } \\
\text { mellitus only }\end{array}$ & Not stated & Ultrasound & Not stated & 1.8 & Low \\
\hline $\begin{array}{l}\text { Malaguarn- } \\
\text { era } 2012\end{array}$ & $\begin{array}{l}\text { Prebiotics/Probiotics/Synbiotics }(n= \\
\text { 34) vs No active intervention }(n=32)\end{array}$ & $\begin{array}{l}\text { All partici- } \\
\text { pants had } \\
\text { NASH }\end{array}$ & Both & $\begin{array}{l}\text { All participants } \\
\text { had supplemen- } \\
\text { tary lifestyle } \\
\text { modification }\end{array}$ & $\begin{array}{l}\text { Ultrasound } \\
+ \text { transami- } \\
\text { nases }\end{array}$ & $2003-2006$ & 5.5 & High \\
\hline $\begin{array}{l}\text { Manzhalii } \\
2017\end{array}$ & $\begin{array}{l}\text { Prebiotics/Probiotics/Synbiotics }(n= \\
\text { 38) vs No active intervention }(n=37)\end{array}$ & $\begin{array}{l}\text { All partici- } \\
\text { pants had } \\
\text { NASH }\end{array}$ & $\begin{array}{l}\text { No participants } \\
\text { had diabetes } \\
\text { mellitus }\end{array}$ & $\begin{array}{l}\text { All participants } \\
\text { had supplemen- } \\
\text { tary lifestyle } \\
\text { modification }\end{array}$ & $\begin{array}{l}\text { Ultrasound } \\
+ \text { transami- } \\
\text { nases }\end{array}$ & Not stated & 2.8 & High \\
\hline Mofidi 2017 & $\begin{array}{l}\text { Prebiotics/Probiotics/Synbiotics }(n= \\
21) \text { vs No active intervention }(n=21)\end{array}$ & Not stated & Not stated & $\begin{array}{l}\text { All participants } \\
\text { had supplemen- } \\
\text { tary lifestyle } \\
\text { modification }\end{array}$ & $\begin{array}{l}\text { Elastog- } \\
\text { raphy }+ \\
\text { transami- } \\
\text { nases }\end{array}$ & Not stated & 6.5 & High \\
\hline Nabavi 2016 & $\begin{array}{l}\text { Prebiotics/Probiotics/Synbiotics }(n= \\
\text { 36) vs No active intervention }(n=36)\end{array}$ & Not stated & Not stated & Not stated & Ultrasound & Not stated & 1.8 & Low \\
\hline Orr 2015 & $\begin{array}{l}\text { Prebiotics/Probiotics/Synbiotics }(\mathrm{n}= \\
\text { not stated) vs No active intervention } \\
\text { ( } \mathrm{n}=\text { not stated) }\end{array}$ & Not stated & Not stated & Not stated & Not stated & Not stated & 2.8 & High \\
\hline Poparn 2020 & $\begin{array}{l}\text { Prebiotics/Probiotics/Synbiotics }(n= \\
\text { 18) vs No active intervention }(n=19)\end{array}$ & Not stated & Not stated & Not stated & Fibroscan & 2016 & 2.8 & Low \\
\hline $\begin{array}{l}\text { Sadrkabir } \\
2020\end{array}$ & $\begin{array}{l}\text { Prebiotics/Probiotics/Synbiotics }(n= \\
\text { 33) vs No active intervention }(n=28)\end{array}$ & Not stated & $\begin{array}{l}\text { No participants } \\
\text { had diabetes } \\
\text { mellitus }\end{array}$ & $\begin{array}{l}\text { All participants } \\
\text { had supplemen- }\end{array}$ & Ultrasound & Not stated & 2 & High \\
\hline
\end{tabular}




\begin{tabular}{|c|c|c|c|c|c|c|c|c|}
\hline & & & & $\begin{array}{l}\text { tary lifestyle } \\
\text { modification }\end{array}$ & & & & \\
\hline Sayari 2018 & $\begin{array}{l}\text { Prebiotics/Probiotics/Synbiotics }(n= \\
70) \text { vs No active intervention }(n=68)\end{array}$ & Not stated & Not stated & $\begin{array}{l}\text { All participants } \\
\text { had supplemen- } \\
\text { tary lifestyle } \\
\text { modification }\end{array}$ & $\begin{array}{l}\text { Ultrasound } \\
+ \text { transami- } \\
\text { nases }\end{array}$ & $2015-2017$ & 3.7 & High \\
\hline $\begin{array}{l}\text { Schatten- } \\
\text { berg } 2017\end{array}$ & $\begin{array}{l}\text { Prebiotics/Probiotics/Synbiotics }(n= \\
\text { 15) vs No active intervention }(n=14)\end{array}$ & $\begin{array}{l}\text { All partici- } \\
\text { pants had } \\
\text { NASH }\end{array}$ & $\begin{array}{l}\text { Participants } \\
\text { with and with- } \\
\text { out diabetes } \\
\text { mellitus }\end{array}$ & $\begin{array}{l}\text { All participants } \\
\text { had supplemen- } \\
\text { tary lifestyle } \\
\text { modification }\end{array}$ & $\begin{array}{l}\text { Ultrasound } \\
+ \text { M30 (bio- } \\
\text { marker of } \\
\text { liver in- } \\
\text { jury) or liver } \\
\text { biopsy }\end{array}$ & Not stated & 3 & High \\
\hline $\begin{array}{l}\text { Scorletti } \\
2020\end{array}$ & $\begin{array}{l}\text { Prebiotics/Probiotics/Synbiotics }(n= \\
45) \text { vs No active intervention }(n=44)\end{array}$ & Not stated & $\begin{array}{l}\text { Participants } \\
\text { with and with- } \\
\text { out diabetes } \\
\text { mellitus }\end{array}$ & Not stated & $\begin{array}{l}\text { biopsy or } \\
\text { imaging evi- } \\
\text { dence of liv- } \\
\text { er fat }\end{array}$ & Not stated & 12 & High \\
\hline $\begin{array}{l}\text { Sepideh } \\
2016\end{array}$ & $\begin{array}{l}\text { Prebiotics/Probiotics/Synbiotics }(n= \\
21) \text { vs No active intervention }(n=21)\end{array}$ & Not stated & Not stated & Not stated & Ultrasound & 2013 & 1.8 & High \\
\hline $\begin{array}{l}\text { Shavakhi } \\
2013\end{array}$ & $\begin{array}{l}\text { Prebiotics/Probiotics/Synbiotics }(n= \\
\text { 31) vs No active intervention }(n=32)\end{array}$ & $\begin{array}{l}\text { All partici- } \\
\text { pants had } \\
\text { NASH }\end{array}$ & Not stated & $\begin{array}{l}\text { All participants } \\
\text { had supplemen- } \\
\text { tary lifestyle } \\
\text { modification }\end{array}$ & Liver biopsy & 2010-2012 & 6 & High \\
\hline Vajro 2011 & $\begin{array}{l}\text { Prebiotics/Probiotics/Synbiotics }(n= \\
\text { 10) vs No active intervention }(n=10)\end{array}$ & Not stated & $\begin{array}{l}\text { No participants } \\
\text { had diabetes } \\
\text { mellitus }\end{array}$ & Not stated & $\begin{array}{l}\text { Elastog- } \\
\text { raphy }+ \\
\text { transami- } \\
\text { nases }\end{array}$ & Not stated & 1.8 & High \\
\hline Wong 2013a & $\begin{array}{l}\text { Prebiotics/Probiotics/Synbiotics }(n= \\
10) \text { vs No active intervention }(n=10)\end{array}$ & $\begin{array}{l}\text { All partici- } \\
\text { pants had } \\
\text { NASH }\end{array}$ & $\begin{array}{l}\text { Participants } \\
\text { with and with- } \\
\text { out diabetes } \\
\text { mellitus }\end{array}$ & Not stated & $\begin{array}{l}\text { Liver biopsy } \\
+ \text { transami- } \\
\text { nases }\end{array}$ & 2009 & 6 & High \\
\hline Argo 2015 & $\begin{array}{l}\text { PUFA }(n=17) \text { vs No active interven- } \\
\text { tion }(n=17)\end{array}$ & $\begin{array}{l}\text { All partici- } \\
\text { pants had } \\
\text { NASH }\end{array}$ & $\begin{array}{l}\text { Participants } \\
\text { with and with- } \\
\text { out diabetes } \\
\text { mellitus }\end{array}$ & Not stated & Liver biopsy & $2007-2010$ & 12 & High \\
\hline
\end{tabular}




\begin{tabular}{|c|c|c|c|c|c|c|c|c|}
\hline Boyraz 2015 & $\begin{array}{l}\text { PUFA }(n=56) \text { vs No active interven- } \\
\text { tion }(n=52)\end{array}$ & Not stated & $\begin{array}{l}\text { Participants } \\
\text { with and with- } \\
\text { out diabetes } \\
\text { mellitus }\end{array}$ & $\begin{array}{l}\text { All participants } \\
\text { had supplemen- } \\
\text { tary lifestyle } \\
\text { modification }\end{array}$ & $\begin{array}{l}\text { Ultrasound } \\
+ \text { transami- } \\
\text { nases }\end{array}$ & 2010-2012 & 12 & High \\
\hline Byrne 2014 & $\begin{array}{l}\text { PUFA }(n=51) \text { vs No active interven- } \\
\text { tion }(n=52)\end{array}$ & Not stated & Not stated & Not stated & Not stated & Not stated & 16.5 & High \\
\hline Chen 2008 & $\begin{array}{l}\text { PUFA }(n=30) \text { vs No active interven- } \\
\text { tion }(n=16)\end{array}$ & $\begin{array}{l}\text { Participants } \\
\text { with and } \\
\text { without } \\
\text { NASH }\end{array}$ & Not stated & Not stated & $\begin{array}{l}\text { Clinical ex- } \\
\text { amination } \\
+ \text { transami- } \\
\text { nases }\end{array}$ & Not stated & 5.5 & High \\
\hline Climax 2020 & $\begin{array}{l}\text { PUFA }(n=63) \text { vs No active interven- } \\
\text { tion }(n=30)\end{array}$ & $\begin{array}{l}\text { Participants } \\
\text { with and } \\
\text { without } \\
\text { NASH }\end{array}$ & $\begin{array}{l}\text { Participants } \\
\text { with and with- } \\
\text { out diabetes } \\
\text { mellitus }\end{array}$ & Not stated & $\begin{array}{l}\text { Imaging or } \\
\text { histology }\end{array}$ & 2016-2019 & 3.7 & High \\
\hline $\begin{array}{l}\text { Dasarathy } \\
2015\end{array}$ & $\begin{array}{l}\text { PUFA }(n=18) \text { vs No active interven- } \\
\text { tion }(n=19)\end{array}$ & $\begin{array}{l}\text { All partici- } \\
\text { pants had } \\
\text { NASH }\end{array}$ & $\begin{array}{l}\text { All participants } \\
\text { had diabetes } \\
\text { mellitus only }\end{array}$ & Not stated & Liver biopsy & Not stated & 11.1 & High \\
\hline $\begin{array}{l}\text { Eriksson } \\
2018\end{array}$ & $\begin{array}{l}\text { PUFA }(n=42) \text { vs No active interven- } \\
\text { tion }(n=42)\end{array}$ & Not stated & $\begin{array}{l}\text { All participants } \\
\text { had diabetes } \\
\text { mellitus only }\end{array}$ & Not stated & MRI & 2015 & 2.8 & Low \\
\hline $\begin{array}{l}\text { EUCTR } \\
2008-008275-34 \\
\text { GB }\end{array}$ & $\begin{array}{l}\text { PUFA }(n=24) \text { vs No active interven- } \\
\text { 4tion }(n=25)\end{array}$ & Not stated & $\begin{array}{l}\text { Participants } \\
\text { with and with- } \\
\text { out diabetes } \\
\text { mellitus }\end{array}$ & Not stated & $\begin{array}{l}\text { Clinical + ul- } \\
\text { trasound }+ \\
\text { abnormal } \\
\text { LFTs }\end{array}$ & $2010-2011$ & 9 & Low \\
\hline $\begin{array}{l}\text { Janczyk } \\
2015\end{array}$ & $\begin{array}{l}\text { PUFA }(n=30) \text { vs No active interven- } \\
\text { tion }(n=34)\end{array}$ & Not stated & $\begin{array}{l}\text { No participants } \\
\text { had diabetes } \\
\text { mellitus }\end{array}$ & Not stated & $\begin{array}{l}\text { Ultrasound } \\
+ \text { transami- } \\
\text { nases }\end{array}$ & $2008-2011$ & 5.5 & High \\
\hline Li 2016 & $\begin{array}{l}\text { PUFA }(n=39) \text { vs No active interven- } \\
\text { tion }(n=39)\end{array}$ & $\begin{array}{l}\text { All partici- } \\
\text { pants had } \\
\text { NASH }\end{array}$ & Not stated & $\begin{array}{l}\text { All participants } \\
\text { had supplemen- } \\
\text { tary lifestyle } \\
\text { modification }\end{array}$ & Liver biopsy & Not stated & 6 & High \\
\hline NCT00845845 & $\begin{array}{l}\text { PUFA }(n=3) \text { vs No active interven- } \\
\text { tion }(n=6)\end{array}$ & $\begin{array}{l}\text { All partici- } \\
\text { pants had } \\
\text { NASH }\end{array}$ & $\begin{array}{l}\text { Participants } \\
\text { with and with- }\end{array}$ & $\begin{array}{l}\text { All participants } \\
\text { had supplemen- }\end{array}$ & Liver biopsy & 2006-2009 & 5.5 & High \\
\hline
\end{tabular}




\begin{tabular}{|c|c|c|c|c|c|c|c|c|}
\hline & & & $\begin{array}{l}\text { out diabetes } \\
\text { mellitus }\end{array}$ & $\begin{array}{l}\text { tary lifestyle } \\
\text { modification }\end{array}$ & & & & \\
\hline NCT00941642 & $\begin{array}{l}\text { PUFA }(n=\text { not stated) vs No active in- } \\
\text { tervention }(n=\text { not stated) }\end{array}$ & $\begin{array}{l}\text { Participants } \\
\text { with and } \\
\text { without } \\
\text { NASH }\end{array}$ & $\begin{array}{l}\text { No participants } \\
\text { had diabetes } \\
\text { mellitus }\end{array}$ & Not stated & Liver biopsy & Not stated & Not stated & High \\
\hline Nobili 2013 & $\begin{array}{l}\text { PUFA }(n=40) \text { vs No active interven- } \\
\text { tion }(n=20)\end{array}$ & Not stated & Not stated & $\begin{array}{l}\text { All participants } \\
\text { had supplemen- } \\
\text { tary lifestyle } \\
\text { modification }\end{array}$ & Ultrasound & Not stated & 24 & High \\
\hline $\begin{array}{l}\text { Nogueira } \\
2016\end{array}$ & $\begin{array}{l}\text { PUFA }(n=27) \text { vs No active interven- } \\
\text { tion }(n=23)\end{array}$ & $\begin{array}{l}\text { All partici- } \\
\text { pants had } \\
\text { NASH }\end{array}$ & Not stated & Not stated & Liver biopsy & Not stated & 6 & High \\
\hline Orang 2020 & $\begin{array}{l}\text { PUFA }(n=22) \text { vs No active interven- } \\
\text { tion }(n=22)\end{array}$ & Not stated & $\begin{array}{l}\text { All participants } \\
\text { had diabetes } \\
\text { mellitus only }\end{array}$ & Not stated & Not stated & Not stated & 2.8 & High \\
\hline $\begin{array}{l}\text { Oscarsson } \\
2018\end{array}$ & $\begin{array}{l}\text { PUFA }(n=23) \text { vs No active interven- } \\
\text { tion }(n=23)\end{array}$ & Not stated & $\begin{array}{l}\text { No participants } \\
\text { had diabetes } \\
\text { mellitus }\end{array}$ & Not stated & Liver MRI & Not stated & 2.8 & High \\
\hline $\begin{array}{l}\text { Pacifico } \\
2015\end{array}$ & $\begin{array}{l}\text { PUFA }(n=25) \text { vs No active interven- } \\
\text { tion }(n=26)\end{array}$ & Not stated & $\begin{array}{l}\text { No participants } \\
\text { had diabetes } \\
\text { mellitus }\end{array}$ & Not stated & Liver biopsy & $2012-2014$ & 6 & High \\
\hline Sanyal 2014 & $\begin{array}{l}\text { PUFA }(n=168) \text { vs No active interven- } \\
\text { tion }(n=75)\end{array}$ & $\begin{array}{l}\text { All partici- } \\
\text { pants had } \\
\text { NASH }\end{array}$ & $\begin{array}{l}\text { Participants } \\
\text { with and with- } \\
\text { out diabetes } \\
\text { mellitus }\end{array}$ & Not stated & Liver biopsy & $2011-2012$ & 12 & Low \\
\hline $\begin{array}{l}\text { Scorletti } \\
2014\end{array}$ & $\begin{array}{l}\text { PUFA }(n=51) \text { vs No active interven- } \\
\text { tion }(n=52)\end{array}$ & Not stated & $\begin{array}{l}\text { Participants } \\
\text { with and with- } \\
\text { out diabetes } \\
\text { mellitus }\end{array}$ & Not stated & Not stated & $2010-2011$ & 15 & High \\
\hline Song 2020 & $\begin{array}{l}\text { PUFA }(n=21) \text { vs No active interven- } \\
\text { tion }(n=21)\end{array}$ & Not stated & $\begin{array}{l}\text { No participants } \\
\text { had diabetes } \\
\text { mellitus }\end{array}$ & $\begin{array}{l}\text { All participants } \\
\text { had supplemen- } \\
\text { tary lifestyle } \\
\text { modification }\end{array}$ & $\begin{array}{l}\text { CT scan + } \\
\text { abnormal } \\
\text { serum lipid } \\
\text { profile or } \\
\text { high BMI }\end{array}$ & 2018 & 2.8 & High \\
\hline
\end{tabular}


Table 2. Characteristics of included studies (ordered by comparisons) (Continued)

\begin{tabular}{|c|c|c|c|c|c|c|c|c|}
\hline $\begin{array}{l}\text { Spadaro } \\
2008\end{array}$ & $\begin{array}{l}\text { PUFA }(n=18) \text { vs No active interven- } \\
\text { tion }(n=18)\end{array}$ & Not stated & Not stated & $\begin{array}{l}\text { All participants } \\
\text { had supplemen- } \\
\text { tary lifestyle } \\
\text { modification }\end{array}$ & $\begin{array}{l}\text { Ultrasound } \\
+ \text { transami- } \\
\text { nases }\end{array}$ & Not stated & 6 & High \\
\hline Yari 2016 & $\begin{array}{l}\text { PUFA }(n=25) \text { vs No active interven- } \\
\text { tion }(n=25)\end{array}$ & Not stated & $\begin{array}{l}\text { No participants } \\
\text { had diabetes } \\
\text { mellitus }\end{array}$ & $\begin{array}{l}\text { All participants } \\
\text { had supplemen- } \\
\text { tary lifestyle } \\
\text { modification }\end{array}$ & $\begin{array}{l}\text { Elastogra- } \\
\text { phy }\end{array}$ & Not stated & 2.8 & High \\
\hline Zhu 2008 & $\begin{array}{l}\text { PUFA }(n=66) \text { vs No active interven- } \\
\text { tion }(n=68)\end{array}$ & Not stated & Not stated & Not stated & Ultrasound & $2006-2008$ & 5.5 & High \\
\hline Song 2020 & $\begin{array}{l}\text { PUFA }(n=21) \text { vs Other supplements } \\
(n=16)\end{array}$ & Not stated & $\begin{array}{l}\text { No participants } \\
\text { had diabetes } \\
\text { mellitus }\end{array}$ & $\begin{array}{l}\text { All participants } \\
\text { had supplemen- } \\
\text { tary lifestyle } \\
\text { modification }\end{array}$ & $\begin{array}{l}\text { CT scan + } \\
\text { abnormal } \\
\text { serum lipid } \\
\text { profile or } \\
\text { high BMI }\end{array}$ & 2018 & 2.8 & High \\
\hline Basu 2012 & $\begin{array}{l}\text { Vitamin } E(n=40) \text { vs No active inter- } \\
\text { vention }(n=35)\end{array}$ & $\begin{array}{l}\text { All partici- } \\
\text { pants had } \\
\text { NASH }\end{array}$ & $\begin{array}{l}\text { No participants } \\
\text { had diabetes } \\
\text { mellitus }\end{array}$ & Not stated & Not stated & Not stated & 6 & High \\
\hline Bril 2019 & $\begin{array}{l}\text { Vitamin } E(n=36) \text { vs No active inter- } \\
\text { vention }(n=32)\end{array}$ & $\begin{array}{l}\text { All partici- } \\
\text { pants had } \\
\text { NASH }\end{array}$ & $\begin{array}{l}\text { All participants } \\
\text { had diabetes } \\
\text { mellitus only }\end{array}$ & $\begin{array}{l}\text { All participants } \\
\text { had supplemen- } \\
\text { tary lifestyle } \\
\text { modification }\end{array}$ & Liver biopsy & $2010-2016$ & 18 & Low \\
\hline Dufour 2006 & $\begin{array}{l}\text { Vitamin } E(n=10) \text { vs No active inter- } \\
\text { vention }(n=11)\end{array}$ & $\begin{array}{l}\text { All partici- } \\
\text { pants had } \\
\text { NASH }\end{array}$ & $\begin{array}{l}\text { Participants } \\
\text { with and with- } \\
\text { out diabetes } \\
\text { mellitus }\end{array}$ & Not stated & Liver biopsy & $1999-2002$ & 24 & High \\
\hline Ekhlasi 2016 & $\begin{array}{l}\text { Vitamin } E(n=15) \text { vs No active inter- } \\
\text { vention }(n=15)\end{array}$ & Not stated & $\begin{array}{l}\text { No participants } \\
\text { had diabetes } \\
\text { mellitus }\end{array}$ & Not stated & $\begin{array}{l}\text { Ultrasound } \\
+ \text { transami- } \\
\text { nases }\end{array}$ & $2012-2013$ & 1.8 & High \\
\hline $\begin{array}{l}\text { Gherghere- } \\
\text { hchi } 2013\end{array}$ & $\begin{array}{l}\text { Vitamin } E(n=17) \text { vs No active inter- } \\
\text { vention }(n=16)\end{array}$ & Not stated & Not stated & $\begin{array}{l}\text { All participants } \\
\text { had supplemen- } \\
\text { tary lifestyle } \\
\text { modification }\end{array}$ & $\begin{array}{l}\text { Ultrasound } \\
+ \text { transami- } \\
\text { nases }\end{array}$ & $2008-2009$ & 6 & High \\
\hline
\end{tabular}




\begin{tabular}{|c|c|c|c|c|c|c|c|c|}
\hline $\begin{array}{l}\text { Kugelmas } \\
2003\end{array}$ & $\begin{array}{l}\text { Vitamin } E(n=9) \text { vs No active inter- } \\
\text { vention }(n=7)\end{array}$ & $\begin{array}{l}\text { All partici- } \\
\text { pants had } \\
\text { NASH }\end{array}$ & Not stated & $\begin{array}{l}\text { All participants } \\
\text { had supplemen- } \\
\text { tary lifestyle } \\
\text { modification }\end{array}$ & Liver biopsy & Not stated & 2.8 & High \\
\hline Lavine 2011 & $\begin{array}{l}\text { Vitamin } E(n=50) \text { vs No active inter- } \\
\text { vention }(n=47)\end{array}$ & $\begin{array}{l}\text { Participants } \\
\text { with and } \\
\text { without } \\
\text { NASH }\end{array}$ & $\begin{array}{l}\text { No participants } \\
\text { had diabetes } \\
\text { mellitus }\end{array}$ & Not stated & Liver biopsy & $2005-2010$ & 27.7 & High \\
\hline $\begin{array}{l}\text { Magosso } \\
2013\end{array}$ & $\begin{array}{l}\text { Vitamin } E(n=43) \text { vs No active inter- } \\
\text { vention }(n=44)\end{array}$ & Not stated & Not stated & Not stated & Ultrasound & $2008-2009$ & 12 & High \\
\hline NCT02690792 & $\begin{array}{l}\text { Vitamin } E(n=\text { not stated }) \text { vs No ac- } \\
\text { tive intervention }(n=\text { not stated) }\end{array}$ & Not stated & Not stated & Not stated & Not stated & $2009-2016$ & 4 & High \\
\hline $\begin{array}{l}\text { Palamaru } \\
2017\end{array}$ & $\begin{array}{l}\text { Vitamin } E(n=20) \text { vs No active inter- } \\
\text { vention }(n=20)\end{array}$ & Not stated & Not stated & $\begin{array}{l}\text { All participants } \\
\text { had supplemen- } \\
\text { tary lifestyle } \\
\text { modification }\end{array}$ & Not stated & $2016-2017$ & 5.5 & High \\
\hline Pervez 2018 & $\begin{array}{l}\text { Vitamin } E(n=31) \text { vs No active inter- } \\
\text { vention }(n=33)\end{array}$ & Not stated & Not stated & Not stated & Ultrasound & $2015-2016$ & 2.8 & High \\
\hline Pervez 2020 & $\begin{array}{l}\text { Vitamin } E(n=35) \text { vs No active inter- } \\
\text { vention }(n=36)\end{array}$ & Not stated & Not stated & Not stated & Ultrasound & $2015-2016$ & 5.5 & High \\
\hline Sanyal 2010 & $\begin{array}{l}\text { Vitamin } E(n=84) \text { vs No active inter- } \\
\text { vention }(n=83)\end{array}$ & $\begin{array}{l}\text { All partici- } \\
\text { pants had } \\
\text { NASH }\end{array}$ & $\begin{array}{l}\text { No participants } \\
\text { had diabetes } \\
\text { mellitus }\end{array}$ & Not stated & Liver biopsy & $2005-2007$ & 27.7 & Low \\
\hline Wang 2008 & $\begin{array}{l}\text { Vitamin } E(n=19) \text { vs No active inter- } \\
\text { vention }(n=38)\end{array}$ & $\begin{array}{l}\text { All partici- } \\
\text { pants had } \\
\text { NASH }\end{array}$ & Not stated & Not stated & Not stated & Not stated & Not stated & High \\
\hline Basu 2013 & $\begin{array}{l}\text { Vitamin } E(n=20) \text { vs Other supple- } \\
\text { ments }(n=20)\end{array}$ & Not stated & Not stated & Not stated & Not stated & Not stated & 12 & High \\
\hline Ekhlasi 2016 & $\begin{array}{l}\text { Vitamin E }(n=15) \text { vs Prebiotics/Pro- } \\
\text { biotics/Synbiotics }(n=15)\end{array}$ & Not stated & $\begin{array}{l}\text { No participants } \\
\text { had diabetes } \\
\text { mellitus }\end{array}$ & Not stated & $\begin{array}{l}\text { Ultrasound } \\
+ \text { transami- } \\
\text { nases }\end{array}$ & $2012-2013$ & 1.8 & High \\
\hline
\end{tabular}




\begin{tabular}{|c|c|c|c|c|c|c|c|c|}
\hline Amiri 2017 & $\begin{array}{l}\text { Vitamin } D(n=74) \text { vs No active inter- } \\
\text { vention }(n=36)\end{array}$ & Not stated & $\begin{array}{l}\text { No participants } \\
\text { had diabetes } \\
\text { mellitus }\end{array}$ & Not stated & Ultrasound & 2015-2016 & 2.8 & High \\
\hline $\begin{array}{l}\text { Barchetta } \\
2016\end{array}$ & $\begin{array}{l}\text { Vitamin } D(n=26) \text { vs No active inter- } \\
\text { vention }(n=29)\end{array}$ & Not stated & $\begin{array}{l}\text { All participants } \\
\text { had diabetes } \\
\text { mellitus only }\end{array}$ & Not stated & $\begin{array}{l}\text { Ultrasound } \\
+ \text { MRI + } \\
\text { transami- } \\
\text { nases }\end{array}$ & Not stated & 5.5 & High \\
\hline $\begin{array}{l}\text { Boonyagard } \\
2016\end{array}$ & $\begin{array}{l}\text { Vitamin } D(n=30) \text { vs No active inter- } \\
\text { vention }(n=30)\end{array}$ & Not stated & $\begin{array}{l}\text { Participants } \\
\text { with and with- } \\
\text { out diabetes } \\
\text { mellitus }\end{array}$ & Not stated & Not stated & 2015 & 4.6 & High \\
\hline $\begin{array}{l}\text { Boonyagard } \\
2020\end{array}$ & $\begin{array}{l}\text { Vitamin } D(n=30) \text { vs No active inter- } \\
\text { vention }(n=30)\end{array}$ & Not stated & $\begin{array}{l}\text { Participants } \\
\text { with and with- } \\
\text { out diabetes } \\
\text { mellitus }\end{array}$ & Not stated & $\begin{array}{l}\text { Ultrasonog- } \\
\text { raphy and } \\
\text { increased } \\
\text { levels of } \\
\text { alanine } \\
\text { transami- } \\
\text { nase }\end{array}$ & $2015-2018$ & 5 & High \\
\hline $\begin{array}{l}\text { Dabbagh- } \\
\text { manesh } \\
2018\end{array}$ & $\begin{array}{l}\text { Vitamin } D(n=59) \text { vs No active inter- } \\
\text { vention }(n=32)\end{array}$ & Not stated & $\begin{array}{l}\text { No participants } \\
\text { had diabetes } \\
\text { mellitus }\end{array}$ & Not stated & Ultrasound & $2011-2013$ & 2.8 & High \\
\hline $\begin{array}{l}\text { Foroughi } \\
2014\end{array}$ & $\begin{array}{l}\text { Vitamin } D(n=30) \text { vs No active inter- } \\
\text { vention }(n=30)\end{array}$ & Not stated & Not stated & Not stated & Ultrasound & Not stated & 2.3 & High \\
\hline Geier 2018 & $\begin{array}{l}\text { Vitamin } D(n=8) \text { vs No active inter- } \\
\text { vention }(n=10)\end{array}$ & $\begin{array}{l}\text { All partici- } \\
\text { pants had } \\
\text { NASH }\end{array}$ & $\begin{array}{l}\text { Participants } \\
\text { with and with- } \\
\text { out diabetes } \\
\text { mellitus }\end{array}$ & Not stated & Liver biopsy & Not stated & 11.1 & High \\
\hline $\begin{array}{l}\text { Hoseini } \\
2020\end{array}$ & $\begin{array}{l}\text { Vitamin } D(n=20) \text { vs No active inter- } \\
\text { vention }(n=20)\end{array}$ & Not stated & Not stated & $\begin{array}{l}50 \% \text { of partici- } \\
\text { pants (factorial } \\
\text { trial design) }\end{array}$ & Not stated & Not stated & 1.8 & High \\
\hline $\begin{array}{l}\text { Hosseini } \\
2018\end{array}$ & $\begin{array}{l}\text { Vitamin } D(n=37) \text { vs No active inter- } \\
\text { vention }(n=38)\end{array}$ & Not stated & $\begin{array}{l}\text { No participants } \\
\text { had diabetes } \\
\text { mellitus }\end{array}$ & Not stated & Ultrasound & $2015-2016$ & 1 & High \\
\hline
\end{tabular}




\begin{tabular}{|c|c|c|c|c|c|c|c|c|}
\hline NCT01083992 & $\begin{array}{l}\text { Vitamin } D(n=\text { not stated }) \text { vs No ac- } \\
\text { tive intervention }(n=\text { not stated })\end{array}$ & Not stated & Not stated & $\begin{array}{l}\text { All participants } \\
\text { had supplemen- } \\
\text { tary lifestyle } \\
\text { modification }\end{array}$ & $\begin{array}{l}\text { Liver biopsy } \\
+ \text { elevated } \\
\text { enzymes }\end{array}$ & Not stated & 6 & High \\
\hline NCT01623024 & $\begin{array}{l}\text { Vitamin } D(n=\text { not stated }) \text { vs No ac- } \\
\text { tive intervention }(n=\text { not stated })\end{array}$ & $\begin{array}{l}\text { All partici- } \\
\text { pants had } \\
\text { NASH }\end{array}$ & Not stated & $\begin{array}{l}\text { All participants } \\
\text { had supplemen- } \\
\text { tary lifestyle } \\
\text { modification }\end{array}$ & Liver biopsy & Not stated & Not stated & High \\
\hline Sakpal 2017 & $\begin{array}{l}\text { Vitamin } D(n=51) \text { vs No active inter- } \\
\text { vention }(n=30)\end{array}$ & $\begin{array}{l}\text { Participants } \\
\text { with and } \\
\text { without } \\
\text { NASH }\end{array}$ & $\begin{array}{l}\text { Participants } \\
\text { with and with- } \\
\text { out diabetes } \\
\text { mellitus }\end{array}$ & $\begin{array}{l}\text { All participants } \\
\text { had supplemen- } \\
\text { tary lifestyle } \\
\text { modification }\end{array}$ & Ultrasound & Not stated & 6 & High \\
\hline Sharifi 2014 & $\begin{array}{l}\text { Vitamin } D(n=27) \text { vs No active inter- } \\
\text { vention }(n=26)\end{array}$ & Not stated & Not stated & Not stated & Ultrasound & $2012-2013$ & 4 & High \\
\hline $\begin{array}{l}\text { Taghvaei } \\
2018\end{array}$ & $\begin{array}{l}\text { Vitamin } D(n=20) \text { vs No active inter- } \\
\text { vention }(n=20)\end{array}$ & Not stated & $\begin{array}{l}\text { No participants } \\
\text { had diabetes } \\
\text { mellitus }\end{array}$ & Not stated & $\begin{array}{l}\text { Elastog- } \\
\text { raphy + } \\
\text { transami- } \\
\text { nases }\end{array}$ & Not stated & 6 & High \\
\hline Zanko 2020 & $\begin{array}{l}\text { Vitamin } D(n=201) \text { vs No active inter- } \\
\text { vention }(n=110)\end{array}$ & Not stated & $\begin{array}{l}\text { Participants } \\
\text { with and with- } \\
\text { out diabetes } \\
\text { mellitus }\end{array}$ & $\begin{array}{l}\text { All participants } \\
\text { had supplemen- } \\
\text { tary lifestyle } \\
\text { modification }\end{array}$ & $\begin{array}{l}\text { Ultrasound } \\
\text { and tran- } \\
\text { sient elas- } \\
\text { tography }\end{array}$ & 2015-2019 & 12 & High \\
\hline Basu 2012 & $\begin{array}{l}\text { Other antioxidants }(n=40) \text { vs No ac- } \\
\text { tive intervention }(n=35)\end{array}$ & $\begin{array}{l}\text { All partici- } \\
\text { pants had } \\
\text { NASH }\end{array}$ & $\begin{array}{l}\text { No participants } \\
\text { had diabetes } \\
\text { mellitus }\end{array}$ & Not stated & Not stated & Not stated & 6 & High \\
\hline Chan 2017 & $\begin{array}{l}\text { Other antioxidants }(n=49) \text { vs No ac- } \\
\text { tive intervention }(n=50)\end{array}$ & $\begin{array}{l}\text { All partici- } \\
\text { pants had } \\
\text { NASH }\end{array}$ & Not stated & $\begin{array}{l}\text { All participants } \\
\text { had supplemen- } \\
\text { tary lifestyle } \\
\text { modification }\end{array}$ & Liver biopsy & $2012-2014$ & 11.1 & Low \\
\hline Chen 2015b & $\begin{array}{l}\text { Other antioxidants }(n=30) \text { vs No ac- } \\
\text { tive intervention }(n=30)\end{array}$ & Not stated & $\begin{array}{l}\text { No participants } \\
\text { had diabetes } \\
\text { mellitus }\end{array}$ & $\begin{array}{l}\text { All participants } \\
\text { had supplemen- } \\
\text { tary lifestyle } \\
\text { modification }\end{array}$ & Ultrasound & $2012-2013$ & 2.8 & Low \\
\hline
\end{tabular}




\begin{tabular}{|c|c|c|c|c|c|c|c|c|}
\hline $\begin{array}{l}\text { Cheragh- } \\
\text { pour } 2019\end{array}$ & $\begin{array}{l}\text { Other antioxidants }(n=25) \text { vs No ac- } \\
\text { tive intervention }(n=24)\end{array}$ & Not stated & $\begin{array}{l}\text { No participants } \\
\text { had diabetes } \\
\text { mellitus }\end{array}$ & $\begin{array}{l}\text { All participants } \\
\text { had supplemen- } \\
\text { tary lifestyle } \\
\text { modification }\end{array}$ & $\begin{array}{l}\text { Elastogra- } \\
\text { phy }\end{array}$ & $2017-2018$ & 2.8 & High \\
\hline $\begin{array}{l}\text { Farhangi } \\
2014\end{array}$ & $\begin{array}{l}\text { Other antioxidants }(n=20) \text { vs No ac- } \\
\text { tive intervention }(n=21)\end{array}$ & Not stated & $\begin{array}{l}\text { No participants } \\
\text { had diabetes } \\
\text { mellitus }\end{array}$ & Not stated & Ultrasound & Not stated & 0.9 & High \\
\hline Farsi 2016 & $\begin{array}{l}\text { Other antioxidants }(n=20) \text { vs No ac- } \\
\text { tive intervention }(n=21)\end{array}$ & Not stated & $\begin{array}{l}\text { No participants } \\
\text { had diabetes } \\
\text { mellitus }\end{array}$ & Not stated & $\begin{array}{l}\text { Ultrasound } \\
+ \text { transami- } \\
\text { nases }\end{array}$ & Not stated & 2.8 & High \\
\hline $\begin{array}{l}\text { Gianturco } \\
2013\end{array}$ & $\begin{array}{l}\text { Other antioxidants }(n=104) \text { vs No } \\
\text { active intervention }(n=92)\end{array}$ & Not stated & $\begin{array}{l}\text { No participants } \\
\text { had diabetes } \\
\text { mellitus }\end{array}$ & $\begin{array}{l}\text { All participants } \\
\text { had supplemen- } \\
\text { tary lifestyle } \\
\text { modification }\end{array}$ & Liver biopsy & Not stated & 12 & High \\
\hline $\begin{array}{l}\text { Gonciarz } \\
2012\end{array}$ & $\begin{array}{l}\text { Other antioxidants }(n=30) \text { vs No ac- } \\
\text { tive intervention }(n=12)\end{array}$ & $\begin{array}{l}\text { All partici- } \\
\text { pants had } \\
\text { NASH }\end{array}$ & Not stated & $\begin{array}{l}\text { All participants } \\
\text { had supplemen- } \\
\text { tary lifestyle } \\
\text { modification }\end{array}$ & Liver biopsy & $2008-2010$ & 8.3 & High \\
\hline $\begin{array}{l}\text { Hashemi } \\
2009\end{array}$ & $\begin{array}{l}\text { Other antioxidants }(n=50) \text { vs No ac- } \\
\text { tive intervention }(n=50)\end{array}$ & $\begin{array}{l}\text { All partici- } \\
\text { pants had } \\
\text { NASH }\end{array}$ & Not stated & Not stated & $\begin{array}{l}\text { Ultrasound } \\
\text { + transami- } \\
\text { nases }\end{array}$ & $2007-2008$ & 5.5 & High \\
\hline Kanoni 2021 & $\begin{array}{l}\text { Other antioxidants }(n=35) \text { vs No ac- } \\
\text { tive intervention }(n=52)\end{array}$ & $\begin{array}{l}\text { Participants } \\
\text { with and } \\
\text { without } \\
\text { NASH }\end{array}$ & Not stated & Not stated & $\begin{array}{l}\text { Magnetic } \\
\text { resonance } \\
\text { imaging }\end{array}$ & $2017-2019$ & 6 & High \\
\hline Ruan 2010 & $\begin{array}{l}\text { Other antioxidants }(n=30) \text { vs No ac- } \\
\text { tive intervention }(n=30)\end{array}$ & Not stated & Not stated & $\begin{array}{l}\text { All participants } \\
\text { had supplemen- } \\
\text { tary lifestyle } \\
\text { modification }\end{array}$ & Not stated & $2008-2009$ & 5.5 & High \\
\hline Solhi 2014 & $\begin{array}{l}\text { Other antioxidants }(n=33) \text { vs No ac- } \\
\text { tive intervention }(n=31)\end{array}$ & $\begin{array}{l}\text { All partici- } \\
\text { pants had } \\
\text { NASH }\end{array}$ & $\begin{array}{l}\text { No participants } \\
\text { had diabetes } \\
\text { mellitus }\end{array}$ & $\begin{array}{l}\text { All participants } \\
\text { had supplemen- } \\
\text { tary lifestyle } \\
\text { modification }\end{array}$ & $\begin{array}{l}\text { Elastog- } \\
\text { raphy + } \\
\text { transami- } \\
\text { nases }\end{array}$ & Not stated & 1.8 & High \\
\hline Yari 2020 & $\begin{array}{l}\text { Other antioxidants }(n=22) \text { vs No ac- } \\
\text { tive intervention }(n=21)\end{array}$ & Not stated & Not stated & $\begin{array}{l}\text { All participants } \\
\text { had supplemen- }\end{array}$ & $\begin{array}{l}\text { Elastogra- } \\
\text { phy }\end{array}$ & $2018-2019$ & 2.8 & High \\
\hline
\end{tabular}




\begin{tabular}{|c|c|c|c|c|c|c|c|c|}
\hline & & & & $\begin{array}{l}\text { tary lifestyle } \\
\text { modification }\end{array}$ & & & & \\
\hline Deng 2005 & $\begin{array}{l}\text { Other antioxidants }(n=48) \text { vs Other } \\
\text { supplements }(n=48)\end{array}$ & Not stated & $\begin{array}{l}\text { No participants } \\
\text { had diabetes } \\
\text { mellitus }\end{array}$ & Not stated & Not stated & Not stated & 3 & High \\
\hline Basu 2012 & $\begin{array}{l}\text { Other antioxidants }(n=40) \text { vs Vita- } \\
\min E(n=40)\end{array}$ & $\begin{array}{l}\text { All partici- } \\
\text { pants had } \\
\text { NASH }\end{array}$ & $\begin{array}{l}\text { No participants } \\
\text { had diabetes } \\
\text { mellitus }\end{array}$ & Not stated & Not stated & Not stated & 6 & High \\
\hline $\begin{array}{l}\text { Khoshbaten } \\
\text { 2010b }\end{array}$ & $\begin{array}{l}\text { Other antioxidants }(n=15) \text { vs Vita- } \\
\min C(n=15)\end{array}$ & Not stated & $\begin{array}{l}\text { Participants } \\
\text { with and with- } \\
\text { out diabetes } \\
\text { mellitus }\end{array}$ & Not stated & $\begin{array}{l}\text { Ultrasound } \\
\text { or transami- } \\
\text { nases }\end{array}$ & 2008 & 3 & High \\
\hline Yari 2020 & $\begin{array}{l}\text { Other antioxidants }(n=22) \text { vs Other } \\
\text { supplements }(n=24)\end{array}$ & Not stated & Not stated & $\begin{array}{l}\text { All participants } \\
\text { had supplemen- } \\
\text { tary lifestyle } \\
\text { modification }\end{array}$ & $\begin{array}{l}\text { Elastogra- } \\
\text { phy }\end{array}$ & 2018-2019 & 2.8 & High \\
\hline $\begin{array}{l}\text { Abdelmalek } \\
2009\end{array}$ & $\begin{array}{l}\text { Amino acids }(n=17) \text { vs No active in- } \\
\text { tervention }(n=18)\end{array}$ & $\begin{array}{l}\text { All partici- } \\
\text { pants had } \\
\text { NASH }\end{array}$ & $\begin{array}{l}\text { Participants } \\
\text { with and with- } \\
\text { out diabetes } \\
\text { mellitus }\end{array}$ & Not stated & Liver biopsy & $2003-2005$ & 12 & High \\
\hline $\begin{array}{l}\text { Amiri- } \\
\text { Moghadam } \\
2015\end{array}$ & $\begin{array}{l}\text { Amino acids }(n=36) \text { vs No active in- } \\
\text { tervention }(n=32)\end{array}$ & $\begin{array}{l}\text { All partici- } \\
\text { pants had } \\
\text { NASH }\end{array}$ & Not stated & $\begin{array}{l}\text { All participants } \\
\text { had supplemen- } \\
\text { tary lifestyle } \\
\text { modification }\end{array}$ & $\begin{array}{l}\text { Ultrasound } \\
\text { + transami- } \\
\text { nases }\end{array}$ & $2013-2014$ & 2.8 & High \\
\hline Bae 2015 & $\begin{array}{l}\text { Amino acids }(n=39) \text { vs No active in- } \\
\text { tervention }(n=39)\end{array}$ & Not stated & $\begin{array}{l}\text { All participants } \\
\text { had diabetes } \\
\text { mellitus only }\end{array}$ & Not stated & CT scan & 2011-2012 & 2.8 & Low \\
\hline $\begin{array}{l}\text { Eghtesadi } \\
2016\end{array}$ & $\begin{array}{l}\text { Amino acids }(n=36) \text { vs No active in- } \\
\text { tervention }(n=32)\end{array}$ & $\begin{array}{l}\text { All partici- } \\
\text { pants had } \\
\text { NASH }\end{array}$ & Not stated & Not stated & Not stated & Not stated & 2.8 & High \\
\hline $\begin{array}{l}\text { Fabbrini } \\
2010\end{array}$ & $\begin{array}{l}\text { Amino acids }(n=9) \text { vs No active in- } \\
\text { tervention }(n=9)\end{array}$ & Not stated & $\begin{array}{l}\text { No participants } \\
\text { had diabetes } \\
\text { mellitus }\end{array}$ & Not stated & Not stated & Not stated & 1.8 & High \\
\hline
\end{tabular}




\begin{tabular}{|c|c|c|c|c|c|c|c|c|}
\hline $\begin{array}{l}\text { Malaguarn- } \\
\text { era } 2010\end{array}$ & $\begin{array}{l}\text { Amino acids }(n=36) \text { vs No active in- } \\
\text { tervention }(n=38)\end{array}$ & $\begin{array}{l}\text { All partici- } \\
\text { pants had } \\
\text { NASH }\end{array}$ & Not stated & $\begin{array}{l}\text { All participants } \\
\text { had supplemen- } \\
\text { tary lifestyle } \\
\text { modification }\end{array}$ & Liver biopsy & 2004-2006 & 5.5 & High \\
\hline $\begin{array}{l}\text { Naganuma } \\
2016\end{array}$ & $\begin{array}{l}\text { Amino acids }(n=10) \text { vs No active in- } \\
\text { tervention }(n=10)\end{array}$ & Not stated & Not stated & $\begin{array}{l}\text { All participants } \\
\text { had supplemen- } \\
\text { tary lifestyle } \\
\text { modification }\end{array}$ & Not stated & Not stated & 3 & High \\
\hline Somi 2014 & $\begin{array}{l}\text { Amino acids }(n=40) \text { vs No active in- } \\
\text { tervention }(n=40)\end{array}$ & Not stated & $\begin{array}{l}\text { No participants } \\
\text { had diabetes } \\
\text { mellitus }\end{array}$ & Not stated & Ultrasound & $2012-2014$ & 5.5 & High \\
\hline Uygun 2000 & $\begin{array}{l}\text { Amino acids }(n=78) \text { vs No active in- } \\
\text { tervention }(n=23)\end{array}$ & $\begin{array}{l}\text { All partici- } \\
\text { pants had } \\
\text { NASH }\end{array}$ & Not stated & $\begin{array}{l}\text { All participants } \\
\text { had supplemen- } \\
\text { tary lifestyle } \\
\text { modification }\end{array}$ & Liver biopsy & Not stated & 12 & High \\
\hline Aller 2015 & $\begin{array}{l}\text { Vitamin E plus other antioxidants ( } n \\
=18) \text { vs No active intervention }(n= \\
18)\end{array}$ & $\begin{array}{l}\text { Participants } \\
\text { with and } \\
\text { without } \\
\text { NASH }\end{array}$ & $\begin{array}{l}\text { No participants } \\
\text { had diabetes } \\
\text { mellitus }\end{array}$ & $\begin{array}{l}\text { All participants } \\
\text { had supplemen- } \\
\text { tary lifestyle } \\
\text { modification }\end{array}$ & Liver biopsy & Not stated & 3 & High \\
\hline Basu 2012 & $\begin{array}{l}\text { Vitamin E plus other antioxidants ( } \mathrm{n} \\
=40) \text { vs No active intervention }(n= \\
35)\end{array}$ & $\begin{array}{l}\text { All partici- } \\
\text { pants had } \\
\text { NASH }\end{array}$ & $\begin{array}{l}\text { No participants } \\
\text { had diabetes } \\
\text { mellitus }\end{array}$ & Not stated & Not stated & Not stated & 6 & High \\
\hline $\begin{array}{l}\text { Bonfrate } \\
2015\end{array}$ & $\begin{array}{l}\text { Vitamin E plus other antioxidants ( } \mathrm{n} \\
=\text { not stated) vs No active interven- } \\
\text { tion ( } \mathrm{n}=\text { not stated) }\end{array}$ & Not stated & $\begin{array}{l}\text { Participants } \\
\text { with and with- } \\
\text { out diabetes } \\
\text { mellitus }\end{array}$ & Not stated & Not stated & Not stated & 6 & High \\
\hline $\begin{array}{l}\text { Amirkhizi } \\
2018\end{array}$ & $\begin{array}{l}\text { Vitamin E plus other antioxidants ( } \\
=23) \text { vs Vitamin } E(n=22)\end{array}$ & Not stated & $\begin{array}{l}\text { No participants } \\
\text { had diabetes } \\
\text { mellitus }\end{array}$ & Not stated & Ultrasound & Not stated & 2.8 & High \\
\hline Basu 2012 & $\begin{array}{l}\text { Vitamin E plus other antioxidants ( } \\
=40) \text { vs Vitamin } E(n=40)\end{array}$ & $\begin{array}{l}\text { All partici- } \\
\text { pants had } \\
\text { NASH }\end{array}$ & $\begin{array}{l}\text { No participants } \\
\text { had diabetes } \\
\text { mellitus }\end{array}$ & Not stated & Not stated & Not stated & 6 & High \\
\hline
\end{tabular}




\begin{tabular}{|c|c|c|c|c|c|c|c|c|}
\hline Basu 2012 & $\begin{array}{l}\text { Vitamin E plus other antioxidants ( } \\
=40) \text { vs Other antioxidants }(n=40)\end{array}$ & $\begin{array}{l}\text { All partici- } \\
\text { pants had } \\
\text { NASH }\end{array}$ & $\begin{array}{l}\text { No participants } \\
\text { had diabetes } \\
\text { mellitus }\end{array}$ & Not stated & Not stated & Not stated & 6 & High \\
\hline NCT04411862 & $\begin{array}{l}\text { Phospholipids }(n=50) \text { vs No active } \\
\text { intervention }(n=50)\end{array}$ & Not stated & Not stated & $\begin{array}{l}\text { All participants } \\
\text { had supplemen- } \\
\text { tary lifestyle } \\
\text { modification }\end{array}$ & $\begin{array}{l}\text { Ultrasound, } \\
\mathrm{CT}, \mathrm{MRI}\end{array}$ & 2016-2019 & 6 & High \\
\hline Tan 2011 & $\begin{array}{l}\text { Phospholipids }(n=10) \text { vs No active } \\
\text { intervention }(n=5)\end{array}$ & $\begin{array}{l}\text { All partici- } \\
\text { pants had } \\
\text { NASH }\end{array}$ & Not stated & $\begin{array}{l}\text { All participants } \\
\text { had supplemen- } \\
\text { tary lifestyle } \\
\text { modification }\end{array}$ & Liver biopsy & Not stated & 6 & High \\
\hline Li 2010 & $\begin{array}{l}\text { Phospholipids }(n=43) \text { vs Other sup- } \\
\text { plements }(n=45)\end{array}$ & Not stated & Not stated & Not stated & CT scan & $2007-2008$ & 6 & High \\
\hline Wang 2018 & $\begin{array}{l}\text { Phospholipids }(n=50) \text { vs Prebi- } \\
\text { otics/Probiotics/Synbiotics }(n=150)\end{array}$ & Not stated & Not stated & Not stated & Not stated & $2010-2015$ & 1 & High \\
\hline Basu 2013 & $\begin{array}{l}\text { Vitamin E plus other supplements ( } \mathrm{n} \\
=20 \text { ) vs Other supplements }(n=20)\end{array}$ & Not stated & Not stated & Not stated & Not stated & Not stated & 12 & High \\
\hline $\begin{array}{l}\text { Aliashrafi } \\
2014\end{array}$ & $\begin{array}{l}\text { Vitamin E plus other supplements ( } \mathrm{n} \\
=29) \text { vs Vitamin } E(n=26)\end{array}$ & Not stated & Not stated & Not stated & $\begin{array}{l}\text { Ultrasound } \\
+ \text { transami- } \\
\text { nases }\end{array}$ & 2011-2012 & 1.8 & High \\
\hline Basu 2013 & $\begin{array}{l}\text { Vitamin E plus other supplements ( } n \\
=20) \text { vs Vitamin } E(n=20)\end{array}$ & Not stated & Not stated & Not stated & Not stated & Not stated & 12 & High \\
\hline Panahi 2012 & $\begin{array}{l}\text { Vitamin E plus other supplements ( } \mathrm{n} \\
=21) \text { vs Vitamin } E(n=33)\end{array}$ & $\begin{array}{l}\text { Participants } \\
\text { with and } \\
\text { without } \\
\text { NASH }\end{array}$ & $\begin{array}{l}\text { Participants } \\
\text { with and with- } \\
\text { out diabetes } \\
\text { mellitus }\end{array}$ & $\begin{array}{l}\text { All participants } \\
\text { had supplemen- } \\
\text { tary lifestyle } \\
\text { modification }\end{array}$ & Ultrasound & 2009 & 3 & High \\
\hline $\begin{array}{l}\text { Youshari } \\
2017\end{array}$ & $\begin{array}{l}\text { Vitamin E plus other supplements ( } \\
=20) \text { vs Vitamin } E(n=21)\end{array}$ & Not stated & Not stated & $\begin{array}{l}\text { All participants } \\
\text { had supplemen- } \\
\text { tary lifestyle } \\
\text { modification }\end{array}$ & Not stated & Not stated & 1.8 & High \\
\hline $\begin{array}{l}\text { Barbakadze } \\
2020\end{array}$ & $\begin{array}{l}\text { Vitamin E plus vitamin C }(n=52) \text { vs } \\
\text { No active intervention }(n=20)\end{array}$ & $\begin{array}{l}\text { All partici- } \\
\text { pants had } \\
\text { NASH }\end{array}$ & Not stated & Not stated & Not stated & Not stated & 12 & High \\
\hline
\end{tabular}




\begin{tabular}{|c|c|c|c|c|c|c|c|c|}
\hline $\begin{array}{l}\text { Harrison } \\
2003\end{array}$ & $\begin{array}{l}\text { Vitamin E plus vitamin } C(n=23) \text { vs } \\
\text { No active intervention }(n=22)\end{array}$ & $\begin{array}{l}\text { All partici- } \\
\text { pants had } \\
\text { NASH }\end{array}$ & $\begin{array}{l}\text { Participants } \\
\text { with and with- } \\
\text { out diabetes } \\
\text { mellitus }\end{array}$ & Not stated & Liver biopsy & $2000-2002$ & 6 & High \\
\hline Nobili 2006 & $\begin{array}{l}\text { Vitamin E plus vitamin } C(n=45) \text { vs } \\
\text { No active intervention }(n=43)\end{array}$ & $\begin{array}{l}\text { Participants } \\
\text { with and } \\
\text { without } \\
\text { NASH }\end{array}$ & Not stated & $\begin{array}{l}\text { All participants } \\
\text { had supplemen- } \\
\text { tary lifestyle } \\
\text { modification }\end{array}$ & Liver biopsy & 2003-2005 & 12 & High \\
\hline Pour 2020 & $\begin{array}{l}\text { Other supplements }(n=38) \text { vs No ac- } \\
\text { tive intervention }(n=38)\end{array}$ & Not stated & $\begin{array}{l}\text { No participants } \\
\text { had diabetes } \\
\text { mellitus }\end{array}$ & $\begin{array}{l}\text { All participants } \\
\text { had supplemen- } \\
\text { tary lifestyle } \\
\text { modification }\end{array}$ & Ultrasound & 2016-2017 & 2.8 & High \\
\hline Rafie 2020 & $\begin{array}{l}\text { Other supplements }(n=23) \text { vs No ac- } \\
\text { tive intervention }(n=23)\end{array}$ & Not stated & $\begin{array}{l}\text { No participants } \\
\text { had diabetes } \\
\text { mellitus }\end{array}$ & $\begin{array}{l}\text { All participants } \\
\text { had supplemen- } \\
\text { tary lifestyle } \\
\text { modification }\end{array}$ & $\begin{array}{l}\text { High levels } \\
\text { of liver en- } \\
\text { zymes (> } 30 \\
\mathrm{U} / \mathrm{L} \text { in men, } \\
>19 \mathrm{U} / \mathrm{L} \text { in } \\
\text { women), ul- } \\
\text { trasound, } \\
\text { and } 24.9< \\
\text { BMI }<35.19\end{array}$ & Not stated & 2.8 & High \\
\hline Nelson 2009 & $\begin{array}{l}\text { MUFA }(\mathrm{n}=\text { not stated }) \text { vs PUFA }(\mathrm{n}= \\
\text { not stated) }\end{array}$ & Not stated & Not stated & Not stated & Not stated & Not stated & 1.8 & High \\
\hline Tobin 2018 & MUFA $(n=86)$ vs PUFA $(n=81)$ & $\begin{array}{l}\text { No partici- } \\
\text { pants had } \\
\text { NASH }\end{array}$ & $\begin{array}{l}\text { Participants } \\
\text { with and with- } \\
\text { out diabetes } \\
\text { mellitus }\end{array}$ & $\begin{array}{l}\text { All participants } \\
\text { had supplemen- } \\
\text { tary lifestyle } \\
\text { modification }\end{array}$ & Ultrasound & 2015-2017 & 5.5 & High \\
\hline NCT00977730 & $\begin{array}{l}\text { Other antioxidants plus other sup- } \\
\text { plements }(n=\text { not stated) vs No ac- } \\
\text { tive intervention ( } n=\text { not stated) }\end{array}$ & $\begin{array}{l}\text { All partici- } \\
\text { pants had } \\
\text { NASH }\end{array}$ & $\begin{array}{l}\text { No participants } \\
\text { had diabetes } \\
\text { mellitus }\end{array}$ & Not stated & Liver biopsy & $2008-2011$ & 12 & High \\
\hline Yari 2020 & $\begin{array}{l}\text { Other antioxidants plus other sup- } \\
\text { plements }(n=25) \text { vs No active inter- } \\
\text { vention }(n=21)\end{array}$ & Not stated & Not stated & $\begin{array}{l}\text { All participants } \\
\text { had supplemen- } \\
\text { tary lifestyle } \\
\text { modification }\end{array}$ & $\begin{array}{l}\text { Elastogra- } \\
\text { phy }\end{array}$ & 2018-2019 & 2.8 & High \\
\hline
\end{tabular}




\begin{tabular}{|c|c|c|c|c|c|c|c|c|}
\hline Yari 2020 & $\begin{array}{l}\text { Other antioxidants plus other sup- } \\
\text { plements }(n=25) \text { vs Other supple- } \\
\text { ments }(n=24)\end{array}$ & Not stated & Not stated & $\begin{array}{l}\text { All participants } \\
\text { had supplemen- } \\
\text { tary lifestyle } \\
\text { modification }\end{array}$ & $\begin{array}{l}\text { Elastogra- } \\
\text { phy }\end{array}$ & 2018-2019 & 2.8 & High \\
\hline Yari 2020 & $\begin{array}{l}\text { Other antioxidants plus other sup- } \\
\text { plements }(n=25) \text { vs Other antioxi- } \\
\text { dants }(n=22)\end{array}$ & Not stated & Not stated & $\begin{array}{l}\text { All participants } \\
\text { had supplemen- } \\
\text { tary lifestyle } \\
\text { modification }\end{array}$ & $\begin{array}{l}\text { Elastogra- } \\
\text { phy }\end{array}$ & $2018-2019$ & 2.8 & High \\
\hline Qin 2015 & $\begin{array}{l}\text { PUFA plus vitamin } E(n=36) \text { vs No } \\
\text { active intervention }(n=34)\end{array}$ & Not stated & Not stated & $\begin{array}{l}\text { All participants } \\
\text { had supplemen- } \\
\text { tary lifestyle } \\
\text { modification }\end{array}$ & Ultrasound & $2012-2013$ & 3 & High \\
\hline Gomez 2009 & $\begin{array}{l}\text { Vitamin C plus other antioxidants ( } \mathrm{n} \\
=30 \text { ) vs No active intervention ( } \mathrm{n}= \\
\text { 30) }\end{array}$ & Not stated & $\begin{array}{l}\text { No participants } \\
\text { had diabetes } \\
\text { mellitus }\end{array}$ & $\begin{array}{l}\text { All participants } \\
\text { had supplemen- } \\
\text { tary lifestyle } \\
\text { modification }\end{array}$ & Liver biopsy & 2007 & 5.5 & High \\
\hline $\begin{array}{l}\text { Ebrahi- } \\
\text { mi-Mameghani } \\
2016\end{array}$ & $\begin{array}{l}\text { PUFA plus vitamin } E(n=19) \text { vs Vita- } \\
\min E(n=19)\end{array}$ & Not stated & Not stated & $\begin{array}{l}\text { All participants } \\
\text { had supplemen- } \\
\text { tary lifestyle } \\
\text { modification }\end{array}$ & Ultrasound & $2014-2015$ & 1.8 & High \\
\hline Ashraf 2017 & $\begin{array}{l}\text { Vitamin C plus other antioxidants ( } \\
=25) \text { vs Vitamin } E(n=27)\end{array}$ & $\begin{array}{l}\text { All partici- } \\
\text { pants had } \\
\text { NASH }\end{array}$ & $\begin{array}{l}\text { No participants } \\
\text { had diabetes } \\
\text { mellitus }\end{array}$ & $\begin{array}{l}\text { All participants } \\
\text { had supplemen- } \\
\text { tary lifestyle } \\
\text { modification }\end{array}$ & $\begin{array}{l}\text { Elastogra- } \\
\text { phy }\end{array}$ & Not stated & 3 & High \\
\hline Miglio 2000 & $\begin{array}{l}\text { Amino acids plus vitamin } C(n=96) \\
\text { vs No active intervention }(n=95)\end{array}$ & Not stated & Both & $\begin{array}{l}\text { All participants } \\
\text { had supplemen- } \\
\text { tary lifestyle } \\
\text { modification }\end{array}$ & Ultrasound & Not stated & 1.8 & High \\
\hline $\begin{array}{l}\text { Celinski } \\
2014\end{array}$ & $\begin{array}{l}\text { Amino acids plus PUFA }(n=51) \text { vs } \\
\text { PUFA }(n=23)\end{array}$ & $\begin{array}{l}\text { Participants } \\
\text { with and } \\
\text { without } \\
\text { NASH }\end{array}$ & $\begin{array}{l}\text { Participants } \\
\text { with and with- } \\
\text { out diabetes } \\
\text { mellitus }\end{array}$ & Not stated & Liver biopsy & Not stated & 14 & High \\
\hline Poulos 2021 & $\begin{array}{l}\text { Amino acids plus vitamin E plus oth- } \\
\text { er antioxidants }(n=14) \text { vs No active } \\
\text { intervention }(n=11)\end{array}$ & Not stated & Not stated & Not stated & $\begin{array}{l}\text { Liver biop- } \\
\text { sy or radi- } \\
\text { ographic }\end{array}$ & Not stated & 4.1 & High \\
\hline
\end{tabular}




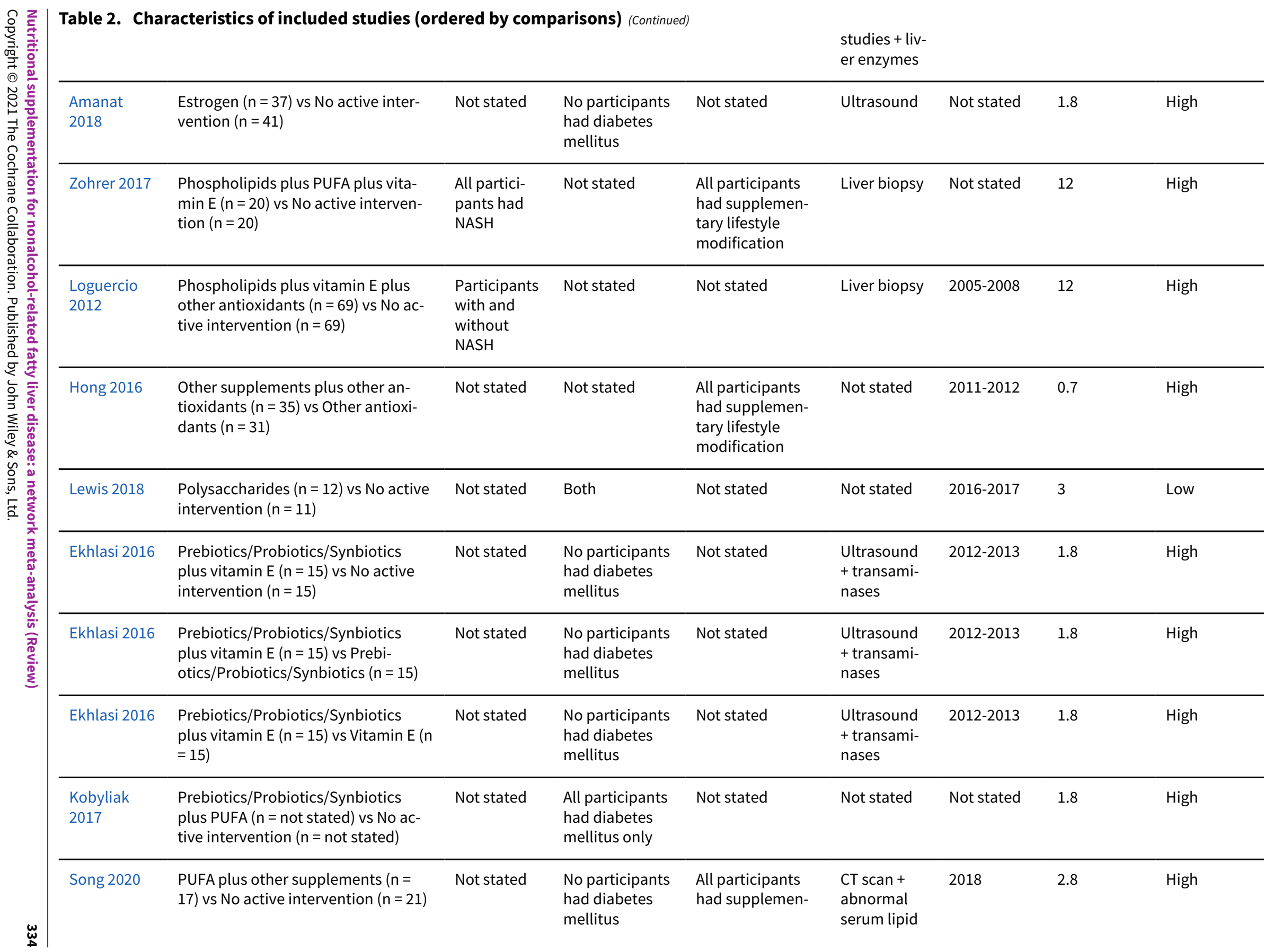




\begin{tabular}{|c|c|c|c|c|c|c|c|c|}
\hline & & & & $\begin{array}{l}\text { tary lifestyle } \\
\text { modification }\end{array}$ & $\begin{array}{l}\text { profile or } \\
\text { high BMI }\end{array}$ & & & \\
\hline $\begin{array}{l}\text { Mor- } \\
\text { varidzadeh } \\
2021\end{array}$ & $\begin{array}{l}\text { Prebiotics/Probiotics/Synbiotics } \\
\text { plus vitamin } D(n=44) \text { vs Prebi- } \\
\text { otics/Probiotics/Synbiotics }(n=44)\end{array}$ & Not stated & Not stated & Not stated & Ultrasound & Not stated & 2.8 & High \\
\hline Song 2020 & $\begin{array}{l}\text { PUFA plus other supplements }(n= \\
17) \text { vs Other supplements }(n=16)\end{array}$ & Not stated & $\begin{array}{l}\text { No participants } \\
\text { had diabetes } \\
\text { mellitus }\end{array}$ & $\begin{array}{l}\text { All participants } \\
\text { had supplemen- } \\
\text { tary lifestyle } \\
\text { modification }\end{array}$ & $\begin{array}{l}\text { CT scan + } \\
\text { abnormal } \\
\text { serum lipid } \\
\text { profile or } \\
\text { high BMI }\end{array}$ & 2018 & 2.8 & High \\
\hline Song 2020 & $\begin{array}{l}\text { PUFA plus other supplements }(n= \\
\text { 17) vs PUFA }(n=21)\end{array}$ & Not stated & $\begin{array}{l}\text { No participants } \\
\text { had diabetes } \\
\text { mellitus }\end{array}$ & $\begin{array}{l}\text { All participants } \\
\text { had supplemen- } \\
\text { tary lifestyle } \\
\text { modification }\end{array}$ & $\begin{array}{l}\text { CT scan + } \\
\text { abnormal } \\
\text { serum lipid } \\
\text { profile or } \\
\text { high BMI }\end{array}$ & 2018 & 2.8 & High \\
\hline $\begin{array}{l}\text { Della Corte } \\
2016\end{array}$ & $\begin{array}{l}\text { PUFA plus vitamin } D(n=18) \text { vs No } \\
\text { active intervention }(n=23)\end{array}$ & $\begin{array}{l}\text { Participants } \\
\text { with and } \\
\text { without } \\
\text { NASH }\end{array}$ & Not stated & Not stated & Liver biopsy & $2014-2015$ & 12 & High \\
\hline Dallio 2020 & $\begin{array}{l}\text { Vitamin D plus vitamin E plus other } \\
\text { antioxidants }(n=60) \text { vs No active in- } \\
\text { tervention }(n=30)\end{array}$ & Not stated & Not stated & Not stated & Not stated & Not stated & 6 & High \\
\hline Afzali 2020 & $\begin{array}{l}\text { Vitamin E plus other antioxidants } \\
\text { plus other supplements }(n=60) \text { vs } \\
\text { Vitamin E plus other antioxidants ( } n \\
=57 \text { ) }\end{array}$ & Not stated & Not stated & Not stated & Ultrasound & $2018-2019$ & 6 & High \\
\hline
\end{tabular}

ALT: alanine aminotransferase.

BMI: body mass index.

LFT: liver function test.

MUFA: monounsaturated fatty acid.

NASH: non-alcoholic steatohepatitis.

PUFA: polyunsaturated fatty acid. 
Table 3. Summary of risk of bias

Domain Classification

Allocation (selection bias)

A total of 106 trials were at low risk of selection bias due to lack of random sequence generation (Miglio 2000; Harrison 2003; Deng 2005; Dufour 2006; Spadaro 2008; Khoshbaten 2010b; Li 2010; Malaguarnera 2010; Sanyal 2010; Aller 2011; Loguercio 2012; Malaguarnera 2012; Ghergherehchi 2013; Gianturco 2013; Illnait 2013; Magosso 2013; Nobili 2013; Wong 2013a; Wong 2013b; Aliashrafi 2014; Alisi 2014; Chachay 2014; Eslamparast 2014; Martinez-Rodriguez 2014; Sanyal 2014; Scorletti 2014; Sharifi 2014; Aller 2015; Argo 2015; Bae 2015; Chen 2015a; Chen 2015b; Dasarathy 2015; Faghihzadeh 2015; Janczyk 2015; Pacifico 2015; Qin 2015; Yan 2015; Zhang 2015; Asgharian 2016; Barchetta 2016; Della Corte 2016; Heeboll 2016; Hong 2016; Nabavi 2016; Nogueira 2016; Pezeshki 2016; Rahimlou 2016; Amiri 2017; Chan 2017; Ebrahimi-Mameghani 2017; Famouri 2017a; Hussain 2017; Manzhalii 2017; Mofidi 2017; Shahmohammadi 2017; Tabatabaee 2017; Zohrer 2017; Amanat 2018; Amirkhizi 2018; Asghari 2018; Bakhshimoghaddam 2018; Dabbaghmanesh 2018; Eriksson 2018; Geier 2018; Hosseini 2018; Kobyliak 2018; Lewis 2018; Oscarsson 2018; Taghvaei 2018; Tobin 2018; Wang 2018; Zamani 2018; Bril 2019; Cheraghpour 2019; Duseja 2019; Jazayeri-Tehrani 2019; Abhari 2020; Afzali 2020; Babaei 2020; Boonyagard 2020; Cai 2020; Cerletti 2020; Fathi 2020; Fernandez-Travieso 2020; Ferro 2020; Hormoznejad 2020; Hosseinabadi 2020; Kazemi 2020; Kooshki 2020; Mansour 2020; Orang 2020; Pasdar 2020; Poparn 2020; Pour 2020; Sangouni 2020; Scorletti 2020; Song 2020; Tutunchi 2020; Yari 2020; Zanko 2020; Hong 2021; Izadi 2021; Morvaridzadeh 2021; Soleimani 2021; EUCTR 2008-008275-34-GB); the remaining 96 trials, which did not provide sufficient information, were at unclear risk of selection bias due to lack of random sequence generation (Uygun 2000; Kugelmas 2003; Chande 2006; Chou 2006; Nobili 2006; Chen 2008; Wang 2008; Zhu 2008; Abdelmalek 2009; Gomez 2009; Hashemi 2009; Nelson 2009; Fabbrini 2010; Ruan 2010; Lavine 2011; Tan 2011; Vajro 2011; Basu 2012; Della Corte 2012; Gonciarz 2012; Panahi 2012; Basu 2013; Saxena 2013; Shavakhi 2013; Askari 2014; Byrne 2014; Celinski 2014; Farhangi 2014; Foroughi 2014; Solhi 2014; Somi 2014; Akbarzadeh 2015; Amiri-Moghadam 2015; Bonfrate 2015; Boyraz 2015; Orr 2015; Boonyagard 2016; Ebrahimi-Mameghani 2016; Eghtesadi 2016; Ekhlasi 2016; Farsi 2016; Ferolla 2016; Guo 2016; Li 2016; Naganuma 2016; Panahi 2016; Rahmani 2016; Sepideh 2016; Yari 2016; Ashraf 2017; Behrouz 2017; Chongsrisawat 2017; Gavrilescu 2017; Jameshorani 2017; Javadi 2017; Jeong 2017; Kobyliak 2017; Navekar 2017; Palamaru 2017; Sakpal 2017; Schattenberg 2017; Wang 2017; Youshari 2017; Ahn 2018; Bomhof 2018; Daneshi-Maskooni 2018; Ghaffari 2018; Javanmardi 2018; Pervez 2018; Sayari 2018; Afsharinasab 2020; Bahrami 2020; Barbakadze 2020; Climax 2020; Dallio 2020; Farzin 2020; Hoseini 2020; Khutsishvili 2020; Moradi 2020; Parsi 2020; Pervez 2020; Rafie 2020; Sadrkabir 2020; Soleimani 2020; Chiou 2021; Kanoni 2021; Poulos 2021; EUCTR 2009-017080-41-GB; NCT00816465; NCT00845845; NCT00941642; NCT00977730; NCT01083992; NCT01623024; NCT02690792; NCT04411862)

In all, 94 trials were at low risk of selection bias due to lack of allocation concealment (Miglio 2000; Harrison 2003; Chande 2006; Dufour 2006; Nobili 2006; Gomez 2009; Khoshbaten 2010b; Malaguarnera 2010; Sanyal 2010; Malaguarnera 2012; Ghergherehchi 2013; Gianturco 2013; Illnait 2013; Magosso 2013; Nobili 2013; Wong 2013b; Alisi 2014; Askari 2014; Chachay 2014; Eslamparast 2014; Sanyal 2014; Scorletti 2014; Sharifi 2014; Amiri-Moghadam 2015; Argo 2015; Bae 2015; Chen 2015a; Chen 2015b; Faghihzadeh 2015; Janczyk 2015; Pacifico 2015; Qin 2015; Zhang 2015; Asgharian 2016; Barchetta 2016; Della Corte 2016; Heeboll 2016; Nabavi 2016; Nogueira 2016; Pezeshki 2016; Rahimlou 2016; Sepideh 2016; Amiri 2017; Chan 2017; Ebrahimi-Mameghani 2017; Famouri 2017a; Javadi 2017; Manzhalii 2017; Mofidi 2017; Shahmohammadi 2017; Tabatabaee 2017; Zohrer 2017; Amanat 2018; Amirkhizi 2018; Asghari 2018; Bakhshimoghaddam 2018; Bomhof 2018; Dabbaghmanesh 2018; Daneshi-Maskooni 2018; Eriksson 2018; Geier 2018; Ghaffari 2018; Kobyliak 2018; Lewis 2018; Oscarsson 2018; Pervez 2018; Tobin 2018; Zamani 2018; Bril 2019; Cheraghpour 2019; Duseja 2019; Jazayeri-Tehrani 2019; Abhari 2020; Babaei 2020; Boonyagard 2020; Cerletti 2020; Fathi 2020; Fernandez-Travieso 2020; Hosseinabadi 2020; Mansour 2020; Moradi 2020; Orang 2020; Pervez 2020; Pour 2020; Rafie 2020; Sangouni 2020; Scorletti 2020; Soleimani 2020; Song 2020; Tutunchi 2020; Yari 2020; Zanko 2020; Soleimani 2021; EUCTR 2008-008275-34-GB); the remaining 108 trials, which did not provide sufficient information, were at unclear risk of selection bias due to lack of allocation concealment (Uygun 2000; Kugelmas 2003; Deng 2005; Chou 2006; Chen 2008; Spadaro 2008; Wang 2008; Zhu 2008; Abdelmalek 2009; Hashemi 2009; Nelson 2009; Fabbrini 2010; Li 2010; Ruan 2010; Aller 2011; Lavine 2011; Tan 2011; Vajro 2011; Basu 2012; Della Corte 2012; Gonciarz 2012; Loguercio 2012; Panahi 2012; Basu 2013; Saxena 2013; Shavakhi 2013; Wong 2013a; Aliashrafi 2014; Byrne 2014; Celinski 2014; Farhangi 2014; Foroughi 2014; Martinez-Ro- 
Table 3. Summary of risk of bias (Continued)

driguez 2014; Solhi 2014; Somi 2014; Akbarzadeh 2015; Aller 2015; Bonfrate 2015; Boyraz 2015; Dasarathy 2015; Orr 2015; Yan 2015; Boonyagard 2016; Ebrahimi-Mameghani 2016; Eghtesadi 2016; Ekhlasi 2016; Farsi 2016; Ferolla 2016; Guo 2016; Hong 2016; Li 2016; Naganuma 2016; Panahi 2016; Rahmani 2016; Yari 2016; Ashraf 2017; Behrouz 2017; Chongsrisawat 2017; Gavrilescu 2017; Hussain 2017; Jameshorani 2017; Jeong 2017; Kobyliak 2017; Navekar 2017; Palamaru 2017; Sakpal 2017; Schattenberg 2017; Wang 2017; Youshari 2017; Ahn 2018; Hosseini 2018; Javanmardi 2018; Sayari 2018; Taghvaei 2018; Wang 2018; Afsharinasab 2020; Afzali 2020; Bahrami 2020; Barbakadze 2020; Cai 2020; Climax 2020; Dallio 2020; Farzin 2020; Ferro 2020; Hormoznejad 2020; Hoseini 2020; Kazemi 2020; Khutsishvili 2020; Kooshki 2020; Parsi 2020; Pasdar 2020; Poparn 2020; Sadrkabir 2020; Chiou 2021; Hong 2021; Izadi 2021; Kanoni 2021; Morvaridzadeh 2021; Poulos 2021; EUCTR 2009-017080-41-GB; NCT00816465; NCT00845845; NCT00941642; NCT00977730; NCT01083992; NCT01623024; NCT02690792; NCT04411862)

Blinding (performance bias and detection bias)

\begin{abstract}
A total of 138 trials were at low risk of performance bias as participants and healthcare providers were blinded (Miglio 2000; Harrison 2003; Chande 2006; Dufour 2006; Nobili 2006; Chen 2008; Abdelmalek 2009; Fabbrini 2010; Malaguarnera 2010; Sanyal 2010; Aller 2011; Lavine 2011; Vajro 2011; Della Corte 2012; Loguercio 2012; Ghergherehchi 2013; Gianturco 2013; Illnait 2013; Nobili 2013; Saxena 2013; Shavakhi 2013; Wong 2013b; Aliashrafi 2014; Alisi 2014; Askari 2014; Byrne 2014; Chachay 2014; Eslamparast 2014; Farhangi 2014; Foroughi 2014; Sanyal 2014; Scorletti 2014; Sharifi 2014; Akbarzadeh 2015; Amiri-Moghadam 2015; Argo 2015; Bae 2015; Bonfrate 2015; Chen 2015a; Chen 2015b; Dasarathy 2015; Faghihzadeh 2015; Janczyk 2015; Orr 2015; Pacifico 2015; Qin 2015; Zhang 2015; Asgharian 2016; Barchetta 2016; Della Corte 2016; Eghtesadi 2016; Ekhlasi 2016; Farsi 2016; Heeboll 2016; Nabavi 2016; Nogueira 2016; Pezeshki 2016; Rahimlou 2016; Rahmani 2016; Sepideh 2016; Amiri 2017; Behrouz 2017; Chan 2017; Chongsrisawat 2017; Ebrahimi-Mameghani 2017; Famouri 2017a; Javadi 2017; Jeong 2017; Kobyliak 2017; Mofidi 2017; Navekar 2017; Shahmohammadi 2017; Tabatabaee 2017; Youshari 2017; Zohrer 2017; Ahn 2018; Amanat 2018; Amirkhizi 2018; Asghari 2018; Dabbaghmanesh 2018; Daneshi-Maskooni 2018; Eriksson 2018; Geier 2018; Ghaffari 2018; Javanmardi 2018; Kobyliak 2018; Lewis 2018; Oscarsson 2018; Pervez 2018; Sayari 2018; Tobin 2018; Zamani 2018; Bril 2019; Cheraghpour 2019; Duseja 2019; Jazayeri-Tehrani 2019; Abhari 2020; Afsharinasab 2020; Afzali 2020; Babaei 2020; Bahrami 2020; Boonyagard 2020; Cerletti 2020; Climax 2020; Farzin 2020; Fathi 2020; Fernandez-Travieso 2020; Ferro 2020; Hormoznejad 2020; Hosseinabadi 2020; Kazemi 2020; Kooshki 2020; Mansour 2020; Moradi 2020; Orang 2020; Parsi 2020; Pasdar 2020; Pervez 2020; Poparn 2020; Pour 2020; Rafie 2020; Sangouni 2020; Scorletti 2020; Soleimani 2020; Song 2020; Tutunchi 2020; Zanko 2020; Chiou 2021; Izadi 2021; Kanoni 2021; Morvaridzadeh 2021; Soleimani 2021; EUCTR 2008-008275-34-GB; EUCTR 2009-017080-41GB; NCT00816465; NCT00845845; NCT00977730; NCT02690792); 46 trials, which did not provide sufficient information, were at unclear risk of performance bias (Uygun 2000; Deng 2005; Spadaro 2008; Wang 2008; Zhu 2008; Gomez 2009; Hashemi 2009; Nelson 2009; Khoshbaten 2010b; Li 2010; Tan 2011; Gonciarz 2012; Malaguarnera 2012; Magosso 2013; Wong 2013a; Celinski 2014; Martinez-Rodriguez 2014; Solhi 2014; Somi 2014; Aller 2015; Boyraz 2015; Boonyagard 2016; Ebrahimi-Mameghani 2016; Ferolla 2016; Guo 2016; Hong 2016; Naganuma 2016; Panahi 2016; Gavrilescu 2017; Hussain 2017; Jameshorani 2017; Palamaru 2017; Sakpal 2017; Wang 2017; Bomhof 2018; Hosseini 2018; Taghvaei 2018; Wang 2018; Barbakadze 2020; Cai 2020; Dallio 2020; Hoseini 2020; Khutsishvili 2020; Sadrkabir 2020; Hong 2021; Poulos 2021); the remaining 18 trials were at high risk of performance bias as it is clear that either participants or healthcare providers or both were not blinded (Kugelmas 2003; Chou 2006; Ruan 2010; Basu 2012; Panahi 2012; Basu 2013; Yan 2015; Li 2016; Yari 2016; Ashraf 2017; Manzhalii 2017; Schattenberg 2017; Bakhshimoghaddam 2018; Yari 2020; NCT00941642; NCT01083992; NCT01623024; NCT04411862)
\end{abstract}

In all, 142 trials were at low risk of detection bias (Miglio 2000; Harrison 2003; Chande 2006; Dufour 2006; Nobili 2006; Chen 2008; Abdelmalek 2009; Fabbrini 2010; Malaguarnera 2010; Sanyal 2010; Aller 2011; Lavine 2011; Vajro 2011; Della Corte 2012; Loguercio 2012; Malaguarnera 2012; Ghergherehchi 2013; Gianturco 2013; Illnait 2013; Magosso 2013; Nobili 2013; Saxena 2013; Shavakhi 2013; Wong 2013b; Aliashrafi 2014; Alisi 2014; Askari 2014; Byrne 2014; Chachay 2014; Eslamparast 2014; Farhangi 2014; Foroughi 2014; Sanyal 2014; Scorletti 2014; Sharifi 2014; Somi 2014; Akbarzadeh 2015; Amiri-Moghadam 2015; Argo 2015; Bae 2015; Bonfrate 2015; Boyraz 2015; Chen 2015a; Chen 2015b; Dasarathy 2015; Faghihzadeh 2015; Janczyk 2015; Orr 2015; Pacifico 2015; Qin 2015; Zhang 2015; Asgharian 2016; Barchetta 2016; Della Corte 2016; Eghtesadi 2016; Ekhlasi 2016; Farsi 2016; Heeboll 2016; Nabavi 2016; Nogueira 2016; Pezeshki 2016; Rahimlou 2016; Rahmani 2016; Sepideh 2016; Amiri 2017; Behrouz 2017; Chan 2017; Chongsrisawat 2017; Ebrahimi-Mameghani 2017; Famouri 2017a; Javadi 2017; Jeong 2017; Kobyliak 2017; Mofidi 2017; Navekar 
Table 3. Summary of risk of bias (Continued)

2017; Shahmohammadi 2017; Tabatabaee 2017; Youshari 2017; Zohrer 2017; Ahn 2018; Amanat 2018; Amirkhizi 2018; Asghari 2018; Dabbaghmanesh 2018; Daneshi-Maskooni 2018; Eriksson 2018; Geier 2018; Ghaffari 2018; Javanmardi 2018; Kobyliak 2018; Lewis 2018; Oscarsson 2018; Pervez 2018; Sayari 2018; Tobin 2018; Zamani 2018; Bril 2019; Cheraghpour 2019; Duseja 2019; Jazayeri-Tehrani 2019; Abhari 2020; Afsharinasab 2020; Afzali 2020; Babaei 2020; Bahrami 2020; Boonyagard 2020; Cerletti 2020; Climax 2020; Farzin 2020; Fathi 2020; Fernandez-Travieso 2020; Ferro 2020; Hormoznejad 2020; Hosseinabadi 2020; Kazemi 2020; Kooshki 2020; Mansour 2020; Moradi 2020; Orang 2020; Parsi 2020; Pasdar 2020; Pervez 2020; Poparn 2020; Pour 2020; Rafie 2020; Sangouni 2020; Scorletti 2020; Soleimani 2020; Song 2020; Tutunchi 2020; Zanko 2020; Chiou 2021; Izadi 2021; Kanoni 2021; Morvaridzadeh 2021; Soleimani 2021; EUCTR 2008-008275-34-GB; EUCTR 2009-017080-41-GB; NCT00816465; NCT00845845; NCT00977730; NCT02690792); 44 trials, which did not provide sufficient information, were at unclear risk of detection bias (Uygun 2000; Kugelmas 2003; Deng 2005; Spadaro 2008; Wang 2008; Zhu 2008; Gomez 2009; Hashemi 2009; Nelson 2009; Khoshbaten 2010b; Li 2010; Tan 2011; Gonciarz 2012; Wong 2013a; Celinski 2014; Martinez-Rodriguez 2014; Solhi 2014; Aller 2015; Boonyagard 2016; Ebrahimi-Mameghani 2016; Ferolla 2016; Guo 2016; Hong 2016; Naganuma 2016; Panahi 2016; Gavrilescu 2017; Hussain 2017; Jameshorani 2017; Palamaru 2017; Sakpal 2017; Wang 2017; Bakhshimoghaddam 2018; Bomhof 2018; Hosseini 2018; Taghvaei 2018; Wang 2018; Barbakadze 2020; Cai 2020; Dallio 2020; Hoseini 2020; Khutsishvili 2020; Sadrkabir 2020; Hong 2021; Poulos 2021); the remaining 16 trials were at high risk of detection bias as it is clear that outcome assessors were not blinded (Chou 2006; Ruan 2010; Basu 2012; Panahi 2012; Basu 2013; Yan 2015; Li 2016; Yari 2016; Ashraf 2017; Manzhalii 2017; Schattenberg 2017; Yari 2020; NCT00941642; NCT01083992; NCT01623024; NCT04411862)

Incomplete outcome data (attrition bias)
A total of 60 trials were at low risk of attrition bias as there were no post-randomisation dropouts or an intention-to-treat analysis was used (Deng 2005; Chande 2006; Wang 2008; Gomez 2009; Li 2010; Sanyal 2010; Vajro 2011; Malaguarnera 2012; Illnait 2013; Magosso 2013; Nobili 2013; Wong 2013a; Wong 2013b; Chachay 2014; Foroughi 2014; Martinez-Rodriguez 2014; Sanyal 2014; Scorletti 2014; Somi 2014; Aller 2015; Bae 2015; Chen 2015a; Chen 2015b; Dasarathy 2015; Zhang 2015; Ferolla 2016; Heeboll 2016; Li 2016; Nabavi 2016; Yari 2016; Chan 2017; Famouri 2017a; Hussain 2017; Manzhalii 2017; Sakpal 2017; Schattenberg 2017; Shahmohammadi 2017; Asghari 2018; Bomhof 2018; Eriksson 2018; Kobyliak 2018; Lewis 2018; Taghvaei 2018; Bril 2019; Jazayeri-Tehrani 2019; Afsharinasab 2020; Hoseini 2020; Kazemi 2020; Mansour 2020; Moradi 2020; Parsi 2020; Pervez 2020; Poparn 2020; Pour 2020; Tutunchi 2020; Zanko 2020; Soleimani 2021; EUCTR 2008-008275-34-GB; EUCTR 2009-017080-41-GB; NCT00845845); 129 trials were at unclear risk of attrition bias (Miglio 2000; Uygun 2000; Harrison 2003; Kugelmas 2003; Chou 2006; Nobili 2006; Chen 2008; Spadaro 2008; Zhu 2008; Hashemi 2009; Nelson 2009; Fabbrini 2010; Khoshbaten 2010b; Malaguarnera 2010; Ruan 2010; Aller 2011; Lavine 2011; Tan 2011; Basu 2012; Della Corte 2012; Gonciarz 2012; Panahi 2012; Basu 2013; Ghergherehchi 2013; Gianturco 2013; Saxena 2013; Shavakhi 2013; Aliashrafi 2014; Alisi 2014; Askari 2014; Byrne 2014; Celinski 2014; Eslamparast 2014; Sharifi 2014; Solhi 2014; Akbarzadeh 2015; Amiri-Moghadam 2015; Argo 2015; Bonfrate 2015; Boyraz 2015; Faghihzadeh 2015; Janczyk 2015; Orr 2015; Pacifico 2015; Qin 2015; Yan 2015; Asgharian 2016; Barchetta 2016; Boonyagard 2016; Della Corte 2016; Ebrahimi-Mameghani 2016; Eghtesadi 2016; Ekhlasi 2016; Farsi 2016; Guo 2016; Hong 2016; Naganuma 2016; Nogueira 2016; Panahi 2016; Pezeshki 2016; Rahimlou 2016; Rahmani 2016; Sepideh 2016; Amiri 2017; Ashraf 2017; Behrouz 2017; Chongsrisawat 2017; Ebrahimi-Mameghani 2017; Gavrilescu 2017; Jameshorani 2017; Javadi 2017; Jeong 2017; Kobyliak 2017; Mofidi 2017; Navekar 2017; Palamaru 2017; Tabatabaee 2017; Wang 2017; Youshari 2017; Zohrer 2017; Ahn 2018; Amanat 2018; Amirkhizi 2018; Bakhshimoghaddam 2018; DaneshiMaskooni 2018; Geier 2018; Ghaffari 2018; Hosseini 2018; Javanmardi 2018; Oscarsson 2018; Pervez 2018; Sayari 2018; Tobin 2018; Wang 2018; Zamani 2018; Cheraghpour 2019; Babaei 2020; Bahrami 2020; Barbakadze 2020; Boonyagard 2020; Cai 2020; Cerletti 2020; Climax 2020; Dallio 2020; Farzin 2020; Fernandez-Travieso 2020; Ferro 2020; Hosseinabadi 2020; Khutsishvili 2020; Kooshki 2020; NCT04411862; Orang 2020; Rafie 2020; Sadrkabir 2020; Sangouni 2020; Soleimani 2020; Song 2020; Yari 2020; Hong 2021; Izadi 2021; Kanoni 2021; Morvaridzadeh 2021; Poulos 2021; NCT00816465; NCT00941642; NCT00977730; NCT01083992; NCT01623024; NCT02690792), as it is not clear whether there were post-randomisation dropouts or whether post-randomisation dropouts were related to outcomes (if there were post-randomisation dropouts); the remaining 13 trials were at high risk of attrition bias (Dufour 2006; Abdelmalek 2009; Loguercio 2012; Farhangi 2014; Dabbaghmanesh 2018; Duseja 2019; Abhari 2020; Afzali 2020; Fathi 2020; Hormoznejad 2020; Pasdar 2020; Scorletti 2020; Chiou 2021), as post-randomisation dropouts probably were related to the intervention and to outcomes 


\section{Table 3. Summary of risk of bias (Continued)}

Selective reporting (reporting bias)
In all, 48 trials were at low risk of selective outcome reporting bias (Nobili 2006; Zhu 2008; Li 2010; Sanyal 2010; Vajro 2011; Loguercio 2012; Malaguarnera 2012; Magosso 2013; Wong 2013a; Wong 2013b; Martinez-Rodriguez 2014; Sanyal 2014; Aller 2015; Bae 2015; Chen 2015b; Faghihzadeh 2015; Janczyk 2015; Zhang 2015; Asgharian 2016; Ferolla 2016; Heeboll 2016; Nabavi 2016; Chan 2017; Manzhalii 2017; Schattenberg 2017; Asghari 2018; Bakhshimoghaddam 2018; Eriksson 2018; Kobyliak 2018; Lewis 2018; Oscarsson 2018; Pervez 2018; Zamani 2018; Bril 2019; Cheraghpour 2019; Duseja 2019; Jazayeri-Tehrani 2019; Abhari 2020; Boonyagard 2020; Cerletti 2020; Climax 2020; Fernandez-Travieso 2020; Mansour 2020; Poparn 2020; Tutunchi 2020; Yari 2020; Soleimani 2021; EUCTR 2008-008275-34-GB), as important clinical outcomes expected to be reported in such trials were reported; the remaining 154 trials were at high risk of selective outcome reporting bias (Miglio 2000; Uygun 2000; Harrison 2003; Kugelmas 2003; Deng 2005; Chande 2006; Chou 2006; Dufour 2006; Chen 2008; Spadaro 2008; Wang 2008; Abdelmalek 2009; Gomez 2009; Hashemi 2009; Nelson 2009; Fabbrini 2010; Khoshbaten 2010b; Malaguarnera 2010; Ruan 2010; Aller 2011; Lavine 2011; Tan 2011; Basu 2012; Della Corte 2012; Gonciarz 2012; Panahi 2012; Basu 2013; Ghergherehchi 2013; Gianturco 2013; Illnait 2013; Nobili 2013; Saxena 2013; Shavakhi 2013; Aliashrafi 2014; Alisi 2014; Askari 2014; Byrne 2014; Celinski 2014; Chachay 2014; Eslamparast 2014; Farhangi 2014; Foroughi 2014; Scorletti 2014; Sharifi 2014; Solhi 2014; Somi 2014; Akbarzadeh 2015; Amiri-Moghadam 2015; Argo 2015; Bonfrate 2015; Boyraz 2015; Chen 2015a; Dasarathy 2015; Orr 2015; Pacifico 2015; Qin 2015; Yan 2015; Barchetta 2016; Boonyagard 2016; Della Corte 2016; Ebrahimi-Mameghani 2016; Eghtesadi 2016; Ekhlasi 2016; Farsi 2016; Guo 2016; Hong 2016; Li 2016; Naganuma 2016; Nogueira 2016; Panahi 2016; Pezeshki 2016; Rahimlou 2016; Rahmani 2016; Sepideh 2016; Yari 2016; Amiri 2017; Ashraf 2017; Behrouz 2017; Chongsrisawat 2017; Ebrahimi-Mameghani 2017; Famouri 2017a; Gavrilescu 2017; Hussain 2017; Jameshorani 2017; Javadi 2017; Jeong 2017; Kobyliak 2017; Mofidi 2017; Navekar 2017; Palamaru 2017; Sakpal 2017; Shahmohammadi 2017; Tabatabaee 2017; Wang 2017; Youshari 2017; Zohrer 2017; Ahn 2018; Amanat 2018; Amirkhizi 2018; Bomhof 2018; Dabbaghmanesh 2018; Daneshi-Maskooni 2018; Geier 2018; Ghaffari 2018; Hosseini 2018; Javanmardi 2018; Sayari 2018; Taghvaei 2018; Tobin 2018; Wang 2018; Afsharinasab 2020; Afzali 2020; Babaei 2020; Bahrami 2020; Barbakadze 2020; Cai 2020; Dallio 2020; Farzin 2020; Fathi 2020; Ferro 2020; Hormoznejad 2020; Hoseini 2020; Hosseinabadi 2020; Kazemi 2020; Khutsishvili 2020; Kooshki 2020; Moradi 2020; Orang 2020; Parsi 2020; Pasdar 2020; Pervez 2020; Pour 2020; Rafie 2020; Sadrkabir 2020; Sangouni 2020; Scorletti 2020; Soleimani 2020; Song 2020; Zanko 2020; Chiou 2021; Hong 2021; Izadi 2021; Kanoni 2021; Morvaridzadeh 2021; Poulos 2021; EUCTR 2009-017080-41GB; NCT00816465; NCT00845845; NCT00941642; NCT00977730; NCT01083992; NCT01623024; NCT02690792; NCT04411862), as outcomes were changed from the protocol published prior to recruitment without sufficient justification, or trials did not report reasonably expected clinical outcomes, if no protocol was published prior to recruitment
Other potential sources of bias
A total of 196 trials were at low risk of other bias (Miglio 2000; Uygun 2000; Harrison 2003; Kugelmas 2003; Deng 2005; Chande 2006; Chou 2006; Dufour 2006; Nobili 2006; Chen 2008; Spadaro 2008; Wang 2008; Zhu 2008; Abdelmalek 2009; Gomez 2009; Hashemi 2009; Nelson 2009; Fabbrini 2010; Khoshbaten 2010b; Li 2010; Malaguarnera 2010; Ruan 2010; Sanyal 2010; Aller 2011; Lavine 2011; Tan 2011; Vajro 2011; Basu 2012; Della Corte 2012; Gonciarz 2012; Loguercio 2012; Malaguarnera 2012; Panahi 2012; Basu 2013; Ghergherehchi 2013; Gianturco 2013; Illnait 2013; Magosso 2013; Nobili 2013; Saxena 2013; Shavakhi 2013; Wong 2013a; Wong 2013b; Aliashrafi 2014; Alisi 2014; Askari 2014; Byrne 2014; Celinski 2014; Chachay 2014; Eslamparast 2014; Farhangi 2014; Foroughi 2014; Martinez-Rodriguez 2014; Sanyal 2014; Scorletti 2014; Sharifi 2014; Solhi 2014; Somi 2014; Akbarzadeh 2015; Aller 2015; Amiri-Moghadam 2015; Argo 2015; Bae 2015; Bonfrate 2015; Boyraz 2015; Chen 2015a; Chen 2015b; Dasarathy 2015; Faghihzadeh 2015; Janczyk 2015; Orr 2015; Pacifico 2015; Qin 2015; Yan 2015; Zhang 2015; Asgharian 2016; Barchetta 2016; Boonyagard 2016; Della Corte 2016; Ebrahimi-Mameghani 2016; Eghtesadi 2016; Ekhlasi 2016; Farsi 2016; Ferolla 2016; Guo 2016; Heeboll 2016; Hong 2016; Li 2016; Nabavi 2016; Naganuma 2016; Nogueira 2016; Panahi 2016; Pezeshki 2016; Rahimlou 2016; Rahmani 2016; Sepideh 2016; Yari 2016; Amiri 2017; Ashraf 2017; Behrouz 2017; Chan 2017; Chongsrisawat 2017; Ebrahimi-Mameghani 2017; Famouri 2017a; Gavrilescu 2017; Hussain 2017; Jameshorani 2017; Javadi 2017; Jeong 2017; Kobyliak 2017; Manzhalii 2017; Mofidi 2017; Navekar 2017; Palamaru 2017; Sakpal 2017; Schattenberg 2017; Shahmohammadi 2017; Tabatabaee 2017; Wang 2017; Youshari 2017; Zohrer 2017; Ahn 2018; Amanat 2018; Amirkhizi 2018; Asghari 2018; Bakhshimoghaddam 2018; Bomhof 2018; Dabbaghmanesh 2018; Daneshi-Maskooni 2018; Eriksson 2018; Geier 2018; Ghaffari 2018; Hosseini 2018; Javanmardi 2018; Kobyliak 2018; Lewis 2018; Oscarsson 2018; Pervez 2018; Sayari 2018; Taghvaei 2018; Wang 2018; Bril 2019; Duseja 2019; Abhari 2020; Afsharinasab 2020; Afzali 2020; Bahrami 2020; Bar- 
Table 3. Summary of risk of bias (Continued)

bakadze 2020; Boonyagard 2020; Cai 2020; Cerletti 2020; Climax 2020; Dallio 2020; Farzin 2020; Fathi 2020; Fernandez-Travieso 2020; Ferro 2020; Hormoznejad 2020; Hoseini 2020; Hosseinabadi 2020; Kazemi 2020; Khutsishvili 2020; Kooshki 2020; Mansour 2020; Moradi 2020; Orang 2020; Parsi 2020; Pasdar 2020; Pervez 2020; Poparn 2020; Pour 2020; Rafie 2020; Sadrkabir 2020; Sangouni 2020; Scorletti 2020; Soleimani 2020; Song 2020; Tutunchi 2020; Zanko 2020; Chiou 2021; Hong 2021; Izadi 2021; Kanoni 2021; Morvaridzadeh 2021; Poulos 2021; Soleimani 2021; EUCTR 2008-008275-34-GB; EUCTR 2009-017080-41-GB; NCT00816465; NCT00845845; NCT00941642; NCT00977730; NCT01083992; NCT01623024; NCT02690792; NCT04411862); the remaining 6 trials were at unclear risk of other bias (Tobin 2018; Zamani 2018; Cheraghpour 2019; Jazayeri-Tehrani 2019; Babaei 2020; Yari 2020), as there were baseline differences in important prognostic factors 


\begin{tabular}{|c|c|c|c|c|c|c|c|c|c|}
\hline Study name & $\begin{array}{l}\text { Intervention } 1 \text { (number of participants) } \\
\text { vs intervention } 2 \text { (number of partici- } \\
\text { pants) }\end{array}$ & $\begin{array}{l}\text { Sequence } \\
\text { genera- } \\
\text { tion }\end{array}$ & $\begin{array}{l}\text { Allocation } \\
\text { conceal- } \\
\text { ment }\end{array}$ & $\begin{array}{l}\text { Blind- } \\
\text { ing of pa- } \\
\text { tients and } \\
\text { health- } \\
\text { care } \\
\text { providers }\end{array}$ & $\begin{array}{l}\text { Blinding } \\
\text { of out- } \\
\text { come as- } \\
\text { sessors }\end{array}$ & $\begin{array}{l}\text { Missing } \\
\text { outcome } \\
\text { bias }\end{array}$ & $\begin{array}{l}\text { Selective } \\
\text { outcome } \\
\text { reporting }\end{array}$ & $\begin{array}{l}\text { Other } \\
\text { bias }\end{array}$ & $\begin{array}{l}\text { Overall } \\
\text { risk of } \\
\text { bias }\end{array}$ \\
\hline $\begin{array}{l}\text { Afshari- } \\
\text { nasab } 2020\end{array}$ & $\begin{array}{l}\text { Other supplements ( } n=21) \text { vs No active in- } \\
\text { tervention }(n=21)\end{array}$ & Unclear & Unclear & Low & Low & Low & High & Low & High \\
\hline $\begin{array}{l}\text { Asghari } \\
2018\end{array}$ & $\begin{array}{l}\text { Other supplements }(n=30) \text { vs No active in- } \\
\text { tervention }(n=30)\end{array}$ & Low & Low & Low & Low & Low & Low & Low & Low \\
\hline Askari 2014 & $\begin{array}{l}\text { Other supplements ( } n=23) \text { vs No active in- } \\
\text { tervention }(n=22)\end{array}$ & Unclear & Low & Low & Low & Unclear & High & Low & High \\
\hline Babaei 2020 & $\begin{array}{l}\text { Other supplements ( } n=13) \text { vs No active in- } \\
\text { tervention }(n=11)\end{array}$ & Low & Low & Low & Low & Unclear & High & Unclear & High \\
\hline $\begin{array}{l}\text { Bahrami } \\
2020\end{array}$ & $\begin{array}{l}\text { Other supplements ( } n=24) \text { vs No active in- } \\
\text { tervention }(n=21)\end{array}$ & Unclear & Unclear & Low & Low & Unclear & High & Low & High \\
\hline Cerletti 2020 & $\begin{array}{l}\text { Other supplements }(n=55) \text { vs No active in- } \\
\text { tervention }(n=58)\end{array}$ & Low & Low & Low & Low & Unclear & Low & Low & High \\
\hline $\begin{array}{l}\text { Chachay } \\
2014\end{array}$ & $\begin{array}{l}\text { Other supplements ( } n=10) \text { vs No active in- } \\
\text { tervention }(n=10)\end{array}$ & Low & Low & Low & Low & Low & High & Low & High \\
\hline $\begin{array}{l}\text { Chande } \\
2006\end{array}$ & $\begin{array}{l}\text { Other supplements ( } n=5) \text { vs No active in- } \\
\text { tervention }(n=3)\end{array}$ & Unclear & Low & Low & Low & Low & High & Low & High \\
\hline Chen 2015a & $\begin{array}{l}\text { Other supplements }(n=30) \text { vs No active in- } \\
\text { tervention }(n=30)\end{array}$ & Low & Low & Low & Low & Low & High & Low & High \\
\hline Chiou 2021 & $\begin{array}{l}\text { Other supplements ( } n=15) \text { vs No active in- } \\
\text { tervention }(n=13)\end{array}$ & Unclear & Unclear & Low & Low & High & High & Low & High \\
\hline Chou 2006 & $\begin{array}{l}\text { Other supplements ( } n=28) \text { vs No active in- } \\
\text { tervention }(n=28)\end{array}$ & Unclear & Unclear & High & High & Unclear & High & Low & High \\
\hline $\begin{array}{l}\text { Daneshi- } \\
\text { Maskooni } \\
2018\end{array}$ & $\begin{array}{l}\text { Other supplements ( } n=43) \text { vs No active in- } \\
\text { tervention }(n=44)\end{array}$ & Unclear & Low & Low & Low & Unclear & High & Low & High \\
\hline
\end{tabular}




\begin{tabular}{|c|c|c|c|c|c|c|c|c|c|}
\hline $\begin{array}{l}\text { Ebrahi- } \\
\text { mi-Mameghani } \\
2017\end{array}$ & $\begin{array}{l}\text { Other supplements }(n=29) \text { vs No active in- } \\
\text { i tervention }(n=26)\end{array}$ & Low & Low & Low & Low & Unclear & High & Low & High \\
\hline $\begin{array}{l}\text { EUCTR } \\
\text { 2009-017080-4 } \\
\text { GB }\end{array}$ & $\begin{array}{l}\text { Other supplements }(n=20) \text { vs No active in- } \\
\text {-1tervention }(n=5)\end{array}$ & Unclear & Unclear & Low & Low & Low & High & Low & High \\
\hline $\begin{array}{l}\text { Faghi- } \\
\text { hzadeh } 2015\end{array}$ & $\begin{array}{l}\text { Other supplements }(n=24) \text { vs No active in- } \\
\text { tervention }(n=24)\end{array}$ & Low & Low & Low & Low & Unclear & Low & Low & High \\
\hline Farzin 2020 & $\begin{array}{l}\text { Other supplements ( } n=25) \text { vs No active in- } \\
\text { tervention }(n=25)\end{array}$ & Unclear & Unclear & Low & Low & Unclear & High & Low & High \\
\hline Fathi 2020 & $\begin{array}{l}\text { Other supplements }(n=25) \text { vs No active in- } \\
\text { tervention }(n=25)\end{array}$ & Low & Low & Low & Low & High & High & Low & High \\
\hline $\begin{array}{l}\text { Fernan- } \\
\text { dez-Travieso } \\
2020\end{array}$ & $\begin{array}{l}\text { Other supplements ( } n=50) \text { vs No active in- } \\
\text { tervention }(n=50)\end{array}$ & Low & Low & Low & Low & Unclear & Low & Low & High \\
\hline Ferro 2020 & $\begin{array}{l}\text { Other supplements }(n=45) \text { vs No active in- } \\
\text { tervention }(n=41)\end{array}$ & Low & Unclear & Low & Low & Unclear & High & Low & High \\
\hline $\begin{array}{l}\text { Ghaffari } \\
2018\end{array}$ & $\begin{array}{l}\text { Other supplements ( } n=64) \text { vs No active in- } \\
\text { tervention }(n=21)\end{array}$ & Unclear & Low & Low & Low & Unclear & High & Low & High \\
\hline $\begin{array}{l}\text { Heeboll } \\
2016\end{array}$ & $\begin{array}{l}\text { Other supplements ( } n=15) \text { vs No active in- } \\
\text { tervention }(n=13)\end{array}$ & Low & Low & Low & Low & Low & Low & Low & Low \\
\hline Hong 2021 & $\begin{array}{l}\text { Other supplements ( } n=43) \text { vs No active in- } \\
\text { tervention }(n=44)\end{array}$ & Low & Unclear & Unclear & Unclear & Unclear & High & Low & High \\
\hline $\begin{array}{l}\text { Hormozne- } \\
\text { jad } 2020\end{array}$ & $\begin{array}{l}\text { Other supplements ( } n=20) \text { vs No active in- } \\
\text { tervention }(n=21)\end{array}$ & Low & Unclear & Low & Low & High & High & Low & High \\
\hline $\begin{array}{l}\text { Hosseinaba- } \\
\text { di } 2020\end{array}$ & $\begin{array}{l}\text { Other supplements ( } n=21) \text { vs No active in- } \\
\text { tervention }(n=23)\end{array}$ & Low & Low & Low & Low & Unclear & High & Low & High \\
\hline $\begin{array}{l}\text { Hussain } \\
2017\end{array}$ & $\begin{array}{l}\text { Other supplements ( } n=40) \text { vs No active in- } \\
\text { tervention }(n=40)\end{array}$ & Low & Unclear & Unclear & Unclear & Low & High & Low & High \\
\hline
\end{tabular}




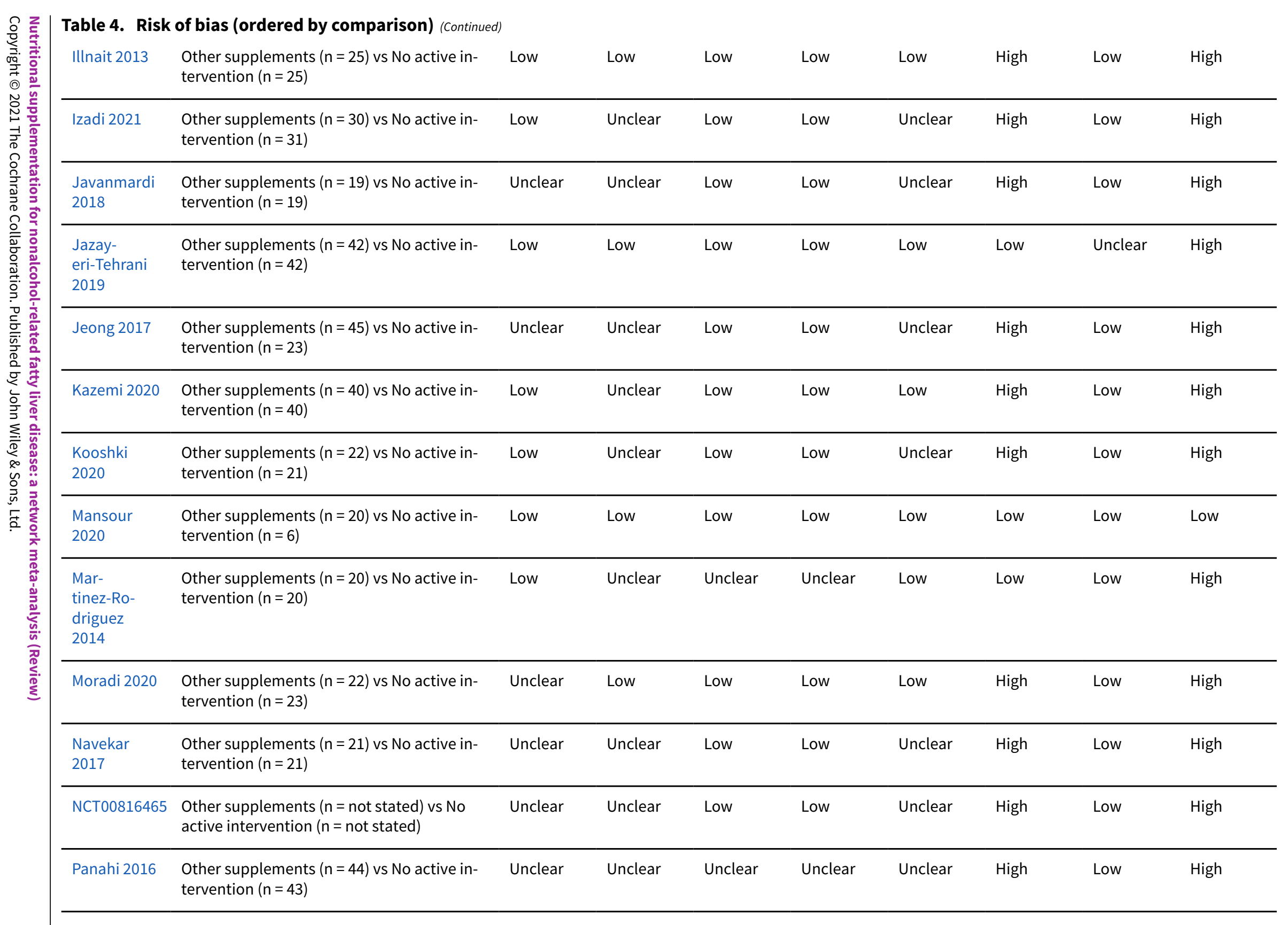




\begin{tabular}{|c|c|c|c|c|c|c|c|c|c|}
\hline Parsi 2020 & $\begin{array}{l}\text { Other supplements ( } n=30) \text { vs No active in- } \\
\text { tervention }(n=30)\end{array}$ & Unclear & Unclear & Low & Low & Low & High & Low & High \\
\hline Pasdar 2020 & $\begin{array}{l}\text { Other supplements ( } n=39) \text { vs No active in- } \\
\text { tervention }(n=39)\end{array}$ & Low & Unclear & Low & Low & High & High & Low & High \\
\hline $\begin{array}{l}\text { Pezeshki } \\
2016\end{array}$ & $\begin{array}{l}\text { Other supplements ( } n=35) \text { vs No active in- } \\
\text { tervention }(n=36)\end{array}$ & Low & Low & Low & Low & Unclear & High & Low & High \\
\hline $\begin{array}{l}\text { Rahimlou } \\
2016\end{array}$ & $\begin{array}{l}\text { Other supplements ( } n=23 \text { ) vs No active in- } \\
\text { tervention }(n=21)\end{array}$ & Low & Low & Low & Low & Unclear & High & Low & High \\
\hline $\begin{array}{l}\text { Rahmani } \\
2016\end{array}$ & $\begin{array}{l}\text { Other supplements ( } n=37) \text { vs No active in- } \\
\text { tervention }(n=40)\end{array}$ & Unclear & Unclear & Low & Low & Unclear & High & Low & High \\
\hline $\begin{array}{l}\text { Sangouni } \\
2020\end{array}$ & $\begin{array}{l}\text { Other supplements ( } n=45) \text { vs No active in- } \\
\text { tervention }(n=43)\end{array}$ & Low & Low & Low & Low & Unclear & High & Low & High \\
\hline Saxena 2013 & $\begin{array}{l}\text { Other supplements ( } n=26) \text { vs No active in- } \\
\text { tervention }(n=24)\end{array}$ & Unclear & Unclear & Low & Low & Unclear & High & Low & High \\
\hline $\begin{array}{l}\text { Shahmo- } \\
\text { hammadi } \\
2017\end{array}$ & $\begin{array}{l}\text { Other supplements ( } n=22) \text { vs No active in- } \\
\text { tervention }(n=22)\end{array}$ & Low & Low & Low & Low & Low & High & Low & High \\
\hline $\begin{array}{l}\text { Soleimani } \\
2020\end{array}$ & $\begin{array}{l}\text { Other supplements ( } n=47) \text { vs No active in- } \\
\text { tervention }(n=51)\end{array}$ & Unclear & Low & Low & Low & Unclear & High & Low & High \\
\hline $\begin{array}{l}\text { Soleimani } \\
2021\end{array}$ & $\begin{array}{l}\text { Other supplements ( } n=27) \text { vs No active in- } \\
\text { tervention }(n=27)\end{array}$ & Low & Low & Low & Low & Low & Low & Low & Low \\
\hline Song 2020 & $\begin{array}{l}\text { Other supplements ( } n=16) \text { vs No active in- } \\
\text { tervention }(n=21)\end{array}$ & Low & Low & Low & Low & Unclear & High & Low & High \\
\hline $\begin{array}{l}\text { Tabatabaee } \\
2017\end{array}$ & $\begin{array}{l}\text { Other supplements ( } n=21) \text { vs No active in- } \\
\text { tervention }(n=24)\end{array}$ & Low & Low & Low & Low & Unclear & High & Low & High \\
\hline $\begin{array}{l}\text { Tutunchi } \\
2020\end{array}$ & $\begin{array}{l}\text { Other supplements ( } n=38) \text { vs No active in- } \\
\text { tervention }(n=38)\end{array}$ & Low & Low & Low & Low & Low & Low & Low & Low \\
\hline Wang 2017 & $\begin{array}{l}\text { Other supplements ( } n=24) \text { vs No active in- } \\
\text { tervention }(n=12)\end{array}$ & Unclear & Unclear & Unclear & Unclear & Unclear & High & Low & High \\
\hline
\end{tabular}




\begin{tabular}{|c|c|c|c|c|c|c|c|c|c|}
\hline Wong 2013b & $\begin{array}{l}\text { Other supplements ( } n=40) \text { vs No active in- } \\
\text { tervention }(n=20)\end{array}$ & Low & Low & Low & Low & Low & Low & Low & Low \\
\hline Yan 2015 & $\begin{array}{l}\text { Other supplements ( } n=55) \text { vs No active in- } \\
\text { tervention }(n=53)\end{array}$ & Low & Unclear & High & High & Unclear & High & Low & High \\
\hline Yari 2020 & $\begin{array}{l}\text { Other supplements }(n=24) \text { vs No active in- } \\
\text { tervention }(n=21)\end{array}$ & Low & Low & High & High & Unclear & Low & Unclear & High \\
\hline $\begin{array}{l}\text { Zamani } \\
2018\end{array}$ & $\begin{array}{l}\text { Other supplements ( } n=45) \text { vs No active in- } \\
\text { tervention }(n=40)\end{array}$ & Low & Low & Low & Low & Unclear & Low & Unclear & High \\
\hline Zhang 2015 & $\begin{array}{l}\text { Other supplements ( } n=37) \text { vs No active in- } \\
\text { tervention }(n=37)\end{array}$ & Low & Low & Low & Low & Low & Low & Low & Low \\
\hline Abhari 2020 & $\begin{array}{l}\text { Prebiotics/Probiotics/Synbiotics }(n=23) \text { vs } \\
\text { No active intervention }(n=22)\end{array}$ & Low & Low & Low & Low & High & Low & Low & High \\
\hline Ahn 2018 & $\begin{array}{l}\text { Prebiotics/Probiotics/Synbiotics ( } n=\text { not } \\
\text { stated) vs No active intervention ( } n=\text { not } \\
\text { stated) }\end{array}$ & Unclear & Unclear & Low & Low & Unclear & High & Low & High \\
\hline $\begin{array}{l}\text { Akbarzadeh } \\
2015\end{array}$ & $\begin{array}{l}\text { Prebiotics/Probiotics/Synbiotics }(n=38) \text { vs } \\
\text { No active intervention }(n=37)\end{array}$ & Unclear & Unclear & Low & Low & Unclear & High & Low & High \\
\hline Alisi 2014 & $\begin{array}{l}\text { Prebiotics/Probiotics/Synbiotics }(n=22) \text { vs } \\
\text { No active intervention }(n=22)\end{array}$ & Low & Low & Low & Low & Unclear & High & Low & High \\
\hline Aller 2011 & $\begin{array}{l}\text { Prebiotics/Probiotics/Synbiotics }(n=14) \text { vs } \\
\text { No active intervention }(n=14)\end{array}$ & Low & Unclear & Low & Low & Unclear & High & Low & High \\
\hline Guo 2016 & $\begin{array}{l}\text { Prebiotics/Probiotics/Synbiotics }(n=42) \text { vs } \\
\text { No active intervention }(n=42)\end{array}$ & Unclear & Unclear & Unclear & Unclear & Unclear & High & Low & High \\
\hline $\begin{array}{l}\text { Asgharian } \\
2016\end{array}$ & $\begin{array}{l}\text { Prebiotics/Probiotics/Synbiotics }(n=38) \text { vs } \\
\text { No active intervention }(n=36)\end{array}$ & Low & Low & Low & Low & Unclear & Low & Low & High \\
\hline $\begin{array}{l}\text { Bakhshi- } \\
\text { moghad- } \\
\text { dam } 2018\end{array}$ & $\begin{array}{l}\text { Prebiotics/Probiotics/Synbiotics }(n=32) \text { vs } \\
\text { No active intervention }(n=28)\end{array}$ & Low & Low & High & Unclear & Unclear & Low & Low & High \\
\hline $\begin{array}{l}\text { Behrouz } \\
2017\end{array}$ & $\begin{array}{l}\text { Prebiotics/Probiotics/Synbiotics }(n=59) \text { vs } \\
\text { No active intervention }(n=30)\end{array}$ & Unclear & Unclear & Low & Low & Unclear & High & Low & High \\
\hline
\end{tabular}




\begin{tabular}{|c|c|c|c|c|c|c|c|c|c|}
\hline $\begin{array}{l}\text { Bomhof } \\
2018\end{array}$ & $\begin{array}{l}\text { Prebiotics/Probiotics/Synbiotics }(n=8) \text { vs } \\
\text { No active intervention }(n=5)\end{array}$ & Unclear & Low & Unclear & Unclear & Low & High & Low & High \\
\hline Cai 2020 & $\begin{array}{l}\text { Prebiotics/Probiotics/Synbiotics }(n=70) \text { vs } \\
\text { No active intervention }(n=70)\end{array}$ & Low & Unclear & Unclear & Unclear & Unclear & High & Low & High \\
\hline $\begin{array}{l}\text { Chongsri- } \\
\text { sawat } 2017\end{array}$ & $\begin{array}{l}\text { Prebiotics/Probiotics/Synbiotics }(n=18) \text { vs } \\
\text { No active intervention }(n=19)\end{array}$ & Unclear & Unclear & Low & Low & Unclear & High & Low & High \\
\hline $\begin{array}{l}\text { Della Corte } \\
2012\end{array}$ & $\begin{array}{l}\text { Prebiotics/Probiotics/Synbiotics ( } n=\text { not } \\
\text { stated) vs No active intervention ( } n=\text { not } \\
\text { stated) }\end{array}$ & Unclear & Unclear & Low & Low & Unclear & High & Low & High \\
\hline Duseja 2019 & $\begin{array}{l}\text { Prebiotics/Probiotics/Synbiotics }(n=10) \text { vs } \\
\text { No active intervention }(n=5)\end{array}$ & Low & Low & Low & Low & High & Low & Low & High \\
\hline Ekhlasi 2016 & $\begin{array}{l}\text { Prebiotics/Probiotics/Synbiotics }(n=15) \text { vs } \\
\text { No active intervention }(n=15)\end{array}$ & Unclear & Unclear & Low & Low & Unclear & High & Low & High \\
\hline $\begin{array}{l}\text { Eslamparast } \\
2014\end{array}$ & $\begin{array}{l}\text { Prebiotics/Probiotics/Synbiotics }(n=26) \text { vs } \\
\text { No active intervention }(n=26)\end{array}$ & Low & Low & Low & Low & Unclear & High & Low & High \\
\hline $\begin{array}{l}\text { Famouri } \\
2017 a\end{array}$ & $\begin{array}{l}\text { Prebiotics/Probiotics/Synbiotics }(n=32) \text { vs } \\
\text { No active intervention }(n=32)\end{array}$ & Low & Low & Low & Low & Low & High & Low & High \\
\hline Ferolla 2016 & $\begin{array}{l}\text { Prebiotics/Probiotics/Synbiotics }(n=27) \text { vs } \\
\text { No active intervention }(n=23)\end{array}$ & Unclear & Unclear & Unclear & Unclear & Low & Low & Low & High \\
\hline $\begin{array}{l}\text { Gavrilescu } \\
2017\end{array}$ & $\begin{array}{l}\text { Prebiotics/Probiotics/Synbiotics ( } n=\text { not } \\
\text { stated) vs No active intervention ( } n=\text { not } \\
\text { stated) }\end{array}$ & Unclear & Unclear & Unclear & Unclear & Unclear & High & Low & High \\
\hline $\begin{array}{l}\text { Jameshorani } \\
2017\end{array}$ & $\begin{array}{l}\text { Prebiotics/Probiotics/Synbiotics }(n=45) \text { vs } \\
\text { No active intervention }(n=45)\end{array}$ & Unclear & Unclear & Unclear & Unclear & Unclear & High & Low & High \\
\hline Javadi 2017 & $\begin{array}{l}\text { Prebiotics/Probiotics/Synbiotics }(n=56) \text { vs } \\
\text { No active intervention }(n=19)\end{array}$ & Unclear & Low & Low & Low & Unclear & High & Low & High \\
\hline $\begin{array}{l}\text { Khutsishvili } \\
2020\end{array}$ & $\begin{array}{l}\text { Prebiotics/Probiotics/Synbiotics }(n=35) \text { vs } \\
\text { No active intervention }(n=38)\end{array}$ & Unclear & Unclear & Unclear & Unclear & Unclear & High & Low & High \\
\hline $\begin{array}{l}\text { Kobyliak } \\
2018\end{array}$ & $\begin{array}{l}\text { Prebiotics/Probiotics/Synbiotics }(n=30) \text { vs } \\
\text { No active intervention }(n=28)\end{array}$ & Low & Low & Low & Low & Low & Low & Low & Low \\
\hline
\end{tabular}




\begin{tabular}{|c|c|c|c|c|c|c|c|c|c|}
\hline $\begin{array}{l}\text { Malaguarn- } \\
\text { era } 2012\end{array}$ & $\begin{array}{l}\text { Prebiotics/Probiotics/Synbiotics }(n=34) \text { vs } \\
\text { No active intervention }(n=32)\end{array}$ & Low & Low & Unclear & Low & Low & Low & Low & High \\
\hline $\begin{array}{l}\text { Manzhalii } \\
2017\end{array}$ & $\begin{array}{l}\text { Prebiotics/Probiotics/Synbiotics }(n=38) \text { vs } \\
\text { No active intervention }(n=37)\end{array}$ & Low & Low & High & High & Low & Low & Low & High \\
\hline Mofidi 2017 & $\begin{array}{l}\text { Prebiotics/Probiotics/Synbiotics }(n=21) \text { vs } \\
\text { No active intervention }(n=21)\end{array}$ & Low & Low & Low & Low & Unclear & High & Low & High \\
\hline Nabavi 2016 & $\begin{array}{l}\text { Prebiotics/Probiotics/Synbiotics }(n=36) \text { vs } \\
\text { No active intervention }(n=36)\end{array}$ & Low & Low & Low & Low & Low & Low & Low & Low \\
\hline Orr 2015 & $\begin{array}{l}\text { Prebiotics/Probiotics/Synbiotics ( } n=\text { not } \\
\text { stated) vs No active intervention ( } n=\text { not } \\
\text { stated) }\end{array}$ & Unclear & Unclear & Low & Low & Unclear & High & Low & High \\
\hline Poparn 2020 & $\begin{array}{l}\text { Prebiotics/Probiotics/Synbiotics }(n=18) \text { vs } \\
\text { No active intervention }(n=19)\end{array}$ & Low & Low & Low & Low & Low & Low & Low & Low \\
\hline $\begin{array}{l}\text { Sadrkabir } \\
2020\end{array}$ & $\begin{array}{l}\text { Prebiotics/Probiotics/Synbiotics }(n=33) \text { vs } \\
\text { No active intervention }(n=28)\end{array}$ & Unclear & Unclear & Unclear & Unclear & Unclear & High & Low & High \\
\hline Sayari 2018 & $\begin{array}{l}\text { Prebiotics/Probiotics/Synbiotics }(n=70) \text { vs } \\
\text { No active intervention }(n=68)\end{array}$ & Unclear & Unclear & Low & Low & Unclear & High & Low & High \\
\hline $\begin{array}{l}\text { Schatten- } \\
\text { berg } 2017\end{array}$ & $\begin{array}{l}\text { Prebiotics/Probiotics/Synbiotics }(n=15) \text { vs } \\
\text { No active intervention }(n=14)\end{array}$ & Unclear & Unclear & High & High & Low & Low & Low & High \\
\hline $\begin{array}{l}\text { Scorletti } \\
2020\end{array}$ & $\begin{array}{l}\text { Prebiotics/Probiotics/Synbiotics }(n=45) \text { vs } \\
\text { No active intervention }(n=44)\end{array}$ & Low & Low & Low & Low & High & High & Low & High \\
\hline $\begin{array}{l}\text { Sepideh } \\
2016\end{array}$ & $\begin{array}{l}\text { Prebiotics/Probiotics/Synbiotics }(n=21) \text { vs } \\
\text { No active intervention }(n=21)\end{array}$ & Unclear & Low & Low & Low & Unclear & High & Low & High \\
\hline $\begin{array}{l}\text { Shavakhi } \\
2013\end{array}$ & $\begin{array}{l}\text { Prebiotics/Probiotics/Synbiotics }(n=31) \text { vs } \\
\text { No active intervention }(n=32)\end{array}$ & Unclear & Unclear & Low & Low & Unclear & High & Low & High \\
\hline Vajro 2011 & $\begin{array}{l}\text { Prebiotics/Probiotics/Synbiotics }(n=10) \text { vs } \\
\text { No active intervention }(n=10)\end{array}$ & Unclear & Unclear & Low & Low & Low & Low & Low & High \\
\hline Wong 2013a & $\begin{array}{l}\text { Prebiotics/Probiotics/Synbiotics }(n=10) \text { vs } \\
\text { No active intervention }(n=10)\end{array}$ & Low & Unclear & Unclear & Unclear & Low & Low & Low & High \\
\hline
\end{tabular}




\begin{tabular}{|c|c|c|c|c|c|c|c|c|c|}
\hline Argo 2015 & $\begin{array}{l}\text { PUFA }(n=17) \text { vs No active intervention }(n= \\
17)\end{array}$ & Low & Low & Low & Low & Unclear & High & Low & High \\
\hline Boyraz 2015 & $\begin{array}{l}\text { PUFA }(n=56) \text { vs No active intervention }(n= \\
52)\end{array}$ & Unclear & Unclear & Unclear & Low & Unclear & High & Low & High \\
\hline Byrne 2014 & $\begin{array}{l}\text { PUFA }(n=51) \text { vs No active intervention }(n= \\
52)\end{array}$ & Unclear & Unclear & Low & Low & Unclear & High & Low & High \\
\hline Chen 2008 & $\begin{array}{l}\text { PUFA }(n=30) \text { vs No active intervention }(n= \\
16)\end{array}$ & Unclear & Unclear & Low & Low & Unclear & High & Low & High \\
\hline Climax 2020 & $\begin{array}{l}\text { PUFA }(n=63) \text { vs No active intervention }(n= \\
30)\end{array}$ & Unclear & Unclear & Low & Low & Unclear & Low & Low & High \\
\hline $\begin{array}{l}\text { Dasarathy } \\
2015\end{array}$ & $\begin{array}{l}\text { PUFA }(n=18) \text { vs No active intervention }(n= \\
\text { 19) }\end{array}$ & Low & Unclear & Low & Low & Low & High & Low & High \\
\hline $\begin{array}{l}\text { Eriksson } \\
2018\end{array}$ & $\begin{array}{l}\text { PUFA }(n=42) \text { vs No active intervention }(n= \\
42)\end{array}$ & Low & Low & Low & Low & Low & Low & Low & Low \\
\hline $\begin{array}{l}\text { EUCTR } \\
2008-008275-34 \\
\text { GB }\end{array}$ & $\begin{array}{l}\text { PUFA }(n=24) \text { vs No active intervention }(n= \\
\text { 425) }\end{array}$ & Low & Low & Low & Low & Low & Low & Low & Low \\
\hline $\begin{array}{l}\text { Janczyk } \\
2015\end{array}$ & $\begin{array}{l}\text { PUFA }(n=30) \text { vs No active intervention }(n= \\
34)\end{array}$ & Low & Low & Low & Low & Unclear & Low & Low & High \\
\hline Li 2016 & $\begin{array}{l}\text { PUFA }(n=39) \text { vs No active intervention ( } n= \\
39)\end{array}$ & Unclear & Unclear & High & High & Low & High & Low & High \\
\hline NCT00845845 & PUFA $(n=3)$ vs No active intervention $(n=6)$ & Unclear & Unclear & Low & Low & Low & High & Low & High \\
\hline NCT00941642 & $\begin{array}{l}\text { PUFA ( } n=\text { not stated) vs No active interven- } \\
\text { tion ( } n=\text { not stated) }\end{array}$ & Unclear & Unclear & High & High & Unclear & High & Low & High \\
\hline Nobili 2013 & $\begin{array}{l}\text { PUFA }(n=40) \text { vs No active intervention }(n= \\
\text { 20) }\end{array}$ & Low & Low & Low & Low & Low & High & Low & High \\
\hline $\begin{array}{l}\text { Nogueira } \\
2016\end{array}$ & $\begin{array}{l}\text { PUFA }(n=27) \text { vs No active intervention }(n= \\
23)\end{array}$ & Low & Low & Low & Low & Unclear & High & Low & High \\
\hline
\end{tabular}




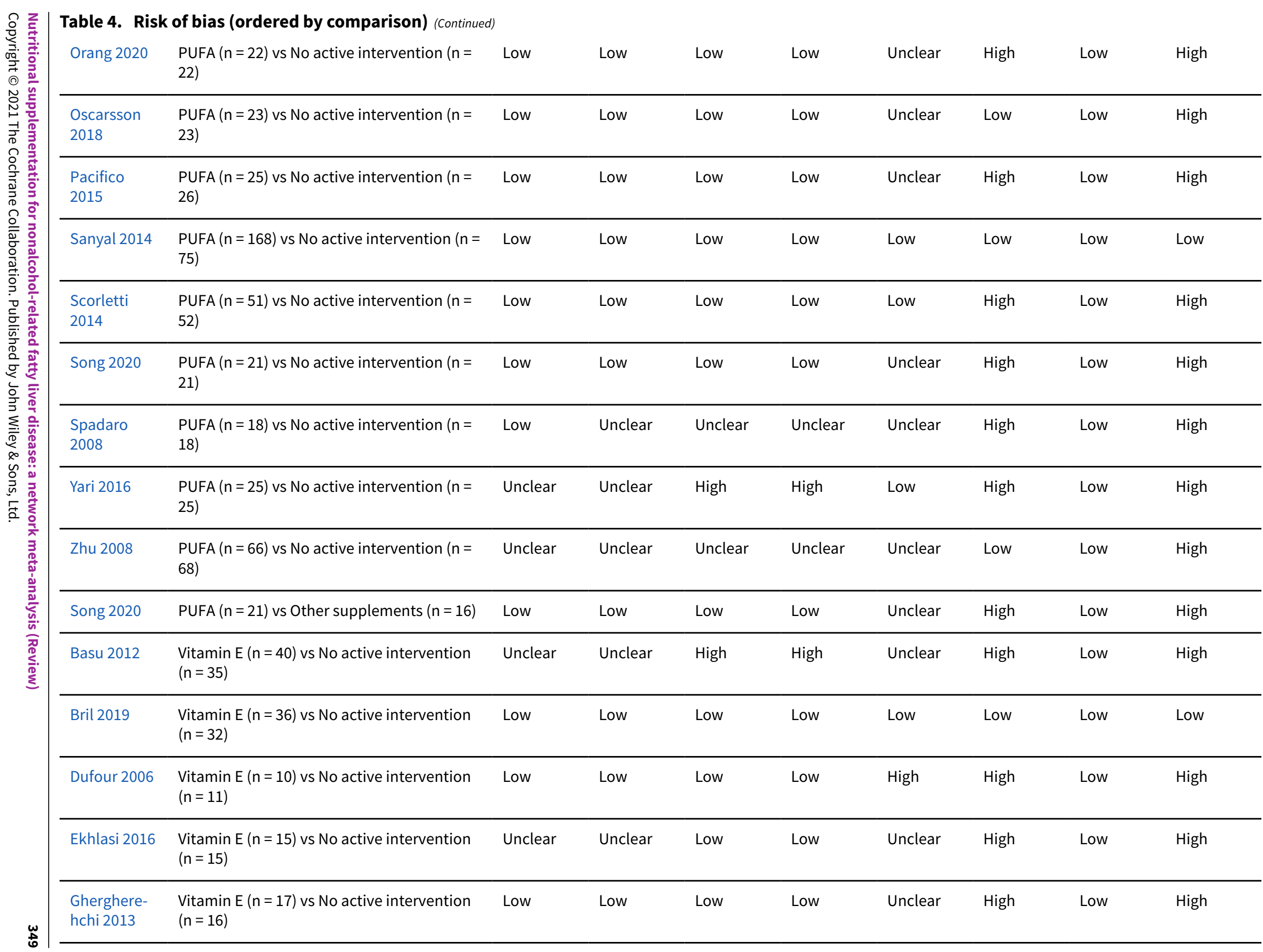




\begin{tabular}{|c|c|c|c|c|c|c|c|c|c|}
\hline $\begin{array}{l}\text { Kugelmas } \\
2003\end{array}$ & $\begin{array}{l}\text { Vitamin } E(n=9) \text { vs No active intervention ( } \\
=7)\end{array}$ & Unclear & Unclear & High & Unclear & Unclear & High & Low & High \\
\hline Lavine 2011 & $\begin{array}{l}\text { Vitamin } E(n=50) \text { vs No active intervention } \\
(n=47)\end{array}$ & Unclear & Unclear & Low & Low & Unclear & High & Low & High \\
\hline $\begin{array}{l}\text { Magosso } \\
2013\end{array}$ & $\begin{array}{l}\text { Vitamin } E(n=43) \text { vs No active intervention } \\
(n=44)\end{array}$ & Low & Low & Unclear & Low & Low & Low & Low & High \\
\hline NCT02690792 & $\begin{array}{l}\text { Vitamin } E(n=\text { not stated }) \text { vs No active inter- } \\
\text { vention }(n=\text { not stated })\end{array}$ & Unclear & Unclear & Low & Low & Unclear & High & Low & High \\
\hline $\begin{array}{l}\text { Palamaru } \\
2017\end{array}$ & $\begin{array}{l}\text { Vitamin } E(n=20) \text { vs No active intervention } \\
(n=20)\end{array}$ & Unclear & Unclear & Unclear & Unclear & Unclear & High & Low & High \\
\hline Pervez 2018 & $\begin{array}{l}\text { Vitamin } E(n=31) \text { vs No active intervention } \\
(n=33)\end{array}$ & Unclear & Low & Low & Low & Unclear & Low & Low & High \\
\hline Pervez 2020 & $\begin{array}{l}\text { Vitamin } E(n=35) \text { vs No active intervention } \\
(n=36)\end{array}$ & Unclear & Low & Low & Low & Low & High & Low & High \\
\hline Sanyal 2010 & $\begin{array}{l}\text { Vitamin } E(n=84) \text { vs No active intervention } \\
(n=83)\end{array}$ & Low & Low & Low & Low & Low & Low & Low & Low \\
\hline Wang 2008 & $\begin{array}{l}\text { Vitamin } E(n=19) \text { vs No active intervention } \\
(n=38)\end{array}$ & Unclear & Unclear & Unclear & Unclear & Low & High & Low & High \\
\hline Basu 2013 & $\begin{array}{l}\text { Vitamin } E(n=20) \text { vs Other supplements }(n= \\
\text { 20) }\end{array}$ & Unclear & Unclear & High & High & Unclear & High & Low & High \\
\hline Ekhlasi 2016 & $\begin{array}{l}\text { Vitamin E }(n=15) \text { vs Prebiotics/Probi- } \\
\text { otics/Synbiotics }(n=15)\end{array}$ & Unclear & Unclear & Low & Low & Unclear & High & Low & High \\
\hline Amiri 2017 & $\begin{array}{l}\text { Vitamin } D(n=74) \text { vs No active intervention } \\
(n=36)\end{array}$ & Low & Low & Low & Low & Unclear & High & Low & High \\
\hline $\begin{array}{l}\text { Barchetta } \\
2016\end{array}$ & $\begin{array}{l}\text { Vitamin } D(n=26) \text { vs No active intervention } \\
(n=29)\end{array}$ & Low & Low & Low & Low & Unclear & High & Low & High \\
\hline $\begin{array}{l}\text { Boonyagard } \\
2016\end{array}$ & $\begin{array}{l}\text { Vitamin } D(n=30) \text { vs No active intervention } \\
(n=30)\end{array}$ & Unclear & Unclear & Unclear & Unclear & Unclear & High & Low & High \\
\hline
\end{tabular}




\begin{tabular}{|c|c|c|c|c|c|c|c|c|c|}
\hline $\begin{array}{l}\text { Boonyagard } \\
2020\end{array}$ & $\begin{array}{l}\text { Vitamin } D(n=30) \text { vs No active intervention } \\
(n=30)\end{array}$ & Low & Low & Low & Low & Unclear & Low & Low & High \\
\hline $\begin{array}{l}\text { Dabbagh- } \\
\text { manesh } \\
2018\end{array}$ & $\begin{array}{l}\text { Vitamin } D(n=59) \text { vs No active intervention } \\
(n=32)\end{array}$ & Low & Low & Low & Low & High & High & Low & High \\
\hline $\begin{array}{l}\text { Foroughi } \\
2014\end{array}$ & $\begin{array}{l}\text { Vitamin } D(n=30) \text { vs No active intervention } \\
(n=30)\end{array}$ & Unclear & Unclear & Low & Low & Low & High & Low & High \\
\hline Geier 2018 & $\begin{array}{l}\text { Vitamin } D(n=8) \text { vs No active intervention ( } n \\
=10)\end{array}$ & Low & Low & Low & Low & Unclear & High & Low & High \\
\hline $\begin{array}{l}\text { Hoseini } \\
2020\end{array}$ & $\begin{array}{l}\text { Vitamin } D(n=20) \text { vs No active intervention } \\
(n=20)\end{array}$ & Unclear & Unclear & Unclear & Unclear & Low & High & Low & High \\
\hline $\begin{array}{l}\text { Hosseini } \\
2018\end{array}$ & $\begin{array}{l}\text { Vitamin } D(n=37) \text { vs No active intervention } \\
(n=38)\end{array}$ & Low & Unclear & Unclear & Unclear & Unclear & High & Low & High \\
\hline NCT01083992 & $\begin{array}{l}\text { Vitamin } D(n=\text { not stated }) \text { vs No active inter- } \\
\text { vention }(n=\text { not stated })\end{array}$ & Unclear & Unclear & High & High & Unclear & High & Low & High \\
\hline NCT01623024 & $\begin{array}{l}\text { Vitamin } D(n=\text { not stated }) \text { vs No active inter- } \\
\text { vention }(n=\text { not stated })\end{array}$ & Unclear & Unclear & High & High & Unclear & High & Low & High \\
\hline Sakpal 2017 & $\begin{array}{l}\text { Vitamin } D(n=51) \text { vs No active intervention } \\
(n=30)\end{array}$ & Unclear & Unclear & Unclear & Unclear & Low & High & Low & High \\
\hline Sharifi 2014 & $\begin{array}{l}\text { Vitamin } D(n=27) \text { vs No active intervention } \\
(n=26)\end{array}$ & Low & Low & Low & Low & Unclear & High & Low & High \\
\hline $\begin{array}{l}\text { Taghvaei } \\
2018\end{array}$ & $\begin{array}{l}\text { Vitamin } D(n=20) \text { vs No active intervention } \\
(n=20)\end{array}$ & Low & Unclear & Unclear & Unclear & Low & High & Low & High \\
\hline Zanko 2020 & $\begin{array}{l}\text { Vitamin } D(n=201) \text { vs No active intervention } \\
(n=110)\end{array}$ & Low & Low & Low & Low & Low & High & Low & High \\
\hline Basu 2012 & $\begin{array}{l}\text { Other antioxidants ( } n=40) \text { vs No active in- } \\
\text { tervention }(n=35)\end{array}$ & Unclear & Unclear & High & High & Unclear & High & Low & High \\
\hline Chan 2017 & $\begin{array}{l}\text { Other antioxidants ( } n=49) \text { vs No active in- } \\
\text { tervention }(n=50)\end{array}$ & Low & Low & Low & Low & Low & Low & Low & Low \\
\hline
\end{tabular}




\begin{tabular}{|c|c|c|c|c|c|c|c|c|c|}
\hline Chen 2015b & $\begin{array}{l}\text { Other antioxidants }(n=30) \text { vs No active in- } \\
\text { tervention }(n=30)\end{array}$ & Low & Low & Low & Low & Low & Low & Low & Low \\
\hline $\begin{array}{l}\text { Cheragh- } \\
\text { pour } 2019\end{array}$ & $\begin{array}{l}\text { Other antioxidants }(n=25) \text { vs No active in- } \\
\text { tervention }(n=24)\end{array}$ & Low & Low & Low & Low & Unclear & Low & Unclear & High \\
\hline $\begin{array}{l}\text { Farhangi } \\
2014\end{array}$ & $\begin{array}{l}\text { Other antioxidants }(n=20) \text { vs No active in- } \\
\text { tervention }(n=21)\end{array}$ & Unclear & Unclear & Low & Low & High & High & Low & High \\
\hline Farsi 2016 & $\begin{array}{l}\text { Other antioxidants }(n=20) \text { vs No active in- } \\
\text { tervention }(n=21)\end{array}$ & Unclear & Unclear & Low & Low & Unclear & High & Low & High \\
\hline $\begin{array}{l}\text { Gianturco } \\
2013\end{array}$ & $\begin{array}{l}\text { Other antioxidants ( } n=104) \text { vs No active in- } \\
\text { tervention }(n=92)\end{array}$ & Low & Low & Low & Low & Unclear & High & Low & High \\
\hline $\begin{array}{l}\text { Gonciarz } \\
2012\end{array}$ & $\begin{array}{l}\text { Other antioxidants }(n=30) \text { vs No active in- } \\
\text { tervention }(n=12)\end{array}$ & Unclear & Unclear & Unclear & Unclear & Unclear & High & Low & High \\
\hline $\begin{array}{l}\text { Hashemi } \\
2009\end{array}$ & $\begin{array}{l}\text { Other antioxidants ( } n=50) \text { vs No active in- } \\
\text { tervention }(n=50)\end{array}$ & Unclear & Unclear & Unclear & Unclear & Unclear & High & Low & High \\
\hline Kanoni 2021 & $\begin{array}{l}\text { Other antioxidants }(n=35) \text { vs No active in- } \\
\text { tervention }(n=52)\end{array}$ & Unclear & Unclear & Low & Low & Unclear & High & Low & High \\
\hline Ruan 2010 & $\begin{array}{l}\text { Other antioxidants }(n=30) \text { vs No active in- } \\
\text { tervention }(n=30)\end{array}$ & Unclear & Unclear & High & High & Unclear & High & Low & High \\
\hline Solhi 2014 & $\begin{array}{l}\text { Other antioxidants ( } n=33) \text { vs No active in- } \\
\text { tervention }(n=31)\end{array}$ & Unclear & Unclear & Unclear & Unclear & Unclear & High & Low & High \\
\hline Yari 2020 & $\begin{array}{l}\text { Other antioxidants ( } n=22) \text { vs No active in- } \\
\text { tervention }(n=21)\end{array}$ & Low & Low & High & High & Unclear & Low & Unclear & High \\
\hline Deng 2005 & $\begin{array}{l}\text { Other antioxidants }(n=48) \text { vs Other supple- } \\
\text { ments }(n=48)\end{array}$ & Low & Unclear & Unclear & Unclear & Low & High & Low & High \\
\hline Basu 2012 & $\begin{array}{l}\text { Other antioxidants }(n=40) \text { vs Vitamin } E(n= \\
\text { 40) }\end{array}$ & Unclear & Unclear & High & High & Unclear & High & Low & High \\
\hline $\begin{array}{l}\text { Khoshbaten } \\
\text { 2010b }\end{array}$ & $\begin{array}{l}\text { Other antioxidants }(n=15) \text { vs Vitamin } C(n= \\
15)\end{array}$ & Low & Low & Unclear & Unclear & Unclear & High & Low & High \\
\hline
\end{tabular}




\begin{tabular}{|c|c|c|c|c|c|c|c|c|c|}
\hline Yari 2020 & $\begin{array}{l}\text { Other antioxidants ( } n=22) \text { vs Other supple- } \\
\text { ments }(n=24)\end{array}$ & Low & Low & High & High & Unclear & Low & Unclear & High \\
\hline $\begin{array}{l}\text { Abdelmalek } \\
2009\end{array}$ & $\begin{array}{l}\text { Amino acids ( } n=17) \text { vs No active interven- } \\
\text { tion }(n=18)\end{array}$ & Unclear & Unclear & Low & Low & High & High & Low & High \\
\hline $\begin{array}{l}\text { Amiri- } \\
\text { Moghadam } \\
2015\end{array}$ & $\begin{array}{l}\text { Amino acids }(n=36) \text { vs No active interven- } \\
\text { tion }(n=32)\end{array}$ & Unclear & Low & Low & Low & Unclear & High & Low & High \\
\hline Bae 2015 & $\begin{array}{l}\text { Amino acids }(n=39) \text { vs No active interven- } \\
\text { tion }(n=39)\end{array}$ & Low & Low & Low & Low & Low & Low & Low & Low \\
\hline $\begin{array}{l}\text { Eghtesadi } \\
2016\end{array}$ & $\begin{array}{l}\text { Amino acids }(n=36) \text { vs No active interven- } \\
\text { tion }(n=32)\end{array}$ & Unclear & Unclear & Low & Low & Unclear & High & Low & High \\
\hline $\begin{array}{l}\text { Fabbrini } \\
2010\end{array}$ & $\begin{array}{l}\text { Amino acids }(n=9) \text { vs No active intervention } \\
(n=9)\end{array}$ & Unclear & Unclear & Low & Low & Unclear & High & Low & High \\
\hline $\begin{array}{l}\text { Malaguarn- } \\
\text { era } 2010\end{array}$ & $\begin{array}{l}\text { Amino acids }(n=36) \text { vs No active interven- } \\
\text { tion }(n=38)\end{array}$ & Low & Low & Low & Low & Unclear & High & Low & High \\
\hline $\begin{array}{l}\text { Naganuma } \\
2016\end{array}$ & $\begin{array}{l}\text { Amino acids }(n=10) \text { vs No active interven- } \\
\text { tion }(n=10)\end{array}$ & Unclear & Unclear & Unclear & Unclear & Unclear & High & Low & High \\
\hline Somi 2014 & $\begin{array}{l}\text { Amino acids }(n=40) \text { vs No active interven- } \\
\text { tion }(n=40)\end{array}$ & Unclear & Unclear & Unclear & Low & Low & High & Low & High \\
\hline Uygun 2000 & $\begin{array}{l}\text { Amino acids }(n=78) \text { vs No active interven- } \\
\text { tion }(n=23)\end{array}$ & Unclear & Unclear & Unclear & Unclear & Unclear & High & Low & High \\
\hline Aller 2015 & $\begin{array}{l}\text { Vitamin E plus other antioxidants }(n=18) \text { vs } \\
\text { No active intervention }(n=18)\end{array}$ & Low & Unclear & Unclear & Unclear & Low & Low & Low & High \\
\hline Basu 2012 & $\begin{array}{l}\text { Vitamin E plus other antioxidants }(n=40) \text { vs } \\
\text { No active intervention }(n=35)\end{array}$ & Unclear & Unclear & High & High & Unclear & High & Low & High \\
\hline $\begin{array}{l}\text { Bonfrate } \\
2015\end{array}$ & $\begin{array}{l}\text { Vitamin E plus other antioxidants ( } n=\text { not } \\
\text { stated) vs No active intervention ( } n=\text { not } \\
\text { stated) }\end{array}$ & Unclear & Unclear & Low & Low & Unclear & High & Low & High \\
\hline $\begin{array}{l}\text { Amirkhizi } \\
2018\end{array}$ & $\begin{array}{l}\text { Vitamin E plus other antioxidants }(n=23) \text { vs } \\
\text { Vitamin } E(n=22)\end{array}$ & Low & Low & Low & Low & Unclear & High & Low & High \\
\hline
\end{tabular}




\begin{tabular}{|c|c|c|c|c|c|c|c|c|c|}
\hline Basu 2012 & $\begin{array}{l}\text { Vitamin E plus other antioxidants }(n=40) \text { vs } \\
\text { Vitamin } E(n=40)\end{array}$ & Unclear & Unclear & High & High & Unclear & High & Low & High \\
\hline Basu 2012 & $\begin{array}{l}\text { Vitamin E plus other antioxidants }(n=40) \text { vs } \\
\text { Other antioxidants }(n=40)\end{array}$ & Unclear & Unclear & High & High & Unclear & High & Low & High \\
\hline NCT04411862 & $\begin{array}{l}\text { Phospholipids ( } n=50) \text { vs No active inter- } \\
\text { vention }(n=50)\end{array}$ & Unclear & Unclear & High & High & Unclear & High & Low & High \\
\hline Tan 2011 & $\begin{array}{l}\text { Phospholipids }(n=10) \text { vs No active inter- } \\
\text { vention }(n=5)\end{array}$ & Unclear & Unclear & Unclear & Unclear & Unclear & High & Low & High \\
\hline Li 2010 & $\begin{array}{l}\text { Phospholipids }(n=43) \text { vs Other supple- } \\
\text { ments }(n=45)\end{array}$ & Low & Unclear & Unclear & Unclear & Low & Low & Low & High \\
\hline Wang 2018 & $\begin{array}{l}\text { Phospholipids }(n=50) \text { vs Prebiotics/Probi- } \\
\text { otics/Synbiotics }(n=150)\end{array}$ & Low & Unclear & Unclear & Unclear & Unclear & High & Low & High \\
\hline Basu 2013 & $\begin{array}{l}\text { Vitamin E plus other supplements }(n=20) \text { vs } \\
\text { Other supplements }(n=20)\end{array}$ & Unclear & Unclear & High & High & Unclear & High & Low & High \\
\hline $\begin{array}{l}\text { Aliashrafi } \\
2014\end{array}$ & $\begin{array}{l}\text { Vitamin E plus other supplements }(n=29) \text { vs } \\
\text { Vitamin } E(n=26)\end{array}$ & Low & Unclear & Low & Low & Unclear & High & Low & High \\
\hline Basu 2013 & $\begin{array}{l}\text { Vitamin E plus other supplements }(n=20) \text { vs } \\
\text { Vitamin } E(n=20)\end{array}$ & Unclear & Unclear & High & High & Unclear & High & Low & High \\
\hline Panahi 2012 & $\begin{array}{l}\text { Vitamin E plus other supplements }(n=21) \text { vs } \\
\text { Vitamin } E(n=33)\end{array}$ & Unclear & Unclear & High & High & Unclear & High & Low & High \\
\hline $\begin{array}{l}\text { Youshari } \\
2017\end{array}$ & $\begin{array}{l}\text { Vitamin E plus other supplements }(n=20) \text { vs } \\
\text { Vitamin } E(n=21)\end{array}$ & Unclear & Unclear & Low & Low & Unclear & High & Low & High \\
\hline $\begin{array}{l}\text { Barbakadze } \\
2020\end{array}$ & $\begin{array}{l}\text { Vitamin E plus vitamin C ( } n=52) \text { vs No ac- } \\
\text { tive intervention }(n=20)\end{array}$ & Unclear & Unclear & Unclear & Unclear & Unclear & High & Low & High \\
\hline $\begin{array}{l}\text { Harrison } \\
2003\end{array}$ & $\begin{array}{l}\text { Vitamin E plus vitamin C }(n=23) \text { vs No ac- } \\
\text { tive intervention }(n=22)\end{array}$ & Low & Low & Low & Low & Unclear & High & Low & High \\
\hline Nobili 2006 & $\begin{array}{l}\text { Vitamin E plus vitamin C }(n=45) \text { vs No ac- } \\
\text { tive intervention }(n=43)\end{array}$ & Unclear & Low & Low & Low & Unclear & Low & Low & High \\
\hline
\end{tabular}




\begin{tabular}{|c|c|c|c|c|c|c|c|c|c|}
\hline Pour 2020 & $\begin{array}{l}\text { Other supplements ( } n=38) \text { vs No active in- } \\
\text { tervention }(n=38)\end{array}$ & Low & Low & Low & Low & Low & High & Low & High \\
\hline Rafie 2020 & $\begin{array}{l}\text { Other supplements ( } n=23) \text { vs No active in- } \\
\text { tervention }(n=23)\end{array}$ & Unclear & Low & Low & Low & Unclear & High & Low & High \\
\hline Nelson 2009 & $\begin{array}{l}\text { MUFA ( } n=\text { not stated) vs PUFA ( } n=\text { not stat- } \\
\text { ed) }\end{array}$ & Unclear & Unclear & Unclear & Unclear & Unclear & High & Low & High \\
\hline Tobin 2018 & MUFA $(n=86)$ vs PUFA $(n=81)$ & Low & Low & Low & Low & Unclear & High & Unclear & High \\
\hline NCT00977730 & $\begin{array}{l}\text { Other antioxidants plus other supplements } \\
\text { ( } \mathrm{n}=\text { not stated) vs No active intervention ( } \mathrm{n} \\
=\text { not stated) }\end{array}$ & Unclear & Unclear & Low & Low & Unclear & High & Low & High \\
\hline Yari 2020 & $\begin{array}{l}\text { Other antioxidants plus other supplements } \\
(n=25) \text { vs No active intervention }(n=21)\end{array}$ & Low & Low & High & High & Unclear & Low & Unclear & High \\
\hline Yari 2020 & $\begin{array}{l}\text { Other antioxidants plus other supplements } \\
(n=25) \text { vs Other supplements }(n=24)\end{array}$ & Low & Low & High & High & Unclear & Low & Unclear & High \\
\hline Yari 2020 & $\begin{array}{l}\text { Other antioxidants plus other supplements } \\
(n=25) \text { vs Other antioxidants }(n=22)\end{array}$ & Low & Low & High & High & Unclear & Low & Unclear & High \\
\hline Qin 2015 & $\begin{array}{l}\text { PUFA plus vitamin } E(n=36) \text { vs No active in- } \\
\text { tervention }(n=34)\end{array}$ & Low & Low & Low & Low & Unclear & High & Low & High \\
\hline Gomez 2009 & $\begin{array}{l}\text { Vitamin C plus other antioxidants }(n=30) \text { vs } \\
\text { No active intervention }(n=30)\end{array}$ & Unclear & Low & Unclear & Unclear & Low & High & Low & High \\
\hline $\begin{array}{l}\text { Ebrahi- } \\
\text { mi-Mameghani } \\
2016\end{array}$ & $\begin{array}{l}\text { PUFA plus vitamin } E(n=19) \text { vs Vitamin } E(n \\
i=19)\end{array}$ & Unclear & Unclear & Unclear & Unclear & Unclear & High & Low & High \\
\hline Ashraf 2017 & $\begin{array}{l}\text { Vitamin C plus other antioxidants }(n=25) \text { vs } \\
\text { Vitamin } E(n=27)\end{array}$ & Unclear & Unclear & High & High & Unclear & High & Low & High \\
\hline Miglio 2000 & $\begin{array}{l}\text { Amino acids plus vitamin } C(n=96) \text { vs No } \\
\text { active intervention }(n=95)\end{array}$ & Low & Low & Low & Low & Unclear & High & Low & High \\
\hline $\begin{array}{l}\text { Celinski } \\
2014\end{array}$ & $\begin{array}{l}\text { Amino acids plus PUFA }(n=51) \text { vs PUFA }(n= \\
23 \text { ) }\end{array}$ & Unclear & Unclear & Unclear & Unclear & Unclear & High & Low & High \\
\hline
\end{tabular}




\begin{tabular}{|c|c|c|c|c|c|c|c|c|c|}
\hline Poulos 2021 & $\begin{array}{l}\text { Amino acids plus vitamin E plus other an- } \\
\text { tioxidants }(n=14) \text { vs No active intervention } \\
(n=11)\end{array}$ & Unclear & Unclear & Unclear & Unclear & Unclear & High & Low & High \\
\hline $\begin{array}{l}\text { Amanat } \\
2018\end{array}$ & $\begin{array}{l}\text { Oestrogen }(n=37) \text { vs No active intervention } \\
(n=41)\end{array}$ & Low & Low & Low & Low & Unclear & High & Low & High \\
\hline Zohrer 2017 & $\begin{array}{l}\text { Phospholipids plus PUFA plus vitamin } E(n= \\
\text { 20) vs No active intervention }(n=20)\end{array}$ & Low & Low & Low & Low & Unclear & High & Low & High \\
\hline $\begin{array}{l}\text { Loguercio } \\
2012\end{array}$ & $\begin{array}{l}\text { Phospholipids plus vitamin E plus other an- } \\
\text { tioxidants }(n=69) \text { vs No active intervention } \\
(n=69)\end{array}$ & Low & Unclear & Low & Low & High & Low & Low & High \\
\hline Hong 2016 & $\begin{array}{l}\text { Other supplements plus other antioxidants } \\
(n=35) \text { vs Other antioxidants }(n=31)\end{array}$ & Low & Unclear & Unclear & Unclear & Unclear & High & Low & High \\
\hline Lewis 2018 & $\begin{array}{l}\text { Polysaccharides }(n=12) \text { vs No active inter- } \\
\text { vention }(n=11)\end{array}$ & Low & Low & Low & Low & Low & Low & Low & Low \\
\hline Ekhlasi 2016 & $\begin{array}{l}\text { Prebiotics/Probiotics/Synbiotics plus vita- } \\
\min E(n=15) \text { vs No active intervention }(n= \\
15)\end{array}$ & Unclear & Unclear & Low & Low & Unclear & High & Low & High \\
\hline Ekhlasi 2016 & $\begin{array}{l}\text { Prebiotics/Probiotics/Synbiotics plus vita- } \\
\min E(n=15) \text { vs Prebiotics/Probiotics/Syn- } \\
\text { biotics }(n=15)\end{array}$ & Unclear & Unclear & Low & Low & Unclear & High & Low & High \\
\hline Ekhlasi 2016 & $\begin{array}{l}\text { Prebiotics/Probiotics/Synbiotics plus vita- } \\
\min E(n=15) \text { vs Vitamin } E(n=15)\end{array}$ & Unclear & Unclear & Low & Low & Unclear & High & Low & High \\
\hline $\begin{array}{l}\text { Kobyliak } \\
2017\end{array}$ & $\begin{array}{l}\text { Prebiotics/Probiotics/Synbiotics plus PUFA } \\
\text { ( } \mathrm{n}=\text { not stated) vs No active intervention ( } \mathrm{n} \\
=\text { not stated) }\end{array}$ & Unclear & Unclear & Low & Low & Unclear & High & Low & High \\
\hline Song 2020 & $\begin{array}{l}\text { PUFA plus other supplements }(n=17) \text { vs No } \\
\text { active intervention }(n=21)\end{array}$ & Low & Low & Low & Low & Unclear & High & Low & High \\
\hline $\begin{array}{l}\text { Mor- } \\
\text { varidzadeh } \\
2021\end{array}$ & $\begin{array}{l}\text { Prebiotics/Probiotics/Synbiotics plus vita- } \\
\text { min } D(n=44) \text { vs Prebiotics/Probiotics/Syn- } \\
\text { biotics }(n=44)\end{array}$ & Low & Unclear & Low & Low & Unclear & High & Low & High \\
\hline
\end{tabular}




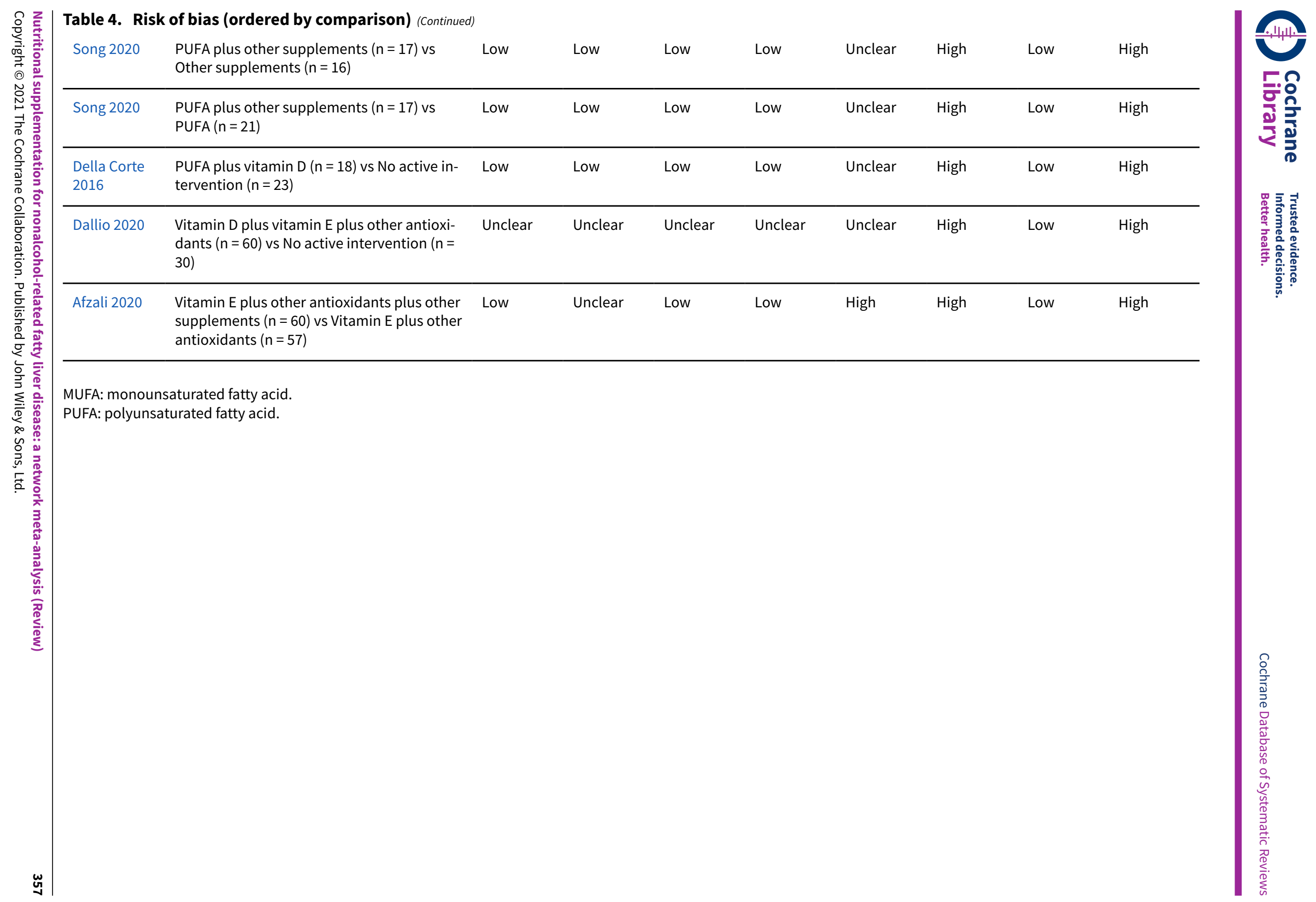


Table 5. Model fit

\begin{tabular}{|c|c|c|c|}
\hline $\begin{array}{l}\text { Serious adverse events (number of peo- } \\
\text { ple) }\end{array}$ & Fixed-effect model & Random-effects model & $\begin{array}{l}\text { Inconsistency } \\
\text { model }\end{array}$ \\
\hline Dbar & 64.08 & 61.87 & - \\
\hline DIC & 77.42 & 77.42 & - \\
\hline $\mathrm{pD}$ & 13.35 & 15.55 & - \\
\hline $\begin{array}{l}\text { Serious adverse events (number of } \\
\text { events) }\end{array}$ & Fixed-effect model & Random-effects model & $\begin{array}{l}\text { Inconsistency } \\
\text { model }\end{array}$ \\
\hline Dbar & 15.77 & - & - \\
\hline DIC & 19.51 & - & - \\
\hline $\mathrm{pD}$ & 3.734 & - & - \\
\hline Any adverse events (number of people) & Fixed-effect model & Random-effects model & $\begin{array}{l}\text { Inconsistency } \\
\text { model }\end{array}$ \\
\hline Dbar & 216.2 & 215 & - \\
\hline DIC & 253.5 & 255.3 & - \\
\hline $\mathrm{pD}$ & 37.23 & 40.3 & - \\
\hline Any adverse events (number of events) & Fixed-effect model & Random-effects model & $\begin{array}{l}\text { Inconsistency } \\
\text { model }\end{array}$ \\
\hline Dbar & 188.3 & 188.3 & - \\
\hline DIC & 206.1 & 206.2 & - \\
\hline pD & 17.85 & 17.9 & - \\
\hline Resolution of fatty liver & Fixed-effect model & Random-effects model & $\begin{array}{l}\text { Inconsistency } \\
\text { model }\end{array}$ \\
\hline Dbar & - & 255.6 & - \\
\hline DIC & - & 297.8 & - \\
\hline pD & - & 42.19 & - \\
\hline Fibrosis score & Fixed-effect model & Random-effects model & $\begin{array}{l}\text { Inconsistency } \\
\text { model }\end{array}$ \\
\hline Dbar & -24.08 & -29.64 & - \\
\hline DIC & 2.946 & 1.69 & - \\
\hline pD & 27.03 & 31.33 & - \\
\hline
\end{tabular}


Table 5. Model fit (Continued)

\begin{tabular}{llcc}
$\begin{array}{l}\text { Non-alcohol-related fatty liver disease } \\
\text { (NAFLD) activity score }\end{array}$ & Fixed-effect model & Random-effects model & $\begin{array}{l}\text { Inconsistency } \\
\text { model }\end{array}$ \\
\hline Dbar & 12.7 & 5.971 & - \\
\hline DIC & 35.64 & 33.69 & - \\
\hline PD & 22.93 & 27.72 & - \\
\hline
\end{tabular}

Dbar: posterior mean of deviance.

DIC: deviance information criteria.

pD: effective number of parameters or leverage.

\section{Table 6. Effect estimates}

This table is too wide to be displayed in RevMan. This table can be found here.

The table provides the effect estimates of each pairwise comparison for the different outcomes: odds ratios for serious adverse events (number of people) and adverse events (number of people), rate ratios for serious adverse events (number of people) and adverse events (number of people), mean differences for fibrosis and non-alcohol-related fatty liver disease activity scores, and hazard ratios for all other outcomes. The top half of the table indicates effect estimates from direct comparisons. The bottom half of the table indicates effect estimates from the network meta-analysis. For network meta-analysis, to identify the effect estimate of a comparison, say A vs B, look at the cell that occupies the row corresponding to intervention $A$ and the column corresponding to intervention $B$ for the effect estimate that is obtained directly (i.e. it provides the odds A/odds B). If that cell is empty (indicated by a '-'), look at the row corresponding to intervention $B$ and the column corresponding to intervention $A$. But this gives odds $B$ /odds $A$. As we are interested in odds $A$ /odds $B$, take the inverse of this number (i.e. 1/number) to arrive at the treatment effect of $A$ versus $B$. For direct comparisons, this is exactly the opposite; look at the cell that occupies the column corresponding to intervention $A$ and the row corresponding to intervention $B$ for the direct effect estimate. If that cell is empty, look at the column corresponding to intervention $B$ and the row corresponding to intervention $A$. Take the inverse of this number to arrive at the treatment effect of $A$ versus $B$. If the cell corresponding to B versus $A$ is also missing in direct comparisons, this means that there was no direct comparison.

Statistically significant results are shown in italics. Green colour indicates that intervention A is better than B, and red colour indicates that intervention $\mathrm{A}$ is worse than $\mathrm{B}$.

Table 7. Sensitivity analysis (worst-best and best-worst scenario analysis)

\section{Any adverse events (number of people)}

\section{Other supplements vs No active intervention}

main analysis: no evidence of difference between groups (OR 1.33, 95\% $\mathrm{Crl} 0.78$ to 2.26 )

best-worst analysis: no evidence of difference between groups (OR $0.75,95 \% \mathrm{Crl} 0.47$ to 1.18 )

worst-best analysis: higher in other supplements than in no active intervention (OR $2.11,95 \% \mathrm{Crl} 1.30$ to 3.46 )

Prebiotics/Probiotics/Synbiotics vs No active intervention

main analysis: no evidence of difference between groups (OR $0.67,95 \% \mathrm{Crl} 0.30$ to 1.46 )

best-worst analysis: lower in prebiotics/probiotics/synbiotics than in no active intervention (OR $0.27,95 \% \mathrm{Crl} 0.12$ to 0.56 )

worst-best analysis: no evidence of difference between groups (OR $1.53,95 \% \mathrm{Crl} 0.77$ to 3.11 )

Vitamin $E$ vs No active intervention

main analysis: no evidence of difference between groups (OR $0.83,95 \% \mathrm{Crl} 0.36$ to 1.91 )

best-worst analysis: lower in vitamin $\mathrm{E}$ than in no active intervention (OR $0.41,95 \% \mathrm{Crl} 0.19$ to 0.87 )

worst-best analysis: no evidence of difference between groups (OR $1.70,95 \% \mathrm{Crl} 0.79$ to 3.71 )

\section{Prebiotics/Probiotics/Synbiotics vs Other supplements}

main analysis: no evidence of difference between groups (OR 0.50, 95\% Crl 0.19 to 1.29)

best-worst analysis: lower in prebiotics/probiotics/synbiotics than in other supplements (OR $0.37,95 \% \mathrm{Crl} 0.15$ to 0.86 )

worst-best analysis: no evidence of difference between groups (OR $0.72,95 \% \mathrm{Crl} 0.32$ to 1.68)

\section{Amino acids vs Other supplements}

main analysis: no evidence of difference between groups (OR 0.46, 95\% Crl 0.18 to 1.14 )

best-worst analysis: lower in amino acids than in other supplements (OR $0.29,95 \% \mathrm{Crl} 0.12$ to 0.71 ) 
Table 7. Sensitivity analysis (worst-best and best-worst scenario analysis) (Continued) worst-best analysis: no evidence of difference between groups (OR $0.80,95 \% \mathrm{Crl} 0.33$ to 1.96) Other antioxidants vs Prebiotics/Probiotics/Synbiotics

main analysis: no evidence of difference between groups (OR 2.51, 95\% Crl 0.72 to 9.04) best-worst analysis: no evidence of difference between groups (OR 1.09, 95\% $\mathrm{Crl} 0.33$ to 3.71 )

worst-best analysis: higher in other antioxidants than in prebiotics/probiotics/synbiotics (OR 6.11, 95\% $\mathrm{Crl} 1.80$ to 21.87 )

Amino acids plus vitamin C vs Prebiotics/Probiotics/Synbiotics

main analysis: no evidence of difference between groups (OR 2.66, 95\% $\mathrm{Crl} 0.72$ to 10.61)

best-worst analysis: no evidence of difference between groups (OR $1.15,95 \% \mathrm{Crl} 0.33$ to 4.32 )

worst-best analysis: higher in amino acids plus vitamin C than prebiotics/probiotics/synbiotics (OR 6.49, $95 \% \mathrm{Crl} 1.82$ to 25.51 )

other antioxidants vs Vitamin $E$

main analysis: no evidence of difference between groups (OR 2.02, 95\% Crl 0.56 to 7.40 )

best-worst analysis: no evidence of difference between groups (OR $0.99,95 \% \mathrm{Crl} 0.28$ to 3.48 )

worst-best analysis: higher in other antioxidants than in vitamin E (OR 4.13, 95\% Crl 1.17 to 14.37 )

Amino acids plus vitamin $C$ vs Vitamin $E$

main analysis: no evidence of difference between groups (OR 2.14, 95\% Crl 0.56 to 8.74)

best-worst analysis: no evidence of difference between groups (OR $1.04,95 \% \mathrm{Crl} 0.28$ to 4.05 )

worst-best analysis: higher in amino acids plus vitamin C than in vitamin E (OR 4.34, 95\% Crl 1.20 to 17.65)

Phospholipids versus Other antioxidants

main analysis: no evidence of difference between groups (OR $0.22,95 \% \mathrm{Crl} 0.02$ to 1.56 )

best-worst analysis: lower in phospholipids than in other antioxidants (OR $0.12,95 \% \mathrm{Crl} 0.01$ to 0.88 )

worst-best analysis: no evidence of difference between groups (OR $0.35,95 \% \mathrm{Crl} 0.03$ to 2.45 )

\section{Oestrogen vs Amino acids}

main analysis: no evidence of difference between groups (OR 1.85, 95\% Crl 0.04 to 80.56)

best-worst analysis: no evidence of difference between groups (OR 1.65, 95\% $\mathrm{Crl} 0.04$ to 71.74 )

worst-best analysis: higher in oestrogen than in amino acids (OR $12.32,95 \% \mathrm{Crl} 1.34$ to 391.90 )

Amino acids plus vitamin C vs Phospholipids

main analysis: no evidence of difference between groups (OR 4.89, 95\% Crl 0.64 to 53.57)

best-worst analysis: no evidence of difference between groups (OR $3.04,95 \% \mathrm{Crl} 0.40$ to 33.08 )

worst-best analysis: higher in amino acids plus vitamin C than in phospholipids (OR $8.70,95 \% \mathrm{Crl} 1.17$ to 95.77)

\section{Resolution of fatty liver}

\section{Vitamin E vs Prebiotics/Probiotics/Synbiotics}

main analysis: no evidence of difference between groups (HR 0.46, 95\% Crl 0.19 to 1.15 )

worst-best analysis: no evidence of difference between groups (HR $0.83,95 \% \mathrm{Crl} 0.30$ to 2.34 )

best-worst analysis: lower in vitamin E than in prebiotics/probiotics/synbiotics (HR 0.38, 95\% Crl 0.15 to 0.97 )

Crl: credible interval.

OR: odds ratio.

\section{AP PEN DICES}

Appendix 1. Search strategies

\begin{tabular}{|c|c|c|}
\hline Database & Time span & Search strategy \\
\hline $\begin{array}{l}\text { Central Register of Con- } \\
\text { trolled Trials (CEN- } \\
\text { TRAL), in the Cochrane } \\
\text { Library }\end{array}$ & Issue 2, 2021 & 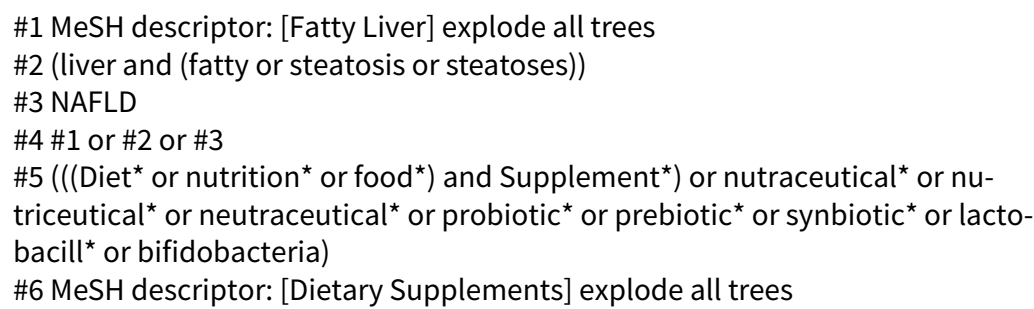 \\
\hline
\end{tabular}


\#7 (vitamin ${ }^{\star}$ or micronutrient ${ }^{\star}$ or (trace near/1 (element ${ }^{\star}$ or mineral $\left.{ }^{\star}\right)$ ) or antioxidant $\left.{ }^{\star}\right)$

\#8 MeSH descriptor: [Vitamins] explode all trees

\#9 MeSH descriptor: [Micronutrients] explode all trees

\#10 MeSH descriptor: [Antioxidants] explode all trees

\#11 (((unsaturated or polyunsaturated) and (fatty near/1 acid $\left.{ }^{\star}\right)$ ) or PUFA or

(linoleic near/1 acid $^{\star}$ ) or (docosahexaenoic near/1 acid ${ }^{\star}$ ) or (eicosapentaenoic near/1 acid))

\#12 MeSH descriptor: [Fatty Acids, Unsaturated] explode all trees

$\# 13 \# 5$ or \#6 or \#7 or \#8 or \#9 or \#10 or \#11 or \#12

\#14 MeSH descriptor: [Exercise] this term only

\#15 MeSH descriptor: [Exercise Therapy] this term only

\#16 MeSH descriptor: [Physical Exertion] this term only

\#17 MeSH descriptor: [Motor Activity] this term only

$\# 18$ MeSH descriptor: [Sports] this term only

$\# 19$ (sport $\left.{ }^{\star}\right)$

\#20 MeSH descriptor: [Physical Education and Training] explode all trees

\#21 (physical near/3 (activit* or education* or exertion* or training))

\#22 (exercise*)

\#23 MeSH descriptor: [Diet Therapy] explode all trees

\#24 ((diet or dieting) near/5 (health ${ }^{\star}$ or weight $\left.^{\star}\right)$ )

\#25 (calorie near/3 (control or reduc* or restriction))

\#26 "food choice*"

\#27 ("fat camp*" or "weight loss camp*")

\#28 "nutrition education"

\#29 MeSH descriptor: [Nutrition Therapy] this term only

\#30 MeSH descriptor: [Behavior Therapy] this term only

\#31 MeSH descriptor: [Cognitive Therapy] this term only

\#32 MeSH descriptor: [Psychotherapy] this term only

\#33 (behavio? ${ }^{\star}$ near/3 (therap* or technique* or modif* or intervention*))

\#34 (cognit* near/3 (therap* ${ }^{\star}$ or technique ${ }^{\star}$ or modif* or intervention $\left.{ }^{\star}\right)$ )

\#35 CBT

\#36 (psychotherap* or psycho-therap*)

\#37 (psycho-social or psychosocial)

\#38 MeSH descriptor: [Health Promotion] explode all trees

\#39 MeSH descriptor: [Health Education] this term only

\#40 (health* near/3 (promot ${ }^{\star}$ or educat* or lifestyle))

$\# 41$ MeSH descriptor: [Life Style] this term only

\#42 (lifestyle $e^{\star}$ or life-style ${ }^{\star}$ )

$\# 43 \# 14$ or \#15 or \#16 or \#17 or \#18 or \#19 or \#20 or \#21 or \#22 or \#23 or \#24 or $\# 25$ or \#26 or \#27 or \#28 or \#29 or \#30 or \#31 or \#32 or \#33 or \#34 or \#35 or \#36 or \#37 or \#38 or \#39 or \#40 or \#41 or \#42

$\# 44 \# 13$ or \#43

$\# 45$ \#4 and \#44
MEDLINE Ovid

January 1947 to 25 Feb-
ruary 2021

1. randomized controlled trial.pt.

2. controlled clinical trial.pt.

3. randomized.ab.

4. placebo.ab.

5. drug therapy.fs.

6. randomly.ab.

7. trial.ab.

8. groups.ab.

9. 1 or 2 or 3 or 4 or 5 or 6 or 7 or 8

10. exp animals/ not humans.sh.

11.9 not 10

12. exp Fatty Liver/

13. (liver and (fatty or steatosis or steatoses)).ti,ab.

14. NAFLD.ti,ab.

15. 12 or 13 or 14 
16. ((( Diet $^{\star}$ or nutrition ${ }^{\star}$ or food $\left.{ }^{\star}\right)$ and Supplement $\left.{ }^{\star}\right)$ or nutraceutical ${ }^{\star}$ or nutriceutical ${ }^{\star}$ or neutraceutical ${ }^{\star}$ or probiotic ${ }^{\star}$ or prebiotic ${ }^{\star}$ or synbiotic ${ }^{\star}$ or lacto-

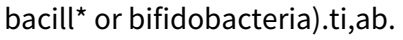

17. exp Dietary Supplements/

18. (vitamin ${ }^{\star}$ or micronutrient ${ }^{\star}$ or (trace adj1 (element ${ }^{\star}$ or mineral $\left.{ }^{\star}\right)$ ) or antioxidant*).ti,ab.

19. exp Vitamins/ or exp MICRONUTRIENTS/ or exp ANTIOXIDANTS/

20. (((unsaturated or polyunsaturated) and (fatty adj1 acid $\left.\left.{ }^{\star}\right)\right)$ or PUFA or (linoleic adj1 acid $\left.^{\star}\right)$ or (docosahexaenoic adj1 acid $\left.^{*}\right)$ or (eicosapentaenoic adj1 acid)).ti,ab.

21. exp Fatty Acids, Unsaturated/

22. 16 or 17 or 18 or 19 or 20 or 21

23. Exercise/ or Exercise Therapy/ or Physical Exertion/ or Motor Activity/ or Sports/

24. sport*.tw.

25. exp "Physical Education and Training"/

26. (physical adj3 (activit ${ }^{\star}$ or education* or exertion* or training)).tw.

27. exercise ${ }^{\star}$.tw.

28. exp diet therapy/

29. ((diet or dieting) adj5 (health* or weight $\left.\left.^{\star}\right)\right)$.tw.

30. (calorie adj3 (control or reduc ${ }^{\star}$ or restriction)).tw.

31. food choice*.tw.

32. (fat camp ${ }^{\star}$ or weight loss camp ${ }^{\star}$ ).tw.

33. nutrition education.tw.

34. Nutrition Therapy/ or behavior therapy/ or Cognitive Therapy/ or psychotherapy/

35. (behavio? $r^{\star}$ adj3 (therap* or technique* or modif* or intervention $\left.\left.{ }^{\star}\right)\right)$.tw.

36. (cognit ${ }^{\star}$ adj3 (therap ${ }^{\star}$ or technique ${ }^{\star}$ or modif* ${ }^{\star}$ or intervention $\left.{ }^{\star}\right)$ ).tw.

37. CBT.tw.

38. (psychotherap* or psycho-therap*).tw.

39. (psycho-social or psychosocial).tw.

40. exp Health Promotion/ or Health Education/

41. (health* adj3 (promot ${ }^{\star}$ or educat* $^{\star}$ or lifestyle)).tw.

42. lifestyle/

43. (lifestyle ${ }^{\star}$ or life-style $\left.{ }^{\star}\right)$.tw.

44.23 or 24 or 25 or 26 or 27 or 28 or 29 or 30 or 31 or 32 or 33 or 34 or 35 or 36 or 37 or 38 or 39 or 40 or 41 or 42 or 43

45. 22 or 44

46. 11 and 15 and 45

Embase Ovid

January 1974 to 25 Feb-
ruary 2021

1. exp crossover-procedure/ or exp double-blind procedure/ or exp randomized controlled trial/ or single-blind procedure/

2. ( ( ((random* or factorial ${ }^{*}$ or crossover ${ }^{\star}$ or cross over ${ }^{\star}$ or cross-over ${ }^{\star}$ or placebo* or double $^{\star}$ ) adj blind ${ }^{\star}$ ) or single ${ }^{\star}$ ) adj blind ${ }^{\star}$ ) or assign ${ }^{\star}$ or allocat ${ }^{\star}$ or volunteer ${ }^{\star}$.af.

3. 1 or 2

4. exp fatty liver/

5. (liver and (fatty or steatosis or steatoses)).ti,ab.

6. NAFLD.ti,ab.

7. 4 or 5 or 6

8. (((Diet ${ }^{\star}$ or nutrition $^{\star}$ or food $\left.^{\star}\right)$ and Supplement $\left.{ }^{\star}\right)$ or nutraceutical ${ }^{\star}$ or nutriceutical $^{\star}$ or neutraceutical ${ }^{\star}$ or probiotic ${ }^{\star}$ or prebiotic ${ }^{\star}$ or synbiotic ${ }^{\star}$ or lactobacill* $^{\star}$ or bifidobacteria).ti,ab.

9. exp dietary supplement/ or probiotic agent/ or prebiotic agent/ or synbiotic agent/

10. $\left(\right.$ vitamin $^{\star}$ or micronutrient ${ }^{\star}$ or (trace adj1 (element ${ }^{\star}$ or mineral $\left.{ }^{\star}\right)$ ) or antioxidant $\left.{ }^{\star}\right)$.ti,ab.

11. exp vitamin/ or exp trace element/ or exp antioxidant/

12. (((unsaturated or polyunsaturated) and (fatty adj1 acid*)) or PUFA or (linoleic adj1 acid $^{\star}$ ) or (docosahexaenoic adj1 acid ${ }^{\star}$ ) or (eicosapentaenoic adj1 acid)).ti,ab. 


\author{
13. exp polyunsaturated fatty acid/ \\ 14. 8 or 9 or 10 or 11 or 12 or 13 \\ 15. exercise/ or kinesiotherapy/ or motor activity/ or sport/ \\ 16. sport*.tw. \\ 17. (physical adj3 (activit* or education* or exertion* or training)).tw. \\ 18. exercise ${ }^{\star}$.tw. \\ 19. exp diet therapy/ \\ 20. ((diet or dieting) adj5 (health* or weight $\left.\left.^{\star}\right)\right)$. tw. \\ 21. (calorie adj3 (control or reduc* or restriction)).tw. \\ 22. food choice*.tw. \\ 23. (fat camp* or weight loss camp $\left.{ }^{\star}\right)$.tw. \\ 24. nutrition education.tw. \\ 25. behavior therapy/or Cognitive Therapy/ or psychotherapy/ \\ 26. (behavio? ${ }^{\star}$ adj3 (therap* or technique* or modif* or intervention $\left.{ }^{\star}\right)$ ).tw. \\ 27. (cognit ${ }^{\star}$ adj3 (therap ${ }^{\star}$ or technique ${ }^{\star}$ or modif* ${ }^{\star}$ or intervention $\left.{ }^{\star}\right)$ ).tw. \\ 28. CBT.tw. \\ 29. (psychotherap* or psycho-therap $\left.{ }^{\star}\right)$.tw. \\ 30. (psycho-social or psychosocial).tw. \\ 31. exp Health Promotion/ or Health Education/ \\ 32. (health ${ }^{\star}$ adj3 (promot ${ }^{\star}$ or educat ${ }^{\star}$ or lifestyle)).tw. \\ 33. lifestyle/ or lifestyle modification/ \\ 34. (lifestyle ${ }^{\star}$ or life-style $\left.{ }^{\star}\right)$.tw. \\ 35.15 or 16 or 17 or 18 or 19 or 20 or 21 or 22 or 23 or 24 or 25 or 26 or 27 or 28 \\ or 29 or 30 or 31 or 32 or 33 or 34 \\ 36. 14 or 35 \\ 37.3 and 7 and 36
}

Science Citation Index Expanded (Web of Science) and Conference Proceedings Citation Index-Science (Web of Science)
January 1945 to 25 February 2021
\#1 TS = ((liver and (fatty or steatosis or steatoses) ) or NAFLD)

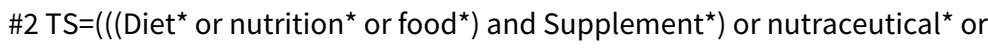
nutriceutical ${ }^{\star}$ or neutraceutical ${ }^{*}$ or probiotic ${ }^{\star}$ or prebiotic ${ }^{*}$ or synbiotic ${ }^{*}$ or lactobacill* or bifidobacterial or vitamin ${ }^{\star}$ or micronutrient ${ }^{\star}$ or (trace near1 (element $^{\star}$ or mineral $\left.{ }^{\star}\right)$ ) or ((unsaturated or polyunsaturated) and (fatty near1 acid $\left.^{\star}\right)$ ) or antioxidant ${ }^{\star}$ or PUFA or (linoleic nearl acid ${ }^{\star}$ ) or (docosahexaenoic near1 acid $^{\star}$ ) or (eicosapentaenoic near1 acid))

\#3 TS=(sport ${ }^{\star}$ or (physical near/3 (activit* $^{\star}$ or education ${ }^{\star}$ or exertion* or training)) or exercise* or ((diet or dieting) near/5 (health* or weight $\left.{ }^{\star}\right)$ ) or (calorie near/3 (control or reduc* or restriction)) or "food choice*" or "fat camp*" or "weight loss camp*" or "nutrition education" or (behavio?r* near/3 (therap* or technique ${ }^{\star}$ or modif* or intervention $\left.{ }^{\star}\right)$ ) or (cognit* near/3 (therap ${ }^{\star}$ or technique $^{\star}$ or modif* or intervention $\left.{ }^{\star}\right)$ ) or CBT or psychotherap* or psycho-ther${a p^{*}}^{*}$ or psycho-social or psychosocial or (health* near/3 (promot* or educat* or lifestyle)) or lifestyle* or life-style* or (alcohol ${ }^{\star}$ near $/ 2$ (drink ${ }^{\star}$ or intoxicat ${ }^{\star}$ or use $^{\star}$ or abus or misus $^{\star}$ or risk or consum $^{\star}$ or withdraw ${ }^{\star}$ or detox or treat $^{\star}$ or therap ${ }^{\star}$ or excess ${ }^{\star}$ or reduc ${ }^{\star}$ or cessation or intervention $\left.{ }^{\star}\right)$ ))

\section{\#4 \#3 OR \#2}

\#5 TS=(random ${ }^{\star}$ OR rct* OR crossover OR masked OR blind* OR placebo* OR meta-analysis OR systematic review* OR meta-analys ${ }^{\star}$ )

\footnotetext{
World Health Organization International Clinical Trials Registry Platform (apps.who.int/trialsearch/Default.aspx)
}

\#6 \#5 AND \#4 AND \#1 
(Continued)

\author{
European Medicines \\ 25 February 2021 \\ "Fatty liver" \\ Agency (www.ema.eu- \\ ropa.eu/ema/) and US \\ Food and Drug Adminis- \\ tration (www.fda.gov)
}

Footnote: This is a common search strategy that was used for this lifestyle interventions review (Buzzetti 2021).

\title{
Appendix 2. Abbreviations
}

AminoAcids: amino acids

Amino acids+PUFA: amino acids plus polyunsaturated fatty acids

Amino acids+VitC: amino acids plus vitamin C

CT scan: computerised tomography scan

Oestrogen: oestrogen

MRS: magnetic resonance spectroscopy

MRI: magnetic resonance imaging

MUFA: monounsaturated fatty acids

NAFLD: non-alcohol-related fatty liver disease

NASH: non-alcohol-related steatohepatitis

NoActivelntervention: no active intervention

OtherAntioxidants: other antioxidants

OtherAntiOx: other antioxidants

OtherAntioxidants+OtherSupplements: other antioxidants plus other supplements

OtherAntiOx+OtherSupp: other antioxidants plus other supplements

OtherSupplements: other supplements

OtherSupp: other supplements

Phospholipids: phospholipids

Phospholipids+PUFA+VitE: phospholipids plus polyunsaturated fatty acids plus vitamin $\mathrm{E}$

Phospholipids+PUFA+VitE+OtherAntioxidants+OtherSupplements: phospholipids plus polyunsaturated fatty acids plus vitamin E plus other antioxidants plus other supplements

Phospholipids+PUFA+VitE+OtherAntiOx+OtherSupp: phospholipids plus PUFA plus vitamin E plus other antioxidants plus other supplements

Phospholipids+VitE+OtherAntioxidants: phospholipids plus vitamin E plus other antioxidants

Phospholipids+VitE+OtherAntiOx: phospholipids plus vitamin E plus other antioxidants

Polysaccharides: polysaccharides

Prebiotics_probiotics_synbiotics+PUFA: prebiotics/probiotics/synbiotics plus polyunsaturated fatty acids

Prebiotics_probiotics_synbiotics+VitE: prebiotics/probiotics/synbiotics plus vitamin E

Prebiotics_Probiotics_Synbiotics: prebiotics/probiotics/synbiotics

PreProSynbiotics+PUFA: prebiotics/probiotics/synbiotics plus PUFA

PreProSynbiotics+VitE: prebiotics/probiotics/synbiotics plus vitamin E

PreProSynbiotics: prebiotics/probiotics/synbiotics

PUFA: polyunsaturated fatty acids

PUFA+VitD: polyunsaturated fatty acids plus vitamin D

PUFA+VitE: polyunsaturated fatty acids plus vitamin $\mathrm{E}$

VitC+OtherAntioxidants: vitamin C plus other antioxidants

VitC+OtherAntiOx: vitamin $C$ plus other antioxidants

VitC+VitE: vitamin C plus vitamin $\mathrm{E}$

VitD: vitamin D

VitE: vitamin E

VitE+OtherAntioxidants: vitamin E plus other antioxidants

VitE+OtherAntiOx: vitamin E plus other antioxidants

VitE+OtherSupplements: vitamin E plus other supplements

VitE+OtherSupp: vitamin E plus other supplements

\section{Appendix 3. Data}

This table is too wide to be displayed in RevMan. This table can be found here. 


\section{H IS T O R Y}

Protocol first published: Issue 10, 2018

\section{CONTRIBUTIONS OFAUTHORS}

\section{Protocol}

Conceiving the protocol: KG.

Designing the protocol: $K G$.

Co-ordinating the protocol: KG.

Designing search strategies: KG.

Writing the protocol: KG.

Providing general advice on the protocol: ET, AM.

Securing funding for the protocol: KG.

All authors approved of the protocol for publication.

Performing previous work that was the foundation of the current study: not applicable.

\section{Review}

Co-ordinating the review: $K G$.

Study selection: KG, OK, EB.

Data extraction: KG, OK, AL, DR, TC, LB.

Writing the review: $K G$.

Providing advice on the review: SF, AJS, NC, EJM, MC, CP, BRD, ET.

Securing funding for the review: $K G$.

All review authors approved the review for publication.

\section{DECLARATIONSOF INTEREST}

None known for any of the review authors.

\section{SOURCES OF SUPPORT}

\section{Internal sources}

- University College London, UK

Writing equipment, software, etc.

\section{External sources}

- National Institute for Health Research, UK

Payment for writing reviews, writing equipment, software

\section{DIFFERENCES BETWEEN PROTOCOL AND REVIEW}

- We have clarified that we did not include trials in which participants without NAFLD were included and no separate data were available on those with NAFLD

- We excluded a trial that included NAFLD patients with chronic kidney disease because this trial does not address the objectives of this systematic review, which refers to people with NAFLD but not with specifically chronic kidney disease

- We have provided additional details on how we constructed the treatment nodes and what we considered a 'decision set'

- We have revised outcomes based on feedback from patients and the public representative for this project, an online survey about outcomes promoted through the Cochrane Consumer Network, and the coreNASH project (Clearfield 2021). This resulted in the addition of liver-related mortality and the MELD score

- We have provided further details on how we translated non-English articles

- We removed the sentence "We excluded such quasi-randomised studies" from the two risk of bias domains on randomisation sequence and concealment. Instead, we made it clear in the beginning of the 'Study design' section that we will exclude quasi-randomised studies

- We removed the 'For profit' bias from risk of bias domains as per the guidance of Cochrane Network editors 
- We did not perform Trial Sequential Analysis (TSA) because the risk of false-positive results with Bayesian meta-analysis is usually less than or at least equivalent to TSA

- We used the latest guidance from the GRADE Working Group - Yepes-Nunez 2019 - rather than previous guidance - Puhan 2014 - in presenting the 'Summary of findings' table

- We used 30,000 iterations (instead of 10,000 iterations) as a minimum for burn-in of the simulation sampler used to estimate quantities in statistical models to ensure convergence of the simulation sampler

- We did not present some information such as ranking probability tables, rankograms, and surface area under the curve (SUCRA) plots because of concern about misinterpretation of results. We have highlighted this clearly within the text of the review, along with reasons for not presenting them

\section{NOTES}

The methods section of this protocol is based on a standard Cochrane Hepato-Biliary Group template with incorporation of advice by the Complex Reviews Support Unit for a network meta-analysis protocol (Best 2018). 